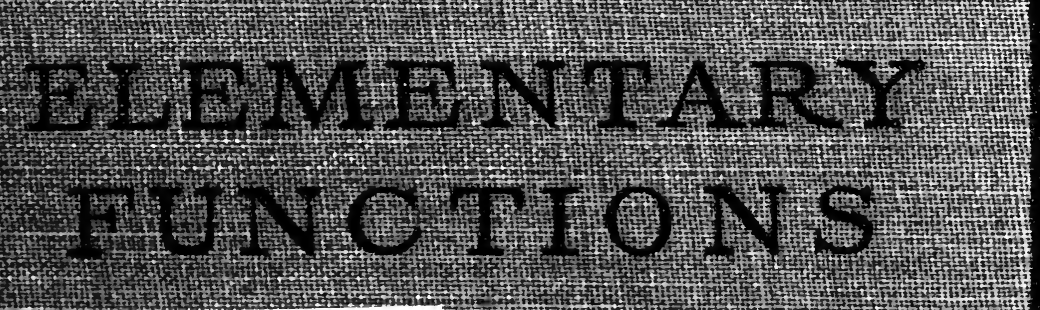

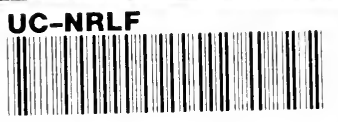

ᄒB 529 36? 

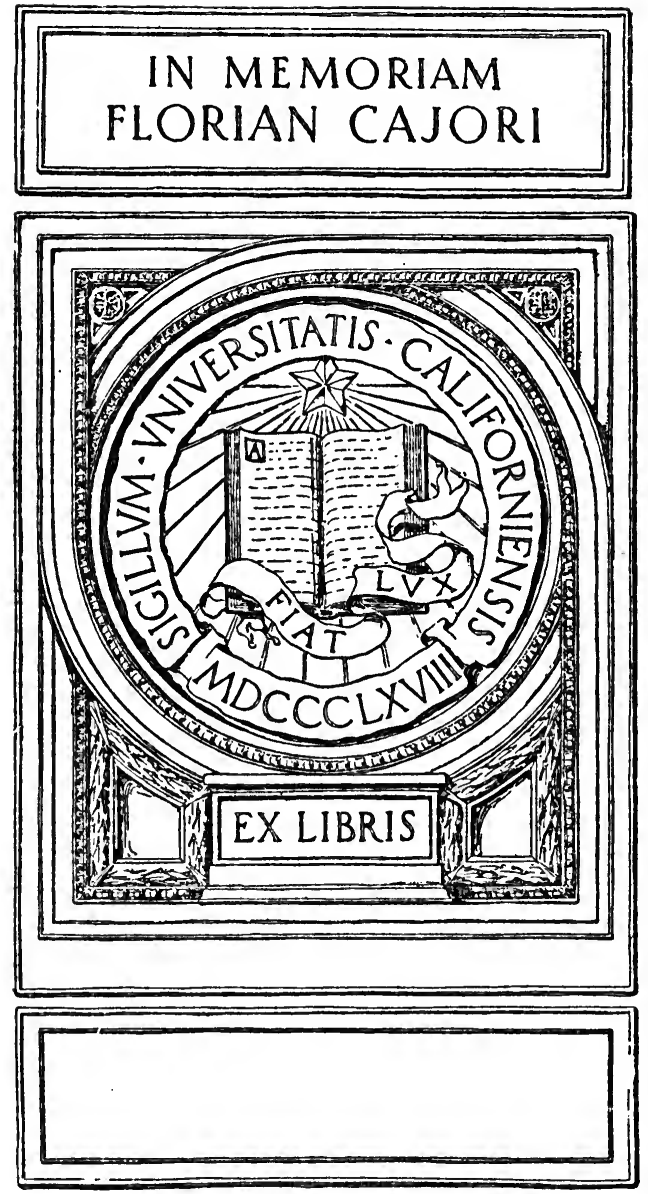


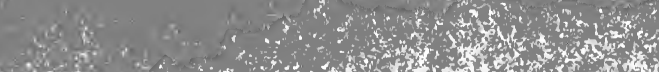

750 s.

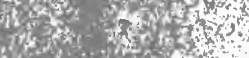

g.t.

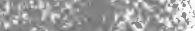

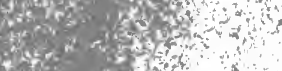

at

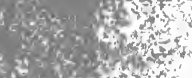

Mrgatos.

3.t. 0 ront.

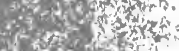

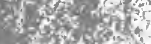

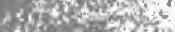

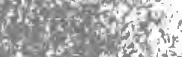

6.

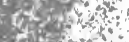

is

$245 \times 5$

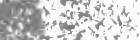

tont

and 3

tond

2018 35

tos

wetsing

is 9040

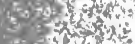

(1)

- $1+x^{2}$

win.

$3 \pi^{2}$

.4. $90 x^{2}$

Hine

vin

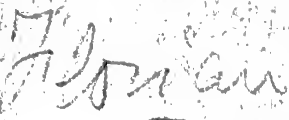

mon

(n)

is

$i+$

isto

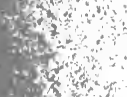

aris i

(5)

$\rightarrow 0$

is

Not $3+1$

s.

4.

A 45,504

By

$3 x$ or 40

2. $\%$, s.

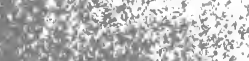
How

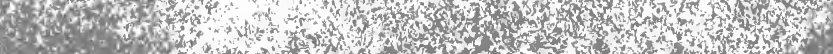

A.

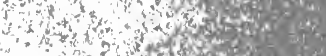

a d t

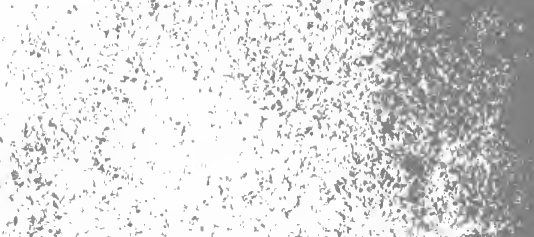

sing ins

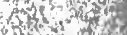

inting

s.3

$+x, y^{1 / 2}$

Antos

$-41069$

ontongen

s. yot

ing 2013

ing

in $240=$

int

w

5.0. $2.3=0$

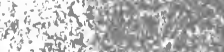

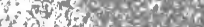

\% 


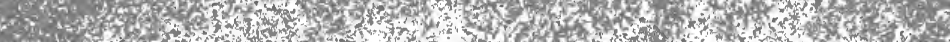

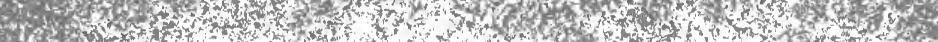

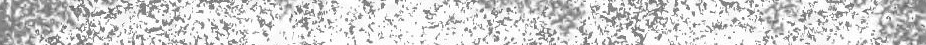

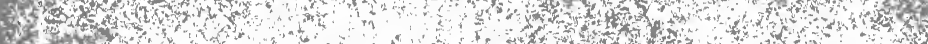

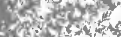

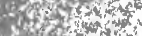

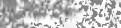

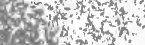

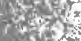

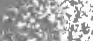

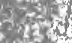

sis

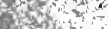

z) 7 th

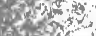

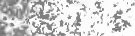

ic

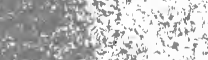

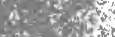

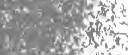

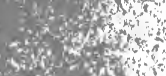

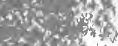

7.6.

sing

+80 is

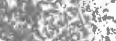

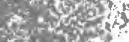

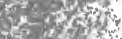

3. 1040

+ 200

$\frac{1}{4}$

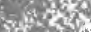

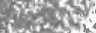

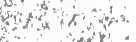

$\frac{x+2 y}{2}$

ond ins

270

(4)

Cot 104

this

aj,

ix 250

$t_{1} \times 5$

73 .

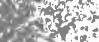

6)

Sorks

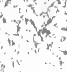

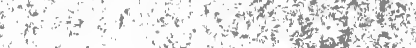

(1)

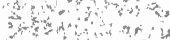

(4)

45

s.t.

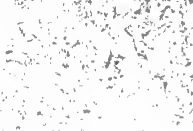

$3:$

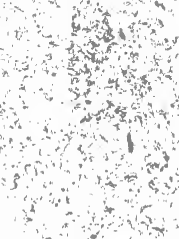

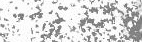

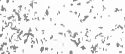

3

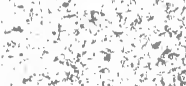

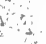

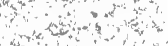

$x^{2}$

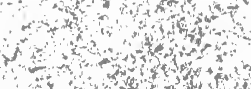

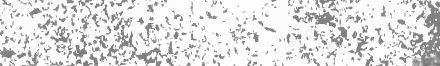

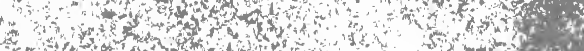

sonds $+4 x^{2}$

S. 30 ing of ands ind

-

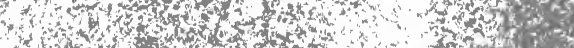

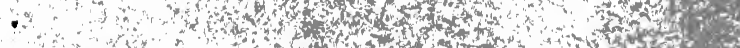

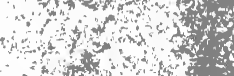

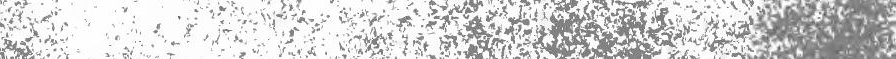

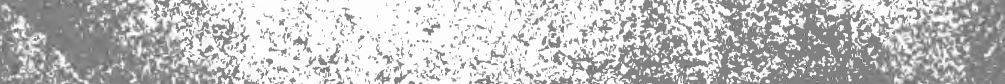

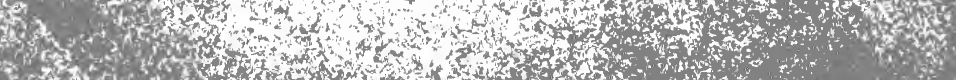




\title{
ELEMENTARY FUNCTIONS
}

AND

\section{A PPLICATIONS}

\author{
BY \\ ARTHUR SULLIVAN GALE, Ph.D. \\ AND
}

CHARLES WILLIAM WATKEYS, A.M. PROFESSORS OF MATHEMATICS IN THE UNIVERSITY OF ROCHESTER

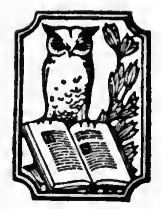

NEW YORK 
44331

93

COPYRIGHT, I920

BY

HENRY HoLT AND COMPANY

CAJORI 


\section{PREFACE}

THrs book presents a coherent year's work in mathematics for college freshmen, consisting of a study of the elementary functions, algebraic and transcendental, and their applications to problems arising in various fields of knowledge. The treatment is confined to functions of one variable, with incidental exceptions, and complex values of the independent and dependent variables are excluded. The subject matter includes the essentials of plane trigonometry and topics from advanced algebra, analytic geometry, and calculus.

The text is the result of experiments beginning in 1907-8. It has been used in the classroom since 1913-14, and each year extensive revisions have been made. Hence the content of the course, the order of topies, and the manner of presentation are based upon the experience of several years.

The unity of the course is gained by an explicit analysis of the functions studied, which enables the student to comprehend the purpose of the course as a whole and the nature of the investigation of properties of functions of a given type. This analysis consists of three parts:

First. Relations between a given function and its graph (see pages 42 and 274). Most of these relations are considered in the first chapter so that at the start the student is made aware of a number of questions which will be investigated in studying a particular type of functions.

Second. Relations between pairs of functions and their graphs (see page 152). These geometric transformations are introduced in connection with simple algebraic functions so that they are familiar tools by the time they are needed for the study of transcendental functions. 
Third. Analogous properties of functions which have no immediate graphical interpretation. Several properties of $x^{n}$ are groubed together on page 153 in order to indicate further questions which should be investiagted in studying transcendental functions.

Emphasis is also placed on characteristic properties which distinguish one class of functions from another.

A very large group of freshmen taking mathematics do not continue the study of this subject in the following years, and the needs of these students have received primary consideration. For the general student, the interpretation of a graph, the fundamental concepts of the calculus, and the usefulness of mathematics are of fundamental importance. Fortunately these matters are also important for the student of mathematics, and experience with the text has shown that it is possible in the second year satisfactorily to complete the usual first courses in analytic geometry and calculus.

To show the usefulness of mathematics, a wide range of problems which deal with matters of interest to the student have been introduced, although exercises which require considerable instruction in other subjects have been avoided. Applications to the solution of problems in mechanics, physics, chemistry, economics, and other subjects have been scattered throughout the course. The analysis of a problem in a field other than mathematics is usually more difficult for a freshman than the solution after the conditions and requirements have been stated in mathematical language. But from the broad standpoint in which mathematics appears as part of an educational system, the training in such analysis is as important as the development of the mathematical processes to which the analysis leads. The obligation resting upon the teacher of mathematics to develop this power of analysis is increased by the proneness of other teachers to tread very lightly on the mathematical aspects of their own subjects, and it is quite possible that this inclination on the part of others is partly due to the failure of mathematicians to emphasize the applications sufficiently. Simple applied problems may furnish 
drill in mathematical technique, with added interest, and with but slightly increased difficulty. Simultaneously, they afford some training in analysis.

Attention is called to the following features:

1. The chapter on the theory of measurements gives an outline of statistical methods which are used in many fields such as economics, biology, physics, education, etc. It is, perhaps, not too much to say that the average college graduate will find more use for this topic than for any included in the traditional freshman course. The treatment given here is intermediate between the books on statistics which presuppose very little mathematical theory and those which the mathematical prerequisites render unsuitable for the average college man.

2. Emphasis is placed upon the determination of a function from a given table of empirical data. In problems of this sort, which illustrate an important method of discovery in science, there is an element of general culture which is too often neglected in elementary courses in the various sciences on account of the mathematics involved.

3. Graphical methods of analyzing problems are used freely.

4. Graphical methods are used in problems which can be solved by straight lines, and an algebraic solution is then obtained by finding the equation of certain lines used in the graphical solution.

5. Use is made of the graphs of the trigonometric functions in tying together and affording the means of recalling many properties of these functions. This use of the graphs is merely a part of the general point of view of the course.

6. Trigonometric analysis, the most abstract topic included, is postponed until late in the course.

7. The introduction of a considerable amount of the elementary portions of the calculus gives the general student a knowledge of the importance and utility of the fundamental ideas of derivative and integral.

8. The average rate of change of a function is introduced at the start, and it is used in studying the linear function. 
The rate of change is introduced informally in connection with the quadratic function, while formal treatment is postponed until later. The difficulties in grasping the concept of a derivative are thus separated, and time for thorough assimilation is afforded.

9. Integration is used to obtain the volumes of a pyramid, cone, and sphere.

10. Tables of squares, square roots etc., that is, tables of functions familiar to the student, are used in advance of other tables. The table of logarithms is introduced as a general tool, and it is not regarded as something to be used primarily with the trigonometric functions. Use is made of various tables throughout a large part of the course, so that the student acquires facility in their use and in the selection of the most suitable table for a given computation.

11. The use of the slide rule, and of logarithmic and semilogarithmic cross section paper, is explained in connection with the logarithmic function.

12. The functions $x^{n}$ and $b^{x}$, which occur frequently in the applications of mathematics, are treated at some length.

13. An effort has been made to render explicit the purpose of the various parts of the course.

14. Formal proofs of a number of theorems are omitted, and some are assumed without proof. The appeal to the intuition underlying most of these assumptions is justified by the belief that the logical presentation of these theorems requires a foundation too abstract for the general student, or too cumbersome for the purpose to be served.

15. The course includes more than a year's work so that teachers have an opportunity for choice of topics, and abundant material is provided for selected sections which progress more rapidly than the average.

16. The course increases in difficulty with a corresponding increase in interest and gain in power on the part of the student.

17. A very wide range of problems, varying in difficulty, makes it possible for the instructor to emphasize different aspects of the subject, to select exercises suitable for students 
of different abilities, and to assign different sets of exercises in different years.

18. Many of the exercises in the later chapters, while dealing with the newer topics, are constructed to afford review of principles presented earlier in the course, and to correlate various parts of the subject.

We thank several friends for the inspiration of their interest and for their suggestions. We also thank the Trustees of The University of Rochester for making it possible for us to use the text in the classroom throughout our experimentation, in which we have participated equally. Combined courses are still to be regarded as in the experimental stage, but it is our conviction that they are fundamentally sound, and we shall feel well repaid if this volume contributes something of value to their development.

\section{Arthur Sullivan Gale \\ Charles William Watkeys}

The University of Rochester

May, 1920. 



\section{CONTENTS}

PAGE

Some Principles of Algebra and Geometry............. $\quad$ xv

\section{CHAPTER I}

SECTION

FUNCTIONS, EQUATIONS, AND GRAPHS

1. Comparison of the Reasoning in Natural Science and in Mathematics........................... 1

2. Example of the Utility of Mathematics in Science....... 2

3. Forms in which Data are Recorded............... 3

4. Variable. Function......................... 4

5. Notation for a Function. ................... 8

6. Determination of the Function which Expresses the Functional Relation between Two Variables ............ 8

7. Graphical Representation. Directed Lines........... 12

8. Rectangular Coördinates....................... 14

9. Graph of a Function...................... 16

10. Discussion of the Table of Values................ 21

11. Functions becoming Infinite. Asymptotes........... 25

12. Variation of a Function...................... 28

13. Average Rate of Change of a Function .............. 34

14. Classification of Functions..................... 38

15. Summary.............................. 41

\section{CHAPTER II}

\section{LINEAR FUNCTIONS}

16. Uniform Rate of Change ..................... 46

17. Characteristic Property of a Straight Line.......... 49

18. Slope of a Straight Line................... 51

19. Graphical Solution of Problems Involving Functions which Change Uniformly .................... 54 
20. Graph of the Linear Function $m x+b \ldots \ldots \ldots \ldots \ldots \ldots$

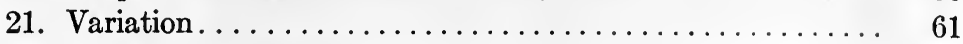

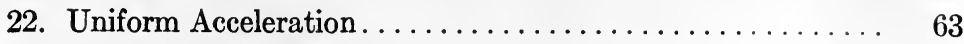

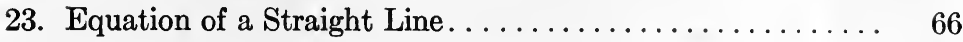

24. Application to the Solution of Problems. . . . . . . . . . 69

25. Remarks on Measurements ............... 72

26. Possible Errors in Arithmetic Calculations. Abridged Mul-

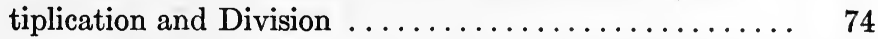

27. Empirical Data Problems.

\section{CHAPTER III}

\section{ALGEBRAIC FUNCTIONS}

28. Introduction. . . . . . . . . . . . . . . . . . 87

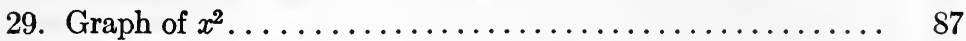

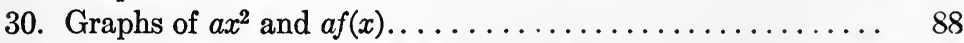

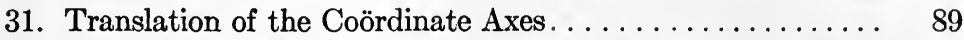

32. Instantaneous Velocity . . . . . . . . . . . . . . . 93

33. Rate of Change. Slope of Tangent Line............. 94

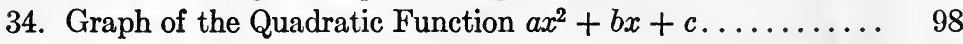

35. Empirical Data Problems....................... 104

36. The Function $x^{n} \ldots \ldots \ldots \ldots \ldots \ldots \ldots \ldots \ldots \ldots \ldots$

37. Tables of Squares, Cubes, Square Roots, Cube Roots, and

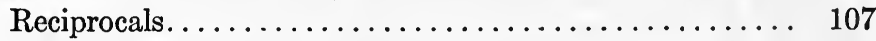

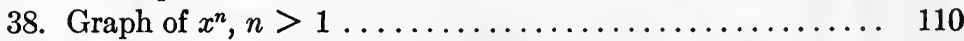

39. Graph of $x^{n}, 0<n<1$. Graphs of Inverse Functions . . 113

40. Graph of $x^{n}, n<0$. Graphs of Reciprocal Functions ... 117

41. Summary of Graph of $x^{n} \ldots \ldots \ldots \ldots \ldots \ldots \ldots \ldots \ldots \ldots \ldots$

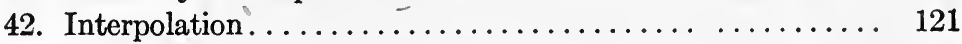

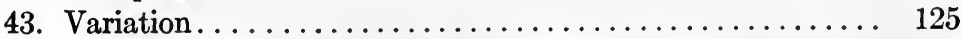

44. Empirical Data Problems.................... 127

45. The Linear Fractional Function $\frac{a x+b}{c x+d} \ldots \ldots \ldots \ldots \ldots 131$

46. Integral Rational Functions. . . . . . . . . . . . . 133

47. The Remainder Theorem . . . . . . . . . . . . . . . 133

48. Synthetic Division. . . . . . . . . . . . . . . . 134

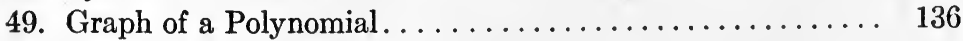

50. Extent of the Tables. . . . . . . . . . . . . . . . 137

51. Solution of Equations. Rational Roots............ 140

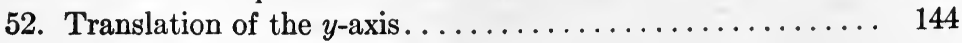


53. Horner's Method of Solution of Equations. . . . . . . . 147

54. Graph of the Function $f(a x) \ldots \ldots \ldots \ldots \ldots \ldots \ldots \ldots \ldots$

55. Related Functions and their Graphs............. 151

56. Some Operations of Algebra regarded as Properties of Functions.

\section{CHAPTER IV}

\section{TRIGONOMETRIC FUNCTIONS}

57. Introduction . . . . . . . . . . . . . . . . . 158

58. Angles of any Magnitude. . . . . . . . . . . . . . 164

59. Trigonometric Functions of any Angle........... 165

60. Radians............................... 170

61. Graphs of the Trigonometric Functions. . . . . . . . . . 172

62. Functions of Complementary Angles.............. 177

63. Tables of Trigonometric Functions. . . . . . . . . . . 178

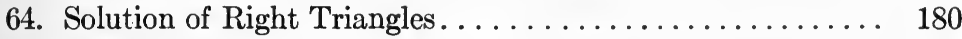

65. Applications.......................... 183

66. Parallelogram Law - Velocities, Accelerations, Forces. . . . 185

67. Conditions of Equilibrium of a Particle. . . . . . . . . . 187

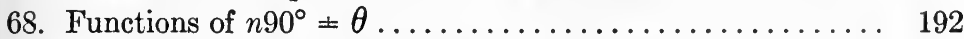

69. Application to the Use of Tables. . . . . . . . . . . . 196

70. Inclination and Slope of a Straight Line. . . . . . . . . 199

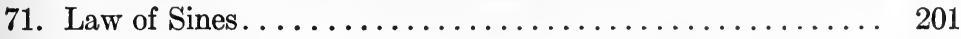

72. Law of Cosines. . . . . . . . . . . . . . . . . . . . 202

73. Solution of Oblique Triangles................. 203

74. Inverse Trigonometric Functions............... 209

\section{CHAPTER V}

\section{EXPONENTIAL AND LOGARITHMIC FUNCTIONS}

75. Introduction . . . . . . . . . . . . . . . . . . 214

76. Graph of the Exponential Function $b^{x}, b>1 \ldots \ldots \ldots 216$

77. Properties of the Exponential Function $b^{x}, b>1 \ldots \ldots 217$

78. Computation by Means of an Exponential Function . . . . . 219

79. The Logarithmic Function, the Inverse of the Exponential Function .......................... 221

80. Graph of the Logarithmic Function. . . . . . . . . . 221

81. Properties of the Logarithmic Function, $\log _{b} x, b>1 \ldots 222$ 
82. Common Logarithms.................... 225

83. Computation by Means of Common Logarithms . . . . . . 229

84. Solution of Triangles. . . . . . . . . . . . . . . . 233

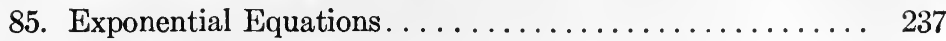

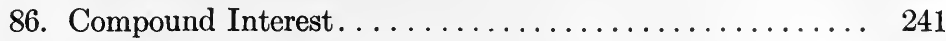

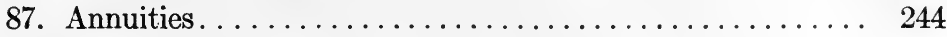

88. Graph of the exponential function $k b^{n x} \ldots \ldots \ldots \ldots \ldots 248$

89. The Logarithmic Scale...................... 249

90. Empirical Data Problems ................... 256

\section{CHAPTER VI}

\section{DIFFERENTIATION OF ALGEBRAIC FUNCTIONS}

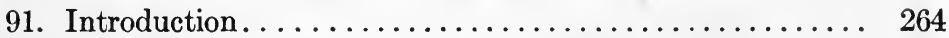

92. Limits............................. 265

93. Derivative of a Function.................. 267

94. Fundamental Formulas for Differentiation........... 269

95. Derivative of a Polynomial................ 271

96. Corresponding Properties of a Function, its Graph and its Derivative.

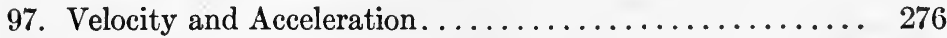

98. Derivative of a Rational Function.............. 278

99. Derivative of an Irrational Function. . . . . . . . . . . 279

100. Equations of Tangent and Normal Lines............ 281

101. Problems in Maxima and Minima............... 285

102. Related Rates......................... 289

103. Small Errors. . . . . . . . . . . . . . . . . . . . . . . 291

104. Approximate value of $f(x+\Delta x) \ldots \ldots \ldots \ldots \ldots \ldots \ldots$

\section{CHAPTER VII}

\section{INTEGRATION}

105. Introduction . . . . . . . . . . . . . . . . . . 301

106. Area under a Curve. . . . . . . . . . . . . . . . . . . . . 304

107. Motion in a Straight Line.................... 308

108. Motion in a Plane ....................... 311

109. Volume of a Right Prism.................. 315

110. Volume of a Right Circular Cylinder............. 318

111. Volume of a Pyramid.................... 320

112. Volume of a Solid of Revolution............... 323 
113. Volume of a Cone of Revolution ............... 325

114. Volume of a Sphere .................... 327

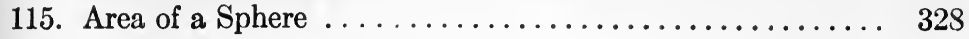

\section{CHAPTER VIII}

PROPERTIES OF TRIGONOMETRIC FUNCTIONS

Logarithmic Solution of Triangles, Cases III and IV

116. Introduction . . . . . . . . . . . . . . . . . 332

117. Fundamental Trigonometric Relations . . . . . . . . . 332

118. Trigonometric Equations. . . . . . . . . . . . . 334

119. Trigonometric Identities . . . . . . . . . . . . . 336

120. Functions of the Sum of Two Angles. . . . . . . . . . 338

121. Functions of the Difference of Two Angles. . . . . . . . 341

122. Functions of Twice an Angle, or the Functions of Any Angle in Terms of Half the Angle............ 342

123. Functions of Half an Angle, or Functions of Any Angle in

Terms of Functions of Twice the Angle........... 343

124. Sum and Difference of the Sines or Cosines of Two Angles 345

125. Logarithmic Solution of Triangles, Case III . . . . . . . . 346

126. Logarithmic Solution of Triangles, Case IV . . . . . . . . 348

127. Miscellaneous Identities and Equations. ........... 351

128. Differentiation of Trigonometric Functions.......... 352

129. Graph of the Function $a \sin (b x+c)$. Harmonic Curves. . 353

130. Empirical Data Problems.................. 361

\section{CHAPTER IX}

\section{THEORY OF MEASUREMENT}

131. Statistical Methods...................... 365

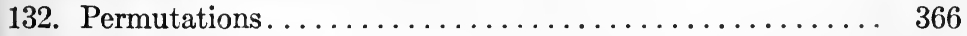

133. Combinations. ........................ 367

134. The Binomial Expansion. .................. 370

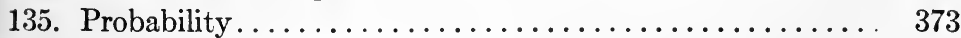

136. Compound Events. . . . . . . . . . . ......... 375

137. Mortality Tables. . . . . . . . . . . . . . . . . . . 379

138. Frequency Distributions. . . . . . . . . . . . . . 382

139. Averages........................... 388 
140. Measures of Variability . . . . . . . . . . . . . 398

141. Equation of the Frequency Curve Representing a Symmetrical Distribution. .................. 404

142. The Probable Error. . . . . . . . . . . . . . . . 411

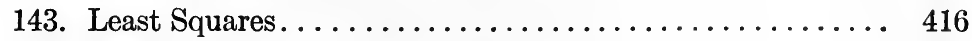

144. Correlation........................ 420 


\section{SOME PRINCIPLES OF ALGEBRA AND GEOMETRY}

1. Classification of the Numbers of Algebra.

Real $\left\{\begin{array}{l}\text { Rational }\left\{\begin{array}{l}\text { Integral }(1,2,-3, \text { etc. }) \\ \text { Fractional }\left(\frac{2}{3},-\frac{1}{2}, \text { etc. }\right)\end{array}\right. \\ \text { Irrational }(\sqrt{2}, \sqrt[3]{3}, \pi, \text { etc. })\end{array}\right.$

Complex (or inaginary), which will not be considered in this course.

2. Laws of Addition and Multiplication.

(a) Commutative laws: $a+b=b+a$. $a b=b a$.

(b) Associative laws: $a+(b+c)=(a+b)+c . \quad a(b c)=(a b) c$.

(c) Distributive law: $a(b+c)=a b+a c$.

3. Zero.

(a) $\quad a \times 0=0$.

(b) If $a \times b=0$, then $a=0$ or $b=0$.

(c) It is impossible to divide by zero, for the quotient of $a$ by zero, if it existed, would be a number $q$ such that $q \times 0=a$. But as $q \times 0=0$, by (a), we have a contradiction, and hence division by zero must be excluded.

\section{Fractions.}

(a) The value of a fraction is unchanged if numerator and denominator are divided by the same number not zero. This enables us to "cancel" a common factor of the numerator and denominator.

(b) The value of a fraction is unchanged if numerato" and denominator are multiplied by the same number, that is, $\frac{a}{b}=\frac{m a}{m b}$.

This gives the rule: To simplify a given complex fraction, multiply numerator and denominator by the least common denominator of the fractions occurring in the numerator and denominator of the given fraction. Thus if we multiply numerator and denominator of the complex

fraction $\frac{\frac{x}{a}+\frac{y}{b}}{c+\frac{d}{m}}$ by $a b m$ we get at once $\frac{b m x+a m y}{a b c m+a b d}$.

(c) Addition and subtraction: $\frac{a}{b}+\frac{c}{d}=\frac{a d+b c}{b d}$. 
(d) Multiplication: $\frac{a}{b} \times \frac{c}{d}=\frac{a c}{b d}$.

(e) Division: $\frac{a}{b} \div \frac{c}{d}=\frac{a d}{b c}$.

(f) The reciprocal of a given number is a second number such that the product of the two is unity. Thus the reciprocal of $a$ is $\frac{1}{a}$.

\section{Rules for Signs.}
(a) $(-a)(-b)=a b$.
(b) $(-a)(b)=-a b$.
(c) $\frac{-a}{-b}=\frac{a}{b}$.
(d) $\frac{-a}{b}=\frac{a}{-b}=-\frac{a}{b}$.
(e) $a-(b-c)=a-b+c$

\section{Factors.}

(a) $a^{2}-b^{2}=(a+b)(a-b)$.

(b) $a^{3}+b^{3}=(a+b)\left(a^{2}-a b+b^{2}\right)$.

(c) $a^{3}-b^{3}=(a-b)\left(a^{2}+a b+b^{2}\right)$.

(d) $a^{n}+b^{n}=(a+b)\left(a^{n-1}-a^{n-2} b+a^{n-3} b^{2}-\ldots+b^{n-1}\right), n$ odd.

(e) $a^{n}-b^{n}=(a-b)\left(a^{n-1}+a^{n-2} b+a^{n-3} b^{2}+\ldots+b^{n-1}\right)$.

(f) $x^{2}+(a+b) x+a b=(x+a)(x+b)$.

7. Binomial Theorem.

$$
(a+b)^{n}=a^{n}+n a^{n-1} b+\frac{n(n-1)}{1 \cdot 2} a^{n-2} b^{2}+\frac{n(n-1)(n-2)}{1 \cdot 2 \cdot 3} a^{n-3} b^{3}
$$
$+\cdots+b^{n}$.

The coefficient of the $(r+1)$ st term is $\frac{n(n-1)(n-2) \cdots(n-r+1)}{1 \cdot 2 \cdot 3 \cdots(r-1) r}$

Special cases: $n=2,(a+b)^{2}=a^{2}+2 a b+b^{2}$.

$$
n=3,(a+b)^{3}=a^{3}+3 a^{2} b+3 a b^{2}+b^{3} .
$$

8. Powers and Roots.
(a) $\quad a^{0}=1$.
(b) $a^{-m}=\frac{1}{a^{m}}$.
(c) $\left(a^{m}\right)^{n}=a^{n n}$.
(d) $a^{\frac{p}{q}}=\sqrt[3]{a^{p}}$.
(e) $a^{m} \cdot a^{n}=a^{m+n}$.
(f) $\frac{a^{m}}{a^{n}}=a^{m-n}$.
(g) $\sqrt{\bar{a}} \sqrt{\bar{b}}=\sqrt{\overline{a b}}$.
(h) $\frac{\sqrt{a}}{\sqrt{b}}=\sqrt{\frac{a}{b}}$.

(i) To rationalize the denominator (or numerator) of a fraction containing a square root, multiply numerator and denominator by the quantity obtained by changing the sign of the radical in the denominator (or numerator).

9. Equations.

(a) If equals be added to, or subtracted from, equals the results are equal.

(b) If equals be multiplied or divided by equals, the results are equal. 
Do not divide both sides of an equation by a quantity until the possibility of division by zero has been excluded.

(c) Like powers, or roots, of equals are equal.

(d) To simplify an equation containing fractions, multiply both sides by the least common denominator of the fractions.

(e) To simplify an equation containing square roots, transpose to one side all the terms except a single radical, and square both sides.

(f) To solve the quadratic equation $a x^{2}+b x+c=0$, factor the lefthand member, set each factor equal to zero, and solve for $x$.

If the left-hand member cannot be easily factored, transform the equation to the form

$$
x^{2}+p x=q,
$$

and complete the square by adding to both sides the square of half the coefficient of $x$. Then extract the square root of both sides of the resulting equation, and solve for $\boldsymbol{x}$.

(g) The roots of the equation

$$
a x^{2}+b x+c=0 \quad \text { are } \quad x=\frac{-b \pm \sqrt{b^{2}-4 a c}}{2 a}
$$

The roots will be real and unequal, real and equal, or imaginary according as the discriminant $b^{2}-4 a c$ is positive, zero, or negative.

(h) Simultaneous equations in two variables $x$ and $y$.

If both equations are linear, to eliminate $y$, multiply each equation by the coefficient of $y$ in the other, and subtract the results.

If one equation is linear and the other quadratic, to eliminate one variable, solve the linear equation for $x$ or $y$ (whichever is easier) and substitute in the quadratic equation.

10. Arithmetical Progression.

In an arithmetical progression,

$$
a, a+d, a+2 d, a+3 d, \ldots,
$$

each term is obtained from the preceding by adding a constant quantity

The $n$th term is

$$
l=a+(n-1) d \text {. }
$$

The sum of $n$ terms is

$$
S=\frac{\pi}{2}(a+l) \text {. }
$$

The arithmetic mean between $a$ and $b$ is $A=\frac{1}{2}(a+b)$.

11. Geometric Progression.

In a geometric progression,

$$
\text { a. } a r, a r^{2}, a r^{3}, \ldots \text {, }
$$

each term is obtained from the preceding by multiplying by a constant quantity. 
The $n$th term is

$$
\begin{aligned}
l & =a r^{n-1} . \\
S & =\frac{r l-a}{r-1} .
\end{aligned}
$$

The sum of $n$ terms is

The geometric mean between $a$ and $b$ is $G=\sqrt{a b}$.

If the numerical value of $r$ is less than unity, as $n$ increases indefinitely the sum of $n$ terms approaches the limit $\frac{a}{1-r}$.

\section{Parallel Lines.}

Two lines are parallel if the alternate-interior angles are equal, or if the exterior-interior angles are equal, and conversely.

\section{Triangles.}

(a) The bisector of the vertex angle of an isosceles triangle bisects the base perpendicularly.

(b) In any plane triangle the sum of the angles is $180^{\circ}$.

(c) The exterior angle of a triangle is equal to the sum of the two opposite interior angles.

(d) In a right triangle the square on the hypotenuse is equal to the sum of the squares on the other two sides.

14. Similar Polygons.

(a) Similar polygons are polygons which have their angles respectively equal and their homologous sides proportional.

(b) If two triangles have the angles of the one equal respectively to the angles of the other, the triangles are similar.

(c) If two triangles have an angle of one equal to an angle of the other, and the including sides proportional, the triangles are similar.

(d) If two triangles have their sides respectively proportional they are similar.

(e) The areas of two similar polygons are to each other as the squares of any two homologous sides.

15. Circles.

(a) An angle at the center of a circle is measured by the intercepted arc.

(b) An inscribed angle of a circle is measured by one half the intercepted arc.

(c) The circumference of a circle is $C=2 \pi r$.

(d) The area of a circle is $\quad A=\pi r^{2}$.

(e) Approximate values of $\pi$ are $\frac{22}{7}$ and 3.1416.

(f) A sector of a circle is a figure formed by two radii and their intercepted arc.

The area of a sector is $A=\frac{1}{2}$ arc $\cdot r$.

(g) A segment of a circle is the figure formed by a chord and its intercepted arc. 
16. Operations on Lines.

(a) Addition and subtraction: These may be performed by the compasses.

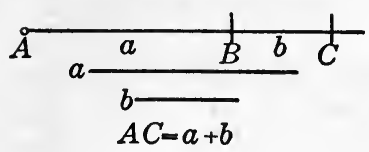

FIg. 1.

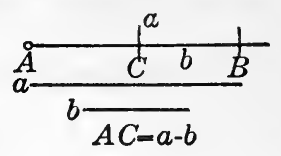

FIg. 2.

(b) Multiplication: On the sides of an angle lay off $O U=1, U A=a$, $O B=b$; join $B$ to $U$ and draw $A C$ through $A$ parallel to $B U$. Then $B C=a b$.

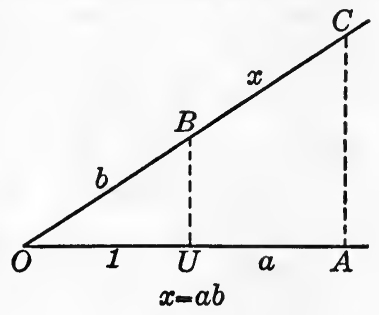

FIg. 3.

(c) Division: Onf the sides of an angle take $O U=1, O B=b, B A=a$; join $B$ to $U$ and draw $A C$ through $A$ parallel to $B U$. Then $U C=a / b$.

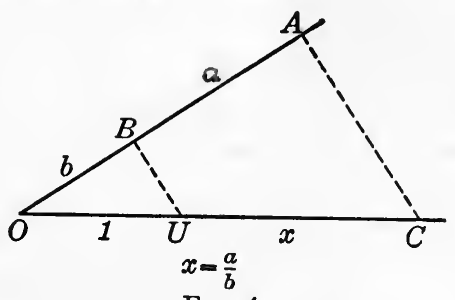

FIg. 4.

(d) Extraction of square root: Take $A B=a,[B C=1$, describe a semicircle on $A C$ as a diameter, and erect $B D \perp A C$. Then $B D=\sqrt{a}$.

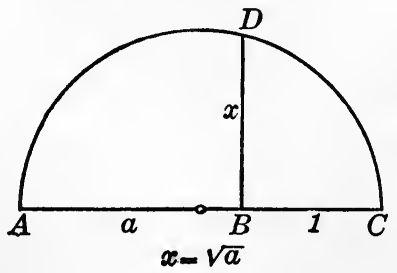

Fra. 5 . 


\section{xx SOME PRINCIPLES OF ALGEBRA AND GEOMETRY}

It follows that an unknown line $x$ can be constructed by ruler and compasses if $x$ can be expressed in terms of known lines $a, b$, $c$, etc., by means of addition, subtraction, multiplication, division, and the extraction of square roots.

Unless $x$ can be so expressed, it cannot be constructed by ruler and compasses. This is proved by a combination of algebra and analytic geometry, and is used to prove that some problems of construction, for example, the trisection of an angle or the squaring of a circle, cannot be solved with ruler and compasses.

17. Some letters from the Greek alphabet.

$\begin{aligned} \alpha & \text { Alpha } & \mu & \mathrm{Mu} \\ \beta & \text { Beta } & \pi & \mathrm{Pi} \\ \gamma & \text { Gamma } & \Sigma, \sigma & \text { Sigma } \\ \Delta, \delta & \text { Delta } & \phi & \text { Phi } \\ \theta & \text { Theta } & \psi & \text { Psi } \\ & \omega & \text { Omega } & \end{aligned}$




\section{ELEMENTARY FUNCTIONS}

\section{CHAPTER I}

\section{FUNCTIONS, EQUATIONS, AND GRAPHS}

1. Comparison of the Reasoning in Natural Science and in Mathematics. In science, the method of procedure in determining the law of phenomena is as follows:

1. Observations are recorded, compared, and classified.

2. An induction is made and the generalization resulting is stated as a hypothesis.

3. Deduction from the hypothesis leads to a conclusion.

4. The conclusions are tested and verified by experiment.

Mathematical reasoning is not inductive, and hence it is not of the nature of stages 1 and 2 of the scientific method. But when a science has advanced to the point where the data are expressed in terms of magnitude, the generalization can be expressed mathematically in simple and compact form, and the deductive process of the scientific method can be carried out by the direct and powerful methods of mathematics.

The reasoning in mathematics is purely deductive in character, and the conclusion reached contains no more than the hypothesis from which it was derived. If the conclusion were more general than the hypothesis, then it would be certain that the deductive reasoning was not performed correctly. While a correctly deduced conclusion states nothing which was not included in the hypothesis, it is in a form which can be more easily comprehended and more readily used. The process of going from hypothesis to conclusion may be likened to the unwrapping of a compact bundle; there is no more pertaining to the bundle at the end of the operation 
than there was at the beginning, but the contents are disclosed to the mind, and can be examined. What is implicit in a hypothesis becomes explicit in the conclusion.

In natural science, if the conclusion when tested does not check with experience, the observations are reëxamined, increased in number, and in some cases after a long interval of time and much work by many men, a new hypothesis is stated, and the rest of the scientific process repeated. In mathematics, the question of the truth or usefulness of the hypothesis may not be raised, but the accuracy of the deduction is an everpresent concern, and should be constantly tested by checks.

The verification in the scientific method involves reference to the field of observation and is not deductive in character, and hence not mathematical.

2. Example of the Utility of Mathematics in Science. A classical example of the scientific method and the part which mathematics plays in natural science is furnished by the steps leading to the discovery of the planet Neptune.

Observations on the motions of the planets of the solar system were recorded in great number by the astronomer Tycho Brahe (1546-1601).

His assistant, Johannes Kepler (1571-1630), generalized from the observations and stated the hypotheses known as Kepler's laws.

Sir Isaac Newton (1642-1727), by means of mathematics, condensed Kepler's three laws into one, the law of gravitation.

Up to 1846 Uranus was the outermost planet of the solar system then known. The irregularities in its orbit led astronomers to suspect that there was another planet outside Uranus which caused these disturbances.

LeVerrier and Adams, independently, using Newton's law and the facts of the disturbances, deduced mathematically the position that an outer planet must occupy to produce these perturbations. Adams spent months in carrying through the intricate calculations necessary and in checking the accuracy of his deductions, which he did by solving the problem a number of times in difierent ways. 
The last stage, the verification, was performed by Galle, who turned his telescope in the direction indicated by LeVerrier, and on September 23, 1846, discovered Neptune. Here was a wonderful verification of Newton's law and a monument to the power and value of mathematics in making deductions.

3. Forms in which Data are Recorded. The observations of the scientist are usually recorded in the form of a table of two related sets of magnitudes. The biologist compares the width of a leaf with the number of specimens examined having that width; the physicist, the expansion of a substance with the temperature; the chemist, the amount of substance in solution with the time.

The following tables illustrate the manner of recording the data.

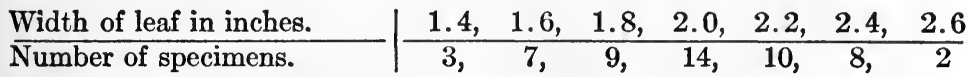

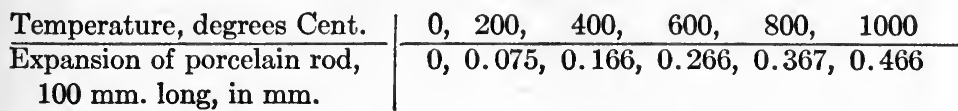

\begin{tabular}{l|ccccc} 
Time in seconds. & 15, & 30, & 45, & 60, & 75 \\
\hline Sugar in solution, grams. & .046, & .088, & .130, & .168, & .206
\end{tabular}

The data are sometimes recorded by an instrument. The temperature and barometer records of the Weather Bureau are so recorded. A pen is connected with the thermometer and rests against a sheet of paper fastened to a revolving cylinder. The cylinder revolves steadily and the pen rising and falling with the temperature traces a curve on the paper, from which the temperature at any time may be determined.

The variation in the tide at an important port is similarly recorded on a chart fastened to a revolving cylinder by a pen attached to a float which rises and falls with the tide (Figure 6). The data may be presented most conveniently in the form of a generalization, expressed either in words or in mathematical symbols. For example, a comparison of the pairs of values in the second table above, neglecting the first two pairs of values, leads to the following generalization, expressed in words: 
For a temperature of $400^{\circ}$, or greater, an increase of $200^{\circ}$ in the temperature causes an expansion of approximately 0.1 of a millimeter in the length of the rod.

The generalization may also be expressed in symbols. If every increase of $200^{\circ}$ causes an expansion of 0.1 of a millimeter,

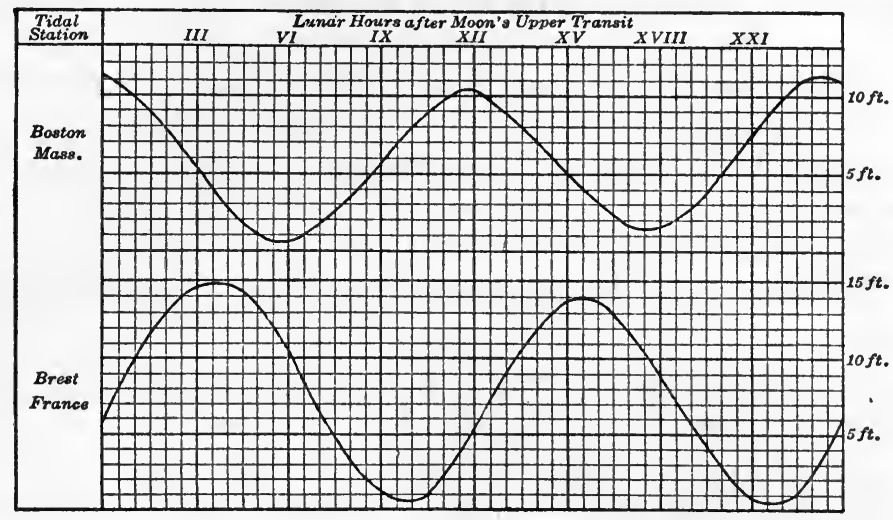

Fig. 6.

then an increase of $1^{\circ}$ would cause an expansion of $0.1 / 200$, or 0.0005 ; hence for an increase of temperature of $t$ degrees the expansion $e$ would be

$$
e=0.0005 t \text {. }
$$

This equation expresses the generalization in more compact form than the sentence above. In this illustration we have considered only a part of the table, a part for which the generalization is very simple.

The determination of the mathematical expression of the generalization from a table of values will be one of the objects of this course. The generalization from a mathematical point of view is considered in the following section.

4. Variable. Function. The following table gives the lengths of an iron bar suspended from one end when carrying different loads.

\begin{tabular}{l|ccccc} 
Load in lbs. & 0, & 500, & 1000, & 1500, & 2000 \\
\hline Length in in. & 30, & 30.67, & 30.91, & 31.23, & 31.52
\end{tabular} 
Any one of the numbers in each of these sets of numbers is conveniently represented by a single symbol, as $x, y, t$, etc. Thus $x$ may be taken to represent one of the numbers $0,500,1000$, 1500,2000 and any other number that might be included in the half of the table giving loads, while $y$ may represent the corresponding number giving lengths in the second part of the table.

The symbols $x$ and $y$ are called variables, in accordance with the

Definition. A variable is a symbol for any one of a set of numbers.

In the experiment giving rise to the above table, the load was changed arbitrarily by the experimenter and the length of the bar for the chosen load measured. In consequence of this order of measurement the variable representing the load is called the independent variable and the one representing the length of the bar the dependent variable.

It is customary to denote the independent variable by $x$ and the dependent variable by $y$.

If the experiment were repeated, under the same conditions, it would be found that for a specified load the length of the bar would be the same, that is, there is a law connecting the load and the length of the bar. This relation between the variables is expressed by saying that the length, $y$, is a function of the load, $x$, in accordance with the

Definition. A function is a variable so related to another variable (called the independent variable) that for every admissible value of the independent variable, one or more values of the function are determined. The function is also called the dependent variable.

The idea of a function arises wherever there is a relation between magnitudes which are changing, and it underlies all magnitude relations which mankind has discovered.

Example 1. The algebraic expression $2 x+3$ is a variable whose value is determined whenever a definite value is assigned to $x$. If $x$ be given the value 1 , then $2 x+3$ has the value of 5 , and if $x$ has the value $2,2 x+3$ has the value 7 . Hence $2 x+3$ is a function of $x$. 
Example 2. A theorem from physics relating to a falling body states that if a heavy object be dropped, the distance it falls in $t$ seconds is $16 t^{2}$. This distance is a function of the variable $t$; for if $t$ be given a definite positive value, the distance is determined. If $t=2$ seconds, the distance fallen is 64 feet; if $t=3$ seconds, the distance is 144 feet. Negative values of $t$ are meaningless and hence not admissible.

EXAmple 3. The formula for the area of a circle is $A=\pi r^{2}$. If the radius $r$ be given a definite value the area is determined. Hence the area $A$ is a function of $r$. The symbol $\pi$ represents the number $3.14159 \ldots$ which remains the same for any pair of corresponding values of $r$ and $A$, and hence is called a constant in accordance with the

Definition. A constant is a symbol for a particular number. It has the same value throughout a discussion.

EXAMPLe 4. A simple equation which occurs frequently in practice is

$$
y=m x,
$$

where $x$ represents the independent variable, $y$ the function, and $m$ is a constant representing a fixed value as $x$ and $y$ vary.

If $x$ represents the number of pairs of shoes of a certain kind sold during a limited time by a dealer, and $y$ the amount of the sales, then $m$ represents the price per pair which remains fixed, for the time considered, while $x$ and $y$ vary.

Example 5. An equation in two variables establishes a functional relation between the variables. For if a value be given to either, the corresponding value or values of the other may be found by substituting the given value of one variable and solving for the other.

Either variable may be regarded as a function of the other, and the form of the function may be found by solving for one variable in terms of the other.

Thus, if the equation $4 y-x^{2}=0$ be solved for $y$ and then for $x$ we have

$$
y=\frac{x^{2}}{4} \quad \text { and } \quad x= \pm 2 \sqrt{y} .
$$

The given equation defines $y$ as a function of $x$ to be the function $x^{2} / 4$, and $x$ as a function of $y$ to be the function $\pm 2 \sqrt{y}$.

Example 6. Some of the elements entering into the cost of a suit of clothes are the supply of cloth, the supply of labor, rent, style, etc. As these elements vary the cost of the suit will vary, so that the cost of the suit is a function of a number of variables.

Considering one of the independent variables at a time, a part of the cost of the suit may be expressed as a function of this variable, e.g., the supply of cloth. 
The law of supply and demand from economics states that the price of an article increases or diminishes as the supply diminishes or increases.

If $x$ represents the supply of cloth and $y$ the price of the suit of clothes, then it is assumed in economics that the functional relation may be expressed by the equation

$$
y=\frac{m}{x},
$$

where $m$ is a constant which can be determined in any concrete case.

In this course we shall confine ourselves to the study of functions of one variable.

Example 7. The temperature at a given place is a function of the time. For at a given time the temperature must have a definite value. But this function is so little understood that the Weather Bureau can only approximate the value for any future time, and that, indeed, only for times in the immediate future.

The data of such departments of human knowledge as physics, astronomy, and engineering are so complete that many of the functions arising there can be identified and studied by mathematical methods. In other subjects, for example, chemistry and economics, the data have only recently been made sufficiently complete to warrant an increasing use of mathematics. But there still remains a countless number of functions which mankind has been unable to represent by a mathematical expression.

\section{EXERCISES}

In the following exercises give the reason for the statement that one variable is a function of another.

1. Mention three variables which are functions of the side of an equilateral triangle of varying size.

2. A train goes from one station to another at a variable rate. Mention two variables of which the rate is a function.

3. What are some of the variables of which the cost of erecting an office building is a function?

4. Mention some variables involved in heating water in a pan on a gas stove. Which are independent variables? Which are functions?

5. Find the functions of $x$ defined by the following equations and tabulate three pairs of values. 

(a) $y-x^{2}-3 x-2=0$.
(b) $x^{3}-3 y^{2}+2 y+4=0$.
(c) $3 x y+6 x-9 y+4=0$.
(d) $2 x^{2}+3 x y+4 y^{2}+6 x-8 y+7=0$

5. Notation for a Function. It is convenient to represent a function of the variable $x$ by the symbol $f(x)$ which is read "function of $x$," "the function $f$ of $x$," or merely " $f$ of $x$." The various parts of the symbol are to be regarded as forming a single compound symbol, never as separate symbols meaning the product of two numbers $f$ and $x$.

This symbol is used to denote either any function or a particular function such as

$$
f(x)=x^{2}+x-1
$$

Similar symbols convenient for distinguishing different functions are

$$
F(x), g(x), \phi(x), \text { etc. }
$$

An advantage of this notation is that the value of a function $f(x)$ for any value of $x$, say $x=a$, may be suggestively represented by $f(a)$. For example, if

$$
\begin{gathered}
f(x)=x^{2}+x-1 \\
f(a)=a^{2}+a-1, f(2)=2^{2}+2-1=5 \\
f(-1)=(-1)^{2}+(-1)-1=-1, f(0)=-1 \\
f(-x)=(-x)^{2}+(-x)-1=x^{2}-x-1, \text { etc. }
\end{gathered}
$$

This notation also enables us to state certain theorems in a more compact form.

\section{EXERCISES}

1. If $f(x)=x^{3}-x^{2}$, find $f(1), f(-3), f(0), f(-x)$.

2. If $F(x)=1 / x^{2}$, find $F(2), F(-1), F(a), F(-x)$.

3. If $\phi(x)=m x+b$, find $\phi(0), \phi(1), \phi\left(x_{2}\right), \phi(-b / m)$.

4. If $f(x)=x^{3}+x$, show that $f(-2)=-f(2)$, that $f(-x)=-f(x)$.

5. If $f(x)=x^{4}+x^{2}$ show that $f(-2)=f(2)$, that $f(-x)=f(x)$.

6. If $f(x)=x^{2}+x+1$, determine whether either $f(-x)=f(x)$ or $f(-x)=-f(x)$ is a true relation.

6. Determination of the Function which Expresses the Functional Relation between Two Variables. The functional relation between two variables is expressed in symbols whenever possible, for the sake of the greater simplicity which this 
gives to the generalization and of the greater ease in making deductions. If $x$ is chosen to represent the independent variable, then an algebraic expression is sought to represent the function.

Example 1. If $x$ represents any one of the numbers in the first column $x \mid f(x)$ of the accompanying table, or the value of the independent $0 \quad \frac{0}{0}$ variable, what function of $x$ will represent the second column?

1 We see by inspection that each number in the second set is $2 \quad 4 \quad$ the square of the corresponding number of the first set.

$3 \quad 9 \quad$ Hence $f(x)=x^{2}$ is the required function.

Check. When $x=0, f(0)=0$; when $x=1, f(1)=1$, etc., for all the table. Example 2. Find the area of an equilateral triangle as a function of the side.

Let $x$ represent the side of the equilateral triangle $A B C$ with the altitude $C D$.

Then

$$
\begin{aligned}
A D & =x / 2 \quad \text { (The altitude bisects the base.) } \\
C D^{2} & =A C^{2}-A D^{2}(A C D \text { is a right triangle. }) \\
& =x^{2}-(x / 2)^{2}=x^{2}-x^{2} / 4 \\
& =\frac{3}{4} x^{2} .
\end{aligned}
$$

Hence

$$
C D=\frac{\sqrt{ } \overline{3}}{2} x .
$$

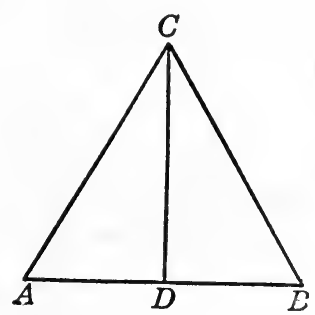

FIG. 7.

Therefore, the area $=\frac{1}{2} A B \cdot D C=\frac{1}{2} x \cdot \frac{\sqrt{3}}{2} x=\frac{\sqrt{3}}{4} x^{2}$, which is the required function of $x$.

Example 3. A man leaves a village $\mathbf{1 0}$ miles directly east of a city and walks east at the rate of 3 miles an hour. Express the distance he is from the city at any time, $t$, after he starts as a function of $t$. Where will he be at the end of 3 hours, and when will he be 25 miles from the city?

\begin{tabular}{l|l|l}
$t$ & $\frac{s}{0}$ & rate \\
\hline 3 & 10 & 3 \\
5 & 25 &
\end{tabular}

Since distance equals the rate multiplied by the time, the distance the man is from the village at any time $t$ after he starts is $3 t$; and the distance he is from the city is $s=3 t+10$, since the village and city are 10 miles apart and he goes away from both.

Hence the required function is $3 t+10$.

When $t=3, s=3 \cdot 3+10=19$; when $s=25,25=3 t+10$, whence $t=5$

The data and results are recorded in the accompanying table.

Example 4. A ball rolls down an inclined plane. The distance $s$ it rolls in the $t$ th second is recorded in the table. Express the distance the ball rolls in any second as a function of $t$. What distance will it roll in the 5th second? 
$t \quad s \quad$ The values of both the independent variable and the function $\overline{1} \overline{4}$ form arithmetic progressions.

$\begin{array}{lll}2 & 12 & \text { The formula for the } n \text {th term of an arithmetic progression is }\end{array}$ $3 \quad 20 \quad l=a+(n-1) d$.

The common difference $d$ of the values of the function is 8 , the first term $a$ is 4 , the number of terms $n$ is $t$, and $l=s$

Hence we have $s=4+(t-1) 8=8 t-4$ which is the required function. When $t=5, f(5)=8 \cdot 5-4=36$.

Check. If the values $1,2,3$ are substituted for $t$ in $8 t-4$ the values 4, 12,20 are obtained, which are the values of $s$ given in the table.

EXAMPLE 5. If $\$ 100$ are placed in a bank at $5 \%$ interest, compounded annually, what is the amount at the beginning of $t$ th year?

The amount at the beginning of the first year is of course $\$ 100$. The interest for the first year is $\$ 5$ and the amount at the beginning of the second year is $\$ 105$.

\begin{tabular}{l|l}
$t$ & \multicolumn{1}{|c}{$A$} \\
\hline 1 & 100 \\
2 & 105 \\
3 & 110.25
\end{tabular}

The interest for the second year is $\$ 5.25$ and the amount at the beginning of the third year is $\$ 110.25$.

The accompanying table gives the data obtained.

gression, for the ratio of the second value to the first is equal to the ratio of the third value to the second, i.e.,

$$
\frac{105}{100}=1.05 \quad \text { and } \quad \frac{110.25}{105}=1.05 \text {. }
$$

The $n$th term of a geometric progression is given by the formula

$$
l=a r^{n-1} .
$$

In this case $l=A, n=t, a=100$ and $r=1.05$. Substituting these values in the above formula, we have for the required function

$$
A=100(1.05)^{t-1} \text {. }
$$

Check. Substituting the values 1, 2, 3, for $t$ in this function we get the values 100105 and 110.25 respectively for $A$, which results agree with the table.

\section{EXERCISES}

1. In each of the following tables let $x$ represent any one of the numbers in the first column (value of the independent variable), and find a function of $x$ which will represent the corresponding number in the second column. Add two additional pairs of values of $x$ and the function to each table.

(a) \begin{tabular}{c|c}
$x$ & $f(x)$ \\
\hline 0 & 0 \\
1 & 1 \\
2 & 8 \\
3 & 27
\end{tabular}

(b) \begin{tabular}{c|c}
$x$ & $f(x)$ \\
\hline 0 & 0 \\
1 & 1 \\
4 & 2 \\
9 & 3
\end{tabular}

(c) \begin{tabular}{r|c}
$x$ & $\frac{f(x)}{1}$ \\
\hline 1 & 1 \\
27 & 3 \\
64 & 4 \\
125 & 5
\end{tabular}

(d) \begin{tabular}{r|r}
$x$ & $f(x)$ \\
\hline 1 & 1 \\
4 & 8 \\
9 & 27 \\
16 & 64
\end{tabular}




(e) \begin{tabular}{r|r}
$x$ & $f(x)$ \\
\hline 1 & 1 \\
8 & 4 \\
27 & 9 \\
64 & 16
\end{tabular}

(f) \begin{tabular}{c|c}
$x$ & $f(x)$ \\
\hline 0 & 0 \\
2 & 3 \\
4 & 6 \\
6 & 9
\end{tabular}

(g) \begin{tabular}{c|c}
$x$ & $f(x)$ \\
\hline 0 & 3 \\
1 & 5 \\
2 & 7 \\
3 & 9
\end{tabular}

(h) \begin{tabular}{r|r}
$x$ & $f(x)$ \\
\hline 1 & 2 \\
2 & 4 \\
3 & 8 \\
4 & 16
\end{tabular}

2. Find the perimeter of an equilateral triangle as a function of the altitude $x$.

3. Express the area of a square as a function of (a) the side, (b) the diagonal.

4. Express the area of a regular hexagon as a function of the side.

5. A regular octagon is formed by cutting off isosceles right triangles from the corners of a square. Express the area as a function of the side of one of the right triangles cut off.

6. Express the side of a regular decagon as a function of the radius of the circumscribed circle.

7. A Norman window consists of a square surmounted by a semicircle. Find the area as a function of the side of the square $x$.

8. (a) A man walks from a certain town toward a second at the rate of three miles an hour. Express the distance traveled as a function of the time.

(b) A second man starts at the same time from the second town, which is $\mathbf{1 0}$ miles from the first, and travels at the rate of 4 miles an hour toward the first town. Express his distance from the first town as a function of the time.

(c) A third man starts from the same town as the first man but two hours later and travels at the rate of $3 \frac{1}{2}$ miles an hour in the same direction. Express his distance from town as a function of the time elapsed since the first man started.

How far will each man be from the first town 4 hours after the first man starts? When will each man be 20 miles from the first town?

9. A man starts from a town $\mathbf{1 5}$ miles directly west of a city and travels east at the rate of 4 miles an hour. A second man starts from the same town at the same time and travels west at the rate of 3 miles an hour. Express the distance of each from the city after $t$ hours as a function of $t$. How far from the city will each be in 3 hours? When will they be 25 miles apart?

10. A ball starting from rest rolls down an inclined plane 4 feet in the first second, 8 feet in the next, 12 feet in the next, etc. Express the distance it rolls in any second $t$ as a function of $t$. How far will it roll in the 8 th second?

11. With the data of the preceding problem and the formula for the sum of an arithmetic progression find the total distance rolled as a function of the time $t$.- When will the ball have rolled 108 feet? 
12. A body starting from rest falls 16 feet in the first second, 48 feet in the next, 80 feet in the next, etc. Find the distance fallen in any second as a function of the time of falling. Find the total distance fallen as a function of the time.

13. $\$ 100$ is placed at simple interest at 4 per cent. Express the amount at the end of $t$ years as a function of $t$.

14. If the interest in the preceding problem is compounded annually, express the amount at the end of $t$ years as a function of $t$.

15. The cross-section of a gutter pipe is in the form of an isosceles trapezoid. The lower base and the inclined sides are each 3 inches long. Find the area as a function of the width across the top.

16. A rectangle is inscribed in a circle 4 inches in diameter. Express the area as a function of one of the sides.

7. Graphical Representation. Directed Lines. The functional relation can be represented by a geometrical figure which furnishes a valuable method for studying the properties of the function, since the whole of the relation is placed before the mind at once. The system of coördinates devised by René Descartes (1596-1650), which is developed in the following section, is the basis of the representation. This system of coördinates rests on the theory of directed lines.

Let $X X^{\prime}$ be any line, and let the direction from $X^{\prime}$ to $X$ be called positive, from $X$ to $X^{\prime}$. negative. These words are used instead of such terms as north and south, to the right and left, up and down, backward and forward. A line upon which a positive direction has been fixed is called a directed line. The positive direction is commonly indicated by an arrow-head.

If $A$ and $B$ be two points on a directed line, the symbol $A B$ is used to denote either:

(1) The line drawn from $A$ to $B$, or

(2) The real number whose numerical value is the number of times the unit of length is contained in the line, and whose sign is positive or negative according as the direction from $A$ to $B$ is positive or negative.

Thus, in the figure, $A B$ and $A^{\prime} B^{\prime}$ denote certain lines.

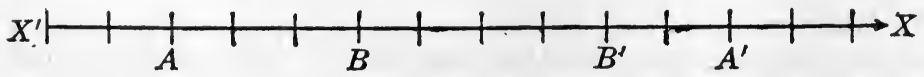

Fig. 8. 
They also denote the numbers $A B=3$ and $A^{\prime} B^{\prime}=-2$, provided the unit of length is a quarter of an inch.

It follows that if $A$ and $B$ are points on a directed line

$$
B A=-A B
$$

Definition. No matter what the position of three points $A, B, C$, on a directed line may be, the sum of $A B$ and $B C$ is defined to be

$$
A B+B C=A C
$$

If $A B$ is thought of as a motion from $A$ to $B$, and $B C$ as a motion from $B$ to $C$, the sum gives a motion from $A$ to $C$. The sum gives the distance from $A$ to $C$ in both magnitude and direction, but not necessarily the total number of units passed over in going from $A$ to $B$ and then from $B$ to $C$.

That this definition agrees with elementary algebra, when $A B, B C$, and $A C$ are regarded as numbers, is seen in the following illustrations, of which the first agrees with arithmetic.
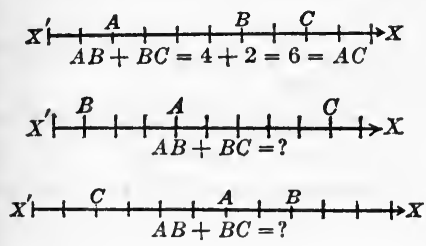
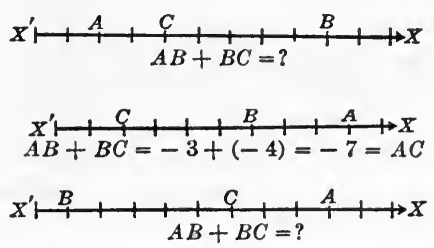

Fig. 9.

An especially important use of directed lines is the following: Let $O$ be any point on a directed line $X^{\prime} X$, and let points be laid off on each side of $O$ at a unit's distance from each other. Then with every point $P$ on $X^{\prime} X$ is associated a real number $O P$, and conversely, with every real number is associated a point on the line.

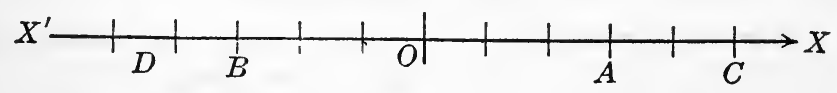

FIG. 10.

In the figure, the numbers associated with the points $A, B$, $C, D$ are respectively $O A=3, O B=-3, O C=5, O D=-4.5$. 
Using this association of points and numbers, if $P_{1}$ and $P_{2}$ are two points on the line, and $x_{1}$ and $x_{2}$ are the numbers associated with them, we have from (2)

$$
O P_{1}+P_{1} P_{2}=O P_{2},
$$

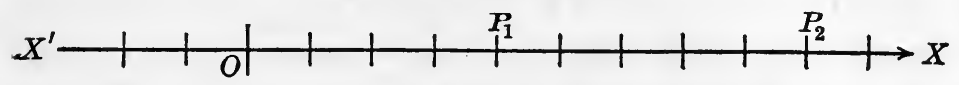

Fig. 11.

whence

$$
P_{1} P_{2}=O P_{2}-O P_{1}=x_{2}-x_{1}
$$

This difference of the values of the $x$ 's is denoted by $\Delta x$, so that

$$
\Delta x=P_{1} P_{2}=x_{2}-x_{1}
$$

Notice that $\Delta x$ is a single symbol (never the product of two numbers $\Delta$ and $x$ ), and that its value is obtained by subtracting the value of $x$ corresponding to the first point from that corresponding to the second.

\section{EXERCISES}

1. Illustrate (3) by numerical examples for the six possible relative positions of $O, P_{1}$, and $P_{2}$. Find $\Delta x$ for each case.

2. Show that $\Delta x$ is positive or negative according as $P_{1}$ lies to the left or right of $P_{2}$, using the definition that $a<b$ if $b-a$ is positive.

3. If $O P=x$, show that $x$ increases or decreases according as $P$ moves to the right or left.

8. Rectangular Coördinates. Let $X^{\prime} X$ and $Y^{\prime} Y$ be two

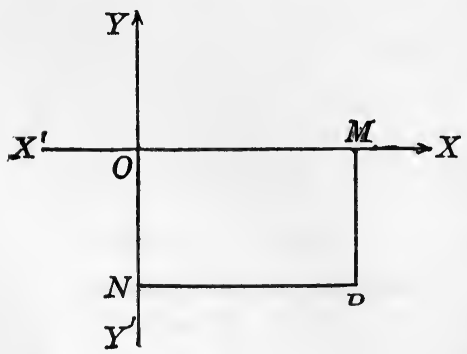

Fig. 12. perpendicular directed lines intersecting at $O$. Let the positive direction on $X^{\prime} X$ and on all lines parallel to it be to the right, and let that on $Y^{\prime} Y$, and on all lines parallel to it, be upward.

Let $P$ be any point in the plane, and draw $P M \perp X^{\prime} X$, and $P N \perp Y^{\prime} Y$. Then the numbers $O M=x$ and $O N=y$ are called rectangular coördinates of $P, x$ the abscissa, and $y$ the 
ordinate. In the figure, the ordinate $y$ is often thought of as $M P$, which equals $O N$ in both magnitude and direction. The directed lines $X^{\prime} X$ and $Y^{\prime} Y$ are called the axes of coördinates and their intersection $O$ the origin.

The abscissa of a point is positive or negative according as the point lies to the right or left of the $y$-axis. The ordinate is positive or negative according as the point lies above or below the $x$-axis. In the figure, the abscissa $x$ is positive, while the ordinate $y$ is negative.

As seen above, any point $P$ determines a pair of real numbers, its coördinates. Conversely, given any pair of real numbers, $x$ and $y$, a point may be plotted, that is, constructed, whose coördinates are $x$ and $y$. For on $X^{\prime} X$ lay off $O M=x$. At $M$ erect a line perpendicular to $X^{\prime} X$ and on it lay off $M P=y$. Then $P$ is the required point.

The symbol $(x, y)$ is used to mean the point whose coördinates are $x$ and $y$. If $P$ is this point, it is indicated by the symbol $P(x, y)$.

Coördinate axes divide the plane into four parts called quadrants. These are numbered as in the figure, which also indicates the signs of the coördinates of a point in every quadrant.

We are frequently concerned with points which are symmetric with respect to the origin, the axes, or a line bisecting the first and third quadrants. Points having these symmetric relations are determined in accordance with the

Definitions. (1) Two points are symmetric with respect to a

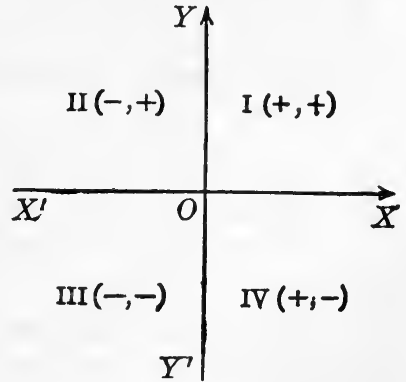

FIG. 13. third point if the line joining the two points is bisected at the third point.

(2) Two points are symmetric with respect to a line, if the line is the perpendicular bisector of the line joining the two points. 


\section{EXERCISES}

1. Plot the points whose coördinates are given below, and determine the nature of the symmetry for the pairs of points in each group.

(a) $(2,1),(-2,1) ;(3,0),(-3,0) ;(-2,-3),(2,-3)$.

(b) $(2,1),(2,-1) ;(0,5),(0,-5) ;(3,-4),(3,4)$.

(c) $(2,1),(-2,-1) ;(-3,1),(3,-1) ;(4,-1),(-4,1)$.

(d) $(2,1),(1,2) ;(1,3),(3,1) ;(3,-5),(-5,3)$.

2. By means of the corresponding parts of Exercise 1, what can be said of the positions of the following pairs of points?
(a) $(x, y)$ and $(-x, y)$.
(b) $(x, y)$ and $(x,-y)$.
(c) $(x, y)$ and $(-x,-y)$.
(d) $(x, y)$ and $(y, x)$.

3. One end of a line bisected by the origin is the point $(-5,2)$. What are the coördinates of the other end?

4. What are the coördinates of the point symmetrical to the point $(-3,4)$ with respect to the $y$-axis? the $x$-axis? the origin? the line bisecting the first and third quadrants?

5. Find the coördinates of the vertex or vertices not given in the regular polygons located as follows:

(a) One vertex of an equilateral triangle is the point $(1,0)$ and the altitude through this vertex, which is $\sqrt{3}$ units long, extends through the origin.

(b) An equilateral triangle has vertices with coördinates $(0,0)$ and $(1,0)$.

(c) A square with opposite vertices having coördinates $(1,0)$ and $(-1,0)$.

(d) A hexagon two of whose opposite vertices have coördinates $(1,0)$ and $(-1,0)$.

(e) An octagon with two opposite vertices having coördinates $(1,0)$ and $(-1,0)$.

6. The coördinates of three vertices of a rhombus are $(-1,0),(0, \sqrt{3})$, $(1,0)$. What are the coördinates of the fourth vertex? (Three solutions.) What are the coördinates of the intersection of the diagonals?

9. Graph of a Function. Values of $x$ and the corresponding values of a function may be exhibited in tabular form.

Table 1 gives the population of the United States in millions for the successive decades from 1830 to 1910 .

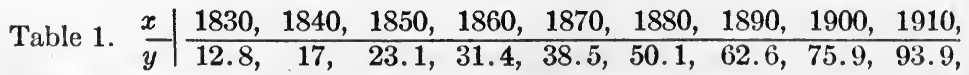

Table 2 gives pairs of values of $x$ and the function $\frac{1}{2} x+\frac{5}{2}$.

Table 2. $\frac{x}{\frac{1}{2} x+\frac{5}{2}} \mid$\begin{tabular}{rrrrrrrr}
-7, & -5, & -2, & -1, & 0, & 1, & 3, & 5 , \\
\hline-1, & 0, & 1.5, & 2, & 2.5, & 3, & 4, & 5 ,
\end{tabular} 
Any such table of values may be strikingly exhibited to the eye by plotting the points whose coördinates are the pairs of numbers in the table, and then drawing a smooth curve through the points so obtained. Proceeding thus, we obtain from Tables 1 and 2 the curves in Figures 14 and 15, which are called the graphs of the functions.

Definition. The graph of $a$ function is a curve such that (1) Any point whose coördinates are corresponding values of $x$ and the function is on the curve, and (2) Conversely, the coördinates of any point on the curve

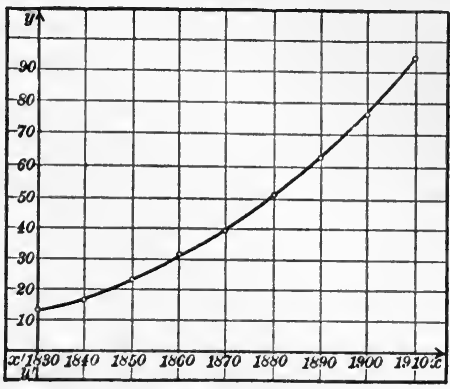

Fig. 14. are a pair of corresponding values of $x$ and the function. Hence the important fact:

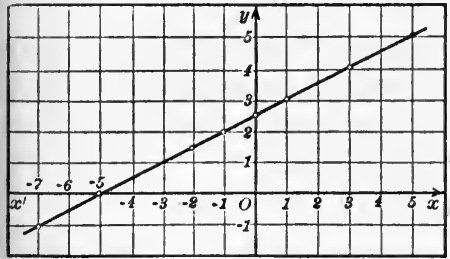

Fia. 15.

The ordinate of any point on the graph represents the value of the function when $x$ equals the abscissa of the point.

In the above definition the word curve is used in a very general sense to mean one or more lines, straight or curved, or parts of such lines. Functions exist which have no graphs, and the graphs of others are merely one or more isolated points, but we shall not encounter them in this course.

The graph of a function may be constructed by the following process:

Construct a table of values of $x$ and the function of $x$.

Plot the points whose coördinates are the pairs of numbers in this table.

Draw a smooth curve through these points.

In constructing a graph, notice that values of $x$ giving imaginary values of the function are discarded, and that the 
number of points plotted must be large enough to indicate without doubt the form of the curve. Whenever it is not clear just how the curve is to be drawn, enlarge the table of values, either by giving more integral values of $x$, or by assuming for $x$ intermediate values such as $2.5,2.8$, etc., as may best serve the purpose. The necessity for this last remark is shown by the fact that three points, situated as in Fig. 16(a), can be connected as in Figs. 16(b), $(c),(d)$ for different types of function, and also in other ways (see Exercise 4 below).

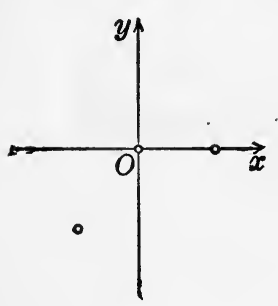

(a)

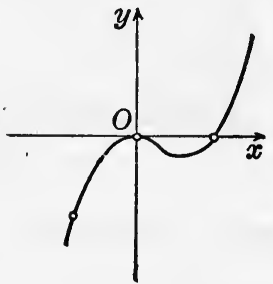

(b)

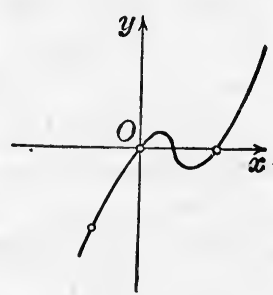

(c)

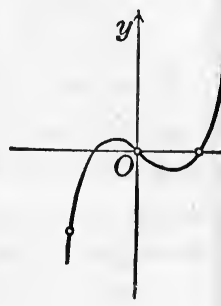

(d)

Fra. 16.

Definition. The graph of an equation in two variables is the curve such that: (1) Every point whose coördinates satisfy the equation is on the curve, and (2) Conversely, the coördinates of any point on the curve satisfy the equation.

To plot the graph of an equation,

Solve the equation for one of the variables in terms of the other, thus obtaining one as a function of the other.

Then proceed as indicated in the rule for the graph of a function.

In many of the applications of the methods of coördinates, the coördinates refer to quantities of different kinds such as time, distance, work, cost, etc., and the graph represents a relation between two of these quantities.

\section{EXERCISES}

1. Construct the graphs of each of the following pairs of functions on the same axes. State a relation that each pair of graphs bear to one another.
(a) $3 x, 3 x+2$.
(b) $-2 x,-2 x+2$.
(e) $-3 x+4,-3 x-5$.
(c) $\frac{1}{2} x, \frac{1}{2} x-3$.
(d) $-3 x,-3 x-2$ 
2. Construct the graphs of the following pairs of functions on the same axes. Can the graph of one member of a set be moved so as to coincide with the graph of the other member? If so, how can this be done?
(a) $2 x^{2}, 2 x^{2}+3$.
(b) $x^{2}+4 x+4, x^{2}+4 x$.
(c) $-x^{2},-x^{2}+4$.

3. Prove the Theorem: The graph of $f(x)+k$ may be obtained by moving the graph of $f(x)$ a distance $k$ in the direction of the $y$-axis.

4. Plot the graphs of each of the functions in the following sets. To insure the proper connection of points obtained from integral values of $x$ use intermediate values of $x$.

(a) $x^{3}-x^{2}, 2 x^{3}-3 x^{2}+x, 2 x^{3}-x^{2}-x$.

(b) $-x^{3}+x^{2},-2 x^{3}+3 x^{2}-x,-x^{3}+2 x^{2}-x$.

5. Plot the graphs of the following equations:
(a) $x-3 y+7=0$.
(b) $y^{2}-4 x=4$.
(c) $x^{2}-y^{2}=16$.
(d) $x^{2}+y^{2}=36$.
(e) $y+2 x^{2}-4=0$.
(f) $x^{2}+9 y^{2}=36$.

6. Distribution of the heights of 12-year-old boys. Plot the graph and state one of its characteristics.

$\left.\begin{array}{l}\text { Heights } \\ \text { in inches. }\end{array}\right\} 49,50,51,52,53,54,55,56,57,58,59,60,61,62$.

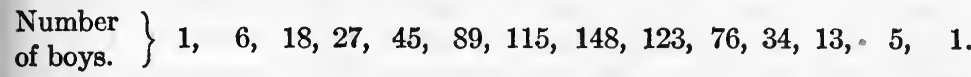

NoтE. In constructing the graphs of some functions it may be desirable to choose different units of length on the $x$ - and $y$-axes.

7. The following tables give the monthly receipts of eggs in the Chicago market in 1910, the price per dozen, and the storage of eggs by months in percentages of the total annual storage by a Chicago firm.

Month. J. F. M. A. M. J. J. A. S. O. N. D.

Eggs in $\left.\begin{array}{c}\text { thousand } \\ \text { cases. }\end{array}\right\} 72,140,160,760,500,400,300,240,180,120,80,48$.

$\left.\begin{array}{l}\begin{array}{l}\text { Storage } \\ \text { of eggs } \\ \text { in per- } \\ \text { centages. }\end{array} \\ \text { Prise }\end{array}\right\} 1.5, .8,9.5,42,19,22,1,0,1, .3, .3,2.6$. $\left.\begin{array}{c}\text { Price per } \\ \text { Pozen, cents. }\end{array}\right\} 7,27,23,21,20,18,17,20,23,26,30,33$.

Let time be the independent variable in each instance, and plot the three graphs on the same set of axes. Let a convenient length from the origin on the $y$-axis represent the three values, 800 thousand cases, $100 \%$, and 40 cents a dozen, then rnark off the subdivisions 50 thousand cases, $5 \%$, and 5 cents a dozen, and multiples of these subdivisions.

State a relation that exists between each two of the three graphs. 
What inferences can be drawn from the graphs with respect to the effect of storing eggs on the price?

8. The following are the monthly statistics for butter received, stored, and the price in the Chicago market in $\mathbf{1 9 1 0 .}$

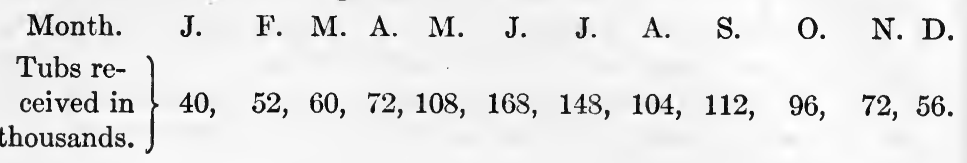

$\left.\begin{array}{l}\text { Storage in } \\ \text { percentage. }\end{array}\right\} \quad 6,3.6,2.8,3.4,14,43,10,2,4.2,2.5,4.5,4$. $\left.\begin{array}{c}\text { Price per } \\ \text { lb. in cents. }\end{array}\right\} 34,28,31,31,26,26,26,28,27,29, \quad 30,29$.

Plot the three graphs on the same set of axes, as in problem 7. State a relation that exists between each two of the three functions. What inferences can be drawn from the graphs regarding the effect of storage of butter on the price?

Note. When a law is stated in such general terms that numerical data representing a concrete situation cannot be derived, a graph which will picture the general situation can be obtained by constructing a table of values with purely arbitrary sets of numbers which conform to the law. Such a graph will indicate the mode of change of the function with respect to the independent variable without representing a concrete situation.

9. The Weber-Fechner law of psychology states that as the intensity of an external stimulus increases in geometric progression, the corresponding sensation increases in arithmetic progression. Construct the graph.

Let $x$ represent the external stimuli, and $y$ the corresponding sensations. Let the geometric progression $1,2,4,8, \ldots$ be the values assumed for $x$, and the arithmetic progression $1,2,3,4, \ldots$ be the values assumed for $y$.

Plot the points whose coördinates are $(1,1),(2,2),(4,3)$, etc., and draw the graph.

At what value of $x$ should the graph begin? Can there be an external stimulus without a corresponding sensation?

The law is said to hold for the senses of touch, hearing and sceing, but not for taste and smell.

10. Water pressure dies away uniformly because of the resistance of the conduits. Construct a graph to show the change in water pressure for points at different distances from a rescrvoir situated on a hill.

11. Malthus' law states that population increases in a geometric progression with reference to time, while subsistence increases in arithmetic progression. Plot and discuss the graphs with reference to the influence these two relations have on one another. The law of diminishing returns states that after a certain point, doubling the cultivation in agriculture will not double the returns. How will this affect the preceding graphs? 
Nоте. In the following problems the functional relation changes in character at two points, and the graph of the function consists of several distinct parts.

12. The amount of heat required to raise through one degree the temperature of one zram of ice is $\frac{1}{2}$ a calorie, of one gram of water is one calorie, of one gram of steam is $\frac{1}{2}$ a calorie, approximately. 80 calories of heat are absorbed without any rise of temperature when the ice is melting, and 537 calories without rise of temperature at the boiling point when the water is vaporizing. If the quantity of heat absorbed is regarded as a function of the temperature $x$, construct a graph representing roughly the change from ice at $10^{\circ}$ below freezing to steam at $5^{\circ}$ above boiling. (Centigrade scale.)

13. In the case of mercury the amount required to raise one gram $1^{\circ}$ in any of the three forms is approximately .033 calorie, the fusing point is $-38^{\circ}$, the heat absorbed in fusing 8.8 calories, the boiling point $675^{\circ}$, and the heat absorbed in vaporization 67.7. Construct a graph for mercury analogous to that for water.

14. A man walks away from his home at the rate of 4 miles an hour for three hours, and then returns at the rate of 2 miles an hour. Construct a graph showing his distance from home at any time.

15. A man rides away from a town at the rate of 6 miles an hour for 2 hours. He then stops for one hour, and walks back at the rate of 3 miles an hour. Construct a graph showing his distance from town at any time.

16. Construct on the same axes the graphs of the functions of $x$ which give the perimeter and area of a square whose side is $x$. Determine from the graphs for what values of $x$ the perimeter is (1) less than the area, (2) equal to the area, (3) greater than the area.

17. Construct on the same axes the graphs of the functions which express the circumference and area of a circle in terms of the radius. Determine from the graph for what values of $r$ the circumference is (1) less than the area, (2) equal to the area, (3) greater than the area.

\section{Discussion of the Table of Values. The considerations} in this section and the section following enable us, in many cases, to abridge the labor of building a table of values, to overcome special difficulties, and to discover properties of the graph.

Example 1. Construct a table of values and the graph of

$$
f(x)=\frac{1}{2} x^{2}-4 .
$$

Symmetry. We shall first see that the table of values need be computed only for positive values of $x$. 
Substituting $-x$ for $x$, we have

$$
f(-x)=\frac{1}{2}(-x)^{2}-4=\frac{1}{2} x^{2}-4=f(x) .
$$

Hence the function has the same value for any two values of $x$ which are equal numerically, but differ in sign, and therefore if $(x, y)$ is a point on the graph, so also is $(-x, y)$. These points are symmetrical with respect to the $y$-axis, and hence the graph is also, in accordance with the

Definition. A curve is said to be symmetrical with respect to a line (or point) if its points by pairs are symmetrical with respect to that line (or point). The line (or point) is called an axis (or center) of symmetry.

Then if the part of the curve to the right of the $y$-axis is plotted, the part on the left may be plotted by means of the symmetry, and hence only positive values of $x$ are needed in the table. Now set

Solving for $x$,

$$
y=f(x)=\frac{1}{2} x^{2}-4
$$

$$
x= \pm \sqrt{(2 y+8)}
$$

Values Excluded. We have agreed to neglect imaginary values of $x$ and $y$. If we substitute any real value of $x$ in (1), we obtain a real value for $y$, and hence no values of $x$ need be excluded. But from (2), we see that all values of $y<-4$, for example $y=-5$, make $x$ imaginary. Hence if a table of values be constructed from (2) by assuming values of $y$, all values of $y$ less than -4 must be excluded.

Graphically, since no values of $x$ are to be excluded, the curve runs off indefinitely to the right and left. Since no positive values of $y$ are excluded the graph runs up indefinitely, but as values less than -4 are excluded, no part of the curve lies more than 4 units below the $x$-axis.

Intercepts. The coördinates of the points in which a graph cuts the axes are usually of special significance, and they should be included in the table of values.

For points on the $x$-axis, $y=0$, and hence the abscissas of the points where the graph cuts the $x$-axis are obtained by set- 
ting $y=0$ in (2), which gives $x= \pm \sqrt{8}= \pm 2.8$. These abscissas are called the intercepts on the $x$-axis in accordance with the

Definition. The intercepts of a curve on the $x$-axis are the abscissas of the points where the graph cuts the $x$-axis, and the intercepts on the $y$-axis are the ordinates of the points of intersection with the $y$-axis.

Since $x=0$ for all points on the $y$-axis, the intercepts on the $y$-axis are found by setting $x=0$ in (1), which gives $y=-4$.

Definition. A zero of a function is a value of $x$ for which the function is equal to zero.

Hence the zeros of $f(x)$ are identical with the roots of the equation $f(x)=0$. All the zeros of a function which are real numbers are represented by the intercepts of the graph on the $x$-axis.

We now build the accompanying table and plot the points $A, B, C, D, E, F$, from it. Then construct $B^{\prime}$ symmetrical to $B$ with respect to the $y$-axis. This is done readily on crosssection paper by counting the squares from $B$ to the $y$-axis and proceeding an equal number of squares beyond. Similarly, construct $C^{\prime}, D^{\prime}, E^{\prime}, F^{\prime}$ symmetrical to $C, D, E, F$ respectively. Then draw the graph.

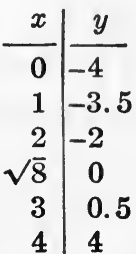

$4 \quad 4$

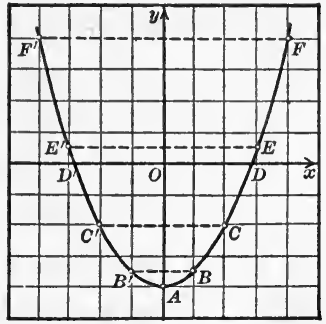

FIg. 17.

The reasoning employed in the illustration of symmetry is general, and may be used with any function or equation. Hence the theorems:

Theorem 1A. The graph of $f(x)$ is symmetrical with respect to the $y$-axis if

$$
f(-x)=f(x) \text {. }
$$

Theorem $1 B$. The graph of an equation is symmetrical with respect to the $y$-axis if the equation obtained by replacing $x$ by $-x$, and simplifying, is identical with the given equation. 
The following theorems, whose proofs are left as exercises, follow from the facts that the points $(-x,-y)$ and $(x,-y)$ are symmetrical to the point $(x, y)$ with respect to the origin and the $x$-axis respectively.

Theorem 2A. The graph of $f(x)$ is symmetrical with respect to the origin if

$$
f(-x)=-f(x)
$$

Theorem 3A. The graph of $f(x)$ is symmetrical with respect to the $x$-axis if its values occur in pairs which are numerically equal but opposite in sign.

Theorem 2B. The graph of an equation is symmetrical with respect to the origin if the equation obtained by replacing $x$ by $-x$ and $y$ by $-y$, and simplifying, is identical with the given equation.

Theorem 3B. The graph of an equation is symmetrical with respect to the $x$-axis if the equation obtained by replacing $y$ by $-y$, and simplifying, is identical with the given equation.

We shall use the phrase to discuss the table of values of a function to mean that the

\section{Symmetry, \\ Values to be excluded, \\ Ir.tercepts, and \\ Asymptotes (see next section)}

are to be determined before building the table of values. For the last three considerations, solve the equation for $y$ in terms of $x$ and for $x$ in terms of $y$. But if the intercepts are desired independently, they may be found by setting either variable equal to zero and solving for the other.

\section{EXERCISES}

1. Does $f(-x)$ always equal either $\pm f(x)$ ?

2. Discuss the table of values (omitting asymptotes) and plot the graph of each of the functions and equations.
(a) $x^{2}-2$.
(b) $x^{3} / 9$.
(c) $3-x^{2}$.
(d) $y=x^{4}$.
(e) $y^{2}-4 x+2=0$.
(f) $y=x^{3}+2$.
(g) $x^{3}-9 x$.
(h) $y^{2}+6 x=0$.
(i) $y^{4}+4 x=0$. 
3. Discuss the table of values (omitting asymptotes) and plot the graph of
(a) $x^{2}+y^{2}=16$.
(b). $x^{2}+y^{2}-6 x=0$.
(c) $x^{2}+y^{2}+4 x=0$.
(d) $4 x^{2}+y^{2}=16$.
(e) $x^{2}-y^{2}=16$.
(f) $x^{2}-y^{2}-6 x=0$.
(g) $4 x^{2}+9 y^{2}=36$.
(h) $9 x^{2}+y^{2}+36 x=0$.
(i) $y=x^{4}-4 x^{2}$.

4. If $f(x)$ is any one of the functions whose graphs are given below, determine whether or not $f(-x)= \pm f(x)$, find the value of $f(0)$, and the values of $x$ for which $f(x)$ is zero, and for which it is imaginary.
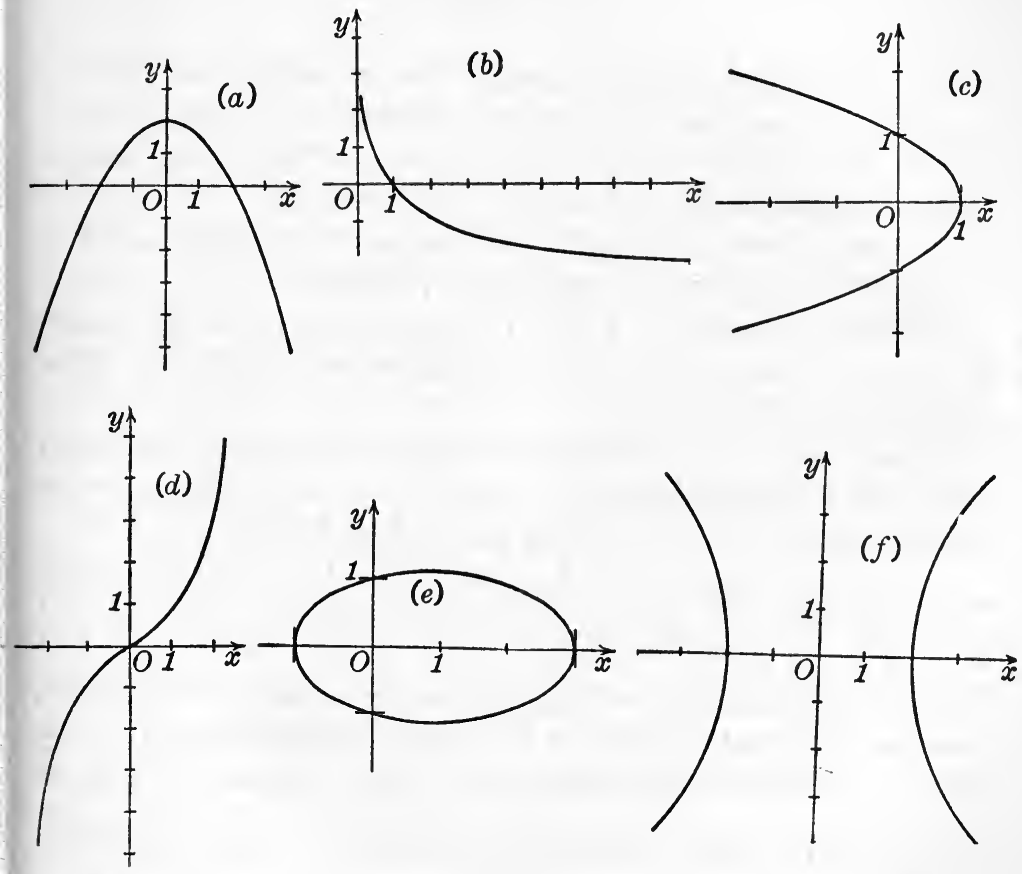

Fig. 18.

11. Functions becoming Infinite. Asymptotes. Continuing the discussion in the preceding section, in the following example we shall need the

Definition. It is said that a function becomes infinite as $x$ approaches $a$ if the numerical value of the function can be made larger than any positive number, however large, by giving $x$ a value sufficiently near to $a$. 
Example. Build a table of values and plot the graph of the function

$$
y=\frac{1}{x-4} \text {. }
$$

Symmetry. The tests for symmetry show that the graph is not symmetrical with respect to either axis or the origin.

Solving (3) for $x$ we get

$$
x=\frac{1+4 y}{y} .
$$

Values excluded. As no radicals occur in (1) and (2), no imaginary values are encountered. Hence no values of $x$ or $y$ need be excluded on this account, and the graph runs indefinitely up and down, and to the right and left.

But the values $x=4$ and $y=0$ must be excluded as it is impossible to divide by zero (see 3 (c), page xv).

Intercepts. Setting $x=0$ in (1), the intercept on the $y$-axis is $y=-\frac{1}{4}$. But as $y=0$ is an excluded value, the curve does not cut the $x$-axis.

Asymptotes. To determine the form of the graph between the points corresponding to $x=3$ and $x=5$, the table includes a number of pairs of values near the excluded value $x=4$.

$\frac{x}{\frac{1}{x-4}} \mid \frac{0,}{\frac{-1}{4},}, \frac{-1}{3}, \frac{-1}{2}, \quad-1,-2,-5, \quad-10, \quad \infty, 10, \quad 5, \quad 2,1, \frac{1}{2}, \frac{1}{3}, \frac{1}{4}$

The numerical value of the function, according to the table, increases as $x$ gets nearer to 4 , and it is readily seen that by giving $x$ values sufficiently near 4 the numerical value of $\frac{1}{x-4}$ can be made larger than any given positive number, however large. Thus to make $\frac{1}{x-4}$ numerically greater than 10 , let $x$ have any value between 3.9 and 4.1 except the value 4 ; to make it greater than 100 , let $x$ have any value between 3.99 and 4.01 except the value 4 ; etc.

Hence the function $\frac{1}{x-4}$ becomes infinite as $x$ approaches 4 . This fact is indicated in the table by the symbols $(4, \infty)$. 
In the figure it is seen that as the graph gets nearer the line perpendicular to the $x$-axis at the point for which $x=4$, it recedes farther and farther from the $x$-axis. Such a line is called an asymptote in accordance with the

Definition. An asymptote of a curve is a straight line such that the distance from a point on the curve to the line approaches zero as the point recedes indefinitely from the origin along the curve.

Similar reasoning establishes the fact that the $x$-axis is also an asymptote, corresponding to the excluded value $y=0$.

The example furnishes an illustration of the general principle:

If a function becomes infinite as $x$ approaches $a$, the line perpendicular to the $x$-axis at $x=a$ is an asymptote of the graph.

We shall consider asymptotes of the graph of an equation or

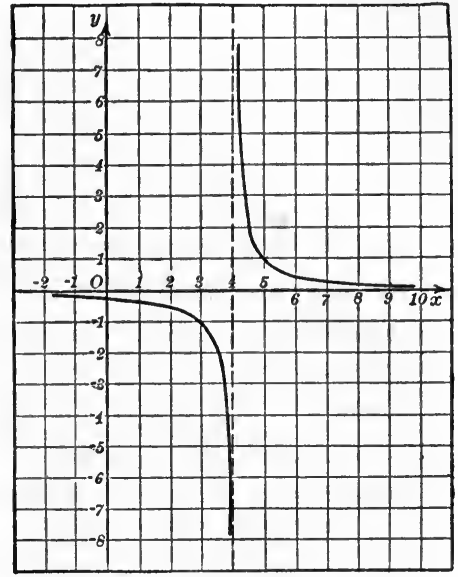

Fig. 19. function only when they are parallel to one of the coördinate axes. Asymptotes parallel to the $y$-axis may be found by solving the equation for $y$; the solution is a function of $x$, and to each value of $x$ for which this function becomes infinite there corresponds a vertical asymptote. Horizontal asymptotes may be found in a similar manner by solving the equation for $x$.

An algebraic function becomes infinite for real, finite values of the variable only if the variable is contained in the denominator, and if the denominator is zero for one or more real values of the variable.

\section{EXERCISES}

1. Discuss the table of values and plot the graph of
(a) $\frac{1}{x}$.
(b) $\frac{1}{x^{2}}$.
(c) $y=\frac{4}{x^{2}}-1$.
(d) $\frac{4}{x-2}$.
(e) $\frac{x^{2}+1}{x}$.
(f) $2 x y-2 x+3 y=0$. 

(g) $2 x y+3 x-5 y=0$.
(h) $x^{2}-2 x y+4=0$.
(i) $x^{2}-4 x y+6=0$.
(j) $\frac{4 x}{x^{2}+1}$.
(k) $\frac{8}{x^{2}+2}$.
(1) $\frac{8}{x^{2}-4}$.
(m) $x^{2}-4 y+2 x=0$.
(n) $x^{2} y+2 y-3 x=0$.
(o) $x^{2} y-4 y-3 x=0$.

2. If a gas is kept at the same temperature, the product of the pressure and the volume is constant. Discuss the table of values and plot the pressure as a function of the volume, assuming a numerical value for the constant.

12. Variation of a Function. Under this heading we consider primarily three things.

First. Sign of the function. If $x$ increases through a given interval, the sign of the function will be positive if, and only if, the graph lies above the $x$-axis; and the sign will be negative if, and only if, the graph lies below the $x$-axis. For the ordinate representing a value of the function lies above or below the $x$-axis according as the function has a positive or negative value.

Second. Changes of the function. If $x$ increases through a given interval, the value of the function increases if, and only if, the graph rises as it runs to the right; and the value decreases if, and only if, the graph falls ass it runs to the right. For the ordinate representing a value of the function increases or decreases according as the curve rises or falls. The motion to the right corresponds to increasing values of $x$.

Third. Average rate of change of the function. This will be considered in the following section.

In order to state in what intervals the function is positive or negative, or is increasing or decreasing, it is necessary to determine the values of $x$ bounding these intervals. These values of $x$, the corresponding values of the function, and the points on the graph representing them, are the remaining important elements of the variation of the function. They are:

First: The real zeros of the function, represented by the intercepts of the graph on the $x$-axis.

Second: The values of $x$ for which the function becomes infinite, which give rise to the vertical asymptotes.

Third: The maximum and minimum values of the function, which we proceed to define. 
Definition. A function $f(x)$ is said to have a maximum value for $x=a$ if $f(a)$ is greater than all other values of $f(x)$ when $x$ is near $x=a$. The point on the graph whose coördinates are $x=a$ and $y=f(a)$ is called a maximum point.

If $x$ increases through $x=a$, the value of $f(x)$ will increase to the value $f(a)$, and then decrease. And the maximum point will be higher than the other points on the graph nearby.

Definition. A function $f(x)$ is said to have a minimum value for $x=a$ if $f(a)$ is less than all other values of $f(x)$ when $x$ is near $x=a$. The point $[a, f(a)]$ on the graph is called a minimum point.

If $x$ increases through $x=a$, the value of $f(x)$ will decrease to the value $f(a)$, and then increase. The minimum point will be lower than other points on the graph nearby.

Example 1. Discuss and plot the graph of the function $2 x^{2}-3 x-9$. Let

$$
y=2 x^{2}-3 x-9
$$

To solve for $x$ in terms of $y$ we first write (1) in the form

$$
2 x^{2}-3 x-9-y=0 \text {. }
$$

Then by the formula for solving a quadratic

$$
x=\frac{3 \pm \sqrt{9+72+8 y}}{4}=\frac{3}{4} \pm \frac{\sqrt{81+8 y}}{4} .
$$

Symmetry. The tests for symmetry show that the graph is not symmetrical with respect to either axis or the origin.

Values excluded. From (1), no values of $x$ need be excluded, so that the graph extends indefinitely to the right and left.

Equation (2) shows that we must exclude all values of $y$ for which $81+8 y<0$. Hence we must exclude $y<-10 \frac{1}{6}$, so that no part of the curve lies more than $10 \frac{1}{8}$ units below the $x$-axis.

Intercepts. Setting $y=0$ in (2), the intercepts on the $x$-axis, or the zeros of the function, are found to be

$$
x=\frac{3}{4} \pm \frac{9}{4}=-1.5 \text { or } 3 .
$$

Setting $x=0$ in (1), the intercept on the $y$-axis is $y=-9$.

Asymptotes. There are no vertical or horizontal asymptotes.

We now proceed to build a table of values and draw the graph.

The variation of the function is readily discussed in connection with the graph. 
Zeros of the function. These are represented by the intercepts on the $x$-axis, $O A$ and $O B$. The curve crosses the axis at $A$ and $B$.

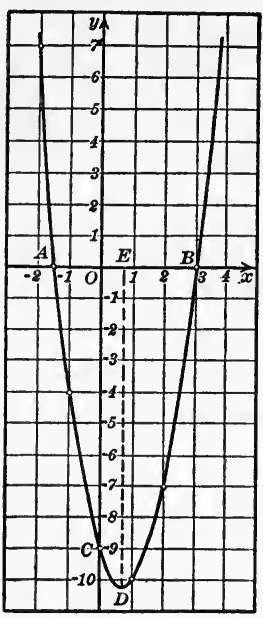

Frg. 20.

Sign of the function. The graph is above the $x$-axis to the left of the point $A$ and to the right of $B$. Hence

$$
2 x^{2}-3 x-9>0 \text { if } x<-1.5 \text {, or } x>3 \text {. }
$$

The graph is below the $x$-axis between $A$ and $B$, and hence

$$
2 x^{2}-3 x-9<0 \text { if }-1.5<x<3 .
$$

The last set of symbols is read " $x$ is greater than - 1.5 and less than 3."

Maximum and minimum values. We saw above that all values of $y$ less than $-10 \frac{1}{8}$ must be excluded. Hence $y=-10 \frac{1}{8}$ is the smallest value of the functions. Substituting this value in (2), the corresponding value of $x$ is seen to be $\frac{3}{4}$. Hence the function has the minimum value $-10 \frac{1}{8}$ when $x=\frac{3}{4}$. This value of the function is represented by the ordinate $E D$, and the point $D\left(\frac{3}{4},-10 \frac{1}{8}\right)$ is a minimum point.

This function does not have a maximum value.

Changes of the function. Since the graph falls to the right at the left of the point $D$,

$$
2 x^{2}-3 x-9 \text { is decreasing as } x \text { increases if } x<\frac{3}{4} \text {. }
$$

And since the curve rises to the right of $D$,

$$
2 x^{2}-3 x-9 \text { is increasing as } x \text { increases if } x>\frac{3}{4} \text {. }
$$

The variation may also be stated as follows, but care must be exercised to see that none of the important elements are omitted.

For a numerically large negative value of $x$ the function is positive. As $x$ increases to $-1.5, f(x)$ decreases to zero. It then becomes negative, and continues to decrease until it assumes its minimum value of $-10 \frac{1}{8}$ when $x=\frac{3}{4}$. As $x$ increases from $\frac{3}{4}$ to 3 , the function is negative and increases to zero. As $x$ increases beyond $x=3, f(x)$ is positive and increasing.

In this example, the function does not change sign unless $x$ increases through a zero of the function. But a function may 
change sign if $x$ increases through a value for which the function becomes infinite. Thus $\frac{1}{x-4}$, whose graph is given on page 27 , changes sign, from negative to positive, if $x$ increases through the value 4 . The intercepts on the $x$-axis and the vertical asymptotes should therefore be determined before considering the sign of a function.

In the example just cited, the vertical asymptote does not separate intervals in which the function increases or decreases, but it may do so. For example, the function $1 / x^{2}$ becomes infinite if $x$ approaches zero, and hence the $y$-axis is a vertical asymptote. As $x$ increases, this function increases if $x$ is negative, and decreases if $x$ is positive. This function also shows that a vertical asymptote need not separate intervals in which the function has opposite signs, for $1 / x^{2}$ is positive for all real values of $x$.

A good order for considering the elements of the variation of a function is as follows:

Zeros of the function,

Function becomes infinite,

Sign of the function,

Maxima and minima,

Changes of the function.

As the zeros and asymptotes are taken up in discussing the table of values, only the last three are new ideas.

These properties of a function may be determined approximately by inspection of the graph of the function. This procedure is especially useful if the table of values or the graph be given and the functional relation itself is unknown. The accuracy of the results will depend upon the choice of units on the axes, the care with which the graph is drawn, and the closeness with which it is read. 
EXAMPLE 2. A thermograph is an instrument which records the temperature continuously by means of such a curve as in the figure. Discuss the variation of the temperature as a function of the time.

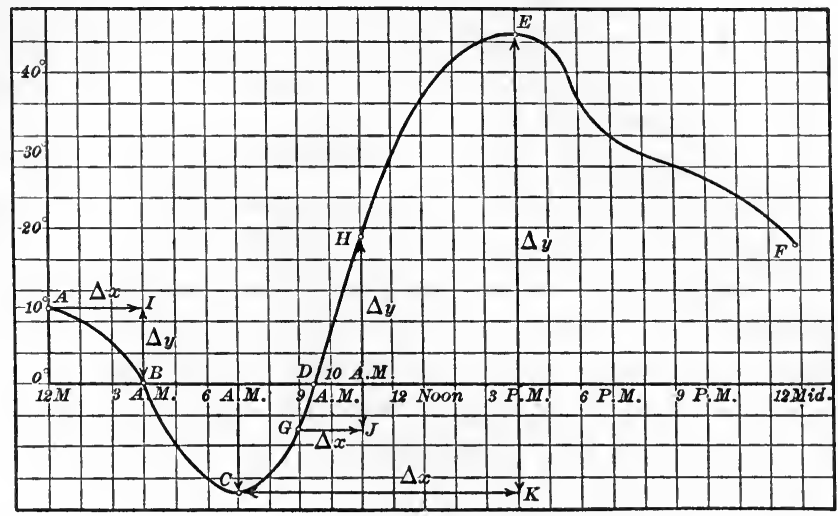

FIg. 21.

Zeros of the function. The graph cuts the time axis at $B$ and $D$, hence the temperature was zero at 3 A.M. and at 8:30 A.M.

Sign of the function. The graph is above the time axis from $A$ to $B$ and from $D$ to $F$ and below from $B$ to $D$. Hence the temperature was above zero from $12 \mathrm{Mid}$. to $3 \mathrm{~A} . \mathrm{M}$. and from 8.30 A.M. to $12 \mathrm{Mid}$. the next night, and below zero from 3 A.M. to 8:30 A.M.

Maximum and minimum values. At $C$ the ordinates cease to decrease and begin to increase. Hence, the temperature had a minimum value of about $-14^{\circ}$ at 6 A.M. At $E$ the ordinates cease to increase and begin to decrease. Hence, the temperature had a maximum value of about $+45^{\circ}$ at 3 P.M.

Changes of the function. The graph rises from $C$ to $E$ and falls from $A$ to $C$ and from $E$ to $F$. Hence, the temperature increased from 6 A.M. to 3 P.M. and decreased from 12 Mid. to 6 A.M. and from 3 P.M. to 12 Mid.

If it is not desired to treat each topic separately, the results might be stated as follows:

The temperature at midnight was $10^{\circ}$. It decreased until it became zero at 3 A.M. and continued to decrease until 6 A.M., when it reached a minimum value of about $-14^{\circ}$. It then increased, becoming zero at $8: 30$ A.M., until 3 P.M., when its maximum value was about $45^{\circ}$. From that time on it decreased to about $20^{\circ}$ at midnight.

The discussion of this function is continued in the next section. 


\section{EXERCISES}

1. Discuss the table of values, plot the graph, and determine the variation of each of the functions:
(a) $y=x / 2$.
(b) $x^{2}$.
(c) $2-x^{2}$.
(d) $y=16 x-x^{2}$.
(e) $x y+6 y=4$.
(f) $x^{2}-4 y+8=0$.
(g) $x^{2}-2 y+4 x=0$.
(h) $2 x y-x-3 y=0$.
(i) $x^{2}-2 x y+1=0$.
(j) $x^{2}-5 x-4 y=0$.
(k) $x^{2}-4 x y+2=0$.
(l) $x^{2}-x y+3=0$.
(m) $x y=6$.
(n) $x^{2} y=12$.
(o) $x+y^{3}=0$.

2. The surplus and shortage of railroad cars in thousands for the months of the years 1915 and 1916 were as follows. Plot the graph and discuss the function.

1915. $180,279, \quad 321,327,291,299,275,265, \quad 185, \quad 78, \quad 26, \quad 38$. 1916. $46,21,-20,3,33,57,52,9,-19,-60,-114,-107$.

3. The following are the data for the surplus reserves of New York banks in millions for the months of the years indicated. Plot the graph and discuss the function. The Federal Reserve System was inaugurated November 16, 1914.

1914. $29,32,20,17,38,40,15,-29,-28,-0.4, \quad 72,117$. 1915. 121, 135, 131, 156, 168, 185, 159, 177, 197, 179, 168, 155.

4. One side of a rectangle whose perimeter is 12 inches is $x$. Find the area as a function of $x$. Construct the graph of the function, discussing the table of values, and find the value of $x$ if the area is a maximum. What is the maximum area?

5. A farmer wishes to fence off a poultry yard whose area is to be 6 square rods. If one dimension is $x$, express the perimeter (the amount of fencing needed) as a function of $x$. Discuss the table of values and plot the graph of the function. What will the dimensions be to require the least amount of fencing? How much should he purchase?

6. There are a number of diseases with continued fever in which the course of the temperature is sufficiently characteristic to furnish the diagnosis. Croupous pneumonia is one of these. (See Fig. 22.)

Discuss the three functions. The zero for temperature would be the normal temperature $98^{\circ} .4$. By comparison of the three functions state in words some of the symptoms of the disease.

7. The data for the temperature curves of measles and scarlet fever 
are given in the following tables. Compare the graphs with the following graph.

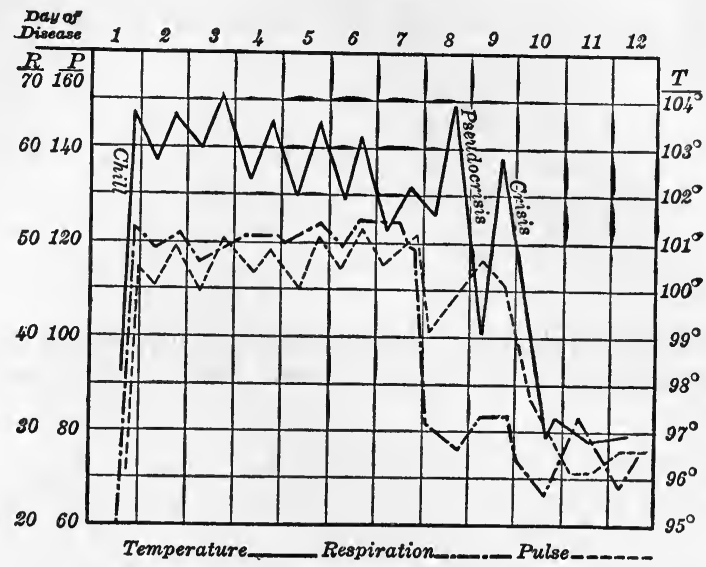

Temperature, pulse, and respiration tables in croupous pneumonia. FIG. 22.

$\begin{array}{lllllllll}\text { Day of fever. } & 1 & 2 & 3 & 4 & 5 & 6 & 7 & 8\end{array}$

T. (Measles) A.M. $\quad 99^{\circ} .4101^{\circ} .2101^{\circ} .2 \quad 101^{\circ} .8103^{\circ} .4102^{\circ} .899^{\circ} \quad 98^{\circ} .4$ P.M. $103^{\circ} .8101^{\circ} .8102^{\circ} .8104^{\circ} .8105^{\circ} .8103^{\circ} .499^{\circ} .8$ normal

T. (Scarlet

fever)

A.M. $\left\{\begin{array}{r}98^{\circ} .4105^{\circ} .2 \\ 103^{\circ} .8104^{\circ}\end{array}\right.$

P.M. $\quad 103^{\circ} .4 \quad 105^{\circ} .8105^{\circ} .2104^{\circ} .4103^{\circ} .4 \quad 103^{\circ} .2 \quad 101^{\circ} .4 \quad 99^{\circ} .4$

Discuss these functions. How do the temperature curves distinguish pneumonia, measles, and scarlet fever, one from another?

13. Average Rate of Change of a Function. The average rate of change of temperature during a given period of time is a familiar idea. For instance, in Example 2 of the preceding section, the temperature rose from $-14^{\circ}$ to $45^{\circ}$ between 6 A.M. and 3 P.M., a total rise of $59^{\circ}$ in 9 hours. Dividing 59 by 9 , we see that the average rate of change in this interval of time was about 6.5 degrees per hour.

On the graph, $C K$ represents the change in time from 6 A.M. to 3 P.M., and $K E$ the corresponding change in temperature. Hence the average rate of change of temperature in this interval, 6.5 degrees per hour, is represented by the ratio $K E / C K$. The graph has its most abrupt rise from about the point $G$ to 
the point $H$, which indicates that the average rate of change of temperature was greatest from about 8 A.M. to 10 A.M. This average rate of change is represented by the ratio $J H / G J$.

Let $y$ denote the temperature at any time $x$, and $\Delta y$ the change in temperature during an interval of time $\Delta x$. Since the average rate of change is the ratio of the change of temperature to the corresponding change in time it

\begin{tabular}{|c|c|c|c|c|}
\hline & $x$ & $y$ & $\Delta y$ & $\Delta u \Delta$ \\
\hline & 12 Mid. & 10 & -10 & -3.3 \\
\hline & 3 A.I & 0 & & \\
\hline & 3 P.M. & $\begin{array}{l}-14 \\
+45\end{array}$ & +59 & +6.5 \\
\hline & $\begin{array}{r}8 \text { A.M. } \\
10 \text { A.M. }\end{array}$ & $\begin{array}{r}-6 \\
+19\end{array}$ & +25 & +12.5 \\
\hline
\end{tabular}
is expressed by $\Delta y / \Delta x$. The computation of the average rate of change for several different intervals may be effected conveniently in tabular form, the values of $y$ for the given values of $x$ being obtained from the graph.

The idea of the average rate of change of a function of the time has been extended to include a function of any variable. This generalization is given in the

Definition. The average rate of change of a function of $x$, for a particular change in $x$, is the ratio of the corresponding change in the function to the change in $x$.

If $y$ denotes the function, and $\Delta y$ the change in $y$ due to a change of $\Delta x$ in $x$, then the average rate of change of $y$ with respect to $x$ is symbolized by $\Delta y / \Delta x$.

If $x$ increases from one given value to another, the average rate of change of $y$ may be found as in the illustration above, except that the values of $y$ would be computed from the given function instead of being read from the graph. The method of finding a general expression for the average rate of change for any interval is illustrated in the

Example. Find the average rate of change of the function $y=\frac{1}{4} x^{2}$ for the intervals of $x$ from 1 to 3 , from 2 to 4 , and from 2 to 6 . Find also the average rate of change for any interval.

The details of the computation for the three given intervals are given in the table on page 36 .

To find the average rate of change of the function for any interval, we start with any pair of corresponding values $x$ and $y$, and let $\Delta y$ be the change n $y$ produced by a change of $\Delta x$ in $x$. Then $x+\Delta x$ and $y+\Delta y$ are cor- 
responding values of the independent variable and the function, an ' hence these values satisfy the given equation.

\begin{tabular}{l|l|l|l|l}
$\Delta x$ & $x$ & $y$ & $\Delta y$ & $\Delta y / \Delta x$ \\
\hline 2 & 1 & $\frac{1}{4}$ & 2 & 1 \\
\hline 2 & 3 & $2 \frac{1}{4}$ & & 1 \\
\hline 4 & 4 & 4 & 3 & $1 \frac{1}{2}$ \\
\hline 2 & 1 & 8 & 2
\end{tabular}
Therefore $y+\Delta y=\frac{1}{4}(x+\Delta x)^{2}$, or $\quad \begin{aligned} y+\Delta y & =\frac{1}{4} x^{2}+\frac{1}{2} x \Delta x+\frac{1}{4} \Delta x^{2} \text {, } \\ \text { But } \quad y \quad & =\frac{1}{4} x^{2} .\end{aligned}$ Subtracting, $\Delta y=\frac{1}{2} x \Delta x+\frac{1}{4} \Delta x^{2}$. This gives the change in $y$ due to a chang of $\Delta x$ in $x$. Dividing by $\Delta x$, the requirec average rate of change for any interval $\Delta x$ is

$$
\frac{\Delta y}{\Delta x}=\frac{1}{2} x+\frac{1}{4} \Delta x \text {. }
$$

The average rate for any particular interval may be obtained from thi result by substitution. For example:

For the first given interval, from 1 to $3, x$ starts with the value $x=1$ and increases by $\Delta x=2$. Substituting these values in (1), we get

$$
\frac{\Delta y}{\Delta x}=\frac{1}{2} \times 1+\frac{1}{4} \times 2=1 .
$$

For the interval from 2 to $4, x=2$, and $\Delta x=2$. Hence

$$
\frac{\Delta y}{\Delta x}=\frac{1}{2} \times 2+\frac{1}{4} \times 2=1 \frac{1}{2} \text {. }
$$

And for the interval from 2 to $6, x=2$, and $\Delta x=4$, so that

$$
\frac{\Delta y}{\Delta x}=\frac{1}{2} \times 2+\frac{1}{4} \times 4=2 \text {. }
$$

As these results agree with those obtained above, we have a desirable check on the correctness of the entire procedure.

The graphical representation of the average rate of change is important The corresponding pairs of values $(x, y)$ and $(x+\Delta x, y+\Delta y)$ are repre sented by two points $P$ and $Q$ on the graph, so that

$$
x=O M, y=M P, x+\Delta x=O N, y+\Delta y=N Q .
$$

Hence $\Delta x=M N=P R$, and $\Delta y=R Q$.

Then $\Delta y / \Delta x$ is represented by the ratio $R Q / P R$. That is, the average rate of change of a function is represented graphically by the ratio of the difference of the ordinates of two points on the graph to the difference of their abscissas.

This interpretation holds for any two points $P$ and $Q$ on the curve. For no matter what the relative positions of the points may be, we have, by the definition on page 13,

\begin{tabular}{r|l}
$x$ & $y$ \\
\hline-2 & 1 \\
-1 & $\frac{1}{2}$ \\
0 & 0 \\
1 & $\frac{1}{2}$ \\
2 & 1 \\
3 & $2 \frac{1}{6}$ \\
4 & 4 \\
5 & $6 \frac{1}{6}$ \\
6 & 9
\end{tabular}

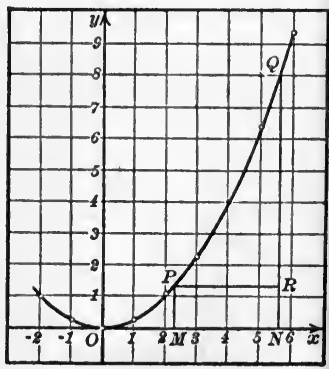

Fra. 23.

$$
O M+M N=O N \quad \text { and } \quad N R+R Q=N Q .
$$




\section{EXERCISES}

1. Find the average rate of change of each of the functions below if $x$ changes from 0 to 1 ; from 1 to 3 ; from 1 to 4 . What can be said of these average rates? Find the average rate for any interval, draw the graph, and interpret the average rate graphically
(a) $2 x-3$.
(b) $2 x$.
(c) $-x+3$
(d) $\frac{1}{2} x+2$.
(e) $-\frac{1}{3} x+3$.
(f) $-2 x+5$.

2. Find the average rate of change of each of the following functions for the intervals from 1 to 3 ; from 1 to -1 ; from 0 to 3 ; for any interval. Check the results. Plot the graph and interpret the average rates.
(a) $-x^{2}+16$.
(b) $x^{2}-2 x$.
(c) $x^{2}-2 x+1$.
(d) $x^{2}+2$.
(e) $x^{2}-5 x+6$.
(f) $x^{3}$.

3. For each of the following functions, discuss the table of values, plot the graph, and determine the variation, including the average rate of change for any interval.
(a) $4-x^{2}$.
(b) $x^{2}-4 x$.
(c) $4 x-x^{3}$.
(d) $x^{2}-4 x+4$.
(e) $x^{2}-3 x+2$.
(f) $x^{3}-3 x^{2}$.

4. Discuss the function represented by the following temperature graph. Find the greatest and the least average rate for the three-hour intervals indicated on the time axis.

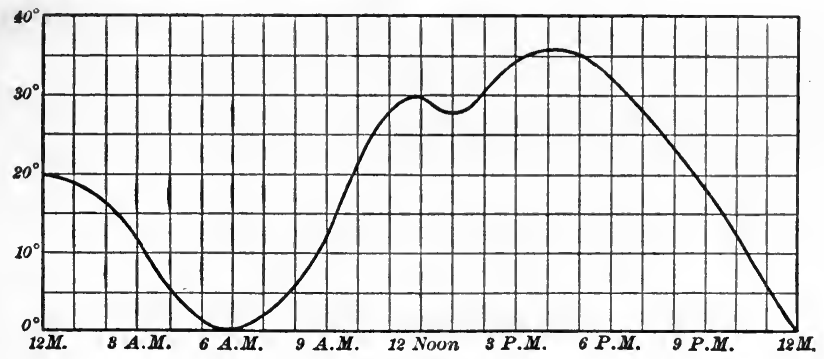

Fig. 24.

5. The daily variation of temperature for a person in normal condition is given in the following table. Plot the graph and discuss the function. For what hour of the day is the average rate the greatest? The least?

Hour 6 A.M.

8

9

10

11

12 м. 1 P.M.

Temperature

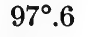

$98^{\circ} .1$

$98^{\circ} .4$

$98^{\circ} .7 \quad 99^{\circ} \quad 98^{\circ} .9$

$98^{\circ} .8 \quad 98^{\circ} .9$

$\begin{array}{cccccccc}2 & 3 & 4 & 5 & 6 & 7 & 8 & 9 \\ 99^{\circ} & 99^{\circ} .1 & 99^{\circ} .2 & 99^{\circ} .3 & 99^{\circ} .2 & 99^{\circ} & 98^{\circ} .8 & 98^{\circ} .7 \\ 10 & 11 & 12 & 1 \text { A.M. } & 2 & & & \\ 98^{\circ} .4 & 98^{\circ} .2 & 98^{\circ} & 97^{\circ} .6 & 97^{\circ} .6 . & & & \end{array}$

The temperature from 2 A.M. to 6 A.M. is stationary. 
14. Classification of Functions. In discussing functions it is convenient to separate them into classes according to the properties they possess or the character of the operations that are involved in calculating them.

The following scheme indicates the important divisions and subdivisions of the functions which we shall study in this course.

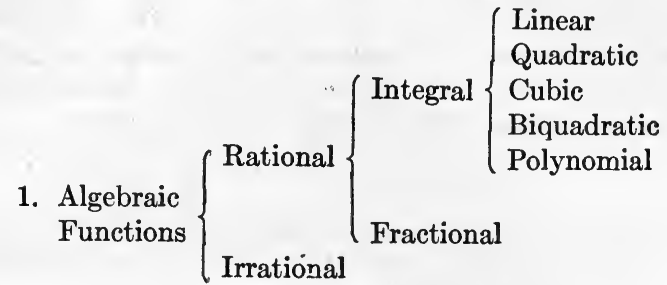
2. Transcen- $\begin{gathered}\text { dental } \\ \text { Functions }\end{gathered}\left\{\begin{array}{l}\text { Elementary }\left\{\begin{array}{l}\text { Exponential } \\ \text { Logarithmic } \\ \text { Trigonometric } \\ \text { Inverse Trigonometric }\end{array}\right. \\ \begin{array}{l}\text { Other transcendental functions studied in higher mathe- } \\ \text { matics. }\end{array}\end{array}\right.$

An algebraic function * is a function whose value may be computed when that of $x$ is given by the application, a finite number of times, of the operations of algebra, namely, addition, subtraction, multiplication, division, involution. and evolution.

The following are examples of algebraic functions:

$$
2 x+3, a x^{2}+b x+c, \sqrt{x^{2}-9}, \frac{2 x+5}{x-3} .
$$

In the work of this course only real numbers will be used. Hence, for us, such an algebraic function as $\sqrt{16-x^{2}}$ is defined only for values of $x$ such that $-4 \leqq x \leqq+4$ (read " $x$ is greater

* This definition is sufficiently general for the purposes of this course. The definition used in higher mathematics, which is more inclusive is as follows: Given a polynomial in $y$,

$$
f(y)=a_{0} y^{n}+a_{1} y^{n-1}+a_{2} y^{n-2}+\ldots+a_{n-1} y+a_{n},
$$

whose coefficients $a_{0}, a_{1}, a_{2}, \ldots a_{n-1}, a_{n}$ are polynomials in $x$, tnen any solution of the equation $f(y)=0$ for $y$ in terms of $x$ is called an algebraic function. 
than or equal to -4 , and less than or equal to +4 "), since for all other values, as $x=5$, the value of the function is imaginary.

An integral rational function (integral function or polynomial) is a function whose value, for a given value of $x$, may be found by the operations of addition, subtraction, and multiplication applied a finite number of times. The following is the general form:

$$
y=a x^{n}+b x^{n-1}+\ldots+k x+l
$$

Polynomials are classified according to the degree of the highest power of $x$ occurring.

$\begin{array}{ll}\text { Linear function } & a x+b . \\ \text { Quadratic function } & a x^{2}+b x+c . \\ \text { Cubic function } & a x^{3}+b x^{2}+c x+d . \\ \text { Biquadratic function } & a x^{4}+b x^{3}+c x^{2}+d x+e .\end{array}$

A rational function is a function whose value, for a given value of $x$, may be found by the four rational operations of addition, subtraction, multiplication, and division, applied a finite number of times. If division involving $x$ in the divisor occurs in the computation, the function may be expressed as the quotient of two polynomials, and it is then called a fractional function. The general form is

$$
y=\frac{a_{0} x^{n}+a_{1} x^{n-1}+\ldots+a_{n-1} x+a_{n}}{b_{0} x^{m}+b_{1} x^{m-1}+\ldots+b_{m-1} x+b_{m}}
$$

An irrational function is a function which involves tne extraction of roots in addition to the four rational operations.

In contrast with algebraic functions, all other functions are called transcendental. This term is merely a synonym for non-algebraic.

Inverse functions. If $y$ be given as a function of $x$ by the relation

$$
y=x^{2}-1,
$$

$x$ is also a function of $y$ obtained by solving this equation for $x$, namely,

$$
x= \pm \sqrt{y+1} .
$$


The two functions $x^{2}-1$ and $\pm \sqrt{y+1}$ are called inverse functions.

The independent variable in the first is, as usual, $x$, but in the second it is $y$. In order to write the inverse function with $x$ as the independent variable we must replace $y$ by $x$. Hence the inverse of $x^{2}-1$ is $\pm \sqrt{x+1}$.

Definition. If $y$ be set equal to $f(x)$, the equation solved for $x$ in terms of $y$, and $y$ replaced by $x$ in the final result, then the function of $x$ so obtained is called the inverse of $f(x)$.

The same result is obtained by interchanging $x$ and $y$ in the equation $y=f(x)$, and then solving for $y$ in terms of $x$.

\section{EXERCISES}

1. What kind of a function is
(a) $3 x+\mathbf{2}$ ?
(b) $x^{2}-4 x+1$ ?
(c) $x^{3}-2 x^{2}+7$ ?
(d) $\frac{4 x-2}{x^{2}+3}$ ?
(e) $\sqrt{x^{2}-4}$ ?
(f) $\frac{2+\sqrt{x}}{5 x+3}$ ?

2. Give a numerical example of a
(a) quadratic function.
(b) fractional function.
(c) cubic function.
(d) irrational function.

3. Find the inverse of each of the following functions; classify each function and its inverse.
(a) $x^{3}+3$.
(b) $\sqrt{x^{2}-4}$.
(c) $\sqrt{\frac{x^{2}}{2-x}}$.
(d) $\sqrt{1+x}+\sqrt{1-x}$.

$$
\text { (e) } y=\sqrt[3]{x+\sqrt{x}} \text {. }
$$

4. Find the inverse functions defined by the following equations and classify them:
(a) $x y+2 y-x-3=0$.
(b) $x^{2}+2 x y+3 y^{2}+1=0$.
(c) $x y^{m}=k$.
(d) $x^{\frac{1}{2}}+y^{\frac{1}{2}}=a^{\frac{1}{2}}$.

15. Summary. Suppose that the data of a law of a science can be recorded in a table of values of two variables, or, by some mechanical device, in the form of a curve. The generalization which expresses the law connecting the two variables is a func- 
tional relation (Sections 1-4). If this relation can be expressed in mathematical symbols, then mathematics becomes, to a considerable extent, the language of that science. An important problem, therefore, is the determination of an expression in mathematical symbols for a function which is given otherwise (Section 6; see also 5 and 6 below).

Function-table of values-graph. These are merely different manifestations of the same concept. A functional relation expresses a law connecting each pair of numbers of two sets; the table gives particular pairs of these numbers; and the graph affords a geometric representation of them.

The fundamental problems arising in connection with this concept are the determinations of any two of these manifestations from the third. They are six in number.

1. Given a function, to build the table of values. This is an easy process for simple algebraic functions.

2. Given a table of values, to plot the graph. This is also easily done if the table is sufficiently extensive.

3. Given a function, to draw the graph. This is done by means of 1 and 2 (Section 9).

4. Given a graph, to construct a table of values. Pairs of values may be read, approximately, directly from the graph.

5 and 6 . Given a table of values, to find the function. This is precisely the problem which confronts the scientist in seeking an unknown law, and it is by far the most difficult of these problems. Methods of solution will be considered in the following chapters (see Sections 25, 44, and 90).

In Sections 10-13 we have considered properties of a function, its table, and its graph, which are important in the study of any function. The correspondence between these properties of a function and its graph may be exhibited compactly as follows:

\section{PROPERTY OF GRAPH}

Length of an ordinate.

Graph symmetrical respect $y$-axis. Graph symmetrical respect origin. Vertical lines do not cut graph.

\section{PROPERTY OF FUNCTION}

A value of the function.

$f(-x)=f(x)$.

$f(-x)=-f(x)$.

Excluded values of $x$. Function imaginary or infinite. 
Intercepts on the $x$-axis.

Intercepts on the $y$-axis.

Vertical asymptote.

Horizontal asymptote.

Graph above $x$-axis.

Graph below $x$-axis.

Ordinate of maximum point.

Ordinate of minimum point.

Graph rises to the right.

Graph falls to the right.

Ratio of difference of ordinates

to difference of abscissas.
Zeros of function, i.e., $f(x)=0$.

Values of $f(0)$.

Function becomes infinite.

Value of function as $x$ becomes infinite.

Function is positive, i.e., $f(x)>0$.

Function is negative, i.e., $f(x)<0$.

Maximum value of function.

Minimum value of function.

$f(x)$ increases as $x$ increases.

$f(x)$ decreases as $x$ increases.

Value of $\Delta y / \Delta x$. Average rate of change of function.

Interpretation of a graph. The properties of the graph may be determined by inspection, if only one has in mind what to look for, and the corresponding properties of the function may then be stated. It is true that the properties of the graph are proved by first establishing the properties of the function. But after the graph is drawn, this interpretation of the graph affords a comprehensive point of view of many properties of the function and furnishes a simple means of stating any particular property.

As we come to study any one class of functions defined in Section 14, we shall take up the properties listed above, and in addition the characteristic properties which distinguish that class of functions from others. A typical graph for each class of functions should be fixed in mind, as it enables one to tie together and recall quickly the characteristics of a function as soon as it is classified. This is of great importance in analyzing problems.

From time to time we shall add other properties of graphs and functions to the list above.

We shall also take up the relations between certain pairs of functions and their graphs, which enable us to obtain the graph of one function from that of the other. Thus the graph of $f(x)+k$ may be obtained from that of $f(x)$ (see Exercise 3, page 19). These two sets of relations constitute the framework of the entire course. 
We turn next to the detailed study of particular classes of functions, beginning with the linear function, which occurs frequently in the applications of mathematics.

\section{MISCELLANEOUS EXERCISES}

1. State as many properties as possible of the functions whose graphs are given below.
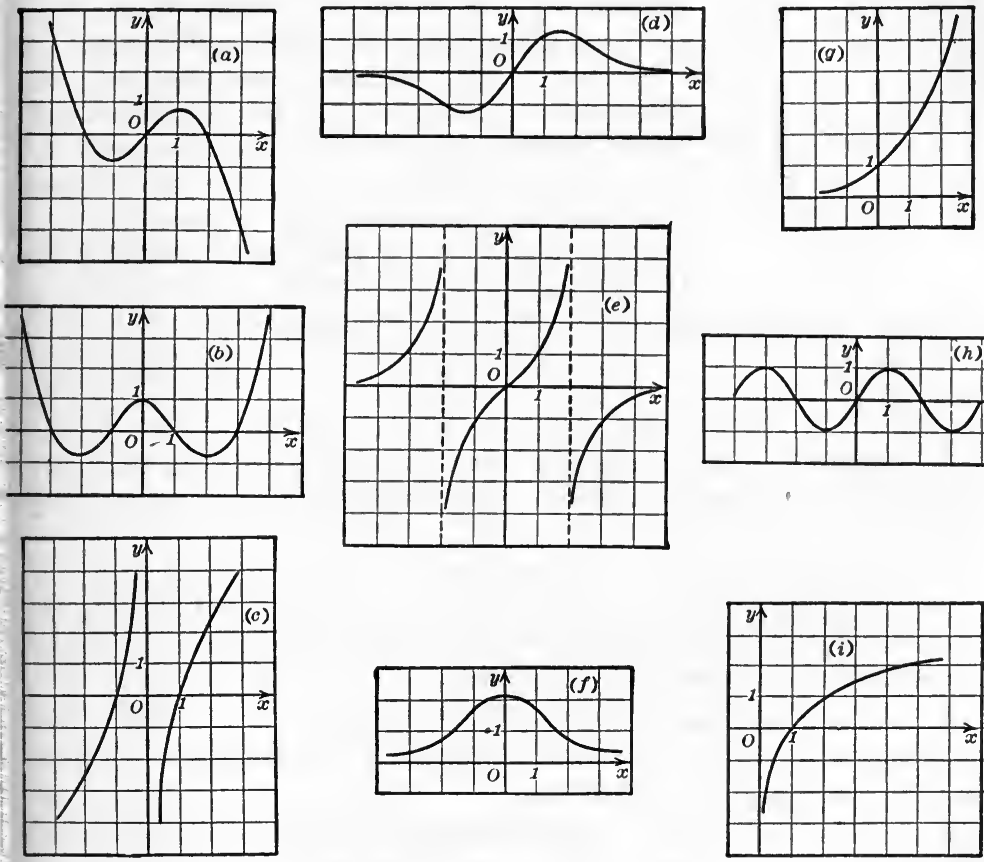

FIG. 25.

2. Discuss the table of values, plot the graph, and determine the variation, including the average rate of change, of:
(a) $\frac{1}{3} x-3$.
(b) $x^{2}-4 y-3=0$.
(c) $x^{3}-16 x+3 y=0$.

3. Discuss the table of values, plot the graph, and determine the variation, omitting the average rate of change, of :
(a) $y^{2}+2 x-9=0$.
(b) $x^{2}+y^{2}-4 x=12$.
(c) $x^{2}+4 y^{2}-4 x=16$.
(d) $x^{2}-2 x y+5=0$.
(e) $x^{2}+4 x y+2=0$.
(f) $3 x y+4 x-2 y=0$. 

(g) $y^{2}=x^{3}$.
(j) $y^{2}(4-x)=x^{2}(4+x)$.
(h) $x^{2} y-4 y-1=0$.
(k) $x^{3}+8 x-16=0$.
(i) $x^{2} y-4 y-x=0$.
(l) $x y^{2}+x-4 y=0$.

4. For a given abscissa, the ordinate of a point on the graph of $y=f(x)+g(x)$ is the sum of the ordinates of the points with the same

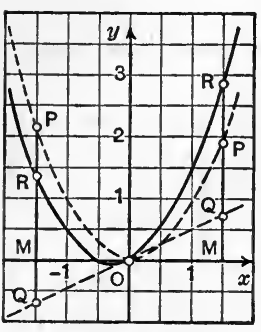

FIG. 26. abscissa on the graphs of $f(x)$ and $g(x)$. Fience the graph of $y$ may be obtained by drawing the graphs of $f(x)$ and $g(x)$ and then adding the ordinates of points with the same abscissa. Using this method, which is called the addition of ordinates, construct the graphs of the following functions.

(a) $y=x^{2}+\frac{1}{2} x$. Solution. Draw the graphs of $x^{2}$ and of $\frac{1}{2} x$. If $M P$ and $M Q$ are the ordinates of points on these graphs with the same abscissa $O M$, then $M R=M P+M Q=M P+P R$ is the ordinate of a point on the graph of the given function.

Several points on the graph may be obtained in this way, and a smooth curve drawn through them. Notice that if $O M$ is negative, so also is $M Q$, so that $M R<M P$.
(b) $x^{2}+x$.
(c) $x^{2} / 3+2 x$.
(e) $x^{3} / 3+x / 3$.
(f) $1 / x+x / 2$.
(d) $x^{2} / 2+x$.
(g) $x^{2}-2 x$.

5. Express the area of a rectangle as a function of one of its sides, assuming that the perimeter is 8 feet. Plot the graph and discuss the variation of the function.

6. A house stands 50 feet from the street. A man is walking along the street. Express his distance from the house as a function of his distance from the entrance to the grounds, which is directly in front of the house. Discuss and plot the graph of the function.

7. A man 6 feet tall walks away from a lamp-post 12 feet high. Express the length of his shadow as a function of his distance from the post, and plot the graph of the function.

8. In Exercise 7, express the distance from the post to the shadow of the man's head as a function of the man's distance from the post. Plot the graph, and show that the average rate of change of the function is constant.

9. A point lies at a distance $r$ from the origin. Find the equation expressing the functional relation between the coördinates $x$ and $y$ of the point.

10. A man walks for 2 hours at the rate of 3 miles an hour, stops an hour and a half for lunch, and walks back at the rate of 2 miles an hour. Construct a graph showing his distance from the starting point at any time. 
Noте. - A solution of two simultaneous equations in $x$ and $y$ consists of a pair of values of these variables which satisfy both equations. If the numbers are real, the point whose coördinates are such a pair of values is on both graphs. Therefore a real solution of two simultaneous equations in $x$ and $y$ is represented by a point of intersection of the graphs. To find the coördinates of the points of intersection of two graphs, solve their equations simultaneously.

11. Plot the graphs of the following equations and find the coördinates of their points of intersection.
(a) $x+2 y-3=0, \quad x+2 y-8=0$.
(b) $2 x+y=0, \quad y^{2}=4 x$.
(c) $x^{2}+y^{2}=25, \quad 3 x+4 y=0$.
(d) $x^{2}+y^{2}=16,2 x-3 y=4$.
(e) $x^{2}+y^{2}=9, \quad y^{2}=4 x$.

12. Plot the graphs of the following equations and from them read approximate values of the solutions of the equations.
(a) $x^{2}+4 y^{2}=16, \quad y^{2}=\frac{4 x}{x-3}$
(b) $x y-y^{2}=6, \quad y^{2}=x^{3}$.
(c) $y=x^{3}-4 x^{2}, 2 x-y+2=0$.
(d) $x^{2}+y=7, \quad y^{2}+x=11$.

13. Two engines in a freight yard are running on parallel tracks. Their distances from a signal tower (in hundred yards) at the time $t$ (in minutes) are given respectively by the equations

$$
s=-\frac{1}{2} t+4 \quad \text { and } \quad s=-\frac{1}{4} t^{2}+4 t+4 .
$$

Plot the graphs, using $t=0,4,8$, etc. Determine when and where the engines are beside each other (two solutions). 


\section{CHAPTER II}

\section{LINEAR FUNCTIONS}

16. Uniform Rate of Change. The position of a body moving on a line, straight or curved, is commonly indicated by its distance $s$ from a given point on the line. The body is said to move uniformly, or at a constant rate, along the line if the ratio of any change in $s$ to the corresponding change in time is constant (that is, if this ratio has the same value for all intervals of time). If $\Delta s$ is the change in $s$ during the time $\Delta t$, then for uniform motion $\Delta s / \Delta t=v$, a constant called the velocity. The value of $v$ gives the change in $s$ during a unit of time.

Since $\Delta s / \Delta t$ is the average rate of change of $s$ during the interval of time $\Delta t$ (see Section 13), the above definition is equivalent to the statement that a body moves uniformly at the rate of $v$ units of distance per unit of time if the average rate of change of $s$ is constant and always equal to $v$.

Example. A farmer lives $\mathbf{1 0}$ miles from town. He starts from home and drives away from town at the uniform rate of 5 miles an hour. Construct and interpret a graph show-

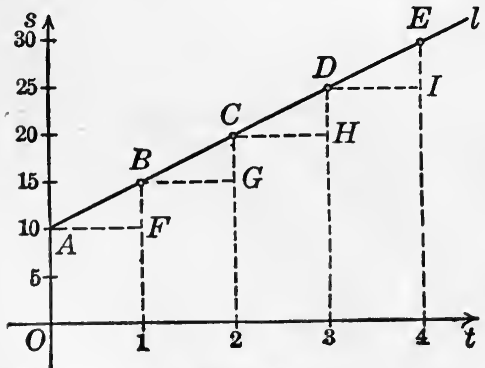

Fia. 27.

whence ing his distance from town at any time.

Let $s$ denote his distance from town $t$ hours after starting. Then $s-10$ denotes the distance he travels in $t$ hours, and hence

$$
\frac{s-10}{t}=5 \text {, }
$$

$$
s=5 t+10 .
$$

In plotting the graph of equation (1) we take values of $t$ as abscissas according to a well-established custom. The pairs of values of $t$ and $s$ in the table are represented by the points $A, B, C, D, E$, which appear to lie on 
a straight line, $l$. We assume that the graph is indeed straight, an assumption which will be justified in Section 20 .

The interpretation of the coördinates of any point on the graph is that the ordinate represents the man's distance from town at the time represented by the abscissa.

The interpretation of the velocity is worthy of detailed consideration. The successive values of $\Delta t$ given in the table are represented by $A F$ = $B G=C H=D I=1$, and those of $\triangle s$ by $F B=G C=H D=I E=5$. Hence $v=\Delta s / \Delta t$ is represented by any one of the ratios

$$
\frac{F B}{A F}=\frac{G C}{B G}=\frac{H D}{C H}=\frac{I E}{D I} .
$$

Now consider any interval of time beginning at $t_{1}$ and ending at $t_{2}$. The distances from town at these times are respectively, from (1),

and

$$
s_{1}=5 t_{1}+10,
$$

$$
s_{2}=5 t_{2}+10 \text {. }
$$

Subtracting,

$$
\Delta s=s_{2}-s_{1}=5\left(t_{2}-t_{1}\right)=5 \Delta t
$$

whence,

$$
v=\frac{\Delta s}{\Delta t}=5 .
$$

Since $A C=\Delta t$ and $C B=\Delta s, v$ is represented by the ratio $C B / A C$.

We may therefore say that the velocity is represented by the ratio of the difference of the ordinates of any two points on the line to the difference of the $a b$ scissas.

Many other examples of uniform change with respect to time will readily suggest themselves. Thus we speak of the

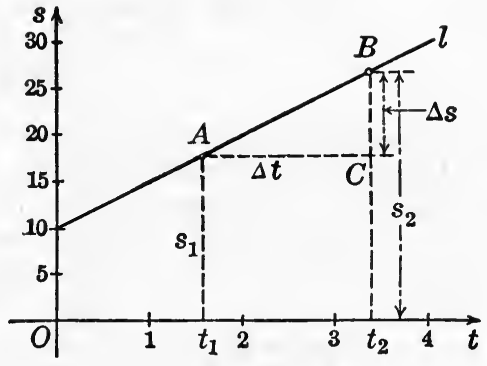

Fig. 28.

\begin{tabular}{c|c|c|c|c}
\hline$\Delta t$ & $t$ & $s$ & $\Delta s$ & $\frac{\Delta s}{\Delta t}$ \\
\hline & & & & \\
1 & 0 & 10 & 5 & 5 \\
1 & 1 & 15 & 5 & 5 \\
1 & 2 & 20 & 5 & 5 \\
1 & 3 & 25 & 5 & 5 \\
& 4 & 30 & & \\
\hline
\end{tabular}
rate at which a tank is filled with water, the rate at which a manufacturer turns out his product, etc.

A generalization of uniform rate of change with respect to time is given in the 
Definition. It is said that any variable $y$ changes uniformly with respect to a second variable $x$, of which $y$ is a function, if the ratio of any change in $y$ to the change in $x$ producing it, $\Delta y / \Delta x$, is equal to a constant, which is called the rate of change of $y$ with respect to $x$. This rate gives the change in $y$ due to a unit change in $x$.

For example, if mercury is poured into a vertical tube, the weight of the mercury in the tube is a function of the height of the column of mercury. The ratio of any change in the weight to the change in height is constant, and equal to the weight of a column of unit height. Hence the weight changes uniformly with respect to the height.

It follows from the definition that if $y$ changes uniformly with respect to $x$, equal changes in $x$ produce equal changes in $y$.

\section{EXERCISES}

It is assumed that the graphs in the following exercises are straight lines.

1. Solve the example in Section 16 if the man starts toward town, considering the motion for 4 hours.

2. A through freight train was 90 miles from a city at 2 o'clock, and 150 miles at 4 o'clock. Construct the graph of its motion. Find its velocity, and interpret it geometrically. From the graph find how far the train was from the city at noon, and when it passed through the city.

3. A tank full of water is being emptied. After 5 minutes it contains 150 gallons, and after 12 minutes 108 gallons. Construct a graph showing the amount of water in the tank at any time. Find the capacity of the tank, the time required to empty it, and the rate at which it is being emptied. What represents each of these quantities graphically?

4. A man walks up a hill inclined at $30^{\circ}$ to the horizon. Find the rate at which his altitude increases with respect to the distance he walks. Is it essential that he walk with a constant velocity?

5. A coal wagon is being filled with coal. Would the rate of change of the weight of the coal in the wagon with respect to the volume of the coal be constant? If so, how would it be measured?

6. Mention some quantity which is changing uniformly with respect to some other quantity, different from time, and state what the rate of change would be.

7. On the same axes, plot graphs showing the number of minute spaces the hands of a clock pass over in $t$ minutes, starting at noon and ending at 1 o'clock. Determine from them how many spaces the hands are apart at 12:30. 
17. Characteristic Property of a Straight Line. Let $P_{1}$ and $P_{2}$ be any two points on a straight line, $M_{1} P_{1}$ and $M_{2} P_{2}$ their ordinates, and let $P_{1} Q$ be perpendicular to $M_{2} P_{2}$. Then $\Delta x=P_{1} Q$ is the difference of the abscissas of $P_{1}$ and $P_{2}$, and $\Delta y=Q P_{2}$ is the difference of the ordinates. The ratio of the difference of the ordinates to that of the abscissas is

$$
\frac{\Delta y}{\Delta x}=\frac{Q P_{2}}{P_{1} Q} \text {. }
$$

For any second pair of points on the line, $P_{3}$ and $P_{4}$, the value of this ratio is

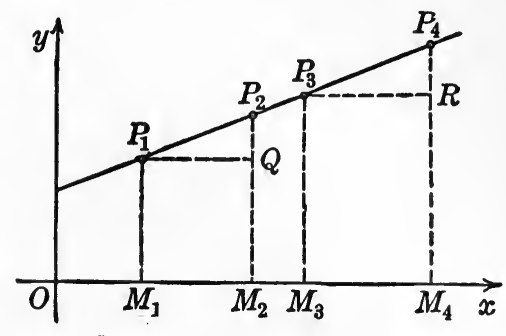

Fig. 29.

$$
\frac{\Delta y}{\Delta x}=\frac{R P_{4}}{P_{3} R} .
$$

Since the triangles $P_{1} Q P_{2}$ and $P_{3} R P_{4}$ are similar (why?), we have

and hence

$$
\frac{R P_{4}}{P_{3} R}=\frac{Q P_{2}}{P_{1} Q}
$$

The ratio of the difference of the ordinates of any two points on a straight line to the difference of the abscissas, $\Delta y / \Delta x$, is the same no matter what two points on the line be chosen.

This fact has been illustrated in the preceding section.

Conversely, a line is a straight line if the ratio of $\Delta y / \Delta x$ is always the same for any two points on the line.

Let $P_{1}$ and $P_{2}$ be two definite points and $P_{3}$ any third point on the given line which is to be proved straight. That the given line is straight will be proved by showing that $P_{3}$ lies on the straight line determined by $P_{1}$ and $P_{2}$. Draw the line through $P_{1}$ parallel to the $x$-axis cutting the ordinates of $P_{2}$ and $P_{3}$ at $Q$ and $R$ respectively. Since, by hypothesis, the values of $\Delta y / \Delta x$ computed for $P_{1}$ and $P_{2}$ and for $P_{1}$ and $P_{3}$ are equal, it follows that

$$
\frac{Q P_{2}}{P_{1} Q}=\frac{R P_{3}}{P_{1} R}
$$


Hence the triangles $P_{1} Q P_{2}$ and $P_{1} R P_{3}$ are similar (why?), and therefore $\angle Q P_{1} P_{2}=\angle R P_{1} P_{3}$. Then $P_{1} P_{3}$ coincides with

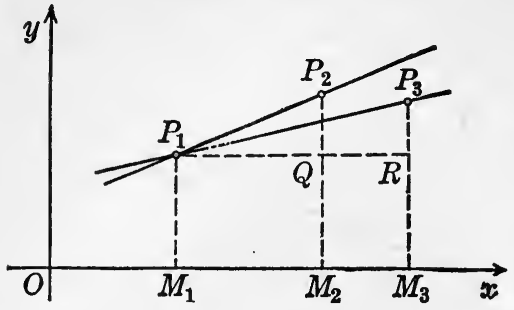

FIG. 30 . $P_{1} P_{2}$, and hence $P_{3}$ lies on the straight line $P_{1} P_{2}$.

Definition. The constant value of the ratio of the difference of the ordinates of any two points on a straight line to the difference of the abscissas is called the slope of the line.

If the slope is denoted by $m$, this definition may be expressed in the symbolic form

$$
m=\frac{\Delta y}{\Delta x} .
$$

For any graph, the ratio of the difference of the ordinates to the difference of the abscissas, $\Delta y / \Delta x$, represents the average rate of change of a function. Hence the facts proved above may be stated as the

Theorem. If $y$ is a function of $x$, the graph of $y$ is a straight line if, and only if, the average rate of change of $y$ with respect to $x$ is constant.

To plot the graph of a function we usually express the functional relation in the form of an equation, build the table of values, and plot the curve. This process is unnecessary if it is known that the average rate of change of the function is constant, for the theorem just proved shows that the graph is a straight line. To draw the graph it is sufficient to obtain two pairs of values, plot the points representing them, and draw the straight line through these points.

Example. Commercial alcohol, 95\% pure, is poured into a bottle. Construct a graph showing the amount of alcohol in the bottle as a function of the amount of liquid in the bottle. Interpret the slope.

Plot the amount of liquid, $l$, on the horizontal axis, and the amount of alcohol, $a$, on the vertical axis. If $\Delta a$ is the amount of alcohol in $\Delta l$ units of the liquid, we have $\Delta a=0.95 \Delta l$, since $95 \%$ of the liquid is alcohol. Hence $\Delta a / \Delta l=0.95$, so that the rate of change of $a$ with respect to $l$ is 
constant. The graph is therefore a straight line. If there is no liquid in the bottle, there is no alcohol, and hence the origin $(0,0)$ is on the line; and if the bottle contains 10 units of liquid, there are 9.5 units of alcohol in the bottle. Hence $(10,9.5)$ is a second point on the line, and these two points are sufficient to determine the graph.

The slope represents the rate of change of $a$ with respect to $l, \Delta a / \Delta l=0.95$, which gives the percentage of alcohol in the liquid.

In plotting the graph by using the point $(10,9.5)$, it is assumed that the unit of volume is some such unit as an ounce or cubic centimeter. If a bottleful were chosen as the unit, which is convenient in

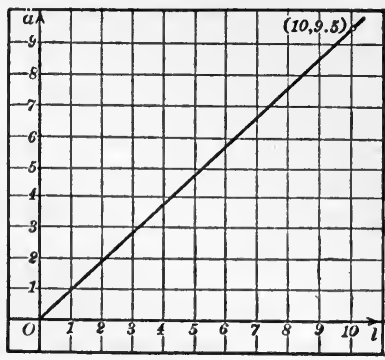

Frg. 31. many problems, it would be better to use the point $(1,0.95)$ and choose the unit on the $l$-axis as large as convenient. For then $l=1$ would mean that the bottle was full.

18. Slope of a Straight Line. "Slope of a line" (definition, Section 17) is the term used technically by mathematicians for what might be called the measure of steepness of the line. It may represent many things. Thus in the example in Section
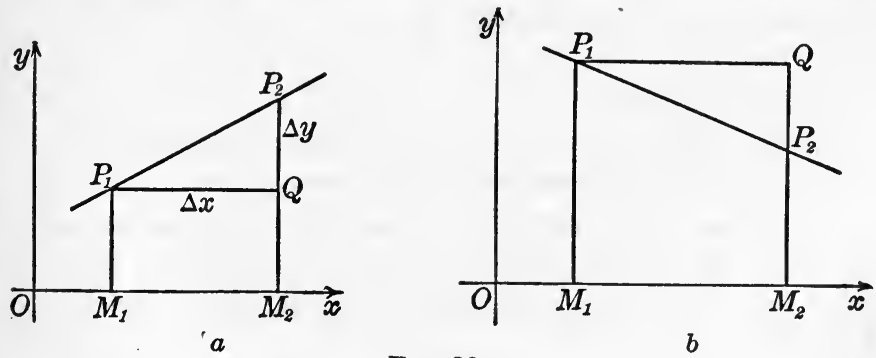

Fig. 32.

$16, \Delta s / \Delta t$ is the slope of the line, and hence the velocity $v$ is represented by the slope.

If the slope $m$ is computed from two points $P_{1}\left(x_{1}, y_{1}\right)$ and $P_{2}\left(x_{2}, y_{2}\right)$ on the line, we have, in either figure,

$$
m=\frac{\Delta y}{\Delta x}=\frac{Q P_{2}}{P_{1} Q}=\frac{M_{2} P_{2}-M_{1} P_{1}}{O M_{2}-O M_{1}}=\frac{y_{2}-y_{1}}{x_{2}-x_{1}}=\frac{y_{1}-y_{2}}{x_{1}-x_{2}} .
$$

In finding $\Delta y$ and $\Delta x$ it is essential that both coördinates of $P_{1}$ be subtracted from those of $P_{2}$, or vice versa. 
Theorem 1. A line runs up to the right or down to the right according as its slope is positive or negative.

For two points $P_{1}$ and $P_{2}$ may be chosen on the line so that $P_{1}$ lies to the left of $P_{2}$, whence $\Delta x$ is positive. Then $\Delta y$ will be positive or negative according as $P_{2}$ is above or below $P_{1}$. Hence $m=\Delta y / \Delta x$ will be positive or negative according as $P_{2}$ is above or below $P_{1}$, that is, according as the line runs up to the right or down to the right.

The precise relation between the value of $m$ and the direction of the line as determined by the angle the line makes with the $x$-axis involves a transcendental function, and will be considered in a later chapter (see also Exercises 3 and 4 below).

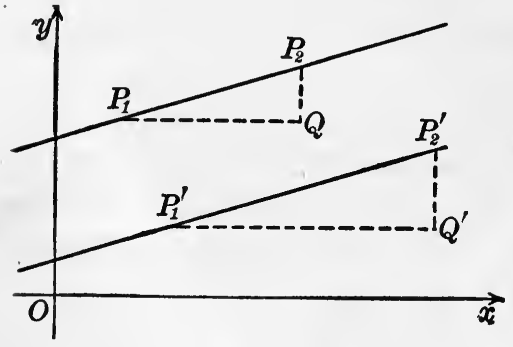

Fra. 33.

Theorem 2. If two lines are parallel their slopes are equal, and conversely.

The triangles $P_{1} Q P_{2}$ and $P_{1}^{\prime} Q^{\prime} P_{2}^{\prime}$ are similar, since their sides are parallel by pairs. Hence

$$
\frac{Q P_{2}}{P_{1} Q}=\frac{Q^{\prime} P_{2}^{\prime}}{P_{1}^{\prime} Q^{\prime}}
$$

that is, the slopes of the two lines are equal.

The proof of the converse is left as an exercise.

Construction. To construct a line through a given point $P_{1}$ whose slope is a given positive fraction $a / b$, take $Q b$ units to the right of $P_{1}$, and $P_{2}$ a units above $Q$; then $P_{1} P_{2}$ is the required line.

For we have

$$
m=\frac{Q P_{2}}{P_{1} Q}=\frac{a}{b} .
$$

If the slope is negative, take $P_{2}$ below $Q$. If the slope is an integer, it may be regarded as a fraction with unit denominator.

If two points are close together, the line through them cannot be drawn as accurately as if they were farther apart. Hence, in this construction, it is sometimes desirable to take $P_{1} Q$ equal to some multiple of $b$ and $Q P_{2}$ equal to the same multiple of $a$. 


\section{EXERCISES}

1. Plot the following pairs of points and the lines determined by them. Find $\Delta x, \Delta y$, and the slope $m$, and indicate the graphical significance of each of these quantities.
(a) $(1,2)$ and $(4,5)$.
(b) $(1,2)$ and $(4,1)$.
(c) $(1,2)$ and $(-2,0)$.
(d) $(1,2)$ and $(3,4)$.
(e) $(3,-2)$ and $(5,2)$.
(f) $(2,5)$ and $(4,2)$.

2. Construct the lines through the points indicated with the given slope.
(a) $P(2,3), m=\frac{1}{2}$.
(b) $P(3,-1), m=-\frac{1}{2}$.
(c) $P(0,4), m=-\frac{2}{3}$.
(d) $P(3,0), m=2$.
(e) $P(-2,4), m=\frac{3}{2}$.
(f) $P(5,2), m=0$.

3. Show that of two lines through the same point the one which makes the greater acute angle with the $x$-axis has the larger slope numerically.

4. Show that the slope of a line parallel to the $x$-axis is zero.

5. If a telegram containing $\mathbf{1 0}$ words or less can be sent to a certain place for $35 k$, and a 24 -word message for $63 \phi$, construct a graph showing the cost of a message containing any number of words, and determine the charge for each additional word above 10 . What represents this charge?

6. Construct a graph to show the cost of any number of eggs at $40 \mathrm{k}$ a dozen. Determine from the graph the number of eggs which can be purchased for 70\&. What does the slope represent?

7. Construct a graph to show the amount of silver in any amount of an alloy containing $25 \%$ of silver. Determine from the graph how much silver there is in 20 pounds of the alloy. What does the slope represent?

8. A pound of an alloy contains 3 parts of silver and 5 of copper. Construct a graph to show the amount of silver in any amount of the alloy. Determine from the graph how much of the alloy contains $4 \frac{1}{2}$ pounds of silver, and how much silver there is in 10 pounds of the alloy. Interpret the slope.

9. Ten ounces of alcohol $95 \%$ pure are poured into a bottle and then 5 ounces of water are added. Construct a graph showing the amount of alcohol in the bottle during the process as a function of the amount of liquid.

10. Solve Exercise 9 if 5 ounces of $50 \%$ alcohol are added instead of 5 ounces of water.

11. Is the slope of the line joining $(3,6)$ to $(6,12)$ the same as the slope of the line joining $(3,6)$ to $(-3,-6)$ ? What can be said of the three points?

12. Are the points $(6,1),(-2,-3)$, and $(0,-2)$ on a straight line?

13. Show that the points $(2,1),(3,7),(5,3)$, and $(0,5)$ are the vertices of a parallelogram.

14. Find the value of $b$ if the line determined by $(2, b)$ and $(-1,3)$ is parallel to the line joining $(-4,-5)$ and $(7,-2)$. 
15. Two boys start from the same point and run in the same direction, one at the rate of 15 feet per second, the other at the rate of 20 feet per second. Construct the graphs showing the distances from the starting point to each boy at any time. What do the slopes represent? Determine from the graphs how far apart the boys are after 3 seconds. Solve the same problem if the boys run in opposite directions.

16. Water is admitted into a tank through two pipes at the rates of 3 and 5 gallons per second. On the same axes, plot graphs showing the amount which has entered through each pipe at any time, and also the total amount. What do the slopes represent?

17. If the cost of setting type for a circular is 50 cents, and if the cost of paper and printing is half a cent a copy, construct a graph showing the cost of any number of copies. From it determine the cost of 500 copies. What does the slope represent? Hint: The cost of setting the type may be regarded as the cost of zero copies.

18. If $\$ 100$ is deposited in a bank to draw simple interest at $6 \%$, construct a graph to show the amount at any time. What does the slope represent? The intercept on the "amount axis"? Determine graphically how long it would take the principal to double itself. What is the relation between the amount and the time?

19. One of the horizontal boards in a flight of stairs is called a tread, and one of the vertical boards a riser. How can the steepness of the stairs be expressed in terms of the widths of the treads and risers?

20. Show that the average rate of change of a function other than a linear function is represented by the slope of a secant line of the graph.

19. Graphical Solution of Problems Involving Functions which Change Uniformly. Graphical methods are used freely

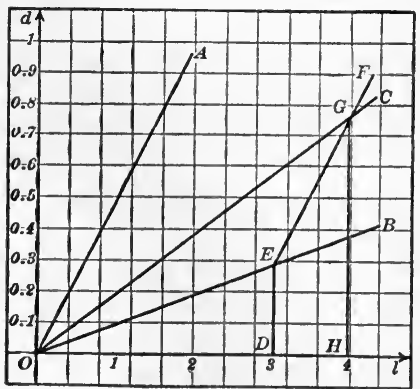

Frg. 34 . by engineers and others because of their relative simplicity. A graphical solution of a problem is also often used as a simple means of checking the accuracy of some other solution. A method of obtaining approximate solutions of many problems by means of graphs is illustrated in the following problems:

EXample 1. A druggist has a $50 \%$ solution of a certain disinfectant and also a $10 \%$ solution. How much of the former must be added to 3 pints of the latter to obtain a $20 \%$ solution? 
The graph representing the amount of disinfectant, $d$, in a quantity, $l$, of the $50 \%$ solution is the straight line $O A$ through the origin whose slope is 0.5 . Similarly, the graphs for $10 \%$ and $20 \%$ solutions are respectively $O B$ and $O C$ whose slopes are 0.1 and 0.2 .

On the $l$-axis take $O D=3$, and draw the ordinate $D E$ to the line $O B$. Then $D E$ represents the amount of disinfectant in 3 pints of the $10 \%$ solution.

Through $E$ draw $E F$ parallel to $O A$. Then the ordinate of a point on $E F$ represents the amount of disinfectant in the mixture as some of the $50 \%$ solution is added to the 3 pints of the $10 \%$ solution.

If $D E$ cuts $O C$ at $G$, and if $G H$ be drawn perpendicular to the $l$-axis, then in $O H$ pints of the combined mixture there are $H G$ pints of disinfectant; and since $G$ lies on $O C$, the strength of the combined mixture is $20 \%$.

Hence we must add $D H$, or 1 pint approximately, of the $50 \%$ solution.

Example 2. A freight train goes from $A$ to $B$ at the rate of 15 miles per hour. Four hours after it starts, an express train leaves $A$ and moves at the rate of 45 miles per hour. The express arrives at $B$ half an hour ahead of the freight. How far is it from $A$ to $B$ and how long does it take each train to go?

Let abscissas denote time in hours measured from the time of departure of the freight train, and ordinates distances from $A$.

The graph representing the distance of the freight at any time is the straight line $O C$

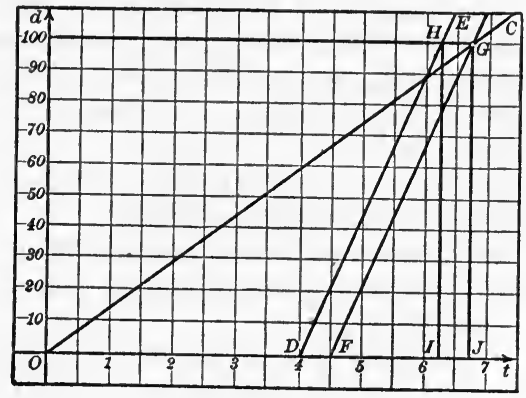

FIG. 35. through the origin with the slope 15 , and that of the express is the line $D E$ through the point $D(4,0)$ with the slope 45 .

At $B$ the express is half an hour ahead of the freight, and hence we must determine a point on $O C$ half a unit to the right of $D E$. To do this, take $F$ on the $t$-axis so that $D F=\frac{1}{2}$. Draw the line through $F$ parallel to $D E$, and let it meet $O C$ at $G$. If the line through $G$ parallel to the $t$-axis cuts $D E$ at $H$, then $H G=\frac{1}{2}$. Hence, the required distance $A B$ is represented by the ordinates $I H=J G=100$ miles approximately.

The time of the freight is represented by $O J=6.7$ hours, approximately, and that of the express by $D I=2.2$ hours, approximately.

The line $F G$ may be interpreted as the graph of a train leaving half an hour later than the express and moving at the same rate of 45 miles an hour. By the conditions of the problem, such a train would overtake the freight at $B$. 


\section{EXERCISES}

Solve each of the following exercises graphically. Use a sharp, hard pencil, and choose the units on each axis so that the figure will be as large as convenient.

1. Two trains start from the same place at the same time and run in the same direction at the rates of 30 and 50 miles per hour respectively. How far apart will they be at the end of two hours? When will they be 100 miles apart?

2. Solve Exercise 2 if the trains run in opposite directions.

3. For printing business cards one firm charges $75 \phi$ for setting the type, and $\frac{1}{4} \&$ apiece for the cards and printing. Another firm makes no charge for typesetting, but charges $\frac{1}{2} \&$ apiece. Which firm will print more cheaply 100 cards? 500 cards? Which will do the more printing for $\$ 1.25$ ? For $\$ 2.00$ ? How many cards will both print at the same cost, and what will the cost be?

4. A man rides horseback at the rate of 8 miles an hour for an hour and a half, and then walks back at the rate of 4 miles an hour. Another man starts from the same place at the same time and walks in the same direction at the rate of 3 miles an hour. When and where will they meet?

5. How much water must be added to 10 gallons of alcohol, $95 \%$ pure, to reduce it to a $66 \frac{2}{3} \%$ solution?

6. A farmer sells three and a half dozen eggs to a grocer at $25 \&$ a dozen, and takes his pay in rice at $8 \&$ a pound. How many pounds of rice will he receive?

7. At what time are the hands of a clock together between three and four o'clock? When are they opposite? Hint: The position of either hand may be determined by the number of minute spaces it has passed over since leaving "12." Plot this number as a function of the number of minutes after three o'clock.

8. Solve Exercise 7 for eight and nine o'clock.

9. The earth revolves around the sun in 52 weeks, and the planet Mars in 98 weeks. How often is the earth between Mars and the sun?

20. Graph of the Linear Function $m x+b$. Let the function be denoted by $y$, so that

$$
y=m x+b .
$$

Let us first determine the average rate of change of $y$ with respect to $x$. Let the independent variable change from any value $x$ to $x+\Delta x$, and suppose that the dependent variable 
changes from $y$ to $y+\Delta y$. Then the corresponding values $x+\Delta x$ and $y+\Delta y$ satisfy (1), so that

$$
y+\Delta y=m(x+\Delta x) \text {. }
$$

Subtracting (1) from (2),

$$
\Delta y=m \Delta x
$$

and dividing by $\Delta x$,

$$
\Delta y / \Delta x=m \text {. }
$$

Hence the average rate of change of $y$ with respect to $x$ is constant, and the graph of (1) is therefore a straight line (Theorem, Section 17).

If the graph is a straight line the value of $\Delta y / \Delta x$ is the slope of the line (Definition, Section 17), and hence the slope of the graph of (1) is $m$. The constant term $b$ is the intercept on the $y$-axis, for if $x=0$, then from (1) $y=b$. The line is thus determined by the point $(0, b)$ and the slope $m$ (Construction, Section 18).

We may therefore express the fundamental theorem of this chapter as follows:

Theorem 1. The graph of the linear function $m x+b$, or of the equation $y=m x+b$, is the straight line whose slope is $m$, and whose intercept on the $y$-axis is $b$.

If $b=0$, the line passes through the origin. Hence the important

Corollary 1. The graph of the function $m x$, or of the equation $y=m x$, is the straight line through the origin whose slope is $m$.

Corollary 2. The graphs of the equations

$$
y=m x+b \quad \text { and } \quad y=m x+b^{\prime}
$$

are parallel lines.

An equation of the first degree in $x$ and $y$ is called linear, and may be reduced to the form

$$
A x+B y+C=0 .
$$

Theorem 2. The graph of any linear equation (3) is a straight line. 
If the equation contains $y$, so that $B \neq 0$ (read " $B$ is not equal to zero"), we may divide by $B$ and solve for $y$, obtaining the linear function

$$
y=-\frac{A}{B} x-\frac{C}{B},
$$

whose graph is the straight line whose slope is $m=-A / B$, and whose intercept on the $y$-axis is $b=-C / B$ (Theorem 1 ).

If $B=0$, equation (3) has the form $A x+C=0$, whence, if $A \neq 0$,

$$
x=-C / A \text {. }
$$

The graph of this equation is a straight line parallel to the $y$-axis. For all points whose abscissas have the same value, $-C / A$, no matter what the ordinates may be, are equidistant from the $y$-axis.

Hence the graph of (3) is always a straight line.

Corollary 1 . The graph of an equation of the form $x=a$ constant is a straight line parallel to the $y$-axis, and that of $y=a$ constant is a line parallel to the $x$-axis.

The proof of the first statement appears explicitly in the preceding proof. Is that of the second included in the proof (see Exercise 4, Section 18)?

Corollary 2. If a linear equation be solved for $y$, then the coefficient of $x$ is the slope of the graph of the equation, and the constant term is the intercept on the $y$-axis.

In constructing the graph of a linear equation it is necessary to plot but two points. The intercepts (Section 10) usually furnish the two most convenient points. The line may be drawn more accurately by choosing points some distance apart than by taking them close together.

The graph of $y=m x$ may be drawn by plotting but one point and joining it to the origin.

\section{EXERCISES}

1. Construct the graphs of the following equations:
(a) $y=2 x+5$.
(b) $y=-2 x+\varepsilon$.
(c) $y=x+3$.
(d) $y=-x+7$.
(e) $y=3 x$.
(f) $y=2 x-4$.
(g) $x-2 y+6=0$.
(h) $2 x+3 y-12=0$.
(i) $2 x+3 y+12=0$.
(j) $y=\frac{2}{3} x$.
(k) $y=\frac{3}{4} x+2$.
(l) $y=-\frac{1}{2} x+5$. 
2. In experiments on the temperature at various depths in a mine, the temperature $t$ (in degrees Centigrade) was found to be connected with the depth $d$ (in feet) by the equation $t=20+0.01 d$. Construct the graph. What is represented by the ordinate and abscissa of a point on the graph? by the intercept on the $t$-axis? by the slope?

3. In experiments on a pulley block the pull $P$, in pounds, required to lift a weight of $W$ pounds was found to be $P=0.3 W+0.5$. Construct the graph. What does the slope represent? The intercept on the $P$-axis? What is the value of the rate of increase of $P$ with respect to $W$ ?

4. The readings, $T$, of a gas meter being tested, were found in comjarison with those of a standard meter, $S$, and the two sets of readings iatisfied the equation $T=300+1.2 \mathrm{~S}$. Draw the graph. What do the ibscissa and ordinate of a point on it represent? The slope? The inercept on the $T$-axis? Has the consumer whose meter is being tested jeen getting more or less gas than the meter indicated?

5. Plot the graph of the equation $s=-3 t+12$. Describe the motion or the first 6 hours if $s$ represents the distance of a man from town, in niles, at the time $t$, in hours. Interpret the slope and the intercepts on oth axes.

6. The ordinate of a point on one of the graphs in the figure represents he distance $s$ (in feet) of a moving body from a certain station at the time

(in seconds) repreented by the abscissa. )escribe the motion epresented by each of he graphs $(a),(b),(c)$, d), from $t=-6$ to $=12$.

Solution for (a). From he graph, $s=7$ when $=-6$. Hence the body iarts at a point 7 feet om the station on the ositive side of it. As $t$ icreases, $s$ decreases, nce the graph is falling

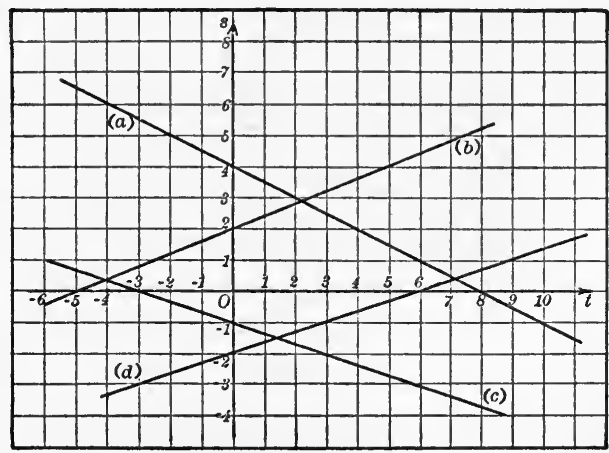

FIg. 36. 3 it runs to the right hanges of the function). Hence the body is approaching the station. Then $t=0$, the distance from the station is $s=4$ (interpretation of the itercept on the $s$-axis). The body passes through the station when $=8$, for then $s=0$ (interpretation of the intercept on the $t$-axis). Four conds later, that is, when $t=12$, the body has reached a point 2 feet eyond the station in the negative direction; for when $t=12, s=-2$. The rate of change of $s$ with respect to $t$, namely, the velocity, is con- 
stant since the graph is a straight line. Computing its value from the points $(0,4)$ and $(8,0)$, we get

$$
v=\frac{\Delta s}{\Delta t}=\frac{0-4}{8-0}=-\frac{1}{2}
$$

The negative sign indicates that the total change in $s$ in any interval of time $\Delta t$ is negative, and hence the body is moving in the negative direction at the uniform rate of half a foot per second.

7. In the following equations, $s$ denotes the number of feet, measured along the road, at the time $t$ (in seconds), from a tree to a steam roller. Construct the graph of each equation, and from it describe the motion of the steam roller from $t=-5$, to $t=10$.
(a) $s=2 t+8$.
(b) $s=-\frac{1}{2} t+3$.
(c) $s=-2 t-4$.

8. Construct and interpret the graph of each of the equations below, in which $W$ denotes the part of a piece of work that a man can do in $D$ days.

(a) $20 W-D-5=0$.

Solving the equation for $W$, we get $W=\frac{1}{20} D+\frac{1}{4}$. Hence the graph is the straight line whose slope is $\frac{1}{20}$ and whose intercept on the $W$-axis is $\frac{1}{4}$. Since $W=1$ indicates that the piece of work has been completed, the

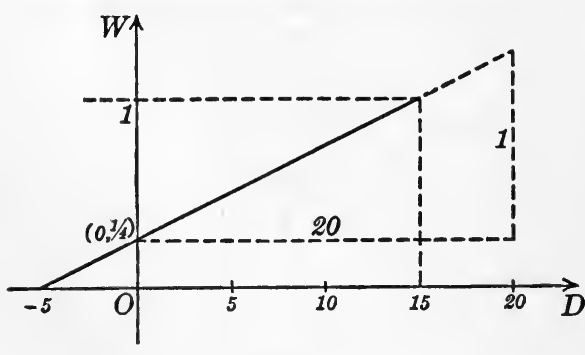

FIG. 37. graph need be plotted only from $W=0$ to $W=1$.

The intercept on the $W$-axis indicates that one fourth of the job has been completed when we begin to consider the problem, and the slope represents the rate at which the man works, namely, $\frac{1}{20}$ of the piece of work per day.

When $W=1$, the grapl shows that $D=15$ (which may be verified from the equation), that is, it will take the man 15 days tc complete the work.

If we care to interpret negative values of $D$, the graph shows that wher $W=0, D=-5$. Hence if we regard the whole piece of work as performec by the man in question, he started 5 days ago.
(b) $12 W-D-6=0$.
(c) $10 W-3 D=0$.
(d) $10 \mathrm{~W}-2 D+4=0$.
(e) $30 W-5 D+10=0$.

9. Mention some function of the form $m x$ arising in daily life, and dis cuss it graphically. 
10. Mention some function of the form $m x+b$ arising in daily life, and discuss it graphically.

11. Plot the graphs of the equations below, using a single set of coordinate axes for each case. Each figure will contain several lines corresponding to the values of the constants indicated.
(a) $y=m x$
for
$m=\frac{1}{4}, \frac{1}{2}, 1,2,4$.
(b) $y=2 x+b$
$b=-3,-2,0,2,5$.
(c) $y=m x+2$
for
$m=\frac{1}{3}, 1,3,-3,-1,-\frac{1}{3}$.

12. Exhibit in tabular form as many as possible of the corresponding properties of a straight line and the function $f(x)=m x+b$ of which the line is the graph (see Section 15). Indicate special properties of this function which distinguish it from all other functions.

21. Variation. We have already had a number of illustrations of the important relation $y=m x$, in which one variable is a constant times the other. For example, the price paid for eggs is the price per dozen times the number of dozen bought; if a man walks at a uniform rate, the distance is the rate times the time; etc. Two technical terms are used in this connection.

Definition. A variable is said to vary as, or to be proportional to, a second variable if the first is equal to a constant times the second. Symbolically, $y$ varies as $x$, or $y$ is proportional to $x$, if $y=m x, m$ being a constant.

This use of the word proportional is justified by the fact that two values of $y$ are proportional to the corresponding values of $x$. For if $y_{1}=m x_{1}$, and $y_{2}=m x_{2}$ then by dividing the first equation by the second, we obtain the proportion $\frac{y_{1}}{y_{2}}=\frac{x_{1}}{x_{2}}$.

The phrase "variation of a function" used in Section 12, should not be confused with "variation" as a heading under which we discuss proportional variables. A more general use of the latter term will be taken up in Chapter III.

The mass of a body is the amount of matter in it. If the nature of the material in the body is the same throughout, the mass varies as the volume. That is, if $M$ denotes the mass and $V$ the volume, $M=m V$, where $m$ is a constant called the density of the substance of which the body is composed. The value of $m$ is the mass of a unit volume. 
Example. If the mass of an aluminum object is 13 , and its volume is 5 , find the mass of an aluminum object whose volume is 8.6, and the volume of an object whose mass is 17.4 .

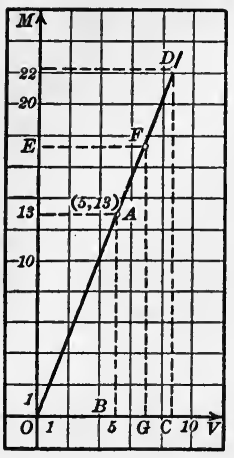

Fig. 38.

Let $M$ denote the mass and $V$ the volume of any aluminum object. Then since $M$ varies as $V$,

$$
M=m V \text {. }
$$

Graphical solution. The graph is a straight line through the origin (Corollary 1 to Theorem 1, Section 20). It also passes through the point $A(5,13)$, since $M=13$ when $V=5$. It is therefore the line $O A$ in the figure.

From the graph, if $V=8.6$, the value of $M$ is $C D=22.4 ;$ and if $M=17.4$, the value of $V$ is $O G=$ 7. These are the required values.

The slope of the line, computed from $O$ and $A$, is

$$
m=\frac{\Delta M}{\Delta V}=\frac{B A}{O B}=\frac{13}{5}=2.6 .
$$

Since $\frac{13}{8}$ is the mass of a unit volume of aluminum, or the density, the slope represents the density.

Algebraic solution. Substituting the given values $M=13$ and $V=5$ in (1), we obtain $13=5 m$, whence the density $m=\frac{13}{5}=2.6$.

Substituting the value of $m$ in (1), we have the relation between the mass and volume of any aluminum object,

Then if $V=8.6$,

$$
\begin{aligned}
M & =2.6 \mathrm{~V} . \\
M & =2.6 \times 8.6=22.36 . \\
17.4 & =2.6 \mathrm{~V}, \\
V & =17.4 / 2.6=6.69 .
\end{aligned}
$$

And if $M=17.4$, whence

The graph has the advantage of exhibiting the relation to the eye, and if it is constructed carefully, it is sufficiently accurate for the purpose of a " ready reckoner." It is very useful when a number of values of either variable are desired and great accuracy is not essential, and in any case it furnishes a valuable check on the accuracy of the computation.

When we are given the fact that a variable $y$ is proportional to a second variable $x$, the general form of the law connecting $x$ and $y$ is $y=m x$. The exact form of the law involves a particular value of the constant $m$. This value of $m$ may be found from a pair of corresponding values of $x$ and $y$, other than $(0,0)$, as in the algebraic solution of the example above 
22. Uniform Acceleration. The motion of a body is said to be accelerated if the velocity is variable. It is said to be uniformly accelerated if the change in velocity is proportional to the change in time. The rate of change of velocity is called acceleration, and it is measured by the number of feet per second by which the velocity changes in each second.

A freely falling body moves with a uniformly accelerated motion, if the resistance of the air is neglected. The acceleration, which is called the acceleration due to gravity and which is denoted by $g$, is approximately 32 feet per second per second. If the positive direction is downward, $g=32$, but if the positive direction is upward, $g=-32$.

Since the rate of change of velocity of a uniformly accelerated body is constant, $v$ is a linear function of $t$, and the graph is a straight line. The slope of the line represents the acceleration, and the intercept on the $v$-axis represents the velocity when $t=0$, which is called the initial velocity.

\section{EXERCISES}

1. Hooke's law says that the amount of stretching in a stretched elastic string is proportional to the tension. If a 2-lb. weight will stretch a string 3 feet, what tension arises when the string is stretched 1 foot? Draw the graph.

2. Prove graphically, that if $y$ varies as $x$, then any two values of $y$ are proportional to the corresponding values of $x$.

3. Is the definition near the end of Section 16 equivalent to the statement following? A variable $y$ changes uniformly with respect to $x$ if the change in $y$ is proportional to the change in $x$ producing it.

4. The mass of a body varies as its volume. On the same axes construct the graphs showing the relation between mass and volume for bodies composed of the following substances whose densities are given:

Lithium, 0.6; alcohol, 0.8; India rubber, 1.0; magnesium, 1.7; diamond, 3.5; silver, 10.5. How are the various densities represented?

5. Wihelmy's law for chemical reactions states that the amount of chemical change in a given time is proportional to the quantity of reacting substance in the system. Construct the graph.

6. The velocity acquired in $t$ seconds by a body falling freely from rest is given by the equation $v=32 t$. Plot the graph and from it determine how fast the body would be falling after 4 seconds. What does the slope represent? 
7. If a ball is dropped from a high building, how fast will it be moving at the end of one second?, at the end of 2 seconds? at the end of 4 seconds? If a ball is thrown vertically upward, how will its velocity be affected in any second? If it is thrown up with an initial velocity of 96 feet per second, how long will it rise?

8. A train starting from rest acquires a velocity of 50 feet per second in 15 seconds. Find the average acceleration.

9. An automobile is moving at the rate of 40 feet per second, when the power is shut off and the brakes applied. If it moves thereafter with a uniform acceleration of -5 feet per second per second, how long before it will stop?

10. Name two quantities arising in every-day experiences which are proportional, and give the value of the constant involved in the relation between them.

11. The pressure of a liquid is proportional to the depth. If the pressure per square inch on the suit of a diver is $5.2 \mathrm{lbs}$. for a depth of $12 \mathrm{ft}$. how deep can he go safely if $78 \mathrm{lbs}$. per square inch is an allowable pressure? Construct the graph.

12. For small changes in altitude, atmospheric pressure varies as the altitude. If the change in the reading of a barometer is 0.1 of a unit for each $90 \mathrm{ft}$. ascent, construct the graph of this relation and determine the difference in readings of two places with a difference in altitude of $1000 \mathrm{ft}$.

13. In a spring balance the extension of the spring is proportional to the weight. If a weight of $2 \mathrm{lbs}$. lengthens the spring 1 inch, construct a graph and determine the extension of the spring for weights of 5,8 , and 17 pounds.

14. The stock of a corporation yields an annual dividend of $5 \%$. What is the relation between the total amount a stockholder receives in dividends and the time the stock is held? If the graph of this relation is drawn, what does the slope of the line represent? What is the value of the slope if the par value of the stock held is $\$ 10,000$ ?

15. The rent charged to each department of a store is proportional to the percentage of the total floor space which the department occupies. If the store pays a rent of $\$ 12,000$, construct a graph to show the rent charged to a department of any size, using as large a scale as convenient. From the graph, determine the rent charged to each department if the various departments occupy $17,25,10,8,5,12$, and 23 per cent of the total floor space.

16. An automobile moving at the rate of 22 feet per second (15 miles per hour) begins to coast down a hill. If its velocity after $t$ seconds is given by $v=10 t+22$, find from the graph of $v$ how fast it would be going after 5 seconds, and how long it would take it to acquire a velocity of 60 miles per hour. What is the acceleration? 
17. If a ball is thrown vertically upward with a velocity of 100 feet per second, its velocity after $t$ seconds is given by the equation $v=-32 t$ +100 . Plot the graph, and describe the motion. What is represented by the intercept on the $v$-axis? the intercept on the $t$-axis? the slope?

18. The ordinate of a point on one of the graphs in the figure represents the velocity $v$ of a moving body at the time $t$ represented by the abscissa. Describe the motion of a body whose velocity is represented by each of the graphs, from $t=-6$ to $t=12$.

Solution for (a). From the graph, the intercept on the $t$-axis is $t=6$, when $v=0$, so that the body is not moving at this instant. The intercept on the $v$-axis is $v=-2$, which gives the velocity when

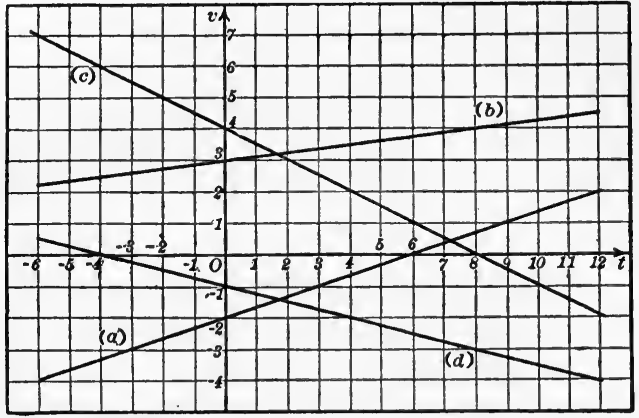

Fra. 39. $t=0$.

The line is below the $t$-axis if $t<6$, so that during this time $v$ is negative and the body is moving in the opposite of a certain direction which has been assumed as positive, i.e., in the negative direction. The line is above the $t$-axis if $t>6$, so that $v$ is positive and the body is moving in the positive direction.

The slope of the line, computed from the points $(6,0)$ and $(0,-2)$ is the acceleration $m=\frac{\Delta v}{\Delta t}=\frac{-2-0}{0-6}=\frac{1}{3} \mathrm{ft}$. per sec. per sec.

Since the line rises to the right, the velocity constantly increases, which agrees with the fact that the acceleration is positive.

From the graph, when $t=-6, v=-4$, and when $t=12, v=2$.

Discarding the technical terms positive and negative, the motion may be described as follows: At the start the body is moving in a certain direction at the rate of 4 feet per second. During the next 12 seconds it slows down uniformly and comes to rest for an instant, and then begins to move in the opposite direction with a constantly increasing speed. After 6 seconds it has acquired a velocity of 2 feet per second. The velocity changes by $\frac{1}{3}$ of a foot per second in each second of the motion.

Notice that where the body is at any time, and in particular where it comes to rest, cannot be determined from the graph of the velocity.

19. The velocity of an automobile, in feet per second, at the time $t$, 
in seconds, is given by one of the following equations. Construct the graph, and describe the motion, from $t=0$ to $t=6$.
(a) $v=4 t$.
(b) $v=3 t-6$.
(c) $v=0.5 t-2$.
(d) $v=-2 t+5$.

20. Describe the motion of a ball rolling on the side of a hill if its velocity is given by one of the following equations, by interpreting the graph. What is the acceleration in each case?
(a) $v=10 t$.
(b) $v=8 t+5$.
(c) $v=6 t-8$.
(d) $v=-4 t+12$.
(e) $v=-6 t+12$.
(f) $v=3 t-12$

21. Construct the graph of the equation $y=-2 x+4$. From it describe the motion of a body (a) if $y$ represents the distance of a body from a certain station at the time $x$; (b) if $y$ represents the velocity of a body at the time $x$.

23. Equation of a Straight Line. We have seen that the graph of $y=m x+b$ is the straight line whose slope is $m$ and whose intercept on the $y$-axis is $b$. Conversely, if a line is given whose slope is, say, 2 and whose intercept on the $y$-axis is, say, 3 , then the equation of which it is the graph is $y=2 x+3$; for the graph of this equation is known to be the given line.

The equation of which a given line or curve is the graph is called the equation of the line or curve.

To find the equation of a line whose slope and intercept on the $y$-axis are given, substitute the given values of $m$ and $b$ in the equation

$$
y=m x+b \text {. }
$$

This is called the slope-intercept form of the equation of a straight line.

To find the equation of a line determined by its slope and any point on it we apply the

Theorem. The equation of the line whose slope is $m$ and which passes through the point $P_{1}\left(x_{1}, y_{1}\right)$ is

$$
y-y_{1}=m\left(x-x_{1}\right) \text {. }
$$

Let $P(x, y)$ be any point on the given line. Since the value of $\Delta y / \Delta x$ for any two points on a straight line is constant (page 49), and equal to $m$ (definition page 50 ), we have 
and hence

$$
\frac{\Delta y}{\Delta x}=\frac{y-y_{1}}{x-x_{1}}=m
$$

$$
y-y_{1}=m\left(x-x_{1}\right) \text {. }
$$

This is called the point-slope form of the equation of a straight line.

To find the equation of a straight line determined by two points, find the slope and then apply (2).

Example 1. Find the equation of the line determined by the points $A(1,4)$ and $B(2,3)$.

The slope of $A B$ is

$$
m=\frac{3-4}{2-1}=-1 \text {. }
$$

Substituting $x_{1}=1, y_{1}=4$, and $m=-1$ in we get

$$
\text { or } \quad \begin{aligned}
& y-4=-1(x-1), \\
& x+y-5=0 .
\end{aligned}
$$

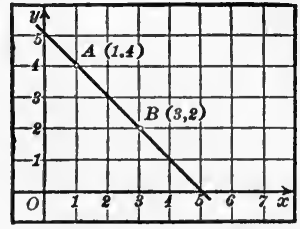

FIG. 40.

Check. The coördinates of $A$ and of $B$ satisfy this equation.

Example 2. Find the equation of the line through the point $A(5,2)$ which is parallel to the line $x-3 y+6=0$.

Solving the given equation for $y$ we get

$$
y=\frac{1}{3} x+2,
$$

and hence the slope of the given line is $m=\frac{1}{3}$, the coefficient of $x$ (Corollary 2 , to Theorem 2, Section 20). Hence the slope of the required line is $\frac{1}{3}$ (Theorem 2, Section 18).

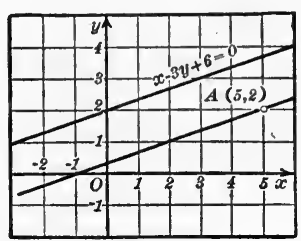

Fig. 41.

Then by (2) the required equation is

$$
\begin{aligned}
y-2 & =\frac{1}{3}(x-5), \\
x-3 y+1 & =0 .
\end{aligned}
$$

Example 3. The freezing point on a Centigrade thermometer is $0^{\circ}$, and on a Fahrenheit thermometer it is $32^{\circ}$. The boiling point on the first is $100^{\circ}$ and on the second is $212^{\circ}$. Find the relation between any two corresponding readings on the two thermometers.

Since the interval between the freezing and boiling points is divided into equal parts on each thermometer, a change of one degree on one of them will always produce a definite, constant change on the other. Hence the graph will be a straight line. 
In plotting, let abscissas denote readings on the Centigrade scale, and ordinates the corresponding readings on the Fahrenheit scale. Then the

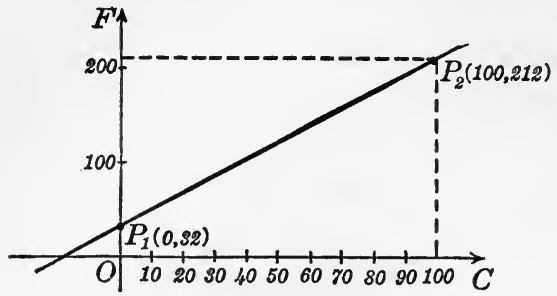

Frg. 42.

readings for the freezing and boiling points are represented respectively by the points $P_{1}(0,32)$ and $P_{2}(100,212)$. The required relation is the equation of the straight line $P_{1} P_{2}$.

The slope of the line is

$$
m=\frac{212-32}{100-0}=\frac{180}{100}=1.8 \text {. }
$$

The intercept on the $F$-axis $\mathrm{i}_{\S} b=32$. Hence, using (1), the relation is

$$
F=1.8 C+32 \text {. }
$$

The slope of the line, 1.8 , is the rate of change of a reading on the Fahrenheit scale with respect to the Centigrade scale. As the rate of change is measured by the change on the Fahrenheit scale due to a unit change on the Centigrade scale, this means that $1^{\circ} .8$ Fahrenheit $=1^{\circ}$ Centigrade.

\section{EXERCISES}

1. Construct each of the lines indicated below. Find its equation and reduce it to the form $A x+B y+C=0$. Check the result by substituting the coördinates of the given point or points.

(a) With the slope $\frac{1}{3}$ and the intercept on the $y$-axis -4 .

(b) Passing through the point $(3,2)$ with the slope $-\frac{1}{2}$.

(c) Determined by the points $(2,5)$ and $(-1,2)$.

(d) Through the point $(3,-4)$ parallel to the $x$-axis; the $y$-axis.

(e) Passing through the points $(0,4)$ and $(3,0)$.

(f) Through the point $(1,3)$ parallel to the line $y=2 x-4$.

(g) Through the point $(3,0)$ parallel to the line determined by the points $(1,2)$ and $(5,4)$.

(h) Through the point $(-2,-3)$ parallel to the line $x+2 y-6=0$.

2. What is the general form of the equation of a line parallel to the $x$-axis? the $y$-axis?

3. What is the value of $A$ if the line $A x-3 y+5=0$ is parallel to the line $3 x+4 y-12=0$ ? If its intercept on the $x$-axis is 10 ?

4. A medicine is $40 \%$ alcohol. Construct a graph showing the amount of alcohol in any amount of the medicine, and find its equation.

5. In an experiment in stretching a brass wire, it is assumed that the elongation $E$ is connected with the tension $T$ by a linear relation. If 
$T=18$ pounds when $E=0.1 \mathrm{inch}$, and $T=58$ pounds when $E=0.3$ inches, construct the graph and find the relation.

6. The population of a town in 1900 was 1200 and in 1910 it was 1400 . Assuming that the growth in population was uniform, construct the graph showing the population at any time, and find the relation between the population and the time.

7. A sum of money at simple interest amounts to $\$ 94.00$ in 5 years, and $\$ 108.00$ in 10 years. Find the sum of money and the rate of interest.

8. It is observed that the boiling point of water at sea level is $212^{\circ} \mathrm{F}$., and that at an altitude of 2299 feet it is $208^{\circ} \mathrm{F}$. What is the boiling point at an altitude of 5000 feet?

9. Find the coördinates of the point of intersection of each of the pairs of lines given below.

(a) $x+y=5$, and $x-y=1$.

Let $A$ be the point of intersection. Then since $A$ is on both graphs, its coördinates must satisfy both equations, and hence these coördinates may be found by solving the equations simultaneously.

Adding the equations, we get $2 x=6$, whence $x=3$.

Substituting in either equation we find that $y=2$. Therefore the coördinates of $A$ are $(3,2)$.

(b) $x+2 y-6=0$ and $x-y+3=0$.

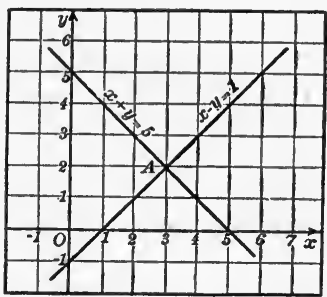

Fig. 43.

(c) $3 x+y+3=0$ and $x-3 y-5=0$.

(d) $0.3 x-0.8 y+5.3=0$, and $y=1.5 x$.

10. Find the coördinates of the point of intersection of the line determined by the points $(2,1)$ and $(4,3)$ and the line through the point $(1,5)$ whose slope is $-\frac{1}{2}$.

24. Application to the Solution of Problems. In the following examples and exercises it is seen how the graphical solution of problems solvable by functions which change uniformly may be used to suggest a method of algebraic solution.

Example 1. Solve Example 1, Section 19, algebraically.

The graphical solution, page 54, shows that the value of $D H$ must be found. But $D H=O H-O D$. It is known that $O D=3$, and since $O H$ is the abscissa of $G$, it may be found by solving the equations of $O C$ and $E F$ for $x$.

Since $O C$ passes through the origin and has the slope 0.2 , its equation is

$$
y=0.2 x \text {. }
$$


The abscissa of $E$ is $x=3$, and since $E$ lies on the line $O B$, whose equation is $y=0.1 x$, its ordinate is $y=0.1 \times 3=0.3$. Hence the coördinates of $E$ are $(3,0.3)$. Since $E F$ was drawn parallel to $O A$ its slope is $m=0.5$, and therefore its equation has the form (Theorem, Section 23)

or

$$
\begin{aligned}
y-0.3 & =0.5(x-3), \\
y & =0.5 x-1.2 .
\end{aligned}
$$

To find $O H$, the abscissa of $G$, solve (1) and (2) for $x$. Eliminating $y$, we have

whence

and therefore

$$
0.2 x=0.5 x-1.2
$$

Then

$$
\begin{aligned}
0.3 x & =1.2, \\
O H & =x=4 .
\end{aligned}
$$$$
D H=O H-O D=4-3=1 .
$$

Example 2. Solve Example 2, Section 19, algebraically.

The time of the freight train and the distance from $A$ to $B$ are represented by the coördinates of the point $G$ (Figure 35, page 55), the point of intersection of the lines $O C$ and $F G$. The equations of these lines are found to be respectively

and

$$
\begin{aligned}
& O C: d=15 t, \\
& F G: d=45 t-202.5
\end{aligned}
$$

Solving these equations we get

$$
\begin{aligned}
& t=6 \frac{3}{4} \text { hours, the time of the freight; and } \\
& d=101 \frac{1}{4} \text { miles, the distance } A B .
\end{aligned}
$$

The time of the express train is represented by

$$
D I=F J=O J-O F=6 \frac{3}{4}-4 \frac{1}{2}=2 \frac{1}{4} \text { hours. }
$$

\section{EXERCISES}

Solve each of the exercises below both graphically and algebraically using the graphic solution as the basis of the algebraic solution.

1. Two trains start toward each other from Buffalo and New York, respectively, 440 miles apart. The one from New York travels at the rate of 50 miles an hour, and the one from Buffalo at the rate of 40 miles an hour. When and where will they meet?

2. A man walks at the rate of 3 miles an hour. Three hours after he starts, another man starts from the same place and travels in the same direction at the rate of $\mathbf{1 0}$ miles per hour. When and where will the latter overtake the former?

3. An express train starts from a city and moves with a velocity of $\mathbf{4 0}$ miles per hour. A freight train is $\mathbf{9 0}$ miles ahead of the express at the start and is moving at the rate of 25 miles an hour. When and where will the express overtake the freight? 
4. When and where will the express train in Exercise 3 be 30 miles behind the freight?

5. Two trains leave a city at the same time, traveling at the rates of 30 and 45 miles an hour respectively. One arrives at another city 3 hours ahead of the first. Find the distance between the cities and the time in which each train made the trip.

6. How much vinegar must be added to a barrel of vinegar $60 \%$ pure to make it an $80 \%$ solution?

7. A cough medicine is $50 \%$ paregoric. How much paregoric should be added to 4 ounces of the medicine to make it $75 \%$ paregoric?

8. Brass is an alloy of copper and zinc. How many cubic centimeters of zinc, density 6.9 , must be combined with 100 cubic centimeters of copper, density 8.8 , to form brass whose density is 8.3 ?

9. Coinage silver is an alloy of copper and silver. How many cubic centimeters of copper, density 8.8 , must be added to 10 cubic centimeters of silver, density 10.6, to form coinage silver whose density is 10.4 ?

10. At what time between 2 and 3 are the hands of a clock together? opposite?

11. At what time between 7 and 8 are the hands of a clock together? opposite?

12. In a clock which is not keeping true time, it is observed that the interval between successive coincidences of the hour and minute hands is 66 minutes. What is the error of the clock?

13. The planet Mercury makes a circuit around the sun in 3 months, and Venus in $7 \frac{1}{2}$ months. Find the time between two successive times when Mercury is between Venus and the sun.

14. A shoe dealer buys 100 pairs of shoes at $\$ 2.00$ a pair, and sells 75 pairs at $\$ 2.50$ a pair. To sell the remaining shoes he marks them down so as to make $20 \%$ profit on the whole. What price per pair will give this result?

15. $A$ and $B$ can do a piece of work in 10 days, but at the end of 7 days A falls sick and B finishes the work in 5 days. How long would it take each man to do the work?

16. One man can do a piece of work in 5 days which it takes a second man 7 days to do. How long will it take the two men working together?

17. A piece of work can be done by A in 10 days, and by B in 12 days. If $\mathbf{A}$ starts the work and works alone for $\mathbf{4}$ days, how long will it take $\mathbf{A}$ and $\mathrm{B}$ working together to complete it?

18. A pound of a certain alloy contains two parts of silver to three parts of copper. How much copper must be melted with this alloy to obtain one which contains three parts of silver to seven of copper?

19. When weighed in water, silver loses 0.09 of its weight, and copper 0.11 of its weight. If a 12-pound mass of silver and copper loses 1.17 pounds, find the weight of the silver and of the copper in the mass. 
20. The crown of Hiero of Syracuse, which was part gold and part silver, weighed 20 pounds, and lost $1 \frac{1}{4}$ pounds when weighed in water. How much gold and how much silver did it contain, if $19 \frac{1}{4}$ pounds of gold and $10 \frac{1}{2}$ pounds of silver each lose 1 pound in water?

21. The admission to an entertainment was $50 \notin$ for adults and $25 \notin$ for children. The proceeds from 125 tickets were $\$ 51.25$. How many adults and how many children were admitted?

22. A tank can be filled by one pipe in 20 minutes, and by another pipe in 30 minutes. How long will it take to fill the tank if both pipes are opened?

23. A tank can be filled by one pipe in 5 hours and emptied by another in 8 hours. If the tank is half full, and both pipes are opened, how long will it take to fill the tank?

25. Remarks on Measurements. This section and the one following contain considerations which are of value to all who make measurements and computations based upon them. In particular, they are important for the statistician. Some of them find application in connection with tables of values of several functions which we shall study.

The operation of making a measurement is counting. It is the determination of the number of units of a certain kind required to equal a magnitude of the same kind.

In measuring a length with a scale graduated to tenths of an inch, the length is recorded to the nearest tenth. For instance, if a line appears to be $7.8 \frac{1}{4}$ inches, the length is recorded as 7.8 inches; if it is about $7.8 \frac{3}{4}$ inches it is recorded as 7.9 inches. If the length is so near the mid point of a subdivision that it is impossible for the observer to decide which point of division is nearer it is customary to choose the submultiple which is even. Thus $7.8 \frac{1}{2}$ inches is recorded as 7.8 inches, and $7.3 \frac{1}{2}$ as 7.4 inches. An experienced observer determines the length to the hundredth part of an inch by making a mental subdivision of the tenth of an inch. If a higher degree of accuracy is desired, instruments which measure more precisely are employed, and various indirect methods of measurement are used.

If a change of units is made the figures of a measurement may be preceded or followed by ciphers which are not deter- 
mined by observation. Thus the same length may be recorded as 0.054 meter, 54 millimeters, or 54,000 microns. The digits 5, 4 are called the significant figures. The ciphers are non-significant.

Ciphers in a number are not always non-significant. A length between the limits 7.795 inches and 7.805 would be recorded as 7.80 inches.

The distance to the sun is sometimes given as $93,000,000$ miles. This statement is ambiguous as there is nothing to indicate how many of the figures are significant and how many of the ciphers merely serve to locate the decimal point with reference to the unit employed. If all the ciphers were significant then the statement would mean that the distance was between the limits $92,999,999.5$ and 93,000,000.5 miles. If the figures 9,3 alone were significant, then the limits would be $92,500,000$ and 93,500,000 miles. In a notation used to remove such ambiguities, known as the standard form, the sun's distance given to two significant figures would be written $9.3 \times 10^{7}$ miles. If the first three figures were significant the distance in standard form would be $9.30 \times 10^{7}$, which would indicate that the true distance is between 92,950,000 and $93,050,000$ miles.

A number like $\pi$ or $\sqrt{2}$ can be calculated to any degree of accuracy desired, but there is a limit to the number of significant figures which can be obtained by measurement.

The relative error in a measurement is the ratio of the possible error to the measurement. It is usually expressed as a percentage.

The relative errors in the two distances $9.3 \times 10^{7}$ and $9.30 \times 10^{7}$ are respectively

$$
\frac{0.05}{9.3}=0.005=0.5 \% \text { and } \quad \frac{0.005}{9.30}=0.0005=0.05 \% \text {. }
$$

This illustrates the fact that the relative error depends upon the number of significant figures and not upon the position of the decimal point. Let $x$ be a number obtained by measurement, expressed in terms of the smallest unit used. Then $x$ 
differs from the true value by not more than $\frac{1}{2}$, and the possible relative error is $\frac{\frac{1}{2}}{x}=\frac{1}{2 x}$. The possible relative error for different numbers of significant figures is given in the following table.

Number of significant figures

One

Two

Three

Four

\section{Range of} numbers

$1, \ldots, 9$

$10, \ldots, 99$

$100, \ldots, 999$

$1000, \ldots, 9999$
Range of possible relative error.

$$
\frac{1}{2} \text { to } \frac{1}{18} \text { or } 50 \% \text { to } 5.56 \%
$$$$
\frac{1}{20} \text { to } \frac{1}{198} \text { or } 5 \% \text { to } 0.505 \%
$$$$
\frac{1}{200} \text { to } \frac{1}{1998} \text { or } 0.5 \% \text { to } 0.050 \%
$$

$$
\frac{1}{2000} \text { to } \frac{1}{19998} \text { or } 0.05 \% \text { to } 0.005 \%
$$

The illustrations following show how great an accuracy has been obtained in some measurements. The ratio of the mean solar day to the sidereal day has been ascertained to the eighth place of decimals. Balances have been constructed which respond to one part in a million. The accuracy attained in measuring a base line of a survey is usually about one part in 60,000 , or an inch in a mile, but accuracy to one part in a million is claimed for some surveys.

Three significant figures are sufficient for most engineering and commercial calculations, four significant figures for most physical and chemical computations, while some astronomical and geodetic calculations require measurements to six or seven significant figures.

26. Possible Errors in Arithmetic Calculations. Abridged Multiplication and Division. As magnitudes determined by measurements are not exact, it is important to be able to estimate the possible error, or the limit of error, in a result calculated from such measurements.

Theorem 1. The possible error of the sum or difference of two measurements is equal to the sum of the possible errors of the individual measurements.

If $a$ and $b$ are two measurements with possible errors $\pm \Delta a$ 
and $\pm \Delta b$, then the correct value of the sum of the measurements lies between the limits

$$
\text { and } \quad \begin{aligned}
& (a+\Delta a)+(b+\Delta b)=(a+b)+(\Delta a+\Delta b), \\
& (a-\Delta a)+(b-\Delta b)=(a+b)-(\Delta a+\Delta b) .
\end{aligned}
$$

Hence the possible error of the sum $a+b$ is $\Delta a+\Delta b$.

The case for the difference of two measurements follows from the fact that the correct value of the difference lies between the limits

$$
(a+\Delta a)-(b-\Delta b)=(a-b)+(\Delta a+\Delta b),
$$

and $\quad(a-\Delta a)-(b+\Delta b)=(a-b)-(\Delta a+\Delta b)$.

Notice that in either case the possible error is half the difference of the limits.

If several numbers representing measurements of different degrees of precision are added together, the significant figures are retained in the numbers and the sum is rounded off to the number of significant figures in the least accurate of the individual numbers.

Example 1. The value of the imports of Alaska from the United States in 1901, 1903, 1904 are reported as in the table. Find the sum.

The first number is rounded off to thousands so that the sum should be rounded off to thousands. This may be done as the numbers are added.

$$
\begin{array}{r}
\$ 13,457,000 \\
9,509,701 \\
10,165,110 \\
\$ 33,132,000
\end{array}
$$

Theorem 2. The possible relative error of the product or quotient of two measurements is approximately equal to the sum of the relative errors of the individual measurements.

The product will lie between the limits

$$
\text { and } \quad \begin{aligned}
(a+\Delta a)(b+\Delta b) & =a b+\Delta a \Delta b+a \Delta b+b \Delta a, \\
(a-\Delta a)(b-\Delta b) & =a b+\Delta a \Delta b-a \Delta b-b \Delta a .
\end{aligned}
$$

The possible error in the product is assumed to be, approximately, half the difference between these limits, so that the error in the product is

$$
\Delta a b=a \Delta b+b \Delta a .
$$


Hence the possible relative error, that is, the ratio of the possible error to the product, is

$$
\frac{\Delta a b}{a b}=\frac{\Delta b}{b}+\frac{\Delta a}{a} \text {. }
$$

The relative error of $a b$ is therefore equal to the sum of the relative errors of $a$ and $b$.

The case for the quotient is proved similarly, assuming that the possible error is half the difference of the limits

$$
\frac{a+\Delta a}{b-\Delta b} \text { and } \frac{a-\Delta a}{b+\Delta b}
$$

with the further assumption that $(\Delta b)^{2}$ is small in comparison with $\Delta a$ and $\Delta b$ and may be neglected.

Since the possible relative error of a product is greater than that of either factor, there can be no more significant figures in the product than in the factor with the smaller number of significant figures.* In practice, factors are rounded off to the same number of significant figures before multiplying, and only that number of significant figures retained in the product.

In division, the dividend, divisor, and quotient are treated. similarly.

Abridged methods of multiplication and division, which do away with the labor of retaining non-significant figures during either operation, are illustrated in the examples following.

ExAMPLE 2. Find the circumference of a circle whose measured diameter is 45.38 inches.

Since the diameter is given to four significant figures, the value of $\pi$ should be taken to four significant figures. This value is $\pi=3.142$.

(a)

\begin{tabular}{r|l}
45.38 \\
3.142 \\
$\overline{9} \mid 076$ \\
181 & 52 \\
453 & 8 \\
13614 & \\
\hline 142.58 & $\overline{396}$
\end{tabular}

(b) 45.38

3.142

$\overline{13614}$

4538

18152

$\frac{9}{142.58} \frac{076}{396}$ (c) 45.38

3.142

$\overline{13614}$

454

1

$$
\overline{142.59}
$$

The value of the circumference, $\pi d$, to four significant figures is therefore 142.6.

* There may be less. For example, if two factors with three significant figures are close to 100 , such as 123 and 114, the possible relative 
Using ordinary multiplication first we have (a), which gives the arithmetically correct product. The last partial product contributes most to the significant figures of the product, and may be written first, as in (b), where the order of all the partial products is reversed. The figures to the right of the vertical lines in (a) and (b) pertain to the non-significant figures of the product, and may be discarded, as in (c), which shows the abridged multiplication.

In (c), the partial product obtained from the first figure on the left of the multiplier, 3 , is entered first. Then the last number on the right of the multiplicand, 8 , is canceled, and the second figure of the multiplier, 1, is used, the amount carried over from the canceled figure being added in. The other partial products are rounded off similarly.

Example 3. Find the density of a mass of 7.643 grams whose volume is 3.564 cubic centimeters.

To obtain the density divide the mass by the volume. As there are four significant figures in both dividend and divisor, neither need be rounded off before the division is performed, and the quotient should be obtained to four significant figures.

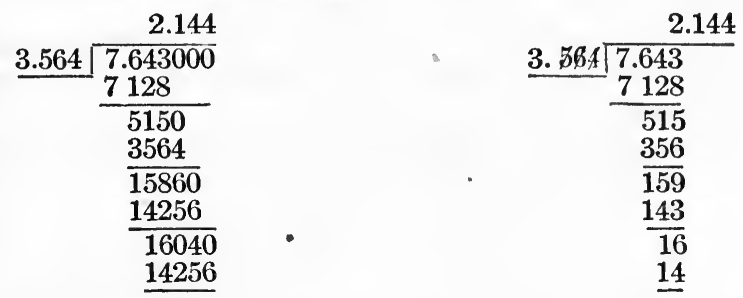

Ordinary long division, given on the left, shows that the density is 2.144 .

The abridged division is shown on the right. After the first figure in the quotient is obtained, instead of adding a cipher to the dividend, the last figure of the divisor, 4 , is canceled. The next partial product is rounded off in accordance error in each is about $0.5 \%$ (by the table in the preceding section), and the possible relative error in the product is therefore approximately $1 \%$, which indicates that only two significant figures should be retained in the product. 
with the amount carried over from the multiplication of the canceled number by the second digit in the quotient, etc. Ordinarily, in finding the partial products it is sufficient to consider only the nearest canceled figure of the divisor, but it is sometimes necessary to consider two canceled figures to determine the amount to be carried over.

\section{EXERCISES}

1. Write the following numbers in standard form and determine the number of significant figures and the percentage of error in each: 3.1416, $0.00732,259.34,678943,0.0020$.

2. The value of $\pi$ to nine figures is 3.14159265 . Round off the value to one significant figure; two; three; four; five. Determine the number of significant figures and the percentage of error of the approximations $3 \frac{1}{7}$ and ${ }_{11}^{3} \frac{5}{3}$. Correct such of the following rounded off values of $\pi$ as are incorrect: $3.141,3.15,3.14160$.

3. Add the following numbers obtained by measurements: 3.4785 , $16.743,253.78,36.583$.

4. The dimensions of a rectangle found by measurement are 24.78 inches and 19.8 inches. Find the area and determine the number of significant figures and the relative error in the area.

5. The diameter of a circle is found by measurement to be $\mathbf{1 5 1 . 6}$ millimeters. Find the circumference. Is $3 \frac{1}{2}$ a sufficiently accurate approximation for $\pi$ in this instance?

6. If one square meter is equivalent to 1.196 square yards, to how much are 5 square meters equivalent? If 5 square meters are equivalent to 5.98 square yards, to what is one square meter equivalent?

7. If 1 centimeter is equivalent to 0.3937 inches, convert a measurement of 3.85 inches to centimeters. Convert 42.83 centimeters to inches.

8. If the length of the year is 365.24 days and the average distance of the earth from the sun is $9.31 \times 10^{7}$ miles, find approximately the velocity of the earth in miles per hour, assuming that the orbit is a circle with the sun at the center.

27. Empirical Data Problems. It is frequently possible to measure corresponding pairs of values of two related variables when the law connecting them is unknown. An important problem presented by such empirical data is to determine a law which represents approximately the relation between the two variables. 
If the pairs of values be plotted, it may happen that the points so obtained lie very nearly on a straight line. If such is the case, it is reasonable to assume that the graph of the relation is a straight line, and hence that the relation connecting the two variables is a linear equation.

When graphical methods are being used, all that is needed is the graph of the relation. Any straight line which passes through or near to each of the points will serve approximately as the graph. A method frequently used by engineers to get the line giving the best approximation is to stretch a rubber band over two pins stuck in the drawing board, and move the pins about until the stretched band appears to make the average distance from the band to each of the points as small as possible.

When algebraic methods are employed, a method of obtaining the equation of a line which answers well as the graph is illustrated in the following examples.

Example 1. In an experiment dealing with friction of wood on wood, a block of wood with various weights on it was placed on a horizontal board. A string fastened to the block ran over a pulley at the end of the board, and

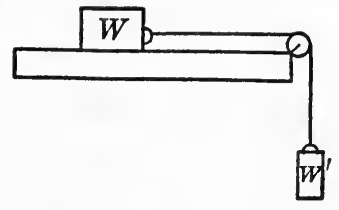

FIg. 44. a pan was tied to the hanging end. Weights were placed in the pan until the block was just on the point of moving. Using $W$ to represent the combined weight of the block and the weights on it, and $W^{\prime}$ for the

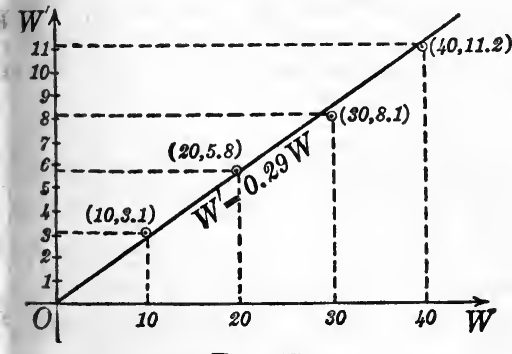

Fig. 45. weight of the pan and of the weights in it, corresponding values of $W$ and $W^{\prime}$ were found as indicated in the table,

$\frac{W}{W^{\prime}} \mid \begin{array}{rrrr}10, & 20, & 30, & 40 \\ 3.1, & 5.8, & 8.1, & 11.2\end{array}$

the unit of weight being the gram. Determine approximately the relation between $W$ and $W^{\prime}$.

Plot the points whose coördinates are the pairs of values of $W$ and $W^{\prime}$, using values of $W$ as abscissas. The four points obtained lie very nearly on a straight line through the origin, and hence we assume that the graph of the relation is a straight line through the origin. 
(That the graph ought to pass through the origin is clear, for if $W=0$, so also must $W^{\prime}=0$.) The required relation must therefore be of the form $W^{\prime}=m W$, where $m$ is the slope of the line.

The slopes of the lines joining the origin to each of the four points are respectively

$$
\frac{3.1-0}{10-0}=0.31 ; \frac{5.8}{20}=0.29 ; \frac{8.1}{30}=0.27 ; \frac{11.2}{40}=0.28 \text {. }
$$

From these values it appears that $m$ may have any value between 0.27 and 0.31 , and the line $W^{\prime}=m W$ would be a fair approximation to the graph required. We shall choose as a good value of $m$ the average of the four values of $m$, namely,

$$
m=\frac{1}{4}(0.31+0.29+0.27+0.28)=\frac{1.15}{4}=0.29 .
$$

In dividing 1.15 by 4 , the second decimal figure is 8 , but the quotient is nearer to 0.29 than to 0.28 ; hence the former value is chosen.

Hence the relation desired is represented approximately by the equation

$$
W^{\prime}=0.29 W \text {. }
$$

The accuracy with which this equation represents the given data may

\begin{tabular}{|c|r|r|r|c|}
\hline$W$ & $W^{\prime}$ & $0.29 W$ & Error & $\begin{array}{c}\% \text { of } \\
\text { error }\end{array}$ \\
\cline { 1 - 3 } 10 & 3.1 & 2.9 & -0.2 & -6.4 \\
20 & 5.8 & 5.8 & 0.0 & 0 \\
30 & 8.1 & 8.7 & +0.6 & +7.4 \\
40 & 11.2 & 11.6 & +0.4 & +3.5 \\
\hline
\end{tabular}
be determined by constructing the table adjoined. The first two columns give the observed values of $W$ and $W^{\prime}$, the third, the values of $W^{\prime}$ computed by means of equation (1) from the observed values of $W$; the fourth, the error in the computed values of $W^{\prime}$, which are obtained by subtracting the second column from the third; and the fifth, the percentage of error. The percentage of error is found by dividing the error by the observed value of $W^{\prime}$. Thus $0.2 / 3.1=0.064$, or $6.4 \%$.

Note the part played by mathematics in this illustration of the scientific method. The given data are obtained by observation. The principles of graphic representation enable us to put the data in a form which makes reasonable the hypothesis that the graph of the law under investigation is a straight line, and that the law is represented by a linear equation. By deductive processes we determine the numerical values of the coefficients of the equation, and the accuracy of the representation of the given data by the equation found. The verification 
would consist, in part, of repeating the experiment a number of times, varying the values of $W$, and seeing if the law remained approximately the same. But a more satisfactory verification would be to deduce from this law some other law which could be verified by an experiment of a different sort. This will be done in a later section.

The law obtained in Example 1 can be stated in a more convenient form. The force tending to make the block slide is equal to $W^{\prime}$, because the tension of the string is the same at all points. This force acts in the direction of the surfaces in contact, and when motion is about to begin, it is numerically equal to the force of friction which is preventing the block from moving. $W$ is the pressure on the board acting perpendicularly to the surfaces in contact. Hence the result obtained may be stated as follows, using the language of Section 21:

The friction of wood on wood varies as the pressure perpendicular to the surfaces in contact.

If different kinds of wood, or other substances, be used in the experiment, the value of $m$ obtained would not be the same. But extensive experiments have shown that we are reasonably justified in stating the law:

When motion is about to take place, the friction between two surfaces varies as the pressure perpendicular to the surfaces.

Hence if $F$ denotes the force of friction and $P$ the perpendicular pressure

$$
F=m P \text {. }
$$

The constant $m$ is called the coefficient of friction, and it is equal to the ratio of the friction to the pressure perpendicular to the surfaces in contact. The coefficient of friction for the block and board in Example 1 is 0.29 .

Example 2. In an experiment with a block and tackle, the pull $P$ necessary to raise a weight $W$, both measured in pounds, was found for

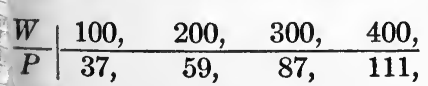
each of the values of $W$ in the table. Find approximately the equation expressing $P$ as a function of $W$.

Plotting the points representing the pairs of numbers in the table, using values of $W$ as abscissas, it is seen that they lie very nearly on a 
straight line. This line ought not to pass through the origin, because if $W=0$, a certain force $P$ is necessary to raise the lower block. Hence $P$ is approximately a linear function of $W$ of the form

$$
P=m W+b .
$$

A good value of $m$ is the average of the slopes of the lines determined by each pair of points.

Denoting the points by $A, B, C, D$ respectively, the slope of the line $A B$ is

$$
m=\frac{59-37}{200-100}=0.22 .
$$

In like manner we find the value of $m$ for each of the lines determined by two of the points $A, B, C, D$. These values are given in the table following.

$$
\frac{\text { Line }}{m} \mid \begin{array}{cccccc}
A B, & A C . & A D, & B C, & B D, & C D \\
\hline 0.22, & 0.25, & 0.25, & 0.28, & 0.26, & 0.24
\end{array}
$$

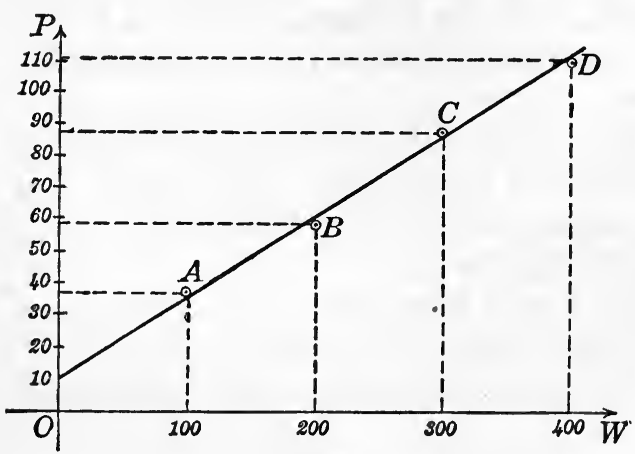

FIg. 46.

The average value of $m$ is found to be $m=$ 0.25 . Hence the function required has the form, approximately,

$$
P=0.25 W+b \text {. }
$$

If the graph of (2) passes through the point $A$, the coördinates of $A$ must satisfy (2), and hence

$$
37=0.25 \times 100+b \text {, }
$$
whence

$$
b=12 .
$$

In like manner, we find the value of $b$ on the assumption that each of the points $B, C, D$, lies on the graph of (2). The results are given in the table.

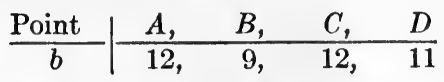

The average of these values of $b$ is $b=11$. Substituting this value in (2), the function required is, approximately,

$$
P=0.25 W+11 \text {. }
$$

The accuracy with which this equation represents the observed data is shown by the table below. The first two columns give the observed values of $W$ and $P$. The third column gives the values of $P$ computed 
from the observed values of $W$ by means of (3). The fourth gives the error in the computed value of $P$ as compared with the observed value, while the last column gives the percentage of error, which is obtained from the second and fourth columns.

\begin{tabular}{|c|c|c|c|c|}
\hline$W$ & $\begin{array}{c}\text { Observed } \\
P\end{array}$ & $\begin{array}{c}\text { Computed } \\
P\end{array}$ & Error & $\begin{array}{c}\text { Per cent } \\
\text { of error }\end{array}$ \\
\cline { 1 - 2 } 100 & 37 & 36 & -1 & -2.7 \\
200 & 59 & 61 & +2 & +3.3 \\
300 & 87 & 86 & -1 & -1.1 \\
400 & 111 & 111 & 0 & 0 \\
\hline
\end{tabular}

A property of a linear function is illustrated in the table, which gives \begin{tabular}{r|r|r|r}
$\frac{\Delta x}{1}$ & $\frac{x}{0}$ & $\frac{y}{3}$ & $\frac{\Delta y}{}$ \\
1 & & & 2 \\
1 & 1 & 5 & \\
1 & 3 & 9 & 2 \\
1 & & & 2
\end{tabular} values of $x, y, \Delta x$, and $\Delta y$ for the function $y=2 x+3$. The values of $x$ being such that the successive values of $\Delta x$ are equal, it appears that the successive values of $\Delta y$ are also equal. That this is always the case follows from the fact that the value of $\Delta y / \Delta x$ is always the same for points on a straight line.

Now suppose, as in the examples above, the points representing a given table of values appear to lie on a straight line. To test the accuracy of this assumption, find the successive values of $\Delta x$ and $\Delta y$. If the values of $\Delta x$ are equal, and if those of $\Delta y$ are nearly equal, we are justified in believing that $y$ is indeed a linear function of $x$.

The labor involved in computing an average may be lessened by means of the following rule: Assume a number $x$ which appears to be a reasonable "guess" for the average desired. Subtract $x$ from each of the given numbers, find the average of the differences, and add it to $x$, paying due regard to signs throughout.

85

70

80

90

75

95

$$
8
$$$$
\frac{6 \mid \frac{-30}{-15}}{-2.5}
$$

0

- 15 numbers in the first column, the value of $x$ being

- 5 chosen as 85 . The differences obtained by subtracting 85 from each of the numbers are given in

- 10 the second and third columns. Their. average is obtained by adding them and dividing by 6 , the number of numbers to be averaged, and is found to be -2.5 . This must be added to 85 , which gives 82.5 as the average.

The advantage of the method lies in the fact that $85+(2.5)=82.5$ it involves only relatively small numbers, which decreases the probability of error, and also enables one to handle simple cases mentally. 
The proof of the correctness of the rule, for 4 numbers $a, b, c, d$, follows from the fact, which is readily verified, that the equation

$$
\frac{a+b+c+d}{4}=x+\frac{(a-x)+(b-x)+(c-x)+(d-x)}{4}
$$

is true no matter what the value of $x$ may be.

\section{EXERCISES}

1. In an experiment on friction of metal on metal, values of $W$ and $W^{\prime}$ (see Example 1) were found as in the following table. Find the equation connecting $W$ and $W^{\prime}$. What is the value of the coefficient of friction?

$$
\frac{W}{W^{\prime}} \mid \begin{array}{lllr}
10, & 20, & 30, & 40, \\
\hline 1.9, & 4.2, & 6.1, & 7.8,
\end{array}
$$

2. In an experiment on the friction of leather on metal, values of $W$ and $W^{\prime}$ (see Example 1) were determined as in the table. Find the equa$W \mid \begin{array}{lll}10, \quad 20, \quad 30, \quad 40, \\ W^{\prime}\end{array}$ $\overline{W^{\prime}} \mid \frac{1}{6.2,11.7,17.9,24.3,}$ the coefficient of friction?

3. The table gives corresponding values of $P$ and $W$ (see Example 2) $\frac{W}{P} \mid \begin{array}{cccc}100, & 200, & 300, & 400, \\ 41.2, & 74.6, & 107.6, & 141.5,\end{array} \begin{aligned} & \text { obtained in an experiment on a block } \\ & \text { and tackle. Find the relation be- } \\ & \text { tween } P \text { and } W \text {. }\end{aligned}$

4. In an experiment on a system of pulleys in which all but one were

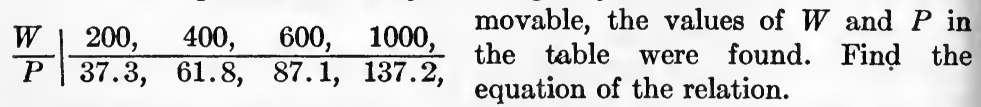

5. The table gives the length, in inches, of an iron rod at different tem-

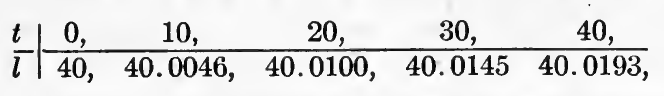
peratures, in degrees $l$ 40, 40.0046, length $l$ at any temperature $t$. (Assume that the length when $t=0^{\circ}$ is exact.)

6. The table gives the length in centimeters of a brass rod at various

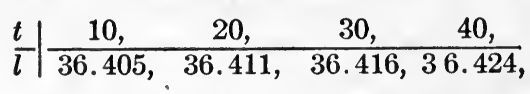
temperatures in degrees Centigrade. Find the length of the rod at $0^{\circ}$ Centigrade, and the rate of change of the length of the rod with respect to the temperature.

7. Find the volume of alcohol at any temperature from the table

$$
\frac{t}{v} \mid \begin{array}{cccc}
10, & 20, & 40, & 60, \\
\hline 101.1, & 101.9, & 103.9, & 106.0,
\end{array}
$$

8. Determine the density (see page 61) of brass from the table, which

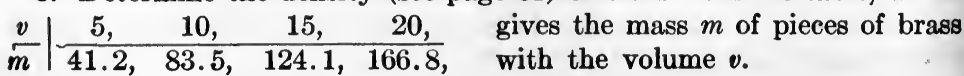


9. In order to graduate a spring balance, the extension $e$ in inches was measured for different weights $w$, w $\quad 2, \quad 5, \quad 7, \quad 10, \quad$ as in the table. Find to hundredths of an inch the extension due to one pound.

10. The pressure $p$, per square foot, at various depths $d$, in feet, under water is given in the table. Find the d $20, \quad 40,60, \quad 80, \quad$ pressure at a depth of one foot, and the $\frac{d}{p} \mid 1240,2500,3760,4980, \quad$ rate of change of the pressure with respect to the depth.

11. Barometer readings $b$ were made simultaneously at various heights $b \mid 29.00,28.88,28.79,28.45$, th in feet, above a certain point. $\bar{h} \mid \frac{29.00,28.88,28.79,28.45,}{0} \quad 98, \quad 201, \quad 495, \quad$ Find the law giving the change in in barometer for small changes in altitude.

\section{MISCELLANEOUS EXERCISES}

1. Construct the line through the point $(3,2)$ whose slope is $-\frac{1}{3}$, and find its equation.

2. Construct the line determined by the points $(2,1)$ and $(4,5)$, and find its equation.

3. Construct the graph of the equation $y=2 t+3$. Discuss and interpret the graph if $y$ represents the distance from a station to a moving body at the time $t$; if $y$ represents the velocity of a moving body at the time $t$.

4. The volume of a cake of ice is proportional to the volume of the water from which the ice was obtained. If 55 cubic centimeters of ice are made by freezing 50 cubic centimeters of water, how much water will be required to make 500 cubic centimeters of ice? Interpret the solution graphically.

5. A train is moving at the rate of 35 miles an hour when the brakes are applied. Six seconds later the train's rate is 17 miles an hour. Construct the graph of the velocity as a function of the time, and find its equation. How long after the brakes are applied will the train come to rest? Check the result graphically.

6. A 12-pound specimen of copper ore lost 2.5 pounds when weighed in water. How much copper did it contain if 8.9 pounds of copper and 2.7 pounds of the material with which the copper was combined each lost 1 pound when weighed in water?

7. How many cubic centimeters of cork, density 0.3 , must be combined with 75 cubic centimeters of steel, density 0.9 , in order that the combined mass will just float in water? Hint: The density of the combination must equal that of water, which is unity. 
8. Specific gravity is the ratio of the density of a body to that of another body taken as a standard. Water which is taken as the standard has a density of unity in the metric system. Hence the mass of a substance is equal to the specific gravity times the volume.

How much water must be added to 25 cubic centimeters of concentrated hydrochloric acid, specific gravity 1.20 , to reduce the specific gravity to 1.12 ?

9. How much water must be added to 30 cubic centimeters of ammonia, specific gravity 0.90 , to raise the specific gravity to 0.96 ?

10. It is desired to reduce the specific gravities of quantities of sulphuric acid, specific gravity 1.84, and of nitric acid, specific gravity 1.42 . How much water must be added to 80 cubic centimeters of each to reduce the specific gravities to 1.18 and 1.20 respectively?

11. What mass of hydrogen (molecular weight 2 ) will be displaced by 10 grams of zinc (molecular weight 65) acted upon by hydrochloric acid if the amounts exchanged are proportional to the molecular weights?

12. Find the money value of pure gold in a $\$ 20$ gold piece, if one ounce of gold is worth $\$ 20.66$ and the coin weighs 516 grains and is $\frac{9}{10}$ pure.

13. A tank can be filled by one pipe in 3 hours, and emptied by a second in 2 hours, and by a third in 4 hours. How long will it take to empty the tank when it is full if all the pipes are opened?

14. The report of a gun was heard in 3 seconds at a place 3189 feet distant, toward which the wind was blowing; and in 2 seconds at a place 2074 feet distant, from which the wind was blowing. Find the velocity of sound and of the wind.

15. A railway passenger observed that a train moving in the opposite direction passes him in 2 seconds, but when moving in the same direction it passed him in 30 seconds. Compare the rates of the trains.

16. Two cities are 39 miles apart. If A leaves one city two hours earlicr than $B$ leaves the other, they meet two and a half hours after B starts. Had B started at the same time as A, they would have met three hours after they started. How many miles an hour does each walk?

17. The accuracy with which one can weigh an object on a balance depends on the number of spaces on the scale which the pointer moves $\frac{W}{D} \mid$\begin{tabular}{cccc}
5, & 25, & 50, & 100, \\
\hline 1.99, & 1.72, & 1.76, & 0.95,
\end{tabular} when a small weight is added. The table gives the deflection, $D$, of the pointer with a weight $W$ in the pan when one milligram is added to $W$. Determine $D$, approximately, as a function of $W$. 


\section{CHAPTER III}

\section{ALGEBRAIC FUNCTIONS}

28. Introduction. In this chapter we shall study the properties (Sections 10 to 13) of certain types of algebraic functions (Section 14).

Among these types are the quadratic function, $a x^{2}+b x+c$, which occurs in the solution of quadratic equations, and the function $a x^{n}$. The latter is an integral rational function if $n$ is a positive integer, a rational fractional function if $n$ is a negative integer, and an irrational function if $n$ is a fraction. Other types are the linear fractional function, $(a x+b) /(c x+d)$, and polynomials, which are studied in order to obtain a method for solving equations of higher degree than the second, an extension of the solution of linear and quadratic equations.

These functions find frequent application in many fields. For example, the quadratic function appears in the theory of falling bodies, and among the laws which can be represented by a function of the type $a x^{n}$ are Newton's law of gravitation, and Boyle's law connecting the pressure and volume of a gas.

In the study of quadratic functions we shall proceed from special cases to the general case by methods which are important in other connections.

29. Graph of $x^{2}$. Since $(-x)^{2}=x^{2}$, the graph is symmetrical with respect to the $y$-axis (Theorem 1A, page 23). Hence the $y$-axis

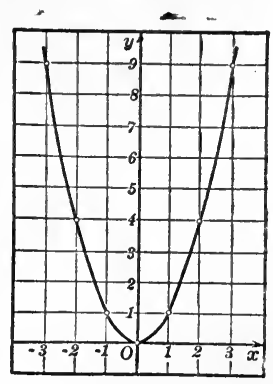

FIG. 47.

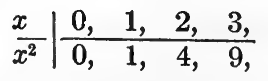
is called an axis of symmetry. The table of values need include only positive values of $x$. 
It is readily seen that the intercepts are $x=0$ and $y=0$. Hence the curve passes through the origin, but does not cut the axes elsewhere.

Since $x^{2}$ is positive if $x$ is real, no part of the curve lies below the $x$-axis.

As $x$ increases, so also does $x^{2}$, and hence the curve broadens out as it rises. If $x>1, x^{2}$ increases more rapidly than $x$, so that, to the right of $x=1$, the curve rises more rapidly than it broadens out. The origin is a minimum point.

30. Graphs of $a x^{2}$ and $a f(x)$. Consider the graph of the function $2 x^{2}$. The ordinate of any point on the graph is twice

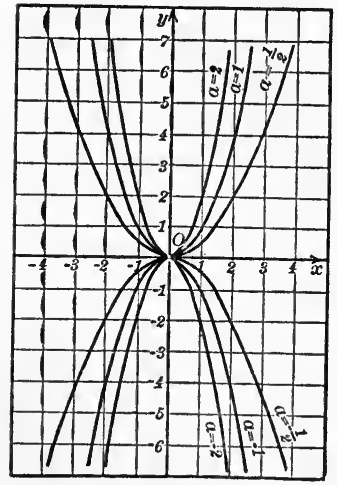

FrG. 48. that of the point with the same abscissa on the graph of $x^{2}$. Hence the graph of $2 x^{2}$ may be plotted by doubling the ordinates of a number of points on the graph of $x^{2}$, and drawing a smooth curve through the points so obtained.

In like manner, the graph of $3 x^{2}$ may be obtained by trebling the ordinates of points on the graph of $x^{2}$, of $\frac{1}{2} x^{2}$ by bisecting them, etc.

The ordinates of points on the graph of $-2 x^{2}$ are numerically twice those of points on the graph of $x^{2}$, but as they are negative, the points on the graph lie below the $x$-axis. The graphs of $2 x^{2}$ and $-2 x^{2}$ are symmetrical to each other with respect to the $x$-axis.

From the method of constructing these graphs we see that

The $y$-axis is an axis of symmetry of the graph of $a x^{2}$. The curve runs up or down, and the origin is a minimum or maximum point, according as $a$ is positive or negative.

If $a$ is positive, the graph of $a x^{2}$ rises more or less rapidly than that of $x^{2}$, for which $a=1$, according as $a$ is greater or less than unity. The larger the value of $a$, the more rapidly the curve rises, and the smaller the value of $a$ is, the less rapidly it rises.

Any one of these curves is called a parabola. The axis of symmetry of a parabola is called its axis. 
The reasoning employed above may be used to prove the

Theorem. The graph of af $(x)$ may be obtained by multiplying by a the ordinates of points on the graph of $f(x)$. Corresponding points on the two graphs lie on the same or opposite sides of the $x$-axis according as $a$ is positive or negative.

If $a=-1$ the graph is symmetrical to that of $f(x)$ with respect to the $x$-axis.

This theorem is the second one we have considered belonging to the set of relations between pairs of functions and their graphs (see the last paragraph but one on page 43).

31. Translation of the Coördinate Axes. Consider a system of coördinate axes with the origin $\mathrm{O}$, and a second system, parallel to the first, with origin $\mathrm{O}^{\prime}$. Replacing the first system by the second is called translating the axes. By the equations for translating the axes we mean equations which express the coördinates of a point referred to the first, or old, axes in terms of the coördinates of the same point referred to the second, or new, axes.

Let the old and new coördinates of any point $P$ be respectively $(x, y)$ and $\left(x^{\prime}, y^{\prime}\right)$, and let the old coördinates of the new origin, $\mathrm{O}^{\prime}$, be $(h, k)$. From the figure we readily obtain

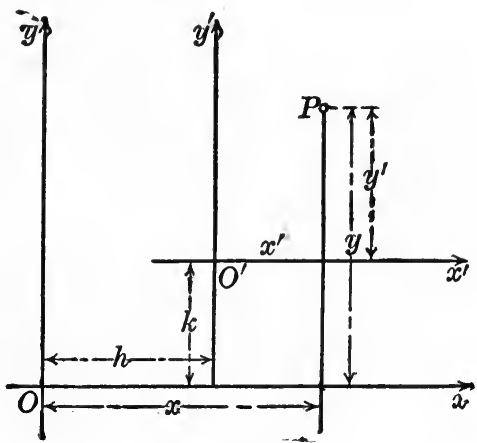

FIG. 49.

Theorem 1. The equations for translating the axes are

$$
\left.\begin{array}{l}
x=x^{\prime}+h, \\
y=y^{\prime}+k,
\end{array}\right\}
$$

where $(h, k)$ are the coördinates of the new origin.

Suppose that the graph of an equation in $x$ and $y$ has been plotted. To find the equation of this graph referred to new axes parallel to the old axes we substitute in the given equation the values of $x$ and $y$ given by (1). The following examples illustrate the utility of the theorem. 
Example 1. Given the equation .

$$
y=x^{2}-6 x+13
$$

translate the axes so that the new origin is the point $(3,4)$ and find the equation in the new coördinates which has the same graph as (2). Plot the graph, and state its most noteworthy properties.

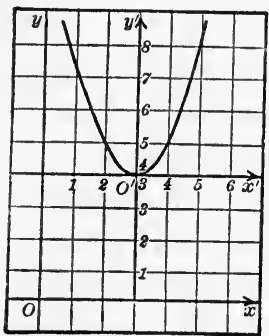

FIg. 50.

By Theorem 1 we have

Substituting these values in (2), we have

$$
y^{\prime}+4=\left(x^{\prime}+3\right)^{2}-6\left(x^{\prime}+3\right)+13 \text {. }
$$

Removing the parentheses,

or

$$
\begin{gathered}
y^{\prime}+4=x^{\prime 2}+6 x^{\prime}+9-6 x^{\prime}-18+13, \\
y^{\prime}=x^{\prime 2} .
\end{gathered}
$$

The graph of (5), plotted on the new axes, is the same as the graph of (2), referred to the old axes. But the graph of (5) is a parabola which is easily plotted on the new axes. It is shown in the figure. This curve is also the graph of (2) when plotted on the old axes. From it we see that the axis of symmetry of the parabola, the $y^{\prime}$-axis, is the line $x=3$, and that the minimum point -is the new origin $(3,4)$.

In this example it turned out that the equation obtained by translating the axes was much simpler than the given equation. The question arises: If an equation is given, how can we determine how to move the axes so as to obtain a simpler equation with the same graph? The method of doing this is illustrated in

Example 2. Simplify the equation

$$
y=2 x^{2}-8 x+11
$$

by translating the axes, and construct the graph.

First solution. Substituting in (6) the values of $x$ and $y$ given by (1), we get

whence

$$
\begin{aligned}
y^{\prime}+k & =2\left(x^{\prime}+h\right)^{2}-8\left(x^{\prime}+h\right)+11 \\
& =2 x^{\prime 2}+4 h x^{\prime}+2 h^{2}-8 x^{\prime}-8 h+11,
\end{aligned}
$$

This equation will be very simple if the coefficient of $x^{\prime}$ and the constant term are zero, that is, if

$$
\left.\begin{array}{r}
4 h-8=0 \\
2 h^{2}-8 h+11-k=0
\end{array}\right\}
$$


Solving these equations for $h$ and $k$, we get

$$
\text { and } \left.\quad \begin{array}{l}
h=2, \\
k=2 \times 2^{2}-8 \times 2+11=3 .
\end{array}\right\}
$$

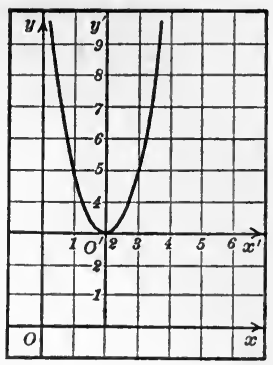

Fig. 51.

Second solution. The form of the given equa-

tion (6) may be changed as follows:

Hence, if the axes be translated so that the origin is moved to the point $(2,3)$, equation (6) assumes the simpler form (10). Plotting the graph of (10) on the new axes we get the curve in the figure.

$$
y^{\prime}=2 x^{\prime 2} \text {. }
$$

$$
\begin{aligned}
y & =2 x^{2}-8 x+11 \\
& =2\left(x^{2}-4 x\right)+11 \\
& =2\left(x^{2}-4 x+4-4\right)+11 \\
& =2\left(x^{2}-4 x+4\right)-8+11 \\
& =2(x-2)^{2}+3, \\
y-3 & =2(x-2)^{2} .
\end{aligned}
$$

whence

It is seen by inspection of this equation that if we set

and

$$
\left.\begin{array}{l}
x=x^{\prime}+2, \\
y=y^{\prime}+3,
\end{array}\right\}
$$

we will obtain the simpler equation

$$
y^{\prime}=2 x^{\prime 2} \text {, }
$$

which is identical with (10). Equations (11) are the equations for translating the axes so that the origin is moved to the point $(2,3)$ (by Theorem 1).

Let the graph of any function $y=f(x)$ be given. In order to move the $x$-axis up $k$ units, leaving the $y$-axis unchanged, we set $y=y^{\prime}+k$, obtaining $y^{\prime}+k=f(x)$, or

$$
y^{\prime}=f(x)-k \text {. }
$$

The graph of (12) is identical with the given graph, if the equation is plotted on the new axes.

Now suppose that the graph of (12) is plotted on the original axes. It may be obtained by moving the given graph down $k$ units (see the Theorem in Exercise 3, page 19). Hence the graph of (12) may be interpreted in two ways, which are essentially identical since the effect of moving the $x$-axis up $k$ 
units, and erasing the original $x$-axis, is the same as moving the graph down $k$ units and erasing the original curve.

In like manner, if we set $x=x^{\prime}+h$, and leave $y$ unchanged, we get

$$
y=f\left(x^{\prime}+h\right) \text {. }
$$

The graph of this equation is identical with the graph of $f(x)$, if plotted on the original $x$-axis and a new $y$-axis $h$ units to the right of the old. But the effect of moving the $y$-axis $h$ units to the right and erasing the old $y$-axis gives the same figure as moving the curve $h$ units to the left and erasing the old curve. Hence the graph of (13) referred to the original axes may be obtained by moving the graph of $f(x) h$ units to the left.

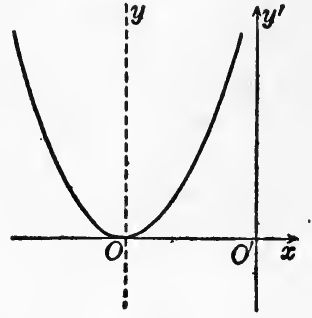

$A$

FIG. 52.

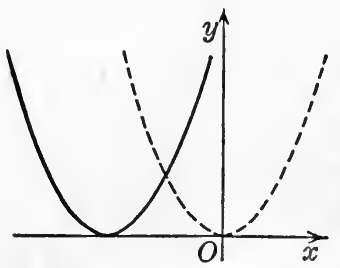

B

In plotting (13) on the original axes it is convenient to write $x$ in place of $x^{\prime}$. We thus obtain

Theorem 2. The graph of $f(x+h)$ may be obtained by moving the graph of $f(x) h$ units to the left. The motion will be to the right if $h$ is negative.

This theorem, for which we find application in later chapters, should be associated with the last paragraph but one on page 43.

\section{EXERCISES}

1. Plot on the same axes the graphs of:
(a) $x^{2}, 3 x^{2}, \frac{1}{3} x^{2},-\frac{1}{3} x^{2}$.
(b) $x^{2}-4 x, 2\left(x^{2}-4 x\right), \frac{1}{2}\left(x^{2}-4 x\right)$.
(c) $x^{2}+3,2\left(x^{2}+3\right), \frac{1}{3}\left(x^{2}+3\right)$.
(d) $(x-2)^{2}, 3(x-2)^{2}, \frac{1}{4}(x-2)^{2}$.

2. Find the value of $a$ if the graph of $y=a x^{2}$ passes through the point $(2,3)$, and construct the graph. 
3. Show that the points $(2,1),\left(3,2 \frac{1}{4}\right)$, and $(4,4)$ lie on one of the parabolas $a x^{2}$.

4. Show that one of the curves $a x^{2}$ passes through any point $P_{1}\left(x_{1}, y_{1}\right)$.

5. Find the average rate of change of $y$ with respect to $x$, for the intervals from $x=0$ to $x=1$, from $x=1$ to $x=2$, from $x=2$ to $x=3$, and for any interval if (a) $y=x^{2}$;
(b) $y=2 x^{2}$;
(c) $y=\frac{1}{2} x^{2}$
(d) $y=a x^{2}$.

6. Translate the axes to the new origin indicated, and construct both pairs of axes and the graph:
(a) $y=x^{2}+3, \mathrm{O}^{\prime}(0,3)$.
(c) $y=-x^{2}, \mathrm{O}^{\prime}(2,-4)$.
(b) $y=x^{2}-4 x, O^{\prime}(2,-4)$.
(d) $y=1 / x, \mathrm{O}^{\prime}(3,2)$.

7. Using Theorem 2 , Section 31 , construct on the same axes the graphs of $x^{2},(x+2)^{2}$, and $(x-3)^{2}$.

8. Construct the graphs of $x^{2}$ and $x^{2}+8 x+16$ on the same axes.

9. On the same axes construct the graphs of $y=x^{2}, y=2 x^{2}, y=(x+2)^{2}$, $y=x^{2}+2$.

10. Simplify the following equations by translating the axes. In each case, construct both pairs of axes and the graph. Determine the axis of symmetry and the maximum or minimum point in the given coordinates.
(a) $x^{2}+6 x+4$.
(c) $2 x^{2}-16 x+35$.
(b) $4 x-x^{2}$.
(e) $-x^{2}+2 x+5$.
(d) $3 x^{2}-12 x+5$.
(f) $-2 x^{2}-12 x-20$.

32. Instantaneous Velocity. If a ball is dropped, its velocity changes continually. An approximate value of what we mean when we speak of its velocity at some given instant is given by the average velocity in an interval of time $\Delta t$ beginning at that instant. The smaller the value of $\Delta t$ is, the more accurate is the approximation. A precise notion of the velocity at an instant is given by the

Definition. The velocity of a body at an instant, or its instantaneous velocity, is the limit * of the average velocity in an interval $\Delta t$ beginning at that instant, as the interval $\Delta t$ approaches zero.

The computation of an instantaneous velocity is illustrated in the following example.

* The limit of a variable is a constant such that the numerical value of the difference between the variable and the constant becomes and remains less than any assigned positive number, however small. 
ExAmple. If a ball is dropped, its distance from the starting point at any time $t$, in seconds, is given by

$$
s=16 t^{2} .
$$

Find the velocity at any time.

The distance $s$ at any time $t$ is given by (1). If the distance changes by $\Delta s$ in the next $\Delta t$ seconds, the distance at the time $t+\Delta t$ will be $s+\Delta s$. These corresponding values of the distance and time must satisfy (1), and hence

$$
s+\Delta s=16(t+\Delta t)^{2}=16 t^{2}+32 t \Delta t+16 \Delta t^{2} .
$$

Subtracting (1) from (2) we obtain

$$
\begin{aligned}
& \Delta s=32 t \Delta t+16 \Delta t^{2}, \\
& \text { and dividing by } \Delta t, \quad \frac{\Delta s}{\Delta t}=32 t+16 \Delta t \text {. }
\end{aligned}
$$

This is the average velocity during an interval of $\Delta t$ seconds beginning at the time $t$. By the definition above, the velocity at the time $t$ is the limit of (3) as $\Delta t$ approaches zero. Denoting it by $v$, we get

$$
v=32 t,
$$

since $16 \Delta t$ approaches zero when $\Delta t$ approaches zero.

33. Rate of Change. Slope of Tangent Line. A generalization of the idea of instantaneous velocity, which may be described as the rate of change of the distance $s$ with respect to the time $t$, is given in the

Definition. The rate of change of $y$ with respect to $x$ for a given value of $x$ is the limit, as $\Delta x$ approaches zero, of the average rate of change of $y$ with respect to $x$ in the interval from $x$ to $x+\Delta x$.

If $y$ is a function of $x$,

$$
y=f(x),
$$

the rate of change of $y$ with respect to $x$ may be determined as follows:

Substitute $x+\Delta x$ for $x$ and $y+\Delta y$ for $y$ in (1). The result,

$$
y+\Delta y=f(x+\Delta x)
$$

is true because $\Delta y$ is the change in $y$ due to a change of $\Delta x$ in $x$, and hence $x+\Delta x$ and $y+\Delta y$ are corresponding values of the independent variable and the function. 
Subtract (1) from (2), which gives $\Delta y$, and divide by $\Delta x$, which gives $\Delta y / \Delta x$, the average rate of change of $y$ in the interval $\Delta x$. Then find the limit of the average rate of change as $\Delta x$ approaches zero. To interpret this process graphically we shall need the

Definition. If $P$ is a given point on a curve and $Q$ any other point on it, the line tangent to the curve at $P$ is the limiting position of the secant $P Q$ as $Q$ moves along the curve and approaches $P$.

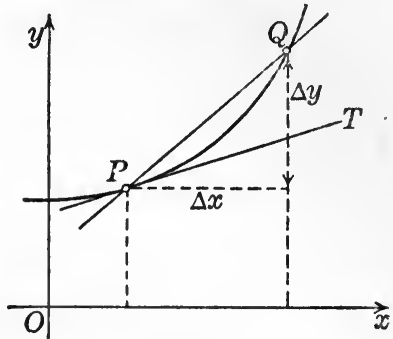

Fig. 53.

The points $P(x, y)$ and $Q(x+\Delta x, y+\Delta y)$ are on the graph of (1), and $\Delta y / \Delta x$ is the slope of the secant $P Q$. As $\Delta x$ approaches zero, $\Delta y / \Delta x$ approaches the rate of change of $y$; also, $Q$ approaches $P$, the secant $P Q$ approaches the tangent $P T$, and the slope of $P Q$ approaches that of $P T$. Hence,

The rate of change of $y$ with respect to $x$ is represented by the slope of a line tangent to the graph of $y$.

The tangent to the graph of $y$ at a maximum or minimum point is horizontal, and hence its slope is zero, and hence, also the rate of change of $y$.

This fact affords a general method of finding maximum and minimum points.

The slope of the tangent line and the rate of change of the function should be added to the table of properties of graphs and functions on page 42 .

ExAmple 1. Find the rate of change of

$$
y=a x^{2},
$$

and discuss its meaning for the graph.

Replacing $x$ and $y$ by $x+\Delta x$ and $y+\Delta y$ respectively, we get

$$
y+\Delta y=a(x+\Delta x)^{2}=a x^{2}+2 a x \Delta x+a \Delta x^{2} .
$$

Subtracting (3) from (4),

$$
\Delta y=2 a x \Delta x+a \Delta x^{2} \text {. }
$$

Dividing by $\Delta x$,

$$
\frac{\Delta y}{\Delta x}=2 a x+a \Delta x \text {, }
$$

which is the average rate of change of $y$ in any interval $\Delta x$. 
Letting $\Delta x$ approach zero, the limit of (5) is

$$
m=2 a x,
$$

the required rate of change, or the slope of the tangent line at any point.

If $x=0$, we have $m=0$, so that the parabola which is the graph of (3) is tangent to the $x$-axis at the origin.

For a given value of $x$, the larger the value of $a$, the larger also is the slope of the tangent line. This justifies the statement made in Section $\mathbf{3 0}$ that " the larger the value of $a$ the more rapidly the curve rises."

If $a=1$, so that $y=x^{2}$, we have $m=2 x$. Hence the slope of the tangent line is less or greater than 1 according as $x$ is less or greater than $\frac{1}{2}$. Therefore, to the right of $x=\frac{1}{2}$, the curve rises more rapidly than it broadens out. (Compare with the statement in Section 29).

For any given value of $a$, the larger the value of $x$ the larger is the value of $m$. Hence the curve rises more and more rapidly as it runs to the right.

Example 2. Find the slope of the line tangent at any point to the graph of

$$
y=x^{2}-4 x .
$$

Find the slope at the point for which $x=3$, construct the line, and find its equation. Find also the coördinates of the maximum or minimum points.

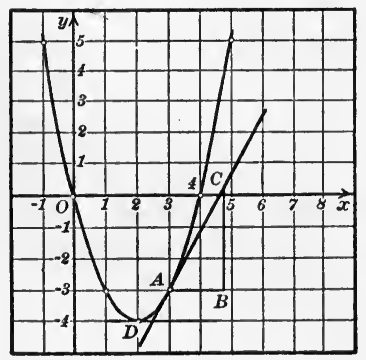

Frg. 54.

The table of values and the graph are readily constructed.

Replacing $x$ by $x+\Delta x$ and $y$ by $y+\Delta y$ in (7) we get

$$
\text { or } \begin{aligned}
y & =(x+\Delta x)^{2}-4(x+\Delta x) \\
y & =x^{2}+2 x \Delta x+\Delta x^{2}-4 x-4 \Delta x .
\end{aligned}
$$

Subtracting (7) from (8),

$$
\Delta y=2 x \Delta x+\Delta x^{2}-4 \Delta x .
$$

Dividing by $\Delta x$,

$$
\frac{\Delta y}{\Delta x}=2 x+\Delta x-4 \text {. }
$$

Passing to the limit as $\Delta x$ approaches zero, the slope of the tangent line at any point is

$$
m=2 x-4 .
$$

At the point $A(3,-3)$ the slope $\frac{x}{y} \mid \frac{-1,0,1, \quad 2, \quad 3,4,5,}{45,0,-3,-4,-3,0,5,}=2 \times 3-4=2$, and the line may be drawn by the construction on page 52 . 
Using the point-slope form of the equation of a straight line (page 66), the equation of the line tangent at $A$ is

or

$$
\begin{aligned}
y+3 & =2(x-3), \\
y & =2 x-9 .
\end{aligned}
$$

As a check, notice that the intercept on the $y$-axis, -9 , agrees with the line as constructed.

At a maximum or minimum point the tangent line is horizontal, and hence the slope $m$ given by (10) must be zero. Hence

whence

$$
\begin{aligned}
2 x-4 & =0, \\
x & =2 .
\end{aligned}
$$

Substituting this value in (7), the minimum value of $y$ is $y=-4$. Hence the minimum point is the point $D(2,-4)$.

\section{EXERCISES}

1. A ball is rolled down an inclined plane. Its distance from the starting point is given by $s=\frac{1}{2} t^{2}+4 t$. Find the velocity at any time; at the instant $t=3$. Plot the graphs of $s$ and $v$ on the same axes, and from them describe the motion.

2. Prove that a line tangent to a circle, as defined in Section 33 , is perpendicular to the radius drawn to the point of contact, using the form of reasoning of elementary geometry.

3. The distance from a fixed station to a moving body is given by $8=t^{2}-3 t$. Find the velocity at any time, and show that the acceleration is constant. Plot the graphs of $s$ and $v$ on the same axes, and describe the motion from $t=0$ to $t=5$.

4. Find the slope of the line tangent at any point to the graph of each of the functions below. Find the slope of the line tangent at the point for which $x=2$, construct the line, and find its equation. Find the coördinates of the maximum or minimum points.
(a) $4-x^{2}$.
(b) $y=3 x-x^{2}$.
(c) $y=x^{2}-1$.
(d) $x^{2}-4 y+2 x=0$.

5 Tabulate the values of the slope of the line tangent to the graph of $x^{2}$ at the points for which $x=-3,-2,-1,0,1,2,3$. What can be said of the value of $m$ as $x$ increases? Does the value of $m$, the slope of the tangent line, always increase as $x$ increases if the graph is concave upward? Is the converse true?

If a curve is concave downward, how does the slope of the tangent line change as $x$ increases? Is the converse true?

6. Find the maximum and minimum points of $y=x^{3}-x$, and then plot the graph.

7. Find the equation of the line tangent to the graph of $x^{2}$ at the point for which $x=1$; for which $x=2$; for which $x=4$. Find the intercept 
of each line on the $y$-axis. How does the intercept compare with the ordinate of the point of contact?

8. Prove that if two lines are perpendicular the slope of one is the negative reciprocal of the slope of the other.

Definition. The line perpendicular to a line tangent to a given curve at the point of tangency is called a normal to the curve.

The slope of the normal at any point may be found from that of the tangent by the preceding exercise.

9. Find the equations of the tangent and normal to the graph of $y=x^{2}+2 x$ at the point $(1,3)$. Construct the figure.

10. Find the equation of the line normal to the graph of $x^{2}$ at the point for which $x=1$; for which $x=3$; for which $x=5$. Find the intercept of each line on the $y$-axis. How does the intercept compare with the ordinate of the point at which the normal cuts the curve?

11. Find the acceleration of a moving body if its velocity is given by $v=2 t^{2}-5 t$. Plot the graphs of $v$ and $a$ on the same axes and discuss the variation of both functions.

12. If the position of a moving body is given by the equation $s=t^{3}-9 t$, find the velocity and acceleration. Plot the graphs of $s, v$, and $a$ on the same axes.

13. Find the acceleration of a body if its position is given by $s=4 t^{2}-t^{3}$.

14. If a ball is dropped the velocity after the ball has fallen $s$ feet is given by $v^{2}=2 g s$. Find the rate of change of $s$ with respect to $v$.

34. Graph of the Quadratic Function $a x^{2}+b x+c$. Denote the function by $y$ so that

$$
y=a x^{2}+b x+c .
$$

In order to determine the form of the graph we shall first show that it always has a single maximum or minimum point, and then translate the axes so as to have this point for the new origin.

To find the maximum or minimum point we need first the slope of the tangent line at any point. In (1) replace $x$ by $x+\Delta x$ and $y$ by $y+\Delta y$. This gives

or

$$
y+\Delta y=a(x+\Delta x)^{2}+b(x+\Delta x)+c,
$$

$$
y+\Delta y=a x^{2}+2 a x \Delta x+\Delta x^{2}+b x+b \Delta x+c .
$$

Subtracting (1) from (2),

$$
\Delta y=2 a x \Delta x+\Delta x^{2}+b \Delta x,
$$


and dividing by $\Delta x$,

$$
\frac{\Delta y}{\Delta x}=2 a x+\Delta x+b .
$$

Passing to the limit as $\Delta x$ approaches zero, the slope of the tangent line at any point is

$$
m=2 a x+b .
$$

The tangent line will be horizontal if $m=0$, that is, if $2 a x+b=0$, whence

$$
x=-\frac{b}{2 a} .
$$

Substituting this value in (1) the corresponding value of $y$ is

$$
\begin{aligned}
y & =a\left(-\frac{b}{2 a}\right)^{2}+b\left(-\frac{b}{2 a}\right)+c \\
& =\frac{b^{2}}{4 a}-\frac{b^{2}}{2 a}+c=\frac{4 a c-b^{2}}{4 a} .
\end{aligned}
$$

The point $\mathrm{O}^{\prime}\left(-\frac{b}{2 a}, \frac{4 a c-b^{2}}{4 a}\right)$ is either a maximum or a minimum point. To translate the axes so that $\mathrm{O}^{\prime}$ is the new origin we set

$$
x=x^{\prime}-\frac{b}{2 a}, \quad y=y^{\prime}+\frac{4 a c-b^{2}}{4 a} .
$$

Substituting these values in (1),

$$
\begin{aligned}
y^{\prime}+\frac{4 a c-b^{2}}{4 a} & =a\left(x^{\prime 2}-\frac{b x^{\prime}}{a}+\frac{b^{2}}{4 a^{2}}\right)+b\left(x^{\prime}-\frac{b}{2 a}\right)+c, \\
& =a x^{\prime 2}-b x^{\prime}+\frac{b^{2}}{4 a}+b x^{\prime}-\frac{b^{2}}{2 a}+c, \\
& =a x^{\prime 2}+\frac{4 a c-b^{2}}{4 a} . \\
y^{\prime} & =a x^{\prime 2} .
\end{aligned}
$$

or

The graph of this equation is a parabola (Section 30) congruent to the graph of $a x^{2}$. Its axis of symmetry is vertical, and the curve runs up or down according as $a$ is positive or negative. 
Then since the graph of (1), plotted on the old axes, is identical with that of (6), plotted on the new axes, we have the

Theorem. The graph of the quadratic function $a x^{2}+b x+c$ is a parabola, with vertical axis, which is congruent to the graph of $a x^{2}$. It runs up or down according as the coefficient of $x^{2}$ is positive or negative.

EXAMPLE. If a ball is thrown vertically upward with a velocity of 80 feet per second, its height $s$, in feet, after $t$ seconds, is given by the equation

$$
s=-16 t^{2}+80 t .
$$

Find when the ball will be highest, how high it will rise, and compare the time of rising with that of falling. Construct the graph.

At the highest point the velocity of the ball is zero, and we therefore seek first the velocity at any time. Replacing $t$ by $t+\Delta t$ and $s$ by $s+\Delta s$ we get

or

$$
\begin{aligned}
& s+\Delta s=-16(t+\Delta t)^{2}+80(t+\Delta t), \\
& s+\Delta s=-16 t^{2}-32 t \Delta t-16 \Delta t^{2}+80 t+80 \Delta t .
\end{aligned}
$$

Subtracting (7) from (8),

and dividing by $\Delta t$,

$$
\Delta s=-32 t \Delta t-16 \Delta t^{2}+80 \Delta t,
$$

$$
\frac{\Delta s}{\Delta t}=-32 t-16 \Delta t+80 \text {. }
$$

Passing to the limit as $\Delta t$ approaches zero, the velocity at any time is

$$
v=-32 t+80,
$$

since the limit of the average velocity $\Delta s / \Delta t$ is the instantaneous velocity.

At the highest point reached by the ball $v=0$, and hence $t=\frac{80}{32}=\frac{5}{2}$ seconds. Substituting this value in (7) the maximum value of $s$ is

$$
s=-16 \times \frac{25}{4}+80 \times \frac{5}{2}=-100+200=100 \text { feet. }
$$

To translate the axes to the maximum point set

$$
t=t^{\prime}+\frac{5}{2}, \quad s=s^{\prime}+100 .
$$

Substituting in (7),

whence

$$
\begin{aligned}
s^{\prime}+100 & =-16\left(t^{\prime}+\frac{5}{2}\right)^{2}+80\left(t^{\prime}+\frac{5}{2}\right), \\
& =-16 t^{\prime 2}-80 t^{\prime}-100+80 t^{\prime}+200, \\
s^{\prime} & =-16 t^{\prime 2} .
\end{aligned}
$$

The graph of this equation plotted on the new axes is identical with that of (7) plotted on the old axes. The figure shows the graph, which is readily plotted on the new axes. It passes through the old origin since 
$8=0$ when $t=0$. From the symmetry of the graph with respest to the $s^{\prime}$-axis the other intercept on the $t$-axis is $t=5$, so that the ball returns to the hand after 5 seconds. Hence the time of rising is equal to the time of falling.

The figure also shows the graph of $v$ [equation (9)]. As $v$ is a linear function of $t$, the slope -32 gives the acceleration, which is constant. A simultaneous discussion of the variation of $s$ and $v$ describes the motion fully. At the start $s=0$ and $v=80$. As $t$ increases, $s$ increases but the velocity decreases, until when $t=5$ the ball reaches its maximum height of 100 feet and

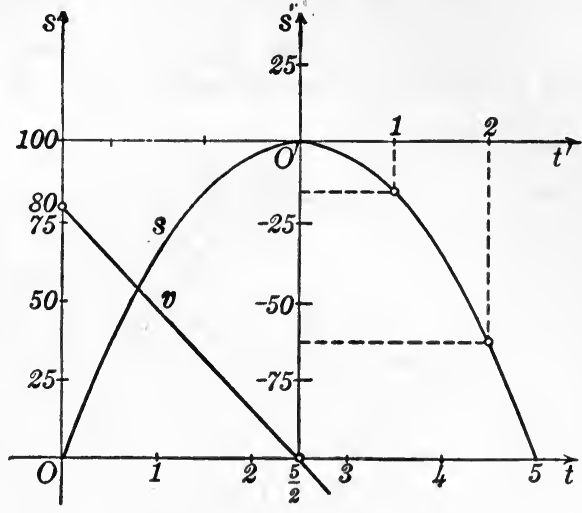

Fig. 55. the velocity becomes zero. The ball then begins to fall, since the velocity changes sign, and its height decreases to zero at $t=5$, when the velocity is -80 , i.e., 80 feet per second downward.

The position of the parabola which is the graph of a quadratic function with reference to the $x$-axis is determined by the discriminant $(9(f)$ page xvii) of the function, for the discriminant determines the nature of the roots of the equation

$$
a x^{2}+b x+c=0 \text {, }
$$

which are identical with the zeros of the function and which are represented by the intercepts on the $x$-axis.

If the discriminant $b^{2}-4 a c$ is positive the roots are real and distinct, if the discriminant is zero the roots are equal, and if the discriminant is negative the roots are imaginary.

It follows that the graph of a quadratic function cuts the $x$-axis twice if the discriminant is positive. If the discriminant is zero, the graph has but one point in common with the $x$-axis, and hence the graph is tangent to the $x$-axis. The point of tangency is the maximum or minimum point. If the discriminant is negative, the curve does not meet the $x$-axis at all. 
A nethod of using the zeros of a quadratic function in constructing the graph of the function is illustrated in the

Alternative solution for the example above. The graph of equation (7) is a parabola running downward, since $s$ is a quadratic function of $t$ in

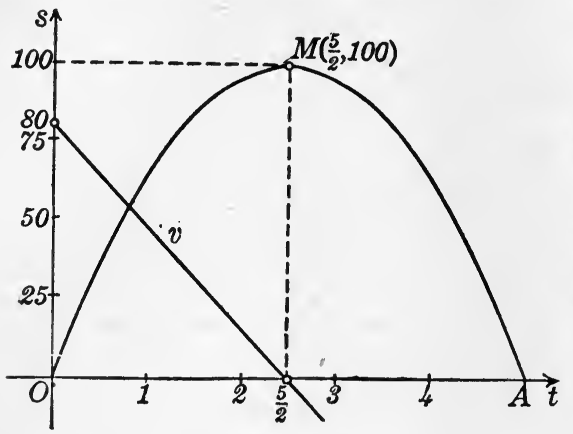

Fig. 56. which the coefficient of $t^{2}$ is negative.

To find the intercepts on the $t$-axis set $s=0$, which gives $-16 t^{2}+80 t=0$. Dividing by -16 , and factoring, $t(t-5)=0$, whence $t=0$ or 5. Hence the origin and the point $A(5,0)$ are on the graph. These intercepts give the time of starting and when the ball returns to the hand.

The axis of symmetry is vertical. Hence it must bisect $O A$, and it is therefore the line $x=\frac{5}{2}=2.5$.

Since the maximum point lies on the axis of symmetry, the ball reaches its greatest height when $t=\frac{5}{2}$ seconds. Substituting this value in (7), the maximum height is $-100+200=100$ feet.

Hence the point $M(2.5,100)$ is the maximum point.

In order to plot the curve from $O$ to $M$ we need only to compute the values of $s$ for $t=1$ and 2 . The other half of the curve is obtained by means of the symmetry.

\section{EXERCISES}

1. The Theorem, Section 34 , shows that the graph of $y=6 x-x^{2}$ is a parabola, with vertical axis, running downward. Construct the graph:

(a) By translating the axes, as in Section 31.

(b) By finding the maximum point as in Section 33 and translating the axes.

(c) By finding the intercepts on the $x$-axis, determining the axis of symmetry from the intercepts, and the maximum value of $y$ by means of the axis of symmetry.

(d) By finding the values of $y$ to be excluded, from which the maximum value of $y$ may be found, and determining the axis of symmetry by means of the maximum value.

In (c) and (d) it will be necessary to obtain a few pairs of values of 2 
and $y$. The most desirable values of $x$ to be assumed may be determined after finding the coördinates of the maximum point.

2. Construct the graph of each of the functions below. Find the axis of symmetry, and the maximum or minimum point. Find the zeros of each function, and state how they are represented graphically.
(a) $x^{2}+2 x-8$.
(b) $2 x^{2}+3 x-2$.
(c) $-x^{2}+3 x$.
(d) $-x^{2}-x+2$.
(e) $x^{2}-4 x+4$.
(f) $-x^{2}-2 x-1$.
(g) $x^{2}+x+1$.
(h) $4 x^{2}+2 x-1$.
(i) $-6 x^{2}+x+5$.
(j) $x^{2}-x+1$.
(k) $5 x^{2}+2 x+1$.
(l) $-3 x^{2}+2 x-2$.

3. Without solving the following equations, determine whether the roots are real or imaginary, equal or unequal. From this, what can be said of the graph of the quadratic function in the left-hand member?
(a) $x^{2}+3 x-5=0$.
(b) $2 x^{2}-3 x+4=0$.
(c) $4 x^{2}+12 x+9=0$.
(d) $x^{2}-{ }_{3}^{2} x+3=0$.
(e) $4 x^{2}-x+1=0$.
(f) $x^{2}-7 x-2=0$.

4. A special quadratic function is one in which one or more of the coefficients are zero. Discuss and plot the graph of the special functions below, and state the special properties of the graph which distinguish it from the general case.
(a) $x^{2}-4$.
(b) $x^{2}-4 x$.
(c) $x^{2} / 3$.
(d) $9-x^{2}$.
(e) $3 x-x^{2}$.
(f) $-2 x^{2}$.
(g) $a x^{2}+\mathrm{c}$.
(h) $a x^{2}+b x$.
(i) $a x^{2}$.

5 If $f(x)=a x^{2}+b x+c$, what must be true of the coefficients if $f(-x)=$ $f(x)$ ?

6. If a ball is thrown vertically upward with a velocity of 50 feet per second, its height after $t$ seconds is given by the equation

$$
s=-16 t^{2}+50 t .
$$

Construct the graph. Find when the ball will be highest, how high it will rise, and when it will return to the hand.

7. Construct the graphs of $x$ and $x^{2}$ from $x=0$ to $x=1$. What is the numerical difference of the ordinates of points on these graphs with the same abscissa? Construct the graph of this difference, and find the value of $x$ for which it is greatest. What is the greatest difference?

8. A Norman window consists of a rectangle (width $2 x$ and height $y$ ) surmounted by a semi-circle. If the perimeter of the window is 16 feet, find the area in terms of the half width $x$, and construct its graph. Find the dimensions which make the area (and hence the amount of light admitted) a maximum. 
9. A cliff 40 feet high overhangs a river. A man on the cliff throws a stone vertically upward with a velocity of $30 \mathrm{ft}$. per second. If the height of the stone above the cliff after $t$ seconds is given by

$$
s=-16 t^{2}+30 t,
$$

find how high the stone rises, and when it will strike the water.

10. If the perimeter of a rectangle is 8 feet, find the area as a function of one of the dimensions. Construct the graph of the area, and find the dimensions and area of the largest rectangle of this sort.

11. Solve Exercise 10 if the perimeter is any constant $p$. [The parcel post regulations require that the sum of the length and girth (greatest perimeter of a section at right angles to the length) of a package shall not exceed 6 feet. By means of this Exercise, what can be said of the shape of the largest rectanglar box which can be mailed?]

12. The equation of the path of a ball thrown into the air at an angle of $60^{\circ}$ with the horizon with a velocity of 32 feet per second is

$$
y=1.7 x-x^{2} / 16
$$

Construct the graph, find the greatest height attained, and where the ball will hit the ground.

13. If a body is projected vericically upward with an initial velocity of $v$ feet per second, its height $s$ after $t$ seconds is given by the equation

$$
s=-16 t^{2}+v t \text {. }
$$

Find how long the body will rise and its maximum height. Prove that the time of rising equals the time of falling.

14. A farmer estimates that if he digs his potatocs now he will have 100 bushels worth $\$ 1.25$ a bushel; but that if he waits, the crop will increase 16 bushels a week, while the price will drop $8 \&$ a week. Find the value of his crop as a function of the time in weeks, and draw the graph. When should he dig to get the greatest cash returns?

15. The amount of wheat obtained per acre depends on the intensity of cultivation. A farmer finds that he can cultivate 15 acres with sufficient intensity so that the return will be 30 bushels per acre; 20 acres so that the return will be 25 bushels per acre; 25 acres so that the return will be 20 bushels per acre; etc. Find the law giving the total return as a function of the number of acres under cultivation, and plot the graph. What is the best size acreage for him to cultivate in order to get the largest gross returns?

35. Empirical Data Problems. The table of values of a linear function is such that if the successive values of $\Delta x$ are equal so also are the successive values of $\Delta y$.

Any quadratic function has a somewhat analogous property, which is illustrated in the table for $y=2 x^{2}+3 x+4$. The 
values of $\Delta x$ being equal, those of $\Delta y$, sometimes called the first differences of $y$, are not equal. But the successive differences of $\Delta y$, called the second differences of $y$ and denoted by $\Delta^{2} y, \quad 1$ are equal. Every quadratic function pos- 1 sesses this property, that if the values of $x$ are such that the successive values of $\Delta x$ are equal, then the successive values of the second differences of $y, \Delta^{2} y$, are equal.

\begin{tabular}{r|r|r|r|r}
$\frac{\Delta x}{1}$ & $\frac{x}{0}$ & $\frac{y}{4}$ & $\frac{\Delta y}{}$ & $\underline{\Delta^{2} y}$ \\
1 & 1 & 9 & 5 & 4 \\
1 & 2 & 18 & 9 & 4 \\
1 & 3 & 31 & 13 & 4 \\
& 4 & 48 & 17 &
\end{tabular}

This property enables us to tell whether a given table of values may be represented approximately by a quadratic function, and also to determine the coefficients of the function.

Example. Find a quadratic function which represents approximately the law connecting the values of $x$ and $y$ given in the table.

\begin{tabular}{r|r|r|r}
$x$ & \multicolumn{1}{c|}{$y$} & \multicolumn{1}{c|}{$\Delta y$} & \multicolumn{1}{c}{$\Delta^{2} y$} \\
\hline 4.1 & 11.2 & 8.1 & \\
4 & 26.9 & 15.7 & 7.6 \\
8 & 51.0 & 24.1 & 8.4 \\
8 & $\frac{83.4}{5 \frac{175.6}{35.12}}$ & $\frac{32.4}{4}$ & 8.3 \\
\hline 10.3 & $\frac{80.08}{20.3}$
\end{tabular}

Inspection of the values of $x$ shows that the successive values of $\Delta x$ are equal. Computing the successive values of $\Delta y$ and $\Delta^{2} y$, it is found that the latter are nearly equal. Hence the values of $y$ resemble those of a quadratic function. the values of $x$ in the given table.

$$
y=a x^{2}+b x+c
$$

be the required function. The second table gives the values of $y, \Delta y, \Delta^{2} y$ computed from (1) for

If the given values of $y$ were exactly the values of some quadratic func-

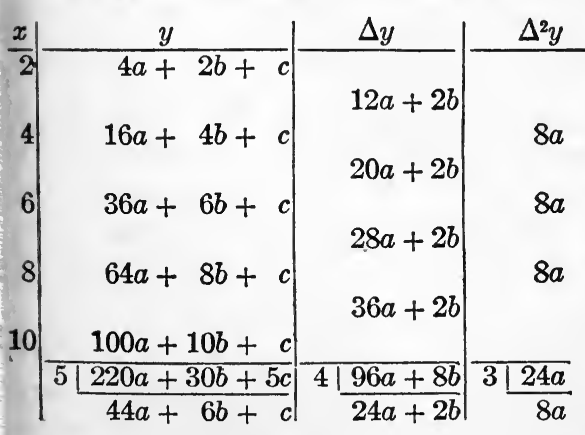
tion, then for the proper values of $a, b, c$, all the numbers of one table would equal the corresponding numbers in the other. In particular, the average values of $y, \Delta y$, and $\Delta^{2} y$ found in the second table would equal those in the first. As it is, these averages are equal for values of $a, b, c$, for which (1) represents the required law approximately. 
Equating the average values of $\Delta^{2} y, 8 a=8.1$, whence $a=1.01$.

Equating the average values of $\Delta y, 24 a+2 b=20.08$. Substituting the value of $a$, and solving for $b$, we get $b=-2.08$.

Equating the average values of $y, 44 a+6 b+c=35.12$. Substituting the values of $a$ and $b$, and solving for $c$, we get $c=3.16$. Substituting the

\begin{tabular}{r|c|c|c|c}
$x$ & Observed $y$ & Computed $y$ & Error & \% error \\
\hline 3.1 & $\frac{1.04}{3.04}$ & -0.06 & -1.9 \\
4 & 11.2 & 11.00 & -0.20 & -1.8 \\
6 & 26.9 & 27.04 & +0.14 & +0.5 \\
10 & 51.0 & 51.16 & +0.16 & +0.3 \\
10 & 83.4 & 83.36 & -0.04 & -0.05
\end{tabular}
values of $a, b, c$, in (1), the required approximation of the law is $y=1.01 x^{2}-2.08 x+3.16$ The accuracy with which this relation expresses the law is indicated in the table.

If it is desired to find the equation of a parabola with vertical axis which passes through, or near, several points whose coördinates are given, the method used in the example may be employed even though the values of $\Delta x$, and hence also those of $\Delta^{2} y$, are not equal. At least three points must be given.

\section{EXERCISES}

1. Find the equation of the parabola with vertical axis which passes through the points $(1,0),(3,10),(5,28)$. Construct the graph and check the result.

2. In sinking a deep mine, new material as well as labor must be invested each day, and the required depth cannot be determined in advance with accuracy. A company which has set aside $\$ 100,000$ for the cost of sinking a shaft finds the total capital invested as the work increases as $\begin{aligned} & \text { Time in months } \\ & \text { Investment in thousands }\end{aligned} \mid \begin{array}{ll}0,1,2,3,3, & \begin{array}{l}\text { given in the table. Find } \\ \text { the law giving the invest- } \\ \text { ment as a function of the }\end{array}\end{array}$ time. If the work continues to progress according to the same law, when must the work be completed if the cost is not to exceed the amount set aside?

3. A contractor agreed to build a breakwater for $\$ 125,000$. After spending $\$ 33,000$ as indicated in the table, he threw up the job without receiving any pay, because he estimated that the cost would increase according to the same law, and that it would require six months more to complete the work. How much would he have lost if he had finished the breakwater?

Time in months Investment in thousands $\mid \frac{0}{5}$

\begin{tabular}{rr}
0, & 1 \\
\hline 5, & 6.1
\end{tabular}

1, 11.2 20.2 4

4. An electric conductor gives out a definite amount of current in every mile of its length. Let $x$ be the distance of any point in miles from the 
end of the line remote from the generator, and $y$ the voltage there. Assuming that the law is of the form $y=a x^{2}+b$, determine the law from the table. What is the voltage at a point 2.5 miles from the end?

Hint: The value of $a$ may be de$\therefore \quad 0, \quad 1, \quad 2, \quad 3, \quad 4$ termined by the general method; $y$ 200,212.5, 250,312.5, 400 after finding $a$, substitute its value in $y=a x^{2}+b$. Then a value of $b$ may be determined by substituting any pair of values of $x$ and $y$. A better value of $b$ is obtained by determining its value for each pair of values of $x$ and $y$ and averaging the results (compare the method of finding $b$ in Section 27 after $m$ is determined).

36. The Function $x^{n}$. Among the functions represented by $x^{i}$ which may be obtained by assigning a numerical value to $n$ may be mentioned the following:

$$
\begin{aligned}
x^{2}, \text { for } n & =2 . \\
x^{3}, \text { for } n & =3 . \\
x^{3}= \pm \sqrt{x,} \text { for } n & =\frac{1}{2} . \\
x^{3}=\sqrt[3]{x}, \text { for } n & =\frac{1}{3} . \\
x^{-1}=1 / x, \text { for } n & =-1 .
\end{aligned}
$$

If no numerical value is assigned to $n$, the symbol $x^{n}$ may represent either the totality of all functions obtained by assigning a numerical value to $n$, or a particular, but unassigned, one of these functions.

The function $x^{n}$, sometimes called the power function, is considered in the following sections.

37. Tables of Squares, Cubes, Square Roots, Cube Roots, and Reciprocals. These tables are extensive tables of values of the function $x^{n}$ for $n=2,3, \frac{1}{2}, \frac{1}{3}$, and -1 . They are laborsaving devices, for from them we can find, for example, the square of a number without the labor of multiplying it by itself. We shall use Huntington's Four Place Tables, Unabridged Edition.

Tables of squares and cubes. The square of a number $n$, between 1 and 10, may be found from the table on page 2 . In order to economize room, the table is not arranged in two long columns of rows. Instead, the first digits in $n$ are given in the border on the left and the last digit in the border at the 
top of the table. To find the square of 1.56 , look in the row in which 1.5 stands on the left, under " $n$," and in the column headed "6." Here we find 2.434, which is the square of 1.56 to four figures. The exact square, obtained by multiplication, is 2.4336 , which is nearer 2.434 , as given in the table, than to 2.433. The lack of exactness in the tables is usually immaterial, for in most of the applications of mathematics three or four figure accuracy is all that is desired, and in many it is all that can be attained. Thus if 1.56 is the side of a square, obtained by measurement, the area is $1.56^{2}=2.43$. Only three figures are retained in the area since the product cannot contain more significant figures than the factors (Section 26). For such an example the table of squares is more accurate than necessary.

To find the squares of numbers greater than 10 , or less than 1 , we use, respectively, the relations

$$
(10 n)^{2}{ }_{i}=100 n^{2} \quad \text { and } \quad(n / 10)^{2}=n^{2} / 100 .
$$

Since multiplication by 10 or 100 shifts the decimal point one or two places to the right, respectively, while division shifts it to the left, we have the rule given at the top of the table: "Moving the decimal point one place in $n$ is equivalent to moving it two places in $n^{2}$."

Repeated application of this rule enables us to find the square of any number. Thus to find $247^{2}$, we find from the table $2.472,=6.10$, whence, applying the rule twice, $247^{2}=$ 61,000. Similarly, $0.247^{2}=0.0610$.

The table of cubes, on page 4 , is very similar to the table of squares. The rule at the top of the table for shifting the decimal follows from the relations:

$$
(10 n)^{3}=1000 n^{3} \quad \text { and } \quad(n / 10)^{3}=n^{3} / 1000 .
$$

Tables of square roots and cube roots. The table of square roots on page 3 is separated into two parts which give the square roots of numbers from 0.1 to 1 and from 1 to 10 . The reason for this lies in the rule for shifting the decimal point. 
Since $\quad \sqrt{100 n}=10 \sqrt{n}$. and $\quad \sqrt{\frac{n}{100}}=\frac{\sqrt{n}}{10}$,

we have the rule at the top of the table: "Moving the decimal point two places in $n$ is equivalent to moving it one place in $\sqrt{n}$."

If $n$ is greater than 10 or less than 0.1 , by moving the point two places at a time, to the left or right, respectively, we will ultimately obtain a number in which the decimal point either precedes or follows the first significant figure. In the former case the square root is found in the upper part of the table, and in the latter case in the lower part. The square root of the original number is then found by re-shifting the decimal point in accordance with the rule.

For example, to find the square root of 35,700 , we shift the point two places to the left twice in succession, obtaining 3.57 . From the lower part of the table we obtain $\sqrt{3.57}=1.889$. Then applying the rule twice, shifting the point to the right, $\sqrt{35700}=188.9$.

To find the square root of 0.0024 , we shift the point two places to the right, getting 0.24. From the upper part of the table, $\sqrt{0.24}=0.490$, whence, by the rule, $\sqrt{0.0024}=0.0490$. Notice that the zero following the nine is retained in order to show the value of the radical to three figures.

The table of cube roots on page 5 is divided into three parts because in moving the decimal point in any number three places at a time (see rule at the top of the table), we ultimately obtain a number in which the point either follows the first significant figure, precedes it immediately, or is followed by a single cipher.

Table of reciprocals. The reciprocal of $n, 1 / n$, differs from $n^{2}, n^{3}, \sqrt{n}, \sqrt[3]{n}$, in that as $n$ increases the reciprocal decreases. For a reason which will appear in Section 42 , it is desirable to have all the tables so arranged that the numbers in the body of the table increase as we read down the page, and from left to right. The table of reciprocals on page 7 is so arranged. The values of $n$, given in the border of the table, increase as we read up and to the left. 
The rule for shifting the decimal point at the top of the table follows from the fact that the product of $n$ and its reciprocal is unity. Hence if one is multiplied by 10 , the other must be divided by 10 .

To find $1 / 436$, for example, we read up the table, on the right, until we come to 4.3 , then over to the left until we are in the column headed 6, where we find 0.2294 , which is the value of $1 / 4.36$. Then by the rule for shifting the point, $1 / 436=0.002294$.

In using any one of these tables the position of the decimal point may frequently be determined by inspection, and it is well to check the result obtained from the table.

\section{EXERCISES}

1. Find $3.24^{2}, \sqrt{0.47}, \sqrt{4.72}, 7.4^{3}, \sqrt[3]{0.0235}, \sqrt[3]{0.24}, \sqrt[3]{1.84}, 1 / 24$.

2. Find the squares of $25.4,0.86,3540,0.0043$.

3. Find the square roots of $59,590,4300,0.000382$.

4. Find the cubes of $54,0.317,53200,0.0000371$.

5 Find the cube roots of $1540,470,18.3,0.0048,0.0000259$.

6. Find the reciprocals of $23.4,0.478,532,0.0074$.

7. Find the value of $0.07^{4}, 0.06^{6}, \sqrt{14.23}, 1 / 23.4^{2}, \sqrt[3]{0.0247^{2}}$.

8. Find the hypothenuse of a right triangle whose sides are 61 and 74 .

9. Find the mean proportional between 6 and 34 .

38. Graph of $x^{n}, n>1$. It will be seen in this section, and those following, that a part of the graph of $x^{n}$ lies in the first quadrant, and that the remaining part may be found by means of the symmetry of the curve. Hence particular attention should be made to fix in mind the various forms of the part of the graph in the first quadrant. The general appearance of this part of the graph varies according as $n>1,0<n<1$, or $n<0$, so that these cases are considered separately. These groups of values include all values of $n$ except $n=0$ and $n=1$, which separate the groups, and which are exceptional in that they are the only values for which the graph is a straight line.

$n=0$. If $n=0, y=x^{n}$ becomes $y=1$, since $x^{\circ}=1$, whose graph is the straight line parallel to the $x$-axis and one unit above it.

$n=1$. For $n=1$ we have $y=x$, whose graph is the straight line through the origin whose slope is unity, that is, the bisector of the first and third quadrants. 
$n>1$. In this case the graph of

$$
y=x^{n}
$$

is tangent to the $x$-axis at the origin $(0,0)$, rises to the right and passes through the point $(1,1)$, at which the slope of the tangent line is $n$. That it passes through these points is seen by substituting their coördinates in (1).

To find the slope of the line tangent to the graph at any point, when $n$ is a positive integer, replace $x$ by $x+\Delta x$ and $y$ by $y+\Delta y$ in (1), which gives $y+\Delta y=(x+\Delta x)^{n}$.

Expanding the right hand member by the binomial theorem, $y+\Delta y=x^{n}+n x^{n-1} \Delta x+\frac{n(n-1)}{2} x^{n-2} \Delta x^{2}+\cdots+\Delta x^{n}(2)$

Subtracting (1) from (2),

$$
\Delta y=n x^{n-1} \Delta x+\frac{n(n-1)}{2} x^{n-2} \Delta x^{2}+\cdots+\Delta x^{n} .
$$

Dividing by $\Delta x$,

$$
\frac{\Delta y}{\Delta x}=n x^{n-1}+\frac{n(n-1)}{2} x^{n-2} \Delta x+\cdots+\Delta x^{n-1} .
$$

Passing to the limit as $\Delta x$ approaches zero, the slope of the tangent line at any point is

$$
m=n x^{n-1} \text {. }
$$

This proof assumes that $n$ is a positive integer, but in Chapter VI it will be shown that the result holds for fractional and negative values of $n$.

At the origin $x=0$, so that, by (3), $m=0$ if $n>1$. Hence the tangent at the origin is the $x$-axis.

At the point $(1,1)$ the slope of the tangent line is $m=n$. Hence the larger the value of $n$ the steeper the curve is at this point, from which it follows that it must be flatter near the origin.

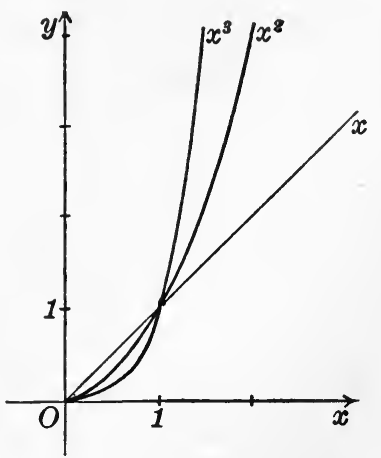

FIG. 57.

If $n$ is a positive integer greater than unity, the part of the graph of $x^{n}$ in the first quadrant is then very much like the graph of $x^{2}$. 
If $n$ is a positive fraction greater than unity the part of the graph in the first quadrant has the same general appearance. To see this, let $r, s$, and $t$ be three values of $n$ such that $r<s<t$. For a positive value of $x$ the value of $x^{8}$ will lie between $x^{r}$ and $x^{t}$, and hence the part of the graph of $x^{s}$ in the first quadrant lies between the graphs of $x^{r}$ and $x^{t}$ (this holds for all values of $r, s$, and $t)$. For example, the graph of $x^{3}$ lies between the graphs of $x$ and $x^{2}$; that of $x^{\frac{5}{2}}$ between those of $x^{2}$ and $x^{3}$; etc.

The figures show the graphs of $x^{2}, x^{3}$, and $x^{\frac{3}{2}}$, which are symmetrical with respect to the $y$-axis, the origin, and the $x$-axis

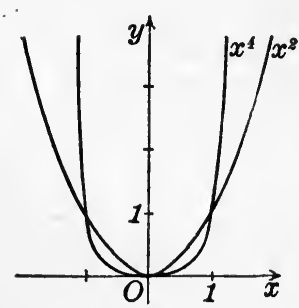

$A$

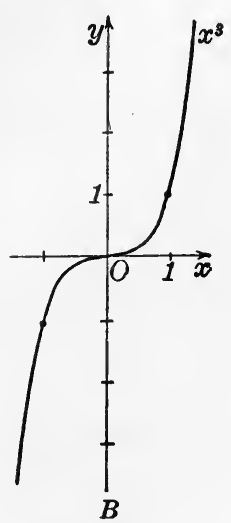

Frg. 58.

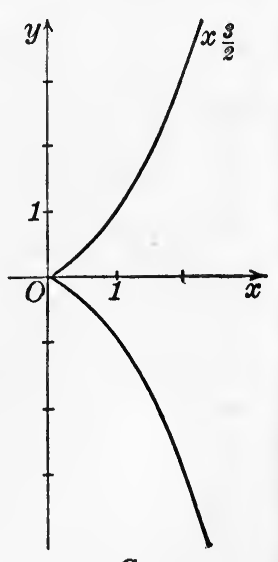

C

respectively. The symmetry of the last curve is seen by writing $y=x^{3}$ in the form $y^{2}=x^{3}$, and applying Theorem $3 B$, page 24 . The graph of $x^{n}, n>1$, always resembles one of these curves. Thus the graph of $x^{4}$ is very much like a parabola, but it is flatter near the origin and steeper elsewhere.

The $x$-axis is tangent to the graph of $x^{3}$ at the origin. This tangent differs from any we have encountered hitherto in that it crosses the curve at the point of tangency.

The graph of $x^{\frac{1}{2}}$ is remarkable in that it has a sharp point $\mathrm{a}^{t}$ the origin. It differs from other curves we have studied in detail in that vertical lines to the right of the $y$-axis cut it 
twice, corresponding to the fact that $x^{\frac{3}{2}}= \pm \sqrt{x^{3}}$ has two values for each positive value of $x$.

39. Graph of $x^{n}, 0<n<1$. Graphs of Inverse Functions. Let us first consider $n=\frac{1}{2}$, or the function $x^{\frac{1}{2}}$. If we set $y=x^{\frac{2}{2}}$, and solve for $x$, we get $x=y^{2}$. This differs from the equation $y=x^{2}$ only in that $x$ and $y$ have been interchanged. Hence if $(x, y)$ is a point on the graph of either equation, the point $(y, x)$ is on the graph of the other. But these points are symmetrical with respect to the bisector of the first and third quadrants, as may be established

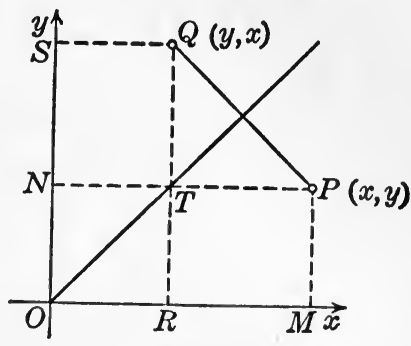

Fig. 59. from the figure. Hence the graphs of the two equations, that is, the graphs of $x^{\frac{1}{2}}$ and $x^{2}$, are symmetrical to each other with respect to this bisector.

The graph of $x^{\frac{1}{2}}$ may therefore be constructed as follows: Construct the graph of $x^{2}$ and the bisector of the first and

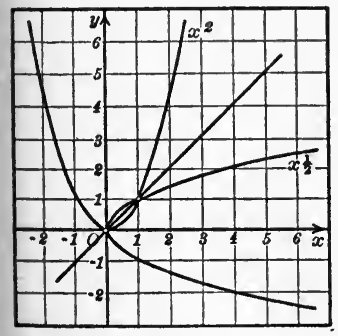

FIG. 60. third quadrants. Choose a number of points on the graph of $x^{2}$, and construct the points symmetrical to them with respect to this bisector. Draw a smooth curve through the points so obtained.

We may now get properties of the function $x^{\frac{1}{2}}$ by interpreting its graph. For example, since the graph of $x^{2}$ is symmetrical with respect to the $y$-axis that of $x^{\frac{1}{2}}$ is symmetrical with respect to the $x$-axis, and hence to each value of $x$ there correspond two values of $x^{\frac{1}{2}}$ which are equal numerically but differ in sign. And since no part of the graph lies to the left of the $y$-axis (why?), the function is imaginary if $x$ is negative. What other properties may be obtained in this way?

If two curves are symmetrical to each other with respect to a line they are congruent, for one may be brought into co- 
incidence with the other by rotating the plane about the line through $180^{\circ}$. Hence the graph of $x^{\frac{1}{2}}$ is a parabola.

Since we obtained the equation $y=x^{2}$ by solving $y=x^{\frac{1}{2}}$ for $x$ and then interchanging $x$ and $y$, the functions $x^{2}$ and $x^{\frac{1}{2}}$ are inverse functions (page 40). The graphical considerations above may be applied to any two inverse functions, and hence we have the

Theorem. The graphs of inverse functions are symmetrical to each other with respect to the bisector of the first and third quadrants.

If the graph of any function is given, then the graph of the inverse function may be obtained readily by this theorem. The distinction between two curves symmetrical to each other with respect to a line and a single curve which is symmetrical with respect to a line should be noted.

To find the inverse of $x^{n}$, set $y=x^{n}$, and interchange $x$ and $y$ which gives $x=y^{n}$; solving for $y$ we get $y=x^{\frac{1}{n}}$. Hence the inverse of $x^{n}$ is $x^{\frac{1}{n}}$, and the graphs of these functions are

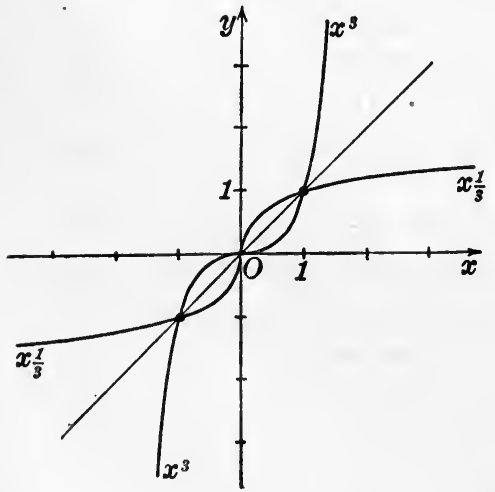

$\boldsymbol{A}$

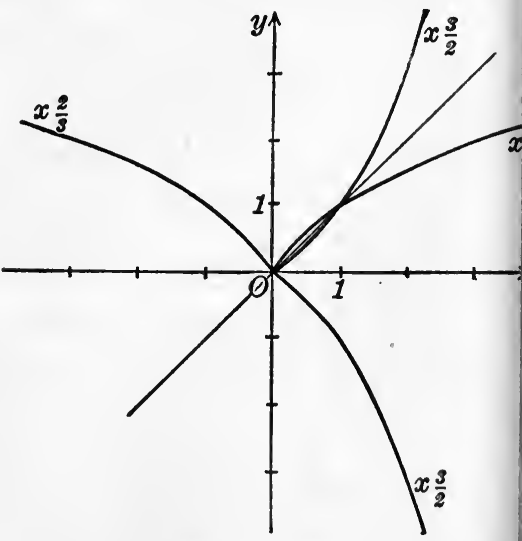

$B$

Fig. 61.

symmetrical to each other with respect to the bisector of the first and third quadrants.

By means of this symmetry, for example, the graph of $x$ 
may be obtained from that of $x^{3}$, and the graph of $x^{3}$ from that of $x^{3}$.

The graph of $x^{n}$, if $0<n<1$, always resembles the graph of one of the functions $x^{\frac{1}{2}}, x^{3}$, and $x^{3}$. In the first quadrant, the graph is tangent to the $y$-axis at the origin, rises to the right, and passes through the point $(1,1)$, at which the slope of the tangent line is $n$. Hence the smaller the value of $n$, the less rapidly the graph rises at this point, from which it follows that it must be steeper near the origin. The remaining part of the graph may be determined by means of the symmetry of the curve with respect to one of the axes or the origin.

\section{EXERCISES}

1. Plot the graph of $x^{n}$ for each of the values of $n$ given below, using the same axes.

(a) $n=2,4,6$. What can be said of the graph if $n$ is an even positive integer?

(b) $n=3,5$. What can be said of the graph when $n$ is an odd positive integer?

(c) $n=1,2,3, \frac{1}{2}, \frac{1}{3}, \frac{3}{2}, \frac{2}{3}$, using as large a scale as possible.

2. Find the slope of the tangent line at any point to the graph of $x^{n}$ for $n=2,3,4,5$. Find and tabulate the slope for each graph at the points for which $x=0,0.3,0.5,0.8,1,2$. As $n$ increases, what can be said of the slope of the tangent line at a point near the origin? Remote from the origin?

3. On separate axes sketch the graphs of $x^{n}$ for $n=\frac{3}{2}, \frac{5}{3}, \frac{4}{3}$. Is there much difference between the parts of these curves in the first quadrants?

4. Find the inverse of each of the functions in Exercise 3, and sketch their graphs.

5. Determine the symmetry of $x^{n}$ when $n$ is a fraction $p / q$, if (a) $p$ is even and $q$ is odd; (b) $p$ is odd and $q$ is even; (c) $p$ and $q$ are both odd. What values of $n$ give typical forms of the graph in these three cases if $p / q>1$ ? If $p / q<1$ ?

6. Plot the graph of $x^{4}$, find the inverse function, and plot its graph.

7. Would it be easy to build a table of values for $x^{\frac{1}{2}}$ ? Construct its graph by first finding the inverse function.

8. Plot the graphs of the following functions, in each case finding the inverse function and its graph.
(a) $y=x^{2}-2$.
(b) $y=x^{2}+2 x$.
(c) $y=-x^{2}+4 x-4$.

9. Using Huntington's Tables, construct the graphs, on the same axes, of $x, x^{2}, x^{3}, x^{\frac{3}{2}}, x^{\frac{1}{3}}$, taking $x=0.2,0.4,0.6, \ldots, 1.4$. Use as large a scale as possible and plot the parts of the graphs in the first quadrant only. 
10. Using Huntington's Tables, construct a table of values of $x^{\frac{3}{2}}$ for $x=$ $0.05,0.10,0.15,0.20,0.25$, obtaining the values of the function to two decimal places. Construct the graph from $x=0$ to $x=0.25$ on as large a scale as possible.

11. Plot the graph of $x^{3}-4 x$. On the same axes sketch the graph of the inverse function, and state several of its properties by interpreting its graph. Can you find the inverse function?

NoтE. In finding the inverse of a function, it is necessary to solve an equation. At any stage of mathematical development, the solution of an equation may be impossible by means of functions already studied. Such an equation defines a new function, whose fundamental properties are determined by the equation.

Thus it is impossible for a student beginning algebra to solve the equation $y^{2}=x$ for $y$. It is first necessary that he should become aequainted with the, to him, new function $x^{\frac{3}{2}}= \pm \sqrt{x}$.

A person unacquainted with the solution of cubic equations cannot find the inverse of the cubic function in Exercise 11. But the theorem on the graphs of inverse functions enables us to get the graph and some of its properties, even though we do not know the function. This point of view will be useful later in studying certain transcendental functions.

The inverse of a cubic or biquadratic function (page 39) is an algebraic function, but the inverse of a polynomial of higher than the fourth degree is usually transcendental.

12. What is the inverse of the function $1 / x$ ? What therefore can be said of the symmetry of the graph of the function?

13. Show that the graph of an equation is symmetrical with respect to the line $y=x$ if the equation is unchanged when $x$ and $y$ are interchanged. Plot the graph of $x y-2 x-2 y=0$.

14. What is the form of an equation in $x$ and $y$ if it defines a function of $x$ which is its own inverse?

15. Plot the graphs of $x^{2}$ and $x^{\frac{1}{2}}$ on the same axes. Show how the following facts are illustrated.
(a) $(\sqrt{x})^{2}=x$.
(b) $\sqrt{x^{2}}=x$.

16. Plot the graphs of $x^{3}$ and $x^{\frac{1}{3}}$ on the same axes. Show how the following facts are illustrated.

(a) $(\sqrt[3]{x})^{3}=x$. (b) $\sqrt[3]{x^{3}}=x$.

17. For what value of $x$ does $x^{2}$ increase at the same rate as $x$ ? For what value does $x^{2}$ increase less rapidly? more rapidly? interpret the results graphically.

18. Find the value of $x$ for which the tangent lines to the graphs of $x^{2}$ and $x^{3}$ are parallel. For what values of $x$ is the graph of $x^{3}$ "flatter" than that of $x^{2}$ ? for what values is it steeper?

19. The horse power of a gas engine is sometimes determined by the equation H.P. $=d^{2} n / 2.5$, where $d$ is the diameter of a cylinder and $n$ is the number of cylinders. 
(a) Plot the graph for four-cylinder engines, taking $d=2.5,3,3.5,4$. On the same axes, plot the graphs for six-, eight- and twelve-cylinder engines.

(b) Plot the graph for $d=3$, taking $n=4,6,8,12$. On the same axes, plot the graph if $d=2.5$, or $d=4$.

40. Graph of $x^{n}, n<0$. Graphs of Reciprocal Functions. Consider first $n=-1$, the function $x^{-1}=1 / x$. Let $y=1 / x$. Replacing $x$ by $-x$ and $y$ by $-y$, and changing the signs of both sides of the equation, we obtain the given equation. Hence the graph is symmetrical with respect to the origin, and the table of values need include only positive values of $x$.

Since the function becomes infinite as $x$ approaches zero, the $y$-axis, $x=0$, is an asymptote. Solving $y=1 / x$ for $x$ we get $x=1 / y$, from which it follows that the $x$-axis, $y=0$, is also an asymptote.

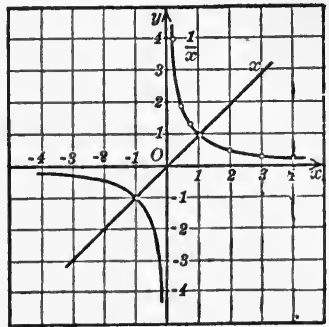

FIG. 62.

The figure shows the graph, which appears to be symmetrical with respect to the line $\frac{x}{1 / x} \mid \frac{0, \frac{1}{4}, \frac{1}{2}, \frac{3}{4}, 1,2,3,4,}{\infty, 4,2, \frac{4}{3}, 1, \frac{1}{2}, \frac{1}{3}, \frac{1}{4}}$ $y=x$, the bisector of the first and third quadrants. That this is the case is seen as follows: The equation $y=1 / x$, or $x y=1$, is unchanged if $x$ and $y$ are interchanged. Hence if $(x, y)$ is on the graph, so also is $(y, x)$. But these points are symmetrical with respect to the line $y=x$ (Section 39), and therefore the graph is also.

The graphs of $x$ and $1 / x$ illustrate the following properties of any two reciprocal variables:

I. Reciprocal variables have the same sign. For both graphs are above the $x$-axis, or both are below, for any value of $x$.

II. If a variable increases, the reciprocal decreases, and vice versa. For as $x$ increases, that is, as the graph of $x$ rises, the graph of $1 / x$ falls; and as $x$ decreases, the graph of $1 / x$ rises.

III. If the numerical value of a variable approaches and becomes unity, so also does that of the reciprocal. For the graphs ntersect at the points $(1,1)$ and $(-1,-1)$. 
IV. If a variable approaches zero, its reciprocal becomes infinite, and vice versa. For both axes are asymptotes.

$n=-2$, the function $x^{-2}=1 / x^{2}$. The function $1 / x^{2}$ is called the reciprocal of the function $x^{2}$ in accordance with the

Definition. Two functions are said to be reciprocal if their product is unity.

The following properties of the graphs of reciprocal functions are proved by the like numbered facts above:

Ia. Corresponding parts of the graphs of reciprocal functions lie on the same side of the $x$-axis.

IIa. If the graph of a function rises, the graph of the reciprocal function falls, and vice versa.

IIIa. If the graph of a function approaches a point on either of the lines $y= \pm 1$, the graph of the reciprocal function approaches the same point from the opposite side of the line. The points of intersection of the graphs lie on these lines.

IVa. If the graph of a function crosses, or is tangent to, the $x$-axis at the point $(a, 0)$, the line $x=a$ is an asymptote of the graph of the reciprocal function, and vice versa.

If the graph of a function has been plotted, these considerations enable us to sketch, roughly, the graph of the reciprocal function, without building a table of values.

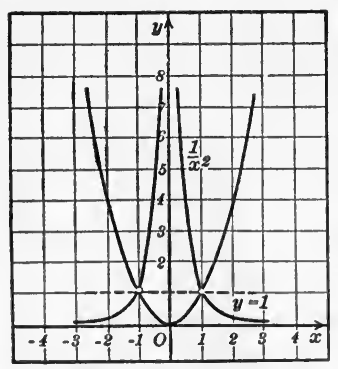

Frg. 63.

The general form of the graph of $1 / x^{2}$ may be obtained from that of $x^{2}$ as follows:

At the extreme left of the figure, the graph of $x^{2}$ lies above the $x$-axis, and is falling. Then the graph of $1 / x^{2}$ lies above the $x$-axis, and is rising (by Ia and IIa). As $x$ increases up to $x=-1$, the graph of $x^{2}$ falls until it reaches the line $y=1$, and hence the graph of $1 / x^{2}$ rises until it reaches this line (by IIIa). From these facts we can sketch the graph of $1 / x^{2}$ from the extreme left of the figure up to $x=-1$.

As $x$ increases from -1 to 0 , the graph of $x^{2}$ falls and be- 
comes tangent to the $x$-axis. Hence the graph of $1 / x^{2}$ rises indefinitely, and approaches the $y$-axis as an asymptote (by IIa and IVa).

As $x$ increases from 0 to 1 , the graph of $x^{2}$ rises to the line $y=1$, and hence that of $1 / x^{2}$ falls to this line. As $x$ increases from 1 on, the graph of $x^{2}$ rises indefinitely, and that of $1 / x^{2}$ continues to fall, but remains above the $x$-axis. The right hand half of the figure might also be obtained from the symmetry with respect to the $y$-axis.

Notice that the graph of $1 / x^{2}$ lies between the graph of $1 / x$ and the $x$-axis to the right of $x=1$, while between $x=0$ and $x=1$, the graph of $1 / x$ lies between it and the $y$-axis.

41. Summary of Graph of $x^{n}$. The figure shows the typical form of the graph in the first quadrant for each of the cases $n>1,0<n<1$, and $n<0$. This part of the graph always lies in the parts of the plane shaded alike. The following properties are noteworthy:

The graph passes through the point (1, 1), at which the slope of the tangent line is $n$.

The rest of the graph may be determined from the part in the first quadrant by means of the symmetry with respect to one of the axes or the origin.

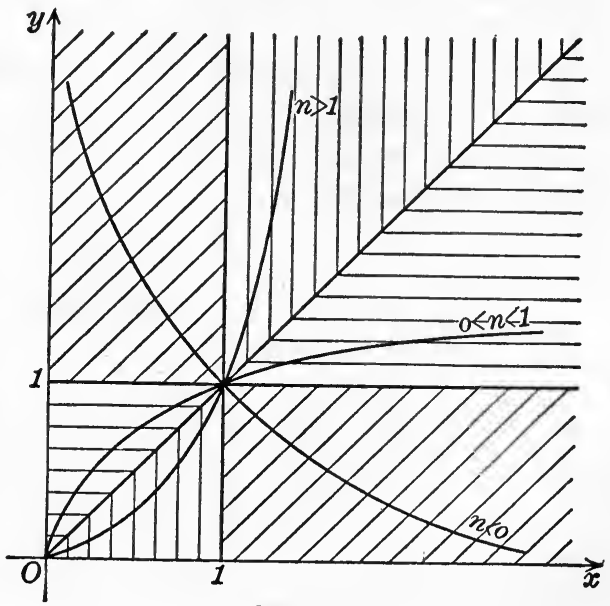

Fig. 64.

The following remarks apply only to the part of the graph in the first quadrant.

$n>1$. The graph is tangent to the $x$-axis at the origin, and rises as it runs to the right. The larger the value of $n$, the flatter the graph is near the origin, and the steeper it is elsewhere. 
$0<n<1$. The graph is tangent to the $y$-axis at the origin, and rises as it runs to the right. The smaller the value of $n$ the more rapidly it rises near the origin, and the less rapidly elsewhere.

The curves of these two groups are separated by the graph of $y=x \quad(n=1)$; the graphs for two reciprocal values of $n$ are the graphs of inverse functions and are symmetrical to each other with respect to this line.

$n<0$. Both axes are asymptotes of the graph, which falls as it runs to the right. The smaller the numerical value of $n$, the closer the graph lies to the $y$-axis, between $x=0$ and $x=1$, and the farther it lies from the $x$-axis.

The curves of this last group are separated from those of the first two groups by the line $y=1(n=0)$, the graphs for two values of $n$ which are numerically equal but differ in sign being the graphs of reciprocal functions.

For three values of $n$ in any one of these three groups, the graph corresponding to the intermediate value of $n$ always lies between the graphs for the other values of $n$.

The graph of $a x^{n}$ may be obtained from that of $x^{n}$ by the theorem in Section 30 .

\section{EXERCISES}

1. Find the reciprocal of each of the following functions, and sketch the graphs of both functions:
(a) $x^{3}$.
(b) $x^{\frac{1}{2}}$.
(c) $x^{\frac{3}{3}}$.
(d) $x^{\frac{3}{2}}$.
(e) $x^{3}$.

2. Find the inverse and the reciprocal of each of the functions below, and sketch the graphs of the three functions on the same axes.
(a) $x^{2}$.
(b) $x^{3}$.
(c) $x^{4}$.
(d) $x^{4}$.

3. On the same axes, plot the graph of the function indicated below for the given values of $a$.

(a) $a x^{3}$ for $a=1, \frac{1}{4}, \frac{1}{2}, 2,3$.

(b) $a x \frac{1}{2}$ for $a=1, \frac{1}{2}, 2,3$.

(c) $a x^{3}$ for $a=1,-1,-\frac{1}{2},-2$.

(d) $a / x$ for $a=1,4,8,-1$.

(e) $a / x^{2}$ for $a=1,2,4,6$.

4. On the same axes, using as large a scale as possible, construct the graph of $x^{n}$ for $n=-2$, and -4 . What can be said of the form of the graph if $n$ is an even negative integer? 
5. On the same axes, using as large a scale as possible, construct the graph of $x^{n}$ for $n=-1$ and -3 . What can be said of the graph of $x^{n}$ if $n$ is an odd negative integer?

6. Construct the graph of $x^{n}$ if $n=-\frac{1}{2}$ (a) By regarding the function as the reciprocal of the function $x \frac{1}{2}$; (b) By regarding it as the inverse of $x^{-2}$.

7. Construct the graph of (a) $x^{-\frac{3}{2}}$, (b) $x^{-\frac{2}{3}}$, in each case using the properties of the graphs of reciprocal functions. What relation exists between these two functions?

8. The quantity of water which flows from an exit pipe $h$ feet below the surface of a reservoir is given by the equation $q=-8.02 a \sqrt{h}$, where $a$ is the area of the cross section of the pipe. Assume $a=1$, and plot the graph. If one outlet pipe is $\mathbf{1 0}$ feet below the surface and another is twice as deep, is the flow of one twice that of the other? Why?

9. The pressure $p$, the volume $v$, and the temperature $t$ of a gas are connected by the relation $p v=k t$, where $k$ is a constant. Assuming $k=1$, plot the graph if (1) $p$ is constant, (2) $v$ is constant, (3) $t$ is constant.

10. As water runs out of a basin, a depression is formed near the outlet. A vertical section of this depression in the surface of the water is called the curve of whirl. It is given by the equation $y=-h^{2} r^{2} / x^{2}$, where $r$ is the radius of the orifice in the outlet pipe, and $h$ is the head, or depth of water. The head changes from instant to instant, so that the curve of whirl constantly changes. Assuming $r=1$, plot the graph for $h=\frac{1}{2}, 1,2$.

11. Plot the graphs of the functions following, and then sketch the graphs of the reciprocal functions. Find the reciprocal function in each case.
(a) $x-3$.
(b) $2-x$.
(c) $x^{2}-1$.
(d) $x^{2}-4$.
(e) $x /\left(x^{2}+1\right)$.
(f) $x^{2}-7 x+10$.
(g) $x^{3}-4 x$.
(h) $x^{3}-4 x$.

12. What is a simple way of sketching the graph of $1 /(2 x+3)$ ? Of $3 /(2 x+3) ?$

42. Interpolation. The process of finding, for example, the cube of such a number as 2.647 , which lies between two successive numbers 2.64 and 2.65 whose cubes are given in the table, is called interpolation. According to Huntington's Tables, $2.64^{3}=18.40$ and $2.65^{3}=18.61$. The difference between these two values of $y=x^{3}$ is $\Delta y=0.21$, and is called the tabular difference. In general, the tabular difference is the difference between two successive numbers in the body of a table. For the process of interpolation it is essential that the 
successive values of the tabular difference should be very nearly equal.

The table gives one row of Huntington's table of cubes and the successive tabular differences. Since $\Delta y$ changes only slightly while $\Delta x$ is always equal to 0.01 , the average rate of change, $\Delta y / \Delta x$, is nearly constant, and the part of the graph of

\begin{tabular}{c|c|c}
$x$ & $y=x^{3}$ & $\Delta y$ \\
\cline { 1 - 2 } 2.60 & 17.58 & \\
2.61 & 17.78 & .20 \\
2.62 & 17.98 & .20 \\
2.63 & 18.19 & .21 \\
2.64 & 18.40 & .21 \\
2.65 & 18.61 & .21 \\
2.66 & 18.82 & .21 \\
2.67 & 19.03 & .21 \\
2.68 & 19.25 & .22 \\
2.69 & 19.47 & .22 \\
2.70 & 19.68 & .21
\end{tabular}
$x^{3}$ constructed from this table would be very nearly a straight line. Some of the successive values of $\Delta y$ are equal, due to the fact that the values of $x^{3}$ are approximate to four figures and not exact, and hence some of the points plotted from the table would actually lie on a straight line.

For the process of interpolation we assume that the part of the graph lying between two successive points is straight.

To find $2.647^{3}$, consider that part of the graph lying between $x=2.64$ and 2.65, as given in Figure 65. We seek the value of the ordinate $E F$ at the point $E$ for which $x=2.647$. It may be obtained by adding $G F$ as a correction to $A B=2.64^{3}$. To find $G F$, we have the slope of $B F$ is the same as that of $B D$, since the graph is assumed straight. Hence

$$
\frac{G F}{B G}=\frac{H D}{B H} \text { or } \frac{G F}{0.007}=\frac{0.21}{0.01},
$$

whence

$$
G F=\frac{0.007}{0.01} \times 0.21=0.7 \times 0.21=0.147=0.15 .
$$

Then $2.647^{3}=E F=A B+G F=18.40+.15=18.55$.

$E F$ may also be obtained by subtracting $I D$ as a correction from $C D=2.65^{3}$. To find $I D$, we have the slope of $F D$ is equal to that of $B D$, since the graph is assumed straight, so that

$$
\begin{aligned}
& \frac{I D}{F I}=\frac{H D}{B H}, \text { or } \frac{I D}{0.003}=\frac{0.21}{0.01} \\
& I D=\frac{0.003}{0.01} \times 0.21=0.3 \times 0.21=0.06 .
\end{aligned}
$$

and hence 
Then $2.647^{3}=E F=C D-I D=18.61-0.06=18.55$, as before.

The latter procedure is usually preferable if the figure for which we are interpolating, 7 in the illustration, is greater than 5 , while the former is used if it is less than, or equal to, 5 . Notice that the correction $G F$ is found by taking seven-tenths of the tabular difference, $H D=0.21$, and the correction $I D$ by taking three-tenths of the tabular differ-

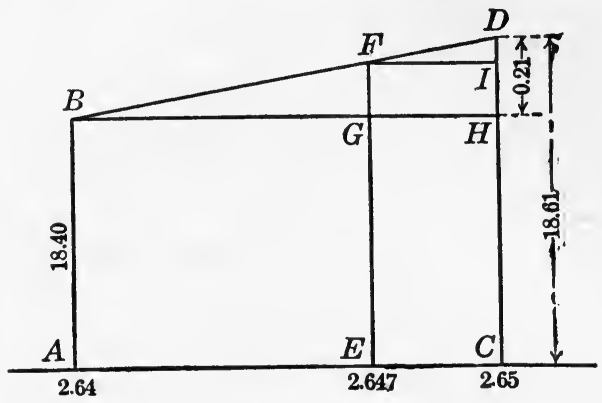

FIG. 65. ence.

The arithmetical operations involved in interpolation may be described as follows:

To find $2.64 a^{3}$, where a stands for the digit for which we are interpolating, apply a-tenths of the tabular difference as a correction to $2.64^{3}$ if $a<5$, while if $a>5$, apply $(10-a)$-tenths of the tabular difference as a correction to $2.65^{3}$. In either case, the correction must be applied (added or subtracted) in such a way that the result lies between $2.64^{3}$ and $2.65^{3}$.

In practice the decimal point is usually omitted in $x, x^{3}$, and the tabular difference, and inserted in the proper place at the end of the operation.

Example 1. Find 26.023. The figure for which we must interpolate is 2 , and the tabular difference is 20. Two-tenths of the tabular difference is 4 , the correction to be applied to 1758 , the cube of 260 . [n order to obtain a result between 1758 and 1778, the cube of 261, he correction must be added, giving 1762 as the first four digits in he required cube. From the rule at the top of the table, we see that $26.02^{3}=17,620$.

Example 2. Find 2.6873. Instead of applying 7-tenths of the tabular lifference as a correction to the cube of 2.68 , we apply 3-tenths of it to he cube of 2.69. Three-tenths of the tabular difference is $6.6=7$, and ience $2.687^{3}=19.40$. 
Except for purposes of explanation, there is no need of writing anything but the desired result.

All the tables in Huntington's Tables are arranged so that the numbers in the body of any table increase as we read from left to right, for the reason that a uniform procedure is obtained for applying the correction. In interpolating, the desired result lies between two numbers in the same row; a correction applied to the left-hand number is always added, while a correction to be applied to the right-hand number is always subtracted.

\section{EXERCISES}

1. Plot the graph of $x^{2}$ from $x=4.50$ to $x=4.60$, using the values of $x^{2}$ given in Huntington's Tables.

2. If we plot the graph of $\sqrt{x}$ for values of $x$ from 2.30 to 3.40 , using the square roots given in the Tables, why will the graph be nearly straight?

3. Find the values of the numbers given below, illustrating the interpolation graphically.

$\begin{array}{lllll}\text { (a) } 3.172^{2} . & \text { (b) } \sqrt{3.478} & \text { (c) } 6.49^{3} . & \text { (d) } \sqrt[3]{0.02814} & \text { (e) } 1 / 926\end{array}$

4. Find the numbers following without illustrating the interpolation graphically. The tabular difference and the necessary number of tenths of that difference should be obtained mentally.

(a) $1.534^{2}, \sqrt{0.468}, 8.43^{3}, \sqrt[3]{2.46}, 1 / 647$.

(b) Squares of $2.784,3762,0.01388,3846000,0.00003728$.

(c) Square roots of $0.634,3.248,42.7,384.3,279,000,0.001876$.

(d) Cubes of $3.143,0.774,1683,0.00004592,4889000$.

(e) Cube roots of $0.02258,0.226,19.34,0.00176,328000$.

(f) Reciprocals of $31.76,0.00647,35990,0.0004325,647$.

5. Find the square of 4732 , and check the result by finding its square root. Find the cube root of 3479 , and check the result by cubing it. Find the reciprocal of 25.63 , and check the result.

6. Find $\sqrt{374.3^{3}}, \sqrt[3]{0.02478^{2}}, 1 / 73 \cdot 26^{2}, 1 / \sqrt{2438}, 1 / 48 \cdot 36^{3}$.

7. Solve the following equations, using the Tables to simplify the com. putations:
(a) $x^{2}+32 x+19=0$.
(b) $2 x^{2}-47 x-27=0$.
(c) $3 x^{2}+29 x-40=0$.
(d) $5 x^{2}+54 x-31=0$.

8. How long an umbrella will go into a trunk measuring $31.5 \times 18$. $\times 22.5$ inches, inside measure, (1) if the umbrella is laid on the bottom (2) if it is placed diagonally between opposite corners of the top an bottom? 
9. A house with a gambrel roof is $\mathbf{2 8 . 5}$ feet wide, the first set of rafters has a slope of $\frac{4}{3}$, and the top set a slope of $\frac{2}{3}$. If the joint in the roof comes 7.8 feet from the side of the building measured horizontally, how long is each set of rafters?

10. If five individuals weigh $120,124,116,112,123$ pounds, respectively, and five others $95,150,132,105,113$ pounds, respectively, then the average weight $M$ of either group is 119 . But one group is distributed very closely around the mean $M$, whereas the other group exhibits marked deviations from it. A measure of the variability or tendency to deviation of measurements is given by the following formula, called the standard deviation.

S.D. $=\sqrt{\frac{d_{1}^{2}+d_{2}^{2}+\cdots+d_{n}^{2}}{n}}$

The average $M$ is found, and each individual measurement is subtracted algebraically from $M$, thus obtaining a series of deviations $d$. The sum of the squares of these deviations is divided by $n$, the number of measurements, and the square root of the quotient is the standard deviation from the average $M$.

The coefficient of variability is defined as the ratio of the standard deviation from the average to the average.

Compare the coefficients of variability for the two sets of measurements.

11. The strength of grip of right hand and left hand, in hectograms, for 10 boys is given in the following table. Compute the standard deviation and coefficient of variability for each.

Right hand. 158, 200, 210, 226, 248, 270, 296, 320, 348, 403. Left hand. 138, 185, 200, 224, 244, 260, 282, 305, 336, 400.

43. Variation. 'Definition. It is said that $y$ varies as, or is proportional to, the nth power of $x$ if $y=k x^{n}, n$ being positive, while $y$ varies inversely as, or is inversely proportional to, the nth power of $x$ if $y=k / x^{n}$. If it is desired to contrast these two forms of variation, the former is called direct variation as opposed to inverse variation.

The case of direct variation in which $n=1$ has already been considered in Section 21, page 61. In any case, the value of the constant $k$ may be determined from a given pair of values of $x$ and $y$, as in that section, by substituting the given values of $x$ and $y$.

The graph of the relation $y=k x^{n}$ may be obtained from one of the curves considered in Sections 38 to 40 , by means of the theorem in Section 30.

The language of variation is used frequently in the applica- 
tions of mathematics. Thus for bodies moving with velocities near that of a rifle ball, the resistance of the air varies as the cube of the velocity. The intensity of light varies inversely as the square of the distance from the source of light, etc.

The language of variation is extended also to apply to relations involving more than two variables. One quantity is said to vary jointly as two or more other quantities, if the first is equal to a constant simes the product of the others: direct and inverse variation may both be involved. For example Newton's law of gravitation states that the attraction of two bodies varies jointly as their masses and inversely as the square of the distance between them. If the masses of the bodies are $m$ and $m^{\prime}$, and if $d$ is the distance between them, then the attraction $A$ is given by $A=\mathrm{kmm}^{\prime} / \mathrm{d}^{2}$.

\section{EXERCISES}

1. If the area of a rectangle is $\mathbf{1 2}$ square inches the altitude varies inversely as the base. Represent the relation graphically. On the figure construct several rectangles of area 12 .

2. A man told a contractor that he wished to have certain work done in the next three days. The contractor replied that it would be impossible, as it would take six men three weeks to do it. Whereupon the man told him that thirty-six men would do it in three days, and to have them on the job the next morning. What relation did he assume exists between the number of men and the number of days in which they could do the work?

3. An assumption which a psychologist has made states that the lawlessness of a mob varies as the square of the number of individuals involved. Illustrate by a graph. Compare the lawlessness of two mobs of 50 men and 500 men.

4. The mean distance of the earth from the sun is $93,000,000$ miles and from the moon is 240,000 . If the mass of the earth is taken as 1 , the masses of the sun and moon are approximately 330,000 and 1/81 respectively.

(a) Does the sun or the earth exert the greater attraction on the moon?

(b) Does the sun or the moon exert the greater attraction on the earth?

(c) At what distance from the earth would a particle be equally attracted by the earth and the moon? By the earth and the sun?

5. The horse power required to propel a boat varies approximately as the cube of its velocity. If a $58 \mathrm{H}$.P. engine will produce a velocity of 
10 feet per second, what horse power will produce a velocity of 20 feet per second? Plot the graph of the relation and illustrate the above data on it.

6. The weight of a metal disk of given thickness and material varies as the square of the diameter. (a) If a disk whose diameter is 1 inch weighs $\frac{1}{2}$ ounce, what is the weight of a disk whose diameter is 4 inches? What is the diameter of a disk weighing 7 ounces? Sketch the graph showing the weight of any such disk. (b) If a given disk weighs 12 ounces, how would its diameter compare with one whose weight is 6 ounces?

7. The volume of a sphere varies as the cube of its diameter. How many shot $\frac{1}{8}$ of an inch in diameter can be made by melting a lead sphere whose diameter is 2 inches?

8. The intensity of light varies inversely as the square of the distance from the source of light. If an object is 20 feet from a light, by how much must it be moved to receive twice as much light? Illustrate graphically.

9. If an object is 3 feet from a light, how far should it be moved to receive one-third as much light?

10. The volume of a cylinder varies jointly as the altitude and the square of the diameter. A preserving kettle 12 inches in diameter is filled to a depth of 8 inches. How many quart jars, $3 \frac{1}{2}$ inches in diameter and 6 inches high, will be needed to hold the preserves in the kettle?

11. In enlarging a photograph, the original negative is projected on a sensitized plate, just as a lantern slide is projected on a screen. The size (area) of the enlargement varies as the square of the distance from the source of light, and is equal to the original if the sensitized plate is placed right against the negative. If a $4 \times 5$ negative is placed ten inches from the source of light, where should the sensitized plate be placed to obtain an enlargement $8 \times 10$ inches?

44. Empirical Data Problems. Since the equation $y=k x^{n}$ may be written in the form $y / x^{n}=k$, the table of values of $x$ and $y$ is such that the quotients obtained by dividing the values of $y$ by the nth powers of the corresponding values of $x$ are equal. This property enables us to determine whether a given table of values, obtained by an experiment, may be represented approximately by $y=k x^{n}$, and to determine the coefficient $k$.

If the points whose coördinates are the pairs of values in the table are plotted, and appear to lie on the graph of $k x^{n}$, we will have $n>1$ if the graph is tangent to the $x$-axis at the origin, $0<n<1$ if the graph is tangent to the $y$-axis at the origin, and $n<0$ if the graph approaches the axes asymptotically (Summary, Section 41). 
If $n>1$, a simple value to choose for $n$ is 2 . By the above property, if the quotients obtained by dividing the values of $y$ by the squares of the corresponding values of $x$ differ by very little, the relation connecting $x$ and $y$ resembles $y=k x^{2}$, and an approximate statement of the law may be given in this form. Each quotient, $y / x^{2}$, is the value of $k$ for which the graph of $y=k x^{2}$ passes through the point $(x, y)$. A very good value to use for $k$ is obtained by finding the average of all the quotients. If, however, the quotients differ widely, it is well to try $n=3$, or $n=\frac{3}{2}$, or some other value of $n$, in order to see if an approximate statement of the law can be found which is better than $y=k x^{2}$.

If $0<n<1$, a simple value to choose for $n$ is $\frac{1}{2}$, but if the result is not satisfactory, other values of $n$ should be tried.

If $n<0$, the values occurring most frequently are $n=-1$ and $n=-2$. These cases may be distinguished, provided that the same unit is used on both axes, by the fact that the graph for $n=-1$ is symmetrical with respect to the bisector of the first and third quadrants. If neither of these values of $n$ proves satisfactory, others should be tried. If $n$ is negative, division by $x^{n}$ is equivalent to multiplication by $x^{-n}$. For example, if $n=-2$, we have $y / x^{-2}=y x^{2}$. The value of $k$ to be chosen is now the average of certain products instead of quotients.

The accuracy with which the equation finally obtained represents the law may be checked as on pages 80, 83, and 106 .

The use of the tables of squares, square roots, etc., is advantageous in finding $k$.

In a later chapter we shall obtain a more satisfactory method of determining $n$.

\section{EXERCISES}

1. In the following table the relation between $x$ and $y$ is $y=k / x$. Con$\frac{x}{y} \mid \frac{1,2, \quad 3, \quad 4,}{4,2.1,1.5,0.9}$, struct the graph and determine $k$. Hint. Find the product of $x$ and $y$ for each pair of values and take the average of these products. Check the accuracy of the result. 
2. In the following table the relation between $x$ and $y$ is $y=k / x^{2}$. $x \mid 1, \quad 2, \quad 3, \quad 4, \quad$ Plot the graph and determine $k$. Check the $y \mid 3,0.78,0.32,0.19$, accuracy of the result.

3. In the table $V$ represents the volume of a vessel containing a gas $V \mid 0.1,0.2,1,2,10, \quad$ and $p$ the pressure on the walls. $\bar{p} 10,5.01,0.99,0.52,0.13$, Find the relation between $V$ and $p$.

4. Determine the law of the attraction $A$ of an electrified rod for a $\frac{d}{A} \mid \frac{0.51,0.98,1.53,1.97,2.56}{5.98,1.48,0.66,0.38,0.23}$, pith ball at a distance $d$ as given in the table. What is the attraction when the distance is 1.2 ?

5. In the following table $W$ represents the effect of the blow when a $\frac{v}{W} \mid \begin{aligned} & 0, \quad 1, \quad 2, \quad 3, \quad 4, \\ & 0,0.23,1,2.37,4.01\end{aligned}$ weight of 16 pounds is dropped and strikes with a velocity $v$. Plot the data, determine the law, and find $W$ if $v=2.5$.

6. The discharge of water through a large pipe line or conduit is meas$\frac{x}{Q} \mid \frac{0, \quad 1.00, \quad 1.98, \quad 2.98, \quad 3.98,}{0,0.0442,0.0637,0.0780,0.0909}$, ured by a Venturi meter, on which we read the difference in height of a column of mercury in the two sides of a $U$-tube. In the table, $x$ denotes the difference in height and $Q$ the discharge. Determine the law.

7. From the following data determine the resistance $R$, in ohms per $\frac{d}{R} \mid 0.083,0.120,0.148,0.220$, mile, of a telegraph wire of diameter $\bar{R} \mid \frac{01,24.42,16.1,7.26,}{d \text {, in inches. }}$

8. Find the relation between the pressure $p$ and the volume $V$ of a gas $\frac{V}{p} \mid \frac{5.03,2.56,1.21,0.80,0.68 \text {, }}{0.51,0.97,2.04,3.03,3.94}$ if the pressure on the walls of a containing vessel for different volumes was found to be as in the table.

9. In the following table, $x$ denotes half the distance between two tele$\frac{x}{y} \mid \begin{gathered}20, \quad 30, \quad 40, \quad 50, \quad 60, \\ 0.98,2.21,3.95,6.19,8.97\end{gathered}$ graph poles, and $y$ the amount the wire sags at the center. Find the law.

10. The table gives the number of revolutions per minute, R.P.M., at $\frac{h}{\text { R.P.M. }} \mid \frac{11.63,62.8,138.8,174.5,}{440,1020,1525,1680,}$ which a certain form of water wheel runs without any load, for different values of the head, $h$ (the height of the surface of the mill pond above the bottom of the wheel). Find the relation.

11. The horse power developed by the water wheel in Exercise 10 is $\frac{h}{\text { H.P. }} \mid \frac{69.8,104.8,139.8,174.5,}{0.64,1.18,1.81,2.56,} \begin{aligned} & \text { given in the table. Find the law } \\ & \text { in the } y=k x^{3} \text {. }\end{aligned}$

12. (a) Economists define supply as the amount of anything which the $\frac{\text { Price }}{\text { Supply }} \mid \frac{0.60,1.00,1.40,1.80}{1, \quad 2, \quad 3,} 4$, producers will offer for sale at a given price. The table gives an assumed set of values of the supply of wheat, in mil- 
lion bushels, at various prices in dollars. Plot the graph and determine the law. What price per bushel will stimulate the producers to furnish 5 million bushels?

(b) The term demand is used to denote the quantity of anything which

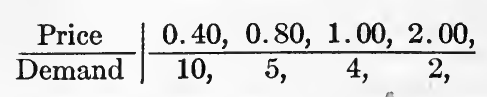
the consumers will purchase at a given price. How does the demand fluctuate as the price rises? As the price falls? The table gives an assumed set of values of the demand for wheat, in million bushels, at various prices, in dollars. Plot the graph and determine the law. What price will stimulate the consumers to demand 7 million bushels?

(c) Assuming the conditions in the preceding parts of this exercise, plot both graphs on the same axes, and find the price and the quantity of wheat transferred from producer to consumer.

13. If the apple crop amounts to 3 million bushels, then, at the harvest $\frac{\text { Price }}{\text { Demand }} \mid \begin{array}{cc}0.50,1,1.25, & 2.50, \\ 5,2.5,2, \quad 2,1,\end{array}$ season, the supply at any price high enough to pay to handle the crop is 3 . The table gives assumed values for the demand in million bushels. Plot both graphs on the same axes, and determine the equations giving the laws. At what price will apples sell under these conditions?

14. Some industries develop approximately to a point where any amount, within certain limits, can be supplied at a constant price. Sup$\frac{\text { Price }}{\text { Demand }} \mid \frac{3,4,5,6,8,10,}{6,5,4,3,2,1,} \begin{aligned} & \text { pose that any number of shoes will be } \\ & \text { supplied the price of } \$ 5 \text { per pair, and }\end{aligned}$ price, in dollars, is as given in the table. Plot both graphs on the same axes, and find the price and the number of shoes sold. Why cannot the price be less than $\$ 5$ ? Assuming competition, why will it not exceed $\$ 5$ ?

(b) If a monopoly arose, with the object of making as much money as possible instead of serving the public to the best of its ability with reasonable profits, the output would be restricted to the point of maximum profits. Assuming the conditions of the preceding part of the problem, suppose that the cost of manufacturing a pair of shoes is $\$ 4$. Construct a table giving the total profits at various prices, plot the graph, and determine the price and profits.

15. Plot the graphs and determine the laws for the data given in the tables. Determine the freight rate and the amount of freight handled.

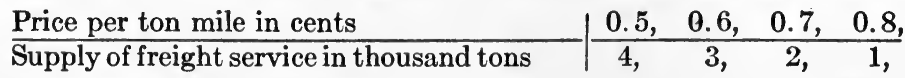

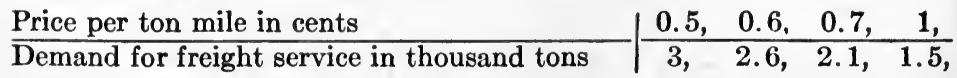




\section{The Linear Fractional Function $\frac{a x+b}{c x+d}$. In order that} this function should really be fractional, it is essential that $c \neq 0$, for if $c=0$, it reduces to the linear function $\frac{a}{d} x+\frac{b}{d}$.

The simplest linear fractional function is $1 / x$, obtained by setting $b=c$, and $a=d=0$, whose graph has been considered in Section 40. If $b \neq c$, while $a=d=0$, we have the function $b / c x$, or $k / x$, where $k=b / c$. Its graph may be obtained by multiplying by $k$ the ordinates of points on the graph of $1 / x$ (Theorem, page 89).

If we set $y=k / x$, whence $x y=k$, it is seen that the graph is symmetrical with respect to the line $y=x$, since the equation is unchanged if $x$ and $y$

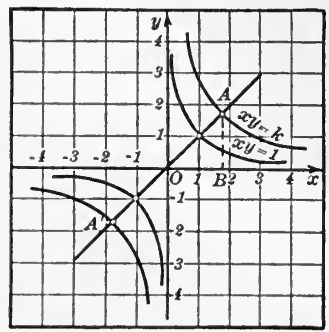

FIG. 66. are interchanged. The graph is also symmetrical with respect to the origin.

The geometric significance of the constant $k$ is obtained by solving the equations $x y=k$ and $y=x$; the solutions are $(\sqrt{k}, \sqrt{k})$ and $(-\sqrt{k},-\sqrt{k})$, which are the coördinates of the points of intersection of the graphs of the equations. The distance from the origin to the point of intersection $A$ is found from the right triangle $O A B$ to be $\sqrt{2 k}$. Hence, for a small value of $k$, the graph lies close to the origin and axes, while if $k$ is large, it lies at some distance from them. If $k$ is negative, the graph will lie in the second and fourth quadrants.

The graph of $x y=k$ is called a rectangular, or equilateral, hyperbola. The former term is derived from the fact that the asymptotes are perpendicular.

In order to determine the form of the graph of the general linear fractional function, set

$$
y=\frac{a x+b}{c x+d}
$$


Multiplying both sides by $c x+b$, and subtracting $a x+b$ from both sides,

$$
c x y-a x+d y-b=0 .
$$

Let us now see if we can simplify this equation by translating the axes (Section 31, page 89). Setting

we get

$$
x=x^{\prime}+h \quad \text { and } \quad y=y^{\prime}+k,
$$

$$
c\left(x^{\prime}+h\right)\left(y^{\prime}+k\right)-a\left(x^{\prime}+h\right)+d\left(y^{\prime}+k\right)-b=0,
$$

or, removing the parentheses and collecting like terms,

$$
c x^{\prime} y^{\prime}+(c k-a) x^{\prime}+(c h+d) y^{\prime}+c h k-a h+d k-b=0 \text {. }
$$

Equating to zero the coefficients of $x^{\prime}$ and $y^{\prime}$, we get

$$
c k-a=0 \text { and } c h+d=0 \text {. }
$$

Solving for $h$ and $k$, which is always possible since $c \neq 0$,

$$
h=-d / c, k=a / c \text {. }
$$

Substituting these values in (5) we get

$$
c x^{\prime} y^{\prime}+c\left(-\frac{d}{c}\right)\left(\frac{a}{c}\right)-a\left(-\frac{d}{c}\right)+d \frac{a}{c}-b=0 .
$$

Simplifying, subtracting the constant term from both sides and dividing by $c$,

$$
x^{\prime} y^{\prime}=\frac{b c-a d}{c^{2}} .
$$

This equation, referred to the new axes, has the same graph as (1). It is of the form $x^{\prime} y^{\prime}=k$, where $k=(b c-a d) / c^{2}$, or $y^{\prime}=k / x^{\prime}$. The graph is therefore a rectangular hyperbola.

Hence we have the

Theorem. The graph of a linear fractional function (1), or of an equation of the form (2), is a rectangular hyperbola whose asymptotes are parallel to the axes of coördinates.

\section{EXERCISES}

1. Plot the graph of $y=(2 x-4) /(x+3)$, (a) by translating the axes; (b) by finding the asymptotes (by the method on page 27), plotting one branch of the curve from a table of values, and the other by means of the symmetry with respect to the point of intersection of the asymptotes. 
2. Plot the graphs of the following equations:
(a) $y=\frac{x+4}{x-5}$.
(b) $y=\frac{3 x+6}{x-2}$.
(c) $y=\frac{x-4}{2 x-3}$.
(d) $x y-2 x+4 y-8=0$.
(e) $2 x y-x+3 y+8=0$.

3. The linear fractional function may be written in the form $a(x+b / a) / c(x+d / c)$. Prove that the linear factors cancel, and the function is a constant, if and only if $b c-a d=0$. For this reason, it is always assumed that $b c-a d \neq 0$.

4. The relation between the radius of curvature of a concave mirror, $R$, the distance from the center of the mirror to the object, $x$, and the distance from the center to the reflected image, $y$, is

$$
\frac{2}{R}=\frac{1}{x}+\frac{1}{y} \text {. }
$$

If $R=2$ feet, construct the graph of the equation.

46. Integral Rational Functions. The general form of an integral rational function or polynomial is (see page 39 )

$$
f(x)=a_{0} x^{n}+a_{1} x^{n-1}+\ldots+a_{n-1} x+a_{n},
$$

where $n$ is an integer, and $a_{0}, a_{1}, \ldots a_{n}$ are constants, positive, negative or zero, except that $a_{0} \neq 0$. It is said to be of degree $n$.

We have already studied polynomials of the first and second degree in considering linear and quadratic functions.

The calculation of a table of values for a polynomial of higher degree than the second by direct substitution presents no new features, but because of the greater number of terms that may be present there is more labor involved. An alternative method which is simpler, in general, than that of direct substitution is developed in the next sections.

\section{The Remainder Theorem.}

Example. Let us divide the polynomial $2 x^{3}-6 x^{2}+11$ by $x-2$, arranging the work as usual.

$$
\begin{aligned}
& \frac{2 x^{3}-6 x^{2}}{2 x^{3}-4 x^{2}}+11 \frac{\mid x-2}{2 x^{2}-2 x-4} \\
& \hline-2 x^{2} \\
& \frac{-2 x^{2}+4 x}{-4 x} \\
& \frac{-4 x+8}{+3}
\end{aligned}
$$


The result may be put in the form

$$
\frac{2 x^{3}-6 x^{2}+11}{x-2}=2 x^{2}-2 x-4+\frac{3}{x-2},
$$

where $2 x^{2}-2 x-4$ is the quotient, denoted by $q(x)$, and the remainder is $R=3$.

Equation (1) is an identity in accordance with the

Definition. An identity is an equation which is satisfied by all values of the variable or variables.

Substituting 2 for $x$ in the polynomial in the example,

$$
f(2)=2.2^{3}-6.2^{2}+11=16-24+11=3 .
$$

Hence the remainder, $R=3$, obtained by dividing the polynomial by $x-2$ is equal to the value of the polynomial when 2 is substituted for $x$.

This result is a verification of a theorem which is proved for any polynomial as follows:

Remainder Theorem. If a polynomial $f(x)$ is divided by $x$ - $a$, the remainder is $f(a)$.

If $f(x)$ be divided by $x-a$, the quotient $q(x)$ is a polynomial of degree one less than that of $f(x)$, and the remainder $R$ is a constant.

The result of the division may be expressed in the form

$$
\frac{f(x)}{x-a}=q(x)+\frac{R}{x-a} \text {. }
$$

Multiplying both members by $x-a$, we obtain the identity

$$
f(x)=(x-a) q(x)+R .
$$

Substituting $a$ for $x$, we have

$$
f(a)=(a-a) q(a)+R .
$$

Since $a-a=0$, we have $(a-a) q(a)=0$.

Hence

$$
f(a)=R \text {. }
$$

48. Synthetic Division. The form of the division in the example of the preceding section is too cumbersome to be of practical use in calculating the table of values of a polynomial. The division may be simplified as follows: 
We note that the operations involved in the division were performed on the coefficients of the dividend, divisor and quotient, the powers of $x$ serving merely to determine the proper positions of these coefficients in carrying out the details of the division. If missing terms are supplied by zero coefficients, the division can be performed by the so-called method of detached coefficients as follows:

$$
\begin{aligned}
& \begin{array}{l|l}
2-6 & +0+11 \frac{1-2}{\mid 2-2}-4 \\
\frac{2-4}{-2} &
\end{array} \\
& \frac{-2+4}{-4} \\
& \begin{array}{r}
-4+8 \\
+3
\end{array}
\end{aligned}
$$

The first term in each partial product is canceled in the subtraction and hence may be omitted. The second term of each partial product may be written immediately under the term in the dividend from which it is subtracted. The first term in the divisor is not now needed, so that the process is further condensed as follows:

$$
\begin{array}{rl}
2^{*}-6+0+11 & -2 \\
-4+4-8 & 2-2-4 \\
\hline-2^{*}-4^{*}+3 &
\end{array}
$$

Noting that the coefficients of the quotient are identical with the numbers marked with an asterisk $(*)$, it is unnecessary to write them under the divisor. As we shall use this process to find the value of $f(2)$, it is convenient to replace -2 in the divisor by +2 and add throughout instead of subtracting. Also bringing down the first coefficient of the dividend, the work is now arranged in the form

$$
\begin{aligned}
& 2-6+0+11\lfloor 2 \\
& +4-4-8 \\
& \hline 2-2-4+3
\end{aligned}
$$

The numbers in the last row are called partial remainders, the last being the remainder 3 (the value of the polynomial 
when 2 is substituted for $x$ ). The preceding partial remainders $2,-2,-4$ are the coefficients of the quotient, the first being the same as the first coefficient of the dividend.

The process of synthetic division may be described as follows:

To divide a polynomial by $x-a$,

Write the coefficients in the order of descending powers of $x$, supplying missing terms with zero coefficients. To the right write $a$.

Bring down the first coefficient of the dividend.

Multiply the first coefficient by $a$, and add the product to the second coefficient.

Multiply the sum so obtained by a, and add the result to the third coefficient, etc.

The last term will be the remainder, $R$, and the preceding partial remainders will be the coefficients of the quotient, $q(x)$, which will be of one degree less than the degree of the polynomial.

The remainder theorem, with the aid of synthetic division, affords a simple method of calculating the value of a polynomial for a given value of $x$.

For example, if $f(x)=3 x^{4}-9 x^{3}-4 x^{2}-17 x-37$, find $f(4)$. By the remainder theorem, $f(4) \quad 3-9-4-17-37\lfloor 4$ is the value of the remainder, $R$, when $f(x)$ is divided by $x-4$. By synthetic $\overline{3+3+8+15+23}$ division we find that $f(4)=23$. Verify this result by substituting 4 for $x$ in the given polynomial.

If the multiplications and additions are performed mentally, the partial products in the second row of numbers omitted, and the partial remainders alone put down, the computation may be arranged still more compactly in the following form:

The second arrangement is more convenient in calculating a table of values of a polynomial for integral \begin{tabular}{l|l}
$3-9-4-17-37$ \\
$4+3+8+15+23$
\end{tabular} values of $x$, while we shall find that the first is better adapted to finding zeros of the function if the zeros are irrational.

49. Graph of a Polynomial. Example. Construct the graph of

$$
f(x)=x^{3}-3 x^{2}-10 x+24 .
$$


The table of values is constructed by means of synthetic division and the remainder theorem, the details of the work and the graph being given below.

$$
\begin{array}{ll}
1-3-10+24 \mid 1 & 1-3-10+24 ! 2 \\
+1-2-12 & \begin{array}{r}
+2-2-24 \\
1-2-12+12
\end{array}
\end{array}
$$

Hence we find that $f(1)=12$, and $f(2)=0$.

The calculations may be arranged compactly by writing the coefficients of the polynomial once for all as in the top row of the following table and by operating on this top row with each value of $x$, performing the multiplications and additions mentally and entering the partial remainders on the same row with

\begin{tabular}{|c|c|c|}
\hline$x$ & & $f(x)$ \\
\hline 0 & $1-3-10$ & 24 \\
\hline 1 & $-2-12$ & 12 \\
\hline & $-1-12$ & 0 \\
\hline & $0-10$ & -6 \\
\hline & $+1-6$ & 0 \\
\hline 5 & +20 & 24 \\
\hline 6 & $+3+8$ & 72 \\
\hline & $-4-6$ & 30 \\
\hline & $-5 \quad 0$ & 24 \\
\hline & $-6+8$ & \\
\hline & $-7+18$ & -48 \\
\hline
\end{tabular}
the value of $x$, under the proper coefficient of the dividend.

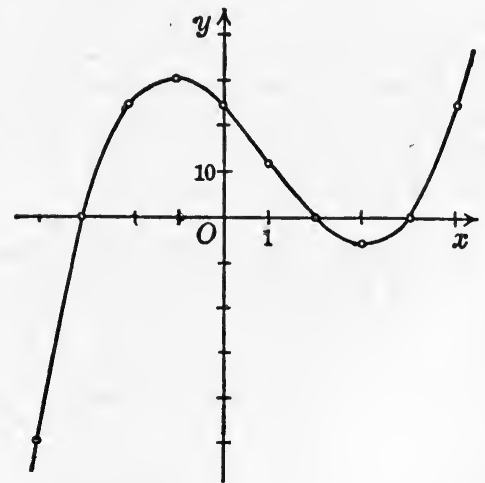

Fig. 67.

50. Extent of the Tables. In the example of the preceding section an examination of the synthetic division by $x-6$ shows that the successive remainders $1,+3,+8,+72$ are all positive. If a larger value of $x$ be used, by the nature of synthetic division, it is evident that the successive remainders will be positive and larger. Hence as $x$ increases beyond 6 $f(x)$ will also increase and the curve will run up to the right indefinitely. The synthetic division by $x+4$ gave remainders $1,-7,+18,-48$ which have alternating signs. As $x$ de- 
creases below $-4, f(x)$ also decreases and hence the curve runs down indefinitely to the left.

The extreme values of $x$ that should be included in the table are determined by the following rule:

The largest value included in the table is such that in the synthetic division the signs of the partial remainders are all the same.

The smallest value (algebraically) included is such that these signs alternate.

\section{EXERCISES}

1. Plot the graphs of each of the polynomials below. From the graph find approximate values of the roots of the equation obtained by equating the polynomial to zero.
(a) $x^{2}-2 x+7$.
(b) $x^{4}-3 x^{3}+2 x^{2}$.
(c) $x^{3}+3 x^{2}-10 x-24$.
(d) $2 x^{3}-8 x^{2}-12 x+17$.
(e) $x^{3}+3 x^{2}+3 x+1$.
(f) $x^{5}-3 x^{4}-x+3$.

2. The graphs of the functions $x^{3}-x^{2}, 2 x^{3}-3 x^{2}+x, 2 x^{3}-x^{2}-x$ pass, through the origin and the point $(1,0)$. Find the slope of the tangent line to each graph at these points, and plot the graphs. How does the slope of the tangent line assist in drawing the graphs?

3. Plot the graph of $x^{4}-4 x^{3}+4 x^{2}$, and show that it is tangent to the $x$-axis at two points.

4. A body moves so that its position, $s$, with reference to a fixed point at any time $t$, is given by one of the following equations. Find the velocity, $v$, and the acceleration, $a$, at any time $t$. Construct the graphs of $s, v$, and $a$ on the same set of axes. When and where is the body at rest? What is the value of the acceleration when the velocity is a minimum? Where is the body at this moment and what is its velocity?
(a) $s=t^{3}-3 t$.
(b) $s=t^{3}+3 t^{2}$.
(c) $s=t^{3}-6 t^{2}+9 t$.
(d) $s=t^{3}+3 t^{2}-t-3$.

Suggestion. To find the acceleration, $a$, at any time $t$, find the limit of the average rate of change of $v$ with respect to $t$.

Note. Rate of change of a polynomial. It was seen on page 111 that the rate of change of $x^{n}$ is $n x^{n-1}$, and it may be shown that the rate of change of $a x^{n}$ is $a n x^{n-1}$. Thus the rate of change of $4 x^{3}$ is $12 x^{2}$, and that of $-2 x^{4}$ is $-8 x^{3}$. It will be show in a later chapter that the rate of change of a polynomial, or the slope of the line tangent to the graph, may be obtained by adding the rates of change of the successive terms, noting that the rate of change of the constant term is zero. 
For example, the rate of change $m$ of the polynomial

is

$$
\begin{aligned}
& y=x^{4}-3 x^{3}+4 x^{2}-5 x+7 \\
& m=4 x^{3}-9 x^{2}+8 x-5
\end{aligned}
$$

5. Find the slope $m$ of a line tangent at any point to the graph of each of the following equations. Find the rate of change of the slope $m$ with respect to $x$. Symbolize the rate of change of $m$ with respect to $x$ by $F$ and plot the graphs of $y, m$ and $F$ on the same axes. Find the ordinate of the point on the graph of $y$, and also of the point on the graph of $F$, which has the same abscissa as the minimum point of $m$. Where is the line tangent to the graph of $y$ horizontal?
(a) $y=x^{3}-6 x^{2}+9 x-2$.
(b) $y=x^{3}-3 x^{2}-9 x+4$.
(c) $y=4 x^{3}-15 x^{2}+12 x$.
(d) $y=x^{3}-12 x+5$.

Note. The point on a graph where $m$ ceases to decrease and begins to increase (or vice-versa) is called a point of inflection. The graph is concave downward on one side of this point and concave upward on the other side. For this value of $x$ the value of $F$ is zero.

Hence, to find the abscissas of the points of inflection on the graph of a function, equate $F$ to zero and solve for $x$. Substitute these values of $x$ in the equation $y=f(x)$ to find the ordinates.

6. Find the coördinates of the point of inflection of the graph of each of the following equations. Translate the axes to this point as a new origin. Show that the graph is symmetric with respect to the point of inflection, and plot the graph of the equation on the new axes.
(a) $y=x^{3}-3 x^{2}-6 x+6$.
(c) $y=x^{3}+6 x^{2}+12 x+8$.
(b) $y=x^{3}-6 x^{2}+12 x-11$.
(d) $y=-x^{3}+3 x^{2}+x-6$.

7. Find $m$ and $F$ for the function $a x^{3}+b x^{2}+c x+d$. If the axes are translated to the point of inflection of the graph of this function, show that the equation of the graph referred to the new origin is $y^{\prime}=a x^{\prime 3}+m^{\prime} x^{\prime}$, where $m^{\prime}$ is the slope of the line tangent to the graph of $y$ at the point of inflection, and that the graph is symmetrical with respect to the point of inflection.

8. Given $s=t^{3}-4 t$, find (a) the average velocity from $t=2$ to $t=4$, (b) the average of the velocities at $t=2$, and $t=4$, (c) the velocity at $t=3$, (d) the value of $t$ at which the velocity is equal to the average velocity for the interval $t=2$ to $t=4$.

9. Find the points of inflection of the following functions and plot their graphs.
(a) $x^{4}-8 x^{3}+3 x^{2}$
(b) $x^{5}-10 x^{2}-8$.
(c) $x^{4}-4 x^{3}$. 
10. For what positive integral values of $n$ does $x^{n}$ have a point of inflection?

51. Solution of Equations. Rational Roots. Any algebraic equation in one variable may be reduced to the form

$$
f(x)=0 \text {, }
$$

where $f(x)$ is a polynomial, by the elementary rules for simplifying an equation involving fractions and radicals. The real roots of the equation (or zeros of the function) are represented graphically by the intercepts on the $x$-axis of the graph of $f(x)$. (See page 23.)

If any integers are roots of (1), they will appear when the table of values for the graph is being constructed. Thus the integral roots of the equation

$$
x^{3}-3 x^{2}-10 x+24=0
$$

obtained by equating to zero the polynomial in the example of Section 49 are 2,4 , and -3 , since the table of values shows that

$$
f(2)=0, \quad f(4)=0, \quad \text { and } \quad f(-3)=0 .
$$

That these are all the roots follows from

Theorem 1. An equation of degree $n$ has $n$ roots, of which some may be imaginary and some equal to each other.

This theorem is assumed without proof.

Returning to equation (1), any real roots other than integers are either fractions or irrational numbers. In this section we shall see how to find the fractional roots of an equation, which with the integral roots, constitute the rational roots.

Theorem 2. If $x=a$ is a root of an equation of degree $n$, $f(x)=0$, then $f(x)=(x-a) q(x)$, where $q(x)$ is of degree $n-1$.

By the definition of a root of an equation $f(a)=0$. Then by the remainder theorem, if $f(x)$ is divided by $x-a$, the remainder is zero, so that $f(x)$ is exactly divisible by $x-a$. Since the degree of the divisor is 1 , the quotient $q(x)$ will be a polynomial of degree $n-1$. Since the dividend is equal to the product of the divisor and quotient, we have the identity

$$
f(x)=(x-a) q(x) \text {. }
$$


Corollary 1. Any root of the given equation $f(x)=0$, except $x=a$, is also a root of the equation $q(x)=0$.

Let $x=b$ be. any root of $f(x)=0$ different from $x=a$; then $f(b)=0$. Substituting $x=b$ in (2)

$$
0=(b-a) q(b) \text {. }
$$

But if the product of two numbers is zero, one of the numbers is zero, and since $b-a$ is not zero, we have $q(b)=0$, and hence $x=b$ is a root of the equation $q(x)=0$.

Corollary 2. Any root of the equation $q(x)=0$ is a root of the equation $f(x)=0$.

If $x=c$ is a root of $q(x)=0$, then $q(c)=0$. Setting $x=c$ in (2) we have

$$
f(c)=(c-a) q(c)=(c-a) \cdot 0=0 .
$$

Hence $x=c$ is a root of the equation $f(x)=0$.

This last theorem and its corollaries simplify the solution of many equations by enabling us, as soon as a rational root is known, to replace the given equation by one of lower degree.

Example 1. Solve the equation $f(x)=$ $12 x^{3}-11 x^{2}-13 x+10=0$. The graph shows that the three roots are all real, and the table of values shows that one root is -1 . Dividing $f(x)$ by $x-(-1)=x+1$, we have from

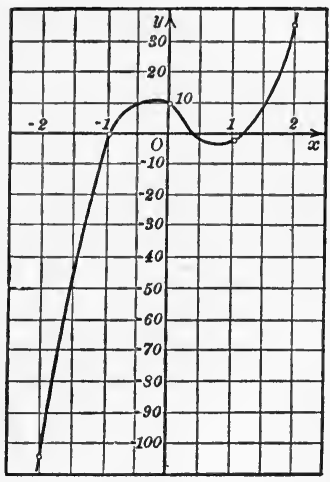

Fig. 68. the table the quotient $q(x)=12 x^{2}-23 x+10$.

\begin{tabular}{r|r|r}
$x$ & & \multicolumn{1}{c}{$f(x)$} \\
\hline 0 & $12-11-13$ & +10 \\
1 & $+1-12$ & -2 \\
2 & $+13+13$ & +36 \\
-1 & $-23+10$ & 0 \\
-2 & $-35+57$ & -104 \\
\hline
\end{tabular}

By Corollary 2, the roots of the equation

$$
q(x)=12 x^{2}-23 x+10=0
$$

are also roots of $f(x)=0$.

Factoring $q(x)$, we have

$$
(3 x-2)(4 x-5)=0 \text {. }
$$

Hence $3 x-2=0$ and $4 x-5=0$, and the roots are therefore

$$
x=\frac{2}{3} \text { and } x=\frac{5}{4} .
$$

The roots of the given equation are therefore $x=-1, \frac{2}{3}$, and 5 . 
We note that the denominators of the fractional roots in Example 1 are both factors of the coefficient of the term of highest degree, and that the numerators are factors of the constant term. This conclusion is a special instance of the following theorem which we assume without proof.

Theorem 3. If $a / b$ is a root of an equation with integral coefficients, then $b$ is a factor of the coefficient of the highest power of $x$ and $a$ is a factor of the constant term (or of the coefficient of the lowest power of $x$ if the constant term is zero).

Corollary. If the coefficient of the highest powers of $x$ is unity and the other coefficients are integers, then the only rational roots are integers.

Fractional roots may be determined by the use of this theorem, synthetic division and the remainder theorem as in

Example 2. Solve the equation $3 x^{4}-5 x^{3}-24 x^{2}+16 x+40=0$. The table of values and the graph are given below.

\begin{tabular}{r|r|l}
$x$ & & $f(x)$ \\
\cline { 2 - 3 } 0 & $3-5-24+16$ & +40 \\
1 & $-2-26-10$ & +30 \\
2 & $+1-22-28$ & -16 \\
3 & $+4-12-20$ & -20 \\
4 & $+7+4+32$ & +168 \\
-1 & $-8-16+32$ & +8 \\
-2 & $-11-2+20$ & 0 \\
-3 & $-14+18-38$ & +154 \\
\hline
\end{tabular}

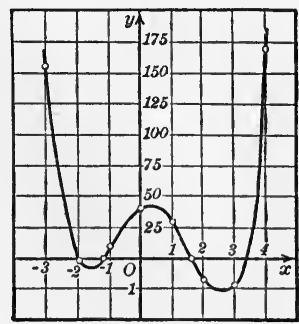

FIg. 69.

The graph shows that there are four real roots. One root is -2 . Dividing $f(x)$ by $x+2$, we have from the table the quotient

$$
q(x)=3 x^{3}-11 x^{2}-2 x+20 .
$$

By Corollary 2, the roots of the equation $q(x)=0$ are the other roots of $f(x)=0$. One root lies between 1 and 2. A fraction between 1 and 2 whose numerator is a factor of 20 and whose denominator is a factor of 3 is $\frac{5}{3}$. Dividing the polynomial by $x-\frac{5}{3}$, it is $\mid 3-11-2+20$ seen that $x=\frac{5}{3}$ is a root and that the quotient is $\frac{5}{3} \mid-6-120$ $3 x^{2}-6 x-12$. Equating this function to zero, dividing by 3, and solving by the quadratic formula we have

$$
x=\frac{2 \pm \sqrt{4+16}}{2}=1 \pm \sqrt{5}=1 \pm 2.236=3.236 \text { or }-1.236 \text {. }
$$

Hence the roots of the given equation are $x=-2, \frac{5}{3}, 3.236,-1.236$. 
Example 3. Solve the equation

$$
6 x^{5}-16 x^{4}+11 x^{3}+37 x^{2}-116 x+60=0 .
$$

\begin{tabular}{|c|c|c|}
\hline$x$ & & $f(x)$ \\
\hline 0 & $6-16+11+37-116$ & +60 \\
\hline 1 & $-10+1+38-78$ & -16 \\
\hline 2 & $-4+3+43-30$ & 0 \\
\hline 3 & $+2+17+88+148$ & +504 \\
\hline-1 & $-22+33+4-120$ & +180 \\
\hline-2 & $-28+67-97+78$ & -96 \\
\hline & $6-4+3+43 \mid-30$ & \\
\hline$\frac{2}{3}$ & $+0+3+45$ & \\
\hline 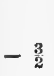 & $\begin{array}{l}2+0+1+15 \\
-3+1 \frac{1}{2}\end{array}$ & \\
\hline
\end{tabular}

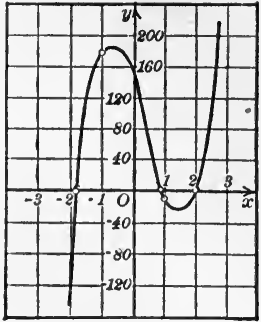

Fig. 70.

An examination of the table of values of the function $f(x)$ shows that $x=2$ is a root, and that the quotient obtained by dividing $f(x)$ by $x-2$ is

$$
q(x)=6 x^{4}-4 x^{3}+3 x^{2}+43 x-30 .
$$

By Corollary 2 all the roots of $q(x)=0$ are identical with the remaining roots of the given equation. The graph shows that one of these is between 0 and 1. If this root is rational, it must be one of the fractions $\frac{1}{2}, \frac{1}{3}, \frac{2}{3}$ (Theorem 3). From the graph it appears that the most probable of these values is $\frac{2}{3}$ and we therefore test it first. As shown in the table, if $q(x)$ is divided by $x-\frac{2}{3}$ the remainder is zero, and therefore $x=\frac{2}{3}$ is a root of $q(x)=0$ and hence of (3).

Applying Corollary 1 to the equation $q(x)=0$, it is seen that the remaining roots of this equation, and hence of the original equation, are identical with the roots of

or the simpler form

$$
\begin{aligned}
& 6 x^{3}+3 x+45=0 \\
& 2 x^{3}+x+15=0 .
\end{aligned}
$$

The graph shows that there must be a real root of (3), and hence of (4), between -1 and -2 . Theorem 1 shows that the only possibility of a rational root between -1 and -2 is $-\frac{3}{2}$. Dividing $2 x^{3}+x+15$ by $x+\frac{3}{2}$ leads to a remainder that is not zero and hence $x=-\frac{3}{2}$ is not a root.

The division of the left member of (4) by $x+\frac{8}{2}$ in the table stopped with the appearance of a fraction among the partial remainders. This is sufficient to show that the final partial remainder cannot be zero. For if a partial remainder is prime to the denominator of a fractional divisor in lowest terms, it will be prime to the powers of that denominator which appear in the further multiplication by the divisor. Hence the remaining 
partial products must be fractions and the last integral coefficient cannot be canceled by a fraction.

The rational roots of (3) are therefore $x=2$ and $\frac{2}{3}$, and the irrational root is, from the graph, nearly - 1.8. The other two roots (Theorem 1) are imaginary.

\section{EXERCISES}

1. Find all the roots of the equations below, in each case constructing the graph of the polynomial on the left.

(a) $x^{3}-x^{2}-7 x+3=0$.

(b) $x^{3}+5 x^{2}+x-12=0$.

(c) $x^{4}-4 x^{3}+8 x-3=0$.

(d) $x^{4}-2 x^{3}+2 x^{2}-x-6=0$.

(e) $6 x^{3}-17 x^{2}+7 x-5=0$.

(f) $3 x^{3}+4 x^{2}-21 x+10=0$.

(g) $6 x^{3}+20 x^{2}+x-20=0$.

(h) $4 x^{4}+4 x^{3}-5 x^{2}-9 x-9=0$.

(i) $6 x^{4}-8 x^{3}-5 x^{2}-4 x-4=0$.

(j) $6 x^{4}+23 x^{3}-7 x^{2}-11 x+4=0$.

2. Find all the rational roots of the equations below, in each case constructing the graph of the polynomial on the left, and estimating the irrational roots, if any, from the graph.

(a) $2 x^{4}-x^{3}+4 x^{2}+24 x-13=0$.

(b) $3 x^{4}+2 x^{3}+3 x^{2}-19 x-14=0$.

(c) $5 x^{5}-7 x^{4}-x^{3}-12 x^{2}+x+6=0$.

(d) $2 x^{5}+5 x^{4}-6 x^{3}-11 x^{2}-3 x+9=0$.

(e) $6 x^{3}-17 x^{2}+7 x-5=0$.

(f) $3 x^{4}+10 x^{3}+7 x^{2}-3 x-7=0$. Using synthetic division, find the irrational root to the nearest tenth of a unit.

3. Show that the graph of a polynomial of degree $n$ cannot have more than $n-1$ maximum and minimum points, and that it cannot have more than $n-2$ points of inflection.

52. Translation of the $y$-Axis. For the purpose of determining approximately an irrational root of an equation $f(x)=0$, where $f(x)$ is a polynomial, a method is used which reduces the roots of an equation by a constant amount $c$.

A translation of the $y$-axis along the $x$-axis in the positive direction diminishes the intercepts on the $x$-axis of the graph of $f(x)$, and hence such a translation represents graphically a diminution of the roots of an equation.

We shall consider the method first from a graphical point of view. 
Example. Construct the graph of

$$
y=x^{3}-9 x^{2}+23 x-15
$$

and find the function whose graph is the same curve referred to a new axis 2 units to the right of the old one.

The graph is constructed by the method of Section 49 .

The equations for translating the axes are (Section 31 , page 89 )

$$
\begin{aligned}
& x=x^{\prime}+2, \\
& y=y^{\prime} .
\end{aligned}
$$

Substituting in (1) we have

$$
\begin{aligned}
y^{\prime}=\left(x^{\prime}+2\right)^{3}-9\left(x^{\prime}+2\right)^{2}+23\left(x^{\prime}+2\right)-15 \\
=x^{\prime 3}+6 x^{\prime 2}+12 x^{\prime}+8 \\
-9 x^{\prime 2}-36 x^{\prime}-36 \\
+23 x^{\prime}+46
\end{aligned}
$$

or

$$
-15
$$

$$
y^{\prime}=x^{\prime 3}-3 x^{\prime 2}-x^{\prime}+3,
$$

which is the required polynomial.

If $f(x)$ represents the function in (1), then $f\left(x^{\prime}+2\right)$ will represent the function in (2). The intercepts on the $x$-axis of the graph referred to the new origin are 2 units less than the intercepts referred to the old origin. Hence the roots of the

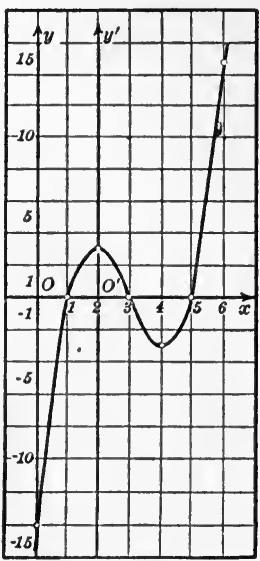

FIG. 71. equation $f\left(x^{\prime}+2\right)=0$, or

$$
x^{\prime 3}-3 x^{\prime 2}-x+3=0
$$

are 2 units less than the roots of the equation $f(x)=0$ or

$$
x^{3}-9 x^{2}+23 x-15=0 .
$$

We shall now derive a method of obtaining the coefficients of (3) which is simpler in general than the preceding method.

Let $\quad f(x)^{0}=a_{0} x^{n}+a_{1} x^{n-1}+\cdots+a_{n-2} x^{2}+a_{n-1} x+a_{n}$.

Substituting $x=x^{\prime}+c$ we have

$$
\begin{aligned}
f\left(x^{\prime}+c\right)=a_{0}\left(x^{\prime}\right. & +c)^{n}+a_{1}\left(x^{\prime}+c\right)^{n-1}+\cdots \\
& +a_{n-2}\left(x^{\prime}+c\right)^{2}+a_{n-1}\left(x^{\prime}+c\right)+a_{n}
\end{aligned}
$$

Expanding the terms on the right by the binomial theorem, collecting like powers of $x^{\prime}$, and representing the coefficients of the powers of $x^{\prime}$ by

$$
b_{0}, b_{1}, b_{2}, \ldots b_{n} \text {, }
$$

we have $f\left(x^{\prime}+c\right)=b_{0} x^{\prime n}+b_{1} x^{\prime n-1}+\cdots+b_{n-2} x^{\prime 2}+b_{n-1} x^{\prime}+b_{n}$.

For any value of $x$ and $x^{\prime}$ connected by the relation $x=x^{\prime}+c$,

$$
x-c=x^{\prime},
$$


we have

$$
\begin{aligned}
& f(x)=f\left(x^{\prime}+c\right) . \\
& \frac{f(x)}{x-c}=\frac{f\left(x^{\prime}+c\right)}{x^{\prime}} .
\end{aligned}
$$

Dividing (8) by (7)

If $f\left(x^{\prime}+c\right)=b_{0} x^{\prime n}+b_{1} x^{\prime n-1}+\cdots \cdot+b_{n-2} x^{\prime 2}+b_{n-1} x^{\prime}+b_{n}$ be divided by $x^{\prime}$, as indicated on the right of (9), the remainder is $b_{n}$ and the quotient is

$$
q_{1}\left(x^{\prime}\right)=b_{0} x^{\prime n-1}+b_{1} x^{\prime n-2}+\cdots \cdot+b_{n-2} x^{\prime}+b_{n-1} .
$$

If the quotient $q_{1}\left(x^{\prime}\right)$ is divided by $x^{\prime}$, the remainder is $b_{n-1}$ and the quotient is

$$
q_{2}\left(x^{\prime}\right)=b_{0} x^{\prime n-2}+b_{1} x^{\prime n-3}+\cdots+b_{n-2} \text {. }
$$

Continuing this process it is seen that the successive remainders are the coefficients of (6) in the order $b_{n}, b_{n-1}, b_{n-2}, \ldots b_{0}$. But if the indicated divisions be performed on both sides of (9), if the respective quotients thus obtained be divided by $x-c$ and $x^{\prime}$, and so on, the successive remainders obtained on the left side of (9) will equal those obtained on the right,

\begin{tabular}{|c|c|c|}
\hline $1-9$ & +23 & -15 \\
\hline+2 & -14 & +18 \\
\hline $1-7$ & +9 & $+3^{*}$ \\
\hline+2 & -10 & \\
\hline $1-5$ & $-1^{*}$ & \\
\hline+2 & & \\
\hline $1^{*}-3$ & & \\
\hline
\end{tabular}
namely, $b_{n}, b_{n-1}, b_{n-2}, \ldots b_{0}$. Hence we have the

Theorem. The coefficients of $f\left(x^{\prime}+c\right)$ may be obtained by dividing $f(x)$ by $x-c$, the quotient by $x-c$, etc.

The computation of the coefficients in the example may be effected by means of this theorem and synthetic division in compact form as indicated. The successive remainders in the divisions, marked with an asterisk, are the coefficients of the function in (3).

\section{EXERCISES}

1. Construct the graph of each of the functions below, and find the function having the same graph referred to a new $y$-axis $c$ units to the right. Verify the result by direct substitution of $x^{\prime}+c$ for $x$.
(a) $x^{3}-3 x^{2}+x-2, c=3$.
(c) $x^{4}+3 x^{3}-7 x^{2}+13 x-5, c=2$.
(b) $2 x^{3}-x^{2}+3, c=1$.
(d) $3 x^{4}-8 x^{3}-9 x-12, c=1$.

2. Show that the second term of the function $y=x^{3}-6 x^{2}+7 x+4$ will be removed if the $y$-axis is translated 2 units to the right.

Deduce a rule for removing the second term of $a x^{3}+b x^{2}+c x+d$.

3. Determine a translation of the $y$-axis so that the graph of $x^{3}+3 x^{2}-4$ in the old system will be the graph of $x^{3}-3 x+2$ in the new system. Plot the graphs of $x^{3}$ and $3 x-2$ on the same axes and from them determine approximately the roots of the equation $x^{3}-3 x+2=0$, and hence of the equation $x^{3}+3 x^{2}-4=0$. 
4. Plot the graph of $x^{4}-2 x^{3}-3 x^{2}+4 x+2$ and determine the coordinates of the maximum and minimum points and of the points of inflection. Find the function which has the same graph referred to a $y$-axis 1 unit to the left.

53. Horner's Method of Solution of Equations. This method enables us to compute irrational roots as accurately as may be desired.

Example. Find, correct to two decimal places, the real root of the equation

$$
f(x)=x^{3}+x-47=0 .
$$

Plotting the graph of $f(x)$ we get the curve in the figure, which shows that there is a real root between 3 and 4 . As the coefficients of $f(x)$ are integers, and that of $x^{3}$ is unity, this root is not fractional, and it therefore must be irrational.

Now move the $y$-axis 3 units to the right.

$$
\begin{array}{r}
1+0+1-47 \\
\frac{3+9+30}{3+10-17} \\
\frac{3+18}{6+28} \\
\frac{3}{9}
\end{array}
$$

The new equation, omitting the primes on the $x$ 's, is

$$
f(x+3)=x^{3}+9 x^{2}+28 x-17=0 .
$$

No confusion should arise from omitting the primes if it be remembered that the graph of the polynomial

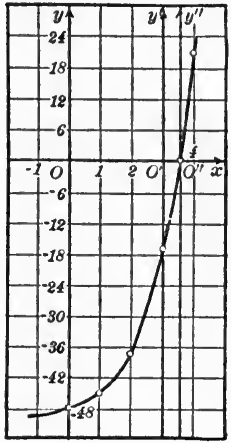

Fig. 72. in (2) is the curve in the figure referred to the axes $O^{\prime} X$ and $O^{\prime} Y^{\prime}$.

Equation (2) has a root between 0 and 1, which from the graph appears to be about 0.5 . Dividing (2) by $x-0.5$, we have

$$
\begin{array}{cl}
1+9+28-17 & 0.5 \\
& \frac{0.5+4.75+16.375}{9.5+32.75-0.625}
\end{array}
$$

As the remainder ${ }_{\perp} \mathrm{S}$ negative, the graph lies below the $x$-axis at $x=0.5$ and hence, from the figure, the root is larger than 0.5. Try $x=0.6$.

$$
\begin{gathered}
1+9+28-17 \\
+0.6+5.76+20.256 \\
\hline+9.6+33.76+3.256
\end{gathered}
$$

The remainder is now positive and hence the graph lies above the $x$-axis at $x=0.6$. Therefore the root of (2) lies between 0.5 and 0.6 . 
Move the $y$-axis 0.5 to the right.

$$
\begin{aligned}
& 1+9+28-17 \\
& \frac{0.5+4.75+16.375}{9.5+32.75-0.625} \\
& \frac{0.5+5}{10+37.75} \\
& \frac{0.5}{10.5}
\end{aligned}
$$

The new equation is

$$
x^{3}+10.5 x^{2}+37.75 x-0.625=0 .
$$

The graph of the polynomial on the left is the same curve referred to the axes $O^{\prime \prime} X$ and $O^{\prime \prime} Y^{\prime \prime}$, and it shows that equation (3) has a root between 0 and 0.1 . As the square and cube of a number less than 0.1 are very small, an approximate value of the root may be obtained by neglecting $x^{3}$ and $x^{2}$ in (3) and solving the resulting linear equation

$$
37.75 x-0.625=0 .
$$

This gives $x=\frac{0.625}{37.75}=0.02$ approximately. Substitute 0.02 in the left member of (3) by dividing by $x-0.02$.

$$
\begin{aligned}
& 1+10.5+37.75-0.625 \\
& +0.02+.2104+0.759208 \\
& \hline+10.52+37.9604+0.134208
\end{aligned}
$$

As the remainder is positive, the graph shows that the root is less than 0.02 . Try 0.01 .

$$
\begin{gathered}
1+10.5+37.75-0.625 \\
+0.01+0.1051+0.378551 \\
\hline+10.51+37.8551-0.246449
\end{gathered}
$$

This remainder is negative, and hence the graph shows that the root is greater than 0.01. Hence the root of (3) lies between 0.01 and 0.02 .

Then the root of equation (2) lies between 0.51 and 0.52 , and that of (1) between 3.51 and 3.52. Hence the real root of (1), correct to two decimal places, is $x=3.51$.

Which is the closer approximation to the root, 3.51 or 3.52 ?hy?

\section{EXERCISES}

Find all the real roots of the equations following, obtaining irrational roots to two decimal places.
1. $x^{3}+2 x-17=0$.
5. $x^{3}-4 x^{2}+3 x+3=0$.
2. $x^{3}+3 x-31=0$.
6. $x^{4}+x^{2}-15 x+2=0$.
3. $2 x^{3}+x-37=0$.
7. $x^{4}-5 x^{3}+2 x^{2}+1=0$.
4. $x^{3}-6 x^{2}+8 x+1=0$. 
Note. To find negative roots by Horner's method, replace $x$ in the equation by $-x$. The graph of $f(-x)$ is symmetrical to that of $f^{\prime}(x)$ with respect to the $y$-axis, and the roots of the equation $f(-x)=0$ will be equal numerically to those of $f(x)=0$, but have opposite signs. Hence the negative roots of an equation $f(x)=0$ may be found by finding the positive roots of $f(-x)=0$, and changing their signs.
8. $x^{3}+2 x+23=0$.
11. $x^{4}-3 x^{3}-4 x^{2}+12 x-10=0$.
9. $x^{3}+x^{2}+7=0$.
12. $x^{4}-2 x^{3}-7=0$.

10. $x^{3}-x^{2}-6 x+1=0$.

Noте. If an equation has both rational and irrational roots, it is advisable to find the rational roots first. Suppose they are $\alpha, \beta, \gamma$, etc. Then divide the equation by $x-\alpha$, the resulting equation by $x-\beta$, the new equation by $x-\gamma$, etc., thus obtaining a simpler equation whose irrational roots are the same as those of the given equation, and then solve this simpler equation by Horner's method.

13. $x^{4}-3 x^{3}+x^{2}+1=0$.

14. $x^{4}+2 x^{3}+3 x^{2}-43 x-98=0$.

15. $3 x^{5}-x^{4}+4 x^{3}-16 x^{2}-33 x+13=0$.

16. $6 x^{4}-31 x^{3}+40 x^{2}+2 x-5=0$.

17. $x^{4}-3 x^{3}-4 x^{2}+14 x-6=0$.

18. $6 x^{5}+17 x^{4}+4 x^{3}-39 x^{2}-297 x+210=0$.

19. Find the fifth root of 279 .

20. A cast iron rectangular girder (breadth $=\frac{1}{2}$ depth) rests upon supports 12 feet apart and carries a weight of 2000 pounds at the center. In order that the intensity of the stress may nowhere exceed 4,000 pounds per square inch, it is determined that the depth $d$ of the girder in inches must satisfy the equation $80 d^{3}-81 d^{2}-17,280=0$. Find $d$ and the crosssectional area.

21. The depth of flotation of a buoy in the form of a sphere is given by the equation $x^{3}-3 r x^{2}+4 r^{3} s=0$, where $r$ is the radius and $s$ is the specific gravity of the material. What is the depth for such a buoy whose radius is 1 foot and specific gravity is 0.786 ?

22. The cross section of the retaining wall of a reservoir is designed as indicated in Fig. 73. The allowable height $x$ of the upper portion is given by the equation $x^{3}+32 x^{2}-69 x-88=0$, where $x$ is expressed in terms of a unit of $\mathbf{1 0}$ feet. Find the allowable height to three significant figures. 
23. The allowable height of the lower portion of the wall in Exercise 22 is given by the equation $y^{4}-2.7 y^{3}+18.4 y^{2}-123 y-1.46=0$, where $y$ is in terms of a unit of 10 feet. Find the height of the lower portion to

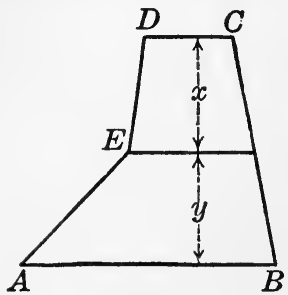

FIG. 73. three significant figures.

24. In Exercise 22 the slope of the water front $B C$ to the vertical is $\frac{1}{10}$, of $D E$ is $\frac{2}{10}$, of $A E$ is $\frac{78}{100}$. The width of the top is 6 feet. What must be the width of the lower portion?

25. The load $P$, concentrated at the center, which a homogeneous elliptical plate can support is given by the formula

$$
P=\frac{3 \pi}{8 m} \cdot \frac{3+2 m^{2}+3 m^{4}}{8+4 m^{2}+3 m^{4}} \cdot h^{2} R,
$$

where $h$ is the thickness of the plate $=\frac{1}{10}$ in., $R$ is the maximum safe unit stress for the material $=16,000$ pounds per square inch, $P=600 \pi$, and $m$ is the ratio of the breadth to the length of the ellipse. Find the value of $m$.

26. The maximum stresses on a parabolic arch of a bridge are given by the roots of the equation $2 r^{5}-5 r^{4}+9 r^{2}+8 r+2=0$, where $r$ is the ratio of the length of the arch occupied by a moving load such as a train. Find $r$.

54. Graph of the Function $f(a x)$. Before proceeding to the summary in the next section, we shall see how the graph of $f(a x)$ may be found from that of $f(x)$. This will complete the study we shall make of pairs of related functions and their graphs. Consider the

Example. If $f(x)=x^{2}-6 x$, then $f(2 x)$ $=(2 x)^{2}-6(2 x)$. Show that the graph of $f(2 x)$ may be obtained by bisecting the abscissas of points on the graph of $f(x)$.

If we substitute 4 for $x$ in the first function, and half of 4 , namely 2 , in the second, the results are both equal to -8 . Hence the point $(4,-8)$ lies on the first graph and $(2,-8)$ on the second, and the abscissa of the latter point is half that of the former.

If we substitute any value for $x$ in the first function, and half that value in the second, the results will be the same, namely $x^{2}-6 x$. Hence if $\left(x_{1}, y_{1}\right)$ is a point on the first graph the point $\left(x_{1} / 2, y_{1}\right)$ will be on the second.

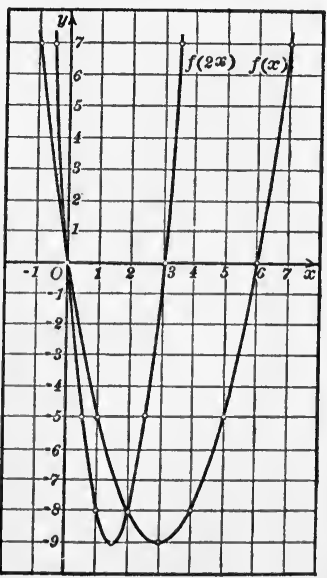

Frg. 74. 
As the latter point may be obtained by bisecting the abscissa of the former, it follows that the graph of $f(2 x)$ may be obtained by bisecting the abscissas of several points on the graph of $f(x)$ and drawing a smooth curve through them.

The reasoning employed in this example may be used to prove the

Theorem. The graph of $f(a x)$ may be obtained by dividing by $a$ the abscissas of points on the graph of $f(x)$. Corresponding points on the two graphs lie on the same or opposite sides of the $y$-axis according as $a$ is positive or negative.

This theorem may also be established as follows: Let $y=f(x)$ and suppose that the result of solving this equation for $x$ is $x=\phi(y)$. The graphs of these two equations are identical. If we solve the equation $y=f(a x)$ for $a x$ the result must be $a x=\phi(y)$, and the graphs of these two equations are identical. But the last equation may be written $x=\frac{1}{a} \phi(y)$, from which it follows that the abscissas of points on this curve are one-ath of the abscissas of the points on the graph of $\phi(y)$.

This theorem will find application in the study of some of the transcendental functions. It is also applied in an alternative method of finding the rational zeros of a polynomial in Exercises 2 to 6 below.

If $a$ has such a value as $\frac{1}{3}$, the division of the abscissas by $\frac{1}{3}$ amounts to multiplication by 3 .

55. Related Functions and their Graphs. We have studied several functions which may be obtained by transforming, or changing, a given function, and whose graphs may be obtained from that of the given function by simple geometric constructions or transformations. These are given in the tables below. In order that the statements may be concise and accurate, it is assumed that the constants involved in the table are positive. The changes necessary if the constants are negative should cause no difficulty. 
The graph of

(1) $f(x)+k$

(2) $f(x+h)$

(3) $\quad a f(x)$

(4)

(5) may be obtained from the graph of $f(x)$ by

moving it up $k$ units (page 19, Exercise 3)

moving it to the left $h$ units (Theorem 2, page 92)

multiplying ordinates by $a$ (Theorem, page 89)

dividing abscissas by $a$ (Theorem, page 151)

by the principles for the graphs of reciprocal functions in Section 40 (page 117).
The graph of

(3a) $-f(x)$

(4a) $f(-x)$

(4b) $-f(-x)$

(6) inverse of $f(x)$ is symmetrical to the graph of $f(x)$ with respect to

the $x$-axis

the $y$-axis

the origin

the bisector of the first and third quadrants (Theorem, page 114).

It should be noticed that $(3 a)$ and $(4 a)$ are the special cases of (3) and (4) respectively, obtained when $a=-1$, and that (4b) may be regarded as a combination of $(3 a)$ and $(4 a)$.

Properties (1) and (2) are closely related to the translation of the axes. If we set (Theorem 1, page 89)

in

$$
\begin{aligned}
x=x^{\prime}+h, y & =y^{\prime}+k \\
y & =f(x), \\
y^{\prime}+k & =f\left(x^{\prime}+h\right) .
\end{aligned}
$$

we get

The graphs of these two functions are identical if $x^{\prime}$ and $y^{\prime}$ are plotted on axes $h$ units to the right and $k$ units above the old axes. But the second equation may be written

$$
y^{\prime}=f\left(x^{\prime}+h\right)-k
$$

and hence, if $x^{\prime}$ and $y^{\prime}$ are plotted on the old axes the graph of $y^{\prime}$ may be obtained by moving that of $y$ to the left $h$ units and $k$ units down (properties (1) and (2)).

Properties (3) or (4) may also be interpreted as giving essentially the same distortion to a curve as is obtained if unequal units are chosen on the coördinate axes.

With these properties might be associated the method of ob- 
taining the graph of $y=f(x) \pm g(x)$ by first plotting the graphs of $f(x)$ and $g(x)$, and then adding or subtracting the corresponding ordinates (see Exercise 4 of the Miscellaneous Exercises following Chapter I).

56. Some Operations of Algebra regarded as Properties of Functions. The identity $\quad(a+b)^{2}=a^{2}+2 a b+b^{2}$

is usually thought of in elementary algebra as a rule for obtaining the square of a binomial. It may also be regarded as a property of the function $x^{2}$, that is, as the means of expressing the value of the function when $x$ is the sum of two given numbers in terms of the squares and first powers of the separate numbers.

It will be well to group together a few such relations, whether we regard them as rules of operation or as properties of functions. As typical of such relations we select the following properties of the function $x^{n}$ :

If $f(x)=x^{n}$, where $n$ is a positive integer, then

$\left.\begin{array}{l}\text { (1) } f(a+b)=(a+b)^{n}=a^{n}+n a^{n-1} b+\cdots+b^{n} \\ \text { (2) } f(a-b)=(a-b)^{n}=a^{n}-n a^{n-1} b+\cdots+b^{n}\end{array}\right\}$ Binomial theorem.

(3) $f(a b)=(a b)^{n}=a^{n} b^{n}$.

(4) $f(a / b)=(a / b)^{n}=a^{n} / b^{n}$.

(5) $f\left(a^{p}\right)=\left(a^{p}\right)^{n}=a^{n p}$.

(6) $f(\sqrt[i]{a})=(\sqrt[i]{a})^{n}=a^{n / r}$.

(7) $f(a)+f(b)=a^{n}+b^{n}=(a+b)\left(a^{n-1}-a^{n-2} b+\cdots+b^{n-1}\right)$, if $n$ is odd.

(8) $f(a)-f(b)=a^{n}-b^{n}=(a-b)\left(a^{n-1}+a^{n-2} b+\cdots+b^{n-1}\right)$.

These relations, or special cases of them, are used constantly in transforming algebraic expressions, for example, in simplifying complex fractions.

Relations analogous to some of these will be derived for each of the transcendental functions to be studied in later chapters. In order to perceive the analogy clearly, it is desirable to associate with these relations the general notation in terms of $f(x)$ given at the beginning of each line.

Essential differences between various functions lie in the differences in analogous properties.

A given function may not possess properties analogous to all of the eight relations above. For example, if $f(x)=x^{2}$, 
then $f(a)+f(b)=a^{2}+b^{2}$, which cannot be factored unless we use imaginary numbers, so that this function has no analogue to (7) in the field of real numbers. Again, if $f(x)=\sqrt{x}$, then $f(a+b)=\sqrt{a+b}$, and there is no other simple form in which this may be expressed, so that $\sqrt{x}$ has no simple property analogous to (1).

The connection between these relations and the graphical study of functions is not as remote as it might at first seem to be. For example, if in (3) we set $b=-1$, we get $f(-a)$ $=(-a)^{n}=(-1)^{n}(a)^{n}= \pm a^{n}= \pm f(a)$ according as $n$ is even or odd. This relation is the one which establishes the symmetry of the graph of $x^{n}$.

If we replace $a$ by $x$ in all the relations above, it is seen that relations (1) and (2) may be associated with property (2) of the preceding section, relations (3) and (4) with property (4), and relations (7) and (8) with property (1). As regards relations (5) and (6), it would be possible to obtain connections between the graph of $f(x)$ and those of $f\left(x^{p}\right)$ and $f(\sqrt[r]{x})$, but they would be complicated and have no important application.

\section{EXERCISES}

1. If $f(x)=x^{2}-1$, find $f(2 x)$ and $f(x / 2)$. Construct the graphs of the three functions on the same axes.

2. If $f(x)=6 x^{2}-x-1$, find $f(x / 6)$, and plot the graphs of both functions on the same axes. What relation will exist between the intercepts of the two graphs on the $x$-axis? between the roots of the two equations $f(x)=0$ and $f(x / 6)=0$ ? Check your answer by solving both equations and comparing their roots.

3. If $f(x)=4 x^{2}-8 x+3$, find $f(x / 2)$, and proceed as in 2 .

4. If $f(x)=3 x^{3}-2 x^{2}+6 x-4$, find $f(x / 3)$ and $9 f(x / 3)$. Plot the graphs on the same axes, obtaining the graph of the second function from the first, and that of the third function from the second. Find the rational root of $9 f(x / 3)=0$, and from it get the rational root of $f(x)=0$. Check the result by substitution.

5. Replace $x$ by $x / a$ in the equation $a x^{3}+b x^{2}+c x+d=0$, and multiply both sides of the resulting equation by $a^{2}$. If $a, b, c, d$ are integers, can the new equation have any fractional roots? If the integral roots of the new equation are found, how can the rational roots of the given equation be determined from them? Can a similar process be used with any equation in the form of a polynomial equal to zero? 
6. Solve equations $1(\mathrm{e})$ to $(\mathrm{j})$, page 144 , by the method indicated in the preceding exercises.

7. Exhibit in tabular form the properties of $x^{2}, x^{3}, 1 / x$, and $\sqrt{x}$ analogous to relations (1) to (8), Section 56 .

\section{MISCELLANEOUS EXERCISES}

1. In the following table, $p$ represents the percentage of alcohol in a $\underline{p} 10,40,70,90,100$, mixture whose specific gravity is $s$. Find $s \mid \frac{1}{982,939,871,823,794}, s$ as a quadratic function of $p$.

2. In the table the quantity of corn, $q$, is assumed to be connected with the price $p$ by a relation of the form

$\frac{q}{p} \mid \frac{1,0.9,0.8,0.7}{1,1.3,1.8,2.6}$

$$
p=\frac{a}{(q-b)^{2}} .
$$

Plot the graph and determine the values of the constants $a$ and $b$. Hint. If two pairs of values of $p$ and $q$ are substituted, the resulting equations may be solved for a pair of values of $a$ and $b$.

3. In doing a certain piece of work, a contractor paid $a$ dollars per day for wages, so that the total amount expended for wages in $t$ days was $W=a t$. The total amount invested in $t$ days increased according to the law $I=a t^{2} / 2$. Plot the graphs of these equations, assuming a numerical value for the wage rate $a$. Find the values of $W$ and $I$ if the work is finished in $t_{1}$ days. What would be the effect on the wage rate if the number of workmen was increased so that the work was finished in $t_{1} / 2$ days? If the amount invested follows the same law as before, i.e., is half the wage rate times the square of the time, what would be the value of $I$ if the work is finished in $t_{1} / 2$ days?

4. It is estimated that the quantity of work done by a man in an hour varies directly as his pay per hour and inversely as the square root of the number of hours he works per day. A man can finish a piece of work in six days when working 9 hours a day at $\$ .50$ per hour. How many days will he take to finish the same piece of work when working 12 hours a day at $\$ .55$ per hour?

5. An automobile manufacturer estimates that if he charges $\$ 800$ for a car he will sell 4000 a month, and that for each decrease of $\$ 100$ in price the sales will increase 1500 cars per month. What price per car will bring the greatest gross returns?

6. The height of a ball thrown vertically upward with a velocity of 64 feet per second is given by $s=-16 t^{2}+64 t$. A boy in a window 16 feet above the ground catches the ball as it falls. With what velocity was it moving when he caught it?

7. An automobile with good brakes moving on a smooth dry pavement at the rate of $v$ miles per hour was stopped in $s$ feet. Find the law con- 


\section{$\frac{v}{s} \mid \begin{array}{rrrr}5, & 10, & 15, \quad 20,\end{array}$}

necting $s$ and $v$ for the corresponding values given in the table. In how many feet should the car stop when moving 30 miles an hour?

8. The magnitude of the pressure on a surface perpendicular to the direction of the wind is proportional to the area of the surface and to the square of the velocity of the wind. If the pressure on the side of a building 20 feet wide and 60 feet high is 648 pounds when the velocity of the wind is 15 feet per second, what is the pressure on a building 30 feet by 80 feet when the velocity is 45 feet per second? Draw a graph to show how the pressure on a particular building changes as the velocity of the wind changes. Draw a graph to show how the pressure on various buildings differs for the same wind.

9. The area of the safety valve for a boiler may be determined by allowing 1 square inch of valve to every 2 square feet of grate surface. Find the diameter of the valve as a function of the area of the grate, and plot the graph. What should the diameter be for a grate 10 feet by 6 feet?

Note. When any thin plane surface is moved through the air so that the direction of the motion makes a small angle with the lower side, the resultant pressure of the air is very nearly normal to the plane surface. The lifting efficiency of the plane is increased by curving it longitudinally, the concave surface being placed so as to meet the air. There is a suction on the upper surface of good wings which amounts to more than twothirds of the total lift.

If a plane surface is presented edgewise to the direction of motion, there is no upward pressure, but if a curved wing is presented edgewise there is upward pressure. If the forward edge of the wing is depressed, the upward pressure decreases. When it is depressed to the point where the upward and downward pressures balance, a horizontal chord of the wing may be drawn through the rear edge. In any other position of the wing, the angle which this chord makes with the horizontal is called the angle of incidence, $\theta$.

The equation connecting the normal pressure $P$, in pounds, the area $S$ of the wing in square feet, the velocity $v$, in feet per second, and the angle of incidence $\theta$, in degrees, is

$$
P=K S v^{2} \theta,
$$

where $K$ is a constant of proportionality which remains constant only for small values of and variations in $\theta$.

If $W$ represents the weight of the aeroplane in pounds, the necessary condition for flight is that the vertical component of the pressure on the plane is equal to $W$. For small values of $\theta$, the vertical pressure is very nearly equal to the normal pressure, so that approximately, for horizontal flight 
710. (a) If $W=2500$ pounds, $S=400$ square feet, and $K=0.000087$, plot the graph of $v$ as a function of $\theta$. In practice $\theta$ varies from about $3^{\circ}$, the lowest safe value, to $12^{\circ}$. For what value of $\theta$ will the aeroplane have the greatest horizontal velocity? If additional power is given by the motor the aeroplane will not increase its horizontal velocity but will rise, and if the motor is shut off, the aeroplane begins to fall, but the horizontal velocity remains the same. Explain.

(b) The term loading is applied to the quantity $W / S$. If $\theta=5^{\circ}$ and $K=0.000087$, plot the graph of $v$ as a function of the loading. How will $v$ change if $S$ is constant and $W$ is quadrupled? How will $v$ change if $W$ is constant and $S$ is reduced by $\frac{1}{2}$ ?

(c) If $W, S$ and $\theta$ have the values used above, how will $v$ vary as $K$, the efficiency coefficient, changes? As the efficiency decreases, will the power required increase or decrease? Why not make aeroplanes with a lifting coefficient that is inefficient and fly very fast?

$>11$. The total resistance to the motion of the aeroplane is made up of two parts, the horizontal component of the pressure on the planes, given by $W \theta$, and the resistance of the struts, body, etc., given by $c s v^{2}$, where $c$ is a constant and $s$ is a theoretical surface which would have the same resistance as the various elements of the aeroplane except the wings. If the value of $v$ from (1) is substituted, the total thrust, $t$, is

$$
t=W \theta+\stackrel{s w c^{2}}{s}=W \theta+\frac{c s W}{K S \theta} .
$$

For a given aeroplane $W, c, s, K, S$ are constant. The single constant $f$, called the fineness, is defined by the equation $1 / f^{2}=c s / K S$. Making this substitution, we have

$$
t=W\left(\theta+\frac{1}{f^{2} \theta}\right) .
$$

If $f=5$, and $W=2500$, plot $t$ as a function of $\theta$. Determine approximately the most efficient value of $\theta$. How does an increase in $S$ affect the ratio $S / s$, hence the fineness, and hence the most efficient angle? How does the thrust vary as the fineness increases? How does the thrust vary with respect to $s$ ? with respect to the plane area, $S$ ? the lifting efficiency?

12. The power required for horizontal flight at velocity is

$$
P=v W\left(\theta+\frac{1}{f^{2} \theta}\right) \text {. }
$$

Substitute the value of $v$ obtained from (1) in (3) and plot $P$ as a function of $\theta$. As $\theta$ decreases and approaches the critical angle $3^{\circ}$, which increases most rapidly, $P, t$, or $\boldsymbol{*}$ ? What can be said of the power required for high speeds at small angles? How is $P$ affected by an increase in $W$ ? a decrease in $S ?$ an increase in $K$ ? an increase in $f$ ? 


\section{CHAPTER IV}

\section{TRIGONOMETRIC FUNCTIONS AND THE SOLUTION OF TRIANGLES (ANGLE MEASUREMENT)}

57. Introduction. One of the most useful applications of mathematics has been the systematic location and relocation of points and lines on the earth's surface, and the measurement of portions of the surface - the art of surveying. In surveying an extensive region, as a state, two points several miles apart, where the intervening surface is level, are selected for the extremities of a so-called base line. The length of this base line is measured with great accuracy. A number of stations are selected to serve as the vertices of a network of triangles stretching across the country, and the angles subtended at each station by every pair of visible stations are carefully measured. From these measurements the sides of all the triangles can be calculated.

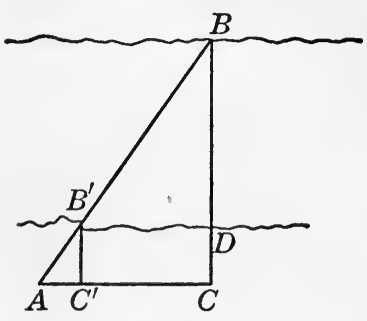

FIg. 75.

The principle underlying the computations of the surveyor is that the homologous sides of similar triangles are proportional. Its use is illustrated in

EXAmple 1. To find the width of a river. Take a base line $A C$ a short distance from one bank, and measure its length. Note the point $B$ directly opposite $C$, so that $\angle A C B$ is a right angle. From a point $B^{\prime}$ on $A B$ draw $B^{\prime} C^{\prime}$ perpendicular to $A C$, and measure $A C^{\prime}$ and $B^{\prime} C^{\prime}$. Since the right triangles $A C B$ and $A C^{\prime} B^{\prime}$ are similar,

$$
\frac{B C}{A C}=\frac{B^{\prime} C^{\prime}}{A C^{\prime}} \text {, or } B C=\frac{B^{\prime} C^{\prime}}{A C^{\prime}} A C .
$$

The width would then be obtained by subtracting from $B C$ the distance $C D$ from $C$ to the bank of the river. 
The solution of the example depends upon finding the ratio of two sides of a right triangle which has the same acute angle $B A C$ as the given triangle. It would be tedious, and sometimes difficult or impossible, if every time we wished to determine an inaccessible side of a triangle we had to construct a similar right triangle whose sides could be measured and their ratio determined. To avoid this difficulty, tables have been constructed which give the ratios of the sides of a right triangle with a given acute angle. Let us consider the construction of such a table.

Suppose that one acute angle of a right triangle is $30^{\circ}$, and that the triangle be placed in the position $O M P$, with the vertex of this angle at the origin of a system of coördinates, the adjacent leg lying along the positive part of the $x$-axis, and the hypotenuse falling in the first quadrant. The triangle is placed in this position so that the procedure will be in accord with fundamental definitions to be given in Section 59 .

Let the coördinates of $P$ be $x=O M$ and $y=M P$, and let $O P=r$. We seek the values of the rations $y / r, x / r$, and $y / x$.

Since the angle at $O$ is $30^{\circ}$, that

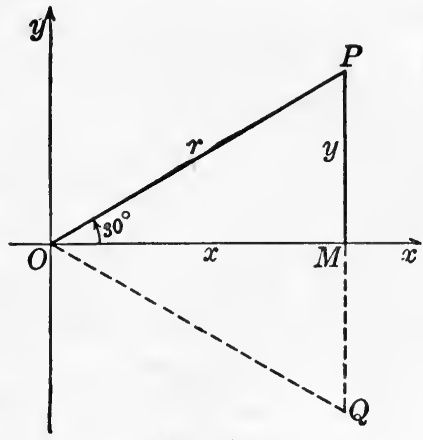

FIG. 76. at $P$ is $60^{\circ}$. Hence $\triangle O M P$ is half an equilateral triangle $O P Q$, and therefore

$$
r=2 y \quad \text { and } \quad x^{2}+y^{2}=r^{2} .
$$

The ratios required may be determined by solving these equations for any two of the sides in terms of the third. We already have $r$ in terms of $y$. Substituting in the second equation, $x^{2}+y^{2}=4 y^{2}$, whence $x=\sqrt{3} y$.

We may now find the values of the ratios:

$$
\frac{y}{r}=\frac{y}{2 y}=\frac{1}{2}, \quad \frac{x}{r}=\frac{\sqrt{3} y}{2 y}=\frac{\sqrt{3}}{2}, \frac{y}{x}=\frac{y}{\sqrt{3} y}=\frac{1}{\sqrt{3}}=\frac{\sqrt{3}}{3} .
$$


As all right triangles whose acute angles are $30^{\circ}$ and $60^{\circ}$ are similar, these results are true for all such triangles.

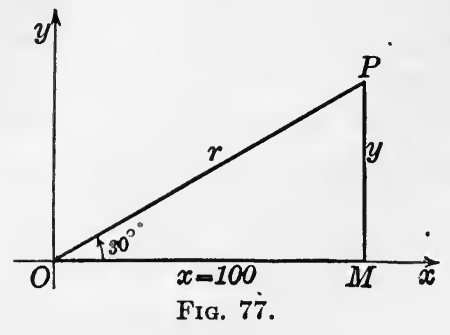

Problems in geometry which can be solved by means of equations (1) may be solved more expeditiously by means of the ratios (2), as in

EXAMPLE 2. Find the height of a flag pole $M P$ if the pole subtends an angle of $30^{\circ}$ at a point $O 100$ feet from the foot $M$.

By the method of plane geometry we obtain the equations

$$
r=2 y, \quad 100^{2}+y^{2}=r^{2} .
$$

The solution is completed by solving these equations for $y$.

Using the third of the ratios (2), which contains the known and required sides, we have

$$
\frac{y}{100}=\frac{\sqrt{3}}{3}, \text { whence } y=\frac{100 \sqrt{3}}{3}=\frac{100 \times 1.732}{3}=57.7 \text { feet. }
$$

The values of the ratios $y / r, x / r$, and $y / x$ for two other angles may be readily obtained by the methods of plane geometry.

If $\angle M O P=45^{\circ}$, we have

$$
y=x \quad \text { and } \quad x^{2}+y^{2}=r^{2} .
$$

These equations may be solved for $y$ and $r$ in terms of $x$, and the values of

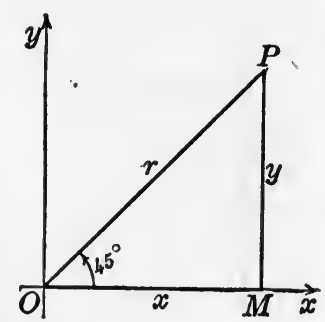

Fig. 78.

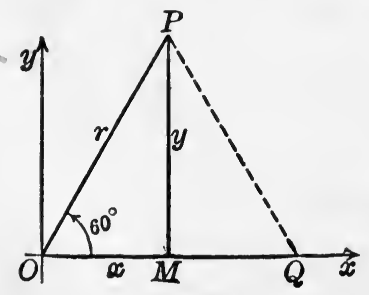

Fra. 79.

the ratios determined.

If $\angle M O P=60^{\circ}$, we have

$$
r=2 x \quad \text { and } \quad y^{2}+x^{2}=r^{2},
$$

which may be solved for $y$ and $r$ in terms of $x$, and the ratios determined. The details of the work are left as exercises. 


\begin{tabular}{c|c|c|c}
$\theta=\angle$ at $O$ & $\frac{y}{r}$ & $\frac{x}{r}$ & $\frac{y}{x}$ \\
\hline $30^{\circ}$ & $\frac{1}{2}=.50$ & $\frac{\sqrt{3}}{2}=0.87$ & $\frac{\sqrt{3}}{3}=0.58$ \\
$45^{\circ}$ & $\frac{\sqrt{2}}{2}=0.71$ & $\frac{\sqrt{2}}{2}=0.71$ & $\frac{1}{1}=1.00$ \\
$60^{\circ}$ & $\frac{\sqrt{3}}{2}=0.87$ & $\frac{1}{2}=0.50$ & $\sqrt{3}=1.73$
\end{tabular}

The values of the ratios for the three angles already considered are collected in the table.

In computing the decimal fractions, we have, for example,

$$
\frac{\sqrt{3}}{2}=\frac{1.732}{2}=0.866 \text {. }
$$

The first two decimal places are 0.86 , but 0.87 is a better approximation to two figures.

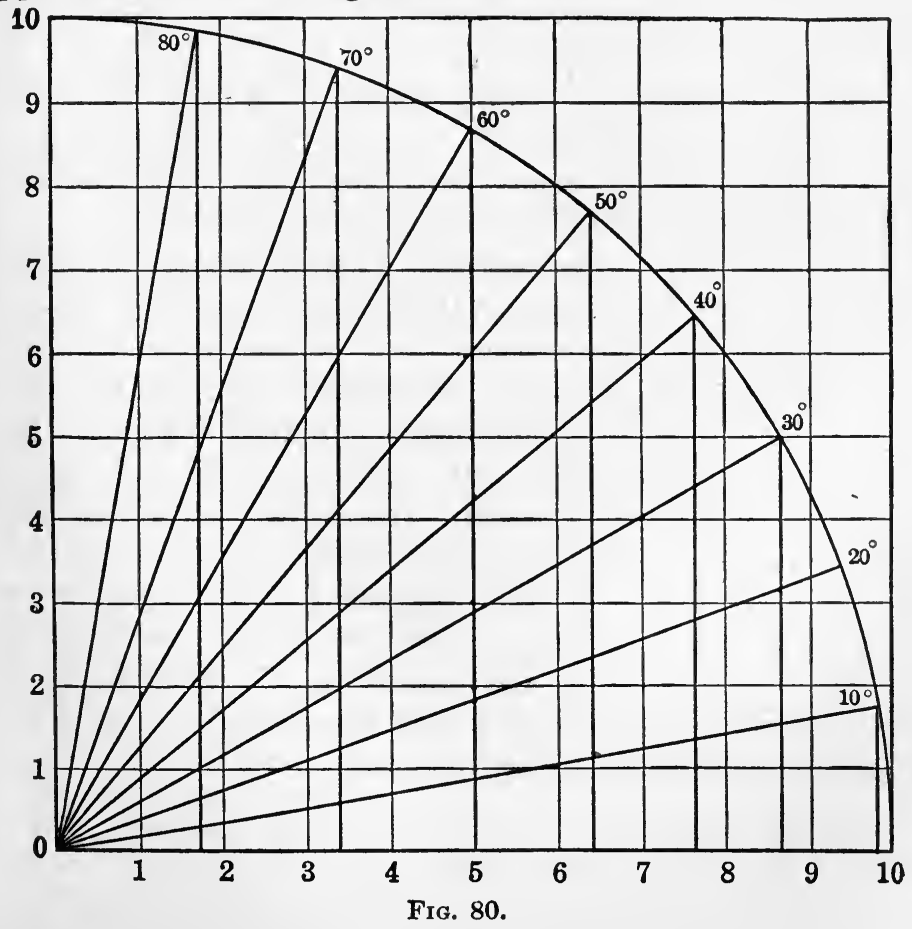


Approximate values of the ratios for any acute angle $\theta$ may be found as follows: By means of a protractor construct the angle $\theta$ so that the vertex is at the origin, one side lying along the $x$-axis, and the other in the first quadrant. From any point $P$ on the latter side drop the line $P M$ perpendicular to the $x$-axis. Measure the lengths of the sides of the triangle $O M P$ to find $x, y$, and $r$, and then divide $y$ by $r, x$ by $r$, and $y$ by $x$. Fig. 80 simplifies and systematizes the process. It consists of a portion of a circle, whose center is the origin and whose radius is 10 , and radii making angles of $10^{\circ}, 20^{\circ}, 30^{\circ}$, etc., with the $x$-axis.

To find the values of the ratios for $\theta=10^{\circ}$, for example, we read off the coördinates of the extremity of the radius making an angle of $10^{\circ}$ with the $x$-axis. They are, approximately, $x=9.8$, and $y=1.7$, while $r=10$. We then have, approximately

$$
\frac{y}{r}=\frac{1.7}{10}=0.17 . \quad \frac{x}{r}=\frac{9.8}{10}=0.98 . \quad \frac{y}{x}=\frac{1.7}{9.8}=0.17 .
$$

The filling in of the remainder of the table, which is to be used in the exercises below, is left as an exercise.

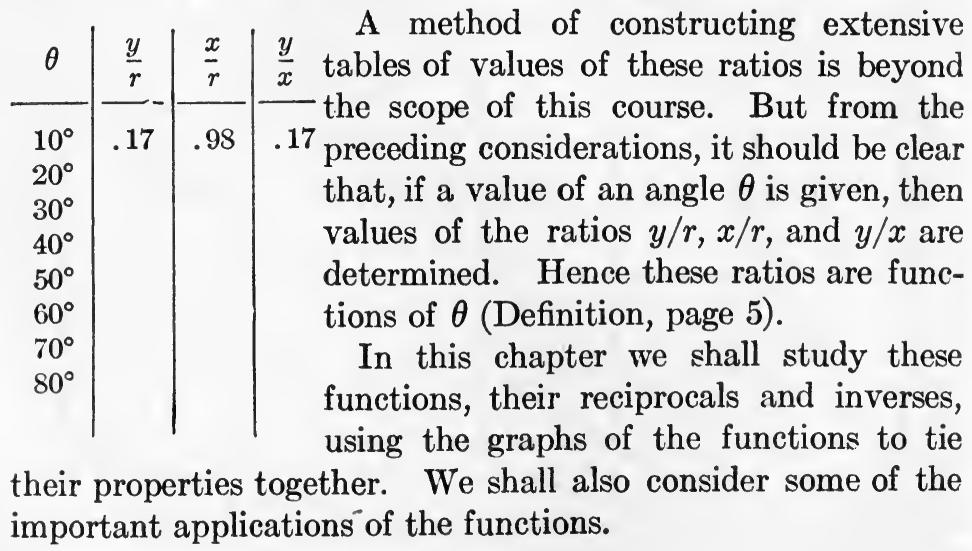




\section{EXERCISES}

1. Find the sides of a right triangle if one acute angle is $30^{\circ}$ and the hypotenuse is 10 .

2. Find the hypotenuse of an isosceles right triangle if one side is $\mathbf{1 2}$.

3. One acute angle of a right triangle is $60^{\circ}$ and the leg adjacent to this angle is 25. Find the other leg and the hypotenuse.

4. Find the side of an equilateral triangle whose altitude is 8 .

5. Find the side of a square if a diagonal is 30 .

6. A path runs up the side of a mountain at an angle of $40^{\circ}$ to the horizon. If a man climbs along the path for 500 yards, how high will he be above the starting point?

7. A canal makes an angle of $20^{\circ}$ with an east and west line. If a barge moves at the rate of 4 miles an hour, how far will it move east in 5 hours? How far north?

8. Two roads cross the canal in the preceding exercise at points 200 rods apart, one running east and west, the other north and south. How large is the triangular field bounded by the canal and the roads?

9. To find the height of a tree, a line 75 feet long is paced off from the foot of the tree. At the end of the line the angle subtended by the tree is $40^{\circ}$. How high is the tree?

10. How high is the sun (i.e., how many degrees above the horizon) if a pole 54 feet high casts a shadow 20 feet long?

11. A balloon is anchored by a rope 1000 feet long which makes an angle of $70^{\circ}$ with the ground. How high is the balloon? If a wrench happened to drop from the balloon, how far from the point of anchorage would it hit the ground?

12. Find the area of a rhombus whose side is 15 inches if one angle is $60^{\circ}$.

13. What is the length of the edge of a hexagonal nut that can be cut from a piece of circular stock one inch in diameter? What is the distance across the flats (i.e., between parallel edges)?

14. Holes are to be drilled through a piece of metal at the vertices of a regular hexagon, 3 inches on a side. The metal is fixed in place with a side $A B$ along the bed of a milling machine. What displacements of the bed of the machine, in the direction of and perpendicular to $A B$, will bring the metal into the proper positions for drilling the holes in rotation?

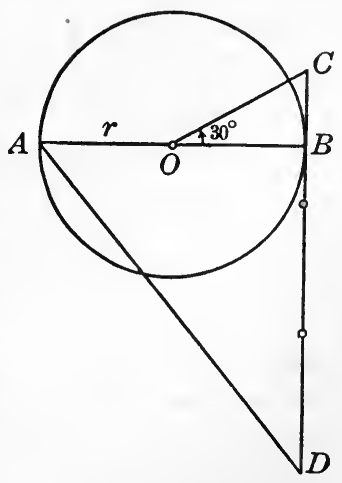

FIg. 81.

15. An approximate geometric method of determining $\pi$ is the following: Draw the diameter $A B$ of a circle and the tangent $C D$ at $B$. Construct 
$\angle B O C$ equal to $30^{\circ}$, and make $C D=3 r$, where $r$ is the radius. Then $A D$ is approximately equal to the semicircumference $\pi r$.

Find to three decimal places the approximate value of $\pi$ given by this construction.

58. Angles of any Magnitude. Let $O X$ and $O P$ be two lines drawn from an initial point $O$. Let a line start from co-

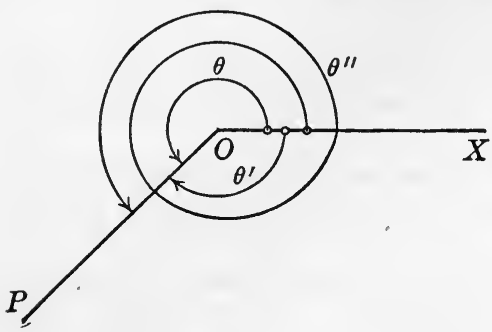

FIG. 82. incidence with $O X$, the initial line, and rotate about $O$, coming to rest finally in coincidence with $O P$, the terminal line. The line may rotate in either direction, and it may make any number of revolutions before coming to rest. The line is said to generate an angle whose magnitude is determined by the amount and direction of the rotation. The numerical value of the magnitude may be given in degrees, right angles, or revolutions.

The sign is positive or negative according as the direction of rotation is counter-clockwise or clockwise, i.e., in the opposite or in the same direction as the hands of a clock rotate.

If the terminal line of a first angle is the initial line of a second the sum of the angles is defined to be the angle whose initial line is that of the first, and whose terminal line is that of the second angle. This is analogous to the sum of two lines (page 13).

Two lines determine a countless number of angles. If $\theta$ is any one of them, the others differ from $\theta$ by an integral

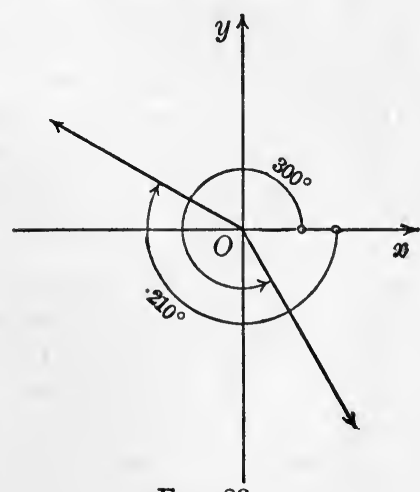

Fra. 83. multiple of $360^{\circ}$. They may all be represented by $\theta+n 360^{\circ}$, where $n= \pm 1, \pm 2, \pm 3, \ldots$ 
The arcs in Fig. 82 indicate the three angles:

$$
\theta=225^{\circ}, \theta^{\prime}=\theta-360^{\circ}=-135^{\circ}, \theta^{\prime \prime}=\theta+360^{\circ}=585^{\circ} \text {. }
$$

If the initial line of an angle coincides with the positive part of the $x$-axis, the angle is said to lie in the quadrant in which the terminal line lies. Thus in Fig. 83, the angle $300^{\circ}$ lies in the fourth quadrant, $-210^{\circ}$ in the second.

The positive direction on the terminal line in such a figure is defined to be away from the origin. For example, the positive direction on the terminal line of an angle of $180^{\circ}$ is to the left.

We shall make an important use of the angles whose terminal lines bound, bisect, or trisect the four quadrants.

59. Trigonometric Functions of any Angle. Let $\theta$ be the number of degrees in any angle whose initial line coincides with the positive part of the $x$-axis, let $P(x, y)$ be any point on the terminal line, and let $O P=r$.

Consider the ratio $y / x$, which is a negative number for the case indicated in the figure, since $x=O M$ is negative and $y=M P$ is positive. The numerical value of $y / x$ may be found approximately by measuring

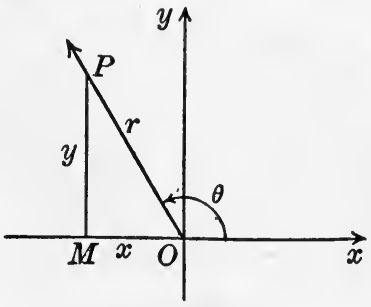

FIg. 84. the lengths of $M P$ and $O M$ and dividing the former by the latter.

If $P^{\prime}\left(x^{\prime}, y^{\prime}\right)$ is any other point on the terminal line, the ratios $y^{\prime} / x^{\prime}$ and $y / x$ have the same sign, and also the same numerical

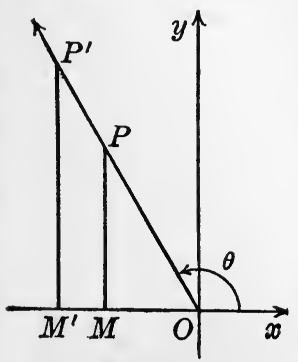

FIg. 85. value, since the triangles $O M P$ and $O^{\prime} M^{\prime} P^{\prime}$ are similar. Hence if $\theta$ is given a definite value, the value of $y / x$ is determined, and therefore the ratio $y / x$ is a function of $\theta$. Similarly, the ratio of any one of the numbers $x, y, r$, to any other is a function of the angle $\theta$. These functions are called trigonometric functions. They are named in accordance with the definitions below, which hold for Fig. $86 A, B, C, D$. 
Definitions. If $\theta$ is any angle whose initial line coincides with the positive part of the $x$-axis, if $P(x, y)$ is any point on the terminal line, and if $O P=r$, then

$$
\begin{array}{ll}
\frac{y}{r}=\operatorname{sine} \text { of } \theta=\sin \theta ; & \frac{r}{y}=\operatorname{cosecant} \theta=\csc \theta ; \\
\frac{x}{r}=\text { cosine of } \theta=\cos \theta ; & \frac{r}{x}=\text { secant of } \theta=\sec \theta ; \\
\frac{y}{x}=\text { tangent of } \theta=\tan \theta ; & \frac{x}{y}=\text { cotangent of } \theta=\cot \theta .
\end{array}
$$

To these are sometimes added

$$
\begin{aligned}
& 1-\frac{x}{r}=1-\cos \theta=\text { versine of } \theta=\text { vers } \theta . \\
& 1-\frac{y}{r}=1-\sin \theta=\text { coversine of } \theta=\operatorname{covers} \theta .
\end{aligned}
$$

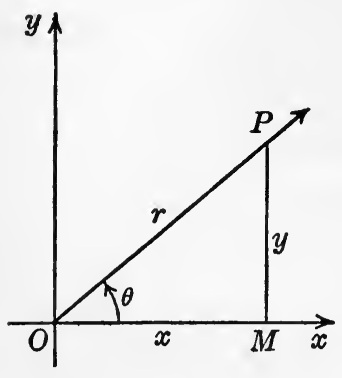

$\boldsymbol{A}$

$\theta$ in quadrant $I$.

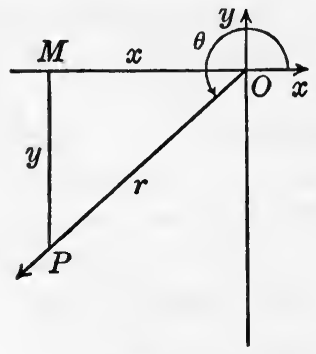

C

$\theta$ in quadrant III.

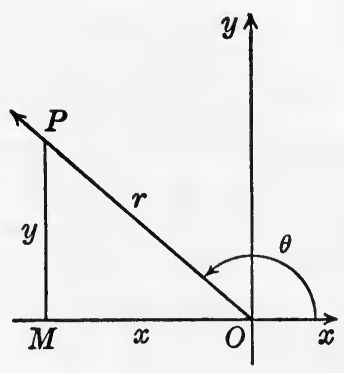

$B$

$\theta$ in quadrant II.

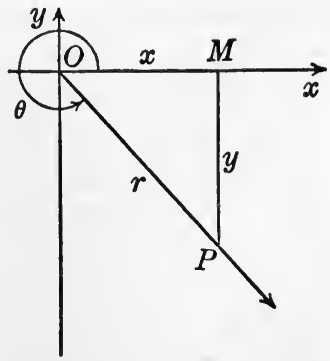

$D$

$\theta$ in quadrant IV.

Fig. 86. 
Since the three ratios on the right are the reciprocals of those on the left, we have the reciprocal relations:

$$
\cot \theta=\frac{1}{\tan \theta}, \quad \sec \theta=\frac{1}{\cos \theta}, \quad \csc \theta=\frac{1}{\sin \theta} .
$$

The first table in Section 57 gives the sine, cosine, and tangent of the angles $30^{\circ}, 45^{\circ}$, and $60^{\circ}$. The values of the sines may be easily remembered by noticing that they are respectively $\frac{1}{2} \sqrt{1}, \frac{1}{2} \sqrt{2}, \frac{1}{2} \sqrt{3}$, while the cosines are the same numbers in the reverse order. The tangent of any one of the angles may be obtained by dividing the sine by the cosine. For we always have

$$
\tan \theta=\frac{y}{x}=\frac{y / r}{x / r}=\frac{\sin \theta}{\cos \theta} .
$$

If $\theta$ is in quadrant I, $x, y$, and $r$ are positive, and the values of the six ratios, or functions, are positive. But if $\theta$ is in one of the other quadrants, either $x$ or $y$, or both, are negative, although $r$ is always positive, and hence these ratios may be negative. For example, if $\theta$ is in the second quadrant, $\tan \theta=y / x$ is a negative number, since $x$ is negative and $y$ is positive.

The reciprocal relations show that the signs of $\cot \theta, \sec \theta$, $\csc \theta$, for a given value of $\theta$, agree respectively with the signs of $\tan \theta, \cos \theta, \sin \theta$. Hence it is necessary to fix in mind the signs of the latter functions only. This is readily done in connection with the graphs (see Section 61).

If the terminal line of $\theta$ bounds, bisects, or trisects one of the quadrants, the functions of $\theta$ may be found directly from the definitions, as in the examples following, by methods of elementary geometry.

Example 1. Find the functions of $0^{\circ}$.

Let $P(x, y)$ be any point on the terminal line, which coincides with the positive part of the $x$-axis, since $\theta=0$. Then $y=0$, and $r=x$. Hence, by the definitions,

$$
\begin{aligned}
& \sin 0^{\circ}=y / r=0 / r=0 . \\
& \cos 0^{\circ}=x / r=x / x=1 . \\
& \tan 0^{\circ}=y / x=0 / x=0 . \\
& \sec 0^{\circ}=r / x=x / x=1 .
\end{aligned}
$$

But the definition of $\cot \theta$ involves division by $y$, and as $y=0$ if $\theta=0, \cot 0^{\circ}$ does

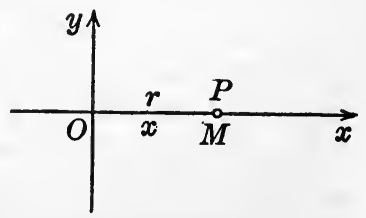

Fra. 87. not exist. However, $\cot \theta$ is the reciprocal of $\tan \theta$, by (1). As $\theta$ ap- 
proaches zero, $\tan \theta$ approaches zero, and hence $\cot \theta$ becomes infinite as $\theta$ approaches zero (IV, page 118). This is sometimes indicated by the symbols $\cot 0^{\circ}=\infty$.

In like manner, as $\theta$ approaches zero, $\csc \theta$ becomes infinite.

Example 2. Find the functions of $210^{\circ}$.

The acute angles of the triangles $O P M$ are $30^{\circ}$ and $60^{\circ}$. Hence the numerical value of $O P$ is twice that of $M P$. If we let the numerical value

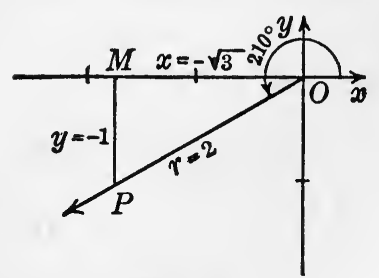

FIg. 88. of $M P$ be 1 , then that of $O P$ is 2 , and hence that of $O P$ is $\sqrt{3}$. But the coördinates of $P$ are negative. Hence we may take

$$
x=-\sqrt{3}, \quad y=-1, \quad r=2 .
$$

Then by the definitions

$$
\begin{aligned}
& \sin 210^{\circ}=y / r=-1 / 2 . \\
& \csc 210^{\circ}=r / y=2 /(-1)=-2 .
\end{aligned}
$$$$
\cos 210^{\circ}=x / r=-\sqrt{3} / 2 \text {. }
$$

sec $210^{\circ}=r / x=2 /(-\sqrt{3})=-2 \sqrt{3} / 3$.

$$
\begin{aligned}
& \tan 210^{\circ}=y / x=-1 /(-\sqrt{3})=\sqrt{3} / 3 . \\
& \cot 210^{\circ}=x / y=-\sqrt{3} /(-1)=\sqrt{3} .
\end{aligned}
$$

Since the functions of two angles with the same terminal line may be defined by means of the same triangle, and since all angles with the same terminal line may be expressed in the form $\theta+n 360^{\circ}$ (Section 58), we have the

Theorem. The trigonometric functions of an angle are unchanged if the angle is increased or decreased by an integral multiple of $360^{\circ}$. Symbolically,

$$
\begin{aligned}
& \sin \left(\theta+n 360^{\circ}\right)=\sin \theta, \\
& \cos \left(\theta+n 360^{\circ}\right)=\cos \theta,
\end{aligned}
$$

where $n= \pm 1, \pm 2, \pm 3$, etc.

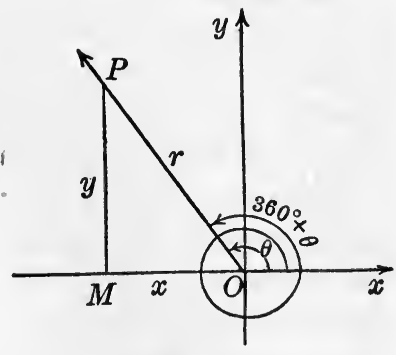

Frg. 89.

Definition. A function is said to be periodic if its value is unchanged when the value of the variable is increased by a constant, that is, if $f(x+c)=f(x)$. If $c$ is the smallest constant of this sort, it is called the period of the function.

For example, the height of the tide at the seashore is a periodic function of the time. For if approximately 12 hours and 25 minutes are added to the time, the height of the tide will be the same. 
The theorem shows that the trigonometric functions are periodic, for if $\theta$ is increased by $360^{\circ}$ the values of the functions are unchanged. It will appear later that $360^{\circ}$ is the period of the sine and cosine, and their reciprocals, while $180^{\circ}$ is the period of the tangent and cotangent. This is a characteristic property of the trigonometric functions, which distinguishes them from all algebraic functions.

\section{EXERCISES}

1. Find all the functions of each of the so-called quadrantal angles: (a) $0^{\circ}$; (b) $90^{\circ}$; (c) $180^{\circ}$; (d) $270^{\circ}$; (e) $360^{\circ}$.

2. Find all the functions of the angles:
(a) $135^{\circ}$.
(b) $330^{\circ}$.
(c) $-45^{\circ}$.
(d) $240^{\circ}$.
(e) $-210^{\circ}$.
(f) $480^{\circ}$.
(g) $315^{\circ}$.
(h) $-150^{\circ}$.
(i) $300^{\circ}$.
(j) $990^{\circ}$.

3. What positive angles less than $360^{\circ}$ have the same functions as (a) $540^{\circ}$, (b) $-60^{\circ}$, (c) $1320^{\circ}$, (d) $-675^{\circ}$, (e) $653^{\circ}$ ?

4. Determine the sign of:
(a) $\cos \theta, \theta$ in quadrant II.
(b) $\tan \theta, \theta$ in quadrant III.
(c) $\cos \theta, \theta$ in quadrant IV.
(d) $\tan \theta, \theta$ in quadrant II.
(e) $\sin \theta, \theta$ in quadrant IV.
(f) sec $\theta, \theta$ in quadrant III.
(g) $\cot \theta, \theta$ in quadrant IV.
(h) $\csc \theta, \theta$ in quadrant II.

5. Determine the sign of each of the functions in each of the quadrants. Tabulate the results.

6. Build a table of values of $\sin \theta$ for $\theta=0^{\circ}, 30^{\circ}, 45^{\circ}, 60^{\circ}, 90^{\circ}, 120^{\circ}$, $135^{\circ}, 150^{\circ}, 180^{\circ}, 210^{\circ}, 225^{\circ}, 240^{\circ}, 270^{\circ}, 315^{\circ}, 300^{\circ}, 330^{\circ}, 360^{\circ}$.

7. Build a table of values, for the angles given in Exercise 6 , for (a) $\cos \theta$; (b) $\tan \theta$; (c) $\cot \theta$; (d) $\sec \theta$; (e) $\csc \theta$.

8. Construct all the positive angles less than $360^{\circ}$ for which $\sin \theta=-\frac{3}{5}$.

Since $\sin \theta=y / r$, the problem is equivalent to constructing right triangles with hypotenuse equal to 5 , and vertical side equal to 3 and lying below the $x$-axis. Hence, describe the circle

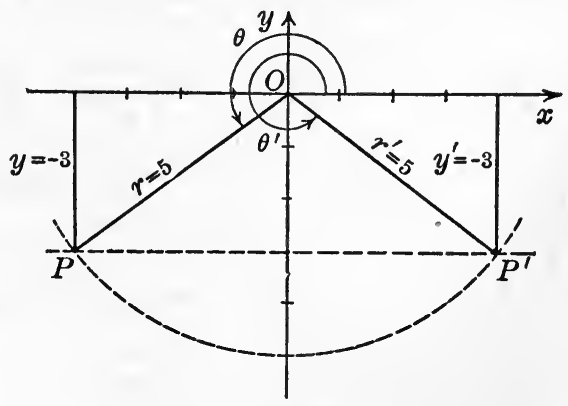

Fig. 90. with center 0 and radius 5 , and draw the line parallel to the $x$-axis and 3 units below it. Let their intersections be $P$ and $P^{\prime}$. Then the lines 
$O P$ and $O P^{\prime}$ are the terminal lines of the required angles. How many such angles are there? How many positive and less than $360^{\circ}$ ?

9. By the method of the preceding exercise, construct all the positive angles less than $360^{\circ}$ for which
(a) $\cos \theta=\frac{1}{2}$.
(b) $\tan \theta=-\frac{5}{8}$.
(c) $\csc \theta=3$.
(d) $\sin \theta=\frac{4}{7}$.
(e) $\cos \theta=-0.3$
(f) $\cot \theta=2$.

10. Find all the functions of $\theta$ given:

(a) $\cos \theta=-\frac{5}{13}$, and $\theta$ in quadrant II.

(b) $\tan \theta=\frac{3}{4}$, and $\theta$ in quadrant III.

(c) $\sin \theta=-\frac{1}{1} \frac{2}{3}$, and $\theta$ in quadrant IV.

(d) $\cot \theta=2$, and $\theta$ in quadrant $I$.

(e) $\sin \theta=\frac{4}{5}$, and $\theta$ in quadrant II.

(f) $\sec \theta=\frac{18}{5}$, and $\theta$ in the fourth quadrant.

11. The intensity of light, $I$, varies inversely as the square of the distance, $d$, from the source. If a street light is at the top of a concrete post 10 feet high, $A B$, express $I$ at a point $C$ on the pavement as a function of $\theta=\angle A C B$.

12. An aeroplane rises along a straight line, which makes an angle $\theta$ with a straight road directly below the path of the aeroplane, at the rate of 50 miles an hour. Express as a function of $\theta$ the speed at which an automobile must move along the road to keep under the aeroplane.

13. If the hypotenuse of a right triangle is 10 , express the area as a function of one of the acute angles.

14. Construct the path of a point on the rim of a wheel rolling on a level road, by rolling a coin along a ruler, without slipping, and marking a number of positions of a point on the edge of the coin. This curve defines $y$, the height of the moving point above the road, as a function of $x$,

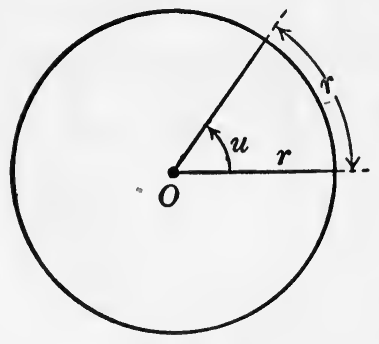

FIG. 91. the distance measured along the road. Show that this function is periodic, and find its period. What can be said of the graph of a periodic function?

60. Radians. To find the number of degrees in the angle $u$ subtended at the center of a circle of radius $r$ by an arc whose length is $r$, we compare the angle with the complete angle about the center. Since angles at the center are proportional to the subtending arcs, and since an angle of $360^{\circ}$ is subtended by the entire circumference, we have 


$$
\frac{u}{360}=\frac{r}{2 \pi r},
$$

and hence

$$
u=180 / \pi=180 / 3.1416=57^{\circ} .295 .
$$

Hence this angle does not depend on $r$, but is the same for all circles, and it may therefore be used as a unit angle.

Definition. A radian is the angle subtended at the center of a circle by an arc whose length is equal to the radius.

Equation (2) enables us to reduce radians to degrees. The most convenient form of this equation is the important relation

\section{$\pi$ radians $=180$ degrees.}

From this we have, for example, that $90^{\circ}=\pi / 2$ radians, and it is customary to speak of $\pi / 2$ radians rather than $3.1416 / 2=1.5708$ radians. Similarly,

It is customary to express in terms of $\pi$ the number of radians in any angle which is a simple multiple or submultiple of $180^{\circ}$.

When the degree or the right angle is used as the unit that fact is usually indicated. Thus we write $\theta=180^{\circ}$, or $\theta=2$ rt. $\measuredangle$. But if the unit is the radian we merely write $\theta=\pi$ without indicating the unit.

The number of radians in an angle is called its circular measure.

It is customary to use the radian as the unit angle in drawing the graphs of the trigonometric functions (see the following section). Another elementary use is given by the

Theorem. The length of an arc of a circle is equal to the radius multiplied by the number of radians in the angle subtended at the center. Symbolically,

\section{Arc $A B=r \times \angle A O B$ (in radians).}

For if $A B$ is any arc, if $\theta$ is the circular measure of the subtended

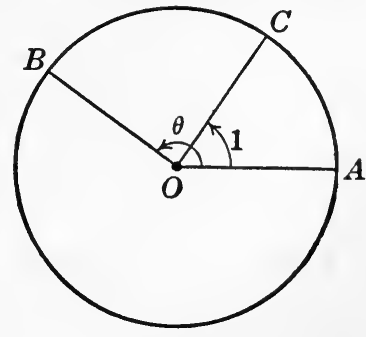

FIG. 92. angle $A O B$, and if arc $A C=r$, so that $\angle A O C=1$, we have $\frac{A B}{r}=\frac{\theta}{1}$, whence $A B=r \theta$. 
The radian is the unit used in all theoretical work in the calculus and higher mathematics.

Tables for converting radians into degrees, and degrees into radians, are to be found on page 32 of Huntington's Tables.

\section{EXERCISES}

1. Reduce the following angles to radians:

(a) The quadrantal angles, namely, $0^{\circ}, 90^{\circ}, 180^{\circ}, 270^{\circ}, 360^{\circ}$.

(b) The angles whose terminal lines bisect the quadrants, namely, $45^{\circ}$, $135^{\circ}, 225^{\circ}, 315^{\circ}$.

(c) The angles whose terminal lines trisect the quadrants, namely, $30^{\circ}, 60^{\circ}, 120^{\circ}, 150^{\circ}, 210^{\circ}, 240^{\circ}, 300^{\circ}, 330^{\circ}$.

2. Reduce the following angles to degrees:

(a) $\pi / 6,3 \pi, 7 \pi / 2,2 \pi / 3$.

(b) $2 \pi / 3, \pi / 4,5 \pi / 3,5 \pi / 4,11 \pi / 3$.

3. Using Huntington's Tables, page 32 , express the following angles in radians, as decimal fractions:

(a) $10^{\circ}, 75^{\circ}, 110^{\circ}, 340^{\circ}, 15^{\circ} .34,3^{\circ} .77$.

(b) The angles in Exercise 1.

4. Using Huntington's Tables, express the following angles in degrees: $0.25,1.13,1.465,0.8327,3.2476$.

5. If the radius of a circle is $\mathbf{1 0}$ inches, find the angle subtended at the center by an arc 15 inches long.

6. If the radius of a circle is 4 inches, find the length of an arc which subtends an angle of $173^{\circ}$ at the center.

7. A strip of tin 8 inches wide is bent into the form of a trough whose cross section is an arc of a circle. Express the angle subtended at the center as a function of the radius.

8. Find the angle at the center of the earth subtended by an arc of the equator one mile long. In doing this, which one of the radii of the earth given on the inside of the back cover of Huntington's Tables should be used?

9. If the radius of a circle is 12 inches, find the length of an arc subtending a central angle of $2 \pi / 3$, and the area of the sector bounded by the arc and the radii drawn to its extremities.

10. Show that the area of a sector of a circle of radius $r$ is $\frac{1}{2} r^{2} \theta$, where $\theta$ is the number of radians in the central angle of the sector. Hint. The area of a sector is one-half the product of the radius and the arc of the sector.

61. Graphs of the Trigonometric Functions. In construct- 
ing these graphs it is customary to use the same unit on both axes, and to measure $\theta$ in radians.

Graph of $\sin \theta$. A sufficiently extensive table of values is obtained by taking the values of $\theta$ for which the terminal line bounds, bisects, or trisects one of the quadrants. The values of $\sin \theta$ for the angles $30^{\circ}=\pi / 6,45^{\circ}=\pi / 4,60^{\circ}=\pi / 3$ are given in the first table in Section 57, and for the angles $0^{\circ}$ and $210^{\circ}=7 \pi / 6$ in the examples in Section 59. The table of values below is identical with that asked for in Exercise 6, following Section 59, except that the angles are now expressed in radians.

To plot the pairs of values in the table, choose a convenient unit on the vertical axis, and on the $\theta$-axis lay off $\pi=3 \frac{1}{7}$ units and $2 \pi=6 \frac{2}{7}$ units. The points $\theta=\pi / 2$ and $\theta=3 \pi / 2$ are obtained by bisecting these segments, giving four segments on the $\theta$-axis corresponding to the four quadrants. The remaining abscissas may be constructed by bisecting and trisecting these segments. Having determined the points on the $\theta$-axis corresponding to the values of $\theta$ in the table, ordinates are erected equal to the respective values of $\sin \theta$, and the curve is then drawn.

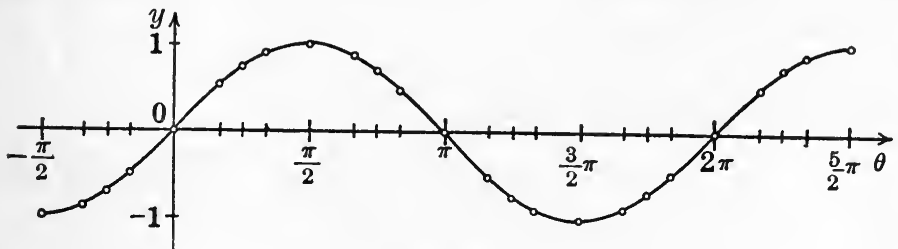

Fig. 93.

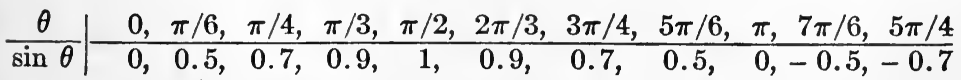

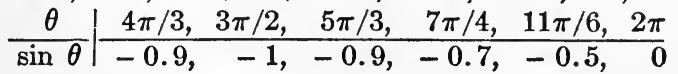

Periodicity of $\sin \theta$. If the terminal line of $\theta$ starts in coincidence with the positive part of the $x$-axis (Section 58), the angle $\theta$ may have any one of the values:

$$
\text { ... }-4 \pi,-2 \pi, 0,2 \pi, 4 \pi, \ldots
$$


As the terminal line makes a complete revolution, $\theta$ may increase through any one of the intervals:

$$
\text { ... }-4 \pi \text { to }-2 \pi,-2 \pi \text { to } 0,0 \text { to } 2 \pi, 2 \pi \text { to } 4 \pi, \ldots
$$

But no matter through which of these intervals $\theta$ increases, $\sin \theta$ will vary through the same set of values (Theorem, Section 59). Hence the part of the graph in each interval is congruent to that in the interval from 0 to $2 \pi$, which was plotted above.

Starting at the origin, the graph does not begin to repeat until $\theta=2 \pi$. Hence $2 \pi$, or $360^{\circ}$, is the smallest constant such that

$$
\sin (\theta+2 \pi)=\sin \theta,
$$

and hence $2 \pi$, or $360^{\circ}$ is the period (Definition, Section 59) of $\sin \theta$.

The part of the graph from 0 to $2 \pi$ should be fixed in mind carefully, and the portion corresponding to each quadrant noted, as the graph affords, a simple means of remembering the following properties of $\sin \theta$ (see page 42).

Zeros of $\sin \theta$. For angles less than $2 \pi, \sin \theta=0$ if $\theta=0$ or $\pi$.

From the periodicity of the function, the other zeros are therefore

and

$$
\begin{aligned}
& 0+2 n \pi= \pm 2 \pi, \quad \pm 4 \pi, \quad \pm 6 \pi, \ldots \\
& \pi+2 n \pi= \pm 3 \pi, \quad \pm 5 \pi, \quad \pm 7 \pi, \ldots
\end{aligned}
$$

Sign of $\sin \theta$. Sin $\theta$ is positive if $\theta$ is in the first or second quadrant, negative if $\theta$ is in the third or fourth quadrant. The periodicity shows that this holds whether or not $\theta$ is positive and less than $2 \pi$.

Maximum and minimum values of $\sin \theta$. The maximum value between 0 and $2 \pi$ is $\sin (\pi / 2)=1$, and the minimum value $\sin (3 \pi / 2)=-1$. The other values of $\theta$ for which $\sin$ $\theta= \pm 1$ may be found by means of the periodicity.

Changes of $\sin \theta$.

As $\theta$ increases from 0 to $\pi / 2, \sin \theta$ increases from 0 to 1 .

As $\theta$ increases from $\pi / 2$ to $\pi, \sin \theta$ decreases from 1 to 0 .

As $\theta$ increases from $\pi$ to $3 \pi / 2, \sin \theta$ decreases from 0 to -1 . 
As $\theta$ increases from $3 \pi / 2$ to $2 \pi$, $\sin \theta$ increases from -1 to 0 .

Notice that the numerical value of $\sin \theta$ cannot exceed unity, and, in particular, that it does not become infinite.

Symmetry of the graph. The graph appears to be symmetrical with respect to the origin. Hence, probably,

$$
\sin (-\theta)=-\sin \theta \text {. }
$$

This may be proved as follows: Construct any angle $\theta$, and then the angle $-\theta$. On their terminal lines take $O P=O^{\prime} P^{\prime}$, so that $r=r^{\prime}$. Then since $P^{\prime}$ is symmetrical to $P$ with respect to the $x$-axis (why?), we have $x=x^{\prime}$ and $y=-y^{\prime}$. Then

$$
\begin{aligned}
\sin (-\theta) & =\frac{y}{r}=\frac{-y^{\prime}}{r^{\prime}} \\
=-\frac{y^{\prime}}{r^{\prime}} & =-\sin \theta .
\end{aligned}
$$

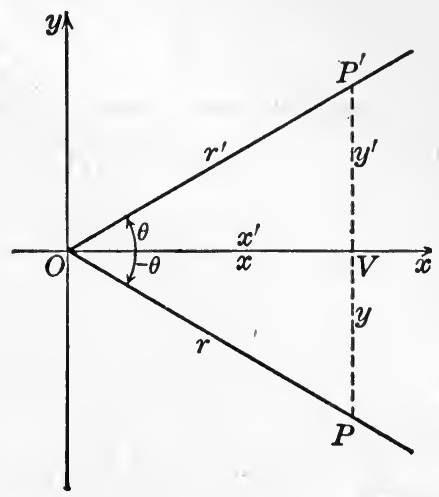

Fig. 94.

The graphs of $\cos \theta$ (Fig. 95) and $\tan \theta$ (Fig. 96) are constructed in like manner, and should be fixed in mind.

The various properties of reciprocal functions (page 118) enable us to get certain properties of $\cot \theta, \sec \theta$, and $\csc \theta$ directly from the graphs of $\tan \theta, \cos \theta$, and $\sin \theta$ respectively. (See also Exercise 2 below.)

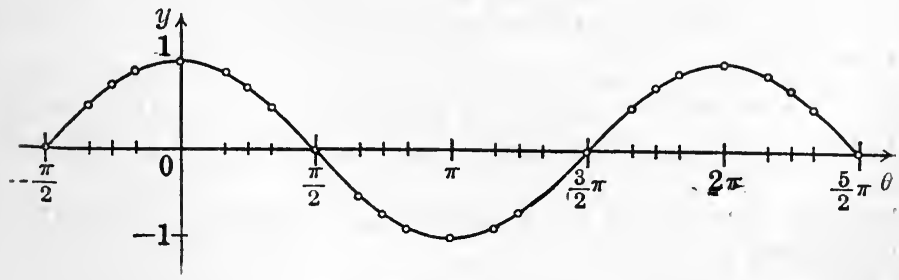

Fig. 95.

$\frac{\theta}{\cos \theta} \mid \begin{array}{lllll}0, \pi / 6, \pi / 4, \pi / 3, \pi / 2, & 2 \pi / 3, \quad 3 \pi / 4, \quad 5 \pi / 6, \quad \pi, & 7 \pi / 6, \quad 5 \pi / 4 \\ 1,0.9,0.7,0.5, & 0,-0.5,-0.7,-0.9,-1,-0.9, & -0.7\end{array}$

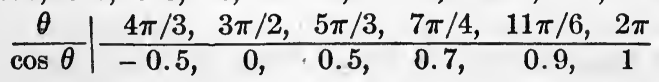




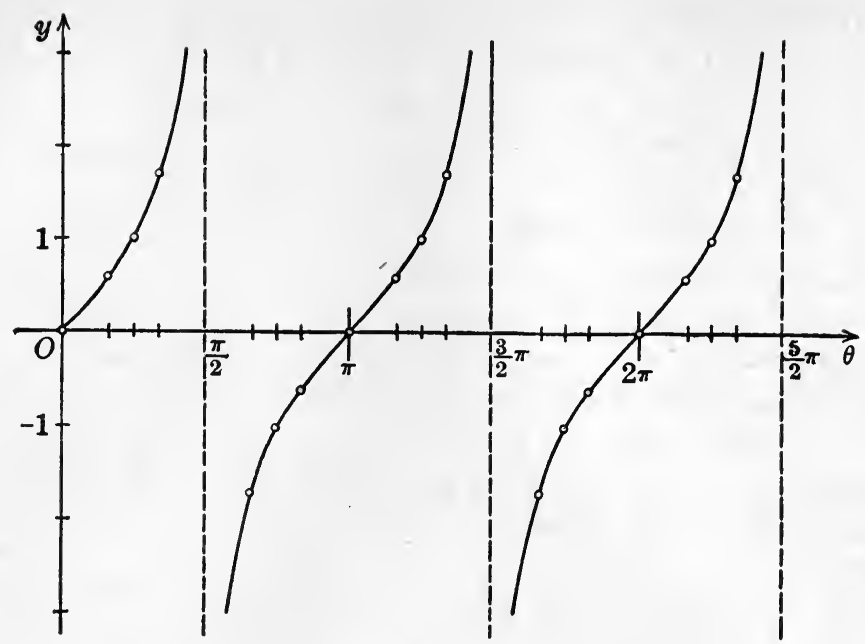

Fig. 96.

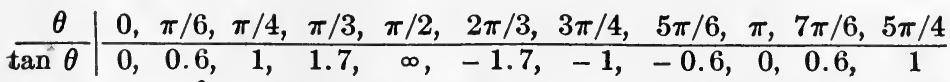

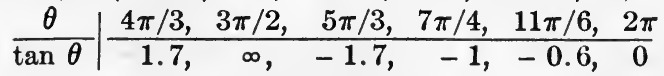

\section{EXERCISES}

1. Discuss the periodicity, zeros, values of $\theta$ for which the function becomes infinite, sign, maxima and minima, changes and symmetry of $\cos \theta$ and $\tan \theta$.

2. Sketch on the same axes the graphs of the pairs of functions following, and discuss the second function with respect to the properties listed in Exercise 1.
(a) $\sin \theta$ and $\csc \theta$.
(b) $\cos \theta$ and $\sec \theta$.
(c) $\tan \theta$ and $\cot \theta$.

3. Construct the graphs of the six trigonometric functions on the same axes.

4. What properties of the functions can be inferred from graphs or: the same axes of
(a) $\sin \theta$ and $\cos \theta$ ?
(b) $\tan \theta$ and $\cot \theta$ ?
(c) $\sec \theta$ and $\csc \theta$ ?

5. Describe the motion of a particle on a straight line if its distance $s$ from a fixed point on the line at any time $t$ is given by $s=\sin t$. Does such a motion approximate any motion occurring in nature?

6. On the same axes sketch the graphs of the functions: 

(a) $\sin \theta$ and $2 \sin \theta$.
(b) $\cos \theta$ and $3 \cos \theta$.
(c) $\tan \theta$ and $\frac{1}{3} \tan \theta$.
(d) $\cot \theta$ and $0.2 \cot \theta$.
(e) $\sec \theta$ and $\frac{1}{2} \sec \theta$.
(f) $\csc \theta$ and $\frac{1}{3} \csc \theta$.

7. Construct and discuss the graph of
(a) vers $\theta=1-\cos \theta$.
(b) $\operatorname{covers} \theta=1-\sin \theta$.

8. By the addition of ordinates (see Exercise 4, page 44) construct the graph of
(a) $\sin x+\cos x$.
(b) $2 \sin x-\cos x$.
(e) $\sin x+3 \cos x$.

62. Functions of Complementary Angles. Construct an acute angle $\theta$ and its complement $90^{\circ}-\theta$ with their initial lines coinciding with the positive part of the $x$-axis. On their terminal lines take $O P=O^{\prime} P^{\prime}$, so that $r=r^{\prime}$. Then $P$ and $P^{\prime}$ are symmetrical with respect to $O A$, the bisector of the first quadrant (why?), and hence $x=y^{\prime}$ and $y=x^{\prime}$. Then

$\sin \left(90^{\circ}-\theta\right)=\frac{y}{r}=\frac{x^{\prime}}{r^{\prime}}=\cos \theta$

and

$\cos \left(90^{\circ}-\theta\right)=\frac{x}{r}=\frac{y^{\prime}}{r^{\prime}}=\sin \theta$.

Using (2), page 167, with (1) and (2), we then have

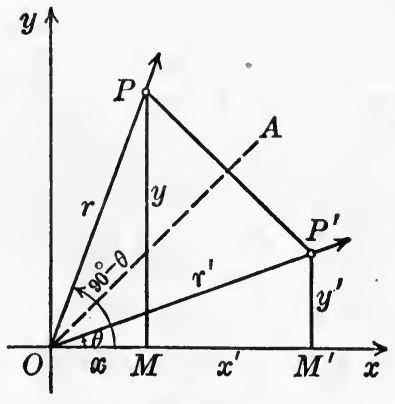

Fig. 97.

$$
\tan \left(90^{\circ}-\theta\right)=\frac{\sin \left(90^{\circ}-\theta\right)}{\cos \left(90^{\circ}-\theta\right)}=\frac{\cos \theta}{\sin \theta}=\frac{1}{\tan \theta}=\cot \theta .
$$

Also, $\cot \left(90^{\circ}-\theta\right)=\frac{1}{\tan \left(90^{\circ}-\theta\right)}=\frac{1}{\cot \theta}=\tan \theta$, and in like manner,

$$
\sec \left(90^{\circ}-\theta\right)=\csc \theta, \csc \left(90^{\circ}-\theta\right)=\sec \theta .
$$

The cosine, cotangent and cosecant of an angle are so named because they are respectively the sine, tangent, and secant of the complementary angle, as is shown by these relations. The former functions are called the cofunctions of the latter, respectively, and vice versa. With this terminology, the six relations (1) to (5) may be stated as the 
Theorem. The functions of any acute angle are equal respectively to the cofunctions of the complementary angle.

A method of extending the proofs of these relations for any value of $\theta$, not necessarily acute, will be given in Section 68 .

63. Tables of Trigonometric Functions. As a consequence of the theorem in the preceding section, tables of values of the trigonometric functions may be printed in very compact form.

Since $\cos \left(90^{\circ}-\theta\right)=\sin \theta$, a table of sines of any set of angles is also a table of cosines of the complementary angles. The complements of $0^{\circ}, 1^{\circ}, 2^{\circ}, \ldots, 88^{\circ}, 89^{\circ}, 90^{\circ}$ are respectively $90^{\circ}, 89^{\circ}, 88^{\circ}, \ldots, 2^{\circ}, 1^{\circ}, 0^{\circ}$. Hence:

1. The table of sines on pages 8 and 9 of Huntington's Tables is also a table of cosines if read backward.

2. The sines and cosines of angles from $0^{\circ}$ to $45^{\circ}$ in the Condensed Table on the inside of the back cover of the Tables are, if read upward, the cosines and sines respectively of the angles from $45^{\circ}$ to $90^{\circ}$.

In like manner, $\tan \theta$ and $\cot \theta$ may be given in one table, and so also may sec $\theta$ and $\csc \theta$.

Thus the theorem on functions of complementary angles makes it practicable to reduce by one-half the space devoted to a table of trigonometric functions.

Fractional parts of a degree, in Huntington's Tables, are given in tenths and hundredths instead of in minutes and seconds. One of the merits of this decimal method of subdivision is that the process of interpolation, in finding a func-

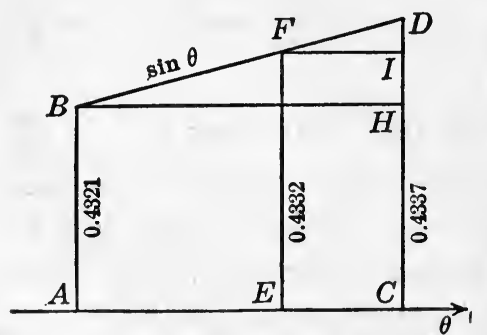

Fig. 98. tion of $\theta$, is identical with that used earlier in the other tables (page 121).

Notice that, in the body of the tables, the decimal point, and any figures preceding it, are usually printed only in the first column. For example, tan $53^{\circ} .32=1.3426$.

The process of finding $\theta$, if a function of $\theta$ is given, is illustrated in the examples following. 
Example 1. Find $\theta$ if $\sin \theta=0.4321$.

Searching through the body of the table of sines, we find that 0.4321 is given in the table, and reference to the margin shows that $\theta=25^{\circ} .6$.

Example 2. Find $\theta$ if $\sin \theta=0.4332$.

A search in the body of the table of sines shows that 0.4332 lies between 0.4321 and 0.4337 , which are the sines of $25^{\circ} .6$ and $25^{\circ} .7$. Fig. 98 shows the graph of $\sin \theta$ between these angles, on the assumption that it is straight (compare the assumption on page 122). To find $\theta$, graphically, is to find the value of $\theta$ at $E$ if $E F=0.4332$.

The slope of the graph is the difference of the ordinates of $B$ and $D$, $H D=0.0016$, divided by the difference of the abscissas, $A C=0.1$ (Definition, page 50). It is also equal to the difference of the ordinates of $F$ and $D, I D=0.0005$, divided by the difference of the abscissas, $E C$.

Hence

whence

$$
\frac{0.0016}{A C}=\frac{0.0005}{E C},
$$

$$
E C=\frac{0.0005}{0.0016} A C=\frac{5}{16} A C=0.3 A C=0.3 \times 0.1=0.03 .
$$

Hence at $E, \theta=25^{\circ} .67$.

The arithmetical processes used in interpolating to find an angle of which a function is given are:

Find the two successive numbers in the proper table between which the given number lies.

Find the difference between the given number and that one of these two numbers to which it is nearer, and divide this difference by the tabular difference.

Apply the result as a correction to the last digit of the angle whose function was used in getting the difference above, in such a way that the result lies between the two angles corresponding to the two numbers in the table.

Example 3. Find $\theta$ if $\cos \theta=0.4815$.

Searching through the body of the table, we find that 0.4815 lies between 0.4818 and 0.4802 , the cosines of $61^{\circ} .2$ and $61^{\circ} .3$. It is nearer the former, the difference being 3 , neglecting the decimal point, while the tabular difference is 16 . The correction to be applied to $61^{\circ} .2$ is therefore $\frac{3}{16}$ of one-tenth of a degree, so that $\theta=61^{\circ} .2 \frac{3}{16}^{6}=61^{\circ} .22$.

In using the table of tenths of the tabular difference, in the margin, find the difference 3 as above. Then look for 3 in the margin. It lies in the column headed 2. Hence 3 is 2 -tenths of the tabular difference, and the digit 2 is annexed to $61^{\circ} .2$, giving $61^{\circ} .22$.

The use of the tables for other than acute angles will be considered in Section 69. 


\section{EXERCISES}

1. Find the cosine, cotangent, and cosecant of $72^{\circ} .43$. Find the sine, tangent, and secant of $17^{\circ} .57$. Compare the two sets of results.

2. Find the six functions of $73^{\circ} .26$, and verify the fact that the last three functions are the reciprocals of the first three in the reverse order. if

3. Find the acute value of $\theta$, illustrating the interpolation graphically,
(a) $\sin \theta=0.9235$.
(d) $\cot \theta=0.3603$.
(b) $\cos \theta=0.3129$.
(e) $\sec \theta=1.5165$.
(c) $\tan \theta=1.1603$.
(f) $\csc \theta=5.515$.

4. The arithmetical operations in this exercise should be performed mentally. Find the acute value of $\theta$ such that

(a) $\tan \theta=0.8134 ; \tan \theta=1.8134 ; \quad \cos \theta=0.3019$.

(b) $\sin \theta=0.8436 ; \sec \theta=4.057 ; \quad \cot \theta=0.6142$.

(c) $\cos \theta=0.0589 ; \csc \theta=1.6397 ; \quad \sin \theta=0.16543$.

(d) $\cos \theta=0.0630 ; \cos \theta=0.06300 ; \tan \theta=1.4628$.

5. Construct the line through the origin whose slope is 2 , and find the angle between the line and the $x$-axis.

6. Construct a table of values of $\theta$ and $\sin \theta$ for values of $\theta$ taken every $15^{\circ}$ from $0^{\circ}$ to $90^{\circ}$, expressing $\theta$ in radians decimally instead of in terms of $\pi$ (see Tables, page 32), and giving the values of $\theta$ and $\sin \theta$ to two decimal places. Construct the graph as accurately as possible from this table, using a large scale. On the same axes draw the graph of $\theta$. What approximate value of $\sin \theta$ for very small angles is suggested by these graphs? Using the Condensed Tables on the inside of the back cover of the Tables, determine for how large an angle this approximation is correct to three decimal places; to four decimal places. What is the limit of $\sin \theta / \theta$ as $\boldsymbol{\theta}$ approaches zero?

7. Solve Exercise 6 replacing $\sin \theta$ by $\tan \theta$.

8. Using the properties of the functions $\sin \theta, \cos \theta$ and $\tan \theta$ suggested by the symmetry of their graphs, find the sine, cosine, and tangent of the negative angles, $-24^{\circ} .32,-48^{\circ} .27,-68^{\circ} .46$.

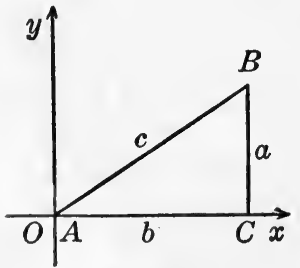

Frg. 99.

64. Solution of Right Triangles. It is customary to denote the magnitude of the angles of a triangle $A B C$ by $A, B, C$ and the lengths of the sides opposite by $a, b, c$ respectively. In a right triangle, the right angle is usually denoted by $C$, and the hypotenuse by $c$. A right triangle $A B C$ may be placed with reference to coördinate axes so that $A$ is at the origin, $A C$ lies along 
the positive $x$-axis, and the hypotenuse $A B$ lies in the first quadrant. Then the definitions on page 166 show that

$$
\left.\begin{array}{rl}
\sin A=a / c, \cos A=b / c, \tan A & =a / b \\
\cot A=b / a, \sec A=c / b, \csc A & =c / a
\end{array}\right\} .
$$

It is not always convenient to place the triangle on coördinate axes, and sometimes other letters must be used, and hence it is desirable to remember these formulas in words, as follows:

The sine of an acute angle of a right triangle is the ratio of the side opposite the angle to the hypotenuse.

The cosine of an acute angle of a right triangle is the ratio of the side adjacent to the angle to the hypotenuse.

The tangent of an acute angle of a right triangle is the ratio of the side opposite the angle to the adjacent side.

Analogous statements for the last three functions are readily obtained by the reciprocal relations (page 166).

The sides and angles of a triangle are called its parts. In order to construct a right triangle, we must be given two parts, in addition to the right angle, of which at least one must be a side. To solve a triangle is to find the unknown parts from the known.

Every right triangle may be solved by means of formulas (1) and the fact that $A+B=90^{\circ}$. But the Pythagorean Theorem is sometimes convenient.

In solving right triangles but two essentially different cases arise:

I. Given a side and an angle, to find the other two sides use those two of equations (1) which contain the unknown sides in the numerators and the given side in the denominator.

II. Given two sides, to find an angle, use that one of the two equations (1) containing the given sides which leads to the simpler division, and then to find the third side use either of the two equations containing the third side in the numerator.

In either case the second angle is found from $A+B=90^{\circ}$.

The use of the equations indicated in these rules will lead to multiplication, and division by a value of $\sin \theta$, for example, is avoided. 
It is desirable that figures be constructed accurately, as the figure may show the absurdity of an incorrect result. A good check on the accuracy of the computation may be obtained by constructing the triangle with scale and protractor, and measuring the unknown parts.

Example 1. Solve the right triangle $A=37^{\circ} .24, b=9$.

Solution. $B=90^{\circ}-A=52^{\circ} .76$.

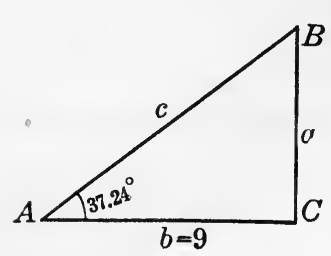

Fig. 100.

$$
\begin{aligned}
& \frac{a}{b}=\tan A \text {, whence } a=b \tan A \text {. } \\
& =9 \times 0.7601 \\
& =6.8409 \text {. } \\
& \begin{array}{l}
\mathbf{c} \\
\bar{b}
\end{array}=\sec A \text {, whence } c=b \sec A \\
& =9 \times 1.2561 \\
& =11.3049 \text {. }
\end{aligned}
$$

Check. The accuracy of the computation may be checked by finding $b$ from $a$ and $c$. By the Pythagorean Theorem, and tables of squares and square roots,

$$
b=\sqrt{c^{2}-a^{2}}=\sqrt{127.7-46.7}=\sqrt{81.0}=9,
$$

which agrees with the given value of $b$.

EXample 2. Solve the right triangle $a=7, b=9$.

The given sides occur in the third of formulas (1). Hence $\tan A=\frac{a}{b}=\frac{7}{9}=0.7778$, whence $A=37^{\circ} .87$, and hence $B=90^{\circ}-37^{\circ} .87$ $=52^{\circ} .13$. To find $c$ we have $\begin{aligned} \csc A=\frac{c}{a}, \text { whence } c & =a \csc A \\ & =7 \times 1.6290=11.403 .\end{aligned}$

Check. Find $b$ from $B$ and $c$. Since $\sin B=b / c$, we have

$$
b=c \sin B=11.403 \times 0.7894=9.0015,
$$

which agrees reasonably well with the given value of $b$.

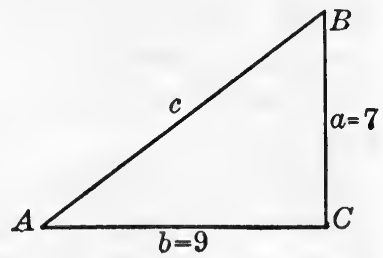

FIG. 101.

It is to be noted that the angles and lines in these examples and in the exercises following have not been measured with the same degree of precision. The angles are given to four figures in order to afford practice in interpolation.

Problems involving isosceles triangles and regular polygons may be solved by means of right triangles, for such figures may be divided into congruent right triangles. 
65. Applications. An instrument known as a transit enables surveyors to measure angles in vertical and horizontal planes.

If $A$ and $B$ are points not in the same horizontal plane, and if $A C$ and $B D$ are horizontal lines in the vertical plane through $A$ and $B$, then $\angle B A C$ is called the angle of depression of $B$ at $A$, and $\angle A B D$ is called the angle of elevation of $A$ at $B$. If the eyes are at $A$, looking horizontally over $B$, the angle of depression of $B$ is the angle through which the eyes must be lowered to see $B$. While if the eyes are at $B$, looking horizontally below $A$, the angle of elevation of $A$ is the angle through which

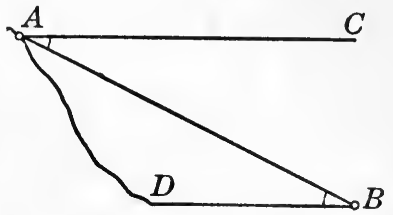

FIg. 102. the eyes must be raised to see $A$.

If $A A^{\prime}$ and $B B^{\prime}$ are the vertical lines through two points $A$ and $B$, meeting the horizontal plane through a third point $C$

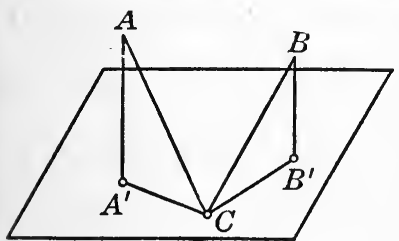

FIG. 103. at $A^{\prime}$ and $B^{\prime}$ respectively, then $\angle A^{\prime} C B^{\prime}$ is called the horizontal angle between $A$ and $B$ at $C$. If we think of $A A^{\prime}$ and $B B^{\prime}$ as two trees, of different heights usually, for an observer at $C$, the horizontal angle between the tree tops $A$ and $B$ is the angle through which one must turn, if one faces first toward the tree $A A^{\prime}$ and then turns to face the tree $B B^{\prime}$.

The bearing of a line is the angle the line makes with some fundamental line of the figure which is called the base line. For example, if the base line is north and south, the bearing of a line running northeast is $45^{\circ}$ east of north.

\section{EXERCISES}

1. In any right triangle, if $c$ and $A$ are given, show that $a=c \sin A$ and $b=c \cos A$. Express these equations in words. If $a$ and $A$ are given, find $b$ and $c$. If $b$ and $A$ are given, find $a$ and $c$. 
2. Solve and check the following right triangles:
(a) $A=22^{\circ} .13, b=5$.
(b) $B=40^{\circ} .28, c=4$.
(c) $b=17, c=30$.
(d) $a=10, \quad b=19$.
(e) $A=66^{\circ} .47,=20$.
(f) $a=30, c=37$.

3. To find the width of a river, two points, $A$ and $C$, are taken on one bank 100 feet apart. If $B$ is the point on the other bank directly opposite $C$, and if $\angle C A B$ equals $72^{\circ} .16$, how wide is the river?

4. From the top of a lighthouse 35 feet high, the angle of depression of a ship is $11^{\circ} .38$. How far is the ship from the lighthouse?

5. What is the angle of elevation of the sun if a pole 49 feet high casts a shadow of 11 feet?

6. An iron wedge for splitting rails is to have a base two inches wide and a vertex angle of $15^{\circ}$. How long will each side be?

7. A rustic summer house, or shelter, is to be built with an octagonal floor, 6 feet on a side. Determine the amount of flooring necessary, making an allowance of $25 \%$ on account of the "tongue and groove" and for waste.

8. Solve Exercise 7 if the floor is to be pentagonal. Solve Exercise 7 if the floor is to be a regular seven-sided polygon. Can this Exercise be solved by plane geometry?

9. If the radius of a regular polygon of $n$ sides is $r$, find (a) the perimeter in terms of $n$ and $r$; (b) the area.

10. If a side of a regular polygon is $a$, express the area as a function of $a$, assuming $n$ to be constant.

11. What is the angle of inclination of an ordinary gable roof, if its pitch (the ratio of its height to its entire width) is $\frac{2}{3}$ ? $\frac{1}{2}$ ?

12. A point $P$ moves with uniform speed around a circle of radius one foot, making a complete revolution every 36 seconds. The projection $M$

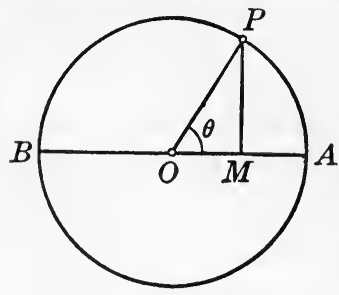

Frg. 104. of $P$ on a diameter $A O B$, moves along the diameter. Find $O M$ for the values $50^{\circ}, 60^{\circ}$, $70^{\circ}$, if $\theta=\angle A O P$. Find the average velocity of $M$ as $\theta$ increases from $50^{\circ}$ to $60^{\circ}$ and from $60^{\circ}$ to $70^{\circ}$. Find the average velocity of $M$ as $\theta$ increases from $59^{\circ}$ to $60^{\circ}$ and from $60^{\circ}$ to $61^{\circ}$. From $59^{\circ} .9$ to $60^{\circ}$ and from $60^{\circ}$ to $60^{\circ} .1$. Approximately what is the velocity of $M$ at the instant when $\theta=60^{\circ}$ ?

13. Two life saving stations are 10 miles apart on a beach running $22^{\circ} .5$, east of north. A lightship anchored off the beach lies $33^{\circ} .75$ north of east from one station, and $11^{\circ} .25$ east of south from the other. How long would it take a boat to run from one station to the other if its speed is 10 miles per hour, and if it passes outside the lightship? How long would it take the boat to go from the ship to the nearest point on the beach?

14. (a) Two sides of a triangle, not a right triangle, are 12 and 20 , 
and the included angle is $28^{\circ} .48$. Find the altitude on the latter side and the area.

(b) Find the area of any triangle $A B C$ in terms of the sides $b$ and $c$ and the included angle $A$.

15. Find the area of a parallelogram in terms of two adjacent sides and the included angle.

16. Two ships are on a line with a lighthouse, which is 40 feet high. At the top of the lighthouse, the angles of depression of the ships are respectively $4^{\circ} .26$ and $6^{\circ} .31$. How far apart are the ships?

17. A regular octagonal tower is 4 feet on each side. The roof is $\mathbf{1 0}$ feet high. How long should the rafters be?

18. A bridge is to be built across a ravine between two points $A$ and $B$ on the same level. Two stations $C$ and $D$ are chosen in the ravine which lie in the vertical plane through $A$ and $B$. At $A$, the angle of depression of $C$ is $42^{\circ} .37$, and $A C=50$ feet. At $C$ the angle of depression of $D$ is $4^{\circ} .52$, and $C D=40$ feet. At $D$ the angle of elevation of $B$ is $37^{\circ} .89$, and $D B=60$ feet. Find the length of the bridge.

19. Check the accuracy of the measurements in Exercise 18, by finding the difference in altitude of $A$ and $D$, and also of $B$ and $D$.

20. To find the width of a river, a tree is selected on one shore. At a point 50 feet from the tree, the angle of elevation of the top is $48^{\circ} .72$. At the point on the other shore directly opposite the tree, the angle of elevation of the top is $17^{\circ} .39$. How wide is the river?

21. From a station $A$ the horizontal angle between a mountain top $C$ and a second station $B, 1000$ feet from $A$ and at the same altitude, is $64^{\circ} .37$. At $B$ the horizontal angle between the mountain top and $A$ is $90^{\circ}$, and the angle

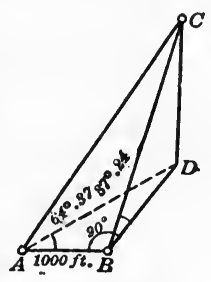

Frg. 105. of elevation of the mountain top is $37^{\circ} .24$. How high is the mountain?

66. Parallelogram Law - Velocities, Accelerations, Forces. The law to be considered in this section is illustrated in

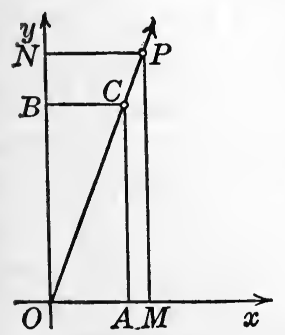

Fig. 106.

Example 1. The current in a river flows at the rate of 2 miles an hour. A man rows across at the rate of 4 miles an hour, keeping his boat at right angles to the shore. Show that the boat moves in a straight line. Find how fast it moves, and in what direction.

Take the starting point for the origin of a system of coördinates, and let the $x$-axis lie along the bank of a river. At the time $t$, the boat will be at a point $P$, whose coördinates give the distance the boat has been carried by the current, $x=2 t$, and the distance the man has rowed from shore, $y=4 t$. Eliminating $t$, we 
obtain $y=2 x$, which is true for all values of $t$. Hence the boat moves in a straight line (Corollary 1, page 57).

Let $C$ denote the position of the boat one hour after starting, and let $O A$ and $O B$ be the coördinates of $C$.

The line $O A$ represents the velocity of the water with reference to the earth, the direction of the line being that in which the water flows, and the length of the line being the number of miles per hour the water moves. Similarly, $O B$ represents, in direction and magnitude, the velocity of the boat with reference to the water. (If the water were at rest, $O B$ would represent the actual motion of the boat, in one hour, with reference to the earth.) With reference to the earth, the boat moves along the line $O C$, and in one hour moves from $O$ to $C$. Hence $O C$ represents, in direction and magnitude, the actual, or resultant, velocity of the boat.

The resultant velocity is readily computed. Its magnitude is

$$
O C=\sqrt{O A^{2}+A C^{2}}=\sqrt{2^{2}+4^{2}}=4.47 \text { miles per hour. }
$$

Its direction may be given by $\angle A O C$. Since

$$
\tan \angle A O C=A C / O A=4 / 2=2 \text {, we have } \angle A O C=63^{\circ} .43 .
$$

This illustration exemplifies the law known as the

Parallelogram of velocities. If a body is subjected to two different velocities, represented in direction and magnitude by two lines $O A$ and $O B$, the actual or resultant velocity is represented by the diagonal $O C$ of the parallelogram determined by $O A$ and $O B$.

If a body is moving through the air in any way, it has an acceleration of 32 feet per second per second directed toward the center of the earth (see Section 22, page 63). This means that its vertical velocity is increased or decreased each second by 32 feet per second, according as it is falling or rising. Its horizontal velocity is uniform, and is not affected by the action of gravity. No account is taken here of the resistance of the air, which would make the discussion very much more complicated.

The acceleration due to gravity may be represented by a vertical line running downward whose length is 32 . In like manner, any acceleration may be represented, in direction and magnitude, by a line.

A force may also be represented, in direction and magnitude, by a line $O A, O$ representing the point of application of the force. Accelerations and forces may also be combined according to the parallelogram law. 
Finding the resultant of two velocities is known as the composition of velocities, the two given velocities being called components of the resultant. The converse problem of determining two component velocities which have a given resultant is called the resolution of velocities. The same terms are also used with reference to accelerations and forces.

If the components are at right angles, the parallelogram is a rectangle, and the composition or resolution may be effected by solving a right triangle. If the parallelogram is not a rectangle, it is necessary to use the methods to be developed in Sections 71-73. Problems of this sort will be found in the exercises following Section 73.

Example 2. A ball rolls down a plane whose inclination is $30^{\circ}$. Resolve the acceleration due to gravity into two components parallet and perpendicular to the plane.

Let $O C=32$ represent the acceleration due to gravity. Through $O$ and $C$ draw lines parallel and perpendicular to the plane $D E$, forming the rectangle $O A B C$. Then $O A$ and $O B$ represent the required components, for the resultant of $O A$ and $O B$ is $O C$ (parallelogram law).

In the triangle $O A C, O C$ $=32$, and $\angle A C O=\angle E D F$ $=30^{\circ}$ (why?).

Hence $\quad O A / 32=\sin 30^{\circ}$ and $\quad A C / 32=\cos 30^{\circ}$. Then $\quad O A=32 \times \frac{1}{2}=16$ and $O B=A C=32 \times \frac{1}{2} \sqrt{3}$ $=16 \sqrt{3}$.

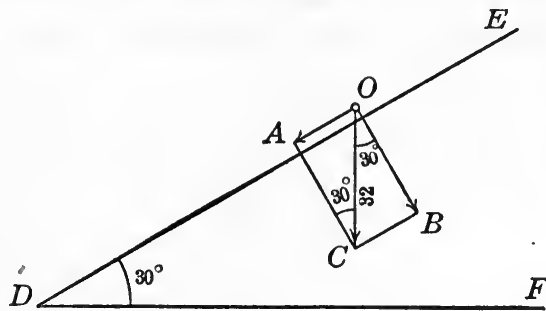

Fig. 107.

As there is no motion in a direction perpendicular to the plane, the component $O B$ is neutralized by the plane. The component parallel to the plane, $O A=16$ feet per second per second, gives approximately the effective acceleration with which the ball rolls down the plane. If the ball starts to roll from rest, how fast will it be moving at the end of one second? At the end of two seconds? At the end of four seconds? If a ball is rolled up the plane, how will its velocity be affected during any second? If it is started up with a velocity of 50 feet per second, how long will it roll up the plane?

67. Conditions of Equilibrium of a Particle. In measuring a force we shall use the pound as the unit. 
If a number of forces act on a particle, which we take as the origin of a system of coördinates, each of the forces may be resolved into two components, one acting along each axis. If the particle is in equilibrium, there is no motion in the direction of the $x$-axis, and hence the algebraic sum of the components along that axis must be zero. Similarly, the sum of the components along the $y$-axis must be zero. And conversely, if each of these sums is zero the particle must be in equilibrium. Hence we have the

Theorem. A particle is in equilibrium under the action of any number of forces if and only if the sum of the components in each of two perpendicular directions is zero.

In applying this theorem, first determine all the forces acting on the particle, and then choose the perpendicular directions. If two of the forces are at right angles to each other, choose these directions.

EXAMPLE 1. A particle weighing 4 ounces is supported on a smooth plane whose inclination is $30^{\circ}$ by a cord parallel to the plane. Find the tension of the cord and the pressure on the plane.

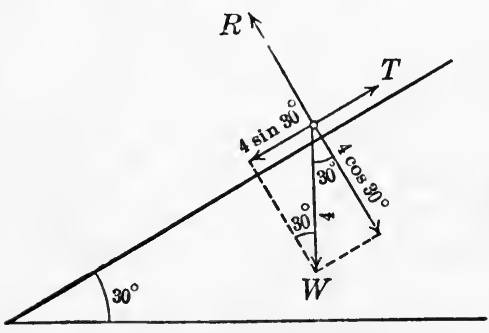

Fig. 108.

The forces acting on the particle are

(1) Its weight, $W=4$, acting vertically.

(2) The tension of the cord, $T$, acting parallel to the plane.

(3) The resistance of the plane, $R$, acting perpendicular to the plane.

Since $R$ and $T$ act at right angles, we proceed to resolve all the forces into components parallel and perpendicular to the plane. Choosing these directions facilitates the work, because $W$ is then the only force whose components must be determined. By the parallelogram law and the solution of a right triangle, the component of $W$ parallel to the plane is found to be $4 \sin 30^{\circ}$, and that perpendicular to the plane is $4 \cos 30^{\circ}$, both acting downward. Then by the theorem, taking the directions of $R$ and $T$ as positive, we have

whence

$$
T-4 \sin 30^{\circ}=0
$$

and

$R-4 \cos 30^{\circ}=0$,

$$
T=2 \text { ounces }
$$

$R=2 \sqrt{3}=3.4$ ounces. 
In pointing out the part played by mathematics in the illustration of the scientific method in Example 1, page 79 (see bottom page 80 ), it was stated that a more satisfactory verification of the law obtained would be indicated later, by deducing from the law some fact that may be verified by an experiment of a different nature. - This deduction will be made in Example 2 below.

Friction between an object and a plane acts parallel to the plane, and in the direction opposite to that in which the object moves or tends to move. When motion is about to take place, the force of friction is equal to the coefficient of friction for the surfaces in contact (see page 81 ) multiplied by the pressure of the object on the plane in the direction perpendicular to the plane. It is this law that we wish to verify.

Example 2. A block of wood weighing 20 grams rests on a horizontal board. If the coefficient of friction is 0.29, the result obtained in Example 1 , page 79 , at what angle may the board be tipped before the block will be on the point of sliding?

The forces acting on the bloc are:

(1) The resistance of the plane, $R$,

(2) The friction, $F$, and

(3) The weight $W=20$.

It is known that

$$
F=0.29 R,
$$

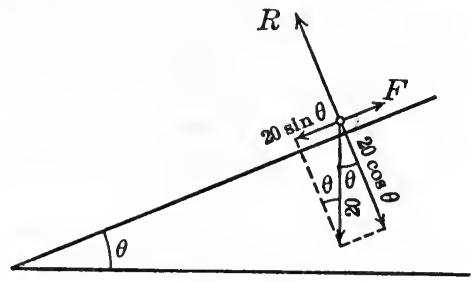

Fig. 109.

since the pressure on the plane is numerically equal to the resistance of the plane.

Resolving $W$ into components parallel and perpendicular to the plane, as in Example 1 above, we get

$$
F=20 \sin \theta, \quad R=20 \cos \theta,
$$

where $\theta$ is the angle at which the block is on the point of sliding.

Substituting in (1) the values of $F$ and $R$ given by (2), we get,

$$
20 \sin \theta=0.29 \times 20 \cos \theta \text {. }
$$

Dividing both sides by $20 \cos \theta$, we obtain

$$
\frac{\sin \theta}{\cos \theta}=0.29 \text {. }
$$


By equation (2) on page $167, \frac{\sin \theta}{\cos \theta}=\tan \theta$.

Hence

whence

$$
\begin{aligned}
\tan \theta & =0.29, \\
\theta & =16^{\circ} .2 .
\end{aligned}
$$

This result may be readily tested by experiment. If it is found that when one end of the board is gradually raised the block begins to slide when the inclination is a little over $16^{\circ}$, then we have a verification of the correctness of the deduction by which $\theta$ was found, and also a verification of the law (1) on which the deduction was based. That is, we have a verification of the law obtained in Example 1, page 79, which was re-stated on page 81 .

\section{EXERCISES}

1. A boy kicks a football so that it would roll across a street at the rate of 15 feet per second, and simultaneously a second boy kicks it so that it would roll along the street with a velocity of 12 feet per second. Find the actual velocity of the ball (magnitude and direction).

2. A man walks across a canal boat. If his velocity with reference to the earth is 7 feet per second in a direction inclined at $30^{\circ}$ to the bank of the canal, find the velocity of the boat and that at which he walks.

3. A ball is thrown into the air at an angle of $40^{\circ}$ with a velocity of 30 feet per second. Find the horizontal and vertical components of the velocity.

4. If a ball is placed on a plane inclined at $20^{\circ}$, find the acceleration with which it rolls down the plane, and how fast it will be moving at the end of 3 seconds.

5. A ball is rolled up a plane inclined at $10^{\circ}$ with an initial velocity of 20 feet per second. How long will it roll up the plane?

6. What is the inclination of a sidewalk if a ball rolls down it with an acceleration of 8 feet per second per second?

7. A cake of ice weighing 200 pounds is held on an ice slide at an ice house by a rope parallel to the slide. If the inclination of the slide is $45^{\circ}$, find the pull on the rope and the pressure on the slide.

8. An automobile weighing 2500 pounds stands on a pavement inclined at $12^{\circ}$. What force do the brakes exert?

9. Will a box slide down a board inclined at $15^{\circ}$ if the friction is 0.3 of the pressure of the box on the plane?

10. A bar of iron weighing 5 pounds rests on a rough board. As one end of the 5 -ard is gradually raised, it is found that the bar is just ready to slide when the inclination is $20^{\circ}$. Find the force of friction and the coefficient of friction (Definition, page 81). 
11. A boy and his sled weigh 50 pounds. What force is necessary to hold them on an icy sidewalk whose inclination is $10^{\circ}$ by means of a rope inclined at $30^{\circ}$ to the walk?

12. (a) A rifle is fired at an angle of $20^{\circ}$ to the horizon. If the muzzle velocity of the ball is 2000 feet per second, find the horizontal and vertical components of the velocity at the muzzle.

(b) How long will a ball rise if it is thrown vertically upward with a velocity of 32 feet per second? of 80 feet per second? How long will the rifle ball in (a) rise? How far will it move horizontally in this time?

13. A rifle with muzzle velocity of 1600 feet per second is fired at an inclination of $30^{\circ}$. Find the horizontal and vertical components of the velocity at the muzzle. How long will it rise? When will it hit the ground (see Exercise 13, page 104)? How far from the point where it is fired will it hit?

14. A rope is tied to a heavy weight lying on the ground. A boy pulls on the rope with a force of 50 pounds in such a way that the rope is inclined at $25^{\circ}$ to the vertical. Find the horizontal and vertical components of his pull. What is the force with which he tends to lift the weight? To drag it? If the weight weighs 100 pounds, what is the pressure of the weight on the ground when the boy pulls?

15. A body weighing 25 pounds rests on a rough plane inclined at $5^{\circ}$. Find the components of the weight parallel and perpendicular to the plane. What is the pressure of the body on the plane? What force is tending to make the body move down the plane? Why does it not move? (The weight of a body is the force with which the earth attracts it.)

16. Three bales of cotton, a total weight of $\mathbf{1 4 0 0}$ pounds, are raised from the hold of a ship by a derrick until they are just above the deck, when they are pulled one side by a horizontali rope. Find the pull of the rope and of the cable on the derrick when the cable is inclined at $12^{\circ}$ to the vertical.

17. A stone weighing 300 pounds is raised to the top of a building by means of a derrick on top of the building, while a man on the ground keeps it away from the side of the building by means of a rope tied to the stone. Find the pull on the cable of the derrick and on the rope if the cable is inclined to the vertical at $5^{\circ}$ and the rope at $60^{\circ}$.

18. Two cords are tied to a weight of 10 pounds. The weight is held by two boys who pull on the cords. If one cord is inclined at $20^{\circ}$ to the vertical and the other at $14^{\circ}$, find the force with which each boy pulls.

19. Two narrow boards are 2 feet long. A boy places their upper ends together and their lower ends on the ground 1 foot apart, and balances a weight of 5 pounds on top of them. Find the pressure along each board. 20. A body weighing 500 pounds is suspended from the center of a horizontal beam. The beam rests on two V-shaped supports, inverted, which are made of pieces of " $2 \times 4$ " each 4 feet long. Find the thrust 
or pressure along each $2 \times 4$, assuming that the effect is the same as if half of the weight were placed directly over each support.

21. Just above the door on the second story of a barn, a beam projects horizontally for 3 feet. Objects are raised to the second story by a rope which passes over a pulley at the end of the beam and enters the barn over a pulley in the wall at a point 4 feet directly above the beam. Find the thrust along the beam when an object weighing 50 pounds is suspended by the rope.

68. Functions of $n 90^{\circ} \pm \theta$. In Section 62 we saw how to express the functions of the complement of $\theta, 90^{\circ}-\theta$, in terms of functions of $\theta$. Let us now consider the functions of $180^{\circ}-\theta$, the supplement of $\theta$, employing the same method.

Construct the angles $\theta$ and $180^{\circ}-\theta$ with their initial lines coinciding with the $x$-axis, and on their terminal lines take $P(x, y)$ and $P^{\prime}\left(x^{\prime}, y^{\prime}\right)$ so that $r=r^{\prime}$. Then $P$ and $P^{\prime}$ are symmetrical with respect to the $y$-axis (why?), and hence $x^{\prime}=-x$,

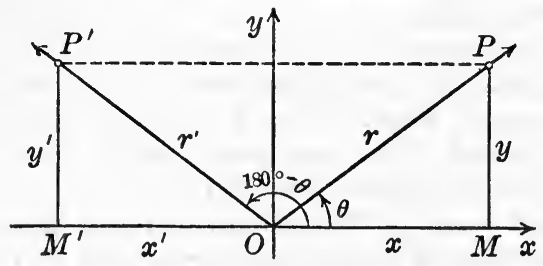

FIG. 110. and $y^{\prime}=y$. Then

$$
\begin{gathered}
\sin \left(180^{\circ}-\theta\right)=\frac{y^{\prime}}{r^{\prime}}=\frac{y}{r} \\
=\sin \theta . \\
\cos \left(180^{\circ}-\theta\right)=\frac{x^{\prime}}{r^{\prime}}=-\frac{x}{r} \\
=-\cos \theta .
\end{gathered}
$$

Dividing (1) by (2), and applying (2), page 167, we obtain

$$
\tan \left(180^{\circ}-\theta\right)=-\tan \theta .
$$

Similar formulas may be obtained for the other functions from these formulas by means of the reciprocal relations.

Inspection of these results shows that

The numerical values of the trigonometric functions of an obtuse angle are equal respectively to the functions of the supplementary angle. In the figure above, $\theta$

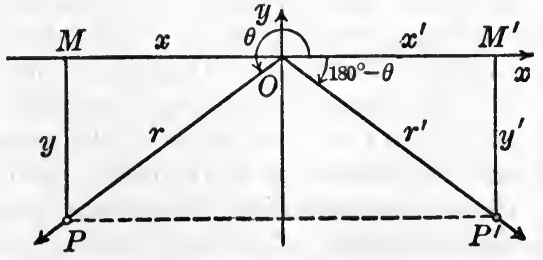

Fig. 111. 
was taken acute. But the proof applies without change to the second figure, in which $\theta$ is in the third quadrant. It would also apply to properly constructed figures in which $\theta$ lies in the second or fourth quadrants, so that these formulas hold for all positive values of $\theta$ less than $360^{\circ}$. The proof may be extended to include all values of $\theta$ by using the periodicity of the functions.

Any function of one of the angles

$$
-\theta, 90^{\circ} \pm \theta, 180^{\circ} \pm \theta, 270^{\circ} \pm \theta, 360^{\circ}-\theta
$$

may be expressed in terms of a function of $\theta$. Any one of these forty-eight formulas may be written down by the following rule:

If any one of the trigonometric functions be denoted by $f(\theta)$, and its co-function (page 177) by $c 0-f(\theta)$, then

$$
f\left(n 90^{\circ} \pm \theta\right)=\left\{\begin{array}{l} 
\pm f(\theta) \text { if } n \text { is zero or even, or } \\
\pm \operatorname{co}-f(\theta) \text { if } n \text { is odd. }
\end{array}\right.
$$

The sign on the right is to agree with that of $f\left(n 90^{\circ} \pm \theta\right)$ when $\theta$ is acute.

A comprehensive survey of these relations is afforded by the figures following. To construct the first figure, construct the

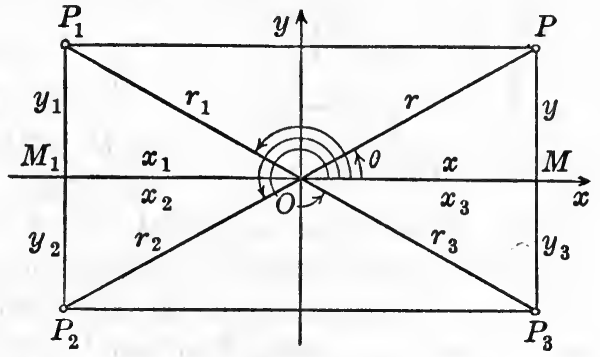

FIG. 112. angles $\theta, 180^{\circ}-\theta, 180^{\circ}+\theta$, and $360^{\circ}-\theta, O X$ being the initial line of each. On their terminal lines take, respectively, $P(x, y), P_{1}\left(x_{1}, y_{1}\right), P_{2}\left(x_{2}, y_{2}\right)$, and $P_{3}\left(x_{3}, y_{3}\right)$, so that

$$
r=r_{1}=r_{2}=r_{3} \text {. }
$$

Then $P_{1}, P_{2}$, and $P_{3}$ are symmetrical to $P$ with respect to the $y$-axis, the origin, and the $x$-axis respectively (why?). Hence the numerical values of $x, x_{1}, x_{2}$, and $x_{3}$ are equal, and so also are those of $y, y_{1}, y_{2}$, and $y_{3}$. Hence the numerical value of any function of one of the angles $180^{\circ}-\theta, 180^{\circ}+\theta$, or $360^{\circ}-\theta$ 
is equal to that of the same function of $\theta$. Whether the signs agree or not will depend on the angle and the function in question. For example:

Since $y_{2}=y_{3}=-y$, we have $\frac{y_{2}}{r_{2}}=\frac{y_{3}}{r_{3}}=-\frac{y}{r}$,

whence $\sin \left(180^{\circ}+\theta\right)=\sin \left(360^{\circ}-\theta\right)=-\sin \theta$.

And since $y_{2}=-y$, and $x_{2}=-x$, so that $y_{2} / x_{2}=y / x$, we have $\tan \left(180^{\circ}+\theta\right)=\tan \theta$.

To construct the second figure, lay off the angles $\theta, 90^{\circ}-\theta$, $90^{\circ}+\theta, 270^{\circ}-\theta$, and $270^{\circ}+\theta$, with $O X$ for the initial line.

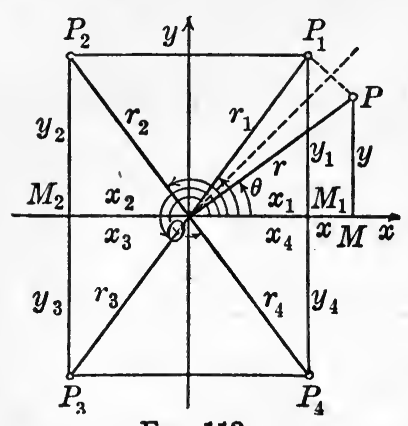

Fig. 113.

On their terminal lines take $P(x, y), \quad P_{1}\left(x_{1}, y_{1}\right), \quad P_{2}\left(x_{2}, y_{2}\right)$, $P_{3}\left(x_{3}, y_{3}\right)$ and $P_{4}\left(x_{4}, y_{4}\right)$, so that $r=r_{1}=r_{2}=r_{3}=r_{4}$.

Since $P_{1}$ is symmetrical to $P$ with respect to the bisector of the first quadrant (why?),

$$
x_{1}=y \text { and } y_{1}=x \text {. }
$$

$\dot{P}_{2}, P_{3}$, and $P_{4}$ are symmetrical to $P_{1}$ with respect to the $y$-axis, the origin, and the $x$-axis respectively. Hence the numerical values of $x_{1}, x_{2}, x_{3}$, and $x_{4}$ are equal to $y$, and those of $y_{1}, y_{2}, y_{3}$, and $y_{4}$ are equal to $x$. Inspection of the definitions on page 166 shows that if $x$ and $y$ are interchanged, each function is replaced by its co-function. Hence the numerical values of any function of one of the angles $90^{\circ} \pm \theta$ or $270^{\circ} \pm \theta$ is equal to that of the co-function of $\theta$.

For example, since $x_{2}=x_{3}=-x_{1}=-y$,

we have

$$
\frac{x_{2}}{r_{2}}=\frac{x_{3}}{r_{3}}=-\frac{y}{r}
$$

whence $\cos \left(90^{\circ}+\theta\right)=\cos \left(270^{\circ}-\theta\right)=-\sin \theta$.

In this survey we have taken $\theta$ acute, so that all the functions of $\theta$ are positive. Hence $f\left(n 90^{\circ} \pm \theta\right)$ will equal $\pm f(\theta)$, or $\pm \operatorname{co}-f(\theta)$, according as $f\left(n 90^{\circ} \pm \theta\right)$ is positive or negative, which can be determined readily in a given case. 
To illustrate the use of the rule, express $\cos \left(270^{\circ}-\theta\right)$ in terms of a function of $\theta$. Since $270^{\circ}=3 \cdot 90^{\circ}$, and 3 is odd, we will obtain the co-function, $\pm \sin \theta$. If $\theta$ is acute, $270^{\circ}-\theta$ is in the third quadrant, in which the cosine is negative (see graph). Hence $\cos \left(270^{\circ}-\theta\right)=-\sin \theta$.

Writing down a formula by the rule does not constitute a proof. The proof of (7), for example, which is identical with part of (6), is given above.

The graphical significance of any one of the formulas is easily found. Consider, for example, formulas (1) and (4), and construct the graph of $\sin \theta$. Let $O A=\theta$, and construct $O B=180^{\circ}-\theta, O C=180^{\circ}+\theta, O D=360^{\circ}-\theta$. Then by (1), the ordinates at $A$ and $B$ are equal, and by (4), the ordinates at $C$ and $D$ are equal numerically to that at $A$, but are opposite in sign.

If $\theta$ increases from $0^{\circ}$ to $90^{\circ}$, it follows that the graph consists of four congruent parts.

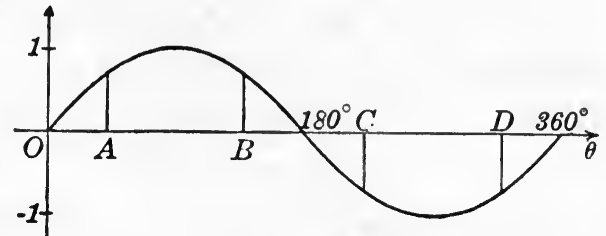

Fig. 114.

Periodicity of $\tan \theta$. From (5) it follows at once that the period of $\tan \theta$ is $180^{\circ}$, or $\pi$.

The following formulas are stated for purposes of reference:

$$
\begin{aligned}
& \sin \left(180^{\circ}+\theta\right)=-\sin \theta . \\
& \cos \left(180^{\circ}+\theta\right)=-\cos \theta . \\
& \tan \left(180^{\circ}+\theta\right)=\tan \theta . \\
& \sin \left(360^{\circ}-\theta\right)=-\sin \theta . \\
& \cos \left(360^{\circ}-\theta\right)=\cos \theta . \\
& \tan \left(360^{\circ}-\theta\right)=-\tan \theta .
\end{aligned}
$$

If $360^{\circ}$ are subtracted from the angle on the left in (11), (12), and (13) we obtain

$$
\left.\begin{array}{r}
\sin (-\theta)=-\sin \theta \\
\cos (-\theta)=\cos \theta \\
\tan (-\theta)=-\tan \theta
\end{array}\right\}
$$

the relations proving the symmetry of the graphs. 
69. Application to the Use of Tables. A positive angle less than $360^{\circ}$ may be put in the form

$$
180^{\circ}-\theta, \quad 180^{\circ}+\theta, \text { or } 360^{\circ}-\theta \text {, }
$$

where $\theta$ is acute, according as the angle lies in the second, third or fourth quadrant. Hence the functions of such an angle may be expressed in terms of the functions of an acute angle $\theta$, and the latter may be found from the tables.

For example:

$$
\begin{aligned}
& \sin 150^{\circ}=\sin \left(180^{\circ}-30^{\circ}\right)=\sin 30^{\circ}=\frac{1}{2} . \\
& \cos 213^{\circ}=\cos \left(180^{\circ}+33^{\circ}\right)=-\cos 33^{\circ}=-0.8387 . \\
& \tan 312^{\circ}=\tan \left(360^{\circ}-48^{\circ}\right)=-\tan 48^{\circ}=-1.1106 .
\end{aligned}
$$

The functions of a positive angle greater than $360^{\circ}$ may be found by using the periodicity of the function, and then proceeding as above; for example,

$$
\begin{aligned}
\sin 985^{\circ} & =\sin \left(2 \cdot 360^{\circ}+265^{\circ}\right)=\sin 265^{\circ}=\sin \left(180^{\circ}+85^{\circ}\right) \\
& =-\sin 85^{\circ}=-0.9962 .
\end{aligned}
$$

The functions of a negative angle are found by first using either the relations

$$
\sin (-\theta)=-\sin \theta, \cos (-\theta)=\cos \theta, \tan (-\theta)=-\tan \theta,
$$
etc., or the periodicity. Thus

$$
\begin{aligned}
\tan \left(-225^{\circ}\right) & =-\tan 225^{\circ}=-\tan \left(180^{\circ}+45^{\circ}\right) \\
& =-\tan 45^{\circ}=-1,
\end{aligned}
$$

or $\tan \left(-225^{\circ}\right)=\tan \left(360^{\circ}-225^{\circ}\right)=\tan 135^{\circ}$

$$
=\tan \left(180^{\circ}-45^{\circ}\right)=-\tan 45^{\circ}=-1 .
$$

The functions of a positive acute angle less than $360^{\circ}$ may also be found by putting the angle in one of the forms

$$
90^{\circ}+\theta, \quad 270^{\circ}-\theta, \text {, or } 270^{\circ}+\theta \text {, }
$$

where $\theta$ is acute. The form (1) is somewhat less confusing because the application of the rule in Section 68 does not involve a change to the co-function.

The examples following show how to find all the angles for which a given function has a given value. The solutions 
depend upon the fact that the numerical value of a function is the same for the three angles (1) as for the acute angle $\theta$.

Example 1. Find all values of $\theta$ for which $\sin \theta=0.4332$.

First find the positive values less than $360^{\circ}$. From the tables, one value is $\theta=25^{\circ} .67$. The graph of $\sin \theta$ shows that a line parallel to the $\theta$-axis and 0.4332 unit above it cuts the graph in two points, one in the first quadrant, corresponding to the value $\theta=25^{\circ} .67$ found from the tables, and one in the second quadrant. Formula (1) Section 68, shows that the second value is $\theta=180^{\circ}-25^{\circ} .67=154^{\circ} .33$.

All values of $\theta$ for which $\sin \theta=0.4332$ are given by

$$
\theta=25^{\circ} .67+n 360^{\circ} \text { and } \theta=154^{\circ} .33+n 360^{\circ} .
$$

Example 2. Find $\theta$ if $\cos \theta=-0.5$.

If we neglect the negative sign and seek an acute angle whose cosine is 0.5 , we know it to be $60^{\circ}$ (table, page 161). The graph of $\cos \theta$ shows that a line parallel to the $\theta$-axis and 0.5 unit below it cuts the graph in two points, one in the second and one in the third quadrant. Then formulas (2) page 192, and (9), page 195, show that the values of $\theta$ corresponding to these points are $\theta=180^{\circ}-60^{\circ}=120^{\circ}$, and $\theta=180^{\circ}+60^{\circ}=240^{\circ}$. All values of $\theta$ are then, by the periodicity of $\cos \theta$,

$$
\theta=120^{\circ}+n 360^{\circ} \text { and } \theta=240^{\circ}+n 360^{\circ} .
$$

If we notice that one of the angles of the second set is, for $n=-1$, $-120^{\circ}$, which may also be obtained from $\theta=120^{\circ}$ by the relation $\cos (-\theta)=\cos \theta$, all the angles may be expressed by the single equation

$$
\theta= \pm 120^{\circ}+n 360^{\circ} \text {. }
$$

Example 3. Find all the values of $\theta$ if $\tan \theta=-\sqrt{3}$.

Neglecting the negative sign, we recognize that one value of $\theta$ is $60^{\circ}$ (table, page 161). A line below the $x$-axis cuts the graph of $\tan \theta$ in two points, one in the second quadrant, and one in the fourth. Hence, by formulas (3) page 192, and (13), page 195, the required values of $\theta$ less than $360^{\circ}$ are $180^{\circ}-60^{\circ}=120^{\circ}$ and $360^{\circ}-60^{\circ}=300^{\circ}$. Then all the required values are

$$
\theta=120^{\circ}+n 360^{\circ} \text { and } \theta=300^{\circ}+n 360^{\circ} .
$$

If we use the fact that the period of $\tan \theta$ is $180^{\circ}$, noticing that $300^{\circ}=120^{\circ}+180^{\circ}$, all these angles may be expressed by the single equation

$$
\theta=120^{\circ}+n 180^{\circ} \text {. }
$$

\section{EXERCISES}

1. Prove formulas (8) - (13), Section 68 , for $\theta$ acute.

2. State, prove, and give the graphical significance of the formula for 

(a) $\cos \left(90^{\circ}+\theta\right)$.
(b) $\cot (-\theta)$.
(c) $\sec \left(180^{\circ}-\theta\right)$.
(d) $\sin \left(270^{\circ}-\theta\right)$.
(e) $\cot \left(360^{\circ}-\theta\right)$.
(g) $\cot \left(180^{\circ}+\theta\right)$.
(h) $\sec (-\theta)$.
(f) $\csc \left(90^{\circ}-\theta\right)$.
(i) $\cos \left(270^{\circ}+\theta\right)$.

3. Prove the six formulas for the functions of the angle:
(a) $-\theta$.
(b) $180^{\circ}-\theta$.
(c) $180^{\circ}+\theta$.
(d) $360^{\circ}-\theta$.
(e) $90^{\circ}-\theta$.
(f) $90^{\circ}+\theta$.
(g) $270^{\circ}-\theta$.
(h) $270^{\circ}+\theta$.

4. By means of the proper formulas and the table on page 161 find

(a) $\sin \left(-60^{\circ}\right), \cos 300^{\circ}, \cos 240^{\circ}, \tan 315^{\circ}$.

(b) $\sin 330^{\circ}, \cos \left(-120^{\circ}\right)$, cot $210^{\circ}$, sec $150^{\circ}$.

(c) $\cos \left(-135^{\circ}\right), \tan 120^{\circ}, \tan \left(-150^{\circ}\right), \sin 225^{\circ}$.

5. By means of the formulas for the sine, cosine, and tangent, and the reciprocal relations, derive the formulas for the cotangent, secant and cosecant of
(a) $-\theta$;
(b) $90^{\circ}-\theta$;
(c) $180^{\circ}-\theta$;
(d) $180^{\circ}+\theta$;
(e) $360^{\circ}-\theta$

6. Find all the functions of $142^{\circ} .30$; of $118^{\circ} .17$.

7. Find all positive values of $\theta$ less than $360^{\circ}$ for which

(a) $\cos \theta=\frac{1}{2} ; \tan \theta=1 ; \sin \theta=-\frac{1}{2}$.

(b) $\sec \theta=-2 ; \cot \theta=-1 ; \csc \theta=\sqrt{2}$.

(c) $\sin \theta=0.3486 ; \cos \theta=-0.8111 ; \tan \theta=0.4770 ; \tan \theta=-1.4770$.

8. Find all the values of $\theta$ for which

(a) $\cos \theta=-\sqrt{3} / 2 ; \cos \theta=0.4761 ; \tan \theta=2$.

(b) $\sin \theta=-0.6460 ; \cos \theta=0.5348 ; \tan \theta=2.638$.

(c) $\sec \theta=1.4788$; $\csc \theta=4.865$; $\cot \theta=33.96$.

9. Express the following as functions of $\theta$.

(a) $\sin \left(\theta-90^{\circ}\right)$.

Solution. Since $\theta-90^{\circ}=-\left(90^{\circ}-\theta\right)$, we have $\sin \left(\theta-90^{\circ}\right)=\sin \left[-\left(90^{\circ}-\theta\right)\right]=-\sin \left(90^{\circ}-\theta\right)=-\cos \theta$.
(b) $\cos \left(\theta-180^{\circ}\right)$.
(c) $\tan \left(\theta-270^{\circ}\right)$.
(d) $\sin \left(\theta-180^{\circ}\right)$.

10. Construct a table of values of $\theta$ and $\sin \theta$ for values of $\theta$ taken every $10^{\circ}$ from $0^{\circ}$ to $360^{\circ}$, expressing $\theta$ in radians decimally instead of in terms of $\pi$ (see Tables, page 32), and giving the values of $\theta$ and $\sin \theta$ to two decimal places. Construct the graph as accurately as possible from this table of values.

11. As in the preceding exercise, construct a table of values and draw the graph of
(a) $\cos \theta$,
(b) $\tan \theta$,
(c) $\cot \theta$,
(d) sec. $\theta$,
(e) $\csc \theta$. 
70. Inclination and Slope of a Straight Line. The function $\tan \theta$ enables us to express precisely the relation between the slope and the direction of a line (see page 52).

The upper, right-hand angle (the northeasterly angle) which a line makes with the $x$-axis is called the inclination of the line.

Parallel lines have the same inclination, and conversely (why?).

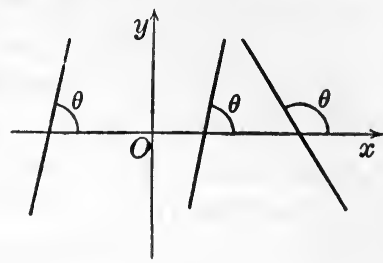

Fig. 115.

Consider any line through the origin. Its slope $m$ is the ratio of the difference of the ordinates of any two of its points to the difference of the abscissas. For these points take the origin and any point $P(x, y)$ on the line, above the $x$-axis. We then have, in either figure,

$$
m=\frac{y-0}{x-0}=\frac{y}{x}=\tan \theta .
$$

Hence the slope of the line through the origin is the tangent of the inclination. As parallel lines have the same slope and the same inclination, the result holds for any line. We thus have

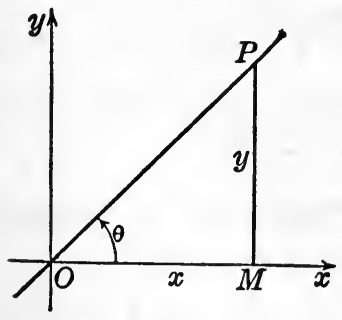

$\boldsymbol{A}$

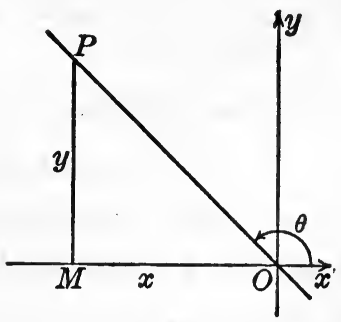

$B$

Fig. 116.

Theorem 1. The slope of a line is the tangent of the inclination, i.e., $m=\tan \theta$.

In most of the applications of the linear equation $y=m x+b$, the slope is of greater importance than the inclination, because the slope is the rate of change of $y$ with respect to $x$. 
The chief importance of the theorem above is in geometry. For from the slope the direction of the line may be found, provided the same unit has been used on the $x$ and $y$-axes.

Let a line start in a horizontal position and rotate about one of its points through half a revolution. Its inclination $\theta$ will increase from $0^{\circ}$ to $180^{\circ}$. The variation of $\tan \theta$ (see graph, page 176) shows that the slope of the line, $m=\tan \theta$, will increase from zero through all positive values, and become infinite as the inclination approaches $90^{\circ}$, when the line becomes vertical. As the inclination increases from $90^{\circ}$ to $180^{\circ}$, the slope is negative, and its numerical value decreases to zero.

EXAmple 1. Find the angle formed by the lines $l$ and $l^{\prime}$ whose equations are $3 x-4 y+12=0$ and $2 x+y-8=0$.

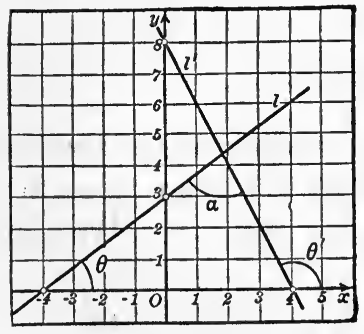

Fig. 117.

Solving the equations of the lines for $y$ we get

$$
y=\frac{3}{4} x+3, \text { and } y=-2 x+8
$$

and hence the slopes of the lines are $m=\tan \theta=\frac{3}{4}=.75$ and $m^{\prime}=\tan \theta^{\prime}=-2$.

From the tables and the rule in Section 69 , the inclinations of the lines are

$$
\theta=36^{\circ} .87 ; \theta^{\prime}=180^{\circ}-63^{\circ} .43=116^{\circ} .57 \text {. }
$$

If $a$ is the angle between the lines, then $a=\theta^{\prime}-\theta$ (why?). Hence

$$
a=116^{\circ} .57-36^{\circ} .87=79^{\circ} .70 .
$$

If two lines are perpendicular, then, using the notation in Example 1, $a=90^{\circ}$, so that $\theta^{\prime}=90^{\circ}+\theta$. Hence

$$
m^{\prime}=\tan \theta^{\prime}=\tan \left(90^{\circ}+\theta\right)=-\cot \theta=-\frac{1}{\tan \theta}=-\frac{1}{m} .
$$

That is, the slope of one is the negative reciprocal of the slope of the other. The converse may be proved by retracing the steps in the reverse order. Hence we have

Theorem 2. Two lines are perpendicular if and only. if the slope of one is the negative reciprocal of the slope of the other. 


\section{EXERCISES}

1. Find the equation of the line passing through the point $(2,3)$ whose inclination is (a) $30^{\circ}$; (b) $135^{\circ}$.

2. Construct each of the lines below, and find its inclination.
(a) $2 x-2 y+7=0$.
(b) $4 x-3 y-12=0$.
(c) $4 x+3 y-12=0$.

3. Find the angle between each of the pairs of lines:

(a) $\sqrt{3} x-3 y+12=0$ and $\sqrt{3} x-y-3=0$.

(b) $5 x-2 y+10=0$ and $x+3 y-6=0$.

4. Find the angles of the triangles formed by the lines below. How can the results be checked?

(a) $x+y-4=0, x-\sqrt{3} y-3=0, \sqrt{3} x-y-3=0$.

(b) $2 x-y-6=0, x+2 y-3=0,2 x+3 y+9=0$.

Definition. The angle between a line and a curve at a point of intersection is the angle between the line and the tangent to the curve at that point. The angle between two curves at a point of intersection is the angle between the tangents to the curves at that point.

5. At what point on the graph of $y=x-x^{2}$ will the tangent line make an angle of $30^{\circ}$ with the $x$-axis? Find the angles at which the curve cuts the $x$-axis.

6. Find the angle made with the $x$-axis by the tangent to the graph of $y=x^{3}-x$ at the point for which $x=1$. At the point of inflection (Definition, page 139).

7. Find the angle between the graphs of $x^{2}$ and $x^{3}$ at the point $(1,1)$; between the graphs of $x^{3}$ and $x^{4}$ at the same point.

8. Find the points on the graph of $y=x^{3}-x^{2}$ at which the inclination of the tangent line is $45^{\circ}$.

9. Find the angles at which the straight line $y=4 x$ cuts the graph of $y=x^{3}$ at the three points of intersection.

10. Find the equation of the line through the origin which is perpendicular to the tangent to the parobola $y=x^{2}$ at the point $(1,1)$.

71. Law of Sines. A theorem of geometry states that if two sides of a triangle are unequal, the angles opposite them are unequal in the same order, and conversely. The exact relation between the sides and angles is given by the

Law of sines. The sides of a triangle are proportional to the sines of the opposite angles. Symbolically,

$$
\frac{a}{\sin A}=\frac{b}{\sin B}=\frac{c}{\sin C} \text {. }
$$


Let $A B C$ be any triangle, and draw the altitude $C D=h$. Then in the right triangles $A D C$ and $B D C$ we have

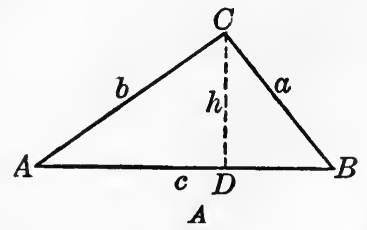

FIG. 118.

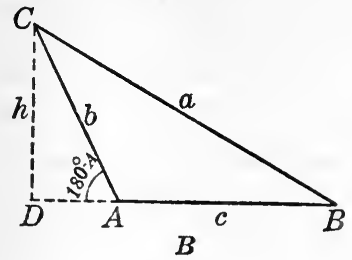

whence

$$
h=b \sin A \text { and } h=a \sin B,
$$

and therefore

$$
b \sin A=a \sin B \text {, }
$$

$$
\frac{a}{\sin A}=\frac{b}{\sin B} \text {. }
$$

If $A$ is obtuse (Fig. $118 B$ ), then $\angle C A D=180^{\circ}-A$, so that as before.

$$
h=b \sin \left(180^{\circ}-A\right)=b \sin A \quad((1), \text { page } 192)
$$

In like manner, by drawing the altitude from $A$ or $B$, we get

$$
\frac{b}{\sin B}=\frac{c}{\sin C} \text { or } \frac{a}{\sin A}=\frac{c}{\sin C} \text {. }
$$

72. Law of Cosines. This law replaces the two theorems in geometry concerning the square of a side of a triangle opposite an acute angle and the square of a side of a triangle opposite an obtuse angle.

Law of cosines. The square of a side of a triangle equals the sum of the squares of the other two sides less twice their product times the cosine of the included angle. Symbolically

$$
\begin{aligned}
& a^{2}=b^{2}+c^{2}-2 b c \cos A . \\
& b^{2}=c^{2}+a^{2}-2 c a \cos B . \\
& c^{2}=a^{2}+b^{2}-2 a b \cos C .
\end{aligned}
$$

If $A$ is acute (Fig. 118A), we have

$$
\begin{aligned}
a^{2} & =h^{2}+D B^{2} \\
& =h^{2}+(c-A D)^{2} \quad(\text { since } D B=c-A D) \\
& =h^{2}+c^{2}-2 c \cdot A D+A D^{2} \\
& =b^{2}+c^{2}-2 b c \cos A \\
& \left(\text { since } h^{2}+A D^{2}=b^{2}, \text { and } A D=b \cos A\right)
\end{aligned}
$$


If $A$ is obtuse (Fig. 118B), we have

$$
\begin{aligned}
a^{2} & =h^{2}+D B^{2} \\
& =h^{2}+(c+A D)^{2} \quad(\text { since } D B=c+A D) \\
& =h^{2}+c^{2}+2 c \cdot A D+A D^{2} \\
& =b^{2}+c^{2}-2 b c \cos A
\end{aligned}
$$

(since $h^{2}+A D^{2}=b^{2}$ and $A D=b \cos \left(180^{\circ}-A\right)=-b \cos A$, (2), page 192.)

\section{EXERCISES}

1. Show that the law of sines reduces to the first two of formulas (1), page 181, if $C=90^{\circ}$. What does the third form of the law of cosines becorne?

2. Prove the first of equations (1), Section 71 , if $B$ and $C$ are both acute.

3. Prove the second of equations (1), Section 71 , if $A$ is acute and $C$ is obtuse.

4. Prove the second form of the law of cosines if $B$ is acute.

5. Prove the third form of the law of cosines if $C$ is obtuse.

6. Find the ratio of two sides of a triangle, $a / b$, if

(a) $A=40^{\circ}$ and $B=20^{\circ}$. Is one side double the other?

(b) $A=60^{\circ}$ and $B=20^{\circ}$. Is $\sin 3 \theta=3 \sin \theta$ ? Can this question be readily answered from the graph of $\sin \theta$ ?

7. If a particle is in equilibrium under three forces, $O A, O B, O C$, prove that $\frac{O A}{\sin B O C}=\frac{O B}{\sin A O C}=\frac{O C}{\sin A O B}$. (The resultant, $O D$, of $O A$ and $O B$ must be equal and opposite to $O C$, and hence the sides of the triangle $O A D$ will represent the forces numerically. Use law of sines.)

73. Solution of Oblique Triangles. An oblique triangle is one none of whose angles is a right angle. The constructions of plane geometry show that an oblique triangle may be constructed if three of its six parts (sides and angles) are given, provided that at least one of them is a side. The laws derived in the last two sections enable us to solve the triangle, that is to compute the three unknown parts from those given. It is necessary to distinguish four cases, according as there are given:

I. A side and two angles.

II. Two sides and the angle opposite one of them.

III. Two sides and the included angle.

IV. Three sides. 
The first two cases may be solved by the law of sines, and the last two by the law of cosines. The last two may also be solved by using first the law of cosines and ihen the law of sines.

CASE I. Given a side and two angles, the third angle is found from $A+B+C=180^{\circ}$, and the other two sides by the law of sines.

Example 1. Two forts by the sea, $A$ and $B$, are 12 miles apart. At $A$ the angle between $B$ and a target $C$ anchored off the coast is $37^{\circ} .24$, and at $B$ the angle between $A$ and $C$ is $42^{\circ} .87$. Find the distance from

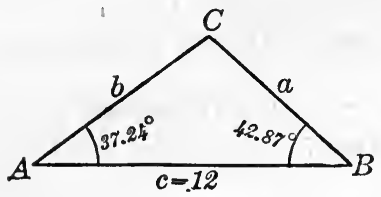

Frg. 119. each fort to the target.

We are given a triangle determined by $A=37^{\circ} .24, B=42^{\circ} .87, c=12$.

We have $C=180^{\circ}-(A+B)=99^{\circ} .69$.

From the law of sines,

$$
a=\frac{c \sin A}{\sin C}, \text { and } b=\frac{c \sin B}{\sin C},
$$

whence $a=\frac{12 \times 0.6052}{0.9857}=7.367$ miles, and $b=\frac{12 \times 0.6803}{0.9857}=8.281$ miles.

Check. Find $c$ from $a, b, C$. By the law of cosines,

$$
\begin{aligned}
c^{2} & =a^{2}+b^{2}-2 a b \cos C \\
& =7.37^{2}+8.28^{2}-2 \times 7.37 \times 8.28 \times(-0.168) \\
& =54.4+68.6+20.5 \\
& =143.5,
\end{aligned}
$$

and therefore $c=11.98$.

This value agrees reasonably well with the given value of $c$, especially when we take into account the fact that the table of squares does not enable us to use all four figures in $a$ and $b$.

CASE II. Given two sides and an angle opposite one of them, the angle opposite the other given side is found by the law of sines, then the third angle is found from the relation $A+B+C=180^{\circ}$, and finally the third side is found by the law of sines.

Example 2. Two straight roads diverge at an angle of $35^{\circ}$. An automobile starts from the fork in the road and runs along one road until a cross road is reached, the odometer showing the distance to be 2.1 miles. It turns into the cross road and after running 1.4 miles comes to the other road. How far is the automobile from the starting point, and ai what angles does the cross road meet the other two? 
If $A$ denotes the fork in the road, $B$ the point 2.1 miles from $A$, and $C$ the third point reached, a triangle is determined by the parts

$$
A=35^{\circ}, c=2.1, a=1.4 .
$$

To construct the triangle, construct angle $A=35^{\circ}$ with one side horizontal, and on the other side lay off $A B=c=2.1$. Then with $B$ as a center, describe the circle with radius $a=1.4$, which, in this problem, cuts the other line in two points $C$ and $C^{\prime}$. Either $B C$ or $B C^{\prime}$ may represent the cross road, so that the problem has two solutions, $A C$ and $A C^{\prime}$. These may be found by solving the triangles $A B C$ and $A B C^{\prime}$.

To find $C$ we have, by the law of

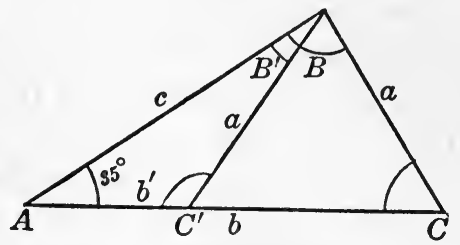

Fig. 120. sines,

$$
\sin C=\frac{c \sin A}{a}=\frac{2.1 \times 0.5736}{1.4}=0.8610,
$$

whence $C=59^{\circ} .43$ or $120^{\circ} .57$ (compare Example 1, page 197). Since $B C C^{\prime \prime}$ is isosceles, $C^{\prime}=\angle A C^{\prime} B^{\prime}=180^{\circ}-C$, and hence $C=59^{\circ} .43$ and $C^{\prime}=120^{\circ} .57$.

Whence

$$
\begin{aligned}
B & =180^{\circ}-(A+C) \text { and } B^{\prime}=180^{\circ}-\left(A^{\prime}+C^{\prime}\right) \\
& =85^{\circ} .57 \\
& =24^{\circ} .43
\end{aligned}
$$

To find $b=A C$ and $b^{\prime}=A C^{\prime}$, we have, by the law of sines,

$$
\begin{aligned}
& \text { in } \triangle A B C \quad b=\frac{a \sin B}{\sin A} \quad \text { and } \quad \text { in } \triangle A B^{\prime} C^{\prime} \quad b^{\prime}=\frac{a \sin B^{\prime}}{\sin A} \\
& =\frac{1.4 \times 0.9969}{0.5736} \\
& =\frac{1.4 \times 0.4136}{0.5736} \\
& =1.009
\end{aligned}
$$

Check. By the law of cosines we have:

In triangle $A B C \quad c^{2}=a^{2}+b^{2}-2 a b \cos C$

$$
\begin{aligned}
& =1.4^{2}+2.433^{2}-2 \times 1.4 \times 2.433 \times 0.5085 \\
& =1.960+5.91-3.464 \\
& =4.406, \text { and hence } c=2.099 .
\end{aligned}
$$

In triangle $A B^{\prime} C^{\prime} c^{\prime 2}=a^{2}+b^{2}-2 a b^{\prime} \cos C^{\prime}$

$$
\begin{aligned}
& =1.4^{2}+1.009^{2}-1 \times 1.4 \times 1.009 \times(-0.5085) \\
& =1.960+1.018+1.437 \\
& =4.415, \text { and hence } c=2.101 .
\end{aligned}
$$

Both of these values agree very well with the given value of $c$. In order to illustrate the method of solution, the computations have been 
carried out with all the accuracy permitted by the tables. Do the given data warrant us in saying that the automobile is either 2.433 miles or 1.009 miles from the fork in the road?

If two sides of a triangle and the angle opposite one of them are given, there can be Two soLutions only if the angle is acute and the side opposite it is less than the other given side. Under these conditions, there will be two solutions unless in solving for the second angle ( $C$, in Example 2), it is found that its sine is equal to or greater than unity ( $\sin C \equiv 1$ ). In the first case there is but one solution, a right triangle; and in the second case there is no solution, since the sine of an angle cannot exceed unity. A complete statement of the possible solutions is given in Exercise 3 below.

CAsE III. Given two sides and the included angle, the third side is found by the law of cosines, and then the remaining angles by either the law of cosines or the law of sines.

Example 3. To find the distance across a bay, $A B$, a point $C$ is taken whose distances from $A$ and $B$ can be measured. It is found that $A C$ is 70 yards, $B C$ is 120 yards, and $\angle A C B$ is $50^{\circ}$. Find $A B$ and also the angles at $A$ and $B$.

A triangle is determined whose given parts are $a=120, b=70, C=50^{\circ}$. By the law of cosines

$$
\begin{aligned}
c^{2} & =a^{2}+b^{2}-2 a b \cos C \\
& =120^{\circ}+70^{2}-2 \times 120 \times 70 \times 0.6428 \\
& =14,400+4900-10,800 \\
& =8500, \quad \text { whence } \quad c=92.2 .
\end{aligned}
$$

To find $A$ and $B$, we use the law of cosines: Substituting the values of

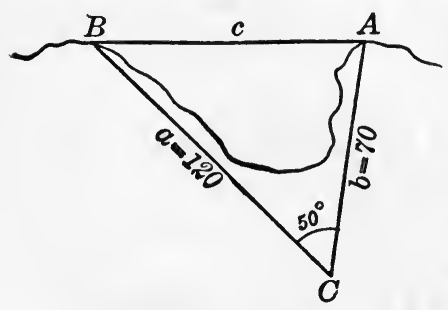

FIg. 121. the sides in $a^{2}=b^{2}+c^{2}-2 b c \cos A$, we get

$$
\begin{aligned}
& 14,400= \\
& 4900+8500-2 \times 70 \times 92.2 \times \cos A, \\
& \text { whence } \cos A=-0.0774 \\
& \text { and hence }
\end{aligned}
$$

$$
A=180^{\circ}-85^{\circ} .55=94^{\circ} .45 .
$$

Substituting the values of the sides in $b^{2}=c^{2}+a^{2}-2 c a \cos B$, we get

$$
4900=8500+14,400-2 \times 92.2 \times 120 \times \cos B,
$$

whence $\cos B=0.8136$

and hence

$$
B=35^{\circ} .55 \text {. }
$$


Check. The angles having been found without using the fact that the sum of the three angles is two right angles, we have

$$
A+B+C=94^{\circ} .45+35^{\circ} .55+50^{\circ}=180^{\circ} \text {. }
$$

Having found $A$ as above, $B$ might have been obtained from $A+B+C=180^{\circ}$. But then it would have been necessary to use the law of sines as a check, and little would have been gained.

CASE IV. Given the three sides, the three angles are found by the law of cosines, or one may be found by the law of cosines and then the others by the law of sines.

Example 4. The lengths of a triangular lot are found by pacing to be 80 feet, 50 feet, and 100 feet. Find the angles at the corners and the area.

A triangle is determined by $a=80, b=50, c=100$. Substituting these values in the three forms of the law of cosines we get

$\begin{array}{rlrl}80^{2} & =50^{2}+100^{2}-2 \times 50 \times 100 \cos A, \\ 50^{2} & =100^{2}+80^{2}-2 \times 100 \times 80 \cos B, \\ 100^{2} & =80^{2}+50^{2}-2 \times 80 \times 50 \cos C, \\ \cos A & =0.6100, \quad \text { whence } \quad A=52^{\circ} .41 \\ \text { and hence } \quad & B=29^{\circ} .69 \\ \cos B & =0.8687, & C=97^{\circ} .90 . \\ \cos C & =-0.1375, & & \end{array}$

Check: $A+B+C=180^{\circ} .00$.

To find the area, draw the altitude from $C$ and denote it by $h$. Then in the right triangle $A C D, h=b \sin A=50 \times 0.7924=39.62$. Then the area is $\frac{1}{2} c h=50 \times 39.62=1981$ square feet.

The lengths of the lines in the examples preceding and in the following exercises have been chosen with but one or two significant figures, in order to simplify the

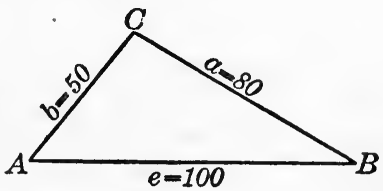

Frg. 122. computations, and the angles to four significant figures for the purpose of obtaining drill in interpolation. In the next chapter, a labor saving device to assist in the computations will be considered, and further exercises given. 


\section{EXERCISES}

1. In Example 4, find $B$ and $C$ by the law of sines. What point in the solution might be overlooked, which one is more likely to notice in using the law of cosines?

2. Is a triangle always determined if values are given for a side and two angles? For two sides and the included angle? For the three sides?

3. If two sides, say $a$ and $b$, and the angle opposite one of them, say $A$, are given, show that

(a) If $A$ is obtuse, there is one solution if $a>b$, and there is no solution if $a \equiv b$.

(b) If $A$ is right, there is one solution if $a>b$, and there is no solution if $a \equiv b$.

(c) If $A$ is acute, there is one solution if $a \geqq b$, there are two solutions if $b \sin A<a<b$, there is one solution if $a=b \sin A$, and there is no solution if $a<b \sin A$.

4. Determine the number of solutions, by Exercise 3, if,
(a) $a=5$,
$b=10$,
$A=30^{\circ}$.
(b) $a=5$,
$b=7$,
$A=30^{\circ}$.
(c) $b=10$,
$c=15$,
$C=20^{\circ}$.
(d) $a=7$,
$c=6$,
$C=40^{\circ}$.

5. Solve the following triangles, and check the solution:

(a) $B=62^{\circ} .74, C=87^{\circ} .20, a=10$.

(b) $b=7, \quad c=5, \quad B=30^{\circ} .17$.

(c) $b=7, \quad c=9, \quad B=30^{\circ} .17$.

(d) $a=3, \quad b=7, \quad C=120^{\circ}$.

(e) $a=3, \quad b=7, \quad c=5$.

(f) $a=10, \quad b=8, \quad B=28^{\circ} .16$.

6. A tunnel is to be built through a hill from a point $A$ to another point $B$. A point $C$, at the same level as $A$ and $B$, is 1000 feet from $A$ and 800 feet from $B$ and $\angle A C B=42^{\circ}$. Find the length of the tunnel.

7. A man starts from camp and walks N.E. for 5 miles, and then $22^{\circ} .5$ east of south until he reaches a point from which the camp is visible in a direction due west. How far has he walked, and how far is he from camp?

8. Two batteries of artillery. $A$ and $B$, are four miles apart. An enemies' battery is located at a point $C$ such that $\angle B A C=64^{\circ} .22$ and $\angle A B C=43^{\circ} .17$. Find the range for each battery.

9. A field is bounded by two roads intersecting at right angles, and by two other straight lines. Find its area if the lengths of the sides, beginning at the corner at the crossing of the roads and measured in order around the field, are $30,60,70$ and 40 rods. 
10. A schooner sails $10^{\circ}$ west of north at the rate of 7 knots an hour across the Gulf Stream at a place where it flows N.E. with a velocity of 4 knots an hour. Find the actual velocity of the schooner in direction and magnitude.

11. The current in a river flows at the rate of 2 miles an hour, and a man rows at the rate of 4 miles an hour. If he desires to cross the river at an angle of $70^{\circ}$ with the bank, in the direction of the current, in what direction should he row?

12. Solve the preceding exercise if the man desires to cross at the same angle with the bank but in the upstream direction.

13. Resolve a velocity of $\mathbf{5 0}$ feet per second into two components inclined at $10^{\circ}$ and $40^{\circ}$ respectively to the direction of the given velocity.

14. A boy in an automobile moving 40 feet per second throws a ball in a horizontal direction inclined at $50^{\circ}$ to the road with a speed of 30 feet per second. At what angle to the road will the ball move?

15. A road runs up a hill at an angle of $20^{\circ}$. At a point on it, 500 feet from the foot, the angle of depression of a horseman on the road leading to the hill is $5^{\circ}$. How far is he from the foot of the hill?

16. To find the width of a river, two points $A$ and $B$ are taken on one bank 100 feet apart. If $C$ is a point on the opposite bank such that $\angle B A C=62^{\circ} .34$ and $\angle A B C=49^{\circ} .82$, find the width of the river.

17. At a certain point the angle of elevation of the top of a mountain is $45^{\circ}$, and at a point 1000 feet nearer the mountain and at the same level as the first, the angle of elevation is $54^{\circ} .13$. How high is the mountain?

18. A ship steams due east at the rate of 25 miles an hour, and the smoke from its funnel is blown in a direction $20^{\circ}$ south of west. The wind gauge shows an apparent velocity of 35 miles an hour for the wind. Find the actual velocity of the wind in direction and magnitude.

74. Inverse Trigonometric Functions. To find the inverse of $\sin x$ (pages 40 and 114) we set $y=\sin x$, and interchange $x$ and $y$, obtaining $x=\sin y$. The solution of this equation for $y$ in terms of $x$ requires the introduction of a new function which is called the angle whose sine is $x$, and which is denoted by $\operatorname{arc} \sin x$. Hence,

$$
\text { if } x=\sin y, \text { then } y=\operatorname{arc} \sin x .
$$

A table of sines may be regarded as a table of angles whose sines are given (see " finding $\theta$ if $\sin \theta$ is given" page 178). Thus Example 2, page 179, might have been stated: find $\theta=\operatorname{arc} \sin 0.4332$, the result being

$$
\operatorname{arc} \sin 0.4332=25^{\circ} .76+n 360^{\circ} \text { or } 154^{\circ} .33+n 360^{\circ} \text {. }
$$


This example illustrates the fact that for a given value of $x$ arc $\sin x$ has not only one but a boundless number of values. This is apparent from the graph of arc sin $x$, which is sym-

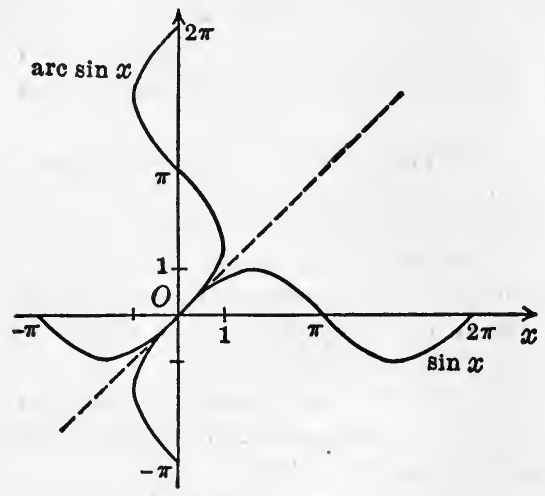

Fig. 123. metrical to that of $\sin x$ with respect to the bisector of the first and third quadrants (see page 114). The graph also shows that the function is defined only for values of $x$ from -1 to +1 inclusively.

The inverse of $\cos x$ is denoted by arc $\cos x$ (read "the angle whose cosine is $x ")$; of $\tan x$, by arc $\tan x$, etc.

Definition. The principal value of any on? of the inverse trigonometric functions for a given value of $x$ is that one of the boundless number of values of the function which is smallest numerically. If two values of the function are equal numerically, but opposite in sign, the positive value is the principal value.

Unless the contrary is indicated, the symbols arc $\sin x$, arc $\cos x$, etc., will be used in this work to denote the principal values only.

Thus $\quad \operatorname{arc} \sin \frac{1}{2}=\pi / 6$,

and $\arcsin (-1)=-\pi / 2$.

The part of the graph which represents the principal values of $\operatorname{arc} \sin x$ is given in the figure.

Inverse trigonometric functions are of much importance, although

we shall use them but little in this course. - They are convenient in stating a general result, as in this

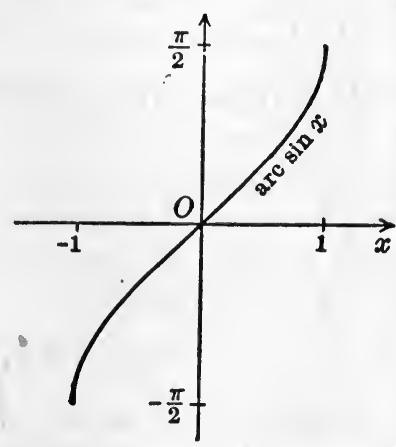

Fra. 124. 
Example. What angle is subtended at the center of a circle of radius $r$ by a chord $c$ units long?

Choose the center of the circle as the origin of a system of coördinates, and let the $x$-axis be perpendicular to the chord. Then the $x$-axis bisects the 'chord and the angle formed by the radii drawn to its extremities. From the figure

whence $\quad \theta / 2=\arcsin (c / 2 r)$

and hence $\quad \theta=2 \arcsin (c / 2 r)$.

If $c=3$ and $r=5$, we would have $\theta=2$ $\operatorname{arc} \sin 0.3=2 \times 17^{\circ} .46=34^{\circ} .92$.

The notation $\sin ^{-1} x, \cos ^{-1} x$, etc., is used sometimes for arc $\sin x$, arc $\cos x$, etc.

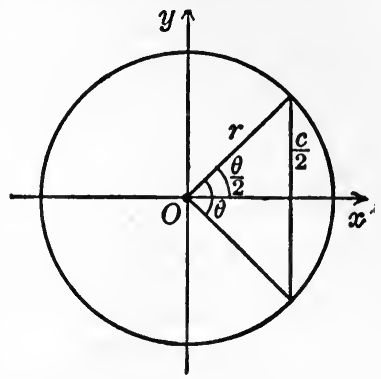

FIG. 125.

\section{EXERCISES}

1. Construct the graph of arc $\sin x$, and indicate on it the part which represents the principal values of the function for all possible values of $x$. State as many properties of the function as can be readily obtained from the graph.

2. Proceed as in Exercise 1 for the function (a) $\operatorname{arc} \cos x$. (b) $\arctan x$. (c) $\operatorname{arccot} c . \quad$ (d) $\operatorname{arcsec} x$. (e) $\operatorname{arccsc} x$.

3. Find all the values of $\operatorname{arc} \sin 0 ; \operatorname{arc} \cos \frac{1}{2} ; \arctan (-1)$.

4. Find the value (noting the convention with regard to principal values) of

(a) $\arccos \left(-\frac{1}{2}\right) ; \arctan 2, \arcsin (-0.3215)$.

(b) $\arcsin \frac{4}{3} ; \operatorname{arcsec} 2 ; 3 \times \arcsin \left(-\sqrt{\frac{3}{2}}\right)$.

5. A rope $l$ feet long is stretched from the top of a building to the ground, the lower end being $d$ feet from the building. Find a general expression for the angle which the rope makes with the ground. What is the angle if $l$ is 50 feet and $d$ is 17 feet?

6. A mountain $h$ feet high is viewed from a point $d$ miles away (horizontally). What angle does the line from the point of observation to the peak make with the ground?

7. What is the value of $\sin (\operatorname{arc} \sin a)$ ? Of $\operatorname{arc} \sin (\sin a)$ ?

8. Recall the method of solution of Exercises 9 and 10, page 170. Find the value of (a) $\sin \left(\arccos \frac{5}{5}\right)$. (b) $\tan \left(\arcsin \frac{5}{18}\right)$. (c) $\cos (\arctan -2)$. (d) $\tan \left[\arccos \left(-\frac{1}{3}\right)\right]$.

9. If the maximum distance from a point on an arc of a circle to the chord of the arc is $d$, show that the central angle subtended by the arc is $2 \times \operatorname{arc} \cos (r-d) / r$, where $r$ is the radius of the circle. 


\section{MISCELLANEOUS EXERCISES}

1. If a creek is 20 feet wide, and if from a point 4 feet above the water's edge on one side the angle of elevation of the top of the bank on the other side, directly opposite, is $7^{\circ} .27$, how high is the bank?

2. An object weighing 60 pounds is supported on a smooth plane whose inclination is $60^{\circ}$ by a man who pushes against it horizontally. Find the force exerted by the man and the pressure on the plane.

3. A board is just strong enough to bear an object weighing 100 pounds at its middle point when the board is supported horizontally at its ends. How heavy an object will it bear at its middle point if it is supported at its ends in a position inclined at $30^{\circ}$, the object being held in position by a rope parallel to the plane?

4. Solve Exercise 3 if the object is held in position by a man pushing against it horizontally.

5. The hatchway into the hold of a ship is $\mathbf{1 6}$ feet wide. To raise an object weighing 200 pounds from the hold, two men on opposite sides of the hatchway pull on a rope which passes through a smooth ring fastened to the object. Find the tension of the rope when the ring is 6 feet below the deck. What force does each man exert? Hint: See the note following.

Note. The tension of a cord or rope which passes over a smooth peg, or over a pulley, or through a smooth ring is assumed to be the same on both sides of the peg, pulley, or ring. It is usually not the same if the rope is tied to the ring.

6. A cord is tied at a point $A$, passes through a smooth ring $B$ weighing 3 pounds, over a pulley $C$ at the same height as $A$, and to the end is tied a weight of 2 pounds. Find $\angle B A C$ when the ring and weight are in equilibrium.

7. A man drags a trunk across a room by pulling on the handle in a direction at $35^{\circ}$ to the horizontal. If the trunk weighs 150 pounds, and if the friction is $\mathbf{0 . 1}$ of the pressure on the floor, find the force he exerts at the instant the trunk is about to move. Note that the pressure on the floor will be less than the weight of the trunk, because a part of the man's effort tends to lift the trunk.

8. Find the force exerted in pushing the trunk in Exercise 7, when the trunk is just on the point of moving, if the man pushes down on the trunk in a direction inclined at $25^{\circ}$ to the horizontal.

9. To find the width of a river, a point $A$ is taken on one bank directly opposite a tree on the other bank, and a point $B$ is taken 100 feet from $A$ in the line of the tree and $A$. At $B$ the angle of elevation of the top of the tree is $32^{\circ} .19$, and at $A$ it is $41^{\circ} .33$. Find the width of the river.

10. If a body on a rough inclined plane is just on the point of moving down the plane, show that the coefficient of friction is equal to the tangent of the angle of inclination of the plane. 
11. Rain drops are falling straight down with a velocity of 20 feet per second. At what angle would they appear to fall to a man walking at the rate of 3 miles per hour? To a man in an automobile moving at the rate of 20 miles per hour? To a man in an express train moving at the rate of 60 miles an hour?

12. Prove that the area of any quadrilateral is equal to one-half the product of the diagonals and the sine of the angle between them.

13. The wind is blowing down a lake 5 miles wide at a rate which would blow a row boat a mile an hour. A man who rows at the rate of 3 miles an hour desires to go straight across. In what direction should he row, and how long will it take him to cross?

14. Two girls hold a traveling bag weighing 40 pounds. One pulls on the handle at an angle of $10^{\circ}$ to the vertical, the other at $15^{\circ}$. What force does each exert?

15. If the distance an ivory ball rolls down a smooth plane inclined at $\frac{t}{s} \mid \frac{1,2,2,3,4}{1.4,5.5,12,5,22.2} \quad 5^{\circ}$ in various times are as given in the table, celeration due to gravity. Analysis of the problem: Find $s$ as a function of $t$ from the table, from this find the velocity at any time, then the acceleration with which the ball rolls down the plane, and finally the acceleration due to gravity.

16. Charles' law states that the rate of increase of the volume of a gas under constant pressure per degree (Centigrade) rise of temperature is $\Sigma_{2} \frac{1}{3}$ of the volume at $0^{\circ}, v_{0}$. Hence the volume $v$ at any temperature $\theta$ is $v=\frac{v_{0}}{273} \theta+v_{0}$. Boyle's law states that the product of the pressure and volume of a gas at constant temperature is constant, $p v=k$. If a quantity of oxygen occupies 200 cubic centimeters at a temperature of $17^{\circ}$ Centigrade under a pressure of $\mathbf{7 4 2}$ millimeters (barometric height), find its volume at $0^{\circ}$ temperature under a pressure of one atmosphere at sea level, or 760 millimeters. Illustrate graphically, plotting the graphs of both laws on the same axes, taking $v$ on the vertical axis for each law. First find the volume $v_{0}$ under a pressure of 742 millimeters; the graph of Charles' law being determined by the given data and the fact that $v=0$ when $\theta=-273^{\circ}$, the " absolute zero." The value of $k$ in Boyle's law is determined by the point whose coördinates are 200 and this value of $v_{0}$.

17. Construct a square 8 inches on a side. Its area is 64 square inches. Cut the square into two rectangles of widths 3 inches and 5 inches. Cut the first rectangle along a diagonal into two triangles. Mark points on the 8 inch sides of the second 5 inches from opposite vertices, and cut along the line joining them. The four parts of the original square may be arranged in the form of a rectangle whose dimensions are 5 inches and 13 inches, and whose area is 65 square inches. Explain the fallacy. 


\section{CHAPTER V}

\section{EXPONENTIAL AND LOGARITHMIC FUNCTIONS}

75. Introduction. The formula for the $n$th term, $l$, of a geometrical progression whose first term is $a$, and whose ratio is $r$, is

$$
l=a r^{n-1} .
$$

If we are given $a=3, r=2, l=96$, then $n$ may be found. Substituting the given values, we have

whence

$$
96=3 \times 2^{n-1},
$$

and hence

$$
2^{n-1}=32=2^{5} \text {, }
$$

from which

$$
\begin{aligned}
n-1 & =5, \\
n & =6 .
\end{aligned}
$$

Equation (2) differs from most of the equations arising in algebra, in that the unknown $n$ appears in an exponent. The possibility of finding $n$ by elementary methods is due to the fact that 32 is recognized as a power of 2 .

If we set $a=3$ and $r=2$ in (1), we get

$$
l=3 \times 2^{n-1} \text {. }
$$

Regarding $n$ as variable, this equation defines $l$ as a function of $n$. For integral values of $n, l$ is the $n$th term of a geometrical progression, but in studying $l$ as a function of $n$ we need not restrict $n$ to integral values. Thus if $n=\frac{3}{2}$,

$$
l=3 \times 2^{3-1}=3 \times 2^{\frac{1}{2}}=3 \times 1.414=4.242 .
$$

Thus the value of $l$ may be found readily by algebraic methods for many fractional values of $n$. But if $n$ is irrational, for example, if $n=\sqrt{2}$ or $n=\pi$, we cannot compute $l$ by algebraic methods. Hence $l$ is a transcendental function of $n$ (definition, page 39). 
Definition. An exponential function is one in which the variable occurs in an exponent. For example, equation (3) defines $l$ as an exponential function of $n$.

The simplest form of an exponential function is $b^{x}$, where $b$ is a constant, other than unity, called the base. It has the property, in common with more general forms of exponential functions, that if $x$ increases in arithmetical progression, the function increases in geometrical progression. For example, values of $x$ in the table are in arithmetical progression, since $\frac{x}{2 x} \mid \frac{1, \quad 2, \quad 3, \quad 4,}{2, \quad 4, \quad 8, \quad 16,}$ successive values of $\Delta x$ are equal, while the values of $2^{x}$ are in geometrical progression, since the ratio of any value to

the preceding is 2 .

Exponential functions are important because in many fields, especially in physics, one variable changes (increases or decreases) in geometrical progression as another, frequently time, changes in arithmetical progression. The former is always an exponential function of the latter. Examples of such changes are Newton's law of cooling of a heated body; the variation of atmospheric pressure with the altitude; the law of chemical reaction; and the law of organic growth. In these examples, and generally in nature, the change proceeds continuously. The amount of a sum of money at compound interest is an exponential function of the time, but the changes come at stated intervals instead of continuously. However, the business of some very large firms, which make many loans a day, approximate a condition of compounding interest every instant. And this property of an exponential function is often called, following Lord Kelvin, the compound interest law. Another name which has been suggested is the snow-ball law. The inverse of the function $b^{x}$ is called the logarithmic function, and a table of values of this function is spoken of as $a$ table of logarithms. Such a table is an invaluable labor-saving device. It enables us to replace the laborious processes of multiplication and division by the simpler operations of addition and subtraction. It also makes it possible to reduce the very tedious operations of computing powers and roots to 
multiplication and division, and through these to addition and subtraction.

For this labor-saving device we are indebted to John Napier (1550-1617), who arrived at it by considering a function which increased in arithmetical progression as the variable decreased in geometrical progression. Following Napier, this tool was put in a more serviceable form by Henry Briggs (1561-1631).

We shall use a table of logarithms for simplifying many computations, especially in connection with the solution of triangles.

The introduction of the exponential and logarithmic functions completes the list of functions to be studied in this course (see Classification, page 38).

76. Graph of the Exponential Function $b^{x}, b>1$. In the following table of values of the exponential function $2^{x}$, the value of the function for $x=0$ is obtained by means of the definition $b^{0}=1$, and the value for any negative value of $x$ is obtained by means of the definition $b^{-n}=\frac{1}{b^{n}}$. By the first definition, we have $2^{0}=1$, and by the second $2^{-2}=\frac{1}{2^{2}}=\frac{1}{4}$.

$\frac{x}{2^{x}} \mid \frac{-2,-1,0,1,2,3}{\frac{1}{4}, \quad \frac{1}{2}, 1,2,4,8}$

The table of values is readily computed and the graph plotted as usual. The process is so simple that it is

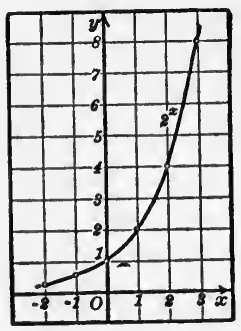

FIG. 126. hardly worth while to discuss the graph in advance. The following properties of the graph are apparent.

1. The intercept on the $y$-axis is 1 .

2. The graph lies entirely above the $x$-axis.

3 . The $x$-axis is an asymptote.

4. Any line parallel to the $y$-axis cuts the graph once and once only.

5. The graph rises to the right more and more rapidly.

The form of the graph of the exponential function $b^{x}, b>1$, is very much like that of $2^{x}$. As indicated in Fig. 127, the graph always cuts the $y$-axis at $y=1$, lies entirely above the 
$x$-axis, which is an asymptote, and runs up to the right. If $b>2$, the curve rises more rapidly to the right of the $y$-axis than in the case of $2^{x}$, and the larger the value of $b$ the more rapid is the rise.

The values of $b$ of most importance in mathematics are 10 and a number denoted by " $e$ " whose value to four figures is $e=2.718$. It was proved in 1844 that there are numbers which are not the roots of any algebraic equation no matter how high the degree, and the first number definitely proved to be of this sort was $e$, in 1873 . In 1882 this same fact was proved about $\pi$, and by means of this it was proved that

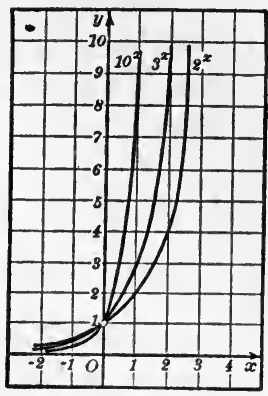

FIG. 127. it is impossible to "square the circle" with ruler and compass.

A table of values of $e^{x}$ is given in Huntington's Tables, page 30 .

77. Properties of the Exponential Function $b^{x}, b>1$. The properties of the function which correspond to the above properties of the graph are as follows:

1. For all exponential functions $b^{0}=1$.

2. The exponential function $b^{x}$ is positive for all real values of $x$.

3. As $x$ decreases indefinitely through negative values $b^{x}$ approaches 0 as a limit.

4. For each value of $x$ there is one and only one value of $b^{x}$.

5. The function $b^{x}$ increases as $x$ increases and the rate of change of $b^{x}$ also increases. Of two exponential functions the one with the larger base has the greater rate of change for $x>0$.

Other important properties of the function which are not so apparent from the graph are the following:

6 . If the values of $x$ be chosen in arithmetical progression, the corresponding values of the function are in geometrical progression.

Let the values of $x$ be

$$
a, a+d, a+2 d, a+3 d \ldots
$$




\section{ELEMENTARY FUNCTIONS}

Then the values of the function are

$$
b^{a}, b^{a+d}, b^{a+2 d}, b^{a+3 d}, \ldots
$$

which form a geometrical progression, for any one of these values may be obtained from the preceeding by multiplying by $b^{d}$.
7. $b^{m} \cdot b^{n}=b^{m+n}$.
8. $\frac{b^{m}}{b^{n}}=b^{m-n}$.
9. $\left(b^{m}\right)^{n}=b^{m n}$.
10. $\sqrt[n]{b^{m}}=b^{m / n}$.

Properties 7, 8, 9, proved in elementary algebra for integral values $m$ and $n$ of $x$, are true for all values of $x$. The definition 10 holds also for all values of $x$.

If $f(x)=b^{x}$, the last four properties may be written, in the reverse order:

$$
\begin{aligned}
& \text { 11. } f(m+n)=b^{m+n}=b^{m} b^{n} \text {. } \\
& \text { 12. } f(m-n)=b^{m-n}=b^{m} / b^{n} \text {. } \\
& \text { 13. } f(m n)=b^{m n}=\left(b^{m}\right)^{n} \text {. } \\
& \text { 14. } f\left(\frac{m}{n}\right)=b^{m / n}=\sqrt[n]{b^{m}} \text {. }
\end{aligned}
$$

In these forms, the analogy of these relations respectively to (1), (2), (3), and (4) on page 153 is apparent. Thus seemingly unrelated rules of elementary algebra are in reality analogous properties of the functions $b^{x}$ and $x^{n}$. 
78. Computation by means of an Exponential Function. The computations in the following examples are considerably

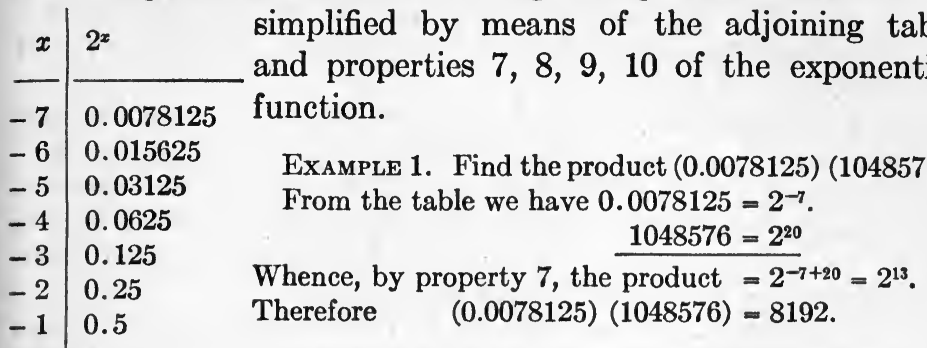

EXample 2. Find the quotient $32768 / 524288$.

From the table we have $32768=2^{15}$.

$\begin{array}{rlrl}524288 & =2^{19} . \\ \text { By property } 8, & \text { the quotient } & =2^{15-19}=2^{-4} \\ \text { Therefore } & 32768 / 524288 & =0.0625 .\end{array}$

EXample 3. Find $8^{6}$.

Since $8=2^{3}$, we have $8^{6}=\left(2^{3}\right)^{6}$.

By property $9, \quad=2^{18}$.

Therefore $\quad 8^{6}=262144$.

9512

$10 \quad 1024$

112048

Example 4. Find $\sqrt[5]{1048576}$.

124096

$13 \quad 8192$

Since $1048576=2^{20}, \sqrt[5]{1048576}=\sqrt[5]{2^{20}}$.

$14 \quad 16384$

By property 10

$=2^{20 / 5}=2^{4}$.

$15 \quad 32768$

Therefore

$\sqrt[5]{1048576}=16$.

These examples are sufficient to show that an extensive table would shorten very appreciably the labor of finding a product, quotient, power, or root. In practice it is found more convenient to use a table of values of the inverse function.

\section{EXERCISES}

1. Plot the graphs of $2^{x}, e^{x}$, and $3^{x}$ on the same set of axes. (See page 30 of the Tables for values of $e^{x}$.) Calculate for each function the value of $\Delta y / \Delta x$ for the intervals -2 to $-1,-1$ to 0,0 to $+1,+1$ to +2 , and determine which has the greatest value of $\Delta y / \Delta x$ and which the least for each interval.

2. Plot the graph of $2^{-x}$ from a table of values. In what two ways is the graph related to that of $2^{x}$ ? 
3. How does $3^{-x}$ change as $x$ increases in arithmetic progression?

4. Plot the graphs of the following functions on the same axes $2^{x},\left(\frac{3}{2}\right)^{x},(1)^{x},\left(\frac{2}{3}\right)^{x},\left(\frac{1}{2}\right)^{x}$. What two relations exist between the graphs of the second and the fourth function? How does the function $b^{x}$ vary if $b>1$ ? if $b=1$ ? if $b<1$ ?

5. Plot the graphs of $e^{x}, e^{-x}, \frac{1}{2}\left(e^{x}+e^{-x}\right)$ and $\frac{1}{2}\left(e^{x}-e^{-x}\right)$ on the same axes for the range $x=-2$ to $x=+2$ at intervals of 0.5 . (See Tables, p. 30.)

The functions $\frac{1}{2}\left(e^{x}+e^{-x}\right)$ and $\frac{1}{2}\left(e^{x}-e^{-x}\right)$, have somewhat the same relation to the equilateral hyperbola that the trigonometric functions have to the circle. They are called respectively the hyperbolic cosine and hyperbolic sine and are symbolized by $\cosh x$ and $\sinh x$.

6. If $x^{\prime}, y^{\prime}$ and $x^{\prime \prime}, y^{\prime \prime}$ are two sets of values satisfying the equation $y=b^{x}$, show that the value of the function corresponding to the arithmetic mean of $x^{\prime}$ and $x^{\prime \prime}$ is the geometric mean of $y^{\prime}$ and $y^{\prime \prime}$.

7. Find the value of $2^{x}$ for $x=1.50$, using Exercise 6 and the values for $x=1$ and $x=2$. Then find the value of $2^{x}$ for $x=1.25$; for $x=1.75$. Arrange the results in tabular form.

8. Referring to the table in Exercise 7, between what two values of $x$ does $x=\sqrt{2}$ lie? The value of $2^{1.41}$ is an approximate value of $2^{\sqrt{2}}$. Find it by applying Exercise 6 several times, using the table in Exercise 7, and choosing the successive values of $x^{\prime}$ and $x^{\prime \prime}$ so that their arithmetic mean approaches 1.41 .

9. By means of the table in Section 78 find the value of each of the following:

(a) (524288) (0.015125).

(b) $4096 / 0.0078125$.

(c) $16^{4}$.

(d) $\sqrt{16384}$.

(e) $(\sqrt{(32768)(0.0625) / 512})^{8}$.

(f) $\sqrt{(4)^{2}}$.

10. A glass marble falls from a height of 4 feet and rebounds one-half the distance fallen, falls again and rebounds one-half the preceding distance fallen, and so on. Express the distance fallen each time as a function of the number of times it has fallen and draw the graph. How far does it fall the eighth time?

11. If the planets are numbered in the order of their distance from the sun the distance from the sun to the $n$th planet is approximately $4+3(2)^{n-2}$, the distance of the earth being represented by 10 . Compare the results of substituting in this formula with the following table.

$\begin{array}{lcccccc}\text { Planet } & \text { Mercury } & \text { Venus } & \text { Earth } & \text { Mars } & \text { Asteroids } & \text { Jupiter } \\ \text { True distance } & 3.9 & 7.3 & 10 & 15.2 & 27.4 & 52 \\ & \text { Saturn } & \text { Uranus } & \text { Neptune } & & \\ & 95.4 & 192 & 300 & & \end{array}$

If there is a planet external to Neptune, at what approximate distance may we expect it to be found? 
12. Show for the exponential function $e^{x}$ that the average rate of change is $\frac{\Delta y}{\Delta x}=e^{x}\left(\frac{e \Delta x-1}{\Delta x}\right)$. Find the value of the second factor if $\Delta x=0.1,0.01$.

Assuming that the limit of the second part is 1 as $\Delta x$ approaches 0 , find $m$ for the exponential $e^{x}$ and determine the angle that the graph makes with the $y$-axis.

13. Plot the graph of $y=k e^{m x}$, first letting $k=1$ and choosing $m$ successively equal to $-2,-1,0,+\frac{1}{2},+1,+2$, and then letting $m=1$ and choosing $k$ successively equal to $-1,+\frac{1}{2},+2$. How do changes in $m$ and $k$ affect the graph?

14. The pull, $P$, needed to check a weight, $W$, being lowered by means of a rope wrapped around an iron drun, is given by the equation $P=W e^{-m \theta}$, where the coefficient of friction is $m=0.3$, and $\theta$, the angle of contact of the rope with the drum, is measured in radians. If $W=500$ pounds plot the graph of $P$ as a function of $\theta$. What is the value of $P$ if the rope is wrapped 4 times around the drum?

79. The Logarithmic Function, the Inverse of the Exponential Function. To find the inverse of $2^{x}$, let $y=2^{x}$ and interchange $x$ and $y$, obtaining $x=2^{y}$. The solution of this equation for $y$ in terms of $x$ is a new function called the logarithm of $x$ to the base 2 , and it is denoted by $\log _{2} x$.

Thus $\quad \mathrm{x}=2^{y}$ and $y=\log _{2} x$

are forms of the same equation, in the one case solved for $x$ and in the other for $y$. In general, we have the

Definition. If $b^{m}=n$, then $m$ is said to be the logarithm of $n$ to the base $b$.

Hence the logarithm of a number to the base $b$ is the exponent of the power to which $b$ must be raised to equal the given number. It is frequently convenient to change from the exponential to the logarithmic form of this relation, and vice-versa.

For example, from $3^{5}=243$, we have $\log _{3} 243=5$.

Again, to find $\log _{5} 625$, we let $\log _{5} 625=m$, whence $5^{m}=625$. Since $5^{4}=625$, we have $m=4$ and hence $\log _{5} 625=4$.

80. Graph of the Logarithmic Function. The graph of $y=\log _{2} x$ may be readily obtained from that of $2^{x}$ by the rule in Section 39, page 113, and from it certain properties of the graph are obvious. 
1. The intercept on the $x$-axis is $x=1$.

2. The graph lies entirely to the right of the $y$-axis.

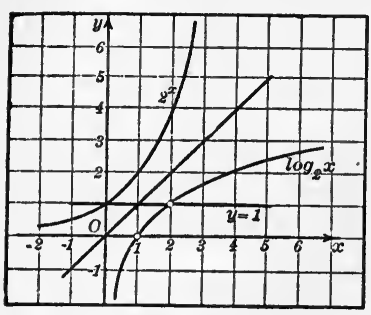

Fig. 128.

3 . The $y$-axis is an asymptote.

4. The graph is above the $x$-axis to the right of $x=1$ and below the $x$-axis to the left of $x=1$.

5. The graph rises to the right but at a decreasing rate.

6. A line parallel to the $x$-axis with an intercept on the $y$-axis at $y=1$ cuts the graph at a point whose abscissa is $x=2$.

As indicated in Fig. 129, which shows the graph of $\log _{b} x$ for $b=2,3,10$, the graph of any logarithmic function,

$$
y=\log _{b} x, b>1,
$$

cuts the $x$-axis at $x=1$, lies entirely to the right of the $y$-axis, which is an asymptote, lies above the $x$-axis to the right of the line $x=1$, and below to the left of $x=1$, rises at a decreasing rate as it moves to the right, and is cut by the line $y=1$ at a point $P$ whose abscissa is $b$.

81. Properties of the Logarithmic Function, $\log _{b} x, b>1$.

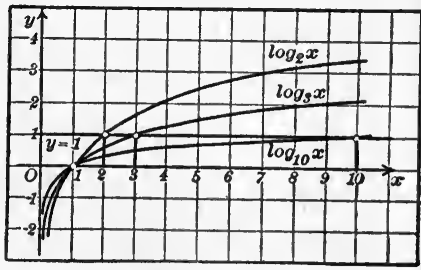

Fia. 129. Corresponding to the properties of the graph are the following properties of the function:

1. For any base, $\log _{b} 1=0$. For $b^{0}=1$.

2. $\log _{b} x$ is a real number for positive values of $x$ only.

3. As $x$ approaches $0, \log _{b} x$ approaches $-\infty$, that is, $\log _{b} 0=-\infty, b>1$.

4. The logarithm of a positive number greater than unity is positive. The logarithm of a positive number less than unity is negative.

5. The function $\log _{b} x$ increases and the rate of change of $\log _{b} x$ decreases as $x$ increases, and of two logarithmic func- 
tions, the one with the larger base has a smaller rate of change for $x>1$.

6. The logarithm of the base itself is unity. For since $b^{1}=b$, we have $\log _{b} b=1$.

Other properties of the function are easily deduced from the corresponding properties of the exponential function as follows:

7. Theorem. The logarithm of the product of two numbers is equal to the sum of the logarithms of the numbers.

Let

$$
p=b^{m}, \quad \text { whence } \quad \log _{b} p=m,
$$

and

$$
q=b^{n}, \quad \text { whence } \quad \log _{b} q=n \text {. }
$$

Then $\quad p q=b^{m} b^{n}=b^{m+n}$.

Therefore

$$
\begin{aligned}
\log _{b} p q & =m+n \\
& =\log _{b} p+\log _{b} q .
\end{aligned}
$$

8. Theorem. The logarithm of the quotient of two numbers is equal to the logarithm of the dividend minus the logarithm of the divisor.

Let $p=b^{m}$ and $q=b^{n}$, whence $\log _{b} p=m$ and $\log _{b} q=n$. Then $p / q=b^{m} / b^{n}=b^{m-n}$.

Therefore

$$
\log _{b} p / q=m-n \quad \text { why? }
$$

$$
=\log _{b} p-\log _{b} q \text {. }
$$

9. Theorem. The logarithm of the nth power of a number equals $n$ times the logarithm of the number.

Let

$$
p=b^{m}, \quad \text { whence } \quad \log _{b} p=m .
$$

Then

$$
p^{n}=\left(b^{m}\right)^{n}=b^{m n} \text {. }
$$

Hence

$$
\log _{b} p^{n}=m n \quad \text { why? }
$$

$$
=n \log _{b} p \text {. }
$$

10. Theorem. The logarithm of the nth root of a number equals the logarithm of the number divided by $n$.

Let

Then

$$
p=b^{m} \text {, whence } \log _{b} p=m \text {. }
$$

Hence

$$
\sqrt[n]{\bar{p}}=\sqrt[n]{b^{m}}=b^{m / n}
$$


If $f(x)=\log _{b} x$, properties $7,8,9,10$, may be written

$$
\begin{aligned}
f(p q) & =\log _{b} p q=\log _{b} p+\log _{b} q . \\
f(p / q) & =\log _{b} p / q=\log _{b} p-\log _{b} q . \\
f\left(p^{n}\right) & =\log _{b} p^{n}=n \log _{b} p . \\
f(\sqrt[n]{p}) & =\log _{b} \sqrt[n]{p}=\frac{1}{n} \log _{b} p .
\end{aligned}
$$

These properties are respectively analogous to those of $x^{n}$ given by (3), (4), (5), (6), page 153 .

If the first two be written in the reverse order, we have

and

$$
f(p)+f(q)=\log _{b} p+\log _{b} q=\log _{b} p q,
$$

which are analogous to (7) and (8), page 153.

By means of the theorems in 7 and 9 the solutions of Examples 1 and 3, page 219, may be written as follows:

EXAMPLE 1. $\log _{2} 0.0078125=-7$

$$
\begin{aligned}
& \log _{2} 1048576=20 \\
& \log _{2} \text { (product) }=13
\end{aligned}
$$

Therefore

$$
\text { product }=8192 \text { Therefore }
$$

Example 3. $\log _{2} 8=3$

$$
\begin{aligned}
\log _{2} 8^{6} & =6 \log _{2} 8 \\
& =18 \\
8^{6} & =262144
\end{aligned}
$$

\section{EXERCISES}

1. Express the following exponential equations in logarithmic form.

$$
2^{3}=8,3^{4}=81,44^{-2}=\frac{1}{16}, 10^{-3}=0.001,16 s=\sqrt{2} .
$$

2. What are the logarithms of $1,2,16,1024,0.125$ with respect to the base 2? Express the answers in exponential and logarithmic form.

3. Find $\log _{2} 2048, \log _{3} 81, \log _{e} 2.718, \log _{\frac{1}{3}} 9, \log _{\sqrt{2}} 16$. Express the answers in exponential form.

4. Find $\log _{b} b^{x}, b^{\log _{b} x}, \log _{2}\left(\log _{4} 16\right), \log _{e}(1 / e), b^{\log _{b} x-\log _{b} y}$.

5. (a) Change the equation $\log _{e} \theta=-k t+\log _{e} \theta_{0}$ to the form $\theta=\theta_{0} e^{-k t}$.

(b) Show that $-\frac{1}{2} \log _{b}\left(1 / x^{2}\right)=\log _{b} x$.

(c) Find $f(x)$ if $\log _{b} f(x)=\log _{b}(1-x)-2 \log _{b} x-\frac{1}{3} \log _{b}(1+x)$.

6. Plot the graphs of $\log _{2} x, \log _{e} x, \log _{3} x$, on the same set of axes. (See page 31 of the Tables for values of $\log _{e} x$.) Calculate the value of $\Delta y / \Delta x$ for each function for the interval 1 to 2 .

7. Plot the graph of $\log _{1 / 0} x$. In what two ways can this graph be obtained from that of $\log _{e} x$ ?

8. Illustrate each of the theorems in $7,8,9,10$, Section 81 , on the graph of $\log _{2} x$. 
9. Write the solutions of Examples 2 and 4, Section 78, in logarithmic forms.

10. Show by Theorems 7 and 8 that $\log _{b}\left(\frac{p q}{r}\right)=\log _{b} p+\log _{b} q-\log _{b} r$.

11. Using the table in Section 78 find (0.03125) $(262144) /(32768)$.

12. Prove the theorem, if the numbers $x, x^{\prime}, x^{\prime \prime}$, etc., are in geometric progression, their logarithms are in arithmetic progression.

13. Plot the graphs of $\log _{3} x, \log _{3} x^{2}, \log _{3} \sqrt{x}$ on the same axes. How can the graphs of the last two be obtained from that of the first function?

14. Plot the graph of $y=\log _{2}(x+k)$ for the values $k=0.1,-1$ on the same axes, and discuss the change effected in the graph by a change in $k$.

15. The equation for the economic law of diminishing utility is $y=k \log _{b}(x / c)$, where $x$ denotes income; $y$ happiness; $c$ the income sufficient for necessities; and $k$ is a constant depending on individuality. Plot a graph of the law, determine the graphical significance of $c$ and $k$, and discuss the law for $x \leqq c$.

82. Common Logarithms. Logarithms to the base $\mathbf{1 0}$ are called common logarithms. The first table of logarithms to the base 10 was published in 1617 by Henry Briggs. It contained logarithms of numbers from 1 to 1000 .

In writing logarithms to the base 10 , the base is usually omitted. Thus $y=\log _{10} x$ is written $y=\log x$, which means that $x=10^{y}$. That is

The common logarithm of a number is the power to which 10 must be raised to obtain the number.

A four-place table of common logarithms of numbers from 1 to 10 is given on pages 16 and 17 in Huntington's Tables. The graph of this table of logarithms is shown in the figure.

The method of interpolation is the same as in the tables already used.

For example, from the tables we find $\log 2.342$ $=0.3696$ which means that $10^{0.3690}=2.342$.

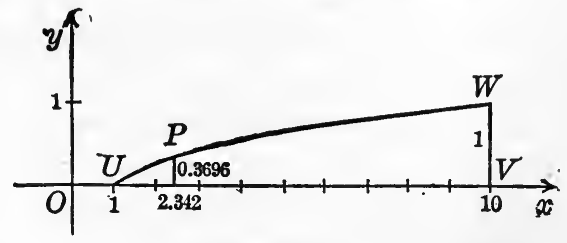

Fig. 130.

The number 2.342, and its logarithm, the number 0.3696 , are the coördinates of a point $P$ on the graph. 
Either from the graph or from the table we see that

The logarithm of any number between 1 and 10 is a positive decimal fraction less than 1.

We proceed to develop, by illustration, the rule for finding from the table the logarithm of any positive number $<1$ or $>10$.

If we translate the exponential equations $10^{-2}=0.01$, $10^{-1}=0.1,10^{0}=1,10^{1}=10,10^{2}=100,10^{3}=1000$, into the equivalent logarithmic equations $\log 0.01=-2, \log 0.1=-1$, $\log 1=0, \log 10=1, \log 100=2, \log 1000=3$, we see that

The logarithm of an integral power of 10 is an integer.

Since $23.42=(2.342)\left(10^{1}\right)$, we have, using the theorem in 7, Section $81, \log 23.42=\log (2.342)\left(10^{1}\right)=\log 2.342+$ $\log 10^{1}=0.3696+1=1.3696$.

Similarly

$234.2=2.342 \cdot 10^{2}$, whence $\log 234.2=\log 2.342+\log 10^{2}=2.3696$, $2342 .=2.342 \cdot 10^{3}$, whence $\log 2342=\log 2.342+\log 10^{3}=3.3696$, $0.2342=2.342 \cdot 10^{-1}$, whence $\log 0.2342=\log 2.342+\log 10^{-1}=$ $0.3696-1$,

$0.02342=2.342 \cdot 10^{-2}$, whence $\log 0.02342=\log 2.342+\log 10^{-2}$ $=0.3696-2$.

The values of $x$ considered above have the same sequence of digits $2,3,4,5$ and the corresponding values of $\log x$ have the same decimal part 0.3696 .

Multiplication of 2.342 by an integral power of 10 merely moved the decimal point without altering the sequence of digits, and corresponding to this operation, the logarithm 0.3696 was increased or diminished by an integer without altering the decimal part.

Any positive number $x<1$ or $>10$ can be expressed in the form $x=x^{\prime} \cdot 10^{n}$ where $x^{\prime}$ is a number in the range $1<x<10$, and $n$ is an integer, positive or negative.

Hence $\log x=\log x^{\prime}+\log 10^{n}=\log x^{\prime}+n$, where $\log x^{\prime}$ is a positive decimal fraction $<1$ which can be obtained from the table of logarithms and $n$ is an integer.

Hence, we see that 
1. The common logarithm of a positive number consists of two parts, a decimal fractional part and an integral part.

2. The decimal part, called the mantissa, depends only on the sequence of digits of the number and is obtained from the table of logarithms of numbers from 1 to 10.

3. The integral part, called the characteristic, is determined by the position of the decimal point in the number with reference to the position immediately following the first significant digit.

4. Moving the decimal point $n$ places to the right (or left) in the number is equivalent to increasing (or diminishing) the logarithm by $n$.

To find the logarithm of a number outside the range of the table we proceed as in Examples 1 and 2.

Example 1. Find $\log 678.4$

$$
\log 678.4=\log (6.784)\left(10^{2}\right)=\log 6.784+2 .
$$

From the table, $\log 6.784=0.8315$.

Therefore $\log 678.4=2.8315$.

EXAMPLE 2. Find log 0.0004867 .

The number 4.867 , lying between 1 and 10 , has the same sequence of digits as the given number.

From the table $\log 4.867=0.6872$.

The given number, 0.0004867 , is obtained from 4.867 by moving the decimal point 4 places to the left. Hence by property 4 above,

$$
\log 0.0004867=0.6872-4 \text {. }
$$

The solution of Example 1 emphasized the reasoning involved in finding the characteristic, while in Example 2 we have applied the rule in 4 . Except for purposes of explanation there is no need of writing anything but the result.

To find a number, given the logarithm of the number, proceed as in Examples 3 and 4.

Example 3. Find $x$ given $\log x=2.5137$.

Disregarding the characteristic 2 for the moment, we find from the table that the number whose logarithm is 0.5137 is 3.264 .

Therefore $x=(3.264)\left(10^{2}\right)=326.4$.

ExAMPLE 4. Find $x$ if $\log x=0.8174-3$. 

6.567 .

We find from the table that the number whose logarithm is 0.8174 is

Since the characteristic of the given logarithm is 3 less than that of 0.8174 , the required number $x$ is obtained from 6.567 by moving the decimal point 3 places to the left (see rule 4 above). Hence

$$
x=0.006567 \text {. }
$$

The graphical significance of the characteristic and mantissa is shown in the following figures, in which the scales on the axes are chosen differently for the sake of clearness.

The first figure represents the graph of $\log x$ for the range $10 \leqq x \leqq 100$, with the scale on the $x$-axis much reduced.
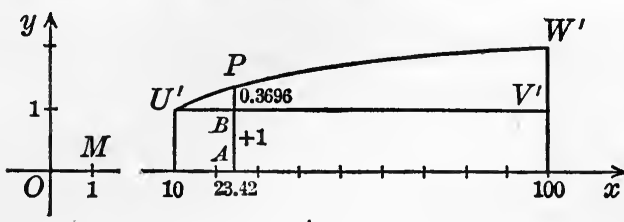

$A$

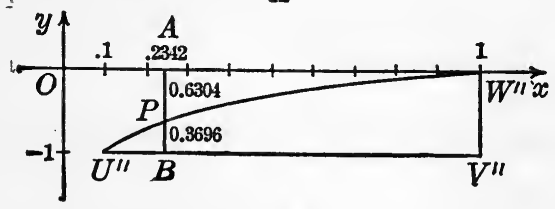

$B$

Fig. 131.

$A P$ is the logarithm of $O A, A B$ is the characteristic, and $B P$ the mantissa. The characteristic $A B$ of the logarithm of any number $O A$ in this range is +1 , while the mantissa $B P$ is the same as that of the number in the range $1 \leqq x \leqq 10$ with the same sequence of digits. The portion $U^{\prime} V^{\prime} W^{\prime}$ corresponds to the portion $U V W$ of the figure in the first part of the section.

The second figure represents the graph of $\log x$ for the range $0.1 \leqq x \leqq 1$, with the scale on the $x$-axis much enlarged. The portion $U^{\prime \prime} V^{\prime \prime} W^{\prime \prime}$ corresponds to the portions $U^{\prime} V^{\prime} W^{\prime}$ and $U V W$ of the other two figures.

We have from the table $\log 0.2342=-1+0.3696$, which is represented in the figure by $A P=A B+B P$.

If the subtraction indicated is performed the logarithm becomes $A P=-0.6304$.

But in this form of the logarithm the rule connecting the mantissa and sequence of digits in the number is not the same 
as that for numbers greater than unity. In order to retain this relation, the subtraction indicated is not performed, and the logarithm is written in the form $0.3696-1$, or $9.3696-10$, or with the new symbol $\overline{1} .3696$, thus retaining the positive mantissa 0.3696 and its connection with the sequence of digits $2,3,4,2$.

It is to the simplicity of the relations between the mantissa of the logarithm and the sequence of digits in the number, the characteristic of the logarithm and the position of the decimal point in the number, that the system of common logarithms owes its superiority to all other systems for purposes of computations. (See, for example, the rule for shifting the decimal point in the system of logarithms to the base $e$, given on page 31 of the Tables.)

83. Computation by means of Common Logarithms. The value of a product, quotient, power or root may be found by logarithms by means of the Theorems 7, 8, 9, 10 in Section 81 . The following examples will illustrate the methods.

To find a product by means of logarithms.

Example 1. Find the distance around the earth at the equator, if the equator is regarded as a circle of radius 3963 .

We have $C=2 \pi 3963$, whence by Theorem $7, \log C=\log 2+\log \pi$ $+\log 3963$. Before turning to the table of logarithms, write out a blank form as indicated on the left. When this is filled in it gives the computation on the right.
$\log 2=$
$\log 2=0.3010$
$\log \pi=$
$\log \pi=0.4971$
$\log 3963=$
$\log C=$
$\log 3963=3.5980$
$C=$
$\log C=4.3961$
$C=24890$
We have from the table, if $\log x=0.3961$
$x=2.489$

To find a quotient by means of logarithms.

EXample 2. Find $x=(0.003468) /(0.4783)$.

We have, by means of Theorem 8 , Section $81, \log x=\log 0.003468$ $-\log 0.4783$.

$$
\begin{aligned}
\log 0.003468=0.5400-3 & =1.5400-4 \\
\log 0.4783=0.6797-1 & =0.6797-1 \\
\log x & =0.8603-3 \\
x & =0.007250
\end{aligned}
$$


The mantissa of the numerator being less than the mantissa of the denominator, we add 1 to and subtract 1 from the characteristic of the numerator in order to avoid a negative mantissa in the difference.

If two measurements are made to four figures their product or quotient should not contain more than four significant figures (Section 26). The product or quotient may be obtained by a four-place table of logarithms to the correct number of significant figures. In general, the use of a table of logarithms in computing products and quotients conforms to the rule for the number of significant figures, if the number of significant figures in the measurements agrees with the number of decimal places in the logarithms.

To find a power by means of logarithms.

Example 3. Find $x=(0.008964)^{4}$.

By Theorem 9, Section 81, $\log x=4 \log (0.008964)$

$$
\begin{aligned}
\log 0.008964 & =0.9525-3 \\
\log x & =\frac{4}{3.8100-12}=0.8100-9 \\
x & =0.000000006457
\end{aligned}
$$

To find a root by means of logarithms.

Example 4. Find $x=(0.6785)^{\frac{4}{3}}$. By Theorem 10, $\log x=\frac{4}{3} \log 0.6785$. $\log \quad 0.6785=0.8315-1$

$\log (0.6785)^{4}=\frac{4}{3.3260-4} \quad$ The characteristic is altered in order to $3|2.3260-3|$ obtain an integral characteristic upon divid$\log x=0.7753-1$ ing by 3 , the index of the root.

$$
x=0.5962
$$

\section{EXERCISES}

1. Compute the values of the following expressions by means of logarithms. In each case, write a blank form for the computation before turning to the tables.
(a) (3.462) (23.14).
(b) $4795 / 2439$.
(c) $32.86^{11}$.
(d) $\sqrt[11]{543.2}$
(e) $\frac{(0.4783)(84.32)}{2.369}$
(f) $\sqrt[5]{32.96 / 12.78}$
(g) $\sqrt{41.26^{3} / 324.6}$

(h) 56.34/973.4. Suggestion: Write the characteristic of the numerator in the form $(3-2)$.

(i) $\sqrt[3]{.03764}$. Suggestion: The characteristic of the given number may be written in the form $(1-3)$. 
2. Find the distance to a tower 59.47 feet high from a point from which the angle of elevation of the top of the tower is $21^{\circ} .87$.

3. Find the area of an isosceles triangle whose base is 21.5 inches and whose vertex angle is $27^{\circ} .16$. Note that it is sufficient to find the logarithm of the altitude and not the altitude itself.

4. Apportion a claim for $\$ 35.55$ damages on freight which traveled on the M.C. 428 miles, N.Y.C. 175 miles, I.C. 235 miles, if each road pays in proportion to the number of miles traveled on it.

5. Find the cost of 1000 rivets weighing 4 ounces each, the output being at the rate of 78 per hour, if the cost of raw material is $\$ 4.50$ a ton, if labor is $\$ 0.375$ an hour, and the overhead expense is $\$ 0.123$ an hour.

6. What is the cost of $674 \frac{1}{4}$ pounds of chromic acid $95 \%$ pure, if acid $90 \%$ pure costs 7 cents a lb.?

7. A cellar 230 feet by 330 feet by $\mathbf{1 5 . 5}$ feet is excavated by means of a scoop with a capacity of $\frac{3}{4}$ cubic yards which makes 15 trips an hour. Estimate the cost of drawing away the dirt if a driver and team cost $\$ 3.75$ a day of 10 hours and draw two loads of $33 \frac{1}{3}$ cubic feet each time.

8. The average daily circulation of a newspaper for six semi-annual periods from 1912 to 1915, expressed in hundred thousands, was 210, $229,230,246,260$ and 298 . Find the percentage of increase in circulation for each period over the preceding.

9. Solve the following quadratic equations using logarithms wherever allowable.
(a) $2.13 x^{2}+4.76 x-3.82=0$.
(b) $32.6 x^{2}-87.5 x+43.7=0$.

10. Find the radius of a parallel of latitude through a point whose latitude is $45^{\circ} \mathrm{N}$., assuming the radius of the earth to be 3963 .

11. The area of a sphere in terms of the radius $r$ is given by the equation $S=4 \pi r^{2}$. Find the area of the surface of the earth considered as a sphere of radius 3963 miles.

12. The volume of a sphere in terms of the radius is $V=\frac{4}{3} \pi r^{3}$. Find the volume of the earth (see 11). If the average density of the earth is 5.2 , and the weight of a cubic foot of water is 62.4, find the weight of the earth in tons.

13. Find the area of the orbit of the earth assuming it to be a circle of radius $92,800,000$ miles. How many miles does the earth travel in a day if we assume a year to be equal to 365 days?

14. Kepler's third law of planetary motion states that for different planets the squares of the times of describing their orbits are proportional to the cubes of the mean distances from the sun. That is $\frac{t^{2}}{T^{2}}=\frac{d^{3}}{D^{3}}$. Given the earth's mean distance, $D=92,800,000$, its periodic time $T=365$, and the periodic time of Mars $t=686$, find the mean distance of Mars from the sun. 
15. If the distance of the earth from the sun changed from $93,000,000$ to $90,000,000$, assuming Kepler's third law, show that the year would be shortened by about $\mathbf{1 7 . 6}$ days. (Let $T=365.3$.) Since no appreciable diminution in the year has been noted from ancient times to the present, what inference can be drawn?

16. In railroad surveying a simple curve is defined as a circular arc joining two tangents.

The degree of curve, $D$, is the angle at the center of the circle subtended by a chord of 100 feet.

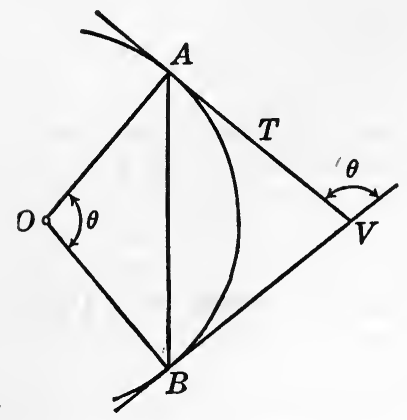

FIG. 132.

(a) Find the degree of curve if the radius is 800 feet.

(b) Find the radius if the degree of curve is $5^{\circ}$.

(c) Find the length of the $\operatorname{arc} A B$ of a $4^{\circ}$ curve if the central angle $\theta=28^{\circ}$.

(d) The exterior angle at $V$ is $52^{\circ}$, and the tangent distance, $T$, is to be 800 feet. Find the radius and the degree of curve. How far will the curve pass from the vertex, $V$ ?

(e) Find $T$ if it is required that the degree of curve shall not exceed $4^{\circ}$ for two tangents with an external angle of $40^{\circ}$.

17. If a body is constrained to move in a curved path, a force directed outward arises which is called the centrifugal force. For example, as a train rounds a curve the pressure of the flanges of the wheels against the outer rail of the curve is a centrifugal force. The magnitude of this force in pounds is $C=\frac{W v^{2}}{g r}$, where $W=$ weight in pounds, $v=$ velocity in feet per second, $r=$ radius of the circle in feet and $g$ is the acceleration due to gravity $=32.2$.

(a) An automobile weighing 2 tons rounds a curve whose radius is 600 feet at a velocity of 25 miles an hour. What is the magnitude of the force tending to make it skid?

(b) The weight of a mass situated at the equator of the earth is decreased by the centrifugal force due to the rotation of the earth on its axis. Find this decrease for a weight of 500 pounds. What would be the decrease in latitude $60^{\circ} \mathrm{N}$.?

(c) At what angle should a circular automobile speedway one mile in circumference be banked for a speed of 100 miles an hour in order that there shall be no tendency to skid?

18. During the war with Spain in 1898 , a chain letter was started for the benefit of the Red Cross. The person starting it wrote to 10 friends 
numbering each letter 1 ; each of these was to write to 10 friends numbering their letters 2 , etc. The chain was to be completed by letters numbered 100. If the chain had not been broken how many letters would have been numbered 100? Approximately how many letters in all would there have been? How does this compare with the population of the world?

84. Solution of Triangles. The formulas (1), page 181, and the law of sines involve multiplication and division only, and hence the computations in the solution of right triangles and in Cases I and II of oblique triangles may be effected expeditiously by means of logarithms.

The law of cosines involves addition and subtraction and hence is not well adapted to logarithms. In Chapter VIII formulas will be derived by means of which the computations in the solution of Cases III and IV of oblique triangles can be effected by means of logarithms.

Example. To find the distance from a point $A$ on the shore of a bay to a point $B$ off shore, a point $C$ is taken on the shore 350 feet from $A$. The angles $B A C$ and $B C A$ are found to be $84^{\circ} .13$ and $72^{\circ} .76$. Find $A B$.

We have $b=350$.

$\begin{aligned} A & =84^{\circ} .13 . \\ C & =72^{\circ} .76 .\end{aligned}$

$$
\begin{aligned}
\log b & =2.5441 \\
\log \sin C & =\frac{0.9800-1}{3.5241-1 .}
\end{aligned}
$$

Then $B=180^{\circ}-(A+C) \quad \log \sin B=0.5939-1$.

$$
=23^{\circ} .11
$$

And

$$
c=\frac{b \sin C}{\sin B}
$$

$\log c=2.9302$

$c=851.7$.

The logarithms of $\sin B$ and $\sin C$ may be obtained directly from page 20 of the Tables.

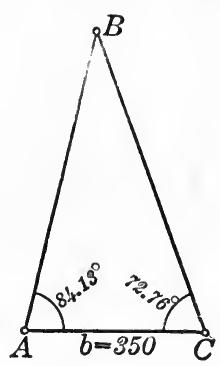

Fig. 133.

Checking the solution of a triangle. In any numerical computation it is of the utmost importance that the accuracy of the results be checked in some way.

If $A B C$ is a right triangle, an excellent check is given by the Pythagorean theorem, $c^{2}=a^{2}+b^{2}$, which can be adapted to logarithmic computation by writing it in the form

$$
a=\sqrt{c^{2}-b^{2}}=\sqrt{(c-b)(c+b)} .
$$

Notice that the possibility of expressing $c^{2}-b^{2}$ as a product is the basis of the adaptation to the use of logarithms, and that 
the property of $x^{2}$ used here is a special case of (8), page 153 . In general, if the difference of two values of a function $f(x)$, namely, $f(a)-f(b)$, can be expressed as a product, this property of the function enables us to compute the difference by means of logarithms.

An excellent check, which is simpler than the law of cosines, for the solution of an oblique triangle by the law of sines is given by the relation

$$
c=a \cos B+b \cos A .
$$

This may be established from the figures in Section 71, page 202 , by the use of right triangles. Similar expressions may be written for $a$ and $b$.

\section{EXERCISES}

1. By means of the relations $\sec A=1 / \cos A$ and $\csc A=1 / \sin A$, show how $\log \sec A$ and $\log \csc A$ can be found.

2. Solve the following triangles and find their areas.

(a) $a=13.75, B=76^{\circ} .23, C=90^{\circ}$.

(b) $a=243.7, \quad c=431.2, \quad C=90^{\circ}$.

(c) $A=34^{\circ} .16, B=92^{\circ} .86, b=32.68$.

(d) $a=14.96, b=12.32, B=49^{\circ} .17$.

3. A level road runs directly toward a hill. From the top of the hill the angles of depression of two milestones on the road are $12^{\circ} .17$ and $9^{\circ} .48$. Find the height of the hill in feet.

4. Two points $A$ and $B, 325$ feet apart, are situated on the edge of a canyon. At $A$ the horizontal angle between $B$ and a point $C$ across the canyon and at the bottom is $76^{\circ} .23$. At $B$, the horizontal angle between $A$ and $C$ is $64^{\circ} .18$ and the angle of depression of $C$ is $69^{\circ} .32$. Find the depth of the canyon.

5. Express the area of a regular polygon of $n$ sides as a function of $n$ and of the side $a$. Use this formula to determine the area of a regular pentagon whose side is 34.8 inches.

6. Show that the area of a quadrilateral is $A=\frac{1}{2} d d^{\prime} \sin \theta$, where $d$ and $d^{\prime}$ are the diagonals and $\theta$ is the angle between them.

7. A farm has the shape of a quadrilateral $A B C D, B C=256$ yards, $\angle A B C=112^{\circ}, \angle D B C=38^{\circ} .5, \angle A C B=42^{\circ}, \angle D C B=124^{\circ}$. Find the selling price at $\$ 85$ an acre. (See Exercise 6.)

8. Two points $A$ and $B$ are on opposite sides of a river 1250 yards wide, $A$ being 450 yards farther upstream than $B$. In what direction relative to the bank should an auto boat be pointed in order to proceed in 
a straight line from $B$ to $A$, if the velocity of the stream is 4.6 miles an hour and that of the boat is $\mathbf{1 6 . 3}$ miles an hour? How long will it take the boat to make the journey?

9. The angle of elevation of a cliff 750 feet high viewed from a ship due east was $18^{\circ} .26$. The ship sailed southwest and from a second point of its course the angle of elevation of the cliff was $22^{\circ} .06$. Find the distance between the two points of observation. What point of the course of the ship was nearest to the cliff?

10. In order to extend a base line beyond an obstacle which could be sighted across, a surveyor chose four stations, as indicated in the figure. The following measurements were made:

$A B=145.6$ feet, $\angle B A D$ $=32^{\circ} .63, \quad \angle C B D=43^{\circ} .88$, $\angle B D C=114^{\circ} .76$. Find $B C$.

11. A monument stands on the top of a hill. From a certain point on the level

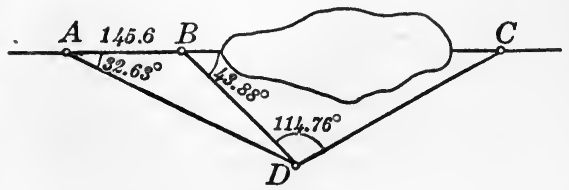

Frg. 134. ground below the hill, the angles of elevation of the top and the base of the monument are $28^{\circ} .37$ and $25^{\circ} .63$, while at a point 325.7 feet nearer the hill the angle of elevation of the top of the monument is $30^{\circ} .32$. Find the height of the monument.

12. To find the horizontal distance to and the height of an observation balloon $A$ anchored behind the enemies lines, a base line, $B C, 478$ feet long is laid off and the horizontal angles between $A$ and $C$ at $B$, and between $B$ and $A$ at $C$ are found to be $74^{\circ} .38$ and $83^{\circ} .27$. At $B$ the angle of elevation of $A$ is $17^{\circ} .85$. Find the distance and the height desired.

\section{ASTRONOMICAL EXERCISES}

The zenith of a point $A$ on the surface of the earth is the point in the heavens in line with $A$ and the center of the earth.

The zenith distance of a heavenly body with respect to a point $A$ is the angle which a line to the body makes with the line to the zenith at $A$.

1. At a point $A$, a star in the zenith is chosen, and a distance $A B$ is measured along the arc of a meridian to a point $B$ where the zenith distance of the star is $1^{\circ}$. If the length of the arc $A B$ is 69.4 miles find the radius of the earth. (See Fig. 135.)

(In view of the great distance of the star from the earth, what assumption can be made with respect to the lines $A S$ and $B S$, and hence what magnitude may be assigned to the angle $A O B$ ?)

2. The north latitude of Berlin is $52^{\circ} 23^{\prime}$, and the south latitude of Cape of Good Hope is $33^{\circ} 5^{\prime}$. Find the chord distance between the two places. Take $r=3963$. (See Fig. 136.) 
3. At Berlin and Cape of Good Hope the zenith distance $z_{1}$ and $z_{2}$ of the moon were measured simultaneously and found to be $z_{1}=53^{\circ} 10^{\prime} 1^{\prime \prime}$, $z_{2}=33^{\circ} 37^{\prime} 32^{\prime \prime}$. Find the

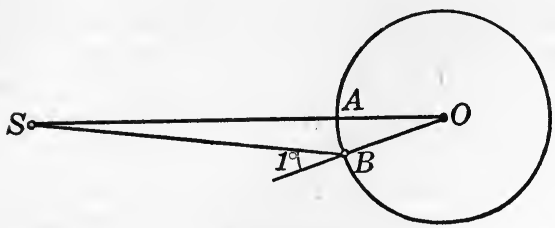

Fig. 135.

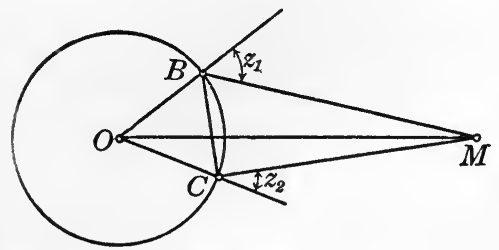

Fig. 136.

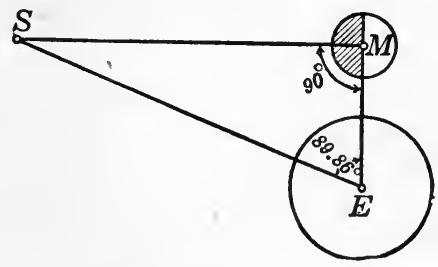

Fig. 137.

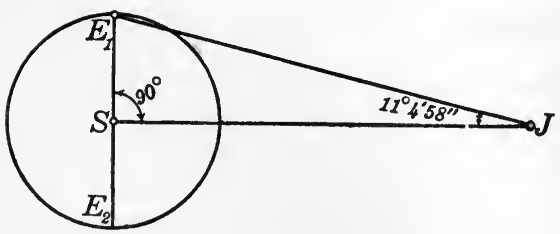

Fig. 138. distances $B M$ and $O M$.

4. When the moon is in the position where we see half the illuminated portion (i.e. half-moon) the angle at the moon is $90^{\circ}$. At such an instant the angle at $E$ was measured and found to be $89.86^{\circ}$. Calculate the distance to the sun using the distance to the moon as a base line.

5. The annual parallax of a star is the angle at the star subtended by the semi-diameter of the earth's orbit. Observations of Jupiter taken from diametrically opposite points of the earth's orbit give the annual parallax of Jupiter as $11^{\circ} 4^{\prime} 58^{\prime \prime}$. Find the distance of the sun from Jupiter.

6. The annual parallax of a Centauri, the nearest fixed star, is $0.916^{\prime \prime}$. Find the distance of the star from the sun. (Note that for very small angles $\tan \theta$ may be replaced by $\theta .{ }^{1}$ Use the value of $1^{\prime \prime}$ in radians found at the bottom of the front cover of the Tables.) If light travels at the rate of 186,320 miles per second, find the length of time necessary for light to traverse the distance.

7. The annual parallax of the North Star is $0.073^{\prime \prime}$. Find the distance to the North Star in light-years.

${ }^{1}$ Inspection of the Condensed Table on the inside of the back cover of the Tables shows that values of $\theta$, in radians, and of $\tan \theta$ agree to three decimal places if $\theta \leqq 6^{\circ}$; to four places if $\theta \leqq 3^{\circ}$. 
8. Find the time that it takes light to reach the earth from the sun; to reach Neptune, the most remote planet, distance $2,788,000,000$ miles.

85. Exponential Equations. An equation in which the unknown enters as an exponent is called an exponential equation.

Exponential equations of the type $a^{f(x)}=b^{F(x)}$, where $f(x)$ and $F(x)$ are linear or quadratic algebraic functions may be solved by equating the logarithms of the two sides of the equation and solving the resulting algebraic equation, as in the following examples.

Example 1. Find the function of the type $y=x^{n}$ whose graph passes through the point $(3,5)$.

Substituting the coördinates of the point in the equation, we have

$$
3^{n}=5 \text {. }
$$

Equating the logarithms of both sides of this equation, we have

or

$$
\log 3^{n}=\log 5
$$

Hence

$$
n \log 3=\log 5 \text {. }
$$

$0.4771 n=0.6990$

$$
\begin{aligned}
n & =\frac{0.6990}{0.4771} & \log 0.6990 & =0.8445-1 \\
& =1.4653 & \log 0.4771 & =0.6786-1 \\
& & \log n & =0.1659
\end{aligned}
$$

Hence the required function is

$$
y=x^{1 \cdot 4653} \text {. }
$$

The value of $n$ is here calculated from the quotient by logarithms rather than by long division. Notice that if 0.4771 were subtracted from 0.6990 , the result would be $\log (5 / 3)$ and not $\log 5 / \log 3$.

Example 2. Find the logarithm to the base $e$ of any positive number $x$ assuming a table of common logarithms to be given. Let

$$
y=\log _{e} x, \quad \text { hence } e^{y}=x .
$$

Equating the common logarithms of both sides of the second equation we have

$$
\log _{10} e^{y}=\log _{10} x, \quad \text { whence } y \log _{10} e=\log _{10} x .
$$

Therefore

$$
y=\frac{1}{\log _{10} e} \log _{10} x .
$$

Since $\log _{10} e=0.4343$, we have $\log _{e} x=\frac{1}{0.4343} \log _{10} x$.

That is, the logarithm to the base $e$ of any number can be found by multiplying the common logarithm of the number by $1 / 0.4343$. 
For instance we have

$$
\log _{e} 6.75=\frac{1}{0.4343} \log _{10} 6.75=\frac{0.8293}{0.4343}=1.91 \text {. }
$$

The constant $\frac{1}{0.4343}$ is called the modulus of the base $e$ with respect to the base 10 .

The figure shows the graphs of $\log _{10} x$ and $\log _{e} x$.

$$
\begin{aligned}
& \text { If } \quad x=O P, \quad \text { then } \log _{10} x=P Q, \quad \text { and } \quad \log _{e} x=P R \text {. } \\
& \text { If }
\end{aligned}
$$

Hence equation (1) may be written in the form $P R=\frac{P Q}{A B}=\frac{1}{0.4343} P Q$

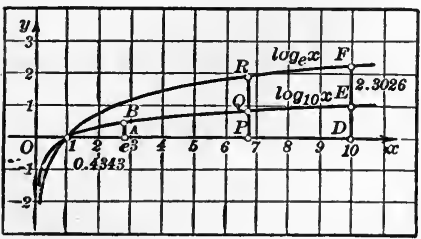

Fig. 139. and the graph of $\log _{e} x$ can be obtained from that of $\log _{10} x$ by the theorem on page 89 .

EXAMPLE 3. The variation of atmospheric pressure $p$ (in millimeters of mercury in a barometer) with respect to the altitude $h$ above sea level (in meters) is given by the equation $\boldsymbol{p}=p_{0} e^{-m h}$, where $p_{0}$ and $m$ are constants and $e$ is the base of the natural system of logarithms. If $p=719$ when $h=450$, and $p=594$ when $h=2000$, find $p$ if $h=8000$.

Before starting the solution, notice that $p_{0}$ is the atmospheric pressure at sea level; for if $h=0, p=p_{0} e^{-m 0}=p_{0} e^{0}=p_{0}$.

A more convenient form of the given equation, for purposes of computation, is obtained by equating the common logarithms of both sides of the equation, which gives:

$$
\log p=\log p_{0}-m h \log e .
$$

Substituting the given data we have the two equations

$$
\begin{aligned}
& \log 719=\log p_{0}-450 \times 0.4343 m, \\
& \log 594=\log p_{0}-2000 \times 0.4343 m .
\end{aligned}
$$

These equations may be solved simultaneously for the constants $m$ and $p_{0}$, as follows:

Subtracting

$\log 719-\log 594=1550 \times 0.4343 m$

Hence $m=\frac{2.8567-2.7738}{1550 \times 0.4343}=\frac{0.0829}{1550 \times 0.4343}$

$$
m=0.0001232
$$

Substituting this value of $m$ in (2) we have

$$
\begin{array}{rlrl}
\log p_{0} & =2.8567+450 \times 0.4343 \times .0001232 \\
& =2.8567+0.02407, \\
\log p_{0} & =2.8808 & \\
p_{0} & =760 . & \log 450 \times
\end{array}
$$

$\log 1550=3.1903$

$\log 0.4343=\frac{0.6378-1}{2.8281}$

$\log 0.0829=0.9186-2$

$\log m=\overline{0.0905-4}$ $\log 450=2.6532$

$\log 0.4343=0.6378-1$ $\log 450 \times 0.4343 m=0.3815-2$ $450 \times 0.4343 m=0.02407$ 
Hence $p=760 e^{-0.0001232 h}$.

$$
\text { If } h=8000, p=760 e^{-0.9856} \text {, }
$$

$\log p=\log 760-0.9856 \log e$.

$=2.8808-0.4282$

$=2.4526$.

Hence $p=283.5$ if $h=8000$.

$$
\begin{aligned}
& \log 0.9856=0.9937-1 \\
& \log 0.4343==0.6379-1 \\
& 0.6316-1
\end{aligned}
$$

$0.9856 \log e=0.4282$

\section{EXERCISES}

1. Solve for $x$,
(a) $7^{x}=4$.
(b) $4^{x-1}=9$.
(c) $4^{2 x}=7$.
(d) $8^{3 x-2}=5^{x-3}$.

2. Each of the persons receiving one of the letters mentioned in Exercise 18, page 232 was requested to forward 10 cents. Find the number on the letters at which the chain should have been completed if the object had been to raise $\$ 1,000,000$.

3. The population of the United States in 1898 was approximately $75,000,000$. Find the number on the letter at which the chain in Exercise 18 , page 232 , should have been completed if it was expected that every man, woman, and child in the country would receive one.

4. Would the method of Section 85 enable one to solve the equation $2^{x}+3^{x}=7 ?$

5. Find a function of the type $y=x^{n}$ which passes through the point $(3,2)$. Find the average rate of change of this function from $x=3$ to $x=4$.

6. Find the common logarithm of any positive number $x$, assuming that a table of logarithms to the base $e$ is given.

7. Find the numerical value of $M=\log _{10} e(e=2.718)$ from the table of common logarithms. By the method of Example 2, Section 85, find the value of $m=\log _{e} 10$. Find the reciprocal of $\mathrm{M}$, and compare it with $m$.

8. Assuming that a table of logarithms to the base $b$ is given, show that the logarithm of any positive number $x$ to the base $a$ is

$$
\log _{a} x=\frac{\log _{b} x}{\log _{b} a}
$$

Hence show that $\log _{e} 10=\frac{1}{\log _{10} e}$, or $\log _{e} 10 \log _{10} e=1$.

9. By means of the preceding exercise show that

$$
\text { and } \begin{aligned}
\log _{e} x & =m \log _{10} x, & \text { where } & m=2.3026, \\
\log _{10} x & =M \log _{e} x, & \text { where } & M=0.4343 .
\end{aligned}
$$

Illustrate these two equations on the graphs of $\log _{e} x$ and $\log _{10} x$.

Note: The system of common logarithms, in which the base is 10 , and that of natural logarithms, in which the base is $e=2.718$, are the most important systems of logarithms. The former is used for numerical 
computations, the latter for most theoretical work. The relations be. tween these two systems obtained in Exercises 6 to 9 may be summed up as follows:

If the common logarithm of a number is given, the natural logarithm is found by multiplying it by

$$
m=\frac{1}{\log _{10} e}=\log _{e} 10=2.3026 .
$$

The constant $m$ is called the modulus for changing from the common to the natural system.

If the natural logarithm of a number is given, the common logarithm is found by multiplying it by

$$
M=\frac{1}{\log _{e} 10}=\log _{10} e=0.4343 .
$$

which is called the modulus for changing from the natural to the common system.

10. Solve Example 3, Section 85, using the table of natural logarithms (page 31 of the Tables).

11. Find $\log _{2} 3, \log _{2} 5, \log _{2} 7$, and then by means of some of the fundamental properties of logarithms (Section 81, properties $7,8,9$ ) construct a table of logarithms to the base 2 for the integers 1 to 10 inclusive.

12. A hawser of a ship is subjected to a strain of 6 tons. How many turns must be taken around a post in order that a man who can not pull more than 200 pounds may keep the hawser from slipping, if the coefficient of friction $m=0.175$ and $S=P e^{m \theta}$, where $S=$ strain in the hawser in pounds, $P=$ pull of the man in pounds, $\theta=$ the angle of contact of the hawser and post in radians.

13. A steamer approaching a dock has a velocity of 20 feet per second at the instant the power is shut off, and 10 feet per second at the end of 1 minute. Find the velocity at the end of 2 minutes if the law of motion is $v=v_{0} e^{-m t}$.

14. In a chemical experiment it was found that at the end of 1 hour there were 35.6 c.c. of a substance in solution and at the end of 3 hours, 18.5 c.c. If the amount $A$ of the substance at any time $t$ is given by the equation $A=k e^{-m t}$, determine the time that elapsed before the amount was reduced to 5 c.c.

15. The intensity of light $I$ after passing through a medium of thickness $T$ is $I=I_{0} e^{-m T}$. If light loses $3 \%$ of its intensity in passing through a lens, what per cent of intensity will remain after passing through four lenses?

16. Radium decomposes so that the amount $A$ remaining after a time $t$ is $A=A_{0} e^{-m \ell}$. If $\frac{3}{4} \%$ disappears in 20 years, how long before one-half of the original amount will be gone? 
17. As a body cools, at any time $t$ the difference in temperature $\theta$ between the body and the surrounding medium is given by the equation $\theta=\theta_{0} e^{-m t}$. If a fireless cooker oven is $430^{\circ}$ above room temperature when $t=0$, and $216^{\circ}$ after one hour, how long before it will be $10^{\circ}$ above room temperature?

86. Compound Interest. Interest is the money paid for the use of borrowed capital which is called the principal. Interest that is paid only on the principal is called simple interest. When interest is paid on unpaid interest, which is added to the principal periodically, it is called compound interest. If unpaid interest is added to the capital at yearly intervals it is said to be compounded annually. If the interest is added every six months or three months it is said to be compounded semiannually or quarterly.

In any problem in compound interest where the interest is compounded annually, four quantities are involved:

$P$, the principal;

$i$, the rate of interest, which is the sum of money in dollars paid for the use of one dollar for one year. Thus, if the rate is $4 \%, i=\$ 0.04$;

$n$, the number of years;

$S$, the amount, which is the sum of the principal and interest for $n$ years.

Theorem 1. The amount, $S$, of a principal of $P$ dollars, interest compounded annually for $n$ years at the rate $i, i s$

$$
\mathbf{S}=\mathbf{P}(\mathbf{1}+\mathbf{i})^{n} \text {. }
$$

The amount of one dollar for one year at the rate $i$ is $1+i$. Hence the amount of $P$ dollars for one year is $P(1+i)$. The amount at the end of the second year is obtained by multiplying the new principal, $P(1+i)$, by the amount of one dollar for one year $1+i$.

Hence the amount at the end of the second year is $P(1+i)(1+i)=P(1+i)^{2}$.

Similarly, the amount at the end of the third year is $P(1+i)^{2}(1+i)=P(1+i)^{3}$. Comparing these results, we see that the exponent of $(1+i)$ is the same as the number of years, and hence 
The amount $S$ at the end of $n$ years is $S=P(1+i)^{n}$.

This equation involves four variables. If any three are given, the fourth may be determined by substituting the given values and solving for the unknown. The value of $S, P$, or $i$ may be determined by algebraic processes while the determination of $n$ involves the solution of an exponential equation.

Example 1. At what rate will $\$ 50$ amount to $\$ 75$ in 7 years? Here $P=50, \quad S=75$, and $n=7$.
Hence
$75=50(1+i)^{7}$,
Then
$(1+i)^{7}=\frac{75}{50}=1.5$
So that
$(1+i)=\sqrt[i]{1.5}$
$\log 1.5=0.1761$.
$\frac{1}{7} \log 1.5=0.0252$.
$=1.0598$,
Hence $\quad i=0.0598$, so that the rate is $5.98 \%$.

If the interest is compounded semi-annually (as in some savings banks), in $n$ years there are $2 n$ periods and the interest on one dollar for each period is $i / 2$, so that the amount in $n$ years is

$$
S=P\left(1+\frac{i}{2}\right)^{2 n}
$$

If the interest is compounded quarterly, the amount in $n$ years is

In general, we have

$$
S=P\left(1+\frac{i}{4}\right)^{4 n}
$$

Theorem 2. If interest is compounded $m$ times a year, at the annual rate $i$, the amount after $n$ years is the same as if the interest were compounded annually at the rate $i / m$, for $m n$ years. That is

$$
S=P(1+i / m)^{m n} .
$$

Example 2. Find the amount of $\$ 50$ for 10 years with interest convertible into principal semi-annually at $4 \%$.

Here $P=50, i=.04, n=10, m=2, i / m=.02$ and $m n=20$.

$$
\text { Hence } \quad \begin{array}{rlrl}
S & =50(1+.02)^{20} & \log 50 & =1.5690 \\
& =50(1.02)^{20} & 20 \log 1.02 & =0.1720 \\
& =\$ 74.30 . & \log S & =1.8710
\end{array}
$$

Definition. The present value, $P$, of a sum of money, $S$, due after $n$ years, is the principal which must be placed at 
compound interest at a given rate $i$, in order to amount to the sum $S$ at the end of $n$ years.

Thus in the formula in Theorem $1, P$ is the present value of $S$.

\section{EXERCISES}

1. A principal of $\$ 100$, deposited in a trust company, bears interest at the rate of $4 \%$ compounded semi-annually. What will the balance be at the end of ten years if no withdrawals are made?

2. A mother promises her twelve year old boy that she will present him with $\$ 100$ on his twenty-first birthday provided he abstains from smoking until that time. How much should she deposit in the trust company of the previous example in order to have an amount of $\$ 100$ when the boy is twenty-one?

3. At what rate would a sum of money double itself in 25 years if interest is compounded annually?

4. In how many years will a sum of money double itself if placed at compound interest if (a) the rate of interest is $5 \%$, compounded annually? (b) the rate is $4 \%$ compounded semi-annually?

5. If $\$ 100$ be deposited in a trust company at $4 \%$, compounded semiannually, how long before it will amount to at least $\$ 150$ ?

6. A building and loan association offers an opportunity for an investment to yield $8 \%$, compounded quarterly.

(a) If $\$ 100$ is invested, to what will it amount in 5 years?

(b) What sum should be invested to amount to $\$ 120$ in 7 years?

(c) How long must $\$ 50$ be invested to amount to $\$ 175$ ?

(d) How long will it take any principal to double itself?

(e) How long will it take for a sum to treble itself?

7. A man borrows $\$ 50$ from a friend, and three years later returns $\$ 65$. What is the equivalent rate of interest, if interest is compounded quarterly?

8. A man bought a diamond for $\$ 150$ in 1900 , and sold it for $\$ 400$ in 1915. What rate of interest did he realize, assuming it to be compounded annually?

9. If a building lot is bought for $\$ 500$, and its value increases by $10 \%$ annually, what will it be worth in 5 years?

10. The number of students in a college increased from 275 to 460 in 10 years. At what rate did the number increase, assuming that the percentage of increase each year was constant?

11. What sum should be deposited in a bank paying $3 \%$ compounded semi-annually in order to pay off a debt of $\$ 500$ due three years later?

12. Construct the graph of the amount of one dollar, interest compounded annually at $6 \%$, as a function of the number of years $n$. Build the table of values for $n=10,20,30,40$. 
13. Verify the fact that the values of $S$ obtained in Exerciso 12 are In geometrical progression.

87. Annuities. Definimion. An annuity consists of a series of equal payments made at equal intervals of time. The first payment of an annuity is made at the end, not at the beginning, of the first period of time, unless otherwise specified.

Annuities are common in commercial life. The rent of a house or store, the premium of an insurance policy, the dividend on a bond, wages and salaries, the payments of interest on a mortgage, are examples of annuities.

Theorem 1. The amount of an annuity of $R$ dollars, payable annually, accumulated for $n$ years at the rate $i$, interest compounded annually is,

$$
\mathbf{K}=\mathbf{R} \frac{(1+\mathbf{i})^{n}-1}{\mathbf{i}}
$$

The compound interest on each payment is obtained by the method of the preceding section.

Since the first payment is made at the end of the first year, the first payment is at interest for $n-1$ years and amounts to $R(1+i)^{n-1}$.

The second payment is at interest for $n-2$ years and amounts to $R(1+i)^{n-2}$, etc.

The next payment to the last is at interest 1 year and amounts to $R(1+i)$.

The last payment bears no interest and amounts to $R$. Adding these amounts in the reverse order we obtain

$$
K=R+R(1+i)+\ldots+R(1+i)^{n-2}+R(1+i)^{n-1} .
$$

The terms on the right form a geometric progression whose sum is obtained by the formula $S=\frac{r l-a}{r-1}$, where the first term is $a=R$, the last term is $l=R(1+i)^{n-1}$, and the ratio is $r=1+i$.

Substituting, we have

$$
K=\frac{(1+i) R(1+i)^{n-1}-R}{1+i-1}=R \frac{(1+i)^{n}-1}{i}
$$


Theorem 2. The amount of an annuity, if $R$ dollars are paid $m$ times a year, accumulated for $n$ years at the rate $i$, interest compounded $m$ times a year, is equivalent to the amount of an annuity of $R$ dollars, payable annually, accumulated for $m n$ years at the rate $i / m$, interest compounded annually.

Definition. The present value of an annuity of $R$ dollars a year for $n$ years is the amount that must be deposited in a bank, interest compounded annually at the rate $i$, so that, if $R$ dollars be withdrawn annually, there will be no balance left after the $n$th withdrawal.

Theorem 3. The present value of an annuity of $R$ dollars payable annually for $n$ years is

$$
A=R \frac{1-(1+i)^{-n}}{i} .
$$

The present value of the annuity is the sum of the present values of $R$ dollars due 1 year hence, $R$ dollars due 2 years hence, etc., for $n$ years. We have from the formula for compound interest that the present value $P$ of a sum $S$ due in $n$ years is $P=S(1+i)^{-n}$. Hence if $A$ denotes the present value of the annuity, we have

$$
A=R(1+\imath)^{-1}+R(1+i)^{-2}+\cdots+R(1+\imath)^{-n} .
$$

The sum on the right is a geometric progression for which $a=R(1+i)^{-1}, l=R(1+i)^{-n}$, and $r=(1+i)^{-1}$. Substituting these values in the formula for the sum of a geometric progression, we have

$$
A=\frac{(1+i)^{-1} R(1+i)^{-n}-R(1+i)^{-1}}{(1+i)^{-1}-1} .
$$

Multiplying the numerator and denominator of the right-hand side by $(1+i)^{+1}$ and simplifying, we have

$$
A=R \frac{(1+i)^{-n}-1}{1-(1+i)}=R \frac{1-(1+i)^{-n}}{i} .
$$

Theorem 4. The present value of an annuity, if $R$ dollars are paid $m$ times a year for $n$ years, at the rate $i$ compounded $m$ times a year, is equivalent to that of an annual annuity of $R$ dollars for $m n$ years at the rate $i / m$ compounded annually. 


\section{EXERCISES}

1. If $\$ 50$ be deposited annually in a building and loan association paying $6 \%$, compounded annually, what will the savings amount to in 10 years?

2. How much must a man save annually, and deposit in a savings and loan company paying $5 \%$, compounded annually, in order to pay off a mortgage for $\$ 2000$ after 5 years?

3. How long will it take to accumulate $\$ 2000$ if $\$ 75$ are deposited annually in a savings and loan company paying $6 \%$, compounded annually?

4. A father buys a bond for $\$ 1000$, due after 18 years, which bears interest at $5 \%$ payable semi-annually, for the college expenses of his infant boy. The interest payments are deposited in a bank paying $4 \%$, compounded semi-annually. How much money will be available 18 years later?

5. A man buys a house and lot, paying $\$ 1000$ down, and agreeing to pay $\$ 1000$ annually for 4 years. What is the equivalent cash price if money is worth $6 \%$ per annum? Note that the first payment is not part of the annuity, since the first payment of an annuity is due at the END of the first period.

6. A piano is sold for $\$ 100$ cash and $\$ 25$ quarterly for 2 years. What is the equivalent cash price, if money is worth $4 \%$ compounded quarterly?

7. A man buys a house, giving back a mortgage for $\$ 5000$ with interest payable annually at $6 \%$. If the mortgage is to be paid off by five equal annual payments, covering principal and interest, what should be the amount of the annual payment? Hint: The annual payments constitute an annuity whose present value is $\$ 5000$.

8. Which is the better offer for a house from the seller's standpoint, $\$ 5000$ down and $\$ 1000$ annually for 4 years, or $\$ 5000$ down and $\$ 2000$ annually for 2 years, if money is worth $4 \%$ ?

9. Six months before a boy enters college, his father wishes to deposit a sum in a savings bank paying $4 \%$, interest compounded semi-annually, which will enable the boy to draw $\$ 300$ every 6 months during his college course. How much should he deposit?

10. It is estimated that a certain mine will be exhausted in 10 years. If the mine yields a net annual income of $\$ 2000$, what would be a fair purchase price, money being worth $5 \%$ ?

11. A man holds a mortgage for $\$ 6000$, due after 3 years, interest $6 \%$ payable quarterly. If he disposed of it how much might he expect to receive, if money is worth $4 \%$ compounded quarterly. Hint: The present value of the mortgage is the sum of the present value of $\$ 6000$ due after 3 years and of a quarterly annuity of $\$ 90$ (one fourth of a year's interest).

12. A bond for $\$ 100$ is to be redeemed at the end of 10 years, and bears interest at 5 per cent, payable semi-annually. At what price for the bond 
will the purchaser realize 4 per cent on his investment? Hint: The present value of the bond consists of the present value of $\$ 100$ due 10 years hence and the present value of the annuity consisting of the semi-annual interest payments.

13. What is the purchase price of the bond in the preceding exercise if the buyer is to realize 6 per cent?

14. An insurance company desires to offer, at the time a policy matures, an option between a 20 -year bond for $\$ 1000$, bearing interest at 5 per cent, payable semi-annually, and an equivalent cash payment. If the computations of the company are on a 3 per cent basis, what should the cash payment be?

15. At the maturity of an insurance policy, the company offers a cash payment of $\$ 1000$ or the option of five equal annual payments, the first to be made one year after the policy matures. If the company assumes that money is worth 3 per cent, what should be the amount of the annual payment?

16. Three months before a boy enters college, his father deposits on the boy's account $\$ 1200$ in a bank paying 4 per cent interest, compounded quarterly. The boy wishes to withdraw the money quarterly, in equal amounts, during the four years of his course. How much should he draw at a time?

17. A city issues 20 -year bonds to the amount of $\$ 100,000$ in order to raise money for the improvement of its water supply. A sinking fund to provide for the extinction of the debt can be accumulated at 4 per cent, interest compounded annually. How much must be deposited in the sinking fund at the end of each year?

18. A man buys an automobile for $\$ 1000$, and estimates that he will be allowed $\$ 400$ for it in purchasing a new car three years later. How much should he save every three months for the purchase of the new car, if he deposits his savings in a bank paying 4 per cent interest, compounded quarterly?

19. A furnace costing $\$ 450$ must be installed in a house every 10 years. How much should the landlord save each year for this purpose, if the savings can be accumulated at 5 per cent, interest compounded annually?

20. A man owes $\$ 2000$ on which he pays $5 \%$ interest. If he pays the debt, principal and interest, in 6 equal annual installments, what is the amount of each payment? How much will he owe at the beginning of the second year? At the beginning of the third year?

21. Find the limit of the present value of an annuity as the number of payments increases indefinitely. Verify the result by finding the sum of the infinite geometric progression whose terms are the present values of the several payments.

22. A college graduate wishes to provide for a scholarship of $\$ 90$ a year. Find the amount which he must present to the college, if collegiate 
funds can be invested at $4 \frac{1}{2} \%$, using the result obtained in the preceding exercise. By what other method can the amount be found?

23. A railroad spends $\$ 900$ annually to provide a watchman for a grade crossing. If money is worth 5 per cent, how much can they reasonably be expected to spend for the elimination of the crossing?

88. Graph of the Exponential Function $k b^{n x}$. The form of the graph of $b^{x}$ was determined on page 216. From it the graph

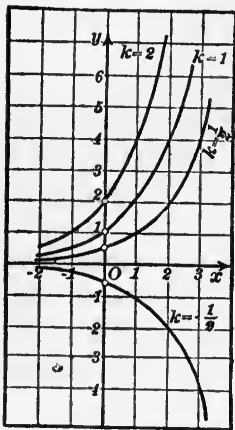

Fig. 140. of $k b^{x}$ may be found by multiplying ordinates by $k$ (Theorem, page 89). Fig. 140 gives the graph of $k 2^{x}$ for several values of $k$. Notice that $y=k$ is the intercept on the $y$-axis.

The form of the graph of the function $b^{n x}$ may be found by dividing by $n$ the abscissas of several points on the graph of $b^{x}$ (Theorem, page 151). The graph of $2^{n x}$ for several values of $n$ is given in Fig. 141 .

The combination of the two theorems quoted shows that the graph of $k b^{n x}$ may be obtained as

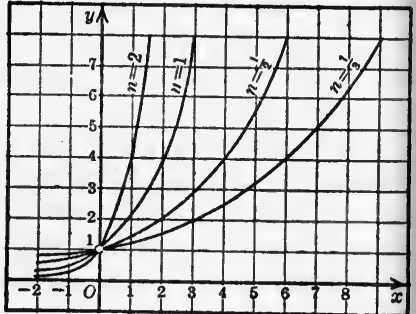

FIG. 141. follows: Plot the graph of $b^{x}$, divide the abscissas of points on it by $n$, which gives the graph of $b^{n x}$, and multiply the ordi-

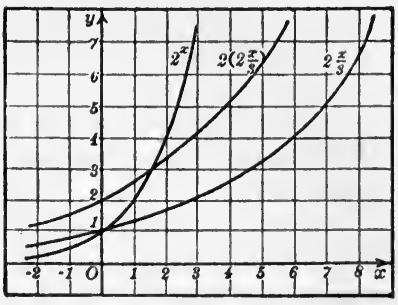

Frg. 142. nates of points on the new graph by $k$.

Example. Sketch the graph of $2\left(2^{x / 3}\right)$. Here $k=2$ and $n=\frac{1}{3}$. Dividing the abscissas by $\frac{1}{3}$ amounts to multiplying them by 3 . Hence we first construct the graph of $2^{x}$ and multiply the abscissas of points on it by 3. The ordinates of points on the curve so obtained are then multiplied by $k=2$.

The form of the graph of $k b^{n x}$ depends on the value of $b$, but for suitable values of $k^{\prime}$ and $n^{\prime}$ the same curve is the graph of $k^{\prime} c^{n x}$. We shall use 
$b=10$ as a standard value, and we shall now show that we can determine a constant $m$ such that the graphs of $k b^{\text {rix }}$ and $k 10^{m x}$ are identical.

The graphs will be identical if and only if

or

$$
\begin{aligned}
k b^{n x} & =k 10^{m x}, \\
b^{n x} & =10^{m x} .
\end{aligned}
$$

This is essentially an exponential equation to be solved for $m$. Equating the logarithms of both sides of the equation,

whence

Then

$$
n x \log b=m x \log 10=m x,
$$$$
m=n \log b .
$$$$
k b^{n x}=k 10^{n x \log b} \text {. }
$$

Hence it is always possible to use the base 10 , and we shall do so in the future unless the contrary is indicated.

The most characteristic properties of the graph of $y=k 10^{m x}$ are that it does not cross the $x$-axis, that it does cross the $y$-axis, and that it always rises or always falls.

\section{EXERCISES}

1. Plot the graphs of the functions:
(a) $\frac{1}{10}\left(2^{2 x}\right)$.
(b) $\frac{1}{2}\left(2^{2 x}\right)$.
(c) $3\left(2^{0.5 x}\right)$.
(d) $\frac{1}{3}\left(2^{0.3 x}\right)$.
(e) $2\left(3^{x / 4}\right)$
(f) $3\left(2^{-0.25 x}\right)$.
(g) $2\left(3^{-0.5 x}\right)$.
(h) $0.25\left(2^{-2 x}\right)$.
(i) $0.5\left(10^{-6 x}\right)$.

2. Plot the graph of $y=e^{-x^{2}}$. How can the graph of $y=k e^{-2 x^{2}}$ be obtained from it? This graph is called the probability curve.

3. Find the inverse of the function $k 10^{m x}$. What are the most important characteristics of the graph of $y=c \log n x$ ?

4. (a) Construct the graph of $k \log _{2} x$ for several values of $k$.

(b) Construct the graph of $\log _{2} n x$ for several values of $n$.

(c) Construct the graph of $\frac{1}{2} \log _{2} 3 x$.

89. The Logarithmic Scale. The ordinates of points on the graph of $\log _{10} x$ corresponding to integral values of $x$ from 1 to 10

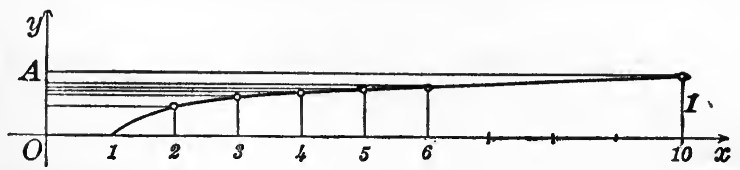

Fig. 143.

inclusive, are a set of segments on the $y$-axis whose lengths from the origin are equal to the logarithms of the corresponding abscissas. 
The line $O^{\prime} A^{\prime}$ is an enlargement of the segment $O A$ on the $y$-axis, the numbers on the right being the logarithms of those on the left.

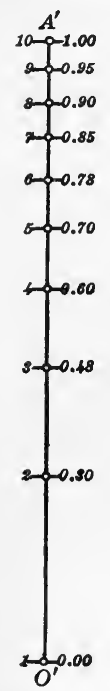

A scale is called a uniform scale if the distance of a number from the point marked 0 is equal to the number, the distance from 0 to 1 being the unit segment.

A scale is called a logarithmic scale if the distance of a number from the point marked 1 is equal to the logarithm of the number, the distance from 1 to 10 being taken as unity.

The logarithms are spaced uniformly along the line $O^{\prime} A^{\prime}$, while the integers are spaced non-uniformly.

The utility of a logarithmic scale lies in the fact that the addition and subtraction of the logarithms of numbers, and hence the multiplication and division of numbers, may be effected mechanically by the addition and subtraction of line segments on the scale. For instance, to find the product $3 \times 2$, we add the segment $1-2$ to the segment $1-3$. For this purpose, place a pair of dividers so that the points are on the extremities of the segment $1-2$, and then with this opening place one end of the dividers on the point 3 and the other will touch the scale exterior to the segment $1-3$ in the point 6 , the required product.

To find the quotient $\frac{3}{2}$, subtract the segment $1-2$ from the segment $1-3$ in a similar manner by means of the dividers.

The logarithmic scale is sometimes called Gunter's scale after Edmund Gunter, who first made use of it for purposes of calculation in 1620 .

The dividers may be dispensed with if two identical logarithmic scales are arranged to slide along one another as shown in Fig. 145, which shows the method of finding the product $3 \times 2$ and the quotient $3 / 2$.

For the product $4 \times 5$, the above method gives a point out- 
EXPONENTIAL AND LOGARITHMIC FUNCTIONS 251

side the scale. To avoid this we divide one of the factors by 10 , then multiply by the other factor and move the decimal point one place to the right in the result.
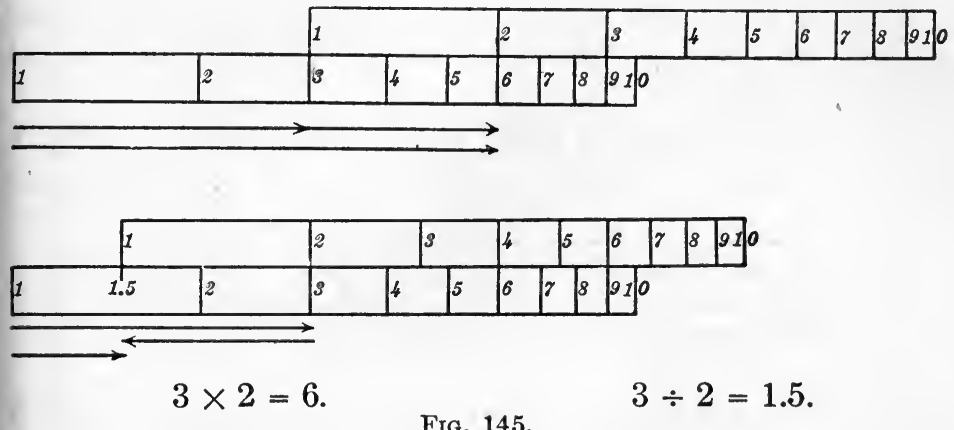

To find the quotient $4 / 5$, divide 10 by 5 , multiply by 4 and move the decimal point one place to the left in the result. The following figures illustrate the methods:
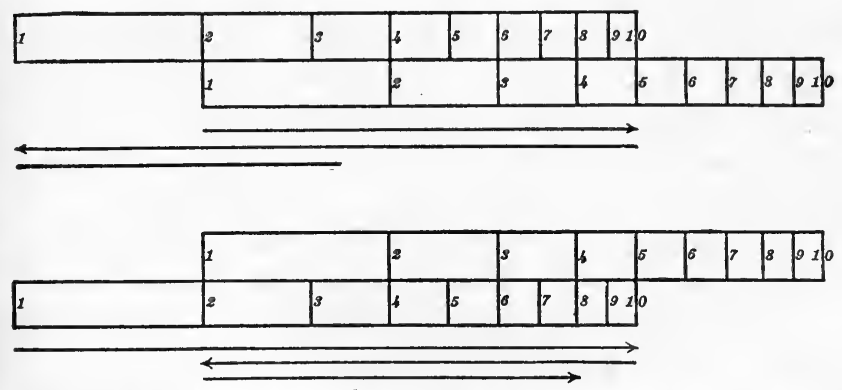

$$
4 \times 5=\left(\frac{5}{10} \times 4\right) 10=20 . \quad \frac{4}{5}=\left(\frac{10}{5} \times 4\right)_{\frac{1}{10}}=0.8 .
$$

Fig. 146.

The logarithmic scale finds practical application in the slide rule, an instrument much used by engineers in calculations.

The slide rule consists of a ruler with a central portion which slides back and forth in grooves on the rule, the slide and rule 
being graduated with equal logarithmic scales which are labeled with the corresponding numbers.

The ordinary slide rule has four scales $A, B, C, D$, as shown in the figure.

Each of the scales $C$ and $D$ is a single logarithmic scale. Each of the scales $A$ and $B$ consists of two logarithmic scales, which are equal.

If we give to the left index on all four scales the value 1 , then the scales $A$ and $B$ extend from 1 to 100 while scales

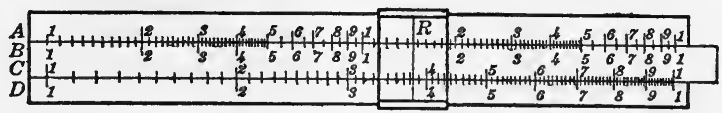

FIG. 147.

$C$ and $D$ extend from 1 to 10 . Hence the scales $A$ and $B$, numerically considered, are twice as long as scales $C$ and $D$, and a number on the upper scales is the square of the number below it on the lower scales. Conversely, a number on the lower scales is the square root of the number directly above it on the upper scales.

The methods employed to keep the result on the scales in calculations of the type $4 \times 5$ and $4 / 5$ are not necessary if the upper scales are used. The lower scales give more accurate results, since the graduations are not so fine as those of the upper scales.

The instrument is provided with a runner, $R$, which enables coinciding points to be found on any of the scales, and also permits of extensive calculations being worked out without the necessity of recording the intermediate results.

The slide rule makes use of matissas only, the characteristic being determined by inspection.

Logarithmic scales are also used on the axes of cross-sectior paper. If the scales on both axes are logarithmic the cross section paper is called logarithmic paper. If the scale on on of the axes is logarithmic and on the other is uniform it is calle semi-logarithmic paper. The methods of using these cross section papers are shown in the following examples. 
Example 1. Plot the graph of the equation obtained by taking logarithms of both sides of the equation $y=3 x^{2}$.

The required equation is $\log y=\log 3+2 \log x$.

Letting $\log y=Y$ and $\log x=X$, we have $Y=2 X+\log 3$, which is a linear equation, and hence its graph is the straight line whose slope is 2 and whose intercept on the $Y$-axis is $\log 3$. The tables give corresponding pairs of values of $x, y$ and of $X, Y$, and the figures show corresponding parts of the graphs. Only the part of the graph of $y=3 x^{2}$ in the first quadrant corresponds to the graph in the $X, Y$ system, as the logarithm of a negative number is not a real number.
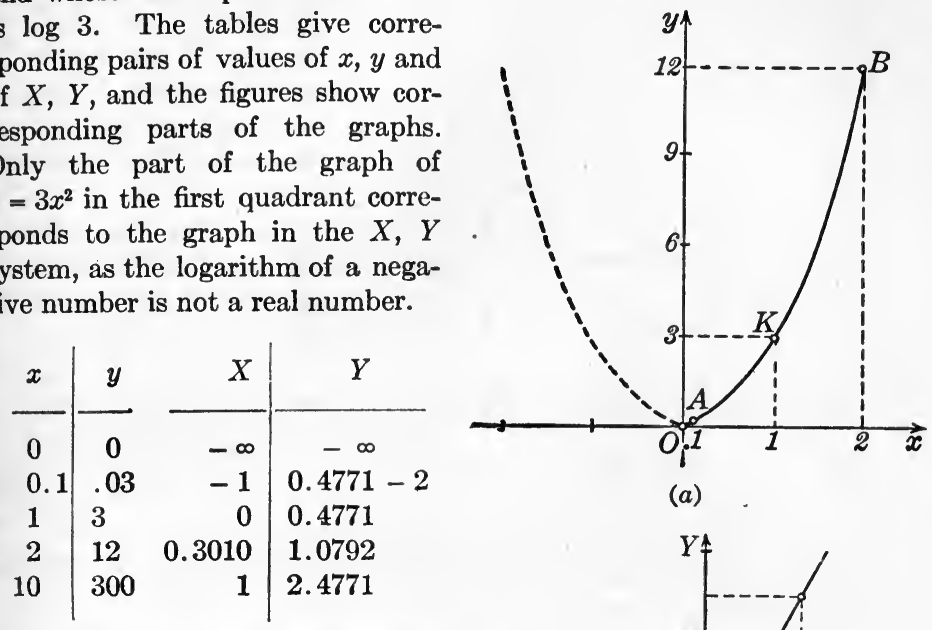

(a)

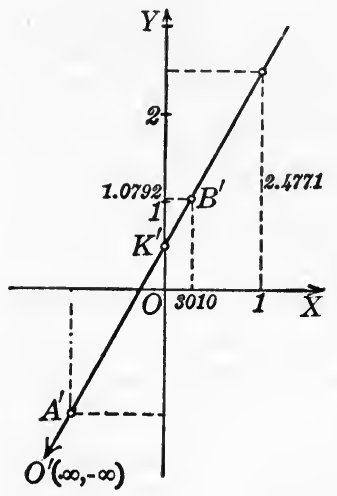

(b)

Fig. 148. axes, the unit lengths 1 to 10 being the same, and $X$ and $Y$ the numbers on the uniform scales attached to the figure, so that $X=\log x$ and $Y=\log y$.

We follow the same procedure as in plotting the graph of the equation on ordinary cross-section paper, except that negative values of $x$ and $y$ are not used, and the lines through the point $(1,1)$ are usually chosen as the axes since $\log 1=0$. 


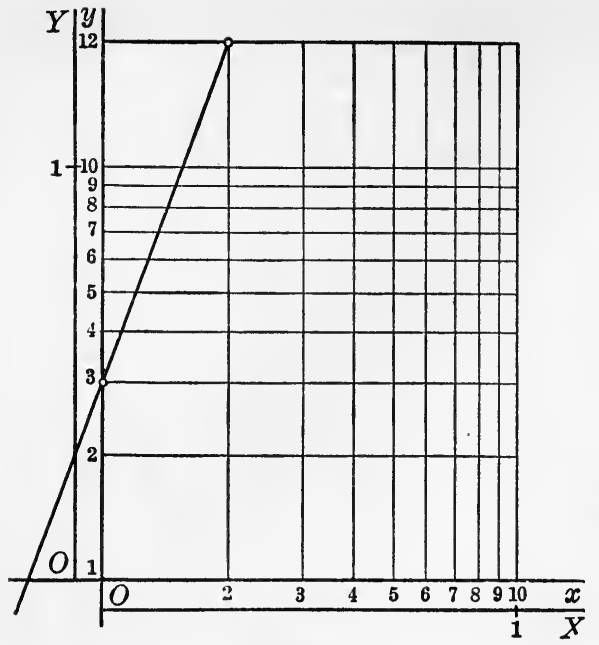

FIG. 149. \begin{tabular}{c|rr}
$x$ & $y$ & $\begin{array}{r}\text { The point }(1,3) \\
\text { will be at the inter- }\end{array}$ \\
- & - & section of the lines \\
1 & 3 & $\begin{array}{l}x=1 \text { and } y=3 . \\
2\end{array}$ \\
12 & The points cor-
\end{tabular} responding to other pairs of numbers are similarly plotted. These points lie on the straight line whose equation $Y=2 X+\log 3$ was obtained in the preceding example.

Note that the uniform scales and not the logarithmic scales determine the slope. Thus

$$
\begin{aligned}
m & =\frac{\log 12-\log 3}{\log 2-\log 1}=\frac{\log 4}{\log 2} \\
& =\frac{\log 2^{2}}{\log 2}=\frac{2 \log 2}{\log 2}=2 .
\end{aligned}
$$

Theorem 1. The graph of the power function, $y=k x^{n}$, plotted on logarithmic paper is the straight line whose slope is $n$ and whose intercept on the $y$-axis is $k$.

Let $X$ and $Y$ represent numbers on uniform scales attached to the axes so that $X=\log x$, and $Y=\log y$.

Taking logarithms of both sides of the equation $y=k x^{n} \quad$ we have $\log y=\log k+n \log x$, or $Y=n X+K$, the graph of which referred to the uniform scales is the straight line with slope $n$ and intercept $K$. Hence,

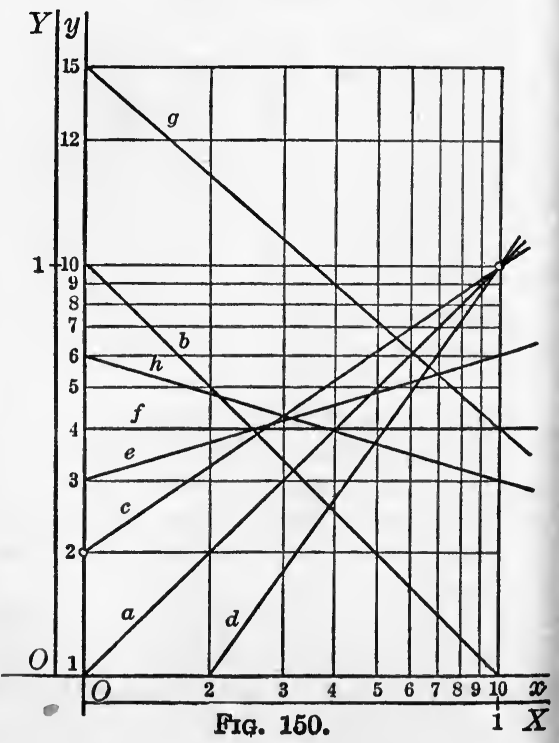


using the notation of the logarithmic scales in place of $X, Y$ and $K$, we have the theorem.

Example 3. Find the equation of the line marked $c$ in Fig. 150.

The intercept on the $y$-axis is 2 . Since the line passes through the points $(1,2)$ and $(10,10)$ the slope is

$$
m=\frac{\log 10-\log 2}{\log 10-\log 1}=\frac{\log 5}{\log 10}=\frac{0.6990}{1}=0.6990 .
$$

Hence the equation is $y=2\left(10^{0.6990 x}\right)$.

EXample 4. Plot the graph of $y=3\left(10^{0.5 x}\right)$ on semi-logarithmic paper with the scale on the $x$-axis uniform and on the $y$-axis logarithmic.

Let $Y$ represent numbers on a uniform scale attached to the $y$-axis, so that $Y=\log y$.

The unit line on the $x$-axis is equal to the unit segment 1 to 10 on the $y$-axis.

Constructing the table of values and plotting the points as usual, we have the graph in the figure.

\begin{tabular}{r|c}
\multicolumn{1}{c|}{} & $y$ \\
\hline 0 & 3.00 \\
0.5 & 5.33 \\
1.0 & 9.49
\end{tabular}

Taking the logarithms of both sides of the equation $y=3 \cdot 10^{0.5 x}$, we obtain the equation

$$
\log y=\log 3+0.5 x
$$

or

$$
Y=0.5 x+\log 3,
$$

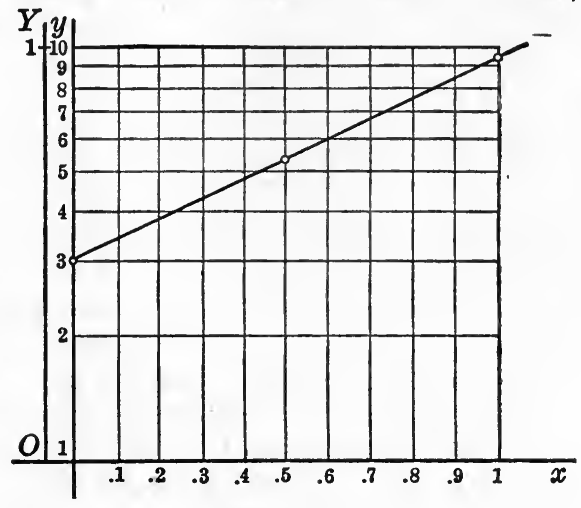

Fig. 151.

whose graph is a straight line referred to the uniform scales on the axes. Hence the graph of $y=3 \cdot 10^{0.5 x}$ plotted on the semi-logarithmic paper is the straight line whose slope is 0.5 and whose intercept on the $y$-axis is $\log 3$.

Note that the uniform scales determine the slope.

Theorem 2. The graph of the exponential function $y=k 10^{m x}$ plotted on semi-logarithmic paper is a straight line whose slope is $m$ and whose intercept on the $y$-axis is $k$.

The proof of this theorem is left as an exercise. 


\section{EXERCISES}

1. Make a paper slide rule by copying the logarithmic scale given in Fig. 144 on two paper rules of an adequate degree of stiffness. With this instrument calculate the following and describe the process.

$$
4 \times 6, \frac{8}{3}, \frac{5}{8}, 6 \times 9,(5 \times 7) /(8 \times 9) .
$$

2. Write the equations of the lines $a, b, c, \ldots g$, in Fig. 150.

3. Write the equations of the lines in Fig. 152.

4. Plot the graphs of the following equations on logarithmic paper.

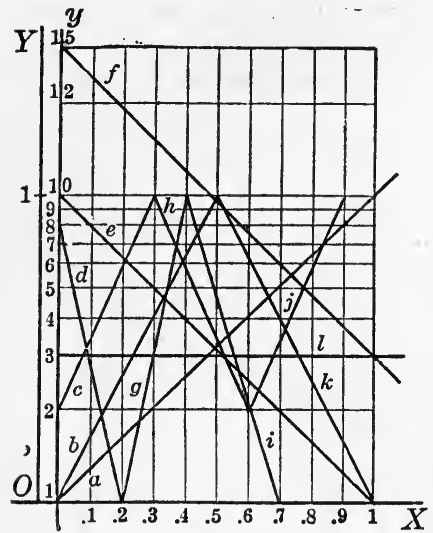

Fig. 152. (a) $y=x^{3}$

(c) $y=2 x^{\frac{1}{2}}$,

(e) $y=3 x$,

(g) $p v=4$, (b) $y=3 x^{2}$,

(d) $y=\frac{1}{2} x^{\frac{3}{2}}$,

$$
y=\frac{4}{x^{2}},
$$

(h) $p v^{\sqrt{2}}=1$.

5. Plot the graphs of the following equations on semi-logarithmic paper.

(a) $y=2\left(10^{2 x}\right)$,

(c) $y=\frac{1}{2}\left(10^{3} x\right)$,

(b) $y=3\left(10^{-2 x}\right)$,

(d) $y=e^{2 x}$.

6. Measure the length of the logarithmic scale in Fig. 144 and calculate the radius of a circle whose circumference is equal to this length. Fasten a circular disk of this radius to a larger disk by means of a pin through their centers so that they may rotate freely. On the circumference of the smaller disk and on the circle of equal radius of the larger copy the logarithmic scale of Fig. 144. Make the calculations of Exercise 1 with this instrument. What advantage has the circular slide rule over the slide rule, of the first exercise?

7. Find the equations of the lines in Fig. 150 if the given ranges of the scales are as follows:
(a) $x$-axis, 10 to 100 ,
(b) 0.1 to 1 ,
(c) 0.01 to 0.1 , $y$-axis, 1 to 10 .
10.0 to 100 .
0.1 to 1.0 .

8. Find the equations of the lines in Exercise 3 if the ranges of the scales are as follows:
$x$-axis,
(a) 10 to 20 ,
(b) -5 to 0 ,
(c) 4 to 8 ,
$y$-axis,
0.01 to 0.1 .
10 to 100 .
100 to 1000 .

90. Empirical Data Problems. If the points representing a given table of empirical data appear to lie on the graph of 
an equation of the form $y=k 10^{m x}$, the constants $k$ and $m$ may be determined as in the

Example. Determine the law representing the table, and find the value of $y$ if $x=2.5$. $\begin{gathered}\text { Plotting the points whose coördinates are } \\ \text { the pairs of values in the table we get a curve }\end{gathered} \mid \frac{1,2, \quad 3, \quad 4}{2.37,3.75,5.97,9.45}$ which appears to be of the form

$$
y=k 10^{m x} \text {. }
$$

To test this assumption, we plot the table on semi-logarithmic paper. As the points obtained lie very nearly on a straight line we conclude that

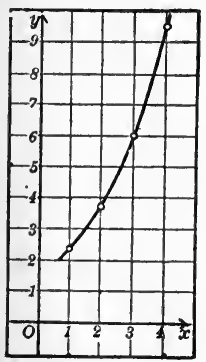

Fig. 153. the law may be represented by an equation of the form (1) with a fair degree of accuracy (Theorem 2, Section 89).

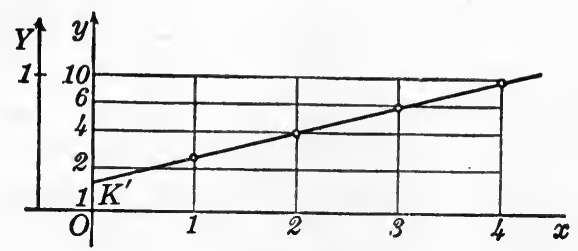

Fig. 154.

To determine $k$ and $m$, equate the logarithms of both sides of (1), which gives

or

$$
\begin{aligned}
\log y & =m x+\log k \\
Y & =m x+K,
\end{aligned}
$$

where

$$
Y=\log y \quad \text { and } \quad K=\log k .
$$

Equation (3) is linear in the variables $x$ and $Y$, and the constants $m$ and $K$ can be determined by the method in Section 27, page 78, from a table of values of $x$ and $Y$. We therefore look up the logarithms of the given values of $y$, which are the values of $Y$. From this table we obtain, by the method referred to, the values $m=0.200$ and $K=0.175$. Since $K=\log k=\frac{x}{Y}$
0.175 , reference to the table shows that $k=1.49$. Substituting the values of $k$ and $m$ in (1), we have the required law,

$$
\text { If } x=2.5, \quad y=1.49\left(10^{0.200} \times 2.5\right)=4.73 .
$$

If the points representing a given table appear to lie on the graph of an equation of the form $y=k x^{n}$ (page 119), a value of $n$ may be chosen by means of the form of the graph, and the 
value of $k$ determined, as in Section 44, page 127 . The method is unsatisfactory in that the only way to tell which of two values of $n$ is the better, for example, whether $n=2$ or $n=\frac{4}{3}$ is the better, is to try them both. The following procedure is preferable.

If the points representing the table appear to lie on the graph of an equation of the form

$$
y=k x^{n},
$$

plot the points on logarithmic paper. If the graph is now approximately straight (Theorem 1 , Section 89 ), we conclude that the law may be well represented by (5). As the logarithms of the two sides of (5) must be equal, we have

$$
\text { or } \quad \begin{aligned}
\log y & =n \log x+\log k, \\
Y & =n X+K,
\end{aligned}
$$

where $Y=\log y, X=\log x$, and $K=\log k$.

With the table of logarithms build the table of values of $X$ and $Y$ corresponding to the given table of values of $x$ and $y$. From it the values of $n$ and $K$ may be found by the method in Section 27, page 78. The value of $k$ is then found from that of $K$.

It should be noticed that if we suspect that the law has the form (1) or (5) it is not essential that the table should be plotted on semi-logarithmic or logarithmic paper, respectively. But this plotting strengthens our feeling of having a suitable law, and approximate values of the constants may be obtained easily directly from the gra ph (see Example 3, Section 89, and Exercises 2 and 3 of the preceding set). Of course, if the new graph is not approximately straight the choice of one of these two laws does not give a very good representation of the given data.

If the points representing the table, plotted on ordinary crosssection paper, appear to lie on the graph of

$$
y=c \log n x,
$$

we interchange $x$ and $y$, and proceed as in the example above.

The graph of (5) may be distinguished from that of (1) or 
(8) by the fact that it passes through the origin, if $n$ is positive, and that it does not cut either axis, if $n$ is negative.

\section{EXERCISES}

1. Find the law of growth of the population of the United States from the following data:

$\frac{\text { Year }}{\text { Population in millions }} \mid$\begin{tabular}{cccc}
1880, & 1890, & 1900, & 1910, \\
\hline 50, & 62, & 76, & 92,
\end{tabular}

Let $x=0$ in 1880. Assuming the law of growth does not change find the population in 1920.

2. Water flows out of a sharp-edged circular opening in the vertical side of a tank. The table gives pairs of values of the head $h$, the height in feet of the surface of the water above the center of the opening, and the discharge $Q$, the number of cubic feet of water flowing through the opening in a second. Determine $Q$ as a function of $h$.

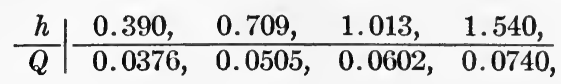

3. A rectangular weir is a rectangular notch in the wall of a reservoir or channel. The table gives pairs of values of the head $h$, the height in feet of the surface of the water above the bottom of the notch, and the discharge $Q$ in cubic feet per second. Find the law. Find $Q$ if $h=0.265$.

$$
\frac{h}{Q} \mid \begin{array}{llll}
0.053, & 0.103, & 0.160, & 0.217, \\
0.0422, & 0.1095, & 0.2065, & 0.3200,
\end{array}
$$

4. A triangular weir is a triangular notch in the side of a tank or channel. The table gives pairs of values of the head, $h$, and the discharge, $Q$. The angle of the notch in this case was $90^{\circ}$. Find the law.

$$
\frac{h}{Q} \mid \begin{array}{lllll}
0.095, & 0.169, & 0.249, & 0.330, & 0.392, \\
\hline 0.00615, & 0.0302, & 0.0780, & 0.1590, & 0.2445,
\end{array}
$$

5. In a chemical experiment it was found that the concentration $x$ of a solution was connected with the amount of precipitation $y$ of a metal as in the table. Determine the law and find $y$ if $x=5$.

$$
\frac{x}{y} \mid \begin{array}{rrrr}
1, & 2,4, \quad 8,
\end{array}
$$

6. Find the velocity, $v$, of a certain chemical reaction as a function of the temperature, $T$, from the data given in the table. Find $v$ if $T=50^{\circ}$.

$$
\frac{T}{v} \mid \begin{array}{ccccc}
0^{\circ}, & 10, & 20, & 30, & 40, \\
\hline 1.00, & 2.08, & 4.32, & 8.38, & 16.19
\end{array}
$$


7. Find the law connecting the maximum speed, $v$, of an electric vehicle and the total weight, $W$, in thousand pounds from the following data. What weight will reduce the maximum to 6 miles per hour?

$$
\frac{W}{v} \mid \begin{array}{ccccc}
2.0, & 3.0, & 4.2, & 6.5, & 8.5 \\
\hline 20, & 14, & 12, & 10, & .8,
\end{array}
$$

8. Find the law connecting the cost, $C$, in cents per miles for tires, repairs, battery, electricity, of electric vehicles of weight, $W$, in thousand pounds. What will be the cost per mile of a vehicle weighing 10,000 ?

$\frac{W}{C} \mid$\begin{tabular}{cccc}
1, & 2, & 4, & 7, \\
\hline 7.9, & 9.1, & 11.5, & 16.0
\end{tabular}

9. From the table find the law connecting the resistance, $R$ (in pounds per ton), which a passenger train encounters at a speed, $v$ (miles per hour). Find $R$ if $v=60$.

$$
\frac{v}{R} \mid \begin{array}{lrrrrr}
5, & 10, & 15, & 20, & 25, & 35, \\
\hline 5.9, & 5.5, & 5.4, & 5.5, & 5.6, & 6.2,
\end{array}
$$

10. Airships are provided with air bags within their hulls for regulating the height of ascent. The total cubic contents of these air bags when filled is $V^{\prime}=m V$ where $V$ is the cubic contents of the airship. Values of $m$ for various heights, $H$, are given in the table. Find the law and the value of $m$ when $H=8$.

$$
\frac{H \text { in thousand ft. }}{m=V^{\prime} / V} \mid \begin{array}{cccc}
1, & 2, & 4, & 10, \\
0.04, & 0.075, & 0.141, & 0.318,
\end{array}
$$

11. Find the law connecting the load, $L$ (in pounds per square foot of wing) of an aeroplane with the area, $A$, of wings (in square feet). Find the safe load if $A=200$.

$$
\frac{A}{L} \mid \begin{array}{llll}
150, & 300, & 400, & 700,
\end{array}
$$

\section{MISCELLANEOUS EXERCISES}

1. The temperature of a body cooling according to Newton's law, $\theta=\theta_{0} e^{-m t}$, fell from $125^{\circ}$ to $94^{\circ}$ in 8 minutes. Find the equation connecting the temperature and the time of cooling.

2. Construct a scale for the function $x^{2}$ following the method used in constructing the logarithmic scale. Find by means of the scale $(2.3)^{2}$ and $\sqrt{11}$.

3. Construct a table of values for the function $y=2 x^{2}+3$ and plot the graph. Let $u=x^{2}$ and hence $y=2 u+3$. Construct on the horizontal axis a uniform scale of $u$ and a corresponding non-uniform scale of $x$. Plot the table of values of $x$ and $y$ using the non-uniform scale of $x$ and the uniform scale of $y$. What is the character of the graph? 
4. Determine scales on the axes so that the graph of $y=3\left(10^{0.01 x^{2}}\right)$ is a straight line.

5. Determine the constants in $y=k 10^{m x^{2}}$ by means of the pairs of values $(2,0.5)$ and $(3,0.8)$. Choose scales on the axes so that the graph of the equation is a straight line. From the graph read the value of $y$ if $x=2.5$.

6. Find the radius of the moon in miles, given that the diameter of the moon subtends an angle of $32^{\prime}$ as seen from the earth, the distance of the moon from the earth being 240,000 . Find the ratio of the mass of the moon to that of the earth if the density of the moon is 0.6 that of the earth.

7. If the distance of the sun from the earth is $92,800,000$ miles and the diameter of the sun subtends an angle of $32.4^{\prime}$ as seen from the earth, find the diameter of the sun, and the ratio of the volume of the sun to that of the earth.

8. If the length, $l$, of a range finder is 20 feet and the distances, $d$, are calculated by the formula $d=l \tan \theta$, find the values of $\theta$ corresponding to the extreme values $400 \mathrm{ft}$. and $20,000 \mathrm{ft}$. on the dial of the instrument. (Use the "log rad" table on page 28 of the Tables.)

9. The following table gives the collegiate running records, the distance $d$ being in yards and the time $t$ in seconds. Find the law and the value of $t$ if $d=600$. Find $t$ if $d=3520$.

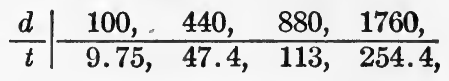

10. An observer on a destroyer moving at the rate of 35 miles an hour notes that the line of sight to a ship makes an angle of $41^{\circ} .56$ with the forward path of the destroyer and that one minute later the angle is $74^{\circ} .83$. He estimates that the ship is moving on a parallel path at the rate of 14 miles an hour. Find the distance between the paths.

11. The economic law of diminishing utility is stated as follows:

The total utility of a thing to any one (that is, the total pleasure or other benefit the thing yields) increases with any increase in one's stock of it but not so fast as the stock increases. If one's stock increases at a uniform rate, the benefit derived from it increases at a diminishing rate.

Another way of stating the law is: The increase in the utility of a thing, or marginal utility, diminishes with every increase in the amount of it any one already has.

Plot the graphs of these two statements of the law.

12. Find approximate values of the real roots of the following equations, which can not be solved by the ordinary methods.

Hint. Plot the graph of the function on the left and from it locate an intersection with the $x$-axis as accurately as possible. Enlarge the table of values until the coördinates of two points are obtained such that the intersection lies between them and such that the part of the graph 
between them may be assumed straight. Then determine an approximate value of the root by interpolation.

(a) $f(x)=2^{-x}-\frac{1}{3} x=0$. Solution. The graph of $f(x)$ shows that there is a root between 1 and 2, near 1.2. Enlarging the table of values, it is seen that the root is between 1.2 and 1.3, since the corresponding values of $f(x)$ have opposite signs.

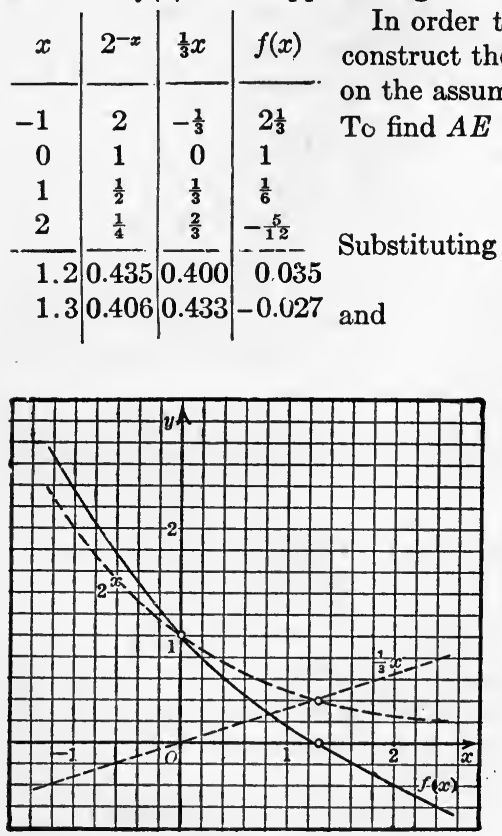

Fig. 155. we get

whence
$A B=0.035$,

$D F=0.027+0.035=0.062$,

Then the abscissa of $E$ is $x=1.2$ $+0.056=1.256$, which is an approximate value of the root of the given equation.

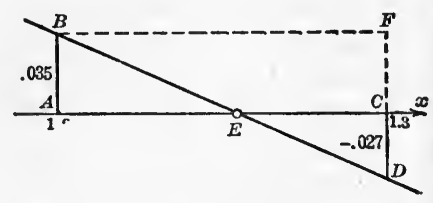

FIG. 156.
(b) $2^{-x}-x=0$.
(c) $3^{-x} / 2-x=0$.
(d) $2^{-x}-x / 2-1=0$.
(e) $4^{-x}-x / 5=0$.
(f) $\sin x-3 x / 4=0$.
(g) $2 \sin x-3 x / 2=0$.
(h) $\cos x-x=0$.
(i) $\tan x-2 x=0$.
(j) $\sin x-x^{2}=0$.
(k) $2^{-x}-\sin x=0$ (smallest positive root).
(l) $\cos x-2^{-x}=0$ (smallest positive root).
(m) $e^{-x}-x=0$.
(n) $e^{-x}-x / 3=0$.
(o) $e^{-x} / 2-x / 3=0$.

13. Some properties of many functions can be expressed entirely in terms of the notation $f(x)$. If analogous properties of two functions can be so expressed an abstract point of view is obtained which gives a deeper insight into the differences between the functions. Establish the following relations: 
(a) If $f(x)=x^{2}$ or $\cos x, \quad f(-a)=f(a)$.

If $f(x)=x^{3}$ or $\sin x$,

$f(-a)=-f(a)$.

If $f(x)=e^{x}$,

$f(-a)=\frac{1}{f(a)}$.

(b) If $f(x)=x^{n}$,

$f(a b)=f(a) \times f(b)$.

If $f(x)=\log x$,

$f(a b)=f(a)+f(b)$.

(c) If $f(x)=x^{n}$,

$\frac{f(a)}{f(b)}=f\left(\frac{a}{b}\right)$.

If $f(x)=e^{x}$,

$\frac{f(a)}{f(b)}=f(a-b)$.

(d) If $f(x)=\sqrt{x}$,

$k f(a)=f\left(k^{2} a\right)$.

If $f(x)=\log x$,

$k f(a)=f\left(a^{k}\right)$.

14. The speed of an aeroplane is 80 miles an hour and the wind is blowing from the north with a velocity of 20 miles per hour. The pilot desires to move S. E. Find the direction in which he should head the machine and how fast he will move.

15. Solve the preceding exercise supposing that the pilot desires to sail $\mathrm{N}$. W. If the aeroplane can stay in the air 4 hours, find the greatest distance the pilot can sail S. E. and be able to return to the starting point.

16. The speed of an aeroplane is $\mathbf{1 0 0}$ miles per hour and the wind is blowing from the west. The plane can stay in the air 5 hours. How far can the pilot sail in the direction $10^{\circ} \mathrm{S}$. of $\mathrm{W}$. and return to the starting point? 


\section{CHAPTER VI}

\section{DIFFERENTIATION OF ALGEBRAIC FUNCTIONS}

91. Introduction. If $y$ is a function of $x$, the average rate of change of $y$ with respect to $x$ in any interval $\Delta x$ is $\Delta y / \Delta x$ (page 35 ). If the average rate of change is constant, its value is the rate of change of $y$ with respect to $x$ (Definition, page 48), and the graph of $y$ is a straight line (Theorem, page 50) whose slope is the rate of change. Numerous applications of uniform rate of change were given in Chapter II.

If the average rate of change, $\Delta y / \Delta x$, is not constant, then the rate of change of $y$ with respect to $x$ is defined to be the limit of $\Delta y / \Delta x$ as $\Delta x$ approaches zero (page 94). It is represented graphically by the slope $m$ of a line tangent to the graph of the function $y$.

In this chapter we shall consider this limit more formally than heretofore, and derive rules for finding it expeditiously if $y$ is an algebraic function of $x$. The applications are based either on the geometric interpretation of the limit of $\Delta y / \Delta x$ as the slope of the tangent line or on the physical interpretation of the limit as the rate of change of $y$ with respect to $x$.

The ideas to be considered in this chapter, and the one following, are among the most fundamental and far-reaching concepts in mathematics. They were developed by the famous Englishman Sir Isaac Newton (1642-1727) and the noted German Gottfried Wilhelm von Liebnitz (1646-1716), and a bitter controversy lasting for many years was waged over the question as to which one of these men should be accorded the honor of the discovery. Leibnitz was the first to publish some of his results, in 1684, but Newton had written a paper on the subject and submitted it to some of his friends in 1669. The 264 
followers of each claimed that the other had been guilty of claiming ideas not his own, but most historians of mathematics are agreed that the work of Newton and Leibnitz was independent. On the basis of the work of these men, there followed a period of rapid and extensive mathematical development.

Let us first consider the underlying concept of the limit of a variable.

92. Limits. By definition (footnote, page 93) the limit of a variable $x$ is a constant such that the numerical value of the difference between $x$ and $a$ becomes and remains as small as we please. This explains what is meant by saying that " $x$ approaches $a$ as a limit," or in more compact form, " $x$ approaches a." It is immaterial whether or not $x$ becomes equal to $a$.

A function of $x$ is a second variable $y$ (Definition, page 5). The functions we have studied are such that if $x$ approaches a limit, so also does $y$, provided that $y$ does not become infinite as $x$ approaches $a$. The notation

$$
\lim _{x \neq a} y=b
$$

is used to indicate that " the limit of $y$, as $x$ approaches $a$, is $b . " \quad T h i s$ means that the numerical value of the difference between $y$ and $b$ can be made as small as we please by taking $x$ sufficiently near to $a$.

Graphically, the difference between the ordinates $y$ and $b$ can be made as small as we please by making the difference between the abscissas $x$ and $a$ sufficiently small. In other words, $\Delta y=b-y$ approaches zero if $\Delta x=a-x$ approaches zero.

For example, the average rate of

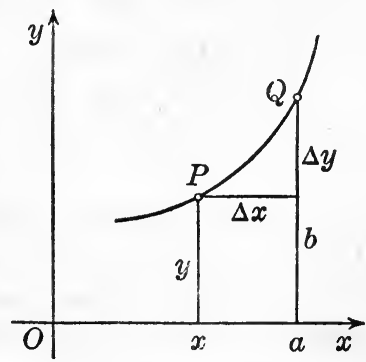

Fig. 157. change of the function $a x^{2}$ in an interval $\Delta x$ beginning at a definite point $x$ is (see (5), page 95)

$$
\frac{\Delta y}{\Delta x}=2 a x+a \Delta x
$$


The rate of change of $y$ with respect to $x$ for a given value of $x$ is therefore

$$
m=\lim _{\Delta x \doteq 0} \frac{\Delta y}{\Delta x}=\lim _{\Delta x \doteq 0}(2 a x+a \Delta x) .
$$

We are thus led to find the limit of $2 a x+a \Delta x$, a function of $\Delta x$, as $\Delta x$ approaches zero. In computing this limit, $x$ has a given value and is regarded as constant.

In order to prove the assumption made earlier that the limit is $2 a x$, it must be shown that $a \Delta x$, the difference between the variable $2 a x+a \Delta x$ and the constant $2 a x$, can be made as small as we please by making $\Delta x$ sufficiently small. This follows readily. For if we wish to make $a \Delta x$ as small as 0.001 , it is sufficient to take $\Delta x=0.001 / a$, which is possible since $\Delta x$ approaches zero and can therefore be made as small as we please.

The limits encountered in computing $\lim _{\Delta x \doteq 0} \frac{\Delta y}{\Delta x}$, where $y$ is a polynomial or a rational function of $x$, can be computed by means of the following theorems, which we assume without proof.

Theorem 1. The limit of the sum of several variables is the sum of their limits.

Theorem 2. The limit of the product of several variables is the product of their limits.

Theorem 3. The limit of the quotient of two variables is the quotient of their limits, provided the limit of the divisor is not zero.

The limit of the difference of two variables may be found by Theorem 1 , since $u-v=u+(-v)$.

In applying these theorems, it is frequently convenient to regard $y=c$, a constant, as a function of $x$, whose limit, as $x$ approaches $a$, is $c$.

EXAMPLE. If $y=x^{3}$, then

$$
\frac{\Delta y}{\Delta x}=3 x^{2}+3 x \Delta x+\Delta x^{2} .
$$

Give the details of the computation of the limit of $\Delta y / \Delta x$ as $\Delta x$ approaches zero. 
DIFFERENTIATION OF ALGEBRAIC FUNCTIONS 267

Denoting the limit by $m$, we have

$$
\begin{aligned}
& m=\lim _{\Delta x \doteq 0} \frac{\Delta y}{\Delta x}=\lim _{\Delta x \dot{=} 0}\left(3 x^{2}+3 x \Delta x+\Delta x^{2}\right) \\
& =\lim _{\Delta x \dot{=} 0} 3 x^{2}+\lim _{\Delta x \dot{=} 0} 3 x \Delta x+\lim _{\Delta x \dot{=} 0} \Delta x \Delta x \text { (Theorem 1) } \\
& =3 x^{2}+\lim _{\Delta x \dot{\doteq} 0} 3 x \lim _{\Delta x \dot{\circ} 0} \Delta x+\lim _{\Delta x \doteq 0} \Delta x \lim _{\Delta x \dot{\doteq} 0} \Delta x
\end{aligned}
$$

(Theorem 2)

$$
=3 x^{2} \text {. }
$$$$
\text { (Since } \lim _{\triangle x \doteq 0} \Delta x=0 \text { ) }
$$

93. Derivative of a Function. Definition. If $y$ is a function of $x$, and if $\Delta y$ is the change in $y$ corresponding to a change of $\Delta x$ in $x$, then the limit of $\Delta y / \Delta x$, as $\Delta x$ approaches zero, is called the derivative of $y$ with respect to $x$. Denoting it by $D_{x} y$ (read "the derivative of $y$ with respect to $x$ "), the definition may be expressed by the equation

$$
D_{x} y=\lim _{\Delta x \doteqdot 0} \frac{\Delta y}{\Delta x} .
$$

The results obtained in Section 33, page 94, may now be stated as follows:

The derivative of $y$ with respect to $x, D_{x} y$, measures the rate of change of $y$ with respect to $x$.

The derivative of $y$ with respect to $x$ is represented graphically by the slope of a line tangent to the graph of $y$. That is

$$
D_{x} y=m=\tan \theta .
$$

The process of finding the derivative of a function is called differentiation, and the succes-

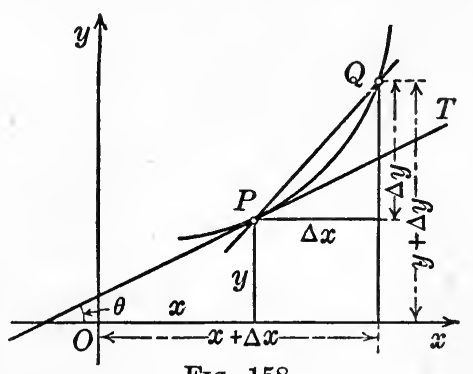

Fig. 158. sive steps in the process have been given in the section cited above.

Example. Differentiate $y=\frac{1}{x^{2}}$.

Substituting $x+\Delta x$ for $x$ and $y+\Delta y$ for $\mathrm{y}$,

$$
y+\Delta y=\frac{1}{(x+\Delta x)^{2}} .
$$


Subtracting the value of $y$,

$$
\Delta y=\frac{1}{(x+\Delta x)^{2}}-\frac{1}{x^{2}}=\frac{x^{2}-(x+\Delta x)^{2}}{x^{2}(x+\Delta x)^{2}}=\frac{-2 x \Delta x-\Delta x^{2}}{x^{2}(x+\Delta x)^{2}} .
$$

Dividing by $\Delta x$,

$$
\frac{\Delta y}{\Delta x}=\frac{-2 x-\Delta x}{x^{2}(x+\Delta x)^{2}}
$$

Passing to the limit as $\Delta x$ approaches zero,

$$
D_{x} y=\lim _{\Delta x \doteq 0} \frac{\Delta y}{\Delta x}=\lim _{\Delta x \doteq 0} \frac{-2 x-\Delta x}{x^{2}(x+\Delta x)^{2}}=\frac{\lim _{\Delta x \doteq 0}(-2 x-\Delta x)}{\lim _{\Delta x \doteq 0} x^{2}(x+\Delta x)^{2}}
$$

by Theorem 3 of the preceding section. Applying Theorem 1 in the numerator and Theorems 1 and 2 in the denominator, we get

$$
D_{x} y=-\frac{2 x}{x^{2} x^{2}}=-\frac{2}{x^{3}} \text {. }
$$

\section{EXERCISES}

1. Evaluate the following limits, indicating in detail the use of the theorems in Section 92 .
(a) $\lim _{x \doteqdot 0}\left(2-3 x+x^{2}\right)$.
(b) $\lim _{x \doteq 2} \frac{x^{2}-3 x}{x+1}$.
(c) $\lim _{\Delta x \doteq 0}(2 x+\Delta x-4)$.
(d) $\lim _{\Delta x \doteqdot 0}\left(3 x^{2}+3 x \Delta x+\Delta x^{2}-6 x-3 \Delta x\right)$.
(e) $\lim _{\Delta x \doteq 0} \frac{x^{2}+x \Delta x+1}{x(x+\Delta x)}$.
(f) $\lim _{\Delta x \doteq 0} \frac{2 x+\Delta x-x^{2}-x \Delta x}{x^{2}(x+\Delta x)^{2}}$.

2. Differentiate the functions:
(a) $y=x^{3}-3 x$.
(b) $y=1 / x$.
(c) $y=(x+1) / x$.
(d) $y=c$.
(e) $y=x$.
(f) $m x+b$.

(g) $y=\sqrt{x}$. Hint. Rationalize the numerator of $\Delta y / \Delta x$ before passing to the limit as $\Delta x$ approaches zero.

3. If $f(x)=\left(x^{2}+3 x\right) /\left(x^{2}-1\right)$, find $\lim _{x \doteq a} f(x)$. Note that the value obtained is $f(a)$, provided $a \neq \pm 1$. What happens if $a= \pm 1$ ?

4. If $f(x)=x^{2}$, prove the relations

$$
\lim _{x \doteq a} f(x)=f(a) \quad \text { and } \quad \lim _{\Delta x \doteq 0} \Delta y=0
$$

and interpret them graphically.

5. Prove that the relations in Exercise 4 are true if $f(x)$ is any quad. ratic function. 
6. If $f(x)$ is any function, and $\Delta x=a-x$, show that the first relation in Exercise 4 is true if and only if the second is true. What is the graphical significance of each relation?

Note. A function is said to be continuous at $x=a$ if the first relation in Exercise 4 is true for the given function. It can be proved that the algebraic and transcendental functions studied in this course are continuous for all values of $x$ for which they do not become infinite. If a function becomes infinite as $x$ approaches $a$, then $f(a)$ has no meaning. Hence $x=a$ is a point of discontinuity. There are other types of points of discontinuity.

7. Prove that (a) any polynomial, (b) any rational function, is continuous for all values of $a$, except, in (b), for the values for which the function becomes infinite.

8. If $u$ is a function of $x$, what is the value of $\lim _{\Delta x \doteq 0} \Delta u$ ? of $\lim _{\Delta x \doteq 0} \frac{\Delta u}{\Delta x}$ ? of $\lim _{\Delta x \doteq 0} \frac{\Delta u^{2}}{\Delta x}=\lim _{\triangle x \doteq 0} \Delta u \frac{\Delta u}{\Delta x} ?$

94. Fundamental Formulas for Differentiation. The rules in this section are useful in differentiating a function without the labor involved in computing the limit considered in Section 93.

Theorem 1. The derivative of a constant is zero; that is,

$$
D_{x} c=0 \text {. }
$$

For the graph of $y=c$ is a straight line parallel to the $x$-axis, whose slope, $m=D_{x} y=D_{x} c$, is zero.

Theorem 2. The derivative of the independent variable is unity, that is,

$$
D_{x} x=1 .
$$

For the graph of $y=x$ is the straight line bisecting the first and third quadrants, whose slope, $m=D_{x} y=D_{x} x$, is unity.

Theorem 3. The derivative of a constant

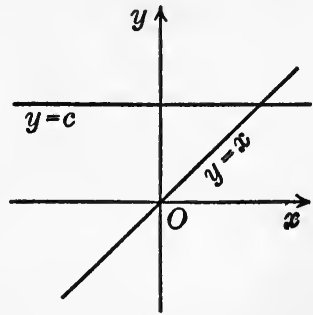

Fig. 159. times a function is equal to the constant times the derivative of the function. Symbolically, if $u$ is any function of $x$,

$$
D_{x} c u=c D_{x} u \text {. }
$$

Let

$$
y=c u \text {. }
$$


Then

and hence, subtracting,

Dividing by $\Delta x$,

$$
\begin{aligned}
y+\Delta y & =c(u+\Delta u), \\
\Delta y & =c \Delta u . \\
\frac{\Delta y}{\Delta x} & =c \frac{\Delta y}{\Delta x} .
\end{aligned}
$$

Hence $D_{x} y=\lim _{\Delta x \neq 0} \frac{\Delta y}{\Delta x}=\lim _{\Delta x \neq 0} c \frac{\Delta u}{\Delta x}=c \lim _{\Delta x \doteq 0} \frac{\Delta u}{\Delta x}=c D_{x} u$.

What is the graphical interpretation of this theorem, in the light of the Theorem on page 89 ?

Theorem 4. The derivative of the sum of two functions is equal to the sum of their derivatives. Symbolically, if $u$ and $v$ are any two functions of $x$

Let

Then

and hence

Dividing by $\Delta x$

$$
D_{x}(u+v)=D_{x} u+D_{x} v
$$

Therefore $\quad D_{x} y=\lim _{\Delta x \doteq 0} \frac{\Delta y}{\Delta x}=\lim _{\Delta x \doteq 0}\left(\frac{\Delta u}{\Delta x}+\frac{\Delta v}{\Delta x}\right)$

$$
=\lim _{\Delta x \doteq 0} \frac{\Delta u}{\Delta x}+\lim _{\Delta x \doteq 0} \frac{\Delta v}{\Delta x}=D_{x} u+D_{x} v .
$$

Corollary. The derivative of the sum of several functions is the sum of their derivatives.

Theorem 5. The derivative of the nth power of a function of $x$ is $n$ times the $(n-1)$ st power of the function times the derivative of the function with respect to $x$. Symbolically, if $u$ is any function of $x$

Let

$$
D_{x} u^{n}=n u^{n-1} D_{x} u \text {. }
$$

Then $y+\Delta y=(u+\Delta u)^{n}$

$$
=u^{n}+n u^{n-1} \Delta u+\frac{n(n-1)}{2} u^{n-2} \Delta u^{2}+\cdots+\Delta u^{n}{ }_{1}
$$

by the binomial theorem. Subtracting,

$$
\Delta y=n u^{n-1} \Delta u+\frac{n(n-1)}{2} u^{n-2} \Delta u^{2}+\cdots+\Delta u^{n} .
$$


DIFFERENTIATION OF ALGEBRAIC FUNCTIONS 271 Hence,

$$
\frac{\Delta y}{\Delta x}=n u^{n-1} \frac{\Delta u}{\Delta x}+\frac{n(n-1)}{2} u^{n-2} \frac{\Delta u}{\Delta x} \Delta u+\cdots+\frac{\Delta u}{\Delta x} \Delta u^{n-1}
$$

and therefore, passing to the limit as $\Delta x$ approaches zero,

$$
D_{x} y=n u^{n-1} D_{x} u \text {. }
$$

Corollary. The derivative of the nth power of $x$ is $n$ times the $(n-1)$ st power of $x$; that is,

$$
D_{x} x^{n}=n x^{n-1} \text {. }
$$

For, by (5), $D_{x} x^{n}=n x^{n-1} D_{x} x=n x^{n-1}$, since $D_{x} x=1$, by (2).

Example 1. Differentiate $y=x^{3}+3 x^{2}+5$.

We have

$$
\begin{aligned}
D_{x} y & =D_{x} x^{3}+D_{x} 3 x^{2}+D_{x} 5 \\
& =3 x^{2}+3 D_{x} x^{2}+0 \\
& =3 x^{2}+3 \cdot 2 x \\
& =3 x^{2}+6 x .
\end{aligned}
$$

Example 2. Differentiate $y=\left(x^{2}+4 x-5\right)^{3}$.

If we think of $x^{2}+4 x-5$ as a function $u$, the given equation has the form $y=u^{3}$. Applying (5).

$$
\begin{aligned}
D_{x} y & =D_{x}\left(x^{2}+4 x-5\right)^{3} \\
& =3\left(x^{2}+4 x-5\right)^{2} D_{x}\left(x^{2}+4 x-5\right) \\
& =3\left(x^{2}+4 x-5\right)^{2}\left[D_{x} x^{2}+D_{x} 4 x+D_{x}(-5)\right] \\
& =3\left(x^{2}+4 x-5\right)^{2}(2 x+4) .
\end{aligned}
$$

95. Derivative of a Polynomial. Any polynomial has the form (page 133)

$$
y=a_{0} x^{n}+a_{1} x^{n-1}+\cdots+a_{n-1} x+a_{n} .
$$

Then $\quad D_{x} y=D_{x}\left(a_{0} x^{n}+a_{1} x^{n-1}+\cdots \cdot+a_{n-1} x+a_{n}\right)$

$$
\begin{aligned}
& =D_{x}\left(a_{0} x^{n}\right)+D_{x}\left(a_{1} x^{n-1}\right)+\cdot \cdot+D_{x}\left(a_{n-1} x\right)+D_{x} a_{n} \\
& \quad \text { by the corollary to Theorem } 4 \text {, Section } 94, \\
& =a_{0} D_{x} x^{n}+a_{1} D_{x} x^{n-1}+\cdots \cdot+a_{n-1} D_{x} x+0
\end{aligned}
$$

by Theorems 3 and 1, Section 94 .

$$
=a_{0} n x^{n-1}+a_{1}(n-1) x^{n-2}+\cdots \cdot+a_{n-1}
$$

Hence,

by (5a), Section 94 .

The successive terms of the derivative of a polynomial may be found from the corresponding terms of the polynomial by multiplying the coefficient by the exponent and decreasing the exponent by 
one. In applying this rule, the constant term, whose derivative is zero, may be regarded as the coefficient of $x^{0}=1$.

For example, if then

$$
\begin{aligned}
y & =3 x^{4}-4 x^{3}+3 x-7, \\
D_{x} y & =12 x^{3}-12 x^{2}+3
\end{aligned}
$$

\section{Corresponding Properties of a Function, its Graph, and} its Derivative. The derivative is useful in expressing some of the properties given on page 42 , and some other properties also.

Rate of Change. The steepness of the graph at any point is measured by the slope of the tangent line, and the rate of change of the function is measured by the value of the derivative.

Changes of the Function. The graph rises (or falls) to the right if and only if the slope of the tangent line is positive (or negative). Hence

A function increases (or decreases) as $x$ increases if and only if its derivative is positive (or negative).

Maxima and Minima. A line tangent to the graph is horizontal if and only if its slope is zero. Hence,

To find the abscissas of the points at which the tangent line is horizontal, set the derivative equal to zero, and solve the resulting equation, $D_{x} y=0$, for $x$.

The roots of this equation may be values of $x$ for which the function has a maximum or minimum value.

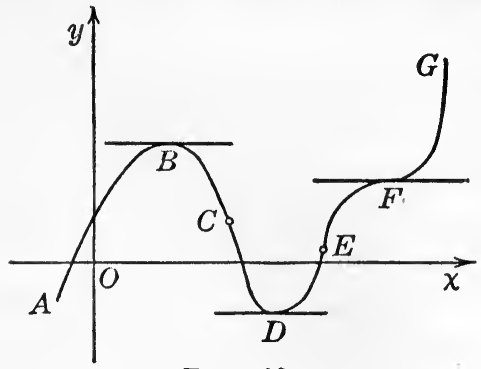

FIG. 160.

At a maximum point, the function ceases to increase and begins to decrease, and hence (see Changes of the Function above), as $x$ increases, the derivative must change sign from positive to negative.

Similarly, at a minimum point, as $x$ increases, the derivative must change sign, from negative to positive.

If, as $x$ increases in the vicinity of a horizontal tangent, the derivative does not change sign, the point of contact is neither a maximum nor a minimum point. 
For example, the graph of $y=x^{3}$ has a horizontal tangent at the origin (page 112), since $D_{x} y=3 x^{2}=0$ if $x=0$. But as $x$ increases through $x=0$, the derivative $3 x^{2}$ does not change sign, and the origin is therefore not a maximum or minimum point.

Concavity. The curve in the figure is concave downward from $A$ to $C$. If the tangent line at $A$ rolls along the curve until the moving point of contact reaches $C$, the slope $m$ decreases, and hence $D_{x} m$ is negative (see Changes of the Function above). Similarly, from $C$ to $E$ the curve is concave upward. The slope of a tangent line rolling from $C$ to $E$ increases, and $D_{x} m$ is positive. Hence

If the graph is concave upward (or downward), $D_{x} m$ is positive (or negative). It is also true that the curve is concave upward (or downward) only if $D_{x} m$ is positive (or negative).

It follows that $a$ point at which the tangent line is horizontal will be a minimum point if $D_{x} m$ is positive, or a maximum point if $D_{x} m$ is negative.

Since $m=D_{x} y$ is the rate of change of $y$, and since $m$ increases or decreases according as $D_{x} m$ is positive or negative, it follows that

The rate of change of a function increases or decreases, as $x$ increases, according as the graph of the function is concave upward or downward.

Points of Inflection. At a point of inflection (Definition, page 139) $m$ ceases to increase and begins to decrease, or vice versa. Hence $m$ has a maximum or minimum value, and therefore $D_{x} m=0$ (see Maxima and Minima above). The curve is concave upward on one side of a point of inflection and concave downward on the other (see Concavity).

Not every root of $D_{x} m=0$ is a point of inflection. It is necessary that as $x$ increases through the root in question $D_{x} m$ shall change sign (see Maxima and Minima). For example, if $y=x^{4}$, then $m=D_{x} y=4 x^{3}$ and $D_{x} m=12 x^{2} ; D_{x} m=0$ if $x=0$, but it does not change sign as $x$ increases through zero. Hence the origin is not a point of inflection. It is, in fact, a minimum point, as is apparent from the graph (page 112), 
or from the fact that $D_{x} y=4 x^{3}$ changes sign, from negative to positive, as $x$ increases through zero.

Since $m$ is the rate of change of $y$,

At a point of inflection on the graph of a function the rate of change of the function has a maximum or minimum value.

The corresponding properties of the graph of a function and the derivative considered in this section may be indicated as follows:

\section{Property of Graph}

Slope of tangent line.

Maximum or minimum point.

Graph rises or falls to the right.

Point of inflection.

Graph concave upward or downward.
Property of Derivative

Value of $D_{x} y$.

$D_{x} y=0$.

$D_{x} y$ is positive or negative.

Derivative of $m=D_{x} y$ is zero.

Derivative of $m$ is positive or negative.

This table supplements the table of corresponding properties of a function and its graph given in Section 15.

EXAMPLe. If

$$
y=x^{4}+x^{3}-3 x^{2}+4
$$

find the points of maxima, minima, and inflection. Plot the graphs of $y$, $m=D_{x} y$ and $D_{x} m$, and discuss their relations to each other.

Differentiating (1) $m=D_{x} y=4 x^{3}+3 x^{2}-6 x$, and differentiating (2) $\quad D_{x} m=12 x^{2}+6 x-6$.

Setting $D_{x} y=0$,

and solving for $x$,

$$
\begin{gathered}
4 x^{3}+3 x^{2}-6 x=0, \\
x=0,0.9,-1.7 .
\end{gathered}
$$

Since $\quad D_{x} m=6\left(2 x^{2}+x-1\right)=6(2 x-1)(x+1)$, the roots of $D_{x} m=0$ are $\quad x=\frac{1}{2}=0.5$, and -1 .

\begin{tabular}{c|c|c|c}
$x$ & $y$ & $D_{x} y$ & $D_{x} m$ \\
\cline { 1 - 3 } 0 & 4 & 0 & -6 \\
0.9 & 2.9 & 0 & 9.1 \\
-1.7 & -1.2 & 0 & 18.5 \\
0.5 & 3.4 & -1.8 & 0 \\
-1 & 1 & 5 & 0 \\
\hline-3 & 31 & -69 & 84 \\
-2 & 0 & -8 & 30 \\
1 & 3 & 1 & 12 \\
2 & 16 & 32 & 54
\end{tabular}

The values of $y, D_{x} y$, and $D_{x} m$ corresponding to the values of $x$ in (5) and (7), which are found by substitution in (1), (2), and (3), are given in the table.

The point $A(0,4)$ is a maximum point since the tangent line is horizontal $\left(D_{x} y=0\right)$ and the curve is concave downward $\left(D_{x} y<0\right)$. While the points $B(0.9,2.9)$ and $C(-1.7,-1.2)$ are minimum points, because the tangent line is horizontal $\left(D_{x} y=0\right)$ and the curve is concave upward $\left(D_{x} m>0\right)$.

As $x$ increases through 0.5 or -1 , in- 
spection of the changes in sign of the factors of (6) shows that $D_{x} m$ changes sign in each case. Hence the points $D(0.5,3.4)$ and $E(-1,1)$ are both points of inflection.

The table of values is then extended to include the necessary integral values (Section 50, page 137), the computation of the entire table being made by means of synthetic division and the remainder theorem. The graphs are then drawn.

The maximum and minimum points of $y, A, B, C$, are directly above or below the points $O, B^{\prime}, C^{\prime}$, at which $D_{x} y$ cuts the $x$-axis. The vertical

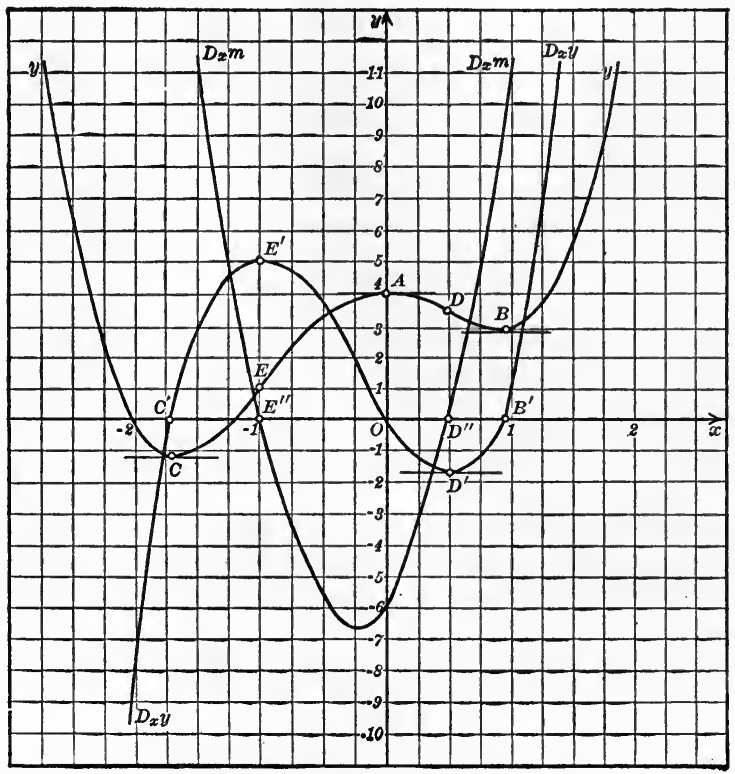

Fig. 161.

line through the maximum point $A$ cuts the graph of $D_{x} m$ below the $x$-axis and the vertical lines through the minimum points $B$ and $C$ cut it above the $x$-axis.

The points of inflection of $y, D$ and $E$, the minimum and maximum points of $D_{x} y, D^{\prime}$ and $E^{\prime}$, and the points at which $D_{x} m$ cuts the $x$-axis, $D^{\prime \prime}$ and $E^{\prime \prime}$, are respectively on the same vertical lines.

The graph of $y$ rises (from $C$ to $A$, and from $B$ to the right) or falls (from the left to $C$ and from $A$ to $B$ ) according as the graph of $D_{x} y$ lies above or below the $x$-axis. And the graph of $D_{x} y=m$ rises or falls according as that of $D_{x} m$ lies above or below the $x$-axis. 
The graph of $y$ is concave upward (from the left to $E$ and from $D$ to the right) or downward (from $E$ to $D$ ) according as the graph of $D_{x} m$ lies above or below the $x$-axis.

What further relations exist between the graphs of $D_{x} y$ and $D_{x} m$ ?

97. Velocity and Acceleration. The velocity of a body moving along a line is the rate of change with respect to the time of its distance $s$ measured along the line from a certain station (Section 32, page 93). Hence,

$$
v=D_{t} s \text {. }
$$

The acceleration $a$ of a moving body is defined to be the rate of change of its velocity $v$, and therefore

$$
a=D_{t} v .
$$

Example. The position of a body moving on a line at the time $t$ is given by

$$
s=t^{3}-5 t^{2}+2 t+8 .
$$

Find the velocity and acceleration at any time. Determine the position, velocity, and acceleration when $t=-1$. When is the body at rest?

Differentiating, we get

and

$$
\begin{aligned}
& v=D_{t} s=3 t^{2}-10 t+2, \\
& a=D_{t} v=6 t-10 .
\end{aligned}
$$

When $t=-1$, we find that $s=0, v=15$, and $a=-16$. Hence at that time the body passed through the station, moving in the positive direction at the rate of 15 feet per second, which was decreasing at the rate of 16 feet per second per second.

The body will be at rest at any instant at which $v=0$; that is when

$$
3 t^{2}-10 t+2=0 \text {. }
$$

Solving for $t$,

$$
t=\frac{5 \pm \sqrt{19}}{3}=0.2 \text { or } 3.2 \text { seconds. }
$$

\section{EXERCISES}

1. Differentiate the functions:
(a) $3 x^{4}-2 x^{3}-4 x^{2}+5 x-3$.
(b) $4 x^{3}-3 x^{2}+2 x-5$.
(c) $x^{3}\left(x^{2}+5 x+6\right)$.
(d) $(x-1)\left(x^{2}+x+1\right)$.
(e) $\frac{x^{3}-7 x^{2}+4}{5}$.
(f) $\frac{x-2}{3} \times \frac{x+1}{4}$.

2. Differentiate $y=\left(x^{2}-3\right)^{2}$ : (a) by applying (5), Section 94 , regarding $u=x^{2}-3$; (b) by first removing the parentheses. 
3. Differentiate the functions:
(a) $y=\left(x^{2}+2 x\right)^{3}$,
(b) $y=\left(2 x^{2}-3 x+7\right)^{2}$,
(c) $y=\left(x^{2}-4\right)^{5}$,
(d) $y=\left(3 x^{3}-5 x-3\right)^{3}$.

4. Find the slope of the line tangent to the graph of $y=x^{2}-4 x$ at any point $P(x, y)$; at the point for which $x=1$. Plot the graph, and draw the tangent at the latter point.

5. The distance fallen in $t$ seconds by a ball thrown downward with a velocity of 48 feet per second is $s=48 t+16 t^{2}$. Find the velocity at any time. How fast will it be moving when $t=3$ ?

6. A billiard ball rolling down a smooth plane inclined at a little less than half a degree moves according to the law

$$
S=\frac{1}{4} t^{2} .
$$

Find the velocity at any time. Plot the graphs of $s$ and $v$ on the same axes, using a large scale, from $t=0$ to $t=5$. From the graphs answer the following:

(a) What is the position and velocity of the ball after 4.5 seconds?

(b) With what velocity will the ball be moving after it has rolled 3 feet?

(c) How far must it roll to acquire a velocity of 2 feet per second?

(d) When is the distance equal to the velocity? How many solutions?

(e) Draw a tangent to the graph of $s$ and measure its slope. Measure the ordinate on the point on the graph of $v$ which has the same abscissa as the point of tangency. How do the two compare?

7. Find the points at which the tangents to the graph of $y=x^{3}-27 x$ are horizontal. Construct the figure.

8. The height after $t$ seconds of a body thrown vertically upward with a velocity of 96 feet per second is $s=96 t-16 t^{2}$. Find the velocity at any time. When will the velocity be zero? How high will the body rise?

9. Find the angles which the graph of $y=-x^{3}+5 x^{2}-6 x$ makes with the $x$-axis.

10. At what angles does the line $y=4 x$ cut the graph of $y=x^{2}$ ?

11. Oil dropped on the floor spreads out in a circle. Find the rate at which the circumference increases with respect to the radius, and the rate at which the area increases with respect to the radius.

12. The kinetic energy of a body of mass $m$ moving with a velocity $v$ is given by

$$
K=\frac{1}{2} m v^{2} .
$$

Find the rate at which the kinetic energy changes with respect to the velocity.

13. If $P$ is the pressure of a body on a surface and $F$ the friction between them, what does the derivative of $F$ with respect to $P$ represent?

14. If $s$ is a quadratic function of $t$, show that the acceleration is uniform (constant). 
15. Find the points of maxima, minima, and inflection on the graphs of each of the equations below. On the same axes, plot the graphs of $y$. $m=D_{x} y$, and $D_{x} m$, and discuss the relations between them.

(a) $y=x^{2}-4 x+5$.

(b) $y=x^{3}+2 x^{2}-4 x-3$.

(c) $y=x^{3}-6 x^{2}+12 x+3$.

(d) $y=x^{3}+3 x^{2}+5 x-2$.

(e) $y=3 x^{4}-4 x^{3}-6 x^{2}+12 x-2$.

(f) $y=x^{4}-x^{3}-2 x+3 x-1$.

(g) $y=x^{3}-5 x^{2}+2 x+8$.

16. The distance from the starting point, after $t$ seconds, to a ball rolled up a plane inclined at a little less than half a degree with an initial velocity of 8 feet per second is

$$
s=-\frac{1}{4} t^{2}+8 t \text {. }
$$

How far will it roll up the plane, and how fast will it be moving when it returns to the starting point?

17. The position of a body moving on a line is given by one of the equations following. Find the velocity and acceleration at any time. On the same axes plot the graphs of $s, v$, and $a$, and discuss the motion with reference to each of them.
(a) $s=t^{2}-4 t+4$.
(b) $s=t^{3}-6 t^{2}+12 t-8$.
(c) $s=t^{3}+t^{2}-6 t$.
(d) $s=2 t^{4}-8 t^{3}-9 t^{2}+54 t$.

18. Find the largest possible number of horizontal tangents to the graph of a polynomial of degree $n$; the largest possible number of points of inflection.

19. If the graph of a function $y$ is concave toward the $x$-axis, show that $y$ and $D_{x} m$ have opposite signs.

20. If $y=a x^{3}+d x^{2}+c x+d$, show that the abscissas of the points on the graph at which the tangent line is horizontal satisfy a quadratic equation. Find the condition that the number of horizontal tangents is two, one, or zero. Apply this condition to determine the number of horizontal tangents in $15, b, c, d$.

21. If $y=u v$, show that $D_{x} y=u D_{x} v+v D_{x} u$.

98. Derivative of a Rational Function. In order to differentiate any rational function we need but one more rule.

Theorem. The derivative of a fraction is a fraction whose numerator is the denominator times the derivative of the numerator less the numerator times the derivative of the denominator, and whose denominator is the square of the denominator. Symbolically,

$$
D_{x} \frac{u}{v}=\frac{v D_{x} u-u D_{x} v}{v^{2}} .
$$

Let.

$$
y=\frac{u}{v}
$$


DIFFERENTIATION OF ALGEBRAIC FUNCTIONS 279

Then

whence

$$
y+\Delta y=\frac{u+\Delta u}{v+\Delta v}
$$

$$
\Delta y=\frac{u+\Delta u}{v+\Delta v}-\frac{u}{v}=\frac{u v+v \Delta u-u v-u \Delta v}{v(v+\Delta v)}=\frac{v \Delta u-u \Delta v}{v(v+\Delta v)} .
$$

Hence

$$
\frac{\Delta y}{\Delta x}=\frac{v \frac{\Delta u}{\Delta x}-u \frac{\Delta y}{\Delta x}}{v(v+\Delta v)} .
$$

Finding the limit as $\Delta x$ approaches zero, we get

Example 1.

$$
D_{x} y=\frac{v D_{x} u-u D_{x} v}{v^{2}} .
$$

$$
\begin{aligned}
D_{x}\left(\frac{3 x-4}{x^{2}+5}\right) & =\frac{\left(x^{2}+5\right) D_{x}(3 x-4)-(3 x-4) D_{x}\left(x^{2}+5\right)}{\left(x^{2}+5\right)^{2}} \\
& =\frac{\left(x^{2}+5\right)(3)-(3 x-4)(2 x)}{\left(x^{2}+5\right)^{2}} \\
& =\frac{-3 x^{2}+8 x+15}{\left(x^{2}+5\right)^{2}} .
\end{aligned}
$$

After one fixes the rule in mind, it becomes easy to write down the second fraction on the right without taking the time to write out the first.

Example 2. Find the derivative of $y=1 / u^{n}$, where $u$ is a function of $x$.

We have

$$
\begin{aligned}
D_{x} \frac{1}{u^{n}} & =\frac{u^{n} D_{x} 1-1 D_{x} u^{n}}{u^{2 n}} \\
& =\frac{0-n u^{n-1} D_{x} u}{u^{2 n}} \text { by (1) and (5), Section } 94 . \\
& =\frac{-n D_{x} u}{u^{n+1}} .
\end{aligned}
$$

Since the result obtained in Example 2 may be written in the form

$$
D_{x}\left(u^{-n}\right)=-n u^{-n-1} D_{x} u,
$$

we see that Theorem 5, Section 94, holds for negative as well as positive, integral values of the exponent $n$.

99. Derivative of an Irrational Function. In order to differentiate an irrational function it is sufficient to show that Theorem 5, Section 94, holds also for a fractional exponent, $n=p / q$. 
Let

$$
y=u^{p / q}
$$

Raising both sides to the $q$ th power,

$$
y^{q}=u^{p} \text {. }
$$

As $y^{q}$ and $u^{p}$ are merely different notations for the same function of $x$, their derivatives are the same. Hence

$$
D_{x} y^{q}=D_{x} u^{p}
$$

Applying Theorem 5, Section 94,

Dividing (4) by (2),

$$
q y^{q-1} D_{x} y=p u^{p-1} D_{x} u \text {. }
$$

$$
q y^{-1} D_{x} y=p u^{-1} D_{x} u .
$$

Multiplying (5) by (1), and dividing by $q$,

$$
\begin{aligned}
& D_{x} y=\frac{p}{q} u^{\frac{p}{q}-1} D_{x} u \\
& \quad=n u^{n-1} D_{x} u, \text { where } n=\frac{p}{q} .
\end{aligned}
$$

Hence we have the

Theorem. If $y=u^{n}$, where in is a fraction, then $D_{x} y=n u^{n-1} D_{x} u$.

EXAMPLE. Differentiate $y=\sqrt{x^{2}-a^{2}}$.

Since

$$
y=\left(x^{2}-a^{2}\right)^{\frac{1}{2}},
$$

and since we may regard $x^{2}-a^{2}$ as a function $u$, we have, by the theorem,

$$
\begin{aligned}
D_{x} y & =\frac{1}{2}\left(x^{2}-a^{2}\right)^{-\frac{1}{2} 2 x} . \\
& =\frac{x}{\sqrt{x^{2}-a^{2}}} .
\end{aligned}
$$

\section{EXERCISES}

1. Find the derivatives of the following functions:
(a) $\frac{x-3}{x+2}$.
(b) $\frac{2 x+7}{3 x-4}$.
(c) $\frac{1}{x^{3}}$.
(d) $\frac{x^{2}-2}{4 x+3}$.
(e) $\frac{2 x}{x^{2}+1}$.
(f) $\frac{2 x^{2}+3}{x^{2}-2 x}$.
(g) $\sqrt{x^{2}-2 x}$.
(h) $\sqrt{1-x^{2}}$.
(i) $\sqrt{x}$.
(j) $x \sqrt{2 x-3}$
Hint: $x \sqrt{2 x-3}=\sqrt{2 x^{3}-3 x^{2}}$.
(k) $x \sqrt{x^{2}-5 x}$.
(l) $x^{3}(2 x+4) \frac{3}{4}$.
(m) $\frac{1}{\sqrt{3 x^{2}-9}}$.
(n) $\left(x^{2}+3 x-5\right)^{\frac{3}{2}}$.
(o) $\frac{\sqrt{x^{2}-1}}{3 x}$.
(p) $x^{2} \sqrt{2 x-x^{2}}$.
(q) $\frac{x^{2}}{\sqrt{x-1}}$. 
2. If the temperature of a gas is constant, the pressure is inversely proportional to the volume. Find the rate of change of the pressure with respect to the volume.

3. The intensity of light varies inversely as the square of the distance from the source. Find the ratio of the intensities at two points, one 2 feet from the source, the other 4 feet. If a body moves away from the source, find the rate at which the intensity changes as the distance increases. Compare the rates of change (find their ratio) at the two points given above.

4. Find the points at which tangents to the graphs of the following equations are horizontal, if any, and construct the figures.
(a) $y=\frac{x+1}{x-2}$
(b) $y=\frac{1}{x^{2}-4}$
(c) $y=\frac{1}{x^{2}+1}$.

5. Construct the graph of $x^{2}-x y=4$, and find the points at which the tangent line is horizontal. Has the curve any points of inflection?

6. Find the points of inflection of the graph of $y=x /\left(x^{2}+1\right)$.

7. The original amount of carbon monoxide in a mixture of formic and sulphuric acids is $a$, and the amount $x$ produced in the time $t$ is given by the equation

$$
\frac{x}{a(a-x)}=k t \text {, where } k \text { is a constant. }
$$

Find the rate at which carbon monoxide is formed.

8. Find the angle at which the graphs of $y=1 / x^{2}$ and $y=x^{\frac{3}{3}}$ intersect.

9. Find the distance from the origin to the point $(x, y)$ in terms of $x$ and $y$, and show that the graph of $x^{2}+y^{2}=16$ is a circle. Find the slope of the tangent line at any point.

10. Show that the graph of $y=+\sqrt{x^{3}}$ is concave upward, and that of $y=-\sqrt{x^{3}}$ is concave downward. Show that both graphs are tangent to the $x$-axis at the origin. What, then, is the form of $y=x^{\frac{3}{2}}= \pm \sqrt{x^{3}}$ near the origin?

100. Equations of Tangent and Normal Lines. The slope of the line tangent to a curve at any point $P_{1}\left(x_{1}, y_{1}\right)$ on it, is the value of the derivative at $P_{1}$, that is, the value of $D_{x} y$ for $x=x_{1}$. Hence the equation of the line tangent at $P_{1}$ may be found by the equation $y-y_{1}=m\left(x-x_{1}\right)$, given on page 66 .

Definition. The normal to the curve at any point on the curve is the line perpendicular to the tangent at that point.

If $m$ is the slope of the tangent line, then the slope of the normal is $-1 / m$, since the slope of one of two perpendicular lines is the negative reciprocal of the slope of the other (page 200). 

of

Example 1. Find the equation of the tangent and normal to the graph

$$
y=\frac{1}{4} x^{2}
$$

at the point for which $x=4$.

The slope of the tangent line at any point is $m=D_{x} y=\frac{1}{2} x$, and hence that of the normal is

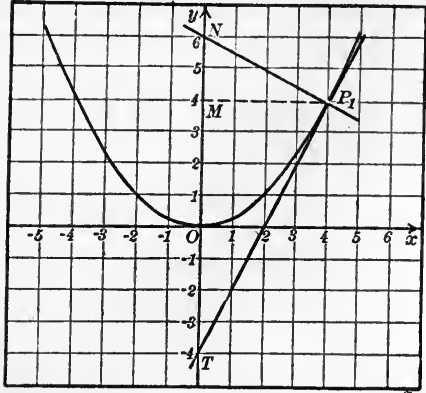

IFIG. 162.

$$
m^{\prime}=-\frac{1}{m}=-\frac{2}{x} \text {. }
$$

At the point $P_{1}$ for which $x_{1}=4$, we have $y_{1}=4, m=2$. Hence the equation of the tangent is

or

$$
\begin{gathered}
y-4=2(x-4) \\
2 x-y-4=0 .
\end{gathered}
$$

At $P_{1}(4,4)$ the slope of the normal is $m^{\prime}=-\frac{1}{2}$. Hence the equation of the normal is

$$
\begin{aligned}
& y-4=-\frac{1}{2}(x-4) \\
& \text { or } \quad x+2 y-12=0 \text {. }
\end{aligned}
$$

Example 2. Find the equation of the tangent and normal to the parabola in Example 1 at any point $\mid P_{1}\left(x_{1}, y_{1}\right)$ on the curve. If $M$ is the projection of $P_{1}$ on the $y$-axis, and if the tangent and normal cut the $y$-axis at $T$ and $N$ respectively, show that $O$ bisects $T M$ and that $M N$ is constant.

At $P_{1}$ the slope of the tangent is $m=x_{1} / 2$, and hence the equation of the tangent is

$$
y-y_{1}=\frac{x_{1}}{2}\left(x-x_{1}\right) \text {. }
$$

Setting $x=0$ in (3) and solving for $y$, the intercept on the $y$-axis is

$$
y=y_{1}-\frac{x_{1}^{2}}{2} \text {. }
$$

Since $P_{1}$ lies on the graph of $y=x^{2} / 4$, its coördinates satisfy this equation so that $y_{1}=x_{1}^{2} / 4$, and hence $x_{1}^{2}=4 y_{1}$. Substituting in (4)

$$
y=y_{1}-\frac{4 y_{1}}{2}=-y_{1} \text {. }
$$

Hence $O T=-y_{1}$, and as $O M=y_{1}$, we have $O T=-O M$, so that $O$ is the middle point of $T M$.

1 The tangent at any point $P_{1}$ may therefore be constructed by laying off on the $y$-axis $O T=-y_{1}$, and joining $T$ to $P_{1}$.

The slope of the normal at $P_{1}$ is $m=-2 / x_{1}$. Hence the equation of the normal is

$$
y-y_{1}=-\frac{2}{x_{1}}\left(x-x_{1}\right) \text {. }
$$


The intercept of (5) on the $y$-axis is

$$
y=y_{1}+2 .
$$

Hence $N$ lies 2 units above $P_{1}$, or $M N=2$, a constant.

The normal at $P_{1}$ may be constructed by drawing the line through $P_{1}$ and the point $N$ which is 2 units above the projection of $P_{1}$ on the $y$-axis.

A frequent source of error in finding the equation of the tangent or normal to a curve at any point $P_{1}$ on the curve is the failure to keep in mind that $x$ and $y$ are variables, the coördinates of any point on the line, while $x_{1}$ and $y_{1}$ are constants, the coördinates of a fixed point. The slope of the tangent or normal line at a given point $P_{1}$ is a constant.

\section{EXERCISES}

1. Obtain equations (1) and (2) above by substituting the coördinates of $P_{1}(4,4)$ in equations (3) and (5).

2. Show that the length of the line joining $P_{1}\left(x_{1}, y_{1}\right)$ and $P_{2}\left(x_{2}, y_{2}\right)$ is $\sqrt{\left(x_{1}-x_{2}\right)^{2}+\left(y_{1}-y_{2}\right)^{2}}$.

3. Show that the coördinates of the middle point of the line $P_{1} P_{2}$ are $x=\left(x_{1}+x_{2}\right) / 2, y=\left(y_{1}+y_{2}\right) / 2$. Hint: If $P$ is the middle point, the values of $\Delta x$ computed for $P_{1}$ and $P$ and for $P$ and $P_{2}$ are equal; and so also are the values of $\Delta y$.

4. Find the equations of the tangent and normal to the following curves at the points indicated. Construct the figure in each case.

(a) $y=x^{2}-4 x,(3,-3)$.

(b) $y=3+2 x-x^{2},(1,4)$.

(c) $y=x^{3},(1,1)$.

(d) $y=x^{3}-4 x+3$, point of inflection.

(e) $x y=4,(4,1)$.

(f) $x y-y=2 x,(2,4)$.

5. Find the equations of the tangent and normal to the parabola $y=x^{2}$ at any point

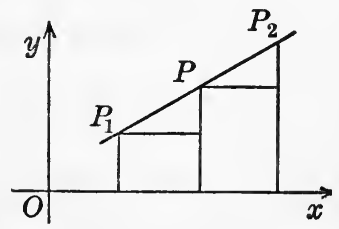

Fig. 163. $P_{1}\left(x_{1}, y_{1}\right)$ on the parabola.

6. Find the equations of the tangent and normal to the hyperbola $x y=4$ at any point $P_{1}\left(x_{1}, y_{1}\right)$.

7. Find the equations of the tangent and normal to the parabola $y=a x^{2}$ at any point $P_{1}\left(x_{1}, y_{1}\right)$. If these lines cut the $y$-axis at $T$ and $N$ respectively, and if $M$ is the projection of $P_{1}$ on the $y$-axis, show that the origin $O$ bisects $T M$, and that $M N$ is constant. State a rule for constructing the tangent and normal at any point on the parabola. 
8. Find the coördinates of the point at which the tangent to the parabola $y=a x^{2}$ at $P_{1}$ cuts the $x$-axis. If the tangent cuts the $x$-axis at $R$, and the $y$-axis at $T$, prove that $R$ is the middle point of $P_{1} T$. Find the equation of the line through $R$ perpendicular to the tangent. Show that this line always cuts the $y$-axis at the same point $F$, no matter what point on the curve $P_{1}$ is.

Definition. The point $F(0,1 / 4 a)$ is called the focus of the parabola $y=a x^{2}$. The focus is the point at which the line in Exercise 8 cuts the $y$-axis.

9. In the figure, $P_{1} A$ is parallel to the $y$-axis. By means of Exercise 8, and methods of plane geometry, show that angle $F P_{1} N=$ angle $N P_{1} A$,

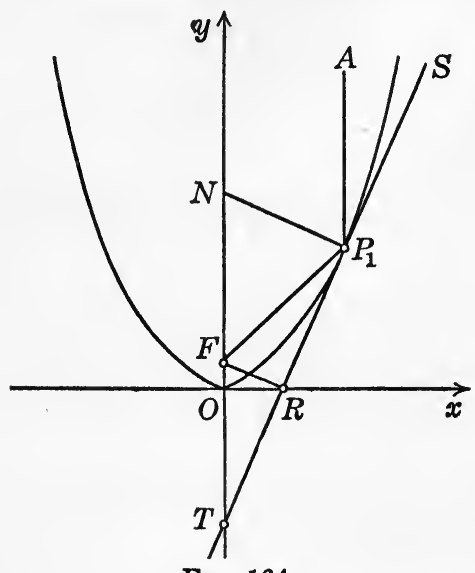

Fig. 164. the notation being the same as in Exercises 7 and 8.

Note: A parabolic reflector, such as is used in a headlight of an automobile, is formed by revolving a parabola about its axis of symmetry. If a source of light is placed at $F$, Exercise 9 shows that the rays will be reflected in parallel lines.

10. Find the equations of the lines tangent to the parabola $y=a x^{2}$ at the two points for which $y=1 / 4 a$. Where do they intersect? At what angle?

11. Find the equation of the line through the focus $F(0,1 / 4 a)$ perpendicular to the tangent to the

parabola at $P_{1}\left(x_{1}, y_{1}\right)$. Show that it intersects the line $x=x_{1}$ on the line $y=-1 / 4 a$.

Definition. The line $y=-1 / 4 a$ is called the directrix of the parabola $y=a x^{2}$.

12. Given the parabola $y=a x^{2}$ and a point $P_{1}\left(x_{1}, y_{1}\right)$ on it, show that the distance from the focus $F(O, 1 / 4 a)$ to $P_{1}$ is $F P_{1}=y_{1}+1 / 4 a$. Show from this that any point on a parabola is equidistant from the focus and the directrix.

13. Find the equation of the tangent to the graph of $y=a x^{3}$ at any point $P_{1}$. If it cuts the $y$-axis at $T$, and if $M$ is the projection of $P_{1}$ on the $y$-axis, show that the origin trisects $T M$.

14. Find the equation of the line tangent to the parabola $y^{2}=x$ at any point $P_{1}$ on the curve. Show that its intercept on the $y$-axis is half the ordinate of the point of contact and that it is perpendicular to the line joining the point of intersection with the $y$-axis to the focus. 
15. Let $F$ be a fixed point on the $x$-axis and $T$ any point on the $y$-axis. Through $T$ draw the line perpendicular to $T F$. Choosing different positions for $T$, draw a number of such lines, enough so that the form of the parabola to which they are tangent becomes apparent.

16. If $P_{1}$ is a point on the equilateral hyperbola $x y=a, M$ its projection on the $y$-axis, and if the tangent line cuts the $y$-axis at $T$, then $M$ is the middle point of $O T$. How can this be used to construct the line tangent at a given point on the curve?

17. Show that the point of contact of a line tangent to the equilateral hyperbola $x y=a$ is the middle point of the segment included between the axes.

18. Find the area included between the axes and the line tangent to the equilateral hyperbola $x y=a$ at any point $P_{1}$. State the result as a theorem.

19. If a normal is drawn to the equilateral hyperbola $x y=a$ at a point $P_{1}$ on it, then $P_{1}$ is the middle point of the segment included between the lines bisecting the coördinate axes.

101. Problems in Maxima and Minima. To solve a problem involving maximum and minimum values, it is necessary first to express the variable $v$ which is to be a maximum or minimum in terms of a single independent variable $x$. The quantity which is to be made a maximum or minimum is usually apparent from the statement of the problem, but there is frequently some choice in the selection of the independent variable. In many problems, as in Example 2 below, the variable $v$ may be expressed at once in terms of two variables, one of which may be eliminated by means of a relation between them. Having found the function $v=f(x)$, one then differentiates $v$ with respect to $x$, sets the derivative equal to zero, and solves for $x$. It must then be shown that at least one of the values of $x$ so found makes $v$ a maximum or minimum, and this maximum or minimum value can be determined by substitution in $v=f(x)$.

Example 1. A box is to be made out of a square piece of cardboard, four inches on a side, by cutting out equal squares from the corners and

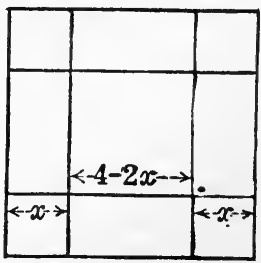

Fig. 165. then turning up the sides. Find the dimensions of the largest box that can be made in this way.

If the squares cut out are small, the box will have a large base and a shallow depth. If the squares are large, the base will be small and the 
box deep. In either case the volume will be small. Somewhere between these two extremes will be a box whose volume is greater than that of any other, that is, a box of maximum volume.

Let $x$ be the side of the square cut out. Then the depth of the box is $x$, and the side of the base is $4-2 x$. Hence the volume, expressed as a function of $x$ is

$$
V=x(4-2 x)^{2}=16 x-16 x^{2}+4 x^{3} .
$$

The graph of $V$ is readily plotted. From it, the value of $x$ which makes $V$ a maximum appears to be somewhat less than unity. By computing

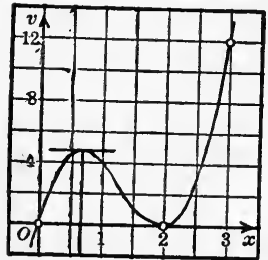

FIG. 166. $V$ for a large number of values of $x$, we could approximate the best value of $x$. By means of the derivative we can avoid this labor, and obtain the exact value.

At the maximum point the tangent line is horizontal, and hence its slope is zero, so that $D_{x} V=0$.

We therefore compute the derivative, set it equal to zero, and find the value of $x$ which produces this result. We then have

$$
\begin{aligned}
D_{x} V=16-32 x+12 x^{2} & =0 \\
3 x^{2}-8 x+4 & =0 . \\
(3 x-2)(x-2) & =0, \\
x & =2 / 3 \text { or } 2 .
\end{aligned}
$$

From the figure, we see that $x=\frac{2}{3}$ makes $V$ a maximum, and $x=2$ gives a minimum. That $x=2$ gives a minimum follows also from the fact that if $x=2$, then $V=0$, that is, the box will not hold anything. The preliminary discussion showed the existence of a maximum, and as $x=\frac{2}{3}$ is the only other possibility it must be the value of $x$ for which $V$ is a maximum. Either of the criteria in Section 96 may also be applied to show that $V$ is a maximum if $x=\frac{2}{3}$.

In order then, to have a box of maximum capacity, we must cut out squares from the corners $\frac{2}{3}$ of an inch on a side. The depth of the box will be $\frac{2}{3}$ of an inch, the side of the base will be $4-2 \times \frac{2}{3}=2 \frac{2}{3}$ inches, and the capacity will be $V=\left(2 \frac{2}{3}\right)^{2} \times \frac{2}{3}=4 \frac{2}{27} \mathrm{cu}$. in.

ExAMPLe 2. As large a rectangular stick of timber as possible is to be sawed from a $\log 10$ inches in diameter at the smaller end, the length of the stick to be the same as that of the log. Find its other dimensions.

Let $V$ denote the volume of the stick of timber, $l$ its length, and $A$ the area of an end. Then $V=l A$, and since $l$ is constant, the volume $V$ will be a maximum if and only if $A$ is a maximum. The end of the stick is a rectangle inscribed in a circle whose diameter is 10 , and the problem reduces to a determination of the dimensions of the maximum rectangle which can be inscribed in this circle. 
The area of the rectangle is

$$
A=x y,
$$

which involves the two variable dimensions $x$ and $y$. These are connected by the relation $x^{2}+y^{2}=100$.

Solving for $y$,

and substituting in (1)

$$
\begin{aligned}
& y=\sqrt{100-x^{2}} . \\
& A=x \sqrt{100-x^{2}} .
\end{aligned}
$$

In order to find the derivative of $A$ we write it in the form

$$
A=\sqrt{100 x^{2}-x^{4}}=\left(100 x^{2}-x^{4}\right)^{\frac{1}{2}} \text {. }
$$

Differentiating,

$$
\begin{aligned}
D_{x} A & =\frac{1}{2}\left(100 x^{2}-x^{4}\right)^{-\frac{1}{2}}\left(200 x-4 x^{3}\right) \\
& =\frac{100 x-2 x^{3}}{\sqrt{100 x^{2}-x^{4}}}=\frac{100-2 x^{2}}{\sqrt{100-x^{2}}}
\end{aligned}
$$

Equating the derivative to zero,

$$
\frac{100-2 x^{2}}{\sqrt{100-x^{2}}}=0 \text {. }
$$

Multiplying both sides by $\sqrt{100-x^{2}}$,

$$
100-2 x^{2}=0,
$$

and solving for $x$,

$$
x= \pm 5 \sqrt{2} \text {. }
$$

The negative value of $x$ has no meaning in this problem. That the value of $A$ is a maximum if $x=5 \sqrt{2}$ may be seen as follows: If $x$ is very small, or very near $10, A$ is very small, while larger rectangles

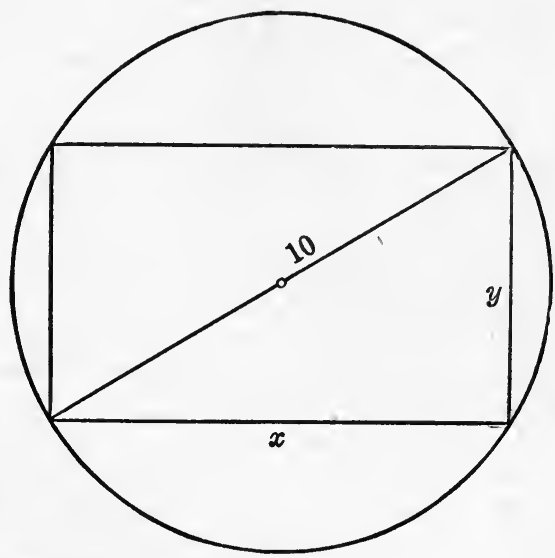

FIG. 167. lie between these extremes. Hence as $x$ increases from 0 to $10, A$ first increases and then decreases, and as $A$ does not become infinite it therefore has a maximum in this interval. But $x=5 \sqrt{2}$ is the only point in this interval at which the tangent to the graph of $A$ is horizontal, and it must therefore give the maximum value.

The corresponding value of $y$, from (2), is $y=5 \sqrt{2}$. Hence the end of the largest stick of timber will be a square whose side is $5 \sqrt{2}$ inches, or very nearly 7 inches. 


\section{EXERCISES}

1. A box is to be made by cutting squares from the corners of a piece of cardboard 6 by 8 inches, and folding up the sides. Find the dimensions if the capacity is to be a maximum.

2. A chicken yard is to be made from 36 feet of poultry fencing, the side of a barn being used for one side of the yard. Find the dimensions in order that the yard may be as large as possible.

3. Find the dimensions and capacity of the largest box which can be made with a square base and no top if the total amount of cardboard in the box is 48 square inches.

4. What should be the dimensions of a rectangular garden plot with a perimeter of 12 rods, in order to have the greatest area possible?

5. A two acre pasture in the form of a rectangle is to be fenced off along the bank of a straight river, no fence being needed along the river. Find the dimensions, in rods, in order that the fence may cost as little as possible.

6. The legs of an isosceles triangle are 6 inches long. How long must the base be in order that the area may be a maximum?

7. The height of a rifle ball fired vertically upward with an initial velocity of 1200 feet per second is $s=1200 t-16 t^{2}$. How high will it rise?

8. By the Parcel Post regulations, the combined length and girth of a package must not exceed 6 feet. Find the dimensions and volume of the largest parcel which can be sent in the shape of a box with square ends.

9. A farmer has 150 rods of fencing. Find the dimensions and area of the largest rectangular field he can enclose and divide into two equal parts by a fence parallel to two of the sides.

10. If the total area of the field in the preceding Exercise is to be $\mathbf{1 5 0}$ square rods, find the dimensions and the amount of fencing needed if the latter is to be a minimum.

11. A rectangular cistern is to be built with a square base and open top. Find the proportions if the amount of material used is to be a minimum.

12. A rectangular piece of ground is to be fenced off and divided into three equal parts by fences parallel to one of the sides. What should the dimensions be in order that as much ground as possible may be enclosed with 16 rods of fence?

13. If the total area enclosed in Exercise $\mathbf{1 2}$ is an acre, find the dimensions in order that the total length of the fence should be a minimum.

14. The number of tons of coal consumed per hour by a certain ship is $0.3+0.001 V^{3}$, where $V$ is the speed in knots. For a voyage of 1000 knots at $V$ knots per hour, find the total consumption of coal. For what speed is the consumption of coal least?

15. Divide a string 16 inches long into two parts, so that the combined area of the square and circle with perimeters equal to the parts shall be $a$ minimum. 
16. Find the coördinates of the maximum or minimum point of $y=$ $a x^{2}+b x+c$.

17. At what height should a light be placed above a writing table in order that a small portion of the table, at a given horizontal distance $d$ from the point directly below the light, may receive the greatest illumination possible? (It is known that the intensity of illumination varies inversely as the square of the distance and directly as the sine of the angle between the line from the light to the point in question and the table. Express the illumination as a function of the height of the light, and find the value of the height which gives the maximum illumination.)

18. A rectangular box is to be made by cutting out the corners of a rectangular piece of cardboard and folding up the sides. If the depth of the box is to be 2 inches, find the dimensions of the smallest piece of cardboard which will make a box to contain 72 cubic inches.

102. Related Rates. If two variables $x$ and $y$ are functions of the time $t$, then their derivatives with respect to $t, D_{t} x$ and $D_{t} y$, measure the rates at which $x$ and $y$ are changing. These rates will be related, that is, connected by a relation, if $y$ is a function of $x$.

For example, if $x$ and $y$ are functions of $t$ such that $y=x^{3}$, then the rates at which $x$ and $y$ change satisfy the equation $D_{t} y=3 x^{2} D_{t} x$, which is obtained by differentiating the given equation with respect to $t$. The rate of change of $y$ depends on the value of $x$ as well as on that of the rate of change of $x$.

In solving problems involving the rates at which two variables change, it is first necessary to determine the relation $y=f(x)$ connecting the variables. Then the relation between the rates of change of $x$ and $y$ is found by differentiating $y=f(x)$ with respect to $t$.

Example 1. Oil dropped on a smooth floor spreads out in the form of a circle. If the radius is increasing at the rate of $\frac{1}{2}$ an inch per second when it is 6 inches long, how fast is the area increasing?

If $r$ denotes the radius and $A$ the area, the question asked may be expressed symbolically as follows: If $D_{t} r=\frac{1}{2}$ when $r=6$, what is the value of $D_{t} A$ ?

In order to answer this question we must have a relation between $D_{t} A$ and $D_{t} r$. And to obtain this relation we must first express $A$ as a function of $r$. From plane geometry we have

$$
A=\pi r^{2} .
$$


Differentiating both sides with respect to $t$, we get

$$
D_{t} A=2 \pi r D_{t} r \text {. }
$$

Substituting the values of $\pi, r$, and $D_{t} r$, the oil covered area is increasing at the rate of $D_{t} A=2 \times 22 / 7 \times 6 \times \frac{1}{2}=18.85$ square inches per second.

EXAmple 2. A man walks along a sidewalk at the rate of three miles an hour (4.4 feet per second), approaching a house which stands back 7

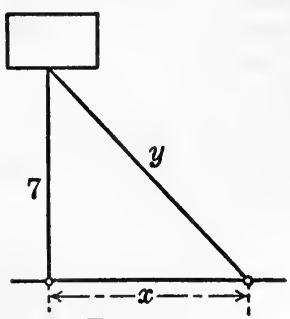

FIG. 168. feet from the walk. When he is 24 feet from the walk leading to the house, how rapidly is he approaching the house?

The rate at which he approaches the house is the derivative of his distance from the house with respect to time. If $y$ denotes his distance from the house, we seek $D_{t} y$.

The rate at which the man walks is the rate of change of his distance from some point on the walk with respect to the time. This point is conveniently chosen as the point on the sidewalk directly in front of the house, because we know this distance at the time we wish to determine $\mathrm{D}_{t} y$. Let his distance from this point be $x$. Then $D_{t} x=4.4$.

To find the relation between these two derivatives we must first express $y$ as a function of $x$. From the figure we have, at any time,

$$
y=\sqrt{\left(x^{2}+49\right)}=\left(x^{2}+49\right)^{\frac{1}{2}},
$$

and hence, by Theorem 5 , page 270 , differentiating with respect to $t$,

$$
D_{t} y=\frac{1}{2}\left(x^{2}+49\right)^{-\frac{1}{2}} D_{t}\left(x^{2}+49\right)=\frac{x D_{t} x}{\left(x^{2}+49\right)^{\frac{1}{2}}} .
$$

We are told that when $x=24, D_{t} x=4.4$, and hence,

$$
D_{t} y=\frac{24 \times 4.4}{(576+49)^{\frac{1}{2}}}=\frac{24 \times 4.4}{25}=4.2 \text { feet per second. }
$$

\section{EXERCISES}

1. In Example 1 above, find the rate at which the circumference is increasing when the radius is 6 inches. Find also the rate at which the area and circumference are increasing when the radius is 10 inches. What essential difference is there in the rates at which the circumference and area increase?

2. Imagine a belt stretched around the earth at the equator (radius 3963 miles). If the radius of the belt increases uniformly at the rate of 3 feet per second, how much will the circumference increase in the first second? 
3. A man 6 feet tall walks directly away from a lamp post 12 feet high at the rate of 4 miles an hour. How fast does the shadow of his head move?

4. A man is walking at the rate of 3 miles an hour along a sidewalk which is 8 feet from the buildings beside it. A light on the other side of the street, 30 feet from the walk, casts a shadow of the man on the buildings. How fast does his shadow move?

5. A cruiser is moving parallel to a straight coast at a distance of 6 miles from it, at the rate of 27 miles an hour. How fast is the cruiser approaching a fort on the shore, 15 miles from the point directly opposite the ship?

6. The pressure and volume of a gas at a constant temperature satisfy the relation $p v=k$. At a certain time the volume is 3 cubic feet, the pressure is 10 pounds per square foot, and the volume is increasing at the rate of 0.2 of a cubic foot per second. Find the rate of change of the pressure.

7. Two ships start from the same place at the same time. One sails east at 12 knots an hour, the other south at 18 knots an hour. How fast will they be separating after half an hour?

8. The height of a ball thrown vertically upward with a velocity of 64 feet per second is given by $s=64 t-16 t^{2}$. The rays of the sun make an angle of $30^{\circ}$ with the horizontal. How fast is the shadow of the ball moving along the ground just before the ball hits the ground?

103. Small Errors. In measuring any quantity directly, we usually have some idea of the accuracy of the measurement. Thus we may measure the length of the edge of a cubical box and feel confident that the length is 10 inches with an error of not more than $\frac{1}{8}$ of an inch. This is indicated by saying that the length is $10 \pm \frac{1}{8}$ inches.

Frequently the size of the error is of less importance than the relative error, which is the ratio of the error to the quantity measured. Thus the relative error in the edge of the cube above is the ratio of $\frac{1}{8}$ to 10 , that is, $\frac{1}{80}=0.0125$ or $1.25 \%$.

The relative error, rather than the magnitude of the error itself, indicates the degree of accuracy of a measurement. Nothing can be said of the comparative accuracy of the measurements of the lengths of two lines if it is known that the error in one case is 2 feet and that in the other it is only half a foot. If the first error occurred in measuring a side of a farm half a mile long, the relative error would be $\frac{{ }^{2}}{26^{2}} \overline{0}=0.000758$, which 
is less than 0.08 of one per cent, while if the second error was made in measuring the frontage of a city lot 50 feet wide the relative error would be $0.5 / 50=0.01$, or one per cent. Under these circumstances the first measurement would be by far the more accurate.

Many quantities are measured indirectly. Thus to find the volume of a cubical box we measure the length of an edge, and compute the volume. If the length of the edge is found to be 10 inches, then the volume is $V=1000$ cubic inches. If an error of $\frac{1}{8}$ of an inch is made in measuring the edge, what is the error in the volume? In the example below we shall develop a method of answering such a question.

Example. The side of a square was found by measuring to be 3 inches. If the measurement is 0.1 of an inch too small, what is the approximate error in the computed area?

First solution. It is obvious that

the computed area $=9$

and the true area $=(3+0.1)^{2}=9+0.6+0.01$.

Since 0.01 is small in comparison with the other terms, it may be disregarded, and the approximate error is 0.6 .

Not only is the term disregarded much smaller than the others, but it is smaller than the change in the approximate error due to a slight change in the estimated error in the measurement of the side. For if the measurement of the side is 0.11 of an inch too small, then

$$
\text { the true area }=(3+0.11)^{2}=9+0.66+0.0121 \text {, }
$$

and the approximate error is now 0.66 . Thus a slight change in the estimated error in the original measurement of the side produces a change of 0.06 in the original approximate error in the area, and this change is 6 times the term originally neglected.

The approximate relative error is the ratio of the approximate error to the computed area, i.e., $0.6 / 9,=0.07$ or $7 \%$.

It is instructive to first solve the problem for any square and then substitute the given numbers.

Let $x$ be the value of the side found by measurement, and $\Delta x$ the estimated error. Let $y$ denote the computed value of the area, and $\Delta y$ the error in $y$. Then the computed area is

and the true area is

$$
y=x^{2}
$$

$$
y+\Delta y=(x+\Delta x)^{2}=x^{2}+2 x \Delta x+\Delta x^{2}
$$


Subtracting (1) from (2), the error in the computed area is seen to be

$$
\Delta y=2 x \Delta x+\Delta x^{2}
$$

When $\Delta x$ is small, $\Delta x^{2}$ is very small compared to $2 x \Delta x$, so that $\Delta x^{2}$ may be neglected, and the approximate error in $y$ is $2 x \Delta x$.

Equation (3) is readily interpreted from the figure. The product $x \Delta x$ is the area of either of the rectangles, and $\Delta x^{2}$ is the area of the small square.

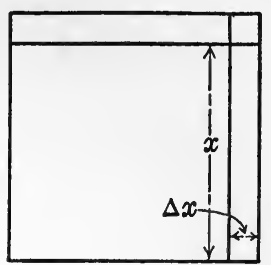

Fig. 169. Obviously, if $\Delta x$ is small compared to $x$, the small square is negligible as compared with the two rectangles. It is also clear that a slight change in $\Delta x$ produces a change in the two rectangles which is greater than the small square disregarded.

Denoting the approximate error by $d y$, we obtain from the above $d y=2 x \Delta x$. The relative error is therefore

$$
\frac{d y}{y}=\frac{2 x \Delta x}{x^{2}}=2 \frac{\Delta x}{x}
$$

so that the relative error in $y$ is twice the relative error in $x$.

Substituting the given values of $x$ and $\Delta x$ we get, as before,

$$
d y=2 \times 3 \times 0.1=0.6 \text {, and } d y / y=2 \times 0.1 / 3=0.07 \text {, or } 7 \% \text {. }
$$

Second solution. The first solution is useful for clarifying the ideas involved, but the method now to be considered is more convenient in all but the simplest examples.

Letting $x$ be the side of any square, the computed area is given by (1), whose graph is shown in Fig. 170. The ordinate $y=M P$ of any point represents the computed area of a square whose side as measured is $x=O M$. If the error in the measurement is $\Delta x=M N$, then the ordinate $N Q$ represents the true area and $\Delta y=R Q$ the true error in $y$.

Construct the line tangent at $P$, and let it cut the ordinate $N Q$ at $S$. Let $d y=R S$. Then $d y$ is an approximate value of the true error $\Delta y$, and if $\Delta x$ is very small this gives a very good approximation. For if $M N$ were less than one-eighth of an inch in this figure, $S$ would be practically coincident with $Q$.

In order to have a more consistent notation set $\Delta x=d x$, so that $d x$ represents the error in $x$.

Then in the right triangle $P R S$, we have $P R=d x$, and

since $\tan \theta=D_{x} y$.

$$
\begin{aligned}
d y & =d x \tan \angle R P S \\
& =d x \tan \theta \\
& =D_{x} y d x,
\end{aligned}
$$


From (1) we have $\mathrm{D}_{x} y=2 x$, and hence, from (4), $d y=2 x d x$, the same result that was obtained in the first solution. The error for the given

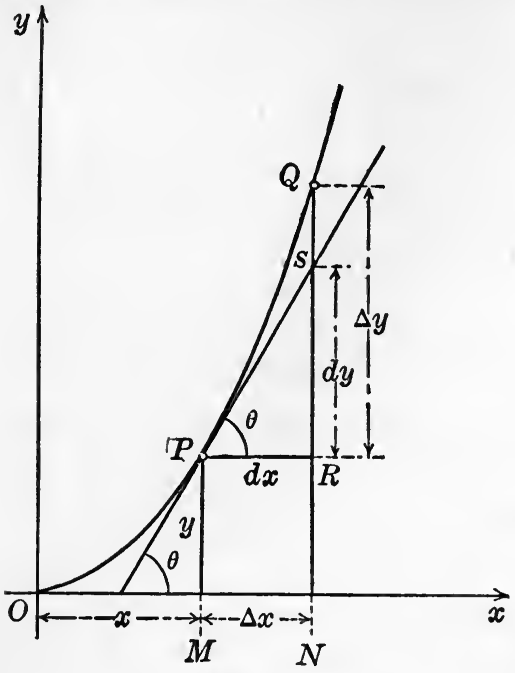

Frg. 170. square, and the relative error, are found as before.

If a magnitude $x$ be measured, and a second magnitude $y$ be computed from it, then $y$ is a function of $x$, $y=f(x)$. The reasoning in the second solution applies equally well to the graph of this function, if we regard the curve in the figure as the graph of any function. Hence:

If $y=f(x)$, an approximate value of the error in $y$ due to an error of $d x$ in the measurement of $x$ is the product of the derivative of $y$ with respect to $x$ and $d x$; i.e.,

$$
d y=D_{x} y d x \text {. }
$$

An approximate value of the relative error is given by $d y / y$.

In what follows we shall use the terms error and relative error to denote these approximations unless the contrary is explicitly mentioned.

In applying this method, it is necessary first to compute $y$ as a function of $x$ without using the given numerical value of $x$. For example, in the illustration above. we must first find the area of any square.

\section{EXERCISES}

1. The edge of a cube is found by measurement to be 8.43 inches with a probable error of 0.005 inches. What is the error in the volume? The relative error?

2. The side of a square is almost exactly 4 inches. What is the allowable error in the measurement of a side if the error in the area is to be determined to within one-tenth of one per cent? 
3. A surveyor measures a square field with la 66 -foot chain and finds the area to be 40 acres. If it is found that his chain has stretched and is one inch too long, find the correct area approximately.

4. Find the side of a cubical box to hold one quart (57.75 cubic inches). How much variation may be allowed on a side if the error in the capacity is not to exceed one per cent?

5. What is the allowable error in the measurement of the largest dimension of a rectangular block, $10 \times 6 \times 4$ inches, if the volume is to be determined to within one-fifth of one per cent? What is the allowable error in the smallest dimension? (Assume in each part of the problem that the other two dimensions are exact.)

6. The intensity of a certain light at a distance $x$ from the source is $I=1 / x^{2}$. What is the error in $I$ if $x=12$, with a probable error of 0.1 ?

7. The quantity of heat necessary to raise the temperature of a certain substance from $0^{\circ} \mathrm{C}$. to $\theta^{\circ} \mathrm{C}$. is $Q=.5290 \theta+.0003 \theta^{2}$. If in an experiment $\theta$ is found to be $20^{\circ}$, with an estimated error of not more than $0^{\circ} .3$, what is the error in $Q$ ? The relative error?

8. The relation between Fahrenheit and Centigrade thermometer readings is $F=1.8 C+32$. A reading of $19^{\circ} .2$ is made on a Centigrade thermometer, and the temperature Fahrenheit computed from it. Find the percentage of error in the computed value, if the error in the reading is $0^{\circ} .1$.

9. The depth of the Colorado Canyon at a certain point was found from the relation $s=16 t^{2}$ by dropping a stone and observing the time of falling. Is the percentage of error in the computed depth less than or greater than the percentage of error in the measurement of the time?

10. The distance to the sun as determined by recent measurements has been stated to be $92,780,000 \pm 500,000$ miles (the notation indicating that the error is probably not greater than 500,000 miles). One method of computing this distance is indicated in the figure, where $a$ is the angle of parallax, 8.811", at the sun subtended by the radius of the earth. What is the allowable

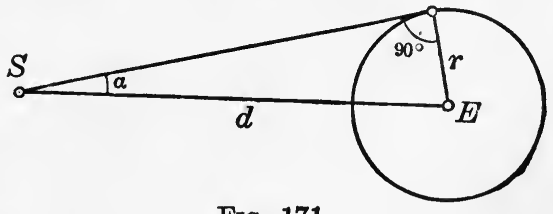

Frg. 171. percentage of error in the radius to give the above result for the distance to the sun?

The radius of the earth is found by measuring an arc of one degree, or 0.017453 radians, on the equatorial or on a meridian circle, and applying the theorem on page 171. Assuming that the angular measurement is exact, what is the allowable percentage of error in the measurement of the $\operatorname{arc}$ ?

An arc of one degree has been measured recently by using a brass rod 
15 feet long with a probable error of 0.00012 inches. Is this sufficiently accurate for the computation of the distance to the sun?

Why is it that the distance to the sun cannot be determined with greater accuracy?

11. If $y$ is a linear function of $x, y=m x+b$, is the percentage of error in the computed value of $y$ ever less than the percentage of error in the measured value of $x$ ?

12. The radius and an arc of a circle were measured, and found to be 6 inches and 15 inches respectively, and the number of radians in the central angle subtended by the arc was computed (Theorem, page 171). Find the error and the relative error in the angle due to an error of onetenth of an inch in the radius; in the arc; assuming in each case that the other measurement is exact.

104. Approximate value of $\mathbf{f}(\mathbf{x}+\Delta \mathbf{x})$. If $y=f(x)$, it is frequently convenient to use $f^{\prime}(x)$ to denote the derivative, instead of $D_{x} y$. The latter notation is used when we are denoting a function by $y$, the former when we are talking of a function $f(x)$, as in this section.

If the graph of $f(x)$ is drawn, and if $x=O M$ and $\Delta x=M N$, then the value of $f(x+\Delta x)$ is represented by the ordinate $N Q$.

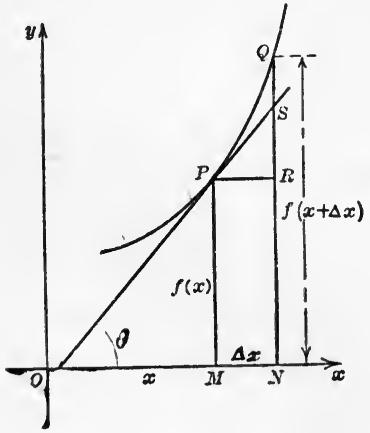

FIG. 172.

In the preceding section it was seen that if $\Delta x$ is small $d y=R S$ is a good approximation of $\Delta y=R Q$. Hence if $\Delta x$ is small $N S$ is a good approximation of $N Q=f(x+\Delta x)$.

Since $R S=\Delta x \tan \theta=\Delta x f^{\prime}(x)$ and since $N R=M P=f(x)$, we have, approximately

$$
\begin{aligned}
f(x+\Delta x) & =N R+R S \\
& =f(x)+f^{\prime}(x) \Delta x .
\end{aligned}
$$

Hence we have the

Theorem. If $\Delta x$ is small, an approximate value of $f(x+\Delta x)$ is given by the relation $f(x+\Delta x)$ $=f(x)+f^{\prime}(x) \Delta x$, where $f^{\prime}(x)$ denotes the derivative of $f(x)$.

In more advanced work in mathematics the error in this approximation is' considered, and also better approximations involving the second and higher powers of $\Delta x$. The utility of the approximation is seen in the examples following. 
Example 1. Find an approximate value of $3.01^{3}$.

Since 3.01 may be put in the form $3+0.01$, we have $3.01=x+\Delta x$, where $x=3$ and $\Delta x=0.01$. And if $f(x)=x^{3}$, the required number is the value of $f(x+\Delta x)$. By the Theorem we have approximately

$$
\text { Then } \begin{aligned}
(x+\Delta x)^{3} & =x^{3}+3 x^{2} \Delta x . \\
3.01^{3} & =3^{3}+3 \times 3^{2} \times 0.01=27+0.27=27.27 .
\end{aligned}
$$

As the exact value is $3.01^{3}=27.270901$, the approximate value obtained by the Theorem is correct to four significant figures.

Example 2. Compute a table of squares, to two decimal places, for the values $x=2.01,2.02,2.03, \ldots 2.10$.

The square of 2.01 may be computed from the square of 2 , the square of 2.02 from that of 2.01, etc., by means of the Theorem which shows that, approximately, $(x+\Delta x)^{2}=x^{2}+2 x \Delta x$.

The computation may be systematized by arranging it in tabular form. The last column in the table gives the squares of the numbers in the first column. The accuracy of the square of the last number in the table

\begin{tabular}{|c|c|c|c|c|}
\hline & $x$ & $\Delta x$ & $x^{2}+2 x \Delta x$ & $(x+\Delta x)^{2}$ \\
\hline 2.01 & 2 & .01 & $+.04=4.04$ & 4.04 \\
\hline 2.02 & 2.01 & .01 & $4.04+.0402=4$. & 4.08 \\
\hline 2.03 & 2.02 & .01 & $4.0802+.0404=4$ & 2 \\
\hline & 2.0 & .01 & $4.1206+$. & \\
\hline 2. & 2.0 & .01 & $12+$ & \\
\hline 2.0 & 2.05 & .01 & 4.20 & t \\
\hline & 2.06 & .01 & 4.24 & \\
\hline 2.0 & 2.0 & .01 & $4.2842+$ & 4.3 \\
\hline 2.0 & 2.08 & .01 & $4.3256+.0$ & 4.3 \\
\hline 2.10 & 2.09 & .01 & $4.3672+.0418=4.4090$ & 4.41 \\
\hline
\end{tabular}
checks the accuracy of the entire computation.

The computation is carried to four decimal places, as these places have an accumulative effect which ultimately changes the value of the tabular difference (at what point in the table?), but very few of the digits in the third and fourth decimal places are accurate. The approximations in the last column are made by taking the nearest figure in the second decimal place.

The approximation given in the Theorem is much used in the computation of tables, as in Example 2. It cannot be used, however, to construct a complete table without making use of the limit of error of the approximation. Thus if the 
table above was continued for $2.11,2.12$, etc., it would be found that the value obtained for $2.16^{2}$ would be too small by 0.01 . In the very simple function $x^{2}$ the accumulative effect of the difference between $(x+\Delta x)^{2}$ and the approximation $x^{2}+2 x \Delta x$ may be seen very clearly (see Exercise 2 below). But for most functions the question of the error involved is much more complicated.

\section{EXERCISES}

1. Compute an approximate value of each of the functions below for the given value of the variable.
(a) $x^{2}, 6.2$.
(b) $x^{3}, 4.1$.
(c) $\sqrt{x}, 2.1$.
(d) $1 / x, 2.1$.

2. Compute the squares of $1.01,1.02$, and 1.03 using (a) the approximation $(x+\Delta x)^{2}=x^{2}+2 x \Delta x$; (b) the exact relation $(x+\Delta x)^{2}=x^{2}+2 x \Delta x+$ $\Delta x^{2}$. Note the error in the approximation of $1.03^{2}$ due to the accumulated effect of neglecting $\Delta x^{2}$ in the approximation.

3. Compute a three-place table of

(a) Squares for $\quad x=3.01,3.02, \cdots, 3.10$.

(b) Cubes for $\quad x=4.01,4.02, \cdots, 4.05$.

(c) Square roots for $x=0.26,0.27, \cdots, 0.30$.

(d) Cubes for $\quad x=2.01,2.02, \cdots$ How far can the computation be carried before the accumulative effect of the error in the approximation becomes evident?

\section{MISCELLANEOUS EXERCISES}

1. The chord of the parabola $y=a x^{2}$ through the focus perpendicular to the axis of symmetry is called the latus rectum. Find its length, and show that the tangents at its extremities are perpendicular.

2. If $P_{1}$ is any point on $y=x^{n}, M$ its projection on the $y$-axis, and $T$ the point of intersection of the $y$-axis and the line tangent at $P_{1}$, show that $T M=n O M$. Illustrate by figures for $n>1,0<n<1, n<0$.

3. If the tangent and normal to the parabola $y=a x^{2}$ at $P_{1}$ cut the $y$-axis at $T$ and $N$ respectively, then the focus $F$ is equidistant from $P_{1}$, $T$ and $N$.

4. If the tangent at $P_{1}$ to the hyperbola $x y=a$ cuts the $x$-axis at $T$, show that $O P_{1}=T P_{1}$.

5. If the normal at $P_{1}$ to the hyperbola $x y=a(a>0)$ cuts the bisector of the first and third quadrants at $B$, then $O P_{1}=B P_{1}$.

6. The line $y=m x+\frac{1}{4} a$ passes through the focus $\left(0, \frac{1}{4} a\right)$ of the parabola $y=a x^{2}$. Find the abscissas of the points at which it intersects the parabola. Show that the tangents at these points are perpendicular, and that they intersect on the directrix. 
7. If $V_{0}$ is the volume of a quantity of water at $0^{\circ}$ Centigrade then the volume at a temperature $\theta^{\circ}$ Centugrade is given by

$$
V=V_{0}\left(1-0.00005758 \theta+0.000007560^{4}-0.0000000351 \theta^{3}\right) \text {. }
$$

Show that the volume is least, and hence the density greatest, when $\theta=3^{\circ} .92 \mathrm{C}$. (Let the decimals be denoted by $a, b, c$ respectively until the end of the computation, and write 0.00005758 in the form $5.758 \times$ $10^{-5}$, etc.)

8. A bandit is walking along a street at the rate of 3 miles an hour toward the intersection with a second street making an angle of $45^{\circ}$ with the first. A tree stands 100 feet from the intersection and 25 feet toward the second street from the first. A timid citizen walks along the second street endeavoring to keep the tree between himself and the bandit. How fast does he walk?

9. There are two sources of light at the points $A$ and $B$. At a distance $x$ from the first the illumination is $I_{1}=8 / x^{2}$, and at a distance $x$ from the second the illumination is $I_{2}=27 / x^{2}$. Find the point on the segment $A B$ where the sum of the illuminations is a minimum.

10. Suppose that $a_{1}, a_{2}, a_{3}, \ldots a_{n}$, are the values of $n$ measurements of a magnitude whose true value is $x$. Then the errors in the measurements are respectively $x-a_{1}, x-a_{2}, x-a_{3}, \ldots x-a_{n}$, some of which are positive, and some of which are negative. The theory of least squares asserts that the most probable value of $x$ is such that the sum of the squares of the errors is a minimum. Show that the most probable value of $x$ is the arithmetic mean (or average).

11. Find the dimensions of the strongest beam which can be sawed from a $\log 12$ inches in diameter, assuming that the strength of the beam varies as the breadth and the square of the depth.

12. Find the dimensions of the stiffest beam which can be cut from a $\log 10$ inches in diameter, if the stiffness is proportional to the breadth and the cube of the depth.

13. Find the dimensions of the rectangle with maximum perimeter which can be inscribed in a circle of radius $r$.

14. A point moves along the straight line $y=2 x+10$. If its abscissa is increasing at the rate of 3 inches per second when $x=1$, find the rate at which its ordinate changes.

15. Two railroad tracks intersect at right angles. A train on one track is 24 miles from the intersection and is approaching it at the rate of 30 miles an hour. A train on the other track is 7 miles from the intersection, and is receding from it at 45 miles an hour. Is the distance between the trains increasing or decreasing? How rapidly?

16. A 16 foot ladder resting against the side of a barn begins to slip. When the foot of the ladder is 10 feet from the barn it is moving 2 feet per second. How fast is the top of the ladder moving? 
17. The side of a square is 39.51 inches, with ap error of not more than 0.01. Find the error in the area of the square. Is the value of $39.51^{2}$ given in Huntington's Tables sufficiently accurate for the area?

18. The intensity of heat at a distance of $x$ feet from a source of heat is $I=100 / x^{2}$. If a body moves directly away from the source at the uniform rate of 3 feet per second, how rapidly is the intensity of the heat changing when $x=5$ feet? When $x=20$ feet?

19. The side of an equilateral triangle is $5.4 \pm 0.1$ inches. What is the percentage of error in the area of the triangle?

20. If a body moves so that $v^{2}=k s$, show that the acceleration is constant. An automobile moving $v$ miles per hour on a slippery pavement should be able to stop in $s$ feet, where $v^{2}=17 s$. Find the acceleration in feet per second per second.

21. A rectangular grain bin, without a cover, is to be divided into two equal parts by a partition parallel to the ends. The bin is to be 3 feet deep and to hold 98 cubic feet. Find its dimensions if the amount of lumber required is a minimum.

22. A rectangular pan is to have its width two-thirds of its length and its capacity is to be $\frac{9}{10}$ of a cubic foot. Find its dimensions if the amount of tin used is a minimum.

23. The thrust of an aeroplane is given by the equation (see Exercise 11 , page 157)

$$
t=W\left(\theta+\frac{1}{f^{2} \theta}\right)
$$

Find the value of $\theta$ for which $t$ is a minimum. Substitute this value of $\theta$ in the given equation, and determine how $t$ varies as the fineness $f$ changes.

24. The power required for oblique flight of an aeroplane up a slope $m$ is

$$
T=v W\left(\theta+\frac{1}{f^{2} \theta}\right)+v W m=v W\left(\theta+\frac{1}{f^{2} \theta}+m\right) .
$$

For gliding flight $T=0$; what relation must eonnect $\theta$ and $m$ for gliding flight? If $\theta=\theta_{1}$ makes $m$ a minimum, $m_{1}$, show that $m_{1}=2 \theta_{1}$. What relation exists between $m$ and the horizontal and vertical distances the aeroplane glides? How does the fineness $f$ affect the greatest horizontal distance the aoroplane can glide? Show that the aeroplane can glide along a given slope for two different values of the angle of incidence $\theta$ and hence for two different velocities (see Note, page 156). 


\section{CHAPTER VII}

\section{INTEGRATION}

105. Introduction. In Chapter VI we considered the problem: Given the function $y$, to find the derivative $D_{x} y$.

In this Chapter we shall consider the inverse problem: Given the derivative $D_{x} y$, to find the function $y$.

Definition. A function whose derivative is a given function is called an integral of the given function and the process of finding it is called integration.

Thus, if $D_{x} f(x)=F(x)$, then $f(x)$ is an integral of $F(x)$.

Example 1. Given $D_{x} y=2 x$, find $y$.

Since $D_{x} x^{n}=n x^{n-1}$, we have $D_{x} x^{2}=2 x$. Hence $x^{2}$ is an integral of $2 x$. But the derivative of each of the functions $x^{2}+5, x^{2}-7, x^{2}+\frac{3}{2}$, is $2 x$. Hence these functions are also integrals of $2 x$. In fact, any function of the form $y=x^{2}+C$, where $C$ is any constant, has the derivative $2 x$, and is therefore an integral of $2 x$.

Thus we see that the process of differentiating the function $x^{2}$ gives rise to the single derivative $2 x$, but that the inverse process of integrating $2 x$ gives rise to an indefinite number of integrals $x^{2}+C$ which differ by a constant.

Theorem. If two functions $f(x)$ and $F(x)$ have the same derivative, their difference is a constant.

Let

Then

$$
y=f(x)-F(x) \text {. }
$$

By hypothesis, $\quad D_{x} f(x)=D_{x} F(x)$.

Hence

$$
D_{x} y=0 \text {. }
$$

But a function whose derivative is equal to zero for all values of the variable is a constant. For the tangent line at every point on the graph is horizontal, so that the graph of the function is a straight line parallel to the $x$-axis. 
Hence or

$$
\begin{aligned}
y & =f(x)-F(x)=C, \\
f(x) & =F(x)+C .
\end{aligned}
$$

That is, every integral can be obtained from a given integral by adding the proper constant.

The constant $C$ which is added to a known integral of a function is called the constant of integration and it should not be omitted in performing the operation of integration.

If $y$ is a function of $x$ whose derivative with respect to $x$ is given, say $F(x)$, then

$$
D_{x} y=F(x) .
$$

The notation employed to indicate that $y$ is an integral of $F(x)$ is

$$
y=\int F(x) d x,
$$

the equation being read " $y$ equals the integral of $F(x) d x . "$ In the illustration above, since $D_{x} y=2 x$, then

$$
y=\int 2 x d x=x^{2}+C .
$$

In a particular problem sufficient data are usually given to determine the constant of integration and to enable us to find

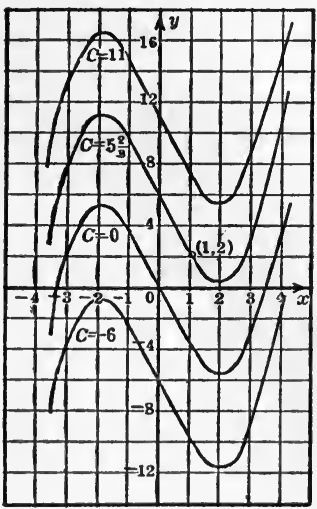

Fig. 173.

a particular integral which satisfies the given conditions. The graphical significance of the constant of integration and the method of determining its numerical value so as to satisfy given conditions is shown in

Example 2. Find $y$ if $D_{x} y=x^{2}-4$ and interpret the result graphically. Find the equation of that one of the resulting curves which passes through the point $(1,2)$.

By differentiating $x^{3}$ we obtain $3 x^{2}$, and hence we must differentiate $\frac{1}{3} x^{3}$ in order to get $x^{2}$. Differentiating $4 x$ gives 4 , and hence if

$$
\begin{aligned}
D_{x} y & =x^{2}-4, \\
y & =\frac{1}{3} x^{3}-4 x+C,
\end{aligned}
$$

where $C$ is the constant of integration.

This represents a set of curves which may be obtained from one of them by moving it up or down (Theorem, page 19). Several curves of this set are shown in the figure. 
These curves have parallel tangent lines at points with the same abscissas, since the derivative of $y$ with respect to $x$ is the same for each function, namely, $D_{x} y=x^{2}-4$.

The intercepts of these curves on the $y$-axis are the respective values of $C$. If $(1,2)$ lies on one of these curves then $2=\frac{1}{3} \cdot 1^{3}-4 \cdot 1+C$, whence $\mathrm{C}=5 \frac{2}{3}$ and the required equation is $y=\frac{1}{3} x^{3}-4 x+5 \frac{2}{3}$.

EXAMPLE 3. If $D_{x} y=a x^{n}$, show that $y=\frac{a}{n+1} x^{n+1}+C$.

The result is correct because if we differentiate it we get

$$
D_{x} y=\frac{a}{n+1}(n+1) x^{n}=a x^{n} .
$$

Hence, to integrate a term of the form ax ${ }^{n}$, increase the exponent by one and divide the coefficient by the new exponent.

In like manner, if $D_{x} y=a u^{n} D_{x} u$, then $y=\frac{a}{n+1} u^{n+1}+C$.

If the integral notation is used, these results may be written

$$
\int a x^{n} d x=\frac{a}{n+1} x^{n+1}+C, \quad \int a u^{n} D_{x} u d x=\frac{a}{n+1} u^{n+1}+C \text {. }
$$

Example 4. (a) Find $y$ if $D_{x} y=4 x^{3}$.

From the preceding example we have $y=\frac{4}{3+1} x^{4}+C=x^{4}+C$.

(b) Integrate $\mathcal{S}\left(3 x^{2}+8 x\right) d x$.

We have $\mathcal{S}\left(3 x^{2}+8 x\right) d x=\int 3 x^{2} d x+\int 8 x d x=x^{3}+4 x^{2}+C$.

(c) Find $y$, if $D_{x} y=\frac{8}{2}\left(x^{2}+3 x\right)^{\frac{1}{2}}(2 x+3)$.

If we think of $x^{2}+3 x$ as a function $u$, then $2 x+3$ is $D_{x} u$, and therefore

$$
y=\frac{3}{2} \frac{\left(x^{2}+3 x\right)^{\frac{3}{2}}}{3 / 2}+C=\left(x^{2}+3 x\right)^{\frac{3}{2}}+C .
$$

Differentiation is a direct process while integration is an inverse process. The former can be carried out according to a general method of procedure but there is no general method of procedure in the case of the latter. Integration in the last analysis is a matter of trial in which attempts are made to reduce the given function to a form the integral of which is known. A thorough knowledge of differentiation is essential for the process of integration.

\section{EXERCISES}

In Exercises 1-12, find $y$ if $D_{x} y$ is the given function, and illustrate the result graphically. Determine the equation of that member of the set of curves which passes through the indicated point. 

1. $x+1,(1,2)$.
2. $x^{2}-3,(-1,3)$.
3. $x^{2}-x,(0,2)$.
4. $x^{3}+x,(-1,11)$.
5. $x^{4},(3,4)$.
6. $\sqrt{x^{3}},(1,3)$.
7. $\sqrt{x+1},(4,-2)$.
8. $\frac{1}{x^{2}},(1,1)$.
9. $\frac{1}{\sqrt{x}},(2,1)$.
10. $\frac{1}{\sqrt{2 x+3}},(0,3)$.
11. $\frac{x}{\sqrt{x^{2}-4}},(2,4)$.
12. $\frac{2 x-3}{\sqrt{\left(x^{2}-3 x\right)^{3}}},(2,0)$.

13. For what value of $n$ does the result in Example 3 above fail?

14. Given $D_{x} y=\left(x^{2}+3 x-2\right)^{2}(2 x+3)$, find $y$.

15. Given $D_{x} y=\left(3 x^{2}+4\right) \sqrt{\left(x^{3}+4 x\right)^{3}}$, find $y$.

16. Find the equation of the curve whose slope at any point is $2 x+3$ and which passes through the point $(2,3)$. Find the equation of the tangent line at this point. Plot the curve.

17. The slope of a curve at any point is $6 x^{2}+4 x$ and it passes through the origin. Find the equation of the curve and the equation of the normal line at the point of inflection. Plot the graphs of the two equations.

18. Find the cubic function whose graph has a maximum point at $(1,4)$ and a minimum point at $(3,-5)$. Find the equation of the tangent line at the point of inflection. Suggestion. $D_{x} y=a(x-1)(x-3)$.

19. The slope of a curve at any point is $4 x-3$ and it passes through the point $(1,2)$. Find the equation of the curve and the coördinates of

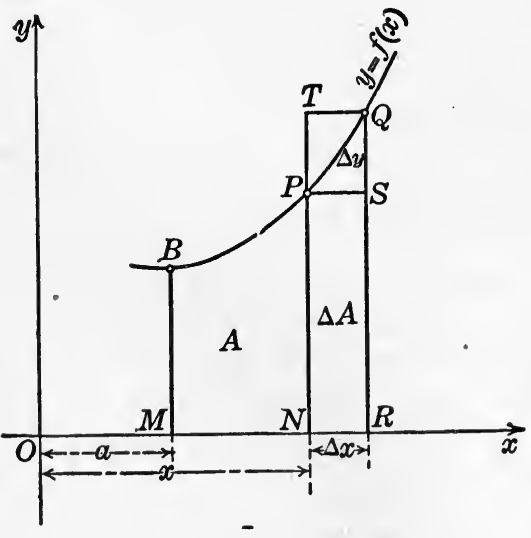

Fig. 174. the minimum point. Plot the graph.

20. Integrate the following:

(a) $\mathcal{S}\left(6 x^{2}+8 x-5\right) d x$.

(b) $\int x_{2}^{3} d x$.

(c) $\int 3 \sqrt{x-2} d x$.

(d) $\int \frac{3}{x^{\frac{2}{3}}} d x$.

(e) $\int 2 \sqrt{4-3 x} d x$.

106. Area under a Curve. Consider the area $A$, bounded by any curve $y=f(x)$, the $x$-axis, the fixed ordinate $M B$ at $x=a$, and the moving ordinate at $N P$.

Since $A$ changes as $x$ changes and is determined when $x$ is fixed, $A$ is a function of $x$.

As $N$ moves to $R, x$ increases by an amount $\Delta x, A$ by an amount $P N R Q=\Delta A$, and $y$ by an amount $S Q=\Delta y$. 
Since $P N R Q$ is less than the rectangle $T N R Q$ and greater than the rectangle $P N R S$, we have

$$
P N R S<\triangle A<T N R Q
$$

or

$$
y \Delta x<\Delta A<(y+\Delta y) \Delta x .
$$

Dividing by $\Delta x$, we have

$$
y<\frac{\Delta A}{\Delta x}<y+\Delta y
$$

As $\Delta x$ approaches zero, $y+\Delta y$ approaches $y$ and $\Delta A / \Delta x$ approaches $D_{x} A$; and as $\frac{\Delta A}{\Delta x}$ lies between the magnitudes $y$ and $y+\Delta y$ we have $D_{x} A=y=f(x)$.

Therefore we have the

Theorem. The rate at which the area $A$ changes with respect to $x$ is equal to the right-hand ordinate of the bounding curve.

Symbolically,

$$
D_{x} A=y \text {. }
$$

The area $A$ can now be found as a function of $x$ by integration, the constant of integration being determined by the fact that $A=0$ when $x=a$. If a fixed right-hand boundary is chosen the area is determined. The method of finding the area is shown in

EXAmple 1. Find the area bounded by the curve $y=x^{2}$, the $x$-axis and the ordinates at $x=1$ and $x=4$.

By the theorem, $D_{x} A=x^{2}$.

$$
\text { Integrating, } \quad A=\frac{x^{3}}{3}+C \text {. }
$$

Since $A=0$ when $x=1$, we have $0=\frac{1}{3}+C$ or $C=-\frac{1}{3}$.

Hence $\quad A=\frac{x^{3}}{3}-\frac{1}{3}$.

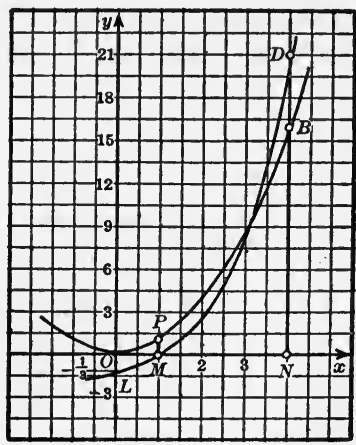

FIG. 175.

Equation (4) gives the area under the curve starting at the ordinate $M P$, at $x=1$, and continuing to any second ordinate. In this case the second ordinate is fixed at $x=4$. Hence substituting $x=4$ in (4) we have

$$
A=\frac{4^{3}}{3}-\frac{1}{3}=\frac{63}{3}=21 \text {. }
$$


The graph of (4) is the curve $L M D$ which crosses the $x$-axis at $x=1$ where the area $M P B N$ begins.

The number of square units in $M B P N$ is the same as the number of linear units in the ordinate $N D$, which is 21 units in length.

Equation (1) may be interpreted thus: Suppose that we had $y=c$, whose graph is a straight line parallel to the $x$-axis.

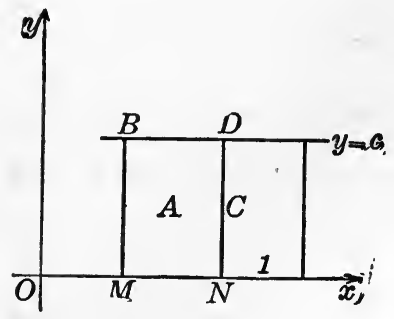

FIg. 176. Then the rate of change of $A$ with respect to $x, D_{x} A$, would be uniform, since $D_{x} A=y=c$. It would be measured by the change in $A$ due to a unit change in $x$ (page 48 ), that is, by a rectangle with base unity and altitude $c$.

Returning to the figure used in proving the theorem, let $N R=1$. Then the area of the rectangle $P N R S=y \times 1=y$ is the amount by which $A$. would increase when $x$ increases by unity, provided that $A$ increased uniformly for $x>O N$.

The method of proving the theorem above may be used to show that an area $A$ bounded by a curve, the $y$-axis, and two abscissas (one fixed, the other not), is such that

$$
D_{y} A=x \text {. }
$$

\section{EXERCISES}

1. Find the area under each of the following curves from $x=1$ to $x=3$.
(a) $y=3 x^{2}$,
(b) $y=2 / x^{2}$,
(c) $y=x^{3}$,
(d) $y=x^{2}+x+7$.

2. Find the area bounded by the curve $y=x^{2}$, the $x$-axis and the line $x=2$, and the area bounded by the curve, the $y$-axis and the line $y=4$. Check by finding the area of the rectangle formed by the axes and the lines $x=2$ and $y=4$.

3. Find the area above the $x$-axis and below the parabola $y=-x^{2}+4$.

4. If the positive intercepts of the parabola $y=-x^{2}+2 x+3$ on the coördinate axes are denoted by $A$ and $B$, and if a tangent parallel to the chord $A B$ cuts the ordinates at $A$ and $B$ in $C$ and $D$, and touches the parabola in $E$, show that the area of the parabolic segment $A B E$ is twothirds that of the parallelogram $A B C D$.

5. If the tangent to the parabola $y=3 x^{2}-6 x+8$, which is parallel to the chord joining the minimum point $A$ to the point $B$ on the curve 
whose abscissa is 2 , cuts the ordinates of $A$ and $B$ in $C$ and $D$, find the relation between the area of the parabolic segment from $A$ to $B$ and the area of the parallelogram $A B C D$.

6. Find the area bounded by the following pairs of curves:
(a) $y=x^{2}, y^{2}=x$,
(b) $y=x^{\frac{3}{2}}, y=x^{\frac{2}{3}}$,
(c) $y=-\frac{7}{8} x^{2}+\frac{15}{8} x, y=\frac{1}{x^{2}}$.

7. Find the area bounded by the curve $y=x^{3}-6 x^{2}+9 x+4$, the tangent to the curve at the point of inflection, and the ordinates at the maximum and minimum points.

8. Show that the area under the parabola $y=a x^{2}+b x+c$ between $x=h$ and $x=k,(k>h)$, is $A=\frac{(k-h)}{6}\left(y_{1}+4 y_{2}+y_{3}\right)$, where $y_{1}, y_{2}, y_{3}$ are the values of $y$ at $x=h, x=\frac{h+k}{2}, x=k$ respectively. Suggestion: Substitute the values of $y$ in the above expression and show that the result is the same as the area under the curve.

9. Find the area under the following parabolas and check the results by means of the formula for the area given in Exercise 8.

(a) $y=2 x^{2}+4 x+1$, from $x=1$ to $x=3$.
(b) $y=-x^{2}+9$; from $x=0$ to $x=3$.
(c) $y=3 x^{2}-4 x+3$, from $x=0$ to $x=2$.

10. Show that the area formula in Exercise 8 is true for any cubic function $y=a x^{3}+b x^{2}+c x+d$.

11. Find the area under the curve $y=x^{3}+12 x+4$ from $x=1$ to $x=3$. Check the result by means of the formula in Exercise 8 .

Definition. The average ordinate of a curve $y=f(x)$ from $x=a$ to $x=b$ is the height of the rectangle with base $b-a$, whose area is equal to the area under the curve from $x=a$ to $x=b$.

If $y$ represents the average ordinate and $A$ the area under the curve from $x=a$ to $x=b$, then

$$
y=\frac{A}{b-a} \text {. }
$$

12. Find the average ordinate of the following curves for the ranges indicated.
(a) $y=2 x+3$, from $x=1$ to $x=4$.
(b) $y=-x^{2}+16$, from $x=0$ to $x=4$.
(c) $y=1 / x^{2}$, from $x=1$ to $x=3$.
(d) $y=x^{\frac{3}{2}}$, from $x=0$ to $x=4$. 
13. Given $\theta=t^{3}-9 t^{2}+15 t+30$, where $\theta$ is the temperature at any time $t$, find the average temperature for the range from maximum to minimum temperature.

14. The pressure $p$ and the volume $v$ of a gas are connected by the equation $p v^{1.2}=k$. and $p=15$ pounds per square inch when $v=0.5$ of a cubic foot. Find the average pressure of the gas in expanding from 1 to 3 cubic feet.

15. The tension $T$ of a spring is connected with the amount of stretching $s$ by the equation $T=\frac{1}{2} s+3$. Find the average tension as $s$ changes from 0 to 3 .

16. Find the percentage of error in the area under the curve $y=x^{2}-2 x+3$ from $x=0$ to $x=2$, due to an error of 1 per cent in the range.

107. Motion in a Straight Line. The fundamental notions involved in the motion of a particle in a straight line are:

The distance, $s$, measured from a convenient station on the line.

The time, $t$, which has elapsed from a fixed time.

The velocity, $v$, given by the equation $v=D_{t} s$.

The acceleration, $a$, given by the equation $a=D_{t} v$.

The motion may be described by an equation involving two or more of the magnitudes $t, s, v, a$.

We have already considered motions described by an equation of the form $s=f(t)$, and obtained the velocity and acceleration by differentiation. We shall now consider two other types in which integration is involved.

If the velocity is given as a function of the time by an equation of the form $v=f(t)$, the acceleration, $a$, is found by differentiating while the space, $s$, is found by integrating this equation. The constant involved in the integration is determined as in the following example.

Example 1. A body moves from rest in a straight line so that its velocity after $t$ seconds is given by the law $v=4 t$. Assuming that a ball placed on a plane inclined at arc $\sin \frac{1}{8}$ would roll according to this law, determine:

(a) How far it will roll in 2 seconds.

(b) Its distance from the upper end of the plane after 1.5 seconds, if it is placed 4 feet from that end.

(c) Its distance from the lower end after 1 second, if it is placed 12 feet from the lower end. 
We know that if $s$ represents distance from a certain point at the time $t$, then $D_{t} s=v$, and hence, in this example,

$$
D_{t} s=4 t \text {. }
$$

The required function, $s$, is therefore one whose derivative with respect to $t$ is $4 t$. One such function is $2 t^{2}$, and adding the constant of integration (page 302), we have

$$
s=2 t^{2}+C .
$$

The value of the constant of integration may be determined if we know a pair of values of $t$ and $s$, and these depend on when and where the ball starts to roll. We shall assume that $t=0$ when the ball begins to roll. The distance $s$ is measured from some chosen station $O$.

(a) The distance the ball will roll in 2 seconds is the same as its distance from the starting point after 2 seconds. Hence for this part of the problem we choose the station $O$ at the starting point, so that $s=0$ when $t=0$. Substituting these values in (1) we find that $C=0$. Hence, substituting this value of $C$ in (1), the distance from the starting point after $t$ seconds is

$$
s=2 t^{2} \text {. }
$$

Then at the end of 2 seconds, $s=2 \times 2^{2}=8$ feet.

(b) Take $O$ at the upper end of the plare. Since the ball starts 4 feet from $O$, we have $s=4$ when $t=0$. Substituting these values in (1), we find that $C=4$. Setting this value of $C$ in (1), the distance from the upper end of the plane to the ball after $t$ seconds is

$$
\begin{aligned}
& s=2 t^{2}+4 . \\
& s=2(1.5)^{2}+4=8.5 \text { feet. }
\end{aligned}
$$

After 1.5 seconds,

That is, the ball is 8.5 feet from the upper end of the plane after 1.5 seconds.

(c) If $O$ is taken at the lower end of the plane and the ball placed 12 feet above it, then $s=-12$ when $t=C$ ( $s$ being negative since the positive direction for $s$ is the same as that of $v$, namely, down the plane). Substituting in (1) we find $C=-12$, so that the distance from the lower end of the plane at any time is

$$
s=2 t^{2}-12 .
$$

If $t=1, s=-10$, that is, after one second the ball is $\mathbf{1 0}$ feet above the lower end of the plane.

Graphically, the given relation $v=4 t$ is represented by the straight line through the origin whose slope is 4 . The graph of (1) is given for $C=0,4,-12$,

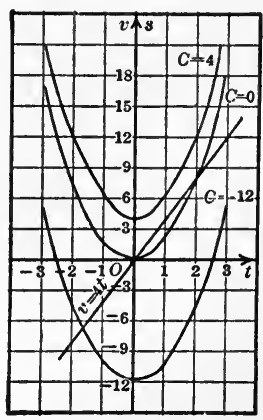

Fig. 177. which are the values of $C$ in (2), (3), (4), respectively. The intercepts of these curves on the $s$-axis are the respective values of $C$, which give the distances from the station $O$ to the ball when $t=0$. 
If the acceleration is given as a function of the time, $t$, by an equation of the form $a=f(t)$, the velocity is obtained by integrating this equation and the distance by integrating the result. Two constants of integration are introduced in the process which are determined by given conditions as in

Example 2. A balloon is ascending with a uniform velocity of 28 feet per second and at the height of 720 feet a ball is dropped. When will the ball strike the ground and with what velocity?

Let $s$ be the height of the ball above the ground, and let $t=0$ when the ball is dropped.

The direction of the acceleration of gravity, being toward the center of the earth, is opposite to the positive direction of $s$, and hence the acceleration is negative.

The initial conditions and required data are collected in the table. As soon as the ball leaves the balloon it is subject to the law

\begin{tabular}{c|c|c|c}
$t$ & $s$ & $v$ & $a$ \\
\hline 0 & 720 & 28 & -32 \\
$?$ & 0 & $?$ & -32
\end{tabular}

$$
a=-32 \text {. }
$$

Integrating (1), $v=-32 t+C_{1}$.

When $t=0, v=28$, and therefore $C_{1}=28$.

Hence $\quad v=-32 t+28$.

Integrating (3), $s=-16 t^{2}+28 t+C_{2}$.

When $t=0, s=720$, hence $C_{2}=720$. Therefore

$$
s=-16 t^{2}+28 t+720 \text {. }
$$

When the ball strikes the ground $s=0$. Substituting this value of $s$ in (5)

or

$$
\begin{gathered}
0=-16 t^{2}+28 t+720, \\
4 t^{2}-7 t-180=0 .
\end{gathered}
$$

Solving for $t$ we have

$$
t=\frac{7 \pm \sqrt{49+2880}}{8}=\frac{54.2}{8}=6.8 \text { seconds, }
$$

the negative value of $t$ having no meaning in this problem. Substituting this value of $t$ in (3)

$$
v=-32(6.8)+28=-189.6 \text { feet per second. }
$$

\section{EXERCISES}

1. A ball is rolled up a plane inclined at an angle of $10^{\circ}$ with an initial velocity of 15 feet per second. Find the distance it rolls up the plane and the time that elapses before it returns to the starting point. (Suggestion: Find the component of the acceleration of gravity acting along the plane.) 
2. Solve Example 2 of the preceding section if the balloon is descending.

3. A high jumper raises his center of gravity 3 feet. How long is he off the ground, and with what velocity does he light?

4. A baseball dropped from one of the windows of the Washington monument, 500 feet from the ground, has been caught. Compare the velocity with which it struck the catcher's hand with the velocity of 120 feet per second which is said to be the maximum velocity that a pitcher has imparted to a ball.

5. A man descending in an elevator whose velocity is 10 feet per second drops a ball from a height of 6 feet above the floor. How far will the elevator descend before the ball strikes the floor of the elevator?

6. In the preceding problem, suppose the elevator is ascending instead of descending.

7. A balloon is ascending with a velocity of 24 feet per second when a ball is dropped from it. The ball reaches the ground in 5 seconds. Find the height of the balloon when the ball was dropped. Determine the highest point that the ball reached.

8. An automobile reduces its speed from 35 miles an hour to 20 miles an hour in 8 seconds. If the retardation is uniform how much longer will it be before it will come to rest, and how far will it travel in this length of time?

9. How high will a ball rise if thrown vertically upward with an initial velocity of 60 feet per second?

10. A street car in going from one stop to another 400 feet distant is uniformly accelerated at the rate of 2 feet per second per second for a distance of 320 feet and then brought to rest with a uniform retardation. Find the time it took to go the 400 feet.

108. Motion in a Plane. If a particle moves along a curve in a plane, two rectangular axes are chosen and the position of the particle at any time determined by means of the coördinates of the particle.

If the coördinates of the particle on the axes are given by the equations

$$
x=f(t) \text { and } y=F(t)
$$

the position of the particle in the plane is determined.

The discussion of the motion of a particle in a plane is thus resolved into the discussion of two motions along straight lines.

The components of the velocity, obtained by differentiating equations (1) are

$$
v_{x}=D_{t} x \quad \text { and } \quad v_{y}=D_{t} y .
$$


They are represented in Fig. 178 by directed lines parallel to the axes.

Since the components are perpendicular the magnitude of the resultant velocity, $v$, is given by the equation

$$
v=\sqrt{v_{x}^{2}+v_{y}^{2}},
$$

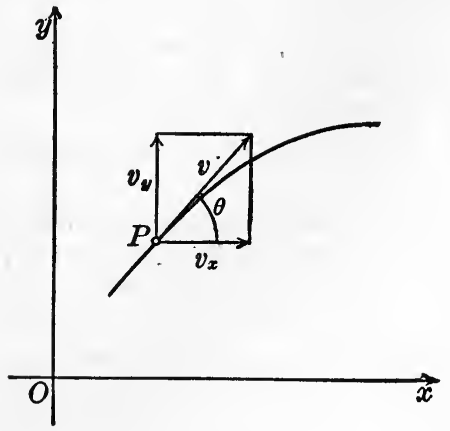

FIG. 178.

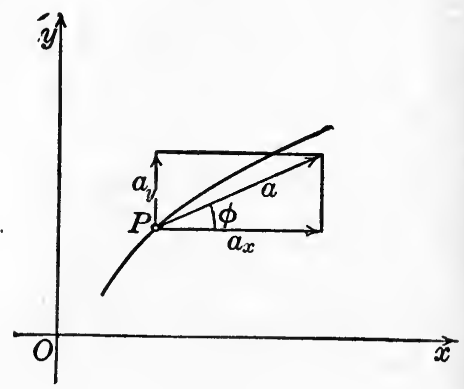

Fig. 179.

and the direction of the velocity can be found from the equation

$$
\tan \theta=v_{y} / v_{x} .
$$

The components of the acceleration, obtained by differentiating equations (2) are

$$
a_{x}=D_{t} v_{x} \quad \text { and } \quad a_{y}=D_{t} v_{y} .
$$

They are represented in Fig. 179 by directed lines parallel to the axes.

The magnitude of the resultant acceleration, $a$, is given by the equation

$$
a=\sqrt{a_{x}^{2}+a^{2}}
$$

and its direction can be found by means of the equation

$$
\tan \phi=a_{y} / a_{x} \text {. }
$$

Example 1. If a particle moves in accordance with the law $x=t^{3}$, $y=t^{2}$, find the equation of the path, the position of the particle when $t=2$, and the magnitude and direction of $v$ and of $a$ at this point.

From the first equation $t=x^{\frac{1}{3}}$ and hence $y=\left(x^{\frac{1}{2}}\right)^{2}=x^{\frac{3}{3}}$, which is the equation of the path shown in Fig. 180. Differentiating the given equar tions with respect to $t$ we obtain 
Hence

$$
v_{x}=3 t^{2}, \quad \text { and } \quad v_{y}=2 t .
$$

$$
v=\sqrt{9 t^{4}+4 t^{2}}=t \sqrt{9 t^{2}+4}
$$

and

$$
\tan \theta=2 t / 3 t^{2}=2 / 3 t .
$$

Differentiating equations obtain,

(1) we

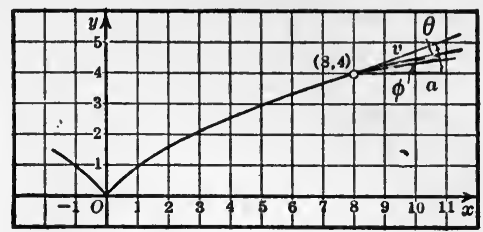

FIg. 180.

$$
a_{x}=6 t, \text { and } a_{y}=2
$$

Hence

$$
a=\sqrt{36 t^{2}+4},
$$

and

$$
\tan \phi=2 / 6 t=1 / 3 t .
$$

Substituting $t=2$ in equations (2), (3), (5), (6), we find $v=12.64$, $\theta=18^{\circ} .43, a=12.17, \phi=9^{\circ} .46$, at the point where $x=8$ and $y=4$.

The inverse problem of determining the path of a particle, given the equations of the component accelerations, is illustrated in

Example 2. Find the equation of the path of a projectile fired at an angle of $30^{\circ}$ to the horizontal with a muzzle velocity of 1200 feet per second.

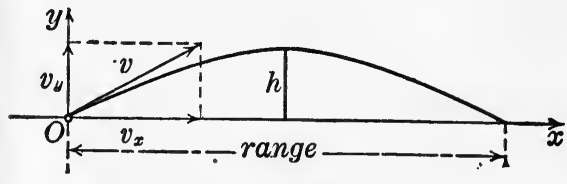

FIG. 181. Find the range of the projectile and the maximum height attained.

Let the origin be taken at the initial point of the path, the $x$-and $y$-axes being horizontal and vertical.

The horizontal and vertical components of the initial velocity are $1200 \cos 30^{\circ}$, and $1200 \sin 30^{\circ}$, respectively.

If the resistance of the air be disregarded there will be no horizontal acceleration.

From the instant the projectile leaves the gun gravity is acting on it vertically in the direction opposite to the positive direction on the $y$-axis.

Hence the acceleration equations of the motion are

$$
a_{x}=0 \text { and } a_{y}=-32 .
$$

Integrating these equations we have

$$
v_{x}=C_{1} \text { and } v_{y}=-32 t+C_{2} .
$$

When $t=0, v_{x}=1200 \cos 30^{\circ}$, and $v_{y}=1200 \sin 30^{\circ}$.

Therefore $C_{1}=1200 \cos 30^{\circ}$, and $\mathrm{C}_{2}=1200 \sin 30^{\circ}$.

Hence $v_{x}=1200 \cos 30^{\circ}$, and $v_{y}=-32 t+1200 \sin 30^{\circ}$. 
Integrating (3),

$$
x=1200 \cos 30^{\circ} \cdot t+C_{8} \text { and } y=-16 t^{2}+1200 \sin 30^{\circ} \cdot t+C_{4} .
$$

To determine the constants we note that when $t=0, x=0, y=0$, and therefore $C_{3}=0$, and $C_{4}=0$.

Hence

$$
x=1200 \cos 30^{\circ} \cdot t \text { and } y=-16 t^{2}+1200 \sin 30^{\circ} \cdot t .
$$

These equations give the position of the particle at any time. Solving the first of these equations for $t$ and substituting in the second we find the equation of the path to be

$$
y=-\frac{16}{1200^{2} \cos ^{2} 30^{\circ}} x^{2}+\tan 30^{\circ} \cdot x,
$$

which is the equation of a parabola.

To find the range, let $y=0$ in (5) and solve for $x$.

Hence $\quad x=\frac{1200^{2} \cos ^{2} 30^{\circ} \tan 30^{\circ}}{16}=38,970$ feet $=7.38$ miles.

At the highest. point $v_{y}=0$, hence from (3), $0=-32 t+1200 \sin 30^{\circ}$. Therefore the highest point is reached when $t=\frac{1200 \sin 30^{\circ}}{32}=18^{\frac{3}{4}}$ seconds. Substituting this value of $t$ in (4), we obtain for the maximum height attained

$$
y=-16\left(\frac{75}{4}\right)^{2}+1200 \sin 30^{\circ} \cdot\left(\frac{75}{4}\right)=5625 \text { feet. }
$$

\section{EXERCISES}

1. In the following exercises find the equation of the path in terms of $x$ and $y$ by eliminating $t$ from the given equations, plot the path, find the magnitudes and directions of $v$ and $a$ for the given value of $t$, and at the point on the path corresponding to this value draw lines representing $v$ and $a$ in magnitude and direction.

(a) $x=2 t, y=t^{2}, t=1$.

(b) $x=t, y=1 / t, t=1$.

(c) $x=3 t^{2}+2, y=2 t^{2}, t=2$.

(d) $x=2 t, y=1 /\left(t^{2}+1\right), t=0$.

(e) $x=3 t^{3}, y=3 t, t=1$.

2. Find the equation of the path of a projectile fired at an angle of $20^{\circ}$ to the horizontal with a muzzle velocity of 1500 feet per second; find the range of the projectile, the maximum height attained and plot the path.

3. Find the equation of the path of a projectile fired at an angle of $\theta^{\circ}$ to the horizontal with an initial velocity of $v_{0}$ feet per second. Find the range and the maximum height attained. Find $v_{0}$ if the range is 24 miles for $\theta=45^{\circ}$.

4. A bomb is dropped from an aeroplane 8000 feet high moving horizontally at a velocity of $\mathbf{1 2 0}$ miles an hour. Determine how far the bomb 
will fall from the point on the ground directly below the aeroplane at the instant it was dropped. Suggestion: If the point on the ground is chosen as the origın with the axes horizontal and vertical, the initial conditions are as given in the table

\begin{tabular}{|c|c|c|c|c|c|c|}
\hline$t$ & $x$ & $y$ & $v_{x}$ & $v_{y}$ & $a_{x}$ & $a_{y}$ \\
\hline 0 & 0 & 8000 & 176 & 0 & 0 & -32 \\
\hline
\end{tabular}

5. A bomb is dropped from an aeroplane $h$ feet high and moving with a velocity of $v_{0}$ feet per second. If $d$ represents the distance from a point on the ground directly below the aeroplane when the bomb was dropped to the point where it strikes the ground, find $d$ as a function of $h$ and $v_{0}$. Plot the graph of $d$ as a function of $v_{0}, h$ being constant, also of $d$ as a function of $h, v_{0}$ being constant. In the first case what is the effect on $d$ of doubling $v_{0}$, and in the second case of doubling $h$ ?

6. A ball rolls off a roof inclined at $30^{\circ}$ to the horizontal. If it starts 20 feet from the eaves, which are 30 feet from the ground, where will the ball strike the ground?

7. The path of a particle is given by the equations $x=t^{2}$ and $y=2 t$. Plot the path, find the acceleration when $t=1$, and the components of the acceleration along the tangent and normal lines to the path at the point in question.

8. Find the range of a rifle ball fired horizontally from a point 5 feet above the ground if the initial velocity is 2000 feet per second. How much is the deviation from the horizontal at 100 yards? 200 yards?

9. A particle moves in a circle whose center is at the origin of a rectangular system of axes. The angle (in radians) through which it turns from an initial position at rest on the $x$-axis is given by the equation $\theta=t^{2}-t$. Find the angular velocity $\omega=D_{t} \theta$ and the angular acceleration $\alpha=D_{t} \omega$ when $t=3$.

10. A wheel revolving at the rate of 120 revolutions per second is retarded uniformly so that in 3 seconds, $\omega=90$ revolutions per second. How long before the wheel will come to rest, and where will a point $P$ on the rim be if when $t=0$ the angle for $P_{0}$ is $\theta=0$ ?

109. Volume of a Right Prism. A prism is a solid bounded by two congruent polygons lying in parallel planes with their corresponding sides parallel and by the parallelograms determined by the pairs of corresponding sides of the polygons. The other sides of these parallelograms are called the lateral edges of the prism, the polygons are called the bases, and the parallelograms the lateral faces of the prism.

A right prism is one whose lateral edges are perpendicular to the planes of the bases. 
A rectangular parallelopiped is a right prism whose base is a rectangle, e.g., an ordinary box.

A polyhedron is a solid bounded by portions of planes. The volume of a polyhedron is the ratio of the polyhedron to a second solid taken as the unit of volume. The unit of volume is usually a cube whose edge is the linear unit.

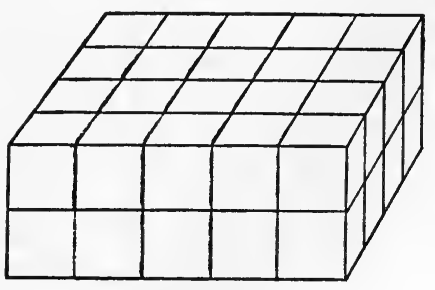

FIG. 182.

Theorem 1. The volume of a rectangular parallelopiped is the product of the area of its base and the length of its altitude.

If the three dimensions of the rectangular parallelopiped are integers, $h, j, k$, the number of unit cubes in it is easily counted.

In any horizontal layer in the figure, there are $k$ rows of cubes, $j$ in each row. Hence by the definition of the multiplication of integers, there are $j k$ cubes in each layer. And since there are $h$ layers, the parallelopiped contains $h j k$ cubes.

Hence the ratio of the parallelopiped to the unit cube is $h j k$, so that the volume is

$$
V=h j k \text {. }
$$

If $b$ is the area of the base, $b=j k$, and hence the volume $V$ is

$$
V=h j k=b h \text {. }
$$

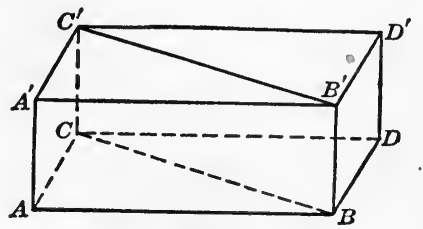

(a)

We shall assume that this result holds when the dimensions are not integers.

A block of wood in the form of a rectangular parallelopiped $A D^{\prime}$ may

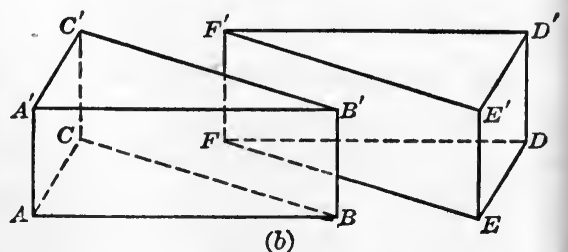

Fig. 183. be sawed into two congruent pieces, as in the figure. These pieces are triangular right prisms, whose bases are right triangles. 
Theorem 2. The volume of a triangular right prism, whose base is a right triangle, is the product of the base and the altitude. If $V$ is the volume of the triangular prism $A B C A^{\prime} B^{\prime} C^{\prime}$, then

$$
\begin{aligned}
V & =\frac{1}{2} \text { volume of } A D^{\prime} \\
& =\frac{1}{2} \text { area } A B D C \times A A^{\prime} \\
& =\text { area } A B C \times A A^{\prime} \\
& =b h,
\end{aligned}
$$

where $b$ is the area of the base, and $h$ is the altitude of the given triangular prism.

Theorem 3. The volume of any triangular right prism is the product of the area of the base and the length of its altitude.

Any triangular right prism whose base $A B C$ is an oblique triangle may be split into two triangular right prisms whose

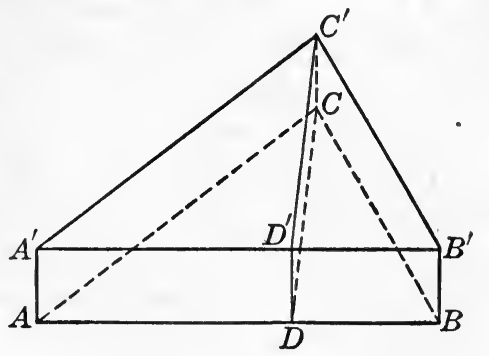

Fig. 184. bases $A D C$ and $B D C$ are right triangles, as indicated in the figure. Then

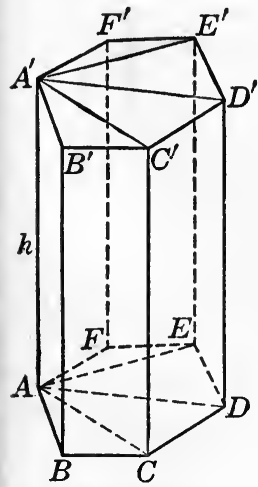

Fra. 185.

$$
\begin{aligned}
V & =A B C A^{\prime} B^{\prime} C^{\prime} \\
& =A D C A^{\prime} D^{\prime} C^{\prime}+B D C B^{\prime} D^{\prime} C^{\prime} \\
& =A D C \times D D^{\prime}+B D C \times D D^{\prime} \\
& =(A D C+B D C) \times D D^{\prime} \\
& =A B C \times D D^{\prime} \\
& =b h,
\end{aligned}
$$

where $b$ is the area of the base and $h$ the length of the altitude of the given prism.

Theorem 4. The volume of a right prism is the product of the area of the base and the length of its altitude.

Let $A D^{\prime}$ be any right prism. It may be divided into triangular right prisms by planes through one of the lateral edges, say $A A^{\prime}$, and each of the non-adjacent lateral edges. If $V$ is the volume, $b$ the area of the base, and $h$ the altitude of the prism, we have 


$$
\begin{aligned}
V & =A D^{\prime}=A B C B^{\prime}+A C D C^{\prime}+A D E D^{\prime}+\cdots \\
& =A B C \times h+A C D \times h+A D E \times h+\cdots \\
& =(A B C+A C D+A D F+\cdots) h \\
& =b h .
\end{aligned}
$$

110. Volume of a Right Circular Cylinder. A cylindrical surface is a surface generated by a straight line which moves parallel to a given line so as to cut a given curve. The various positions of the moving line are called the elements of the surface.

A cylinder is a solid bounded by a cylindrical surface and two parallel planes. The sections of these planes cut out by the surface are called the bases, and the distance between them the altitude of the cylinder.

In a right cylinder the elements are perpendicular to the planes of the bases, and any element is equal to the altitude. If the bases are circles, the solid is called a right circular cylinder.

Let $r$ be the radius of the base and $h$ the altitude of a right circular cylinder.

Construct a right prism whose bases are regular polygons of $n$ sides inscribed in the bases of the cylinder, and a second

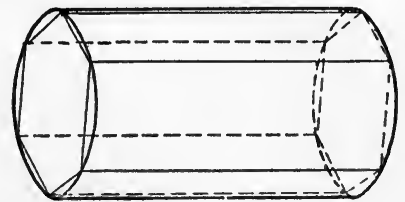

(a)

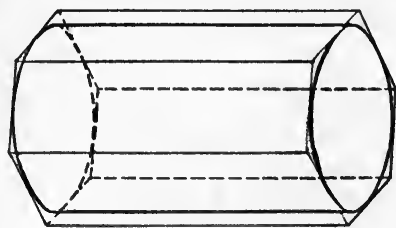

(b)

Fig. 186.

prism whose bases are regular polygons of $n$ sides circumscribed about the bases of the cylinder. Let $V^{\prime}$ and $V^{\prime \prime}$ be the volumes of these prisms, $b^{\prime}$ and $b^{\prime \prime}$ the areas of their bases. Their altitudes are equal to $h$. Then by Theorem 4, Section 109, $V^{\prime}=b^{\prime} h$ and $V^{\prime \prime}=b^{\prime \prime} h$.

Now let $n$ increase indefinitely. Then by a theorem of plane geometry, $b^{\prime}$ and $b^{\prime \prime}$ approach the area of the circle, $\pi r^{2}$, as a limit. Hence, by Theorem 2, page 266, $V^{\prime}$ and $V^{\prime \prime}$ approach the same limit $\pi r^{2} h$. 
This common limit of $V^{\prime}$ and $V^{\prime \prime}$ is defined to be the volume of the cylinder, $V$. Hence, we have the

Theorem. The volume of a right circular cylinder is the product of the area of its base and the length of its altitude; that is,

$$
V=\pi r^{2} h \text {. }
$$

\section{EXERCISES}

1. Find the volume of a right prism with a hexagonal base 6 inches on a side and an altitude of 8 inches. Find the lateral area (area of the lateral faces) and also the total area of the prism.

2. Find the volume of a cylinder if $r=16$ and $h=12$.

3. Theorem. If the radius of a cylinder is $r$ and the altitude is $h$, then the lateral area $S$ is $S=2 \pi r h$, and the total area $T$ is $T=2 \pi r(r+h)$.

Suggestion: The curved surface of a right cylinder may be spread out in the form of a rectangle.

4. A tin can is 3 inches in diameter and 5 inches high. Find the amount of tin needed for its construction and its capacity in quarts. (231 cubic inches equal one gallon.)

5. How much space will be occupied by a ton of furnace coal if 1 cubic foot weighs 52 pounds? How many tons can be placed in a bin 10 by 5 by 7 feet? Measure the dimensions of your bin and calculate its capacity.

6. How many barrels of Portland cement (weight $=425$ pounds) will be required to construct a silo in the form of a hollow cylinder 8 inches thick, 8 feet inside diameter and 24 feet high, if cement weighs 90 pounds a cubic foot?

7. What force will be needed to drag a block of ice 2 by 4 by 6 feet up an incline of $35^{\circ}$, if the density of ice is 0.92 and 1 cubic foot of water weighs 62.4 pounds?

8. Find the area of a cylindrical water tank on top of a building if the tank has an altitude of 6 feet and a diameter of 5 feet. What will it cost to line it with zinc 0.05 of an inch thick, at $\$ 0.20$ a pound (density of zinc $=7.9)$ ? What will be the capacity of the tank in gallons?

9. Find the volume and total area of a right prism if its base is a regular pentagon with a side of 3 inches and its altitude is 20 inches.

10. Find the volume and total area of the cylinder inscribed in the prism in Exercise 9.

11. A maple syrup can is a rectangular parallelopiped with a square base and holds one gallon. Find the most economical proportions for the manufacturer.

12. Find the volume of the largest cylindrical package that can be sent by parcel post (see Exercise 8, page' 288). (As a circle has a greater area 
than any other plane figure with the same perimeter, a cylindrical package is the largest package that can be mailed.)

13. Find the dimensions of a tomato can holding one quart, if the amount of tin used is a minimum (one quart $=57.75$ cubic inches).

14. The length and diameter of a cylindrical bar are nearly 24 inches and 3 inches respectively. Find the error and relative error in the volume due to an error of 0.02 of an inch in measuring the diameter.

15. What is the allowable error in measuring (a) the diameter of the base, 4 inches, (b) the height, 12 inches, of a cylinder if its volume is to be determined within $\frac{1}{5}$ of one per cent? Assume in each case that the other measurement is correct. What is the allowable percentage of error in the measurement in each case?

16. A piston slides freely in a circular cylinder of diameter 6 inches. At what rate is the piston moving when steam is admitted into the cylinder at the rate of 11 cubic feet per second?

17. Assuming that a joint of a bamboo is a cylinder whose diameter is proportional to its length, find the rate of increase of the volume in terms of the rate of increase of the length when the bamboo is growing. Compare the rates when the diameter is $\frac{1}{2}$ inch and when it is $1 \frac{1}{2}$ inches, assuming that the length increases uniformly.

18. A block of granite (density $=2.7$ ) 3 by 2 by 6 feet is being raised by a crane. Find the strain in the guy rope $B C$ and the thrust in the jib $A B$ as the block is about to swing to a landing. The angle of elevation of the jib is $75^{\circ}$, and the angle between the jib and guy rope, $A B C$, is $10^{\circ} .2$.

111. Volume of a Pyramid. A pyramid is a solid bounded by a polygon and a set of triangles with a common vertex whose bases are the sides of the polygon. The polygon is called the base, the triangles the lateral faces, and the common vertex the vertex of the pyramid. The altitude of the pyramid is the perpendicular distance from the vertex to the base.

If the base is a regular polygon, and if the foot of the altitude is the center of the base, the pyramid is said to be regular.

Theorem 1. If a pyramid is cut by a plane parallel to the base, the section formed is to the base as the square of its distance from the vertex is to the square of the altitude.

Let $O-A B C D E$ be the pyramid, and $A^{\prime} B^{\prime} C^{\prime} D^{\prime} E^{\prime}$ the section.

Let the altitude $O P$ cut the section at $P^{\prime}$.

The pairs of triangles $O A B, O A^{\prime} B^{\prime} ; O B C, O B^{\prime} C^{\prime}$; etc., are similar (why?), and hence 


$$
\frac{A^{\prime} B^{\prime}}{A B}=\frac{O B^{\prime}}{O B}=\frac{B^{\prime} C^{\prime}}{B C}=\frac{O C^{\prime}}{O C}=\frac{C^{\prime} D^{\prime}}{C D}=\cdots
$$

The equality of the alternate ratios shows that the corresponding sides of the polygons are proportional.

The corresponding angles of the polygons are equal. To show $\angle B^{\prime} C^{\prime} D^{\prime}=\angle B C D$, for example, draw $B D$ and $B^{\prime} D^{\prime}$. Then since the triangles $O B D$ and $O B^{\prime} D^{\prime}$ are similar (why?)

$$
\frac{O B^{\prime}}{O B}=\frac{B^{\prime} D^{\prime}}{B D}
$$

and by combining this result with (1) it is seen that the corresponding sides of the triangles $B C D$ and $B^{\prime} C^{\prime} D^{\prime}$ are proportional. Hence these triangles are similar, and therefore $\angle B^{\prime} C^{\prime} D^{\prime}$ $=\angle B C D$.

Since the corresponding angles of the polygons are equal, and the corresponding sides proportional, the polygons are similar.

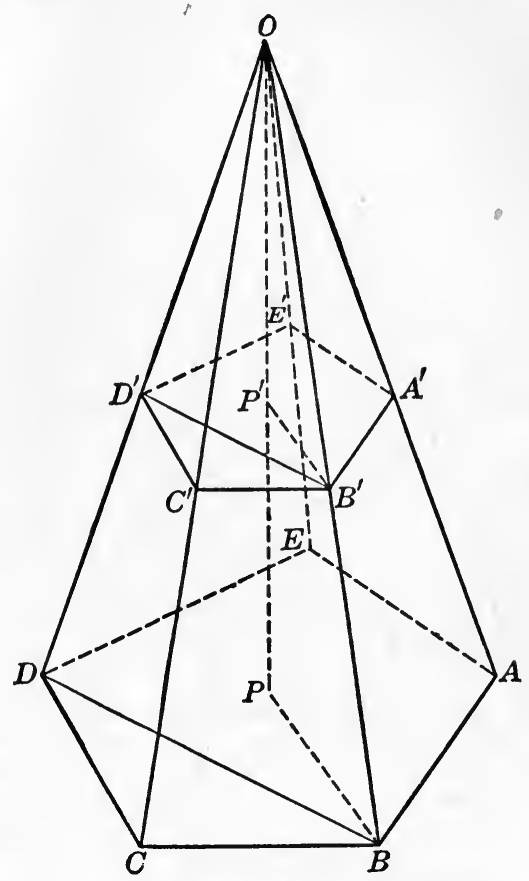

FIG. 187.

Hence their areas are proportional to the squares of any two corresponding sides, so that

$$
\frac{A^{\prime} B^{\prime} C^{\prime} D^{\prime} E^{\prime}}{A B C D E}=\frac{A^{\prime} B^{\prime 2}}{A B^{2}}
$$

But from (1), and from the similar triangles $O B P$ and $O B^{\prime} P^{\prime}$,

$$
\frac{A^{\prime} B^{\prime}}{A B}=\frac{O B^{\prime}}{O B}=\frac{O P^{\prime}}{O P} \text {. }
$$

Hence

$$
\frac{A^{\prime} B^{\prime 2}}{A B^{2}}=\frac{O P^{\prime 2}}{O P^{2}},
$$


so that,

$$
\frac{A^{\prime} B^{\prime} C^{\prime} D^{\prime} E^{\prime}}{A B C D E}=\frac{O P^{\prime 2}}{O P^{2}}
$$

Theorem 2. The volume of a pyramid is one-third the product of the area of the base by the length of the altitude.

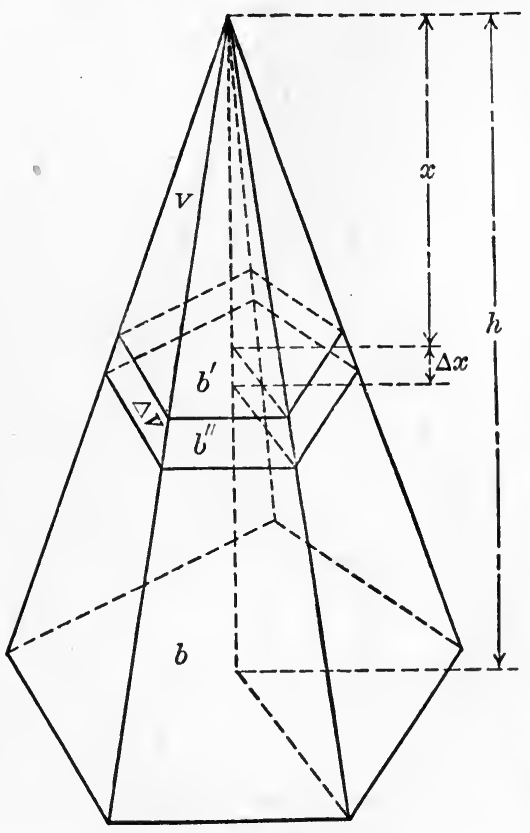

FIG. 188 .

Let $V$ denote the volume of that part of the pyramid between the vertex and a section parallel to the base at a distance $x$ from the vertex.

Let $b$ be the area of the base, $b^{\prime}$ the area of the section, and $h$ the altitude.

Let $b^{\prime \prime}$ be the area of a section parallel to the base at a distance $x+\Delta x$ from the vertex.

When $x$ increases by $\Delta x$, $V$ increases by an amount $\Delta V$ equal to the part of the pyramid between $b^{\prime}$ and $b^{\prime \prime}$. This part of the pyramid is greater than a prism with base $b^{\prime}$ and altitude $\Delta x$, and less than a prism with base $b^{\prime \prime}$ and altitude $\Delta x$.

Hence

$$
b^{\prime} \Delta x<\Delta V<b^{\prime \prime} \Delta x \text {. }
$$

Dividing by $\Delta x$ we have

$$
b^{\prime}<\frac{\Delta V}{\Delta x}<b^{\prime \prime} .
$$

As $\Delta x$ approaches zero, $b^{\prime \prime}$ approaches $b^{\prime}$ and $\frac{\Delta V}{\Delta x}$ approaches $D_{x} V$. Hence, passing to the limit;

$$
D_{x} V=b^{\prime} .
$$


But by Theorem $1, \frac{b^{\prime}}{b}=\frac{x^{2}}{h^{2}}$, or $b^{\prime}=\frac{b}{h^{2}} x^{2}$.

Hence, substituting in (1), $\quad D_{x} V=\frac{b}{h^{2}} x^{2}$.

Integrating

$$
V=\frac{b}{3 h^{2}} x^{3}+C .
$$

As $V=0$ when $x=0$, we have $C=0$, and hence

$$
V=\frac{b}{3 h^{2}} x^{3}
$$

When $x=h$, the value of $V$ is the volume of the given pyramid. Hence the required volume is

$$
V=\frac{b}{3 h^{2}} h^{3}=\frac{1}{3} b h .
$$

112. Volume of a Solid of Revolution. A solid of revolution is a solid generated by revolving an area about an axis. Such a solid may be turned out on a lathe. A section of the solid by a plane perpendicular to the axis is always a circle.

Theorem. The volume $V$ generated by revolving about the $x$-axis the area bounded by the curve $y=f(x)$, the $x$-axis, and the ordinates to the curve at the points whose abscissas are a and $x$ is such that

$$
D_{x} V=\pi y^{2} .
$$

Let the solid $V$ be bounded on the left by the plane perpendicular to the $x$-axis at $A(a, o)$. The radius of the circle bounding $V$ on the right is $y=f(x)$. Denote the area of this section by $b^{\prime}$ (Fig. 189).

If $x$ increases by $\Delta x, V$ increases by an amount $\Delta V$ which is the volume of that part of the solid lying between $b^{\prime}$ and a section $b^{\prime \prime}$ at a distance $\Delta x$ from $b^{\prime}$. If $y$ increases as $x$ increases, then $b^{\prime}<b^{\prime \prime}$, and $\Delta V$ is greater than a cylinder with base $b^{\prime}$ and altitude $\Delta x$, and less than a cylinder with base $b^{\prime \prime}$ and altitude $\Delta x$. Hence:

$$
b^{\prime} \Delta X<\Delta V<b^{\prime \prime} \Delta x \text {. }
$$


Dividing by $\Delta x$,

$$
b^{\prime}<\frac{\Delta V}{\Delta x}<b^{\prime \prime} .
$$

As $\Delta x$ approaches zero, $b^{\prime \prime}$ approaches $b^{\prime}$ and $\frac{\Delta V}{\Delta x}$ approaches

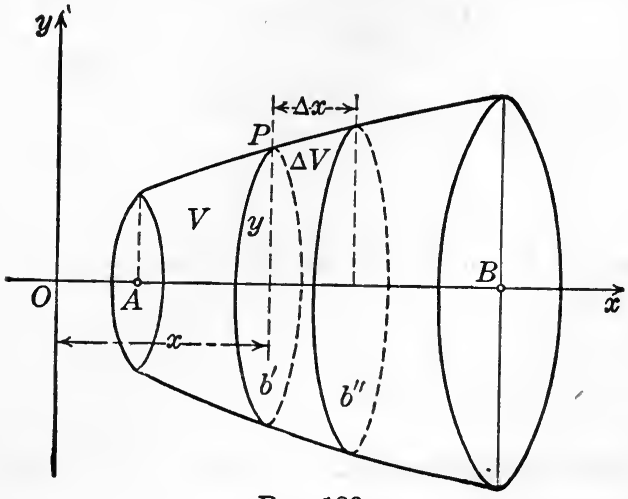

FIG. 189.

$D_{x} V$. Therefore

$$
D_{x} V=b^{\prime} .
$$

Hence,

$$
D_{x} V=\pi y^{2} \text {. }
$$

The volume of the solid between the planes perpendicular to $O X$ at $A$ and a second given point $B(b, o)$, is found by integrating, setting $V=0$ and $x=a$ to determine the constant of integration, and then setting $x=b$.

ExampLe. Find the volume of the solid generated by revolving about the $x$-axis the area bounded by $y=\sqrt{x}$, the $x$-axis, and the ordinates at $x=1$ and $x=4$.

Let $V$ denote the volume between the planes perpendicular to the $x$-axis at $x=1$ and the point whose abscissa is $x$. Then by the theorem

$$
\begin{aligned}
D_{x} V & =\pi y^{2} \\
& =\pi x .
\end{aligned}
$$

Integrating,

$$
V=\frac{\pi}{2} x^{2}+C .
$$

Since $V=0$ when $x=1$,

$$
O=\frac{\pi}{2}+C \text {, so that } C=-\frac{\pi}{2} \text {. }
$$

Hence

$$
V=\frac{\pi}{2} x^{2}-\frac{\pi}{2}
$$

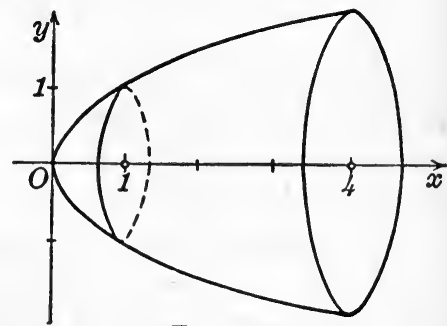

FIG. 190.

The required volume is found by setting $x=4$, which gives

$$
V=\frac{\pi}{2} \cdot 16-\frac{\pi}{2}=\frac{15 \pi}{2}=23.6 \text {. }
$$


113. Volume of a Cone of Revolution. A cone of revolution is a cone that may be generated by revolving a right triangle about one leg as an axis.

Theorem. The volume of a cone of revolution is one third the product of the area of the base and the atitude.

Consider the cone in the figure as generated by revolving the triangle $O A B$ about $O A$. Take the origin at $O$, and the $x$-axis along $O A$.

Let $r=A B$ denote the radius of the base of the cone, and $h=O A$ the altitude.

The slope of $O B$ is $m=A B / O A=r / h$.

And hence the equation of $O B$ is

$$
y=m x=\frac{r}{h} x .
$$

Therefore if $V$ denotes the volume from the vertex $O$ to a section at a distance $x$ from $O$

(Theorem, Section 112),

$$
D_{x} V=\pi y^{2}=\frac{\pi r^{2}}{h^{2}} x^{2}
$$

Integrating

$$
V=\frac{\pi r^{2}}{3 h^{2}} x^{3}+C .
$$

As $\mathrm{V}=0$ when $x=0$, then $C=0$, and hence $V=\frac{\pi r^{2}}{3 h^{2}} x^{3}$.

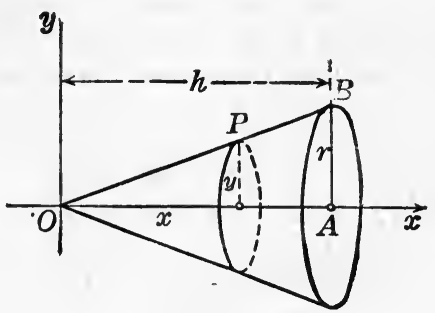

FIG. 191.

The volume of the given cone is obtained when $x=h$. Remembering that the area of the base $b=\pi r^{2}$, we have

$$
V=\frac{\pi r^{2}}{3 h^{2}} h^{3}=\frac{1}{3} \pi r^{2} h=\frac{1}{3} b h .
$$

\section{EXERCISES}

1. Find the volume of a regular octagonal pyramid if a side of the base is 4 inches and a lateral edge is 6 inches.

2. Find the volume of a cone of revolution if the diameter of the base is 12 inches and the altitude is 6 inches. 
Definition. A straight line drawn from the vertex of a cone of revolution to a point in the circumference of the base is called the slant height of the cone. It is one of the positions of the hypotenuse of the generating triangle.

3. Find the volume of a cone of revolution if the slant height is $\mathbf{1 3}$ inches and the diameter of the base is 10 inches.

Definition. The slant height of a regular pyramid is the altitude of one of the equal isosceles triangles forming its lateral faces.

4. Find the lateral area and the total area of the pyramid in Exercise 1.

5. Find the lateral and total area of the cone in Exercise 2.

Hint: The lateral surface of a cone of revolution may be spread out in the form of a sector of a circle.

6. Theorem. The lateral area of (a) a regular pyramid, (b) a cone of revolution, is half the product of the perimeter of the base and the slant height.

7. Find the volume generated by revolving about the $x$-axis the area bounded by the lines $y=3 x, y=0$, and $x=3$. Check the result by the theorem in the preceding section.

8. State and prove a theorem analogous to that on page 323 for the volume of a solid generated by revolving an area about the $y$-axis.

9. Find the volume generated by revolving about each axis the area bounded by the parabola $y=4-x^{2}$ and the positive parts of the axes.

10. Find the volume generated by revolving about the $x$-axis the area bounded by the parabola $y=-x^{2}+4 x-3$ and the $x$-axis.

11. Find the volume of the sphere generated by revolving the circle $x^{2}+y^{2}=16$ about the $x$-axis.

12. Find the volume generated by revolving about the $y$-axis the area bounded by $y=x^{2}, x=0$, and $y=9$.

13. Find the volume of the solid generated by revolving about the $x$-axis the area bounded by $y=\frac{1}{2} x+2$, the $x$-axis, and the ordinates at $x=2$ and $x=6$.

Definition. A frustum of a cone is that part of a cone included between two planes perpendicular to the axis. It may be generated by revolving a trapezoid $A B C D$, such that $A D$ and $B C$ are perpendicular to $A B$, about $A B$. See, for example, the solid obtained in Exercise 13.

14. If that part of the line $y=x+2$ which lies between $x=1$ and $x=4$ is revolved about the $x$-axis, find the volume of the frustum of a cone which is generated.

15. Find the volume of a frustum of a cone if the radii of the bases are 3 and 7 inches and the altitude is 19 inches.

16. The altitude of a conical receptacle is $\mathbf{1 0}$ inches, and the radius of the base is 6 inches. The axis is vertical with the vertex at the bottom. If water is poured into it at the rate of 20 cubic inches per second, how fast is the surface rising when the depth is 5 inches? 
17. The water reservoir of a town is in the form of an inverted conical frustum with the sides inclined at an angle of $45^{\circ}$, the radius of the smaller base being 100 feet. If the depth is decreasing at the rate of 5 feet per day when the water is 20 feet deep, show that the town is being supplied with water at the rate of $72,000 \pi$ cubic feet per day.

18. The diameter of the base of a cone is found to be 6 inches and the altitude 15 inches. If the error in each measurement is not greater than one per cent, find the relative error in the computed volume, assuming first that the measurement of the diameter is exact, and then that the measurement of the altitude is exact.

19. The diameter of the base of a cone is found to be 8 inches and the slant height 10 inches. Assuming that the first measurement is exact, what is the admissible error in the second if the volume is to be determined within 0.1 of one per cent?

20. How many cubic feet of air will there be in the largest conical tent that can be made out of 200 square feet of canvas?

21. What is the least amount of canvas that can be used to make a conical tent of 1200 cubic feet capacity?

114. Volume of the Sphere. A sphere may be generated by revolving a circle about a diameter. Choosing the center of the circle as origin, a point $P(x, y)$ will lie on the circle if and only if $O P=r$, where $r$ is the radius.

From the right triangle $O M P$,

$$
x^{2}+y^{2}=O P^{2},
$$

and hence the equation of the circle is

$$
x^{2}+y^{2}=r^{2} \text {. }
$$

Let this circle be revolved about the $x$-axis, and denote the extremities of the diameter on the $x$-axis by $A(-r, 0)$ and $B(r, 0)$.

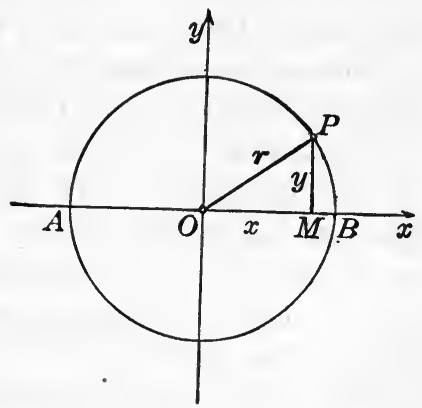

Fig. 192.

Let $V$ denote the volume from $A$ to a section cutting the $x$-axis at a point whose abscissa is $x$.

By the theorem on page 323, we have, from (1),

Integrating

$$
D_{x} V=\pi y^{2}=\pi\left(r^{2}-x^{2}\right) .
$$

$$
V=\pi\left(r^{2} x-x^{3} / 3\right)+C .
$$


Since $V=0$ when $x=-r$,

$$
0=\pi\left(-r^{3}+r^{3} / 3\right)+C \text {, whence } C=2 \pi r^{3} / 3 \text {. }
$$

Hence .

$$
V=\pi\left(r^{2} x-x^{3} / 3\right)+2 \pi r^{3} / 3
$$

When $x=r, V$ is the volume of the sphere, hence the volume of the sphere is

$$
V=\pi\left(r^{3}-r^{3} / 3\right)+2 \pi r^{3} / 3=\frac{4}{3} \pi r^{3} .
$$

Hence the

Theorem. The volume of a sphere of radius $r$ is $\boldsymbol{V}=\frac{4}{3} \pi r^{3}$.

115. Area of a Sphere. Imagine a very large number of planes drawn tangent to the sphere. The area and volume of the polyhedron bounded by these planes would be very nearly equal to the area and volume of the sphere. Planes passed through the center of the sphere and the lines of intersection of the tangent planes would divide the polyhedron into as many little pyramids as there are tangent planes.

These pyramids would all have the radius for altitude, and the sum of their base areas would be the area of the polyhedron.

Hence the volume of the polyhedron would be one-third its area multiplied by the radius.

This would be true no matter how large the number of faces of the polyhedron. If the number of tangent planes is increased indefinitely in such a way that the area of the base of each little pyramid approaches zero, then the volume and area of the polyhedron approach respectively the volume and area of the sphere.

Hence the volume of the sphere is also equal to one-third its area multiplied by the radius.

Denoting the area of the sphere by $S$ and applying the theorem in the preceding section, we have

$$
\frac{1}{3} S r=\frac{4}{3} \pi r^{3}, \text { whence } S=4 \pi r^{2} .
$$

Hence the

Theorem. The area of a sphere of radius $r$ is $S=4 \pi r^{2}$. 


\section{EXERCISES}

1. Find the area and volume of a sphere whose radius is 5 inches.

2. Find the volume of the sphere inscribed in a cone of revolution whose altitude is 12 inches, the radius of whose base is 5 inches.

3. For what values of the radius is the number of units in the volume of a sphere less than the number of units in the area? For what values of the radius is $D_{t} V<D_{w} S$ ? $D_{t} V>D_{W} S$ ? In biology, we learn that a cell subdivides after a certain time. Why?

4. The diameter of a sphere is found by measurement to be 8.3 inches with a probable error of 0.1 inch. What is the error in the computed value of the area of the sphere? In the computed value of the volume? Is the percentage error in the area greater or less than the percentage error in the volume?

5. What is the relation between the rates of increase of the radius and volume of a soap bubble? When the radius is 3 inches and is increasing at the rate of 0.1 inch per second, how fast is the volume increasing? The surface?

6. A washbowl is in the form of a hemisphere of radius 8 inches. How much water is there in it when the water is 5 inches deep?

7. When the depth of the water in the bowl of Exercise 6 is 4 inches the surface is falling at the rate of $\frac{1}{4}$ of an inch per second. How rapidly does the water run off through the drain pipe?

8. Find the area and volume of the earth, assuming that it is a sphere whose radius is 3957 miles. Find the error and relative error in each case if the error in the radius is not more than 7 miles. How does the relative error in the area and in the volume compare with the relative error in the radius?

9. How many lead shot $\frac{1}{4}$ of an inch in diameter can be made from a piece of lead pipe 2 feet long whose outside and inside diameters are respectively 1.25 inches and 1 inch?

\section{MISCELLANEOUS EXERCISES}

1. Find the volume generated by revolving the area bounded by the curves $y=x^{2}$ and $x=y^{2}$ about the $x$-axis.

2. A ball rolls down a smooth plane inclined at an angle of $18^{\circ}$. Find how far it will roll in $t$ seconds.

3. The diameter of a cone is found to be 5 inches, and the altitude 8 inches. If the error in the diameter is 0.06 of an inch and the altitude is exact, find the error in the computed value of the lateral area.

Definition. At the center $O$ of an equilateral triangle $A B C$ erect a line perpendicular to the plane of the triangle. Take a point $D$ on it such that $A D=A B$, and join $D$ to $A, B$, and $C$. The figure so obtained is called a regular tetrahedron [Fig. $193(a)$ ]. 
4. Find the altitude $D O$ and the volume of a regular tetrahedron (a) whose edge $A B$ is 6 inches; (b) whose edge is $e$.

Definition. At the center $O$ of a square $A B C D$ erect a line perpendicular to the plane of the square, and extend it on both sides of the plane to points $E$ and $F$ such that $E A=F A=A B$. Join $E$ and $F$ to the vertices of the square. The resulting figure is called a regular octahedron.

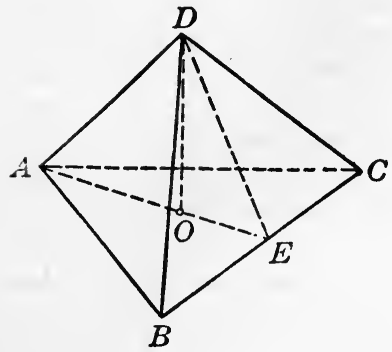

(a)

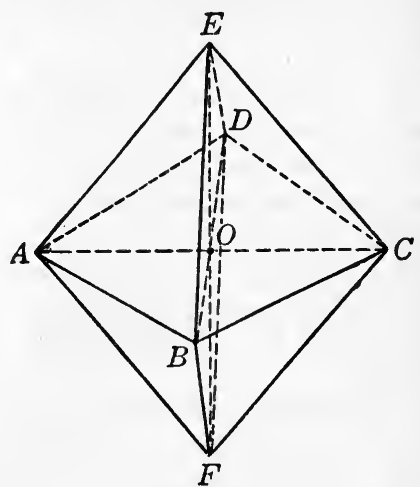

(b)

Fig. 193.

5. Find the volume of a regular octahedron (a) whose edge $A B$ is 6 inches; (b) whose edge is $e$. (Note that $E F$ is a diagonal of the square $A E C F$.)

6. Express the total area of the octahedron in terms of the edge. Do the same for the cube and the regular tetrahedron, and plot the graphs of these three functions of $e$ on the same axes. For the same value of $e$, which solid has the greatest area? Which area increases the most rapidly as $e$ increases?

7. The graph of $x+y \frac{1}{2}=a \frac{1}{2}$ is a parabola tangent to the coördinate axes whose axis of symmetry bisects the first quadrant. Find the area bounded by the curve and the axes.

8. Find the volume of the solid generated by revolving about the $y$-axis the area bounded by the parabola $y=a x^{2}, x=0$, and $y=h$. Show that this volume is one-half the volume of a cylinder whose altitude is $h$ and whose bases are equal to the circle forming the upper surface of the solid.

9. A point moves so that its coördinates at any time $t$ are given by

$$
x=\frac{2 t}{1+t^{2}}, \quad y=\frac{1-t^{2}}{1+t^{2}} .
$$

Find the components parallel to the axes of the velocity and acceleration. Show that the point moves in a circle (to eliminate $t$, square both equations 
and add the results) and determine the part of the circle it describes from $t=0$ to $t=1$. Find the velocity and acceleration (direction and magni- tude) at these times

10. A water resevoir is in the form of the figure generated by revolving the curve $12 y=x^{2}$ about the $y$-axis. How much does it contain if the water is $\mathbf{1 0}$ feet deep at the center? If water is entering at the rate of 10 cubic feet per second when the depth at the center is 12 feet, how rapidly is the surface rising? 


\section{CHAPTER VIII}

\section{PROPERTIES OF TRIGONOMETRIC FUNCTIONS}

\section{LOGARITHMIC SOLUTION OF TRIANGLES, CASES III AND IV}

116. Introduction. In this Chapter we shall derive formulas which express properties of the trigonometric functions analogous to certain properties of algebraic, exponential, and logarithmic functions with which we are already familiar. These properties enable us to change the form of expressions involving trigonometric functions, a process of great importance in those parts of more advanced mathematics where these functions appear. These properties, together with the formulas for the functions of $n 90^{\circ} \pm \theta$ (page 192), are used more, perhaps, than the solution of triangles, except in such fields as surveying.

As an application of these formulas we shall obtain formulas for the solution of triangles, which are adapted to the use of logarithmic tables, for those cases in which we have hitherto used the law of cosines.

In the sections immediately following we shall consider the interdependence of the trigonometric functions of an angle $\theta$, with applications to the solution of equations and the proof of identities.

117. Fundamental Trigonometric Relations. As an immediate consequence of the definitions of the trigonometric functions we obtained the reciprocal relations

$$
\begin{aligned}
& \cot \theta=\frac{1}{\tan \theta} ; \\
& \sec \theta=\frac{1}{\cos \theta} ; \\
& \csc \theta=\frac{1}{\sin \theta} .
\end{aligned}
$$




\section{PROPERTIES OF TRIGONOMETRIC FUNCTIONS}

In the same section, we saw that

$$
\tan \theta=\frac{\sin \theta}{\cos \theta}
$$

To these relations we now add

$$
\sin ^{2} \theta=\cos ^{2} \theta=1,
$$

in which $\sin ^{2} \theta$ denotes the square of $\sin \theta$, or $(\sin \theta)^{2}$, and $\cos ^{2} \theta$ denotes $(\cos \theta)^{2}$.

To prove (5), we have, for any value of $\theta$,

$$
\sin ^{2} \theta+\cos ^{2} \theta=\frac{y^{2}}{r^{2}}+\frac{x^{2}}{r^{2}}=\frac{y^{2}+x^{2}}{r^{2}}=\frac{r^{2}}{r^{2}}=1 .
$$

These five formulas are known as the fundamental formulas of trigonometry. They are independent, that is, no one of them is a consequence of the other four, and they may be solved for any five of the six functions in terms of the sixth. This is illustrated in the

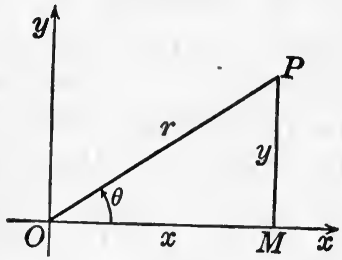

FIG. 194.

Example. Express each of the trigonometric functions in terms of $\sin \theta$. From (5) we obtain $\cos \theta= \pm \sqrt{1-\sin ^{2} \theta}$.

Substituting in (4),

Then by (1), (2), (3),

$$
\tan \theta=\frac{\sin \theta}{ \pm \sqrt{1-\sin ^{2} \theta}}
$$

$$
\cot \theta=\frac{ \pm \sqrt{1-\sin ^{2} \theta}}{\sin \theta}, \sec \theta=\frac{1}{ \pm \sqrt{1-\sin ^{2} \theta}}, \csc \theta=\frac{1}{\sin \theta} .
$$

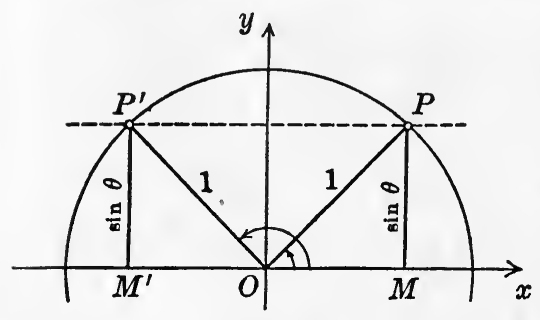

FIG. 195. The sign of the radical must be chosen from a knowledge of the quadrant in which $\theta$ lies. These results may also be obtained from a figure by means of the definitions of the functions (see Exercises 9 and 10, page 170). The given function, $\sin \theta$, may be regarded as a fraction with unit denominator. Describe a circle about the origin with radius $r=1$, and draw the line $y=\sin \theta$, regarding $\theta$ as constant. Let the line cut the circle in $P$ and $P^{\prime}$. Then the angles 
$X O P$ and $X O P^{\prime}$ are such that their sines are equal to $\frac{\sin \theta}{1}$, and hence these angles are values of $\theta$.

From the right triangles, using the Pythagorean Theorem, we get

$$
O M=\sqrt{1-\sin ^{2} \theta} \text { and } O M^{\prime}=-\sqrt{1-\sin ^{2} \theta} .
$$

Then the results obtained above may be written down from the figure. In this figure it is assumed that $\sin \theta$ is positive. If $\sin \theta$ is negative, the angles would lie in the third and fourth quadrants.

This example shows that it would be entirely possible to get along with only one of the trigonometric functions, but frequently it would be inconvenient.

The formulas (1)-(5) are identities (Definition, page 134). There can not be more than five independent equations connecting the six functions, for if there were six, it would be possible to solve them for the six functions, which would then be constants. Hence any other identity involving functions of a single angle $\theta$ must be a consequence of these five, and may be proved by them.

- Three other identities are of sufficient importance to be listed with the fundamental formulas. They are:

$$
\begin{gathered}
\cot \theta=\frac{\cos \theta}{\sin \theta} . \\
1+\tan ^{2} \theta=\sec ^{2} \theta . \\
1+\cot ^{2} \theta=\csc ^{2} \theta .
\end{gathered}
$$

The first two may be proved as follows, the proof of (8) being analogous to that of (7).

$$
\begin{gathered}
\cot \theta=\frac{1}{\tan \theta}=\frac{1}{\frac{\sin \theta}{\cos \theta}}=\frac{\cos \theta}{\sin \theta} \\
1+\tan ^{2} \theta=1+\frac{\sin ^{2} \theta}{\cos ^{2} \theta}=\frac{\cos ^{2} \theta+\sin ^{2} \theta}{\cos ^{2} \theta}=\frac{1}{\cos ^{2} \theta}=\sec ^{2} \theta .
\end{gathered}
$$

The formulas in this and the following sections should be memorized.

118. Trigonometric Equations. No specific rules for the solution of equations involving trigonometric functions can be given, but the following remarks may be of service. 
Express the equation in terms of a single function by means of the formulas in the preceding section, and solve for this function. The values of the angle may then be found from the tables.

If, when all the terms are written on the left of the equality sign, the left-hand member can be factored, equate each factor to zero, and solve the resulting equations. For any solution makes the product of the factors zero, and hence it must make one of the factors zero. Conversely, any solution of one of the resulting equations makes one of the factors zero, and hence also the product.

EXAMPLe 1. Solve $\tan x-2 \sin x=0$.

By (4), Section $117, \frac{\sin x}{\cos x}-2 \sin x=0$.

Multiplying by $\cos x, \sin x-2 \sin x \cos x=0$.

Factoring, $\quad \sin x(1-2 \cos x)=0$.

Equating each factor to zero and solving,

Hence

$$
\begin{array}{rlrlrl}
\sin x & =0, & & \text { or } & \cos x & =\frac{1}{2} . \\
x & =0, \pi & \text { or } & x & =\pi / 3,5 \pi / 3 .
\end{array}
$$

All the other values of $x$ may be obtained by adding $2 n \pi$ to each of these four solutions.

To check the results, we substitute each of the values of $x$ in the given equation. This gives:

$$
\begin{array}{ll}
\text { If } x=0, & \tan x-2 \sin x=0-2 \times 0=0 . \\
\text { If } x=\pi, & \tan x-2 \sin x=0-2 \times 0=0 . \\
\text { If } x=\pi / 3, & \tan x-2 \sin x=\sqrt{3}-2 \times \sqrt{3} / 2=0 . \\
\text { If } x=5 \pi / 3, & \tan x-2 \sin x=-\sqrt{3}-2(-\sqrt{3} / 2)=0 .
\end{array}
$$

Hence the results obtained are correct.

Example 2. Solve the equation $2 \sin x-\cos x=1$.

By (5), Section 117,

Transposing, $2 \sin x \pm \sqrt{1-\sin ^{2} x}=1$. $2 \sin x-1= \pm \sqrt{1-\sin ^{2} x}$

Squaring both sides, $4 \sin ^{2} x-4 \sin x+1=1-\sin ^{2} x$, whence $5 \sin ^{2} x-4 \sin x=0$.

Factoring, so that $\sin x(5 \sin x-4)=0$, and hence $\sin x=0$ or $\sin x=\frac{4}{5}=0.8$, $x=0$ or $\pi$, or $x=53^{\circ} .13=0.9272$ or $126 .{ }^{\circ} 87=2.2144$. 
The values of the last two angles in radians are obtained from page 32 of the Tables.

It is essential that these results be checked, as extraneous roots are frequently introduced when an equation is squared. We have:

$$
\begin{array}{ll}
\text { If } x=0, & 2 \sin x-\cos x=2 \times 0-1=-1 . \\
\text { If } x=\pi, & 2 \sin x-\cos x=2 \times 0-(-1)=1 . \\
\text { If } x=0.9272, & 2 \sin x-\cos x=2 \times 0.8-0.6=1 . \\
\text { If } x=2.2144, & 2 \sin x-\cos x=2 \times 0.8-(-0.6)=2.2 .
\end{array}
$$

The first and last values of $x$ do not satisfy the given equation, and they are therefore discarded. The values $x=\pi$ and $x=0.9272$ do satisfy the equation. All other solutions may be obtained from these by adding $2 n \pi$.

119. Trigonometric Identities. Identities constitute an important part of mathematics. If we encounter a complicated fraction in the solution of a problem, we proceed to simplify it. The reduction of the fraction is essentially a proof of the identity obtained by equating the original fraction to the simpler result which is found.

The logarithmic identity,

$$
\log \frac{a \sin B}{\sin A}=\log a+\log \sin B-\log \sin A,
$$

which is proved by Theorems 7 and 8 , page 223, is the basis of computing $b$, a side of a triangle, if $a, A$, and $B$ are given.

Trigonometric identities are of the same importance in dealing with expressions involving the trigonometric functions as algebraic and logarithmic identities are in working with algebraic and logarithmic functions.

Trigonometric identities involving functions of a single angle may be proved by means of the fundamental formulas in Section 117 and the operations of algebra, by transforming one member of the identity into the other. The proofs of equations (6) and (7) in the section cited are illustrations of the method.

Example 1. Prove the identity,

$$
\sec ^{2} x-\sec x \tan x=\frac{1}{1+\sin x} .
$$


By the formulas in Section 117 we obtain

$$
\begin{aligned}
\sec ^{2} x-\sec x \tan x & =\frac{1}{\cos ^{2} x}-\frac{1}{\cos x} \frac{\sin x}{\cos x} \\
& =\frac{1-\sin x}{\cos ^{2} x} \\
& =\frac{1-\sin x}{1-\sin ^{2} x} \\
& =\frac{1}{1+\sin x} .
\end{aligned}
$$

Example 2. Prove $\frac{\sin x}{1-\cos x}=\frac{1+\cos x}{\sin x}$.

Proof.

$$
\begin{aligned}
\frac{\sin x}{1-\cos x} & =\frac{\sin x(1+\cos x)}{(1-\cos x)(1+\cos x)} \\
& =\frac{\sin x(1+\cos x)}{1-\cos ^{2} x} \\
& =\frac{\sin x(1+\cos x)}{\sin ^{2} x} \\
& =\frac{1+\cos x}{\sin x}
\end{aligned}
$$

Another method of establishing the truth of an identity is to show that both members can be reduced to the same form. Still another is to transform the given equation until it is reduced to some known identity.

\section{EXERCISES}

1. Express formulas (1)-(8), Section 117, in words.

2. By means of the formulas in Section 117 , find all the functions of $\theta$, given
(a) $\cos \theta=\frac{5}{13}$.
(b) $\tan \theta=\frac{3}{4}$.
(c) $\csc \theta=3$.

Check the results by the method used in Exercise 10, page 170.

3. Using both methods in the Example in Section 117, express each of the functions in terms of
(a) $\cos \theta$.
(b) $\tan \theta$.
(c) $\cot \theta$.
(d) $\sec \theta$.
(e) $\csc \theta$.

4. Express
(a) $\frac{\tan \theta+\cot \theta}{\cot \theta-\tan \theta}$ in terms of $\sin \theta$ and $\cos \theta$.
(b) $\frac{\sec \theta-\tan ^{2} \theta}{\sin ^{2} \theta}$ in terms of $\cos \theta$.
(c) $\frac{2 \sin \theta \cos \theta}{\cos ^{2} \theta-\sin ^{2} \theta}$ in terms of $\tan \theta$. 
5. Solve the equations:
(a) $2 \sin ^{2} x+3 \cos x=0$.
(b) $\cot x-2 \cos x=0$.
(c) $\sin x \tan x-\cos x=0$.
(d) $\sin x+\cos x=1$.
(e) $\sin x+3 \cos x=1$.
(f) $\sin x+3 \cos x=2$.

6. Prove the identities:
(a) $\sin ^{2} \theta-\cos ^{2} \theta=2 \sin ^{2} \theta-1$.
(b) $2 \sin ^{2} \theta-1=1-2 \cos ^{2} \theta$.
(c) $\tan \theta+\cot \theta=\sec \theta \csc \theta$.
(d) $\frac{1-\sin x}{\cos x}=\frac{\cos x}{1-\sin x}$.
(e) $\csc ^{2} \theta+\cot \theta \csc \theta=\frac{1}{1-\cos \theta}$.
(f) $(2 \sin x \cos x)^{2}+\left(\cos ^{2} x-\sin ^{2} x\right)^{2}=1$.

120. Functions of the Sum of Two Angles. The relation

$$
(a+b)^{2}=a^{2}+2 a b+b^{2}
$$

expresses in a different form the square of the sum of two numbers. We seek now an analogous expression for the sine of the sum of two angles, $\sin (\theta+\phi)$, where $\theta$ and $\phi$ are any two acute angles. The sum $\theta+\phi$ is constructed by using the terminal line of $\theta$ as the initial line of $\phi$. It may be either an acute or an obtuse angle, as indicated in the figures.

Let $A$ be any point on the terminal line of $\theta$, and $\operatorname{draw} A B$ perpendicular to $O X$. Then

$$
\sin (\theta+\phi)=\frac{B A}{O A} \text {. }
$$

To express this ratio in terms of $\theta$ and $\phi$, draw $A C$ perpendicular to the initial line of $\phi$, and $C D$ perpendicular to $O X$. Then the functions of $\theta$ and $\phi$ may be found from the right triangles $O D C$ and $O C A$ respectively.

Draw $C E$ perpendicular to $A B$. Then the triangles $O D C$ and $A E C$ are similar (why?), so that $\angle E A C=\theta$, and the functions of $\theta$ may also be found from the triangle $A E C$.

We then have 


$$
\begin{aligned}
\sin (\theta+\phi) & =\frac{B A}{O A} \\
& =\frac{D C+E A}{O A} \\
& =\frac{O C \sin \theta+C A \cos \theta}{O A} \\
& =\sin \theta \frac{O C}{O A}+\cos \theta \frac{C A}{O A}
\end{aligned}
$$

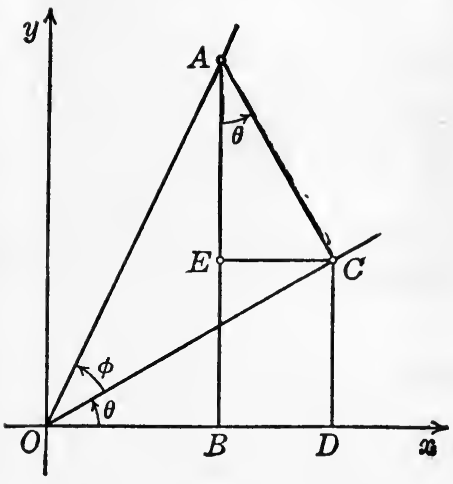

(a)

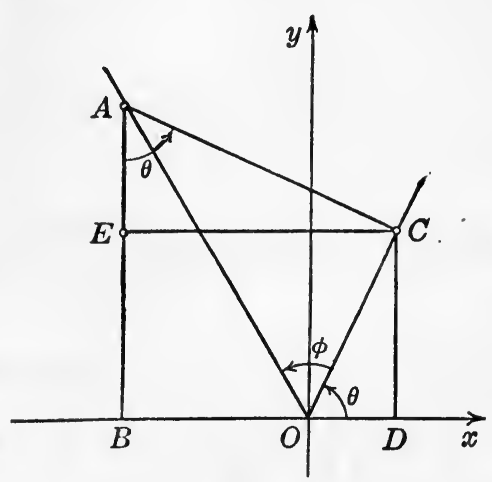

(b)

FIG. 196.

or $\quad \sin (\theta+\phi)=\sin \theta \cos \phi+\cos \theta \sin \phi$.

In like manner,

$$
\begin{aligned}
\cos (\theta+\phi) & =\frac{O B}{O A} \\
& =\frac{O D-E C}{O A} \\
& =\frac{O C \cos \theta-C A \sin \theta}{O A} \\
& =\cos \theta \frac{O C}{O A}-\sin \theta \frac{C A}{O A}
\end{aligned}
$$

so that

$$
\cos (\theta+\phi)=\cos \theta \cos \phi-\sin \theta \sin \phi .
$$


The proofs hold for either figure. In deriving (2) when $\theta+\phi$ is obtuse, it should be noted that $O B$ is negative, so that we will still have $O B=O D-B D$.

We shall assume that these formulas hold for all values of $\theta$ and $\phi$, negative as well as positive. (See Exercises 5-8 below.)

Dividing (1) by (2), and using (4), page 333, we have

$$
\tan (\theta+\phi)=\frac{\sin (\theta+\phi)}{\cos (\theta+\phi)}=\frac{\sin \theta \cos \phi+\cos \theta \sin \phi}{\cos \theta \cos \phi-\sin \theta \sin \phi} \text {. }
$$

Dividing numerator and denominator by $\cos \theta \cos \phi$, we obtain

$$
\tan (\theta+\phi)=\frac{\frac{\sin \theta}{\cos \theta}+\frac{\sin \phi}{\cos \phi}}{1-\frac{\sin \theta}{\cos \theta} \frac{\sin \phi}{\cos \phi}},
$$

whence $\tan (\theta+\phi)=\frac{\tan \theta+\tan \phi}{1-\tan \theta \tan \phi}$.

\section{EXERCISES}

1. Express formulas (1), (2), (3), in words.

2. Using the functions of $30^{\circ}, 45^{\circ}, 60^{\circ}$, as given on page 161 , find all the functions of (a) $75^{\circ}$, (b) $105^{\circ}$. Hint: $\sin 75^{\circ}=\sin \left(45^{\circ}+30^{\circ}\right)$, etc.

3. If $\theta=\arcsin \frac{3}{5}$ and $\phi=\operatorname{arc} \cos \frac{5}{13}$, find the functions of $\theta+\phi$.

4. What force acting parallel to the plane is necessary to support a body weighing 100 pounds on a smooth plane if the inclination is $\theta=\operatorname{arc}$ $\sin \frac{1}{5}$ ? If the inclination is $\phi=\operatorname{arc} \sin \frac{12}{13}$ ? If the inclination is $\theta+\phi$ ?

5. Prove that (1) holds for all positive values of $\theta$ and $\phi$.

Solution: We will first prove that: If (1) and (2) are true for a pair of values $\theta$ and $\phi$, then (1) is true when $\theta$ is increased by $90^{\circ}$.

Let

$$
\theta^{\prime}=90^{\circ}+\theta \text {. }
$$

$$
\text { Then } \begin{aligned}
\sin \left(\theta^{\prime}+\phi\right) & =\sin \left(90^{\circ}+\theta+\phi\right) \\
& =\cos (\theta+\phi) \\
& =\cos \theta \cos \phi-\sin \theta \sin \phi \\
& =\cos \left(\theta^{\prime}-90^{\circ}\right) \cos \phi-\sin \left(\theta^{\prime}-90^{\circ}\right) \sin \phi \\
& =\cos \left(90^{\circ}-\theta^{\prime}\right) \cos \phi+\sin \left(90^{\circ}-\theta^{\prime}\right) \sin \phi \\
& =\sin \theta^{\prime} \cos \phi+\cos \theta^{\prime} \sin \phi .
\end{aligned}
$$

Since (1) holds for all acute values of $\theta$ and $\phi$, by the above it holds for all obtuse values of $\theta$ and all acute values of $\phi$. 
Applying the fact proved above once more, (1) holds for all values of $\theta$ less than $270^{\circ}$ and of $\phi$ less than $90^{\circ}$; etc.

In like manner we may prove that $\phi$ may be increased by $90^{\circ}$ and thence that (1) holds for all positive values of $\theta$ and $\phi$.

6. Prove that (2) holds for all positive values of $\theta$ and $\phi$.

7. Prove that (1) holds if $\phi$ is negative.

Solution: No matter what the value of $\phi$ may be, we can choose an integer $n$ such that $n 360^{\circ}+\phi$ is positive.

Then $\sin (\theta+\phi)=\sin \left(n 360^{\circ}+\theta+\phi\right)=\sin \left[\theta+\left(n 360^{\circ}+\phi\right)\right]$

$$
\begin{aligned}
& =\sin \theta \cos \left(n 360^{\circ}+\phi\right)+\cos \theta \sin \left(n 360^{\circ}+\phi\right) \\
& =\sin \theta \cos \phi+\cos \theta \sin \phi .
\end{aligned}
$$

8. Prove that (2) holds for all negative values of $\phi$.

9. Is it necessary to employ the methods of Exercises 5-8 to prove that (3) holds for all values of $\theta$ and $\phi$, positive and negative?

10. What property of (a) $x^{n}$, where $n$ is a positive integer; (b) $b^{x}$, is analogous to the properties of $\sin x, \cos x$, and $\tan x$ given by formulas (1), (2), and (3)?

11. If $\sin 1^{\circ}$ is known, how complete a table of values of the trigonometric functions can be computed?

12. Prove the identities:

(a) $\operatorname{Sin}\left(x+\frac{\pi}{3}\right)-\cos \left(x+\frac{\pi}{6}\right)=\sin x$.

(b) $\operatorname{Tan}\left(\frac{\pi}{4}+x\right)=\frac{1+\tan x}{1-\tan x}$.

(c) $\operatorname{Sin}(x+y) \cos y+\cos (x+y) \sin y=\sin (x+2 y)$.

13. Solve the equations:

$$
\begin{aligned}
& \text { (a) } \operatorname{Sin}\left(x+\frac{\pi}{4}\right)=\frac{\sqrt{2}}{2} . \\
& \text { (b) } \operatorname{Sin}\left(x+\frac{\pi}{4}\right)-\cos \left(x+\frac{\pi}{4}\right)=\frac{\sqrt{2}}{2} .
\end{aligned}
$$

14. Prove that the force to make a sailboat move forward will be greatest when the direction of the sail bisects the angle between the keel and the apparent direction of the wind.

121. Functions of the Difference of Two Angles. We now seek an expression for the sine of the difference of two angles, analogous to the formula in algebra for the square of the difference of two numbers. 
Since $\theta-\phi=\theta+(-\phi)$, and since the formulas in the preceding section are true for all values of the angles, we have

$$
\begin{aligned}
\sin (\theta-\phi) & =\sin (\theta+(-\phi)) \\
& =\sin \theta \cos (-\phi)+\cos \theta \sin (-\phi) .
\end{aligned}
$$

But $\cos (-\phi)=\cos \phi$ and $\sin (-\phi)=-\sin \phi$. And hence,

$$
\sin (\theta-\phi)=\sin \theta \cos \phi-\cos \theta \sin \phi .
$$

In like manner it may be proved that

and

$$
\begin{aligned}
& \cos (\theta-\phi)=\cos \theta \cos \phi+\sin \theta \sin \phi, \\
& \tan (\theta-\phi)=\frac{\tan \theta-\tan \phi}{1+\tan \theta \tan \phi} .
\end{aligned}
$$

122. Functions of Twice an Angle, or the Functions of Any Angle in Terms of Half the Angle. Since $2 \theta=\theta+\theta$, we have and hence

$$
\begin{aligned}
\sin 2 \theta & =\sin (\theta+\theta) \\
& =\sin \theta \cos \theta+\cos \theta \sin \theta, \quad \text { (1), page } 339,
\end{aligned}
$$

$$
\sin 2 \theta=2 \sin \theta \cos \theta .
$$

In like manner, it may be proved that

$$
\text { and } \quad \begin{aligned}
\cos 2 \theta & =\cos ^{2} \theta-\sin ^{2} \theta, \\
\tan 2 \theta & =\frac{2 \tan \theta}{1-\tan ^{2} \theta} .
\end{aligned}
$$

Since $\sin ^{2} \theta+\cos ^{2} \theta=1$, (2) may be written in the forms

$$
\begin{aligned}
& \cos 2 \theta=\left(1-\sin ^{2} \theta\right)-\sin ^{2} \theta=1-2 \sin ^{2} \theta . \\
& \cos 2 \theta=\cos ^{2} \theta-\left(1-\cos ^{2} \theta\right)=2 \cos ^{2} \theta-1 .
\end{aligned}
$$

The graph of $\sin 2 \theta$ may be obtained by bisecting the abscissas of points on the graph of $\sin \theta$ (Theorem, page 151). The graph suggests that the period (Definition, page 168) of $\sin 2 \theta$ is $\pi=180^{\circ}$. This is, indeed, the case. For if we replace $\theta$ by $\theta+180^{\circ}$, we get

$$
\sin 2\left(\theta+180^{\circ}\right)=\sin \left(2 \theta+360^{\circ}\right)=\sin 2 \theta .
$$

A general expression for the sine of the product of two numbers would be analogous to the theorem giving the log- 
arithm of the product of two numbers (page 223), or the $n$th power of the product of two numbers [(3), page 153]. Formula (1) is the special case of such a theorem obtained when one

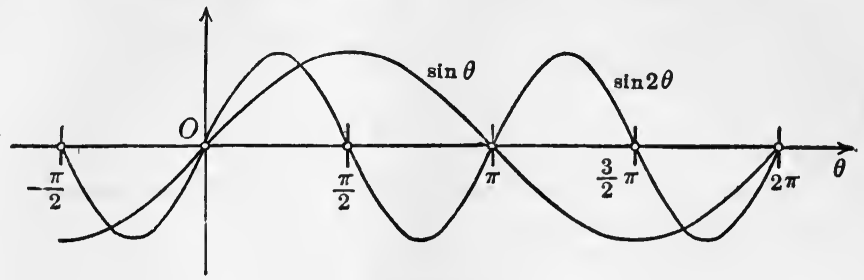

FIG. 197.

of the numbers is 2. Another special case is given in equation (1) of the section following.

123. Functions of Half an Angle, or Functions of Any Angle in Terms of Functions of Twice the Angle. Since all that is essential in the formulas of the preceding section is that the angle on the left be double that on the right, formulas (2a) and $(2 \mathrm{~b})$ may be written

$$
\begin{aligned}
& \cos \theta=1-2 \sin ^{2} \theta / 2, \\
& \cos \theta=2 \cos ^{2} \theta / 2-1 .
\end{aligned}
$$

Solving these equations for $\sin \theta / 2$ and $\cos \theta / 2$ we have

$$
\begin{aligned}
& \sin \theta / 2= \pm \sqrt{\frac{1-\cos \theta}{2}}, \\
& \cos \theta / 2= \pm \sqrt{\frac{1+\cos \theta}{2}} .
\end{aligned}
$$

Dividing (1) by (2) and using the formula (4), page 333,

$$
\tan \theta / 2= \pm \sqrt{\frac{1-\cos \theta}{1+\cos \theta}}
$$

\section{EXERCISES}

1. Express the formulas in Sections 121, 122, and 123 in words. In Section 122 , describe (a) $\theta$ as any angle, (b) $2 \theta$ as any angle. In Section 123, describe (a) $\theta$ as any angle, (b) $\theta / 2$ as any angle.

2. Find all the functions of $15^{\circ}$ from those of $45^{\circ}$ and $30^{\circ}$. 
3. Find the functions of $\pi / 8$ from those of $\pi / 4$; of $\pi / 12$ from those of $\pi / 6$.

The table given by the Hindu Aryabhata (476 A.D. -) gives the values of the sines of angles at intervals of $3^{\circ} 45^{\prime}$. How could this table be obtained?

4. If $\theta=90^{\circ}$, show that (1), (2), (3), Section 121 , reduce to formulas in Section 62, page 177.

5. If $\theta=180^{\circ}$, show that (1), (2), (3), Section 121 , reduce to (1), (2), (3), page 192.

6. State the properties of $x^{3}$ analogous to each of the equations (1) in Sections 120, 121, 122, 123.

7. In the following, find $\sin \theta$ and $\cos \theta$ without finding $\theta$ given
(a) $\operatorname{Tan} 2 \theta=\frac{3}{4}$.
(b) $\tan 2 \theta=-\frac{3}{4}$.
(c) $\tan 2 \theta=\frac{12}{5}$.

Hint: Find $\cos 2 \theta$ from a figure (see Exercise 10, page 170) and then use formulas (1), and (2), Section 123.

8. Express $\sin 4 \theta$ in terms of functions of $2 \theta$; $\sin \theta$ in terms of functions of $\theta / 2 ; \sin 3 \theta$ in terms of functions of $3 \theta / 2$.

9. Transform (3), Section 123 , into the forms

$$
\tan (\theta / 2)=\frac{1-\cos \theta}{\sin \theta}=\frac{\sin \theta}{1+\cos \theta} .
$$

Then transform each of the fractions into $\tan (\theta / 2)$ by expressing $\sin \theta$ and $\cos \theta$ in terms of $\theta / 2$, using the formulas in Section 122. In what respect is the latter procedure preferable?

10. If $\tan 2 \theta=1.4123$, find $\theta$ and $\cos \theta / 2$.

11. A body is placed on a rough plane which is inclined at any angle greater than the angle of friction. (The angle of friction is an angle $\phi$ such that $\tan \phi=m$, the coefficient of friction.) If the body is supported by a force acting parallel to the plane, find the limits between which the force must lie.

Let $\theta$ be the angle of inclination of the plane, $W$ the weight of the body and $R$ the reaction perpendicular to the plane.

(a) Let the body be on the point of moving down the plane, so that the force of friction acts up the plane and is equal to $m R$. Let $P$ be the force required to keep the body at rest.

Resolving $W$ into components parallel and perpendicular to the plane, we have $P+m R=W \sin \theta, R=W \cos \theta$, and therefore $P=W(\sin \theta-m$ $\cos \theta)$. Since $m=\tan \phi$ we have, $P=W(\sin \theta-\tan \phi \cos \theta)$.

$$
\text { Hence } P=W \frac{\sin \theta \cos \phi-\sin \phi \cos \theta}{\cos \phi}=W \frac{\sin (\theta-\phi)}{\cos \phi} \text {. }
$$

(b) Let the body be on the point of motion up the plane. Complete the solution. 
12. What is the maximum and the minimum force which will hold a weight of 12 pounds on a plane inclined at an angle of $40^{\circ}$, if the coefficient of friction is 0.5 , and if the force acts parallel to the plane?

13. A block $W$ rests on a horizontal plane. If an oblique force $P$ acts upon $W$, making an angle $\theta$ with the direction of sliding, and if the coefficient of friction is $m=\tan \phi$, prove that the magnitude of $P$ that will cause the block to slide is

$$
P=W \frac{\sin \phi}{\cos (\theta-\phi)} \text {. }
$$

Find the least pull that will make the block slide.

Suggestion: Show that $P$ will be a minimum when $\theta=\phi$, i.e., the direction of pull is given by the angle of friction.

14. If the inclination of a plane is $\theta=\operatorname{arc} \sin \frac{1}{3}$, what horizontal force would support a body of 50 pounds upon it? What horizontal force would be required if $\theta$ were doubled?

15. On the same axes, plot the graphs of the functions below. Find the period of each function.

(a) $\cos \theta$ and $\cos 2 \theta$.

(b) $\sin \theta$ and $\sin (\theta / 2)$.

(c) $\tan \theta$ and $\tan 2 \theta$.

(d) $\cos \theta$ and $\cos (\theta / 2)$.

(e) $\tan \theta$ and $\tan (\theta / 2)$. (f) $\sin \theta$ and $\sin 3 \theta$.

(g) $\cos \theta$ and $\cos 3 \theta$.

(h) $\sin \theta$ and $2 \sin (\theta / 2)$.

(i) $\cos \theta$ and $3 \cos 2 \theta$.

(j) $\tan \theta$ and $\frac{1}{2} \tan (\theta / 2)$.

16. Solve the equations:

(a) $\sin 2 x+\cos x=0$.

(d) $\sin 2 x-2 \sin x=0$.

(b) $\tan 2 x+\tan x=0$.

(e) $\cos 2 x+\sin x=0$.

(c) $2 \tan ^{2} x-3 \tan x+1=0$.

(f) $\cot 2 x+\tan x=0$.

17. Prove the identities:

(a) $(\sin x+\cos x)^{2}=1+\sin 2 x$.

(b) $\frac{2 \tan x}{1+\tan ^{2} x}=\sin 2 x$.

(c) $\frac{\sin 2 \theta}{1+\cos 2 \theta}=\tan \theta$.

(d) $\cos (x+y) \cos (x-y)=\cos ^{2} x-\sin ^{2} y$.

(e) $\sin \alpha \cos (\beta-\alpha)+\cos \alpha \sin (\beta-\alpha)=\sin \beta$.

124. Sum and Difference of the Sines or Cosines of Two Angles. To express the sum of the sines of two angles, $\sin a$ $+\sin \beta$, as a product, let

and

$$
\begin{aligned}
& \alpha=\theta+\phi \\
& \beta=\theta-\phi .
\end{aligned}
$$


Then $\quad \sin \alpha+\sin \beta=\sin (\theta+\phi)+\sin (\theta-\phi)$

$$
\begin{aligned}
= & \sin \theta \cos \phi+\cos \theta \sin \phi \\
& +\sin \theta \cos \phi-\cos \theta \sin \phi \\
= & 2 \sin \theta \cos \phi .
\end{aligned}
$$

Solving the first two equations for $\theta$ and $\phi$, by adding and subtracting, and dividing by 2 , we have

$$
\theta=\frac{1}{2}(a+\beta), \phi=\frac{1}{2}(a-\beta),
$$

and hence, substituting,

$$
\sin a+\sin \beta=2 \sin \frac{1}{2}(\alpha+\beta) \cos \frac{1}{2}(a-\beta) .
$$

In like manner, prove

$$
\begin{aligned}
& \sin \alpha-\sin \beta=2 \cos \frac{1}{2}(\alpha+\beta) \sin \frac{1}{2}(\alpha-\beta) . \\
& \cos \alpha+\cos \beta=2 \cos \frac{1}{2}(\alpha+\beta) \cos \frac{1}{2}(\alpha-\beta) . \\
& \cos \alpha-\cos \beta=-2 \sin \frac{1}{2}(\alpha+\beta) \sin \frac{1}{2}(\alpha-\beta) .
\end{aligned}
$$

Equations (1) and (2) give properties of the function $\sin x$. What are the analogous properties of $\log x$ ? To what property of $x^{2}$ is (2) analogous?

125. Logarithmic Solution of Triangles, Case III. The law of sines (page 201) may be written

$$
\frac{\sin A}{\sin B}=\frac{a}{b} \text {. }
$$

Applying division and composition to this proportion

$$
\frac{\sin A-\sin B}{\sin A+\sin B}=\frac{a-b}{a+b} \text {. }
$$

Then by (2) and (1), Section 124,

$$
\frac{2 \cos \frac{1}{2}(A+B) \sin \frac{1}{2}(A-B)}{2 \sin \frac{1}{2}(A+B) \cos \frac{1}{2}(A-B)}=\frac{a-b}{a+b} .
$$

From (6), (1), and (4), page 333, we then have

$$
\frac{\tan \frac{1}{2}(A-B)}{\tan \frac{1}{2}(A+B)}=\frac{a-b}{a+b}
$$

whence $\quad \tan \frac{1}{2}(A-B)=\frac{a-b}{a+b} \tan \frac{1}{2}(A+B)$. 


\section{PROPERTIES OF TRIGONOMETRIC FUNCTIONS 347}

If two sides and the included angle of a triangle are given, the other angles may be found by means of (1), as is the

Example. Solve the triangle, given

$$
\begin{aligned}
& a=118, \quad C=34^{\circ} .24 . \\
& b=96, \quad .
\end{aligned}
$$

We have:

$$
\begin{aligned}
a+b & =214 \\
a-b & =22 \\
\frac{1}{2}(A+B) & =\frac{1}{2}\left(180^{\circ}-C\right)=72^{\circ} .88 .
\end{aligned}
$$

Then by (1),

$$
\begin{aligned}
\tan \frac{1}{2}(A-B) & =\frac{22}{214} \tan 72^{\circ} .88 . \\
\log 22 & =1.3424 \\
\log \tan 72^{\circ} .88 & =\frac{0.5115}{2.8539}-1 \\
\log 214 & =\underline{2.3304}
\end{aligned}
$$

$\log \tan \frac{1}{2}(A-B)=0.5235-1$.

Hence $\frac{1}{2}(A-B)=18^{\circ} .46$.

But $\frac{1}{2}(A+B)=72^{\circ} .88$,

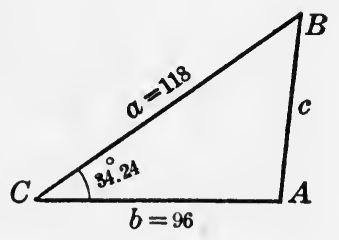

Fig. 198.

$$
\begin{array}{ll}
\text { and hence } & A=91^{\circ} .34 \text { (adding) } \\
\text { and } & B=54^{\circ} .42 \text { (subtracting) }
\end{array}
$$

To find $c$, we have

$$
\begin{aligned}
c & =\frac{b \sin C}{\sin B}=\frac{96 \times \sin 34^{\circ} .24}{\sin 54^{\circ} .42} . \\
\log 96 & =1.9823 \\
\log \sin 34^{\circ} .24 & =\frac{0.7502-1}{2.7325-1} \\
\log \sin 54^{\circ} .42 & =\frac{0.9102-1}{1.8223} \\
\log c & =1.42 . \\
c & =66.42 .
\end{aligned}
$$

Check. Find one of the given sides from $c, A$ or $B$, and $C$.

$$
a=\frac{c \sin A}{\sin C}=\frac{66.42 \times \sin 91^{\circ} .34}{\sin 54^{\circ} .42} \text {. }
$$

$\log 66.42=1.8223$

$$
\begin{aligned}
\log \sin 91^{\circ} .34 & =\frac{0.9999-1}{2.8222-1} \\
\log \sin 34^{\circ} .24 & =\frac{0.7502-1}{\log a}= \\
a & =118.0720
\end{aligned}
$$

which agrees with the given value of $a_{\text {. }}$ 
126. Logarithmic Solution of Triangles, Case IV. We seek an expression for $\tan \frac{1}{2} A, A$ being an angle of a triangle $A B C$, in terms of the sides. We have

$$
\begin{aligned}
& \tan \frac{1}{2} A=\sqrt{\frac{1-\cos A}{1+\cos A}} \quad \text { (by (3), page 343), } \\
& \sqrt{\frac{1-\frac{b^{2}+c^{2}-a^{2}}{2 b c}}{1+\frac{b^{2}+c^{2}-a^{2}}{2 b c}}} \text { (substituting the value of cos } A \\
&=\sqrt{\frac{a^{2}-\left(b^{2}-2 b c+c^{2}\right)}{\left(b^{2}+2 b c+c^{2}\right)-a^{2}}} \text { and by the law of cosines) } \\
& \text { grouping the terms) }
\end{aligned}
$$

Denote half the perimeter by $s$, so that

Subtracting $2 b$,

$$
a+b+c=2 s \text {. }
$$

Subtracting $2 c$,

Subtracting $2 a$,

$$
\begin{array}{ll}
a-b+c=2 s-2 b= & 2(s-b) . \\
a+b-c= & 2(s-c) . \\
b+c-a= & 2(s-a) .
\end{array}
$$

Substituting above,

$$
\begin{aligned}
\tan \frac{1}{2} A & =\sqrt{\frac{2(s-b) 2(s-c)}{2 s 2(s-a)}} \\
& =\sqrt{\frac{(s-a)(s-b)(s-c)}{s(s-a)^{2}}} \\
& =\frac{1}{s-a} \sqrt{\frac{(s-a)(s-b)(s-c)}{s}} .
\end{aligned}
$$

Hence

$\tan \frac{1}{2} A=\frac{r}{s-a}, \quad$ where $\quad r=\sqrt{\frac{(s-a)(s-b)(s-c)}{s}}$.

Interchanging the values of $a, b, c$, does not affect the values of $s$ and $r$ and hence

$$
\tan \frac{1}{2} B=\frac{r}{s-a}, \tan \frac{1}{2} C=\frac{r}{s-c} .
$$


It is proved in most elementary geometries that the area of a triangle in terms of its sides is

whence

$$
A=\sqrt{s(s-a)(s-b)(s-c)},
$$

A simpler proof is indicated in Exercises 6 and 7 below.

Example. Find the angles and area of the triangle $a=34.25, b=42.91$, $c=50.82$.

The angles are found by (1) and (2), the first step in the computation being to find $\log r$ from its value in (1).

$$
\begin{aligned}
& a=34.25 \\
& b=42.91 \\
& \begin{aligned}
& c=50.82 \\
& \hline 2 s=127.98
\end{aligned} \\
& s=63.99 \\
& s-a=29.74 \\
& s-b=21.08 \\
& s-c=13.17 \\
& \log (s-a)=1.4734 \\
& \log (s-b)=1.3239 \\
& \log (s-c)=\frac{1.1196}{3.9169} \\
& \log s=1.8061 \\
& 2 \mid \overline{2.1108} \\
& \log r=1.0554 \\
& \frac{1}{2} A=20^{\circ} .90 \text {, } \\
& A=41^{\circ} .80, \\
& \frac{1}{2} B=28^{\circ} .32 \\
& \frac{1}{2} C=40^{\circ} .78 \text {. } \\
& B=56^{\circ} .64 \\
& C=81^{\circ} .56 \text {. }
\end{aligned}
$$

Check. $A+B+C=180^{\circ} .00$.

By (3), the area is $A=r s$

$$
=727.0 \text {. }
$$

$$
\begin{aligned}
& \log r=1.0554 \\
& \log s=1.8061 \\
& \log A=2.8615
\end{aligned}
$$

\section{EXERCISES}

1. Solve the equations:

(a) $\sin 3 x+\sin x=0$. Hint: Use (1), page 346 .

(b) $\cos 3 x+\cos x=0$.

2. Prove the identities:

$$
\begin{gathered}
\text { (a) } \sin \left(\frac{\pi}{3}+x\right)-\sin \left(\frac{\pi}{3}-x\right)=\sin x . \\
\text { (b) } \frac{\sin 3 x+\sin x}{\cos 3 x+\cos x}=\tan 2 x .
\end{gathered}
$$

3. Solve and check the following triangles, and find the areas.
(a) $a=34.34$,
$b=18.96$,
$C=50^{\circ} .68$.
(b) $a=543.2$,
$b=496.7$,
$c=568.3$,
(c) $b=7634$,
$c=8427$,
$A=102^{\circ} .16$.
(d) $a=1243$,
$b=1497$
$c^{\circ}=2046$. 
4. Two men start to walk from the same point in directions inclined at $45^{\circ}$ to each other. When one has gone 792 yards, the other has gone 846 yards. What is the inclination of the line joining them to their paths, and how far apart are they?

5. The sides of a triangular field are 84.3 rods, 77.5 rods, and 102.1 rods. What are the angles of the field? How many potatoes could be raised on it, if the yield were 200 bushels per acre?

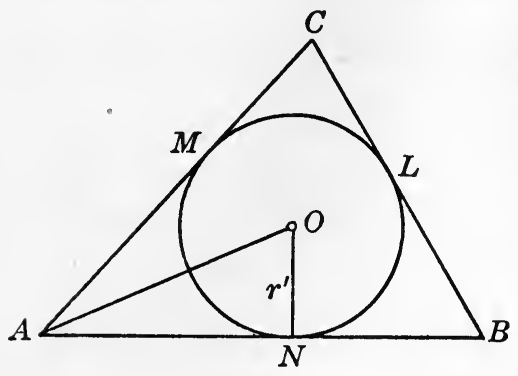

Fig. 199.

6. Find the radius of the circle inscribed in a triangle in terms of its sides.

Let the points of contact with the sides be $L, M, N$. Then

$A N=A M, B L=B N, C L=C M$, hence whence

$$
s=A N+B L+C L=A N+a,
$$

$$
A N=s-a .
$$

Then if $r^{\prime}$ is the radius and $O$ the center of the circle, we have

$$
\begin{aligned}
\tan \frac{1}{2} A & =\frac{r^{\prime}}{A N}=\frac{r^{\prime}}{s-a} . \text { But } \tan \frac{1}{2} A=\frac{r}{s-a}, \\
r^{\prime} & =r=\sqrt{\frac{(s-a)(s-b)(s-c)}{s} .}
\end{aligned}
$$

7. Prove that the area of $A B C$ is $A=r s$. Hint: If $O$ is the center of the inscribed circle, $A B C=O A B+O B C+O C A$.

8. Find the diameter of the circle circumscribed about a triangle. Hint: Draw the diameter $C D$ through $C$, and join $D$ to $B$. What are the values of the angles $C B D$ and $C D B$ ?

9. $A$ and $B$ are two inaccessible points. If $C D=1000$ yards, $\angle A C D$ $=82^{\circ} .34, \angle A D C=58^{\circ} .22, \angle B C D$ $=72^{\circ} .78, \angle B D C=105^{\circ} .13$, find the direction of $A B$ (the angle $A B$ makes with $A C$ ) and the distance from $A$ to $B$.

10. The altitude of a triangular prism is 13.48 inches, and the sides of the base are 11.23 inches, 8.43 inches, and 4.79 inches. Find the volume.

11. The sides of a field measured in succession are $22.5,20.4,18.7$, and

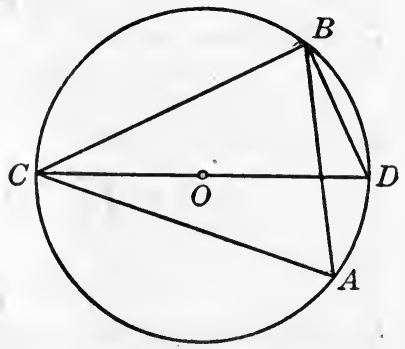

Fig. 200. 21.5 rods. The angle between the first two sides is $84^{\circ} .5$. How many acres does the field contain? 


\section{Miscellaneous Identities and Equations.} have

Example 1. Express $\sin 3 \theta$ in terms of $\sin \theta$. Since $3 \theta=2 \theta+\theta$, we

$$
\begin{aligned}
\sin 3 \theta=\sin (2 \theta+\theta) & =\sin 2 \theta \cos \theta+\cos 2 \theta \sin \theta \\
& =(2 \sin \theta \cos \theta) \cos \theta+\left(\cos ^{2} \theta-\sin ^{2} \theta\right) \sin \theta \\
& =3 \sin \theta \cos ^{2} \theta-\sin ^{3} \theta \\
& =3 \sin \theta\left(1-\sin ^{2} \theta\right)-\sin ^{3} \theta \\
& =3 \sin \theta-4 \sin ^{3} \theta .
\end{aligned}
$$

This identity, like the formula for $\sin 2 \theta$, is a special case of the sine of the product of two numbers (compare (3), page 153, and (7), page 223. It may be used in the proof of the fact that it is impossible to trisect an angle with ruler and compasses, as this phrase was understood by the ancient Greeks.

Example 2. Prove the identity $\frac{\tan 2 x+\tan x}{\tan 2 x-\tan x}=\frac{\sin 3 x}{\sin x}$.

Proof.

$$
\begin{aligned}
\frac{\tan 2 x+\tan x}{\tan 2 x-\tan x} & =\frac{\frac{\sin 2 x}{\cos 2 x}+\frac{\sin x}{\cos x}}{\frac{\sin 2 x}{\cos 2 x}-\frac{\sin x}{\cos x}} \\
& =\frac{\sin 2 x \cos x+\cos 2 x \sin x}{\sin 2 x \cos x-\cos 2 x \sin x}
\end{aligned}
$$

(by multiplying numerator and denominator by $\cos 2 x \cos x$ )

$$
=\frac{\sin (2 x+x)}{\sin (2 x-x)}=\frac{\sin 3 x}{\sin x} \text {. }
$$

EXAMPLE 3. Solve the equation $\cos x-\cos 2 x+\cos 3 x=0$.

Applying the formula for the sum of the cosines of two angles to the first and third terms, we get

Factoring, whence $\quad \cos 2 x=0$

$2 \cos 2 x \cos x-\cos 2 x=0$.

Then $\cos 2 x(2 \cos x-1)=0$,

$$
2 x= \pm \frac{\pi}{2}+2 n \pi \quad \text { or } \quad x= \pm \frac{\pi}{3}+2 n \pi .
$$

Hence

$$
x= \pm \frac{\pi}{4}+n \pi \quad \text { or } \quad x= \pm \frac{\pi}{3}+2 n \pi .
$$

\section{EXERCISES}

1. Prove the following indentities.
(a) $\tan x+\tan y=\frac{\sin (x+y)}{\cos x \cos y}$.
(b) $\cot x+\cot y=\frac{\sin (x+y)}{\sin x \sin y}$. 

(c) $\frac{\sin x+\sin 2 x}{1+\cos x+\cos 2 x}=\tan x$.
(k) $\frac{\sin x}{1-\cos x}=\cot \frac{x}{2}$.
(d) $\frac{\sin x+\sin 2 x+\sin 3 x}{\cos x+\cos 2 x+\cos 3 x}=\tan 2 x$.
(l) $\cot \left(x+\frac{\pi}{2}\right)=\frac{1-\sin 2 x}{\cos 2 x}$.
(e) $\frac{\sin A+\sin B}{\cos A+\cos B}=\tan \frac{1}{2}(A+B)$.
(m) $\cot x+\tan x=2 \csc 2 x$.
(f) $1+\tan 2 x \tan x=\sec 2 x$.
(g) $\frac{\cot \frac{x}{2}-\tan \frac{x}{2}}{\cot \frac{x}{2}+\tan \frac{x}{2}}=\cos x$.
(n) $\sin \left(\frac{\pi}{4}+x\right)=\cos \left(\frac{\pi}{4}-x\right)$
(o) $\tan \left(\frac{x}{2}+\frac{\pi}{4}\right)= \pm \frac{\sqrt{1+\sin x}}{\sqrt{1-\sin x}}$.
(h) $\frac{\sin 2 x}{1+\cos 2 x}=\tan x$.
(i) $\tan x=\frac{2 \cot \frac{x}{2}}{\cot ^{2} \frac{x}{2}-1}$.
(p) $\frac{2 \tan \frac{x}{2}}{1+\tan ^{2} \frac{x}{2}}=\sin x$.
(j) $\frac{1-\tan ^{2} \frac{x}{2}}{1+\tan ^{2} \frac{x}{2}}=\cos x$.
(q) $\frac{\tan 3 x-\tan x}{\tan 3 x+\tan x}=\frac{1}{2 \cos 2 x}$.
(r) $\cos 3 x=4 \cos ^{3} x-3 \cos x$.

(s) $2 \sin \left(x+\frac{\pi}{4}\right) \sin \left(x-\frac{\pi}{4}\right)=\sin ^{2} x-\cos ^{2} x$.

2. Solve the following equations.
(a) $\cos 2 x-2 \cos x-3=0$.
(h) $\sin 2 x+\sin 3 x=0$.
(b) $\cos 2 x-\cos x=0$.
(i) $\sin 3 x+\sin 5 x=0$.
(c) $\cos 2 x-\sin x=0$.
(j) $2 \sin x-3 \cos x=1$.
(d) $\tan 2 x=\sin x \sec x$.
(k) $\sin x+\cos x=\sqrt{2}$.
(e) $12 \sec ^{2} x+7 \tan x-24=0$.
(l) $4 \sin x+3 \cos x=5$.
(f) $\sin x+\sin 2 x+\sin 3 x=0$.
(m) $\tan x+\tan 2 x+\tan 3 x=0$.
(g) $\cos x+\sin 2 x-\cos 3 x=0$.
(n) $\cos ^{2} \frac{x}{2}-\sin ^{2} \frac{x}{2}=1-2 \cos ^{2} x$.

3. In the following identities transform the left member into the right.

(a) $\sin ^{2} x=\frac{1}{2}-\frac{1}{2} \cos 2 x$.

(b) $\cos ^{2} x=\frac{1}{2}+\frac{1}{2} \cos 2 x$.

(c) $\sin ^{4} x=\frac{3}{8}-\frac{1}{2} \cos 2 x+\frac{1}{8} \cos 4 x$.

(d) $\cos ^{4} x=\frac{3}{8}+\frac{1}{2} \cos 2 x+\frac{1}{8} \cos 4 x$.

128. Differentiation of Trigonometric Functions. In order to find the derivative of $\sin x$ we shall need to show that

$$
\lim _{\theta \doteq 0} \frac{\sin \theta}{\theta}=1 \text {. }
$$


For this purpose we construct the figure, which is self-explanatory, and assume that $P^{\prime} P<\operatorname{arc} P^{\prime} N P<Q^{\prime} Q$.

Dividing by 2 ,

$$
M P<\operatorname{arc} N P<N Q \text {. }
$$

Substituting

$$
\begin{aligned}
M P & =O P \sin \theta=r \sin \theta, \\
N Q & =O N \tan \theta=r \tan \theta,
\end{aligned}
$$

and

$$
\operatorname{arc} N P=r \theta \text { (Theorem, page 171), }
$$

we get

$$
r \sin \theta<r \theta<r \tan \theta .
$$

Dividing by $r \sin \theta$,

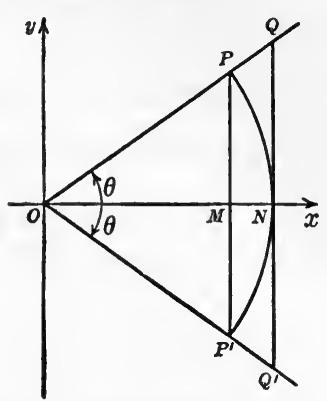

Fiq. 201.

$$
1<\frac{\theta}{\sin \theta}<\frac{1}{\cos \theta} .
$$

As $\theta$ approaches zero, $\cos \theta$ approaches 1 , and so also does $1 / \cos \theta$. Hence $\theta / \sin \theta$, which lies between 1 and $1 / \cos \theta$, approaches 1 , and hence the reciprocal function, $\sin \theta / \theta$, approaches 1 .

Theorem 1. If $u$ is a function of $x$, then the derivative of $\sin u$ with respect to $x$ is cos $u$ times the derivative of $u$ with respect to $x$, that is,

$$
D_{x} \sin u=\cos u D_{x} u .
$$

Let

$$
y=\sin u \text {. }
$$

Then

$$
y+\Delta y=\sin (u+\Delta u)
$$

Subtracting,

$$
\begin{aligned}
\Delta y & =\sin (u+\Delta u)-\sin u \\
& =2 \cos \left(u+\frac{\Delta u}{2}\right) \sin \frac{\Delta u}{2}[(2), \text { page } 346 .]
\end{aligned}
$$

Multiplying and dividing by $\frac{\Delta u}{2}$,

$$
\Delta y=\cos \left(u+\frac{\Delta u}{2}\right) \frac{\sin \frac{\Delta u}{2}}{\frac{\Delta u}{2}} \Delta u .
$$


Dividing by $\Delta x, \frac{\Delta y}{\Delta x}=\cos \left(u+\frac{\Delta u}{2}\right) \frac{\sin \frac{\Delta u}{2}}{\frac{\Delta u}{2}} \frac{\Delta u}{\Delta x}$.

In passing to the limit as $\Delta x$ approaches zero, we notice that $\Delta u$ approaches zero, and so also does $\frac{\Delta u}{2}$. Then the limit of the second factor on the right is unity, by (1) above. Passing to the limit, we get

$$
D_{x} y=\cos u D_{x} u .
$$

Corollary 1. If $y=\sin x$, then $D_{x} y=\cos x$.

Corollary 2. If $D_{x} y=\cos x$, then $y=\sin x+C$, and if

$$
D_{x} y=\cos u D_{x} u \text {, then } y=\sin u+C .
$$

Theorem 2. $D_{x} \cos u=-\sin u D_{x} u$.

By (1), page 177 , we have

$$
y=\cos u=\sin \left(\frac{\pi}{2}-u\right)
$$

and hence

$$
\begin{aligned}
D_{x} y & =D_{x} \sin \left(\frac{\pi}{2}-u\right) \\
& =\cos \left(\frac{\pi}{2}-u\right) D_{x}\left(\frac{\pi}{2}-u\right) \\
& =-\sin u D_{x} u .
\end{aligned}
$$

Corollary 1. If $y=\cos x$, then $D_{x} y=-\sin x$.

Corollary 2. If $D_{x} y=\sin x$, then $y=-\cos x+C$,

and if

$$
D_{x} y=\sin u D_{x} u, \text { then } y=-\cos u+C \text {. }
$$

Example 1. Uniform motion in a circle. If the position of a point $P(x, y)$ at the time $t$, is given by the equations

$$
x=a \cos \omega t, y=a \sin \omega t,
$$

show that $P$ moves in a circle, and find the magnitude and direction of the velocity and acceleration.

Squaring and adding the given equations, we get

$$
x^{2}+y^{2}=a^{2} \cos ^{2} \omega t+a^{2} \sin ^{2} \omega t=a^{2},
$$

which is the equation of a circle (page 327), and the point $P$ therefore moves on a circle. 
The radius $O P$ makes an angle of $\theta=\omega t$ with the $x$-axis. Since $D_{t} \theta=\omega$, the rate of change of $\theta$, or the angular velocity of $O P$, is the constant $\omega$.

The table of values gives the times at which $P$ crosses the axes in its first revolution.

Velocity of $P$. The components of the velocity parallel to the axes are (page 311)

$v_{x}=D_{t} x=D_{t}(a \cos \omega t)$

$=-a \sin \omega t D_{t} \omega t=-a \omega \sin \omega t$, and

$$
v_{\nu}=a \omega \cos \omega t .
$$

Then the magnitude of the velocity is $v=\sqrt{(-a \omega \sin \omega t)^{2}+a^{2} \omega^{2} \cos ^{2} \omega t}=a \omega$.

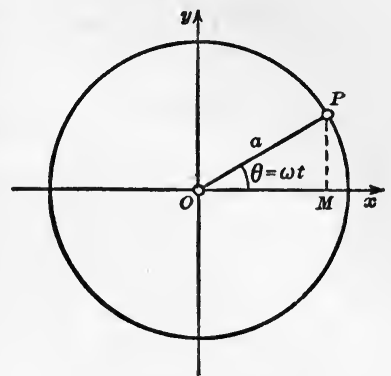

FIG. 202.

As $a \omega$ is constant, the point $P$ moves uniformly in its path. This result may be verified as follows: Since the angular velocity is $\omega$, in one

\begin{tabular}{c|c|c|c}
$t$ & $\theta=\omega t$ & $x$ & $y$ \\
\hline 0 & 0 & $a$ & 0 \\
$\frac{\pi}{2 \omega}$ & $\frac{\pi}{2}$ & 0 & $a$ \\
$\frac{\pi}{\omega}$ & $\pi$ & $-a$ & 0 \\
$\frac{3 \pi}{2 \omega}$ & $\frac{3 \pi}{2}$ & 0 & $-a$ \\
$\frac{2 \pi}{\omega}$ & $2 \pi$ & $a$ & 0
\end{tabular}
second the radius $O P$ will sweep over $\omega$ radians, and hence the arc described by $P$ will be $a \omega$ (Theorem, page 171 ); that is, $P$ will move a distance $a \omega$ in one second.

The slope of the direction of the velocity is

$$
\frac{v_{y}}{v_{x}}=\frac{a \omega \cos \omega t}{-a \omega \sin \omega t}=-\frac{a \cos \omega t}{a \sin \omega t}=-\frac{x}{y}
$$

As this is the negative reciprocal of the slope of $O P$, the direction of the velocity is perpendicular to $O P$, and hence it is along the tangent line.

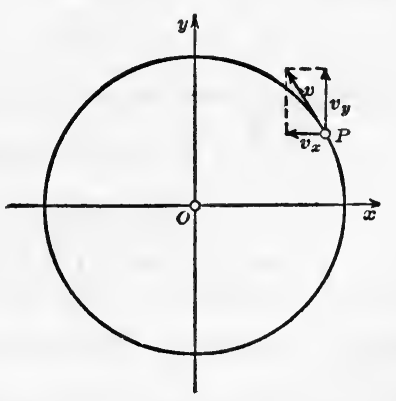

Fig. 203.

Acceleration of $P$. The components of the acceleration are

$$
\begin{aligned}
& a_{x}=D_{t} v_{x}=-a \omega^{2} \cos \omega t, \\
& a_{y}=D_{t} v_{y}=-a \omega^{2} \sin \omega t .
\end{aligned}
$$


Then the magnitude of the acceleration is

$$
a=\sqrt{a^{2} \omega^{4} \cos ^{2} \omega t+a^{2} \omega^{4} \sin ^{2} \omega t}=a \omega^{2} .
$$

The slope of the direction of the acceleration is

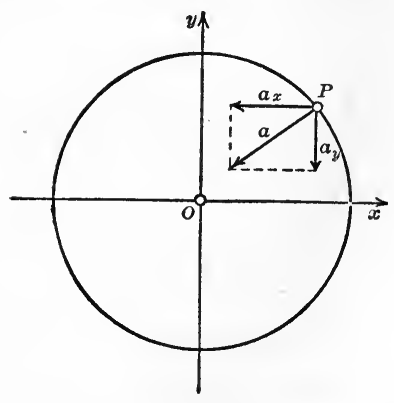

FIG. 204.

$$
\frac{a_{y}}{a_{x}}=\frac{-a \omega^{2} \sin \omega t}{-a \omega^{2} \cos \omega t}=\frac{a \sin \omega t}{a \cos \omega t}=\frac{y}{x} .
$$

As this is the slope of $O P$, the acceleration acts along the radius. It is directed toward the center.

Example 2. Find the area of one arch of the graph of $\sin 2 x$ (page 343 ).

- From page 305 we have

$$
D_{x} A=y=\sin 2 x .
$$

As $2 x$ is a function, $u$, of $x$ whose derivative is 2 , we multiply and divide $\sin 2 x$ by 2 , obtaining

$$
D_{x} A=\frac{1}{2} \sin 2 x \times 2=\frac{1}{2} \sin 2 x D_{x}(2 x) .
$$

Integrating by (9),

$$
A=-\frac{1}{2} \cos 2 x+C \text {. }
$$

As $A=0$ when $x=0$,

$$
0=-\frac{1}{2}+C, \quad \text { whence } \quad C=\frac{1}{2} \text {. }
$$

Hence $\quad A=-\frac{1}{2} \cos 2 x+\frac{1}{2}$.

Substituting $x=\frac{\pi}{2}$, the desired area is

$$
A=-\frac{1}{2} \cos \pi+\frac{1}{2}=-\frac{1}{2}(-1)+\frac{1}{2}=1 .
$$

In finding the derivative of $\sin u$, use was made of the fact that $\lim _{\theta \doteq 0} \frac{\sin \theta}{\theta}=1$, and in finding this limit the angle $\theta$ was measured in radians. Hence,

In applying the rules for differentiating and integrating trigonometric functions it must be remembered that the independent variable is measured in radians.

\section{EXERCISES}

1. Differentiate the functions:
(a) $2 \sin (3 x+4)$.
(b) $3 \cos 4 x$.
(c) $2 \cos (1-\pi x)$.
(d) $3 \sin (2 \pi x-3)$.
(e) $a \sin (b x+c)$.
(f) $\mathrm{c} \cos (m x+r)$.
(g) $\sin \left(x^{2}\right)$.
(h) $\sin \left(x^{2}+3 x\right)$.
(i) $2 \cos \left(x^{3}-x^{2}\right)$. 


\section{PROPERTIES OF TRIGONOMETRIC FUNCTIONS}

2. Find the derivatives of the following functions, by first expressing them in terms of the sine and cosine.
(a) $D_{x} \tan u=\sec ^{2} u D_{x} u$.
(b) $D_{x} \cot u=-\csc ^{2} u D_{x} u$.
(c) $D_{x} \sec u=\tan u \sec u D_{x} u$.
(d) $D_{x} \csc u=-\cot u \csc u D_{x} u$.

3. Find the derivative of each of the functions below, using the fundamental method of replacing $x$ by $x+\Delta x$, etc.
(a) $\cos x$.
(b) $\tan x$.
(c) $\cot x$.
(d) $\sec x$.
(e) $\csc x$.

4. Find the maximum and minimum values of each of the trigonometric functions by the method of differentiation.

5. Differentiate the following functions, using (5) page 270 .
(a) $\sin ^{2} x$.
(b) $\cos ^{2} 2 x$.
(c) $\sec ^{2} x$.
(d) $2 \csc ^{2} 3 x$.

6. Find the coorrdinates of the points of inflection of the graph of
(a) $\sin x$.
(b) $\cos x$.
(c) $\tan x$.
(d) $\cot x$.

7. A point moves so that $x=10 \cos 2 \pi t$ and $y=10 \sin 2 \pi t$. Show that the point moves in a circle, making one revolution per second. Find the components parallel to the axes and the magnitude and the direction of the velocity and acceleration. Where does the point start?

8. As in the preceding exercise, discuss the motion of a point if $x=10$ $\cos (2 \pi t+\pi / 2)$ and $y=10 \sin (2 \pi t+\pi / 2)$. Where does the point start?

9. Discuss the motion of a point if $x=4 \cos (\pi x / 2+\pi / 3), y=4 \sin$ $(\pi x / 2+\pi / 3)$.

10. The position of a point $P$ moving on a circle of radius 5 so that $O P$ rotates through 2 radians per second is given by

$$
x=5 \cos 2 t, y=5 \sin 2 t .
$$

If $M$ is the projection of $P$ on the $x$-axis (see figure for Example 1 of the preceding section), find the position $(s=O M)$, velocity, and acceleration of $M$ at any time. Find when and where the values of $s, v$, and $a$ are greatest and least.

11. A chip on the surface of a pond moves up and down with the waves according to the law $s=\sin \frac{1}{2} t$. Find the velocity and acceleration. Determine the maximum and minimum values of $s, v$, and $a$. Plot the three graphs on the same axes.

12. Find the area of an arch of each of the curves, (a) $\sin x$. (b) $\sin \frac{1}{2} x$. (c) $\cos 2 x$. (d) $3 \sin x$.

13. In a right triangle $A B C$, it was found that $b=10$ inches, and $A=45^{\circ}$. Find $a$ and $c$, and the error in each due to an error of $0^{\circ} .1$ in $A$.

14. In a triangle $a=2, b=4, C=60^{\circ}$. Find $c$ by the law of cosines and the error in $c$ due to an error of $0^{\circ} .2$ in $C$.

15. Given $\sin 30^{\circ}=\frac{1}{2}=.5000$ and $\cos 30^{\circ}=\sqrt{3} / 2=.8660$, find approximate values of $\sin 30^{\circ} .1$ and $\cos 30^{\circ} .1$. 
16. Given $\sin 45^{\circ}=\cos 45^{\circ}=\sqrt{ } \overline{2} / 2=.7071$, find approximate values of $\sin 45^{\circ} .1$ and $\cos 45^{\circ} .1$.

17. Given $\sin 60^{\circ}=\sqrt{3} / 2=.866$ and $\cos 60^{\circ}=\frac{1}{2}=.500$, compute a three-place table of sines and cosines of $60^{\circ} .1,60^{\circ} .2,60^{\circ} .3,60^{\circ} .4,60^{\circ} .5$.

18. A ship is anchored 300 yards from a straight shore, along which a searchlight is played. If the light is turned uniformly at the rate of one radian per minute, how fast will the beam of light be moving along the shore when it makes an angle of $30^{\circ}$ with the shore?

19. A balloon is ascending vertically at the rate of 5 feet per second. An observer stands 200 feet from the point at which the balloon started. How fast is the angle of elevation changing when it equals $60^{\circ}$ ?

20. A level road approaches a hill 300 feet high. An observer on the hill notes that the angle of depression of a motorcycle is $80^{\circ}$ and that the angle decreases $1^{\circ}$ in three seconds. Find approximately the speed of the motorcycle.

129. Graph of the Function $a \sin (b x+c)$. Harmonic Curves. Suppose at first that $c=0$, and consider the special case

$$
y=a \sin b x .
$$

The graph of this function may be obtained from that of $\sin x$ by first dividing the abscissas by $b$, which gives the graph

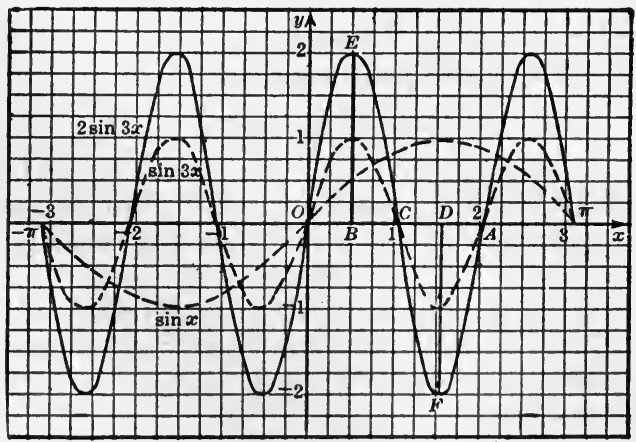

GrAPH OF $a \sin b x, a=2, b=3$.

FIG. 205.

of $\sin b x[(4)$, page 152$]$, and then multiplying the ordinates of points on the graph of $\sin b x$ by $a$ [(3), page 152]. Since the graph of $\sin x$ repeats itself in intervals of $2 \pi$, the graph of (1) will repeat in intervals of $2 \pi / b$, so that the function 
$a \sin b x$ is periodic with the period $2 \pi / b$. And since the maximum and minimum values of $\sin x$ are 1 and -1 , the maximum and minimum values of $a \sin b x$ are $a$ and $-a$. The constant $a$ is called the amplitude of the function.

The form of the graph of (1) is therefore much like that of $\sin x$, but it differs from it in period and amplitude, or in width and height. The graph may be sketched expeditiously, without drawing the graphs of $\sin x$ and $\sin b x$, as follows:

Lay off $O A$ (Fig. 205) on the $x$-axis equal to the period $2 \pi / b$, and divide $O A$ into four equal parts by $B, C$, and $D$. At $B$ erect $B E$ perpendicular to the $x$-axis and equal to $a$, and at $D$ draw the ordinate $D F=-a$. Then the graph cuts the $x$-axis at $O$, $C$, and $A$, has a maximum point at $E$, and a minimum point at $F$. The part of the curve from $O$ to $A$ can be drawn by using these five points, and the rest of the curve may be obtained by the periodicity.

Now consider the general equation

$$
y=a \sin (b x+c) .
$$

It may be written in the form

$$
y=a \sin b\left(x+\frac{\dot{c}}{b}\right)
$$

If we set $x=x^{\prime}-\frac{c}{b}$, which moves the $y$-axis to the new origin $O^{\prime}\left(-\frac{c}{b}, 0\right)$, equation (3) becomes

$$
y=a \sin b x^{\prime} .
$$

Comparing (4) with (1), we see that the graph of (4) may be drawn on the new axes by the method given above. The constant $O O^{\prime}=-c / b$ is called the phase of the function.

The graph of an equation in the form (2) is called a simple harmonic curve, and the function a simple harmonic function.

EXample. Construct the graph of $2 \sin \left(\frac{\pi}{2} x+\pi\right)$.

Here $a=2, b=\pi / 2$, and $c=\pi$. Since the phase is $-c / b=-2$, we lay off $O O^{\prime}=-2$ on the $x$-axis, and choose $O^{\prime}$ as a new origin. The period 
is $2 \pi / b=4$, and hence we lay off $O^{\prime} A=4$, and divide it into four equal parts by $B, C, D$. Since the amplitude is $a=2$, we erect the ordinates

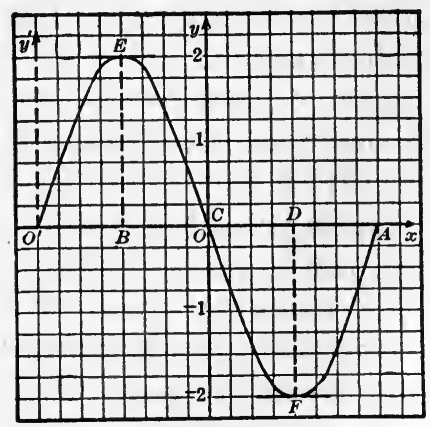

FIg. 206 $B E=2$, and $D F=-2$. The graph is then drawn through $O^{\prime}, E, C, F$, and $B$. It repeats itself every 4 units along the $x$-axis.

A compound harmonic curve is obtained by adding the ordinates of points on two harmonic curves. It is the graph of an equation of the form

$$
\begin{aligned}
y=a & \sin (b x+c) \\
& +d \sin (e x+f) .
\end{aligned}
$$

Fig. 207 shows the compound harmonic curve

$$
2 \sin x+\sin (2 x+\pi) .
$$

Harmonic curves find important applications in such theories as sound and electricity which depend on wave theory. The

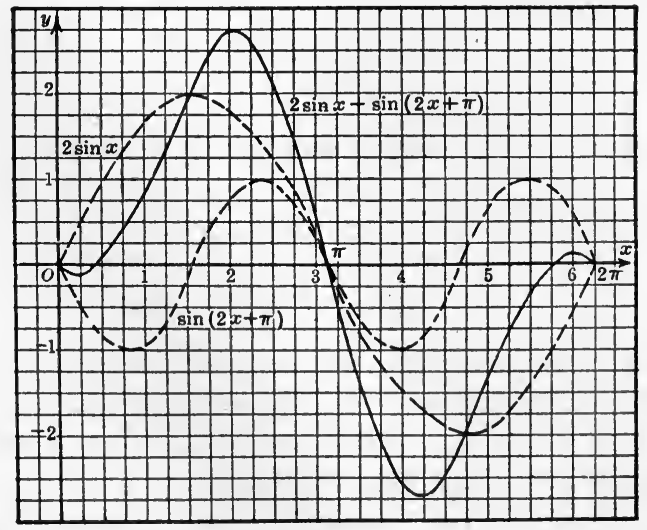

Frg. 207.

period is frequently called the wave length. They are applied, also, in many motions, for example, in the theory of the pendulum. 
130. Empirical Data Problems. We shall content ourselves with pointing out the method of solution of some very simple problems in determining the constants in the equation of a harmonic curve. The treatment of empirical data problems in which the points representing a given table of values appear to lie on a harmonic curve furnishes sufficient material for a book involving considerable higher mathematics.

Example. Determine the constants $a$ and $b$ if the graph of

$$
y=a \sin x+b \sin 2 x
$$

is to pass through the points $(\pi / 6,1+\sqrt{3} / 2)$ and $(\pi / 4,1+\sqrt{2})$.

Since these points are to lie on the graph their coördinates must satisfy the given equation. Hence $1+\frac{\sqrt{3}}{2}=\frac{1}{2} a+\frac{\sqrt{3}}{2} b$,

and

$$
1+\sqrt{2}=\frac{\sqrt{2}}{2} a+b .
$$

Solving these equations for $a$ and $b, a=2$ and $b=1$. Hence the required equation is $y=2 \sin x+\sin 2 x$.

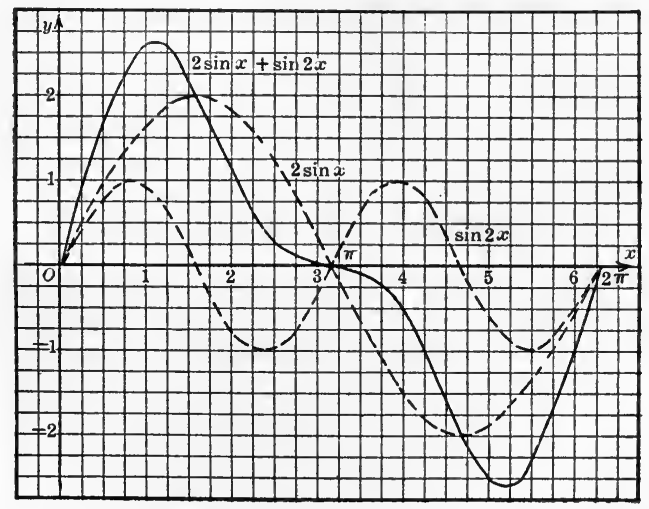

Fig. 208.

If several points are given which appear to lie on or near the graph of an equation of the form given in the example, we may determine a pair of values of $a$ and $b$ from each pair of points. If the values of $a$ agree closely, and also those of $b$, by using the average value of the $a$ 's and that of the $b$ 's we obtain a good approximation of the law connecting the coördinates of the points. 


\section{EXERCISES}

1. Show that the equation $y=a \sin (b x+c)$ may be put in the form

$$
y=A \sin b x+B \cos b x,
$$

where $A=a \cos c$ and $B=a \sin c$.

2. If $y=A \sin b x+B \cos b x$, find the values of $a$ and $c$ for which the given equation has the form $y=a \sin (b x+c)$.

3. Show that $y=a \sin (b x+c)$ may be put in the form $y=$ $a \cos \left(b x+c^{\prime}\right)$, where $c^{\prime}=c-\pi / 2$.

4. On the same axes sketch the graphs of
(a) $\sin x, \sin \frac{1}{2} x, 3 \sin \frac{1}{2} x$.
(b) $\cos x, \cos 2 x, \frac{1}{2} \cos 2 x$.
(c) $\sin x, \sin \pi x, 4 \sin \pi x$.

5. Determine the phase, period, and amplitude of each of the following functions, and sketch the graph.
(a) $3 \sin \pi x$.
(b) $2 \sin \pi x / 2$.
(c) $\frac{1}{2} \sin \pi x / 3$.
(d) $2 \sin (\pi x+\pi)$.
(e) $\frac{1}{3} \sin (x / 2+\pi / 2)$.
(f) $2 \sin (x-1)$.
(g) $3 \sin (\pi x / 3-\pi)$.
(h) $\frac{1}{2} \sin (\pi x / 2+\pi / 2)$.
(i) $4 \sin (\pi x-\pi / 3)$.

6. Sketch the following compound harmonic curves by the addition of ordinates.
(a) $\sin x+2 \sin 2 x$.
(c) $3 \sin 2 x-\sin x / 2$.
(b) $2 \sin x+\sin x / 2$.
(e) $\sin \pi x-\frac{1}{2} \sin \pi x / 2$.
(d) $\frac{1}{2} \sin x+\sin 3 x$.
(g) $2 \sin 2 \pi x / 3+\frac{1}{2} \sin 2 \pi x$.
(f) $2 \sin \pi x / 3+\frac{1}{2} \sin \pi x$.
(h) $1.4 \sin .7 x+.3 \sin 1.2 x$.

(i) $2 \sin (\pi x / 4+\pi / 2)+\frac{1}{2} \sin (2 \pi x+\pi)$.

7. Find the symmetry, intercepts, and the points of maxima, minima, and inflection for each of the curves below. Sketch the curve, and find the area of the arch to the right of the origin.
(a) $2 \sin x+\sin 2 x$.
(b) $\sin x-\sin \frac{1}{2} x$.
(c) $\sin 2 x+\cos 2 x$.

Note. Simple Harmonic Motion. Let a radius of a circle, $O P$, revolve uniformly at the rate $\omega$, in radians per second (see Example 1, Section 128), and let $M$ be the projection of $P$ on a fixed diameter. The motion of $M$ is called simple harmonic motion. The point $M$ moves back and forth along the diameter, making one complete oscillation for every revolution of $O P$.

Let the fixed diameter be chosen for the $x$-axis, and suppose that $P$ starts on the positive $x$-axis. Then the abscissa of $P$, which gives the position of $M$ at any time, is $s=a \cos \omega t$, where $a$ is the radius of the circle. 
If $P$ starts at the point for which $O P$ makes an angle of $\theta_{0}$ with the $x$-axis then

$$
s=a \cos \left(\omega t+\theta_{0}\right) .
$$

If the diameter on which $M$ moves is taken for the $y$-axis, the distance $O M$ is given by

$$
s=a \sin \left(\omega t+\theta_{0}\right) .
$$

8. A point moves according to one of the laws below. Find the time of one oscillation, and when and where the distance, velocity, and acceleration have maximum or minimum values. Describe the motion.
(a) $s=8 \sin 2 t$.
(b) $s=10 \sin \frac{1}{2} t$.
(c) $s=2 \sin \pi t$.
(d) $s=4 \cos \pi t / 3$.
(e) $s=\sin (3 t+\pi / 2)$.
(f) $s=2 \sin (\pi t / 3+\pi / 4)$.

9. If $s=a \sin \left(\omega t+\theta_{0}\right)$ show that (a) the acceleration is proportioned to the distance, (b) that the acceleration and distance have maximum or minimum values when the body is at rest, $(c)$ that the body is at the center of its motion and has no acceleration when it is moving fastest.

10. The wind has left long swells moving along the surface of a lake when a slight breeze produces a ripple. The equations of cross-sections of the waves before and after the breeze are $y=2 \sin x / 4$ and $y=$ $2 \sin x / 4+\frac{1}{2} \sin 2 x$. Plot both curves on the same axes.

11. Determine the constants $a$ and $b$ if the graph of the given function is to pass through the given points. Sketch the graph.
(a) $a \sin x+b \sin x / 2,(\pi / 2,3-\sqrt{2}),(\pi,-2)$.
(b) $a \sin x / 2+b \sin 2 x,(\pi / 3,1-\sqrt{3} / 4),(2 \pi / 3, \sqrt{3}+\sqrt{3} / 4)$.
(c) $a \sin x+b \sin 2 x,(.157, .309),(.332, .632),(.611,1.04)$.
(d) $a \sin x+b \cos x,(.122, .570),(.349,1.20),(.646,1.47)$.

\section{MISCELLANEOUS EXERCISES}

1. $A$ and $B$ are two points on the opposite sides of a hill which are to be connected by a tunnel. Both points are visible from a third point $C$ which is 1356 feet from $A$ and 2582 feet from $B$. If $\angle A C B=47^{\circ} .34$ and if the angles of elevation of $A$ and $B$ at $C$ are respectively $12^{\circ} .35$ and $9^{\circ} .82$, find the length of the tunnel and the inclination of the tunnel.

2. Construct a table of analogous properties (see Section 56) of the functions $e^{x}, \log x$, and $\sin x$.

3. Contrast the formulas for $e^{a+b}$ and $\tan (a+b)$ by expressing each entirely in terms of the notation $f(x)$.

4. Find approximate values of the real roots of the following equations (see Exercise 12, page 261).
(a) $\sin 2 x-x=0$.
(c) $\sin 3 x-e^{-x}=0$. (smallest positive root.)
(b) $\cos \frac{1}{2} x-x^{2}=0$.
(d) $\cos 2 x-2 x=0$.
(e) $\sin \frac{1}{2} x-\log x=0$.
(f) $2 \cos 2 x-e^{x}=0$.
(g) $\tan \frac{1}{2} x-x^{2}=0$. 
5. To find the distance between two towers on opposite sides of a river a base line 300 feet long was laid off. The following angles were measured, $C$ and $D$ representing the towers and $A B$ the base line: $\angle A B C=35^{\circ} .24$, $\angle B A D=29^{\circ} .61, \angle A B D=108^{\circ} .47, \angle B A C=97^{\circ} .59$. Compute $C D$.

6. Find the number of acres in a field in the form of a quadrilateral $A B C D$ if $A B=315$ yards, $\angle D A C=123^{\circ} .6, \angle C A B=54^{\circ} .68, \angle A B D=$ $65^{\circ} .23$, and $\angle A B C=112^{\circ} .4$.

7. If a projectile is fired at an angle $\theta$ with the horizontal with an initial velocity of $v_{0}$, the equation of its path is

$$
y=x \tan \theta-\frac{16 x^{2}}{v_{0}^{2} \cos ^{2} \theta} .
$$

Find the range as a function of $2 \theta$, and the value of $\theta$ which gives the maximum range.

8. A corridor turns at right angles. It is 8 feet wide on one side of the turn and 6 feet wide on the other. How long a beam can be carried horizontally around the corner?

9. The path of a point on the rim of a wheel rolling along a level road is given by the equations

$$
x=a(\omega t-\sin \omega t), \quad y=a(1-\cos \omega t),
$$

where $a$ is the radius of the wheel, $\omega$ is the angular velocity of the wheel about the axle, and $t$ is the time. Plot the equation of the path taking $t=\pi / 6 \omega, \pi / 3 \omega, \pi / 2 \omega$, etc. Find the velocity of the point at any time, and its maximum and minimum values. Where is the point when it is moving slowest? fastest? A bit of mud is thrown from the highest point of a wheel on an automobile. Compare the velocity with which the mud leaves the wheel with that of the automobile.

10. If $a$ is the angle between two lines whose slopes are $m_{1}$ and $m_{2}$; show that

$$
\tan \alpha=\frac{m_{1}-m_{2}}{1+m_{1} m_{2}} \text {. }
$$




\section{CHAPTER IX}

\section{THEORY OF MEASUREMENT}

131. Statistical Methods. The object of an experiment is to replace a complex system in which a number of causes are operating by a simpler system in which causes are controlled and allowed to vary only at the will of the investigator. In physics, for instance, after the isolation of two related variables, an endeavor is made to determine the relation between the variables and to express this relation in the form of a function $y=f(x)$.

In some sciences it is difficult or impossible to isolate two related variables from the complex system in which they oecur. In such cases the variation of each variable is observed independently of the others.

Statistics has to deal with variables affected by a number of causes. Some of the methods of statistics which have been developed for the analysis of such variations are discussed briefly in this chapter, three of the principal ends sought being the determination of

(1) An average value which will represent the values of $\mathbf{a}$ variable.

(2) A measure of the variability of the items with respect to the average value chosen.

(3) A measure of the extent of the relationship between two variables which are associated.

The first few sections are given to some essential prerequisites. To illustrate the fundamental principle on which the following sections are based, consider the

Example. There are five pitchers and three catchers on a base ball team. In how many ways can a battery be chosen for a particular game? 
The position of the pitcher can be filled in 5 ways, and with each of these there is a choice of 3 catchers. Hence the two positions together can be filled in $5 \times 3=15$ ways.

Fundamental Principle. If one act can be done in $A$ ways and a second act in $B$ ways, the total number of ways in which the two acts may be performed in succession is $A \cdot B$.

The following theorem is a generalization of the fundamental principle.

Theorem. If one act can be done in' $A$ ways, a second in $B$ ways, a third in $C$ ways, and so on, they can all be done together in the order stated in $A \cdot B \cdot C$. . . ways.

\section{EXERCISES}

1. If two coins are tossed, in how many ways can they fall?

2. If two dice are thrown, in how many ways can they fall?

3. A committee of three is chosen from seven physicians, eight lawyers, and twelve business men, so that each group is represented. In how many ways can the committee be chosen?

4. If two dice and three coins are tossed, in how many ways can they fall?

132. Permutations. Definition. Each arrangement which can be made of all or part of a number of things is called a permutation.

Thus the permutations of the letters $a, b, c$, taken three at a time are $a b c, a c b, b a c, b c a, c a b, c b a$, and their permutations taken two at a time are $a b, b a, a c, c a, b c, c b$.

The number of permutations of $n$ different things taken $r$ at a time is denoted by ${ }_{n} P_{r}$.

Theorem. The number of permutations of $n$ things taken $r$ at a time is

$$
{ }_{n} P_{r}=n(n-1)(n-2) \ldots(n-r+1) .
$$

There are $r$ places to fill and $n$ things from which to choose. For the first place there is a choice of $n$ things. The second place can be filled with any one of the remaining $n-1$ things. The third can be filled in $n-2$ ways, and so on.

For the $r$ th place there is a choice of $n-(r-1)$ or $n-r+1$ things. 
Hence by the theorem of Section 131,

$$
{ }_{n} P_{r}=n(n-1)(n-2) \ldots(n-r+1) .
$$

Corollary. The number of permutations of $n$ things taken all at a time is

$$
{ }_{n} P_{n}=n(n-1)(n-2) \ldots 2 \times 1=n !
$$

The symbol $n$ ! is read factional $n$, and represents the product of all the integers from 1 to $n$ inclusive.

Example. (a) In how many ways can the letters of the word triangle be arranged? (b) How many of the arrangements will begin with the letters tri in this order? (c) How many arrangements will have the letters tri together in any order? (d) How many arrangements consisting of three letters can be made from the word triangle?

(a) Here $n=8$ and $r=8$, and hence the number of arrangements is ${ }_{8} P_{8}=8 !=40,320$.

(b) The first three places are filled and there remain five letters to be permutated in five places, hence the number of arrangements is ${ }_{5} P_{5}=120$.

(c) The letters tri can be permuted as a group with the remaining five letters, and then the three letters $t r i$ be permuted within their own group. Hence the number of arrangements is ${ }_{8} P_{3} \times{ }_{6} P_{6}=3 ! 6 !=4320$.

(d) Here $n=8$ and $r=3$; hence the number of arrangements is ${ }_{8} P_{3}=$ $8 \times 7 \times 6=336$.

\section{EXERCISES}

1. In how many ways can five flags of different colors be arranged five in a line? Three in a line?

2. How many arrangements of all the letters of the word English will begin with a vowel, and end with a consonant? How many will have the vowels together?

3. How many different numbers less than 1000 can be formed from the digits $1,2,3,4,5,6$ ?

4. In how many ways may first and second prizes be awarded, if there are 12 competitors in a race?

133. Combinations. Definition. A group of things which is independent of the order of the elements is called a combination.

The combinations of $a, b, c$, taken two at a time are $a b, a c$, $b c$.

The selection $b a$ is a different permutation from $a b$, but the same combination. 
The six permutations of the letters $a b c$ taken three at a time, namely, $a b c, a c b, b a c, b c a, c a b, c b a$ are different arrangements of one combination.

The number of combinations of $n$ things taken $r$ at a time is denoted by ${ }_{n} C_{r}$.

Theorem 1. The number of combinations of $n$ things taken $r$ at a time is

$$
{ }_{n} C_{r}=\frac{n(n-1)(n-r) \ldots(n-r+1)}{r !} .
$$

Each combination of $r$ things chosen from the $n$ things can be arranged in $r$ ! ways.

Therefore all the combinations can be arranged in $r !_{n} C_{r}$ ways.

But this is the number of ways in which $n$ things taken $r$ at a time can be arranged, so that

$$
\begin{aligned}
r !_{n} C_{r} & ={ }_{n} P_{r} \\
\therefore{ }_{n} C_{r} & =\frac{{ }_{n} P_{r}}{r !} \\
& =\frac{n(n-1) \ldots(n-r+1)}{r !} .
\end{aligned}
$$

Theorem 2. The number of combinations of $n$ things taken $r$ at a time is the same as the number of combinations of $n$ things taken $n-r$ at a time.

Multiplying numerator and denominator of the formula for ${ }_{n} C_{r}$ by $(n-r)$ ! we have

$$
\begin{aligned}
{ }_{n} C_{r} & =\frac{n(n-1) \ldots(n-r+1)(n-r)(n-r-1) \ldots 2 \cdot 1}{r !(n-r) !} \\
& =\frac{n !}{r !(n-r) !} .
\end{aligned}
$$

By Theorem 1, we have

$$
{ }_{n} C_{n-r}=\frac{n(n-1) \ldots(n-(n-r)+1)}{(n-r) !}=\frac{n(n-1) \ldots(r+1)}{(n-r) !} \text {. }
$$

Multiplying numerator and denominator by $r$ !

$$
\begin{gathered}
{ }_{n} C_{n-r}=\frac{n(n-1) \ldots(r+1) r(r-1) \ldots 1}{(n-r) ! r !}=\frac{n !}{(n-r) ! r !} . \\
\therefore{ }_{n} C_{r}={ }_{n} C_{n-r .}
\end{gathered}
$$


Example 1. A committee of 5 is to be chosen from 7 lawyers and 6 physicians. How many committees will contain (a) just 3 lawyers, (b) at least 3 lawyers?

(a) The number of ways of selecting three lawyers is

$$
{ }_{7} C_{3}=\frac{7 \cdot 6 \cdot 5}{1 \cdot 2 \cdot 3}=35
$$

The number of ways of selecting two physicians is

$$
{ }_{6} C_{2}=\frac{6 \cdot 5}{1 \cdot 2}=15 \text {. }
$$

Hence the number of committees which will contain exactly three lawyers is

$$
{ }_{7} C_{3} \times{ }_{6} C_{2}=35 \times 15=525 .
$$

(b) At least three lawyers are present in each committee of the types: 3 lawyers and 2 physicians, 4 lawyers and 1 physcian, and 5 lawyers.

Hence the number of committees which contain at least three lawyers is

$$
{ }_{7} C_{3} \times{ }_{6} C_{2}+{ }_{7} C_{4} \times{ }_{6} C_{1}+{ }_{7} C_{5}=756 .
$$

Example 2. How many arrangements can be made consisting of two vowels and three consonants chosen from the letters of the word triangle?

The vowels may be selected in ${ }_{3} C_{2}=3$ ways.

The consonants may be selected in ${ }_{5} C_{3}=10$ ways.

Hence the total number of selections consisting of two vowels and three consonants is ${ }_{3} C_{2} \times{ }_{5} C_{3}=30$.

Each of these selections can be arranged in 5! ways. Hence the required number of arrangements is $5 !{ }_{3} C_{2} \times{ }_{5} C_{5}=3600$.

\section{EXERCISES}

1. How many alloys can be made from thirty of the known metals chosen two at a time counting one alloy only for each pair of metals? Solve the problem if there are three metals in each alloy.

2. In how many ways can a basket ball team be selected from 9 candidates? If A plays center in every combination, in how many ways can the team be chosen?

3. How many arrangements can be made of 3 vowels and 4 consonants chosen from 5 vowels and 8 consonants?

4. How many straight lines are determined by (a) 5 points no 3 of which are in the same straight line? straight line?

(b) $n$ points, no 3 of which are in the same

5. In how many ways can a baseball nine be chosen from 13 candidates, provided A, B, C, D are the only battery candidates, and can play in no other position? 
6. In how many ways can a committee of 5 be chosen from 7 democrats and 7 republicans, so that there will be (a) three democrats, (b) no more than three democrats, (c) at least three democrats on the committee?

134. The Binomial Expansion. In finding the product of the binomial factors $\left(x+a_{1}\right)\left(x+a_{2}\right)\left(x+a_{3}\right)$, each partial product is obtained by choosing one and only one term from each factor and multiplying the three terms together. The sum of the partial products gives the desired product.

There is only one term containing $x^{3}$, since the three $x$ 's can be chosen from the three factors in but one way.

The terms of the product containing $x^{2}$ are obtained by choosing $x$ from two of the factors and an $a$ from the third factor, which gives the partial products $x^{2} a_{1}, x^{2} a_{2}, x^{2} a_{3}$. The number of such terms will be the number of ways we can choose an $a$ from the three $a$ 's, or ${ }_{3} C_{1}$.

To obtain the term in $x$, we choose an $x$ from one binomial and two $a$ 's from the two remaining in all possible ways, which gives the partial products $x a_{1} a_{2}, x a_{1} a_{3}, x a_{2} a_{3}$. The number of such products is therefore ${ }_{3} C_{2}$.

There is only one way of choosing the three $a$ 's.

Hence,

$$
\begin{aligned}
\left(x+a_{1}\right)\left(x+a_{2}\right)\left(x+a_{3}\right)=x^{3} & +\left(a_{1}+a_{2}+a_{3}\right) x^{2} \\
& +\left(a_{1} a_{2}+a_{1} a_{3}+a_{2} a_{3}\right) x+a_{1} a_{2} a_{3} .
\end{aligned}
$$

If we let $a_{1}=a_{2}=a_{3}=a$, we have

$$
(x+a)^{3}=x^{3}+3 a x^{2}+3 a^{2} x+a^{3} .
$$

And since, from the preceding, the coefficient of $x^{2}$ is the number of ways we can choose an $a$ from the three $a$ 's, the coefficient of $x$ is the number of ways we can choose two $a$ 's out of three $a$ 's, and so on, we can write the expansion in the form

$$
(x+a)^{3}=x^{3}+{ }_{3} C_{1} a x^{2}+{ }_{3} C_{2} a^{2} x+{ }_{3} C_{3} a^{3} .
$$

In a similar manner it can be shown that when $n$ is a positive integer, we have

The binomial expansion:

$$
\begin{aligned}
(x+a)^{n}=x^{n}+{ }_{n} C_{1} a x^{n-1} & +{ }_{n} C_{2} a^{2} x^{n-2}+\ldots \\
& +{ }_{n} C_{r} a_{r} x^{n-r}+\ldots .+{ }_{n} C_{n} a^{n},
\end{aligned}
$$




$$
\text { where } \begin{aligned}
{ }_{n} C_{1} & =1,{ }_{n} C_{2}=\frac{n(n-1)}{2 !}, \\
{ }_{n} C_{r} & =\frac{n(n-1) \ldots(n-r+1)}{r !}, \\
{ }_{n} C_{n} & =1 .
\end{aligned}
$$

The values of the coefficients are given in the following table for several values of $n$. The table is called Pascal's triangle.

\begin{tabular}{r|rrrrrrrrrrr}
$n$ &. & 1 & 2 & 3 & 4 & 5 & 6 & 7 & 8 & 9 & $10 \mid r$ \\
\hline 1 & 1 & 1 & & & & & & & & & \\
2 & 1 & 2 & 1 & & & & & & & & \\
3 & 1 & 3 & 3 & 1 & & & & & & & \\
4 & 1 & 4 & 6 & 4 & 1 & & & & & & \\
5 & 1 & 5 & 10 & 10 & 5 & 1 & & & & & \\
6 & 1 & 6 & 15 & 20 & 15 & 6 & 1 & & & & \\
7 & 1 & 7 & 21 & 35 & 35 & 21 & 7 & 1 & & & \\
8 & 1 & 8 & 28 & 56 & 70 & 56 & 28 & 8 & 1 & & \\
9 & 1 & 9 & 36 & 84 & 126 & 126 & 84 & 36 & 9 & 1 & \\
10 & 1 & 10 & 45 & 120 & 210 & 252 & 210 & 120 & 45 & 10 & 1
\end{tabular}

The coefficients in each row in the table may be calculated from those in the preceding row by the following rule:

In any row add to a coefficient the following coefficient and place the sum below the latter.

As an application, consider the

Theorem. The total number of combinations of $n$ things taken one at a time, two at a time, and so on up to $n$ at a time is $2^{n}-1$.

In the binomial expansion for $(x+a)^{n}$ let $x=a=1$.

Hence

Therefore

$$
\begin{gathered}
(1+1)^{n}=1+{ }_{n} C_{1}+{ }_{n} C_{2}+\ldots+{ }_{n} C_{n} \\
{ }_{n} C_{1}+{ }_{n} C_{2}+\ldots+{ }_{n} C_{n}=2^{n}-1 .
\end{gathered}
$$

\section{EXERCISES}

1. What is the middle coefficient in the expansion of $(a+b)^{14}$ Using Pascal's triangle write the coefficients of the expansion $(a+b)^{11}$.

2. Plot the terms of the expansion $\left(\frac{1}{2}+\frac{1}{2}\right)^{12}$ as ordinates at equal distances along the $x$-axis.

3. How many compounds consisting of two elements could be made from eighty-three chemical elements? How many consisting of three elements?

4. From eight men, in how many ways can a selection of four men be made (a) which includes two specified men? (b) which excludes two specified men? 
5. How many symbols would be available for a cipher if each symbol is an arrangement of the letters $a, b$, in a group of five. Thus, $A=a a a a a$, $B=a a a a b$, etc.

6. Twelve competitors run a race for three prizes. In how many ways is it possible that the prizes may be given?

7. In how many ways can a baseball nine be arranged if each of the nine players is capable of playing any position? If $\mathrm{A}$ must pitch and $\mathrm{B}$, $\mathrm{C}, \mathrm{D}$, play in the outfield? If A or B must pitch, $\mathrm{B}$ or $\mathrm{C}$ catch, and $\mathrm{D}$, $\mathrm{E}, \mathrm{F}$, play on the bases?

8. How many dominoes are there in a set from double blank to double six?

9. How many melodies consisting of four notes of equal duration can be formed from the eight tones of the major scale? From the thirteen tones of the chromatic scale?

10. A Yale lock contains 5 cylinders, each capable of being placed in 10 distinct positions, and opens for a particular arrangement of the cylinders. How many locks of this kind can be made so that no two shall have the same key?

11. The combination of a safe consists of figures and letters arranged on three wheels, one bearing the numbers 0 to 9 inclusive, another the letters $A$ to $M$ inclusive, and the third the letters $N$ to $Z$ inclusive. If the safe opens for but one of these arrangements, how many different combinations can be used?

12. How many arrangements of all the letters of the word Columbia (a) begin with a vowel? (b) begin with a consonant and end with a vowel? (c) have the vowels together?

13. Show that the number of ways in which $n$ things can be arranged in a circle is $(n-1)$ ! In how many ways can six persons be arranged in a line? In a circle?

14. Show that if of $n$ things $a$ are alike, $b$ others are alike, $c$ others are alike, etc., the number of distinct permutations, taken all at a time, is

$$
\frac{n !}{a ! b ! c ! \ldots}
$$

15. How many distinct arrangements can be made of all the letters of the word Mississippi? International?

16. How many signals can be made by arranging 2 white flags, 3 red, and 1 blue in a row?

17. Prove that ${ }_{n} C_{r}+{ }_{n} C_{r-1}={ }_{n+1} C_{r}$. Compare this with the rule fo: finding numbers in Pascal's triangle.

18. Prove that ${ }_{2 n} C_{n+r+1}={ }_{2 n} C_{n+r}\left(\frac{n-r}{n+r+1}\right)$, a result which will be used 
19. How many different sums can be formed with a penny, a nickle, a dime, a quarter, a half dollar, and a dollar?

20. A set of weights consists of $1,2,4,8$, and 16 ounce weights. How many different amounts can be weighed?

21. If three coins are tossed in how many ways can they fall? Solve the problem for 4 coins.

22. If two dice are thrown in how many ways can they fall?

23. In how many ways can the hands of whist be dealt?

24. How many four-figure numbers can be formed with the digits $0,1,2,3,4,5,6,7,8$, which are (a) divisible by two? (b) divisible by five?

135. Probability. On one of the faces of a cube is placed the letter $A$, on two of the faces the letter $B$, and on the remaining three faces the letter $C$. If the cube is thrown the total number of ways the cube can fall is six, all of which we will assume are equally likely to occur. The number of ways that the letters, $A, B, C$, can turn up are respectively one, two, and three. In a great number of trials the letter $A$ would turn up approximately in $\frac{1}{6}$ th of the total number of trials, the letter $B$ in $\frac{2}{6}$ ths and the letter $C$ in $\frac{3}{6}$ ths. This does not mean that in every set of six trials $A$ turns up once, $B$ twice, $C$ three times, but that in the long run, as the number of trials is increased, the frequency with which $A, B, C$ turn up approximates to $\frac{1}{6}, \frac{2}{6}, \frac{3}{6}$ of the total number of trials.

Definition. The ratio of the number of ways in which a particular form of an event may occur to the total number of ways in which the event can occur (all assumed equally likely) is said to be the probability of the particular event.

Theorem 1. If the probability that an event will happen is $p$ and the probability that it will not happen is $q$, then $q=1-p$.

Let $T$ denote the number of ways the event can happen, $F$ the number of ways in which the favorable form of the event can happen, and $U$ the number of ways in which the favorable form of the event cannot happen.

Then,

$$
p=\frac{F}{T}, q=\frac{U}{T}
$$

Since

$$
F+U=T
$$


we have, dividing by $T$,

or

$$
\begin{gathered}
\frac{F}{T}+\frac{U}{T}=1, \\
p+q=1 . \\
\therefore q=1-p .
\end{gathered}
$$

Corollary. If the favorable form of an event is certain to happen, then $q=0$ and $p=1$.

Example 1. Find the probability of not throwing a sum of five witn two dice.

If the dice fall 1,4 , or 4,1 , or 2,3 , or 3,2 , the sums will be five. Hence, $F=4, T=6 \cdot 6=36, p=\frac{4}{36}=\frac{1}{9}, q=\frac{8}{9}$.

Hence, the probability of not throwing a sum of five with two dice is $\frac{8}{9}$.

If a sum of money $s$ is paid upon the happening of an event whose probability is $p$, the product $s p$ is called the mathematical expectation.

Example 2. According to the experience of the American life insurance companies, of 100,000 children 10 years of age, 749 die within a year. Neglecting interest on money and the cost of administration, what would be the cost of insuring the life of a 10-year old child for $\$ 1000$ for one year?

The probability of a child dying is $p=\frac{749}{100,000}$.

The mathematical expectation is $\frac{749}{100,000} \cdot 1000=7.49$, and hence the required premium is $\$ 7.49$. That is, if each of the 100,000 children paid $\$ 7.49$, then the sum $\$ 749,000$ resulting would be sufficient to make 749 payments of $\$ 1000$ each.

EXAmple 3. From a bag containing 8 white balls and 6 black balls, 5 balls are drawn at random. What is the probability that 3 are white and 2 are black?

From 14 balls, 5 can be selected in ${ }_{14} C_{5}$ ways.

From 8 white balls, 3 can be selected in ${ }_{8} C_{3}$ ways, and from 6 black balls, 2 can be selected in ${ }_{6} C_{2}$ ways. Hence the probability of drawing 3 white and 2 black balls is

$$
p=\frac{{ }_{8} C_{3 \cdot{ }_{6} C_{2}}}{{ }_{14} C_{5}}=\frac{\frac{8 \cdot 7 \cdot 6}{1 \cdot 2 \cdot 3} \cdot \frac{6 \cdot 5}{1 \cdot 2}}{\frac{14 \cdot 13 \cdot 12 \cdot 11 \cdot 10}{1 \cdot 2 \cdot 3 \cdot 4 \cdot 5}}=\frac{60}{143} .
$$




\section{EXERCISES}

1. From a bag containing 3 white balls and 7 black balls one ball is taken at random. What is the probability that it will be white? Black? What is the sum of these probabilities?

2. From a bag containing 4 white balls and 8 black balls 2 balls are drawn at random. What is the probability that they will both be black? both white? one white and one black? What is the sum of these probabilities?

3. If two dice are thrown what is the chance of throwing a sum of seven? double sixes?

4. If the probability of an event is $\frac{5}{1^{2}}$, what is the probability that the event will not happen?

5. Six persons are about to seat themselves in a row. 'What is the probability that two specified persons will be together? Will not be together?

6. If a prize of $\$ 10$ is given for drawing a red ball from a bag containing 2 red balls and 6 white, what is the value of the expectation?

7. If of 92,637 people living at the age of 20 there are 85,441 living at the age of 30 , what should be the premium for insuring the life of a person of age 20 , for $\$ 1000$ for 10 years, neglecting interest and administrative charges.

136. Compound Events. Sometimes it is convenient to consider an event as made up of two or more simpler events. Thus if two balls are drawn from a bag containing 4 white balls and 5 black balls, the double drawing may be regarded as a compound event made up of two single drawings performed in succession.

The component events into which a compound event is resolved are said to be dependent or independent according as the occurrence of one does or does not affect the occurrence of the others.

If two balls are drawn from the bag mentioned above, the probability of drawing a white ball the first time is $\frac{4}{9}$. If a white ball is drawn the first time and not replaced, the probability of drawing a white ball the second time is $\frac{3}{8}$. But if a black ball is drawn the first time and not replaced, the probability of drawing a white ball the second time is $\frac{4}{8}$. Hence if the ball drawn the first time is not replaced the events are dependent, as the form of occurrence of one does affect the occurrence of the other.

If the first ball is drawn and then replased the probability 
of drawing a white ball the second trial is the same as on the first trial. The events are independent as the occurrence of one does not depend on the occurrence of the others.

Several events are said to be mutually exclusive if only one of the events can happen. Thus in drawing two balls from the bag mentioned above there are three ways in which at least one white ball might be drawn. Both balls might be white, the first white and the second black, the first black and the second white. These events are mutually exclusive for the occurrence of one necessarily excludes the occurrence of the others.

Theorem 1. If $p_{1}, p_{2}, \ldots, p_{n}$ are the probabilities of $n$ mutually exclusive events, the probability that one of these events will occur is equal to the sum of their separate probabilities.

Let $T$ be the total number of ways in which the event can happen, $F_{1}$ the number of ways favorable to the first event, $F_{2}$ to the second, and so on. Then

$$
p_{1}=\frac{F_{1}}{T}, p_{2}=\frac{F_{2}}{T}, . ., p_{n}=\frac{F_{n}}{T} .
$$

Since each one of the mutually exclusive events is favorable, the total number of favorable ways is $F_{1}+F_{2}+\ldots+F_{n}$.

Therefore the probability that one of them will happen is

$$
p=\frac{F_{1}+F_{2}+\ldots F_{n}}{T}=\frac{F_{1}}{T}+\frac{F_{2}}{T}+\ldots \frac{F_{n}}{T} .
$$

Hence

$$
p=p_{1}+p_{2}+\ldots+p_{n} .
$$

Example 1. If a cube has the letter $A$ on one face, the letter $B$ on two faces, and the numeral 5 on three faces, what is the probability that a letter will turn up when the cube is tossed?

There are two mutually exclusive events favorable to this contingency.

(1) The turning up of the letter $A$.

(2) The turning up of the letter $B$.

The probability that $A$ will turn up is $\frac{1}{6}$.

The probability that $B$ will turn up is $\frac{2}{6}$.

The probability that a letter will turn up is therefore

$$
\frac{1}{6}+\frac{2}{6}=\frac{3}{6}=\frac{1}{2} \text {. }
$$

Theorem 2. If $p_{1}$ and $p_{2}$ are the probabilities of two independent events the probability that both events happen is the product of their separate probabilities, $p_{1} p_{2}$. 
Let $T_{1}$ and $F_{1}$ be respectively the total and favorable number of ways the first event can happen.

Let $T_{2}$ and $F_{2}$ be respectively the total and favorable number of ways the second event can happen.

Then the total number of ways the compound event made up of these two events can happen is $T_{1} T_{2}$ and the number of the favorable ways for the compound event is $F_{1} F_{2}$.

Hence the probability of the compound event is

$$
p=\frac{F_{1} \cdot F_{2}}{T_{1} \cdot T_{2}}=\frac{F_{1}}{T_{1}} \cdot \frac{F_{2}}{T_{2}}=p_{1} p_{2} .
$$

In general if $p_{1}, p_{2}, \ldots, p_{n}$ are the respective probabilities of $n$ independent events, the probability that all the events will happen is the product of all the separate probabilities.

EXample 2. Find the probability of throwing a six in the first trial only in two throws of a single die.

The probability of throwing a six the first time is $\frac{1}{6}$.

The probability of not throwing a six the second time is $\frac{5}{6}$.

These events are independent, and the probability of throwing a six the first trial only is $\frac{1}{6} \cdot \frac{5}{6}=\frac{5}{36}$.

Example 3. If the probability that $A$ will solve a problem is $\frac{3}{5}$ and the probability that $B$ will solve it is $\frac{4}{7}$, find the probability that the problem will be solved.

The problem will be solved if A and B both succeed, if A succeeds and $B$ fails, or if A fails and B succeeds.

The probability that they will both succeed is $\frac{3}{5} \cdot \frac{4}{7}=\frac{1}{3} \frac{2}{5}$, since A's success or failure is independent of B's success or failure. Likewise, the probability that A succeeds and B fails is $\frac{3}{5} \cdot \frac{3}{7}=\frac{9}{35}$, and the probability that A fails and B succeeds is $\frac{2}{5} \cdot \frac{4}{7}=\frac{8}{35}$.

Since the three possible ways in which the solution of the problem may occur are mutually exclusive the probability that the problem will be solved is

$$
\frac{12}{35}+\frac{9}{35}+\frac{8}{35}=\frac{29}{35} \text {. }
$$

A second method of solving the problem is as follows:

The probability that the problem will not be solved is $\frac{2}{5} \cdot \frac{3}{7}=\frac{6}{35}$.

Hence, by Theorem 1, Section 135, the probability that the problem will be solved is $1-\frac{6}{35}=\frac{2}{3} 9$.

Theorem 3. If there are any number of dependent events and if $p_{1}$ is the probability of the first, $p_{2}$ the probability that when the first has happened the second will follow, $p_{3}$ the probability that 
when the first and second have happened the third will follow, and so on, then the probability that the events will occur in succession in this way is $p_{1}, p_{2} \ldots p_{n}$.

The proof is similar to that of Theorem 2 .

Example 4. If a bag contains 4 white balls and 5 black balls, and if 2 balls are drawn, what is the probability of drawing a white ball and a black ball?

Consider the compound event as resolved into two successive drawings. The probability of drawing a white ball first is $\frac{4}{9}$.

The probability of drawing a black ball second is $\frac{5}{8}$.

The probability of drawing the balls in this order is therefore $\frac{4}{9} \cdot \frac{5}{8}=\frac{5}{18}$. But since the balls may be drawn in 2 ways, the probability of drawing a white ball and a black ball is $\frac{5}{18} \cdot 2=\frac{5}{9}$.

Theorem 4. If the probability of the happening of an event in one trial is $p$ and the probability of its failing is $q$, the probability of its happening exactly $r$ times in $n$ trials is ${ }_{n} C_{r} p^{r} q^{n-r}$.

The probability that the event will happen $r$ times and fail $n-r$ times in a given order (for example, happen the first $r$ times and fail the last $n-r$ times) is $p^{r} q^{n-r}$ (Theorem 2).

The number of ways this can happen is the number of ways that the $r$ trials can be selected from the $n$ trials, which is ${ }_{n} C_{r}$. Hence the event can happen exactly $r$ times in ${ }_{n} C_{r}$ mutually exclusive ways, and the required probability is therefore ${ }_{n} C_{r} p^{r} q^{n-r}$ (Theorem 1).

Example 5. What is the probability of throwing six twice in five throws with a single die?

Here $p=\frac{1}{6}, q=\frac{5}{6}, n=5, r=2, \quad$ and hence

$$
P={ }_{5} C_{2}\left(\frac{1}{6}\right)^{2}\left(\frac{5}{6}\right)^{3}=\frac{5 \cdot 4}{1 \cdot 2} \cdot \frac{1}{36} \cdot \frac{125}{216}=\frac{625}{3888} \text {. }
$$

Theorem 5. If the probability that an event will happen in one trial is $p$, then the probability that it will happen at least $r$ times in $n$ trials is

$$
P=p^{n}+n p^{n-1} q^{1}+\ldots .+{ }_{n} C_{r} p^{r} q^{n-2} .
$$

An event will happen at least $r$ times in $n$ trials, if it happens $n$ times, or $n-1$ times, and so on down to $r$ times. These are mutually exclusive events. 
Hence, applying Theorems 3 and 1, the probability that an event will happen at least $r$ times in $n$ trials is

$$
P=p^{n}+{ }_{n} C_{n-1} p^{n-1} q+{ }_{n} C_{n-2} p^{n-2} q^{2}+\ldots+{ }_{n} C_{r} p^{r} q^{n-r},
$$

which reduces to what is required.

\section{EXERCISES}

1. What is the chance of making a throw with two dice that will be greater than 9 ?

2. A bag contains 3 dimes and 4 quarters. Three coins are drawn. Find the value of the expectation. Suggestion: Find the value of the expectation of drawing 3 quarters, 2 quarters and 1 dime, 1 quarter and 2 dimes, 3 dimes, and add.

3. Johnny may or may not receive a birthday gift of $\$ 1.00$ from each of five relatives. What is the value of the expectation?

4. In a bag are five white and five black balls. If two balls are drawn what is the chance that they will both be white? Both black? one white and one black?

5. In a bag are five white and four black balls. If three balls are drawn in succession what is the chance that they will be white, black, white, in this order, if (a) the balls are not replaced each time, (b) the balls are replaced each time.

6. A man is sent 8 keys on a ring for eight locks. What is the probability that he will be able to unlock the first lock with the first key he tries? With one of the first two keys? With one of the first three?

7. Five cards are drawn from a pack of 52 cards. Find the probability that (a) there is a pair, (b) three of a kind, (c) two pairs, (d) three of a kind and a pair, (e) four of a kind, (f) a flush, (g) a straight, (h) a straight flush.

8. Three dice are thrown. What is the most probable throw? What is the probability of throwing exactly 15 ? At least 15 ?

9. If 5 letters are chosen from a group of 4 vowels and 6 consonants, what is the probability that a set will begin with a consonant and end with a vowel?

10. If there were eight independent chances in youth of growing one inch above 5 feet, find the probabilities of the statures from 5 feet to 5 feet 8 inches.

137. Mortality Tables. An important application of the theory of probability is the application to problems concerned with the duration of human life, such as life insurance, pensions, life annuities, and inheritance tax laws. Such problems are based on tables called mortality tables which show the 
number of deaths that may be expected to take place during a given period, among a given number of persons of a given age. These tables differ for different countries, different races, in the same country, different periods of time, and for the two sexes.

Some tables are constructed from the experience of insurance companies, others from census and vital statistics reports. The American Experience Table is based on the records of the Mutual Life Insurance Company of New York.

If a mortality table is based on a sufficiently large number of observations, the difference between the result furnished by the tables and actual mortality is negligible. But this must be understood to apply to large groups of people and furnishes no surety to an individual.

The following table is a selection from the American Experience Table of mortality, which gives the number of people $l_{x}$ living at age $x$ out of 100,000 living at the age 10 .

\begin{tabular}{c|c}
$\begin{array}{c}\text { Age } \\
x\end{array}$ & $\begin{array}{c}\text { Number living } \\
l_{x}\end{array}$ \\
\hline 10 & 100,000 \\
15 & 96,022 \\
20 & 92,637 \\
25 & 89,032 \\
30 & 85,441 \\
35 & 81,822 \\
40 & 78,106 \\
45 & 74,173 \\
50 & 69,804 \\
55 & 64,563 \\
60 & 57,917 \\
65 & 49,341 \\
70 & 38,569 \\
75 & 26,237 \\
80 & 14,474 \\
85 & 5,485 \\
90 & 847 \\
95 & 3
\end{tabular}

The probability that a person of age $x$ will be alive at the end of $n$ years is denoted by

$$
{ }_{n} p_{x}=\frac{l_{x+n}}{l_{x}}
$$

The probability that a person of age $x$ will not be alive at the end of $n$ years is

$$
{ }_{n} q_{x}=1-{ }_{n} p_{x}=1-\frac{l_{x+n}}{l_{x}}=\frac{l_{x}-l_{x+n}}{l_{x}} .
$$

Example. An inheritance of $\$ 20,000$ is to be paid a boy 10 years of age when he becomes 20 . What is the present value of the inheritance, money being worth 5\% compounded semi-annually?

If the sum were certain to be paid to the boy in ten years the present value would be

$$
P=20,000\left(1+\frac{.05}{2}\right)^{-20}=\$ 12,220 .
$$

Since the payment is contingent upon the probability that the boy will live to receive the 
sum, the present value will be the mathematical expectation of receiving $\$ 12,220$ contingent upon the probability that he will live 10 years.

The probability that a person 10 years old will live at least 10 years is

$$
{ }_{10} p_{10}=\frac{l_{20}}{l_{10}}=\frac{92,637}{100,000}=.92637 .
$$

Hence the present value of the inheritance is $(12,220)(.92637)=\$ 11,320$.

\section{EXERCISES}

1. Plot the graph of the mortality table given in Section 137. By means of the graph estimate your own chance of living to the age of 75 .

2. A man is 45 years of age and his son is 15 . What is the probability that both will be alive 10 years hence? What is the probability that at least one will be alive?

3. A man and his wife are 40 and 35 years old respectively, when their child is 10 years old. What is the probability that all will be alive until the 20th anniversary of the child's birth? What is the probability that at least one will survive?

4. What should be the minimum cost of insuring the life of a person 20 years old for $\$ 1000$ for five years? For insuring a couple against the death of either or both for the same sum and period if the husband is 30 and the wife 25? (Neglect interest, etc.)

5. A man makes a will leaving $\$ 40,000$ to his wife in case she survives him. A son is to inherit the money if he survives both parents. If the ages of husband, wife, and son are 60, 50, 25 respectively, and if money is worth $5 \%$ compounded semi-annually, what is the present value of the expectation (a) that the wife will inherit the money in 10 years? (b) that the son will inherit the money in 15 years?

6. In each of the following exercises plot a graph with the probabilities as ordinates at arbitrary equal intervals along the $x$-axis.

(a) A coin is tossed six times. Find the probabilities of the various ways in which it can turn up heads.

(b) Find the probabilities for the various ways in which two dice can fall in one throw.

(c) In the long run $A$ wins 3 games out of 4 from B at chess. Find the probabilities of the various numbers of games which A might win in 8 successive games.

(d) If a die is tossed six times, find the probabilities of the various ways in which an ace can turn up.

(e) If the quantity of a trait in an individual of a group is the result of a chance combination of seven causes, determine the probabilities of the ways in which the trait may occur. 
138. Frequency Distributions. At an agricultural experimental station 110 apples were classified with respect to the number of seeds each contained, and the number of apples in each class was determined. The results are given in the table.

\begin{tabular}{c|c}
$\begin{array}{c}\text { Number of } \\
\text { seeds }\end{array}$ & $\begin{array}{c}\text { Number of } \\
\text { apples }\end{array}$ \\
\cline { 2 - 2 } 4 & 9 \\
5 & 4 \\
6 & 14 \\
7 & 21 \\
8 & 24 \\
9 & 25 \\
10 & 13
\end{tabular}

Thus 9 apples had 4 seeds each (the minimum number found in this investigation), 13 apples had 10 seeds each (the maximum number), while 25 apples had 9 seeds each (the most frequent number occurring).

Such an arrangement of the individuals of a group, classified with respect to some characteristic which gives the number of individuals in each of the classes is called a frequency distribution and the table in which the classes and frequencies are given a frequency table.

A graphical representation of this analysis, called a frequency polygon, is obtained by plotting the magnitudes of the classes as abscissas, the frequencies as ordinates, and connecting the points by straight lines as in the figure.

The magnitude of each class in this case is an integer and the class intervals are said to vary discretely. In case the

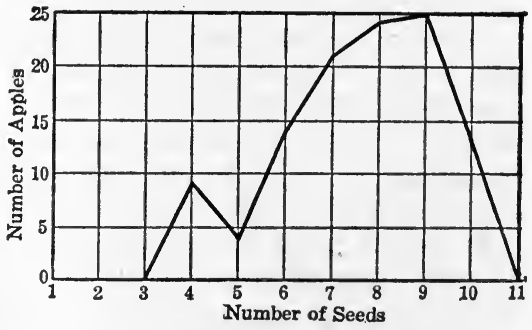

Fig. 209. characteristic measured varies continuously, as for instance the stature of a group of men, the size of the class intervals and their mid-points are chosen arbitrarily.

The magnitude measured may vary discretely but by such small amounts that the number of classes is so great that the variation of the group with respect to the characteristic cannot be easily determined. In such a case the class interval is enlarged by grouping the frequencies in two or more adjacent 
classes and associating the resulting frequency with the midvalue of the resulting larger class.

Example. The grades in geometry of 30 students were $30,42,48,55$, $60,64,68,71,72,74,75,76,77,77,78,78,78,79,80,82,82,83,84,85$, $86,87,88,88,91,95$. Collect the data in frequency tables with class intervals of $5 \%$ and $10 \%$.

TABLE 1

Grades Frequency

28-32 1

33-37 0

38-42 1

43-47 0

48-52 1

$53-57 \quad 1$

58-62 1

63-67 1

$68-72 \quad 3$

$73-77 \quad 5$

78-82 7

$83-87 \quad 5$

88-92 3

93-100 1
TABLe 2

Grades Frequency

30-34 1

35-39

40-44

45-49

50-54

$55-59$

60-64

65-69

$70-74$

75-79

80-84

85-89

90-94

95-100

\section{TABLE 3}

Grades Frequency 30-39 1

$40-49 \quad 2$

50-59 1

60-69 3

$70-79 \quad 11$

80-89 $\quad 10$

90-100 2

Tables 1 and 2 show the data collected in class intervalsı of $5 \%$ with different mid-points. In table 3 the class interval is $10 \%$.

The class interval of $1 \%$ is too small for an adequate presentation of the data. Even class intervals of $5 \%$ leave some classes empty.

The class interval should be chosen so as to avoid empty classes. The smaller the number of measurements the larger the class interval should be, and vice versa. The starting points of the intervals are not so material, but it is convenient to take them so that the mid-points of the intervals are integers. In age distributions the returns usually cluster about the multiples of 5, which are taken as the mid-points of the intervals.

A second method of representing frequency tables graphically is indicated in the Figs. 210-212 representing the tables above. Rectangles are constructed on the class intervals as bases with altitudes equal to the frequencies. Such diagrams are called histograms. The area of a histogram is the sum of the frequencies times the class interval. 
If the number of observations be increased and the class interval decreased the frequency polygon or histogram will
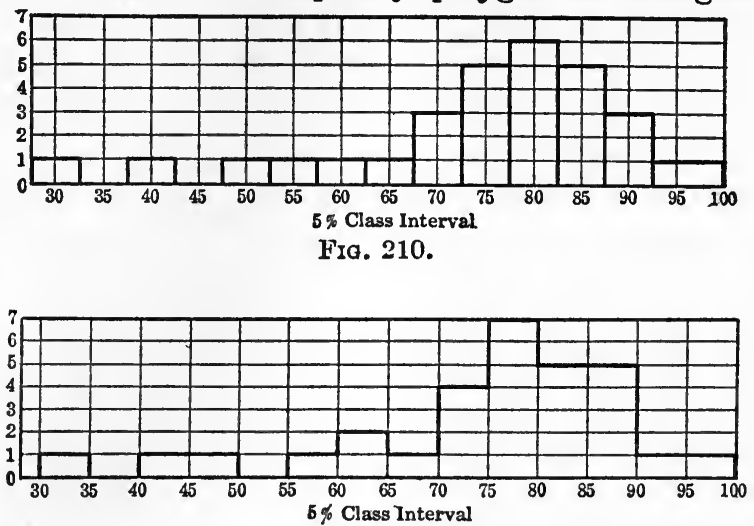

Fig. 211.

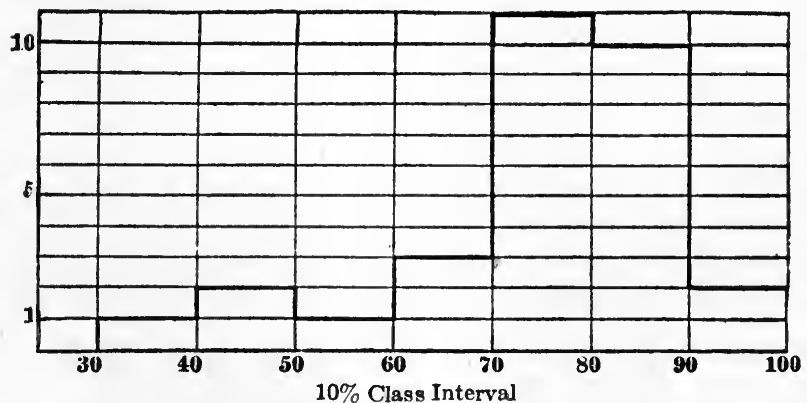

FIg. 212.

approach more and more closely to a smooth curve. Such a curve is called a frequency curve.

A frequency histogram is sometimes smoothed by drawing a curve through the mid-point of the upper base of each rectangle in such a way that the area under the curve is the same as the area of the histogram.

A frequency table is smoothed by replacing the middle frequency of three adjacent frequencies by the average of the three frequencies. The two end values are counted twice and averaged with the adjoining value. 
Thus if $A, B, C, D, E, \ldots$ are the values of the frequencies the smoothed values are

$$
\frac{2 A+B}{3}, \frac{A+B+C}{3}, \frac{B+C+D}{3}, \text { ete. }
$$

If the polygons or histograms corresponding to successive smoothings of the table are plotted we can approximate closely to the frequency curve which best represents the data.

The types of frequency distributions which are most common are:

(a) The symmetrical distribution in which the frequencies decrease to zero symmetrically on either side of a central magnitude.

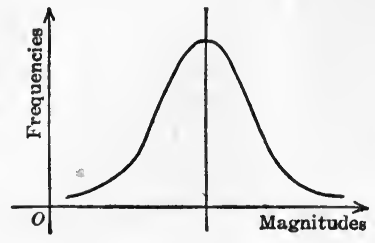

Fig. 213.

It is found in the distribution of errors in chemical and physical measurements and in biological measurements, par-

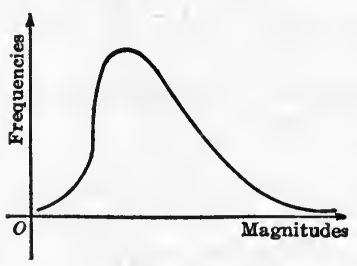

FIG. 214. ticularly the measurements of anthropology.

(b) The moderately asymmetrical distribution in which the frequencies decrease more rapidly on one side of the value of maximum frequency than on the other.

It is the most common of all the distributions occurring in all forms of statistics.

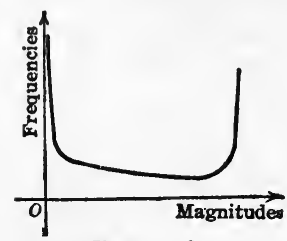

FIG. 216.

(c) The J-shaped distribution in which the frequency con-

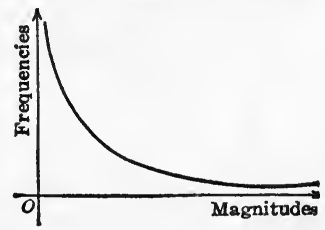

FIG. 215. stantly increases or decreases. It is found in economic statistics and is characteristic of the distribution of wealth.

(d) The U-shaped distribution in which the frequency decreases to a minimum value and then increases. It is rare. It occurs in meteorological statistics and in statistics pertaining to heredity. 


\section{EXERCISES}

1. The following grades were obtained in a spelling test given to thirdgrade pupils; $65,85,55,60,100,23,92,74,73,75,76,94,82,17,10$, $63,97,77,96,75,90,85,90,75,86,82,94,100,90,100,74,100,100$.

Construct the frequency polygon, giving frequencies for class intervals of $5 \%$ arranged along the $x$-axis with multiples of 5 (a) at the first points of the intervals, (b) at the middle points of the intervals. Construct the smoothed curves. Were the words used a good test of the ability of the pupils in spelling?

2. Construct the histogram for the data in Exercise 1, with class intervals of $10 \%$, (a) with mid-points at 55, 65, etc.; (b) with mid-points at 60,70 , etc. Calculate the smoothed values in the first table, and draw a curve through the ordinates resulting.

3. The number of seeds per apple in normal and aphis-injured Rome apples, as determined by an investigation, are given in the table.

Number of seeds per apple.. $0,1,2,3,4,5,6,7,8,9,10,11,12$ Frequencies of normal apples $0,0,0,4,31,30,38,63,64,37,31,1,1$ Frequencies of aphis-injured

apples............ 5, 14, 20, 29, 44, 37, 28, 44, 38, 25, 13, 3, 0

Plot the histograms based on these samples. What is the most probable number of seeds in the normal and in the aphis-injured apple? State two differences between the two variations.

4. The following table gives the degrees of cloudiness of the sky observed at Breslau during the years 1876-1885, on a scale of 10 .

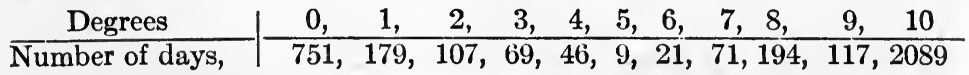

Plot the histogram and the smoothed curve. What is the type of the distribution? Would the arithmetic average of the measurements represent the variation well? What is the probability that a day at Breslau will be clear? Very cloudy?

5. Individuals A and B were tested by hearing a series of 10 letters read at the rate of 1 per second and being required to write as many as they could remember in the proper order of the letters, as soon as the reading was finished. Their scores were:

\begin{tabular}{l|rrrrrrr} 
Letters correct & 4, & 5, & 6, & 7, & 8, & 9, & 10, \\
\hline A frequencies & 1, & 4, & 5, & 8, & 11, & 4, & 3, \\
\hline $\bar{B}$ frequencies & 0, & 2, & 4, & 10, & 16, & 3, & 1,
\end{tabular}

Draw the histograms and the smoothed curves. Which is the better performance? What would be the probability that $A$ and $B$ could each get 8 correct in a particular trial?

6. The following table gives the age distributions for deaths from typhoid, measles, scarlet fever, influenza, tuberculosis, circulatory and respiratory diseases in New York state in 1916. 


\begin{tabular}{|c|c|c|c|c|c|c|c|}
\hline Age & Typhoid & Measles & $\left\{\begin{array}{l}\text { Scarlet } \\
\text { fever }\end{array}\right.$ & Influenza & $\begin{array}{c}\text { Tubercu- } \\
\text { losis }\end{array}$ & $\begin{array}{c}\text { Circula- } \\
\text { tory } \\
\text { diseases }\end{array}$ & $\begin{array}{c}\text { Respira- } \\
\text { tory } \\
\text { diseases }\end{array}$ \\
\hline $0-1$ & 2 & 293 & 9 & 143 & 371 & 165 & 4161 \\
\hline $1-2$ & 3 & 404 & 26 & 58 & 301 & 37. & 1726 \\
\hline $2-3$ & 3 & 136 & 31 & 39 & 194 & 35 & 638 \\
\hline $3-4$ & 4 & 64 & 31 & 11 & 129 & 32 & 274 \\
\hline $4-5$ & 5 & 25 & 30 & 9 & 78 & 39 & 149 \\
\hline $5-9$ & 22 & 54 & 52 & 33 & 285 & 285 & 327 \\
\hline $10-19$ & 112 & 17 & 14 & 43 & 1267 & 603 & 299 \\
\hline $20-29$ & 170 & 8 & 9 & 62 & 3634 & 788 & 837 \\
\hline $30-39$ & 127 & 4 & 5 & 83 & 3754 & 1252 & 1379 \\
\hline $40-49$ & 76 & 12 & 1 & 123 & 2936 & 2222 & 1761 \\
\hline 50-59 & 56 & 3 & $\ldots$ & 193 & 1748 & 3748 & 2084 \\
\hline $60-69$ & 23 & 0 & $\ldots$ & 291 & 889 & 5564 & 2257 \\
\hline $70-79$ & 8 & 0 & $\ldots$ & 448 & 302 & 6464 & 2137 \\
\hline $80-89$ & 0 & 0 & $\ldots$ & 365 & 45 & 3536 & 1203 \\
\hline $90-99$ & 0 & 1 & $\ldots$ & 73 & 2 & 497 & 212 \\
\hline 100 & 0 & 0 & $\ldots$ & 3 & 0 & 11 & 10 \\
\hline Unknown & & & & & & & \\
\hline Age & 0 & 0 & $\ldots$ & $\ldots$ & 2 & 4 & 3 \\
\hline Total & 611 & 1,021 & 208 & 1,977 & 15,937 & 25,282 & 19,457 \\
\hline
\end{tabular}

Plot the curves given by these tables, identify the types of curves, and state characteristics of the diseases.

7. The errors of observation in 471 astronomical measurements made by Bradley were distributed as in the table, in which the mid-values of the magnitudes of the errors are given in decimals of a second of arc.

Mid-values of errors $\mid 0.5,1.5,2.5,3.5,4.5,5.5,6.5,7.5,8.5,9.5,10.5$ Frequencies

Assuming that positive and negative errors occur with equal frequency, plot the histogram and the frequency curve. Discuss their forms.

8. Discuss the form of the frequency curve obtained by plotting the probabilities given by the terms of the expansion $(p+q)^{n}$, for $p=\frac{1}{6}$, $q=\frac{5}{6}, n=6$, at equal intervals along the $x$-axis and drawing a smooth curve. What is the most probable number of times the event will occur in 6 trials? If the distances along the $x$-axis are multiples of unity, what will the area under the curve from the maximum ordinate to the right hand extent of the curve represent? 
9. Plot the graph of the frequency curve $y=e^{-x^{2}}$. Determine approximately by counting squares the abcissa of the point whose ordinate bisects the area of the right branch of the curve.

10. Plot the graph of the frequency curve $y=10\left(1+\frac{x}{3}\right)^{6}\left(1-\frac{x}{4}\right)^{8}$ from $x=-3$ to $x=4$. What is the type?

11. Plot the graph of the frequency curve $y=10 x^{0.5} e^{-0.2 x}$ from $x=0$ to $x=10$, and discuss the type.

139. Averages. The frequency gives the variation of the magnitude measured but it is convenient in most cases to have. a single value to represent the table. The representative values or averages which are commonly used for various kinds of frequency distribution are
(a) the arithmetic mean,
(b) the median,
(c) the mode,
(d) the geometric mean,
(e) the harmonic mean.

The arithmetic mean. The word mean or average alone is generally understood to denote the arithmetic mean. It is defined by the equation

$$
A=\frac{\text { sum of the measurements }}{\text { number of }} \text { measurements }
$$

If there are $n$ measurements $m_{1}, m_{2}, \ldots m_{n}$, then

$$
\boldsymbol{A}=\frac{m_{1}+m_{2}+\ldots+m_{n}}{n}=\frac{\Sigma m}{n},
$$

where the symbol $\Sigma m$ is used to denote the sum of the $m$ 's.

If a measurement $m_{1}$ is obtained $f_{1}$ times, then the sum of these $f_{1}$ measurements is $f_{1} m_{1}$. If the measurements $m_{1}$, $m_{2}, \ldots, m_{n}$ occur with the frequencies $f_{1}, f_{2}, \ldots, f_{n}$, respectively, then the sum of all the measurements is

$$
f_{1} m_{1}+f_{2} m_{2}+\ldots+f_{n} m_{n}=\Sigma f m,
$$

and the total number of measurements is

$$
f_{1}+f_{2}+\ldots+f_{n}=\Sigma f \text {. }
$$


Hence the arithmetic mean of the measurements is

$$
A=\frac{\Sigma f m}{\Sigma f}
$$

This equation defines the weighted arithmetic mean of the numbers $m_{1}, m_{2}, \ldots, m_{n}$ with the weights $f_{1}, f_{2}, \ldots, f_{n}$. It is used whenever the numbers to be averaged are not of equal importance.

Example. If the averages of the weekly wages of three factories are $14,17,18$ dollars and the number of employees are 450, 360, 670, respectively, then the average weekly wage for all three factories is

$$
A=\frac{450 \times 14+360 \times 17+670 \times 18}{450+360+670}=16.6 .
$$

The calculation of the arithmetic mean of a frequency distribution is simplified by considering the measurements of a class as concentrated at the center of the class interval, assuming the class interval as a unit and applying an extension of the rule on page 83 , given by the

Theorem. If $m_{1}, m_{2}, \ldots, m_{n}$ are measurements with frequencies $f_{1}, f_{2}, \ldots, f_{n}$, if $A$ is their true mean, $E$ an estimated value of the mean, and $d_{1}, d_{2}, \ldots, d_{n}$ deviations of the measurements from $E$, then

$$
A=E+\frac{\Sigma f d}{\Sigma f}
$$

We have $m_{1}=E+d_{1}$, so that $f_{1} m_{1}=f_{1} E+f_{1} d_{1}$, and similarly $f_{2} m_{2}=f_{2} E+f_{2} d_{2}$, etc.

Adding

$$
\begin{aligned}
f_{1} m_{1}+f_{2} m_{2}+\ldots+f_{n} m_{n}=E( & \left.f_{1}+f_{2}+\ldots+f_{n}\right) \\
& +\left(f_{1} d_{1}+f_{2} d_{2}+\ldots+f_{n} d_{n}\right) .
\end{aligned}
$$

Using the symbol $\Sigma$ to represent the summations,

$$
\Sigma f m=E \Sigma f+\Sigma f d .
$$

Dividing by $\Sigma f, \quad A=\frac{\Sigma f m}{\Sigma f}=E+\frac{\Sigma f d}{\Sigma f}$. 
Corollary. The sum of the deviation from the arithmetic mean is zero.

For if $E=A$, then the correction $c=\frac{\Sigma f d}{\Sigma d}$ is zero. Hence $\Sigma f d$, the sum of the deviation from $A=E$, is zero.

The computation of the correction $c=\frac{\Sigma f d}{\Sigma f}$ is illustrated in

Example 1. Find the arithmetic mean of the incomes of the group of families in the first two columns of the table.

\begin{tabular}{|c|c|c|c|}
\hline $\begin{array}{c}\text { Incomes } \\
\text { (class intervals) }\end{array}$ & $\begin{array}{c}\text { Number of } \\
\text { families, } \\
f\end{array}$ & $\begin{array}{l}\text { Deviation from } \\
\text { mean estimated, } \\
\qquad d\end{array}$ & ${ }^{f} d$ \\
\hline $\begin{array}{c}\$ 400-\$ 499 \\
500-599 \\
600-699 \\
700-799 \\
800-899\end{array}$ & $\begin{array}{r}8 \\
17 \\
72 \\
79 \\
73\end{array}$ & $\begin{array}{r}-4 \\
-3 \\
-2 \\
-1 \\
0\end{array}$ & $\begin{array}{r}-32 \\
-\quad 51 \\
-144 \\
-\quad 79 \\
0 \\
\end{array}$ \\
\hline $\begin{array}{c}900-999 \\
1000-1099 \\
1100-1199 \\
1200-1299 \\
1300-1399 \\
1400-1499 \\
1500-1599\end{array}$ & $\begin{array}{r}63 \\
31 \\
18 \\
8 \\
8 \\
1 \\
6\end{array}$ & $\begin{array}{l}+1 \\
+2 \\
+3 \\
+4 \\
+5 \\
+6 \\
+7\end{array}$ & $\begin{array}{r}63 \\
62 \\
54 \\
32 \\
40 \\
6 \\
42 \\
\quad+299\end{array}$ \\
\hline
\end{tabular}

Estimated average, $E=850 . \quad c=\frac{-7}{384}=-0.0182$ class intervals.

$$
\begin{aligned}
\therefore A & =850-\frac{7}{384} \cdot 100 \\
& =850-1.8=\$ 848.20
\end{aligned}
$$

In the computation it is assumed that the income of the families in each class is the income at the middle of the class 
interval. With this assumption an income of $\$ 450$ is received by 8 families, of $\$ 550$ by 17 families, etc.

Estimating the mean as $\$ 850$, the deviations of the incomes of the successive classes from $\$ 850$ are $-400,-300$, etc. To simplify the computation we take the class interval $\$ 100$ as a unit, so that the deviations $d$ become $-4,-3$, etc.

To find the correction, $c$, which must be applied to the estimated mean, multiply the pairs of values of $f$ and $d$, obtaining the column headed $f d$, find the sum of the numbers in the columns headed $f$ and $f d$, and divide the latter by the former. As $\$ 100$ was taken as a unit, this quotient must be multiplied by 100 . The correction to be applied to the estimated mean of $\$ 850$ is thus found to be $-\$ 1.8$.

The median. If all the measurements of a series are arranged in order of magnitude, the magnitude of the measurement halfway up the series is called the median. The magnitudes one-quarter and three-quarters up the series are called the first and third quartiles.

The number of the measurement whose magnitude is the median is $\frac{n+1}{2}$, where $n$ is the number of measurements. The numbers of the measurements whose magnitudes are the quartiles are $\frac{1}{4}(n+1)$ and $\frac{3}{4}(n+1)$.

Graphically, the first quartile is the abscissa of the point whose ordinate cuts off one-fourth of the area of the frequency curve of a distribution to the left of it. The median has onehalf the area on either side, the third quartile has one-fourth the area to the right of it.

In a frequency table where the items are given by classes it is assumed for the computation of the median that the measurements are distributed uniformly through the class interval, so that after the class in which the median lies has been determined, its position in that class interval is found by ordinary interpolation.

Example 2. Find the median and quartiles of the incomes of the group of families given in Example 1. 
The table is conveniently arranged in the form given.

\begin{tabular}{r|c}
$\begin{array}{r}\text { Incomes } \\
\text { less than }\end{array}$ & $\begin{array}{c}\text { Number of } \\
\text { families } \\
f\end{array}$ \\
\cline { 2 - 2 }$\$ 500$ & 8 \\
600 & 25 \\
700 & 97 \\
800 & 176 \\
900 & 249 \\
1000 & 312 \\
1100 & 343 \\
1200 & 361 \\
1300 & 369 \\
1400 & 377 \\
1500 & 378 \\
1600 & 384
\end{tabular}

Dividing by two the number of terms plus one, we have $\frac{385}{2}=192.5$, so that the median is the average of the 192nd and 193rd terms. As appears in the table, there are 176 measurements in the first four classes and 249 in the first five classes. Hence the median lies in the 5 th or $800-899$ class, $192.5-176=16.5$ measurements from the lower end.

Hence, the median is

$$
M_{6}=800+\frac{16.5}{73} \cdot 100=822.6 .
$$

This result means that half of the 384 families have incomes less than $\$ 822.6$ and half have larger incomes.

The quartiles are round as follows:

Since $\frac{1}{4}(385)=96.25$, the first quartile, $Q_{1}$, lies between the 96 th and 97 th terms, in the third or $600-699$ class, $96 \frac{1}{4}-25=71 \frac{1}{4}$ measurements from the lower end.

$$
\therefore Q_{1}=600+\frac{71.25}{72} \cdot 100=698.9 \text {. }
$$

And since $\frac{3}{4}(385)=288.75, Q_{3}$ is in the $900-999$ class, $288 \frac{3}{4}-249=39 \frac{3}{4}$ measurements from the lower end.

$$
\therefore Q_{3}=900+\frac{39.75}{63} \cdot 100=963.1 \text {. }
$$

The mode. The mode is the magnitude of the most frequent item of a distribution. It is the abscissa of the maximum ordinate in a smoothed distribution. The average income, the average man of the newspapers usually means the modal income, the modal man.

In Example 1, assuming that the incomes are all at the midpoints of the class intervals, the modal income would be $\$ 750$. Without this assumption, the modal income would be somewhere between $\$ 700$ and $\$ 799$.

The mode or modes of a frequency distribution may not be well defined for the class intervals given in the table. In such cases the mode is roughly located by grouping in larger class intervals as shown in 
Example 3. The distribution of grades in a class in mathematics are given in the following table. Locate the mode roughly.

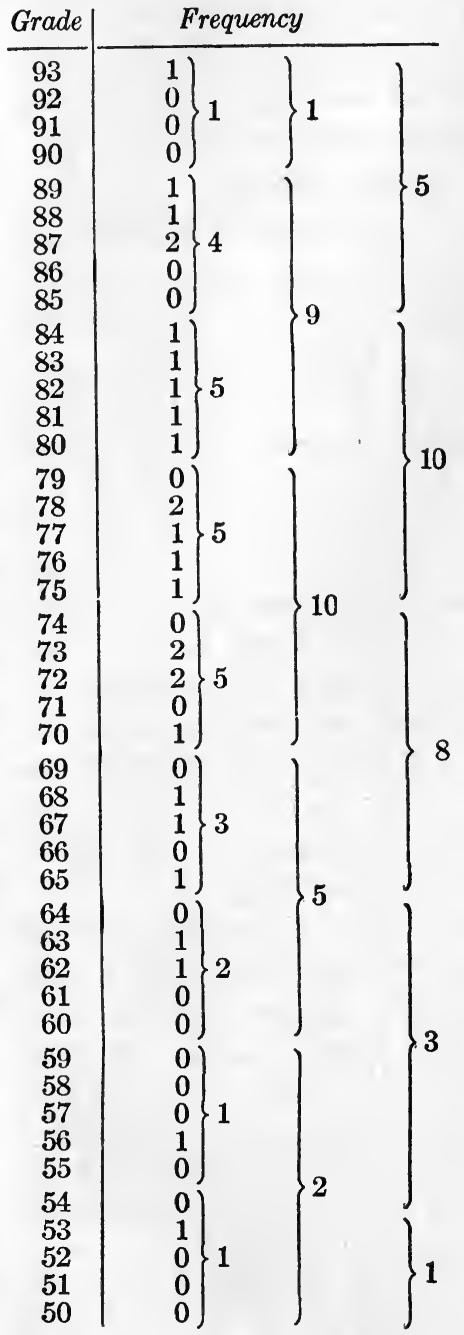

The table gives the frequencies for class intervals of $1 \%$. There is no well-defined maximum frequency, and hence no value of the mode is apparent.

The numbers by the left-hand column of brackets give the frequencies for the class intervals $95-90,89-85$, etc., but the class containing the mode is not yet apparent so that a class interval larger than $5 \%$ must be chosen.

The numbers by the middle column of brackets give the frequencies for class intervals from 99-90, 89-80, etc. The maximum frequency, 10 , shows that the mode lies in the third class, between 79 and 70 .

The numbers by the right-hand brackets give another grouping of the frequencies by $10 \%$ class intervals, from 94-85, 84-75, etc. Here the mode appears in the class interval from 84-75.

As the mode lies between $70 \%$ and $79 \%$ and also between $75 \%$ and $84 \%$, it must therefore lie between $75 \%$ and $79 \%$.

In a distribution of the symmetrical type the arithmetic mean, the median and the mode fall together. In a moder- 
ately asymmetric group the mode, median and mean lie in the order named with the mean toward the longer branch of

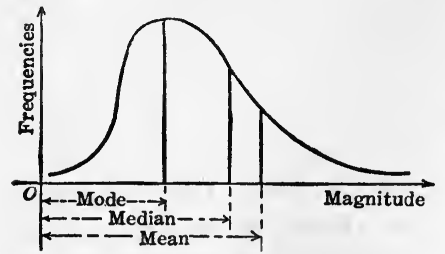

Fig. 217. the curve, as in the figure.

A good approximation to the value of the mode is obtained by the use of the formula

Mode $=$ Mean

$$
\text { - } 3 \text { (Mean - Median). }
$$

This formula is based on the assumption, which has been observed to hold approximately in a large number of cases, that the median lies one third of the distance from the mean toward the mode. The application of this formula shows that the modal income in Examples 1 and 2 is

$$
848.2-3(848.2-822.6)=771.4 \text {. }
$$

The best method of determining the mode is to find the equation of the smooth frequency curve that best fits the data,

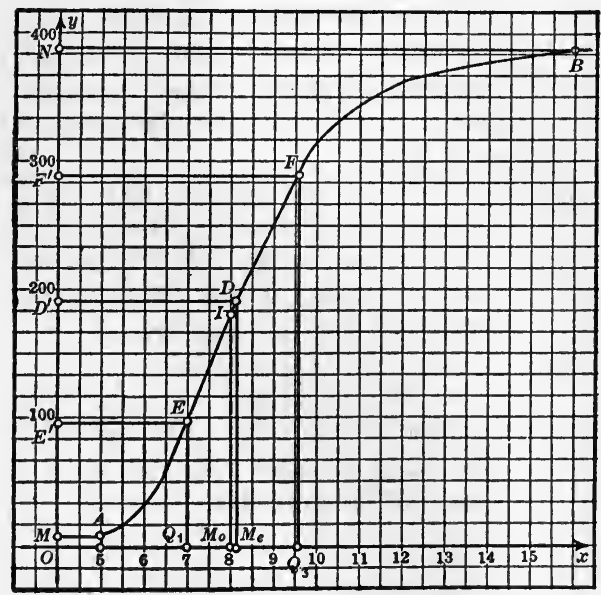

FIG. 218. and then find the abscissa of the maximum point by equating the derivative to zero. The method is tedious and difficult.

A graphic method of determining the median and the mode is given in

EXAMPLE 4. Find graphically the median, the quartiles, and the mode of the distribution in Example 1.

Plot the data in the table in Example 2, using 500,600 , etc., as abscissas, the ordinates being respectively the number of families with incomes less than 500,600 , etc. For convenience in plotting $\$ 100$ is taken as a unit so that the abscissas are 5,6 , etc. 
Let $M$ and $N$ be the projections on the $y$-axis of $A$ and $B$, the end points of the curve. Divide $M N$ into four equal parts by the points $D^{\prime}$, $E^{\prime}, F^{\prime}$. Let the perpendiculars to the $y$-axis at these points cut the curve at $D, E, F$, respectively. Then the abscissa of $D$ is the median, $M_{\mathrm{o}}=820$, approximately, and the abscissas of $E$ and $F$ are the quartiles, $Q_{1}=700$ and $Q_{3}=960$.

The mode is represented graphically by the abscissa of the point of inflection $I$. This point may be determined roughly by inspection, or by placing a ruler tangent to the curve and rolling it along the curve so that it remains tangent. The direction of rotation of the ruler changes when the point of contact coincides with the point of inflection. In this figure the mode, $M_{0}$, appears to be about 800 .

The geometric mean is defined by the equation,

$$
G=\sqrt[n]{m_{1} m_{2} \ldots m_{n}}
$$

It is most easily calculated by using logarithms, since

$$
\log G=\frac{\log m_{1}+\log m_{2}+\ldots+\log m_{n}}{n} .
$$

The geometric mean is used in averaging rates of increase such as arise in the study of the growth of population, growth of skill in an individual, and relative changes in the prices of commodities.

The trend of prices in a series of years is gauged by finding the ratio of the average price of a commodity in any year to that in a particular year which is chosen as a base and given the arbitrary value 100 . Numbers which are determined for the purpose of showing the trend in prices are called index numbers.

If the index numbers of three commodities for one year are $a, b, c$, and for a second year are $a r, b s, c t$, then the ratios for the three commodities are $r, s, t$, and the geometric mean of the ratios is $\sqrt[3]{r s t}$.

The geometric mean of the index numbers for the first year is $\sqrt[3]{a b c}$ and for the second is $\sqrt[3]{a b c r s t}$. The ratio of these two numbers is $\sqrt[3]{r s t}$.

Hence the geometric mean of the ratios of the index numbers of several commodities for two years is equal to the ratio of the 
geometric mean of the index numbers for the second year to that of the first.

This property of the geometric mean is the reason for its use in averaging index numbers.

The harmonic mean is defined by the equation

$$
H=\frac{1}{\frac{1}{n}\left(\frac{1}{m_{1}}+\frac{1}{m_{2}}+\ldots+\frac{1}{m_{n}}\right)} .
$$

It is used in finding the average amount of work performed in a given time, and the average amount of a commodity purchased for a given price.

In the equation $v=\frac{s}{t}$, if $s$ is constant and $t$ varies, then the average time for a given distance would be found by the harmonic mean.

For since

$$
v_{1}=\frac{s}{t_{1}}, v_{2}=\frac{s}{t_{2}}, \ldots v_{n}=\frac{s}{t_{n}},
$$

the average rate $=\frac{v_{1}+v_{2}+\ldots+v_{n}}{n}=\frac{s}{n}\left(\frac{1}{t_{1}}+\frac{1}{t_{2}}+\ldots+\frac{1}{t_{n}}\right)$. Hence the average time $=\frac{s}{\text { average rate }}=\frac{1}{\frac{1}{n}\left(\frac{1}{t_{1}}+\frac{1}{t_{2}}+\ldots+\frac{1}{t_{n}}\right)}$.

The mode determined as in Example 3 can be found roughly more easily than the median or mean can be calculated. The calculation of the precise true mode is more difficult than that of any of the averages. In the case of discrete variation, as for instance the number of seeds in an apple, the mode may be the only average that will mean anything, as the values of the other averages are quite likely not to occur in the series. The mode is the most probable value of a distribution of the asymmetrical type, since it is the value that occurs most frequently in the distribution, and hence it is the best representative value of central tendency. It is the typical case. It is not useful if it is desirable to give weight to extreme variation, since all the items of the group do not enter into its determination. 
The median ranks after the mode in ease of determination, and usually may be located more precisely.

If the unit of measure is difficult or impossible to determine and a ranking of the items is all that can be attained, as in the measurement of scholarship, the median is the best representative value. If the values of some of the items are given vaguely, as for instance if items of an upper class are given as greater than some value, the median can be determined more precisely than the mean.

All the items enter into the determination of the median but extreme cases affect its value but slightly. Changes in the values of extreme cases would affect the value of the mean without disturbing the value of the median.

The arithmetic mean is the average most generally employed. It is the most familiar of the averages, is the most precise when all the items are given, gives weight to extreme deviations, which is desirable in certain cases, and is affected by every item in the distribution. It may be determined if the aggregate and the number of items are known though knowledge of the values of the items is lacking, and conversely the aggregate may be determined if the mean and the number of items is known. This property is not possessed by the other averages.

The harmonic mean and geometric mean are used less frequently than the other averages as they are unfamiliar and more difficult to calculate. The arithmetic mean is sometimes incorrectly employed in place of the harmonic mean in averaging time rates, and sometimes incorrectly in place of the geometric mean in averaging rates of increase. If variations are measured by their ratio to, rather than by their difference from, the average, then the geometric mean is the best average to employ.

\section{EXERCISES}

1. Which average is meant in the following: average student, average wage in an industry, average daily temperature, average stature, average number of potatoes of a given species in a hill, average annual rainfall, average gain per year in height of a child, average ability in arithmetic, 
average rate of increase of population, average time in doing a piece of work?

2. Find the mean, median, and mode of the distribution in the example in Section 138.

3. Ten men in a department can complete a piece of work in the following number of minutes respectively, $45,50,60,60,60,65,65,70,75$, and 85 . What is the average time for the work?

4. The population of a city increased in a decade from 185,000 to 260,000 . What was the average annual rate of increase?

5. By the probable duration of life of a man $m$ years of age is meant the number of years which he has an even chance of adding to his life. By the expectancy of life for a man of $m$ years is meant the arithmetic mean of the number of additional years of life enjoyed by all men $m$ years of age. Find the probable duration and the expectancy of life of a man 20 years old (use the table in Section 137).

6. The following table gives the distribution of wages per week of a group of laborers.

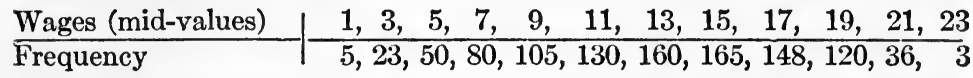

Find the mean, median and mode of the distribution. Which average represents the table best?

7. What is the average age at death for each disease in the tables in Exercise 6, page 387. What average should be used? Determine by inspection of the tables which are diseases of children and which of adults. Which warrants being called a vacation disease? Why?

8. The frequencies of distribution of budgets of a group of college students with a class interval of $\$ 50$ starting at $\$ 350$ and running to $\$ 1800$ inclusive were $4,15,21,26,39,46,52,32,34,24,17,17,14,11,8,10,6$, $6,4,4,3,3,1,1,0,2,1,0,0,3$. Calculate the median and quartiles algebraically and graphically. Which average would best represent the distribution?

9. In 1913 the index numbers for steak, bacon, chickens, eggs, butter, milk, flour, potatoes, sugar were $94,95,97,91,108,100,100,90,100$. In 1918 the numbers in the same commodities were respectively 131, 179, $170,177,151,151,200,188,193$. Calculate the average index numbers in each year and the relative increase in prices.

140. Measures of Variability. Next in importance to selecting an average to represent a group of measurements is the determination of a measure of the extent to which the other values cluster about or are dispersed from the average chosen, that is, a measure of the variability of the group with respect to the average. 
The average obviates the necessity of stating all the measurements from which it is derived, and a measure of variability is a single number characterizing the deviation from the average. A series of measurements can thus be summarized by two numbers which are usually written in the form $a \pm d$, where the first number gives the average value chosen and the second gives a measure of the deviations of the items of the series from the average.

The principal measures of dispersion or variability of a distribution from an average are (a) the quartile deviation, (b) the mean deviation, (c) the median deviation or probable error, (d) the standard deviation.

The quartile deviation is defined by the equation $Q=\frac{Q_{3}-Q_{1}}{2}$, where $Q_{1}$ and $Q_{3}$ are the first and third quartiles respectively. It is the simplest measure of deviation to calculate.

The quartile deviation for the distribution of incomes in Example 2, Section 139, is

$$
Q=\frac{963.1-698.9}{2}=132.1 \text {. }
$$

Hence the group is summarized by the median value of the group and the quartile deviation in the form $822.6 \pm 132.1$. This means that approximately $50 \%$ of the incomes lie between $\$ 822.6-\$ 132.1=\$ 690.5$ and $\$ 822.6+\$ 132.1=\$ 954.7$.

The mean deviation is the arithmetic mean of the numerical values of the deviations from an average. The method of calculation for a frequency distribution is shown in

Example 1. Find the mean deviation from the arithmetic mean of the distribution of incomes in Example 1, Section 139.

In this example the estimated mean is $E=850$, the numerical value of the correction is $c=1.82$ units $=0.0182$ class intervals and the true mean is $A=848.2$.

The sum of the numerical values of the deviations from $E$ is $306+299$ $=605$ class intervals.

The deviation from $A$ of each of the 176 items less than $A$ is 0.0182 class intervals less than the deviation from $E$.

The deviation from $A$ of each of the $73+135=208$ items greater than 
$A$ is 0.0182 class intervals greater than the deviation from $E$. Hence the sum of the numerical values of the deviations from $A$ is

$$
605+208 \times .0182-176 \times .0182=606 .
$$

Dividing by $n=384$, we have

The mean deviation from $A=\frac{606}{384}=1.6$ class intervals $=160$ units.

The median deviation or probable error. If all the deviations from some one of the averages are arranged in order of magnitude without regard to sign, the median deviation is calculated in the same way as the median of the distribution.

The middle $50 \%$ of the items come within the range of the median deviation if the median is the average used to represent central tendency.

Approximately $50 \%$ of the items come within the range of the quartile deviation, but these cases are not necessarily the middle $50 \%$ since the median does not lie exactly halfway between the quartiles except in a symmetrical distribution. The median deviation is usually called the probable error and will be discussed further in Section 142.

The standard deviation is defined by the equation

$$
\sigma^{2}=\frac{d_{1}^{2}+d_{2}^{2}+\ldots d_{n}^{2}}{n}=\frac{\Sigma d^{2}}{n},
$$

where $d_{1}, d_{2}, \ldots, d_{n}$ are the deviations of the measures from the average chosen. If the deviations occur with the frequencies $f_{1}, f_{2}, \ldots, f_{n}$, then the standard deviation is

$$
\sigma=\frac{\sqrt{\Sigma f d^{2}}}{\Sigma f} .
$$

This measure of dispersion gives more weight to extreme cases than the other measures of variability, and while more tedious to calculate is more generally used.

In all deviation measures retain at most two significant figures. The reason for this rule is as follows. Consider a length $l=324.57 \mathrm{~cm}$. with a mean deviation of $.14 \mathrm{~cm}$. The mean deviation indicates that the figure 5 in $l$ in the first position after the decimal point is uncertain by 1 unit and that 
the next figure 7 is uncertain by 14 units. The next figure would be uncertain by at least 140 units and is discarded.

If the first significant figure of the deviation is 8 or 9 , the figure in the corresponding position of the average and the figure following are retained but only one significant figure of the measure of deviation is retained.

The calculation of the standard deviation is simplified by means of the

Theorem. If $x_{1}, x_{2}, \ldots, x_{n}$ are deviations from the arithmetic mean $A$ of a series of measurements with frequencies $f_{1}, f_{2}, \ldots, f_{n}$, if $d_{1}, d_{2}, \ldots, d_{n}$ are the deviations from an estimated mean $E$, and if $A=E+c$, then

$$
\sigma_{x}^{2}=\sigma_{d}^{2}-c^{2}
$$

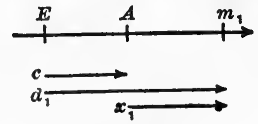

Fig. 219. =

where $\sigma_{x}$ and $\sigma_{d}$ are the standard deviations of the deviations from $A$ and $E$ respectively.

Since $A=E+c$, we have, by the directed lines in the figure, for the first measurement, $d_{1}=x_{1}+c$.

Similarly, $d_{2}=x_{2}+c, \ldots, d_{n}=x_{n}+c$.

Hence the sum of the squares of the deviations from $E$ is

$$
\Sigma f d^{2}=\Sigma f(x+c)^{2}=\Sigma f x^{2}+2 c \Sigma f x+c^{2} \Sigma f .
$$

But $\Sigma f x=0$, since the sum of the deviations from the mean is zero, and hence

$$
\Sigma f d^{2}=\Sigma f x^{2}+c^{2} \Sigma f
$$

Transposing and dividing by $\Sigma f$,

or

$$
\begin{aligned}
\frac{\Sigma f x^{2}}{\Sigma f} & =\frac{\Sigma f d^{2}}{\Sigma f}-c^{2} \\
\sigma_{x}^{2} & =\sigma_{d}^{2}-c^{2} .
\end{aligned}
$$

The value of the correction $c$ is (Theorem, Section 139)

$$
c=\frac{\Sigma f d}{\Sigma f} .
$$

Corollary 1. The sum of the squares of the deviations from the arithmetic mean $A$ is less than the sum of the squares of the deviations from any other number $E$. 
For, from (1), $\Sigma f d^{2}$ is greater than $\Sigma f x^{2}$ by the positive number $c^{2} \Sigma f$.

Corollary 2. The standard deviation of a series of measurements is a minimum when the deviations are measured from the arithmetic mean.

This follows from equation (2).

An application of this theorem is made in

Example 2. Find the arithmetic mean and the standard deviation for the following distribution of grades in geometry.

\begin{tabular}{c|c|c|c|c}
\hline \hline Grade & $\begin{array}{c}\text { Frequency } \\
f\end{array}$ & $\begin{array}{c}\text { Deviation } \\
\text { from } E \\
d\end{array}$ & $f d$ & $f d^{2}$ \\
\hline $95-100$ & 3 & +5 & 15 & 75 \\
$90-94$ & 9 & +4 & 36 & 144 \\
$85-89$ & 12 & +3 & 36 & 108 \\
$80-84$ & 8 & +2 & 16 & 32 \\
$75-79$ & 14 & +1 & 14 & 14 \\
$70-74$ & 9 & 0 & 0 & 0 \\
& & -1 & -17 & 17 \\
$65-69$ & 17 & -2 & -22 & 44 \\
$60-64$ & 11 & -3 & -6 & 18 \\
$55-59$ & 2 & -4 & -8 & 32 \\
$50-54$ & 2 & -5 & -15 & 75 \\
Below 50 & 3 & & $\Sigma f d=49$ & $\Sigma f d^{2}=559$ \\
\hline
\end{tabular}

Let the estimated mean be $E=72.5$ and let the class interval be taken as a unit.

The correction is $c=\frac{\sum f d}{\Sigma f}=+\frac{49}{90}$ class intervals $=\frac{49}{90} \times 5 \%=2.7 \%$.

Hence the mean is $A=E+c=72.5+2.7=75.2$.

The standard deviation of the deviations from $E$ is

$$
\sigma_{d^{2}}=\frac{\Sigma f d^{2}}{n}=\frac{559}{90}=6.2 \text { class intervals. }
$$

Hence

$$
\sigma_{x^{2}}=\sigma_{d}^{2}-c^{2}=6.2-\left(\frac{49}{90}\right)^{2}=6.2-.29=5.91
$$

$$
\therefore \sigma_{x}=2.4 \text { class intervals }=12 \% \text {. }
$$

Hence the average grade in the above distribution is $75.2 \%$ and the standard deviation is $12 \%$. 
The absolute value of two measures of variability may be the same and yet their significance be quite different, for instance, if two averages and their measures of variability are $25 \pm 5$ and $250 \pm 5$, the first indicates greater relative variability than the second.

A measure of the relative variability of a set of measurements is obtained by dividing the measure of deviation $d$ by the average $a$. The quotient $\frac{d}{a}$ is called a coefficient of relative variability. The quantity $v=100 \frac{\sigma}{A}$, which gives the ratio of the standard deviation to the arithmetic mean expressed as a percentage, is called the coefficient of variation.

\section{EXERCISES}

1. (a) Find the median grade and the quartile deviation of the following distribution of grades in algebra, the given grades being at the lefthand ends of the intervals.

\begin{tabular}{l|ccccccccc} 
Grade & 55, & 60, & 65, & 70, & 75, & 80, & 85, & 90, & 95, \\
\hline Frequency & 3, & 7, & 10, & 14, & 22, & 26, & 20, & 16, & 5,
\end{tabular}

(b) Find the arithmetic mean and the standard deviation of the distribution.

(c) Find the mean deviation from the median.

(d) Find the mode and the median deviation from the mode.

(e) Draw the frequency curve of the distribution and show the graphical significance of each of the averages and the corresponding dispersion. Which pair of numbers best represents the distribution and variability?

2. By the use of directed lines show that the mean deviation from a point of reference of a set of seven points placed arbitrarily on the $x$-axis is least when the point of reference is the median point of the set.

3. Compare the coefficients of variability from the arithmetic mean of a group of men and a group of women measured with respect to the number of associations set up by a series of words.

\begin{tabular}{l|rrrrrrrrrr}
$\begin{array}{c}\text { Number of } \\
\text { associations }\end{array}$ & 2, & 4, & 6, & 8, & 10, & 12, & 14, & 16, & 18, & 20, \\
\hline $\begin{array}{l}\text { Frequencies, } \\
\text { men }\end{array}$ & 3, & 7, & 25, & 51, & 55, & 38, & 42, & 20, & 8, & 2, \\
\hline $\begin{array}{l}\text { Frequencies, } \\
\text { women }\end{array}$ & 0, & 8, & 9, & 35, & 57, & 66, & 30, & 18, & 10, & 6,
\end{tabular}


4. The following table gives the errors in minutes in the predictions of high water at Portsmouth during three months in 1897. Find the median deviation, the mean deviation, and the standard deviation. Approximately what fractional part of the standard deviation is the median deviation? the mean deviation?

\begin{tabular}{|c|c|c|c|c|c|c|c|c|}
\hline Errors & $0-5$, & 6-10, & 11-15, & 16-20, & $21-25$ & $26-30$, & $31-35$, & 52 \\
\hline Frequencies & 69 & 50 & 25 & 10 & 11 , & 7 & 4 & 1 \\
\hline
\end{tabular}

\section{Equation of the Frequency Curve Representing a} Symmetrical Distribution. The derivation of the equation is based on the theory of probability.

If a coin is tossed four times, the different ways in which it may fall are heads every time, heads three times and tails once, heads twice and tails twice, heads once and tails three times, and tails every time. The probabilities of the different ways in which it can fall are the terms of the expansion

$$
\begin{aligned}
1=\left(\frac{1}{2}+\frac{1}{2}\right)^{4} & =\left(\frac{1}{2}\right)^{4}+4\left(\frac{1}{2}\right)^{3}\left(\frac{1}{2}\right)^{1}+6\left(\frac{1}{2}\right)^{2}\left(\frac{1}{2}\right)^{2}+4\left(\frac{1}{2}\right)^{1}\left(\frac{1}{2}\right)^{3}+\left(\frac{1}{2}\right)^{4} \\
& =\frac{1}{24}(1+4+6+4+1) \\
& =\frac{1}{16}+\frac{1}{4}+\frac{3}{8}+\frac{1}{4}+\frac{1}{16} .
\end{aligned}
$$

Now consider the adjoined frequency table in which an arbitrary interval $\Delta x$ is chosen, and in which the frequencies $\frac{m}{f} \mid \frac{-2 \Delta x,-\Delta x, 0, \Delta x, 2 \Delta x}{\frac{1}{16}, \frac{1}{4}, \frac{3}{8}, \frac{1}{4}, \frac{1}{16}}$ are the terms of the expansion above. The frequency polygon (page 405) plotted from this table is symmetrical with respect to the $y$-axis.

The standard deviation for this table is

$$
\begin{aligned}
\sigma^{2}=\frac{\Sigma f m^{2}}{\Sigma f} & =\frac{\frac{1}{16}(-2 \Delta x)^{2}+\frac{1}{4}(-\Delta x)^{2}+\frac{3}{8} \cdot 0^{2}+\frac{1}{4}(\Delta x)^{2}+\frac{1}{16}(2 \Delta x)^{2}}{\frac{1}{16}+\frac{1}{4}+\frac{3}{8}+\frac{1}{4}+\frac{1}{16}} \\
& =\frac{\left(\frac{1}{4}+\frac{1}{4}+\frac{1}{4}+\frac{1}{4}\right) \Delta x^{2}}{1}=(\Delta x)^{2} .
\end{aligned}
$$

Therefore $\sigma=\Delta x$.

Plotting the table, using $\Delta x=\sigma$ as the unit on the $x$-axis we obtain the upper frequency polygon in the figure.

If the coin is tossed six times, the probabilities of the ways in which it can fall are given by the terms of the expansion

$$
\left(\frac{1}{2}+\frac{1}{2}\right)^{6}=\frac{1}{2}(1+6+15+20+15+6+1) \text {. }
$$


Let $n$ increase indefinitely. We assume that $n$ increases and $\Delta x$ decreases in such a way that $n(\Delta x)^{2}$ always remains equal to a constant $2 \sigma^{2}$, and that the frequency polygon approaches an ideal symmetrical frequency curve.

To derive the equation of this curve, let $P$ and $Q$ be the successive vertices of the polygon determined by the $r$ th and $(r+1)$ st terms from the middle term of the expansion above. Then the ordinates of these points are

$$
y=\frac{{ }_{2 n} C_{n+r}}{2^{2 n}} \quad \text { and } \quad y+\Delta y=\frac{{ }_{2 n} C_{n+r+1}}{2^{2 n}} .
$$

Since

$$
{ }_{2 n} C_{n+r+1}={ }_{2 n} C_{n+r}\left(\frac{n-r}{n+r+1}\right),
$$

we have $y+\Delta y=\frac{{ }_{2 n} C_{n+r}}{2^{2 n}} \frac{n-r}{n+r+1}=y \frac{n-r}{n+r+1}$.

Hence

$$
\frac{\Delta y}{\Delta x}=\frac{y \frac{n-r}{n+r+1}-y}{\Delta x}=\frac{y}{\Delta x} \frac{-2 r-1}{n+r+1} \text {. }
$$

The abscissa of $P$ is $x=r \Delta x$, and hence $r=\frac{x}{\Delta x}$. Substituting this value of $r$, and simplifying,

$$
\frac{\Delta y}{\Delta x}=\frac{-y}{\Delta x} \frac{2 x+\Delta x}{n \Delta x+x+\Delta x}=-y \frac{2 x+\Delta x}{n(\Delta x)^{2}+x \Delta x+(\Delta x)^{2}} .
$$

Replacing $n(\Delta x)^{2}$ by $2 \sigma^{2}$, in accordance with the assumption made above,

$$
\frac{\Delta y}{\Delta x}=-y \frac{2 x+\Delta x}{2 \sigma^{2}+x \Delta x+(\Delta x)^{2}} .
$$

Passing to the limit as $\Delta x$ approaches zero, the rate of change of $y$ with respect to $x$ is seen to be

whence

$$
\begin{aligned}
& D_{x} y=-y \frac{2 x}{2 \sigma^{2}} \\
& \frac{D_{x} y}{y}=-\frac{x}{\sigma^{2}}
\end{aligned}
$$

In the integral calculus it is shown that the integral of $\frac{D_{x} y}{y}$ 
is $\log _{e} y$. Integrating by means of this result we have

Hence

$$
\log _{e} y=-\frac{x^{2}}{2 \sigma^{2}}+\log _{e} C^{*} \quad \text { or } \quad \log _{e} \frac{y}{C}=-\frac{x^{2}}{2 \sigma^{2}} .
$$

$$
y=C e^{-\frac{x^{2}}{2 \sigma^{2}}}
$$

This is the equation of the curve with the constant of integration still undetermined. The curve is called the probability curve (see Fig. 222, page 409).

Discussion of the Curve. Symmetry. The curve is symmetrical with respect to the $y$-axis, since the equation is unchanged when $x$ is replaced by $-x$.

Intercepts. The intercept on the $y$-axis is $C$, for if $x=0$, then $y=C$.

Asymptotes. The $x$-axis is an asymptote. For $y$ approaches zero as $x$ becomes infinite, since the exponent of $e$ is always negative and increases numerically when $x$ increases.

Points of Inflection. To find the points of inflection, we assume without proof the formula $D_{x} e^{u}=e^{u} D_{x} u$.

Differentiating

$$
y=C e^{-\frac{x^{2}}{2 \sigma^{2}}}
$$

we obtain the slope of the tangent line

$$
m=D_{x} y=C e^{-\frac{x^{2}}{2 \sigma^{2}}}\left(-\frac{x}{\sigma^{2}}\right)=-\frac{C}{\sigma^{2}} x e^{-\frac{x^{2}}{2 \sigma^{2}}}
$$

Differentiating a second time, using the rule for the derivative of a product, $D_{x} u v=u D_{x} v+v D_{x} u$ (Exercise 21, page 278), and equating the result to zero, we have

$$
\begin{gathered}
D_{x} m=-\frac{C}{\sigma^{2}}\left(x e^{-\frac{x^{2}}{2 \sigma^{2}}}\left(-\frac{x}{\sigma^{2}}\right)+e^{-\frac{x^{2}}{2 \sigma^{2}}}\right)=0 . \\
-\frac{C e^{-\frac{x^{2}}{2 \sigma^{2}}}}{\sigma^{2}}\left(-\frac{x^{2}}{\sigma^{2}}+1\right)=0 . \\
x= \pm \sigma .
\end{gathered}
$$

Therefore

* Since any number is the logarithm of some other number the constant of integration may be written in the form $\log _{e} C$. This is convenient here on account of the ease with which $\log _{\ominus} y-\log _{\ominus} C$ may be transformed to $\log _{e} \frac{y}{C}$. 
Hence the value of $\sigma$ gives the abscissas of the points of inflection. The ordinates of these points are both equal to $C e^{-\frac{1}{2}}=C / \sqrt{e}$.

The equation of the probability curve was originally derived in connection with the errors arising in measurement. The difference between a measurement and the unknown true value of the magnitude measured is called an error, and this is to be distinguished from the deviation of one of a set of measurements from the arithmetic mean of the set. It is assumed that as the number of measurements increases the mean approaches the true value, and the deviations from the mean become more nearly the true errors.

It is also assumed that errors are distributed symmetrically about the true value and that they conform to the laws of probability. Hence the law of errors is given by equation (1).

From this point of view, an ordinate $y_{1}$ of the probability curve, or curve of errors, gives the probability of an error equal to the corresponding abscissa $x_{1}$, that is, the probability of an error $x_{1}$ is

$$
y_{1}=C e^{-\frac{x_{1}^{2}}{2 \sigma^{2}}}
$$

In actual measurements there is a lower limit to the size of the unit employed in making the measurements. The error curve can be thought of as a histogram in which the width of a column is $\Delta x$, the magnitude of the unit used, and its height, $y$, is the probability that an error will fall in the corresponding class interval. If all the columns are placed on end the total length is $\Sigma y=1$, since this is the sum of the probabilities of all possible errors. (Theorem 1, Section 136.) Since the area of the histogram is proportional to the sum of the corresponding ordinates, the distribution of errors may be measured by means of the areas of the corresponding columns. Hence we have for the probability curve:

The probability that an error will be between two values $x_{1}$ and $x_{2}$ is given by the area under the curve between these limits.

Determination of the constant $C$. Since all errors will be 
included between the limits $-\infty$ and $+\infty$, the area between the curve and its asymptote ${ }^{*}$. must represent certainty, and hence be equal to unity. It can be shown that the area between the curve

$$
y=e^{-\frac{x^{2}}{2 \sigma^{2}}}
$$

and its asymptote is $\sigma \sqrt{2 \pi}$, a result which we assume. Then the area between the curve

$$
y=C e^{-\frac{x^{2}}{2 \sigma^{2}}},
$$

and its asymptote is $\sigma \sqrt{2 \pi} C$, since the latter curve may be obtained from the former by multiplying the ordinates by $C$.

Hence

$$
\begin{aligned}
\sigma \sqrt{2 \pi} C & =1 . \\
C & =\frac{1}{\sigma \sqrt{2 \pi}} .
\end{aligned}
$$

Whence

Therefore the equation of the probability curve is

$$
y=\frac{1}{\sigma \sqrt{2 \pi}} e^{-\frac{x^{2}}{2 \sigma^{2}}}
$$

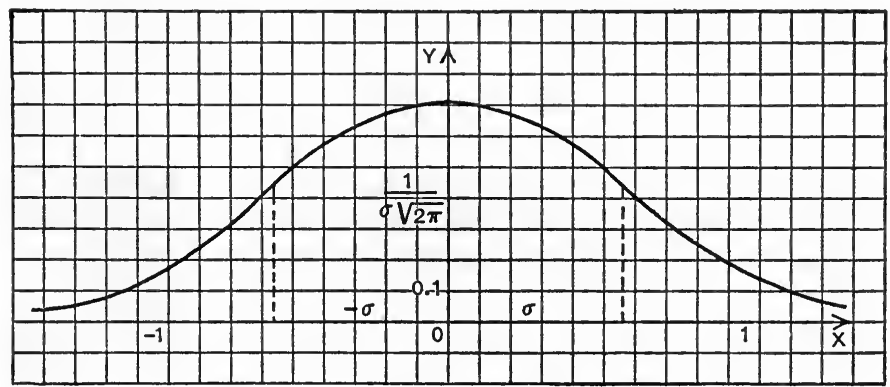

$$
\text { GrAPH of } y=\frac{1}{\sigma \sqrt{2 \pi}} e^{-\frac{x^{2}}{2 \sigma^{2}}}, \sigma=\frac{1}{\sqrt{\pi}}=.56
$$

FIg. 222.

* Let $A$ denote the area bounded by the probability curve, the $x$-axis, and the ordinates $x=-a$ and $x=a$. It can be shown that $A$ approaches a definite limit as $a$ becomes infinite. This limit is called the area between the curve and its amptote. 
In the theory of measurements it is customary to set $h^{2}=\frac{1}{2 \sigma^{2}}$ so that equation (2) becomes

$$
y=\frac{h}{\sqrt{ } \pi} e^{-l^{2} x^{2}}
$$

Example. Find the equation of an ideal frequency curve (probability curve) which fits approximately the upper polygon in the figure at the beginning of the section. Test the accuracy with which the curve fits the polygon by finding the values of $y$ given by the equation for the values of $x$ given in the table and the amounts by which these values differ from the given values of $y$.

\begin{tabular}{r|c|c|c}
$x$ & $\begin{array}{c}\text { Given } \\
y\end{array}$ & $\begin{array}{c}\text { Computed } \\
y\end{array}$ & Difference \\
\hline-2 & $\frac{1}{16}=.063$ & .054 & -.009 \\
-1 & $\frac{1}{4}=.250$ & .242 & -.008 \\
0 & $\frac{3}{8}=.375$ & .399 & +.024 \\
1 & $\frac{1}{4}=.250$ & .242 & -.008 \\
2 & $\frac{1}{16}=.063$ & .054 & -.009
\end{tabular}

Changing the notation by replacing $m$ and $f$ by $x$ and $y$ respectively, and taking $\Delta x=1$, the table at the beginning of the section may be given as in the first two columns of the acompanying table.

The standard deviation for this table is $\sigma=1$, since we saw that $\sigma=\Delta x$, and here $\Delta x=1$. The equation required is found by substituting this value of $\sigma$ in (2) which gives

$$
y=\frac{1}{\sqrt{2 \pi}} e^{-\frac{x^{2}}{2}} .
$$

Substitution of the given values of $x$ in this equation gives the numbers in the third column of the table. The computation requires the use of a table of values of the exponential function, and is effected by the use of logarithms.

\section{EXERCISES}

1. Construct the center frequency polygon in the figure at the beginning of Section 141, find the equation of an ideal frequency curve which approximates the polygon, and plot the curve on the same axes as the polygon. Find the difference between the ordinate of each vertex of the polygon and the corresponding ordinate of the curve.

2. In determining the constant of integration in equation (1) of the preceding section the area between the curve and its agymptote is some- 
times taken equal to $n$, the sum of the frequencies. Show that in this case the equation becomes

$$
y=\frac{n}{\sigma \sqrt{2 \pi}} e^{-\frac{x^{2}}{2 \sigma^{2}}}
$$

What would equation (3) become in this case?

3. If a coin is tossed eight times, find the frequencies of each of the various ways it can fall. Plot these frequencies as ordinates at a unit distance from each other, and draw the frequency polygon. Find the equation of the smoothed frequency curve by means of the preceding exercise.

4. Find the arithmetic mean and the standard deviation for the distribution of grades in trigonometry given in the table, where the mid\begin{tabular}{c|c} 
Grade & Frequency \\
\hline 95 & 8 \\
85 & 22 \\
75 & 24 \\
65 & 17 \\
55 & 6 \\
45 & 3
\end{tabular} values of the class intervals for the grades are given. Plot the frequency polygon. Find the equation of the smoothed frequency curve using Exercise 2. Plot the curve on the same figure as the polygon, using as origin the point on the $x$-axis which represents the mean, and computing the values of $y$ for the values of $x$ corresponding to the mid-values of the class intervals given in the table.

5. Find the arithmetic mean and the standard deviation of the heights of 12-year old boys given in Exercise 6, page 19. Find the equation of the smoothed frequency curve, and plot the frequency polygon and curve in the same figure.

6. Plot the graphs of $y=\frac{8 a^{3}}{x^{2}+4 a^{2}}$ and $y=\frac{a}{e^{x}+e^{-x}}$, and compare them with the probability curve. Might either of them be used in place of the probability curve?

142. Probable Error. The value $r$ of $x$ such that half the errors lie between the limits $-r$ and $+r$ is called the probable error.

The probable error is a measure of the deviation of the measurements from the true value. Since in a symmetrical distribution the mean and median coincide, in this type of distribution the probable error is the same as the quartile deviation $\left(Q_{3}-Q_{1}\right) / 2$. Graphically it is the abscissa on the $x$-axis whose ordinate bisects the area of one branch of the curve.

The area under the curve $y=\frac{h}{\sqrt{\pi}} e^{-h^{2} x^{2}}$ from $-h x$ to $+h x$ is given in the following table for different values of $h x$. 


\begin{tabular}{c|c||c|c||c|c}
$h x$ & \multicolumn{1}{|c||}{$A$} & $h x$ & $A$ & $h x$ & $A$ \\
\hline 0 & 0 & .8 & .742 & 1.6 & .976 \\
.1 & .112 & .9 & .797 & 1.7 & .984 \\
.2 & .223 & 1.0 & .843 & 1.8 & .989 \\
.3 & .329 & 1.1 & .880 & 1.9 & .993 \\
.4 & .428 & 1.2 & .910 & 2.0 & .995 \\
.5 & .521 & 1.3 & .934 & 2.1 & .997 \\
.6 & .604 & 1.4 & .952 & 2.2 & .998 \\
.7 & .678 & 1.5 & .966 & 2.3 & .999
\end{tabular}

From the table it is seen that the area is $A=\frac{1}{2}$ when $h x$ is between 0.4 and 0.5 . The calculation of $h r$ to four places, which is beyond the scope of this course, gives

$$
h r=0.4769 \text {. }
$$

Hence

$$
r=\frac{0.4769}{h}=0.4769(\sqrt{2 \sigma})=0.6745 \sigma .
$$

Therefore

$$
r=0.6745 \sqrt{\frac{\sum x^{2}}{n}} .
$$

The probable error is here expressed in terms of the unknown errors $x$. In a particular case only the deviations from the mean can be calculated. If we change from errors to deviations in the formula, the numerator is diminished, since $\Sigma d^{2}$ $<\Sigma x^{2}$ (the sum of the squares of the deviations from the mean is smaller than from any other value). It is assumed in practice that the best value of the denominator to conform to this change is $n-1$.

Hence

$$
\begin{aligned}
& r=0.6745 \sqrt{\frac{\Sigma d^{2}}{n-1}} \\
& h=\sqrt{\frac{n-1}{2 \Sigma d^{2}}} .
\end{aligned}
$$

If two sets of measurements of unequal precision are com-

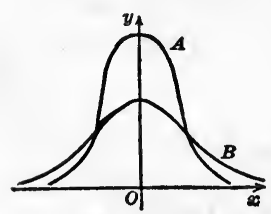

Fra. 223. pared it will be found that while the areas under the probability curves are the same, viz., unity, the measurements in the more precise set will cluster more closely about the mean, the value of $\sigma$ for this set will be smaller, and since $h=1 / \sigma \sqrt{2}$, the value of 
$h$ will be larger. In the figure the curve $A$ represents the set with greater precision.

The value $h$ is called the measure of precision of a set of measurements. Since the intercept on the $y$-axis is $h / \sqrt{\pi}$, this intercept is proportional to the measure of precision.

In comparing different sets of measurements the weight attached to the arithmetic mean of any set (Theorem 3, page 418) is given by $h^{2}$, whose value, from (3), is

$$
h^{2}=\frac{n-1}{2 \Sigma d^{2}}
$$

\section{Probable Errors in Calculations}

Theorem 1. If $y=f(x)$, if the probable error in $x$ is $r$, and if the probable error in $y$ is $R$, then $R=D_{x} y r$.

Let $d x_{1}, d x_{2}, \ldots, d x_{n}$ denote the errors in the measurements of $x$ and $d y_{1}, d y_{2}, \ldots, d y_{n}$, the corresponding errors in $y$.

Then by (1)

$$
R=0.6745 \sqrt{\frac{\sum(d y)^{2}}{n}} \quad \text { and } \quad r=0.6745 \sqrt{\frac{\sum(d x)^{2}}{n}} .
$$

But by the theorem on page 294,

$$
d y_{1}=D_{x} y d x_{1}, d y_{2}=D_{x} y d x_{2}, \ldots, d y_{n}=D_{x} y d x_{n} .
$$

Squaring each of these equations and adding the results,

$$
\Sigma(d y)^{2}=\left[D_{x} y\right]^{2} \Sigma(d x)^{2} .
$$

Extracting the square root of both sides of this equation and multiplying both sides of the result by $0.6745 / \sqrt{n}$,

$$
0.6745 \sqrt{\frac{\sum(\overline{d y})^{2}}{n}}=D_{x} y\left(0.6745 \sqrt{\frac{\sum(d x)^{2}}{n}}\right) .
$$

\section{Hence}

$$
R=D_{x} y r .
$$

Theorem 2. If $y$ is a function of several variables, $y$ $=f(x, z, w)$ and if the probable errors in $x, z, w$, and $y$ are respectively $r_{1}, r_{2}, r_{3}$, and $R$, then

$$
R^{2}=\left(D_{x} y\right)^{2} r_{1}^{2}+\left(D_{z} y\right)^{2} r_{2}^{2}+\left(D_{w} y\right)^{2} r_{3}{ }^{2} .
$$

Let $d x_{1}, d x_{2}, \ldots, d x_{n}, d z_{1}, d z_{2}, \ldots, d z_{n}, d w_{1}, d w_{2}, \ldots, d w_{n}$ 
be the errors in $x, z$, and $w$ for $n$ sets of measurements and $d y_{1}$, $d y_{2}, \ldots, d y_{n}$ the errors in $y$ corresponding to each set of measurements, respectively.

Then $\quad * d y_{1}=\left(D_{x} y\right) d x_{1}+\left(D_{x} y\right) d z_{1}+\left(D_{w} y\right) d w_{1}$

$$
d y_{n}=\left(D_{x} y\right) d x_{n}+\left(D_{z} y\right) d z_{n}+\left(D_{w} y\right) d w_{n} .
$$

Squaring and adding these equations, we have

$$
\Sigma(d y)^{2}=\left(D_{x} y\right)^{2}\left[\Sigma(d x)^{2}\right]+\left(D_{z} y\right)^{2}\left[\Sigma(d z)^{2}\right]+\left(D_{w} y\right)^{2}\left[\Sigma(d w)^{2}\right] \text {. }
$$

The product terms are omitted, because in the long run there will be as many positive products as negative, and as they will be distributed according to the law of error, they will cancel each other. The squared terms are all positive and hence cannot cancel.

Multiplying both sides of the last equation by $(0.6745)^{2} / n$, it follows that

$$
R^{2}=\left(D_{x} y\right)^{2} r_{1}^{2}+\left(D_{z} y\right)^{2} r_{2}^{2}+\left(D_{w} y\right)^{2} r_{3}{ }^{2} .
$$

Example. Find the probable error $R$ in the value of the acceleration of gravity $g$ as determined by a seconds pendulum, if five measurements of the time of vibration $t$, in seconds, are 1.0028, 1.0006, 0.9994, 0.9990, 0.9982 , and five measurements of the length $l$, in centimeters, are 100.18, $100.12,99.90,99.96,99.84$, given that

$$
g=\frac{\pi^{2} l}{t^{2}} .
$$

* The following extension of the theorem on small errors on page 294 is proved in works on the calculus:

If $y$ is a function of several independent variables $x, z, w$, then an approximate value of the error in $y$ due to errors of $d x, d z, d w$, in the independent variables is given by

$$
d y=D_{x} y d x+D_{z} y d z+D_{w} y d w .
$$

In obtaining $D_{x} y, z$ and $w$ are regarded as constants. A different notation for the derivative of $y$ with respect to $x$ alone, namely, $\frac{\partial y}{\partial x}$, is usually used when $y$ is a function of other variables besides $x$, but it seems hardly worth while to introduce it. in this connection. 
The means of $t$ and $l$ and their probable errors are calculated as follows:

\begin{tabular}{r|r|r|r|r|r}
\multicolumn{1}{c|}{$t$} & \multicolumn{1}{c|}{$d$} & \multicolumn{1}{c|}{$d^{2}$} & \multicolumn{1}{c|}{$l$} & \multicolumn{1}{c|}{$d$} & \multicolumn{1}{c}{$d^{2}$} \\
\hline 1.0028 & $+28 \times 10^{-4}$ & $784 \times 10^{-8}$ & 100.18 & $+18 \times 10^{-2}$ & $324 \times 10^{-4}$ \\
1.0006 & $+6 \times 10^{-4}$ & $36 \times 10^{-8}$ & 99.90 & $-10 \times 10^{-2}$ & $100 \times 10^{-4}$ \\
.9994 & $-6 \times 10^{-4}$ & $36 \times 10^{-8}$ & 99.96 & $-4 \times 10^{-2}$ & $16 \times 10^{-4}$ \\
.9990 & $-10 \times 10^{-4}$ & $100 \times 10^{-8}$ & 99.84 & $-16 \times 10^{-2}$ & $256 \times 10^{-4}$ \\
.9982 & $-18 \times 10^{-4}$ & $324 \times 10^{-8}$ & 100.12 & $+12 \times 10^{-2}$ & $144 \times 10^{-4}$ \\
\hline$A_{t}=1.0000$ & \multicolumn{1}{c|}{$\Sigma d^{2}=1280 \times 10^{-8}$} & $A_{l}=100.00 \quad \Sigma d^{2}=840 \times 10^{-4}$
\end{tabular}

By (2) we have:

Probable error $\tau_{c}=0.6745 \sqrt{\frac{1280 \times 10^{-8}}{4}}=0.0012 \mathrm{seo}$

Probable error $r_{l}=0.6745 \sqrt{\frac{840 \times 10^{-4}}{4}}=0.10 \mathrm{~cm}$.

Differentiating the given equation,

$$
\begin{aligned}
D_{l} g & =\frac{\pi^{2}}{t^{2}}, \quad \cdot D_{t} g=-\frac{2 \pi^{2} l}{t^{3}} . \\
\therefore R^{2} & =\left(\frac{\pi^{2}}{t^{2}}\right)^{2} r_{l}{ }^{2}+\left(-\frac{2 \pi^{2} l}{t^{3}}\right)^{2} r_{t}{ }^{2} .
\end{aligned}
$$

(Theorem 2)

Substituting the mean values of $t$ and $l$,

$$
\begin{aligned}
R^{2} & =\left(\frac{\pi^{4}}{1}\right)(0.10)^{2}+\left(-\frac{2 \pi^{2} 100}{1}\right)^{2}(0.0012)^{2} \\
& =0.974+5.61=6.584 \\
\therefore R & =2.6 .
\end{aligned}
$$

The value of $g$ is

$$
g=\frac{\pi^{2} l}{t^{2}}=\frac{\pi^{2} 100}{1}=987.0 \text {. }
$$

Hence

$$
g=987.0 \pm 2.6
$$

\section{EXERCISES}

1. Find the probable error in the circumference of a circle whose diameter is 2.14 centimeters with a probable error of \pm 0.08 .

2. The length and breadth of a rectangle were measured in centimeters and the following values obtained:

\begin{tabular}{l|lllll} 
Length & 54.32, & 54.35, & 54.36, & 54.31, & 54.33 \\
\hline Width & 25.64, & 25.62, & 25.63, & 25.61, & 25.65
\end{tabular}

Find the area and the probable error of the area.

3. The length and diameter of a cylinder were measured in inches and the following values obtained.

\begin{tabular}{llllll} 
Length & 2.745, & 2.747, & 2.742, & 2.744, & 2.743 \\
\hline Diameter & 0.872, & 0.870, & 0.873, & 0.873, & 0.874
\end{tabular}

Find the volume and the probable error in the volume. 
4. A measurement is recorded as 7.50 with a probable error of $r= \pm 0.32$. What is the probability that an error will be less than 0.16 ?

Suggestion. Since $h=0.4769 / r, h x=0.4769 x / r=0.4769 \times 0.16 / 0.32$ $=0.24$. Use the table of areas to find the probability. What is the probability that the error will be less than 0.64 ? greater than 0.64 ?

-5. A line is measured 25 times and the value of the measure of precision found to be 0.2 inch. How many errors are less than 1 inch? greater than 1 but less than 2 inches? greater than 2 inches?

6. What is the probable error of the sum or difference of two magnitudes whose probable errors are $r_{1}$ and $r_{2}$ ? of the product of the two magnitudes? of the quotient?

7. If the sides $a$ and $b$ of a right triangle are $34.26 \pm 0.14$ and $77.81=0.23$ what is the probable error in $\tan A$ ?

8. A length of 1000 feet is measured with a steel tape 100 feet in length. What is the probable error in the length if the probable error in the tape is 0.01 foot?

9. What would be the probable error of an observation if the law of the distribution of errors were

(a) $y=b$, from $x=-a$ to $x=+a$ ? In a seven-place table of logarithms the seventh place is never in error by more than 0.5 ; what is the probable error?

(b) $y=a-x$ from $x=0$ to $x=a$ and $y=a+x$ from $x=-a$ to $x=0$ ?

(c) $y=-x^{2}+a^{2}$ from $x=-a$ to $x=+a$ ?

143. Least Squares. Let $z$ represent the most probable value of a set of $n$ measurements $x_{1}, x_{2}, \ldots, x_{n}$, which are supposed equally precise. Then the deviations from $z$, namely

$$
z-x_{1}, z-x_{2}, \ldots, z-x_{n}
$$

will be distributed according to the law of probability.

The probabilities of the several deviations are (by (3), page 410)

$$
\begin{aligned}
y_{1} & =\frac{h}{\sqrt{\pi}} e^{-h^{2}\left(z-x_{1}\right)^{2}} \\
y_{2} & =\frac{h}{\sqrt{\pi}} e^{-h^{2}\left(z-x_{2}\right)^{2}} \\
\ldots . \cdots & \cdots \\
y_{n} & =\frac{h}{\sqrt{\pi}} e^{-h^{2}\left(z-x_{n}\right)^{2}}
\end{aligned}
$$

As the measurements are independent, the probability of this particular system is (Theorem 2, page 376) 


$$
P=\frac{h^{n}}{\pi^{n / 2}} e^{-h^{2}\left[\left(z-x_{1}\right)^{2}+\left(z-x_{2}\right)^{2}+\cdots+\left(z-x_{n}\right)^{2}\right]} .
$$

The probability $P$ will have a maximum value when the second factor in the exponent,

$$
\left(z-x_{1}\right)^{2}+\left(z-x_{2}\right)^{2}+\ldots\left(z-x_{n}\right)^{2}
$$

has a minimum value. Hence we have the

Principle of least squares. The most probable value of an observed magnitude which has been repeatedly measured gives the least possible value to the sum of the squares of the deviations of the measurements.

To find the value of $z$ for which the sum of the squares above is a minimum, we differentiate with respect to $z$ and equate the derivative to zero. This gives

Hence

$$
2\left(z-x_{1}\right)+2\left(z-x_{2}\right)+\ldots+2\left(z-x_{n}\right)=0 .
$$

and

$$
\begin{aligned}
n z & =x_{1}+x_{2}+\ldots+x_{n} \\
z & =\frac{x_{1}+x_{2}+\ldots+x_{n}}{n} .
\end{aligned}
$$

Hence we have

Theorem 1. The best value to represent a set of equally precise measurements is their arithmetic mean.

The probable error, $R$, of the arithmetic mean, $z$, is found by Theorem 2 of the preceding section to be

$$
R^{2}=\left(\frac{1}{n}\right)^{2} r_{1}{ }^{2}+\left(\frac{1}{n}\right)^{2} r_{2}{ }^{2}+\ldots+\left(\frac{1}{n}\right)^{2} r_{n}{ }^{2},
$$

where the $r$ 's are the probable errors of the measurements. As the measurements are supposed equally precise, let $r$ be the common value of

Hence

$$
r_{1}=r_{2}=r_{3}=\ldots=r_{n} .
$$

so that

$$
R^{2}=n\left(\frac{1}{n}\right)^{2} r^{2}
$$

$$
R=\frac{r}{\sqrt{n}} \text {. }
$$

Hence we have

Theorem 2. The probable error of the arithmetic mean of a set of equally precise measurements varies inversely as the square 
root of the number of measurements. The probable error decreases as the number of measurements increases, but less rapidly.

Theorem 3. The most probable value, $z$, for a set of arithmetic means $x_{1}, x_{2}, \ldots x_{n}$, of unequal precision $h_{1}, h_{2}, \ldots h_{n}$, is the arithmetic mean of the means with the weights $h_{1}{ }^{2}, h_{2}{ }^{2}, \ldots h_{n}{ }^{2}$.

That is

$$
z=\frac{h_{1}^{2} x_{1}+h_{2}^{2} x_{2}+\ldots+h_{n}^{2} x_{n}}{h_{1}^{2}+h_{2}^{2}+\ldots+h_{n}^{2}}
$$

The proof, which is left as an exercise, is analogous to that of Theorem 1. In applying equation (3), page 410 , the value of $h$ is different for the different measurements.

In an empirical data problem, the most probable values of the constants in the desired equation may be calculated by the method of least squares.

Example. Find the most probable values of the coefficients of a linear function which will represent the following empirical table of values.

\begin{tabular}{c|c|c|c}
$x$ & $\begin{array}{c}\text { Empirical } \\
y\end{array}$ & $\begin{array}{c}\text { Theoretical } \\
y\end{array}$ & $\begin{array}{c}\text { Deviation of theoretical } y \\
\text { from empirical } y\end{array}$ \\
\hline 1 & 1.3 & $m+b$ & $m+b-1.3$ \\
2 & 1.6 & $2 m+b$ & $2 m+b-1.6$ \\
3 & 3.1 & $3 m+b$ & $3 m+b-3.1$ \\
4 & 2.8 & $4 m+b$ & $4 m+b-2.8$
\end{tabular}

$\Sigma d^{2}=(m+b-1.3)^{2}+(2 m+b-1.6)^{2}+(3 m+b-3.1)^{2}+(4 m+b-2.8)^{2}$.

Differentiating the sum of the squares of the deviations with respect to $m$ and equating the result to zero, we have

$2(m+b-1.3)+2(2 m+b-1.6) 2+2(3 m+b-3.1) 3+2(4 m+b-2.8) 4=0$ or $\quad(1+4+9+16) m+(1+2+3+4) b=1.3+3.2+9.3+11.2$.

Hence

$$
30 m+10 b=25 \text {. }
$$

Differentiating (1) with respect to $b$ and equating the result to zero we have $2(m+b-1.3)+2(2 m+b-1.6)+2(3 m+b-3.1)+2(4 m+b-2.8)=0$ or $(1+2+3+4) m+4 b=1.3+1.6+3.1+2.8$.

Hence

$$
10 m+4 b=8.8
$$

Solving equations (2) and (3) simultaneously, we have

$$
m=0.6 \quad \text { and } \quad b=0.7 \text {. }
$$

Hence the required function is $y=0.6 x+0.7$.

By the method of Section 27, $y=0.68 x+0.50$. 
For this method the errors are $-0.12,+0.26,-0.56,+0.42$

or

$-9 \%,+16 \%,-18 \%,+15 \%$

and the standard deviation of the errors is $\sigma=0.378$. For the method of least squares the errors are

or $\quad 0 \%,+20 \%,-19 \%,+11 \%$

with a standard deviation of the errors of $\sigma=0.368$.

\section{EXERCISES}

1. If the pairs of values $\left(x_{1}, y_{1}\right),\left(x_{2}, y_{2}\right), \ldots\left(x_{n}, y_{n}\right)$ of an empirical table are connected by the relation $y=m x$ show by the method of least squares that the most probable value of $m$ is $m=\Sigma x y / \Sigma x^{2}$.

2. In a psychological experiment to determine the ability of an individual to determine variation in pressure, the following table was obInitial weight in grams $\mid 10,20,30,40$, tained. Find \begin{tabular}{|l|l}
\hline Just perceptible weight in grams & $15,7.8,9.9,13.3$, \\
$5.15 e$ law, and \\
change in
\end{tabular} weight which is just perceptible when the initial weight is 50 grams.

3. A steel bar was stretched by attaching weights, and the measureTension in pounds $\mid 150,400,500,700$, ments made as given in the Stretching in inches $\frac{150,40,}{32,80,98,1.44}$ table. Determine the law, (Stretching in inches would be stretched by a weight of 800 pounds.

4. The table gives the horse power required for a given load in the Load in tons $50,70,90,100$, case of a locomotive running 40 miles Horse power $81,107,127,142$ an hour. Determine the law, and load of 68 tons.

5. If the values $\left(x_{1}, y_{1}\right),\left(x_{2}, y_{2}\right), \ldots\left(x_{n}, y_{n}\right)$ of an empirical table are connected by the relation $y=m x+b$, show that the most probable values of $m$ and $b$ are given by the equations

$$
m=\frac{n \Sigma(x y)-\Sigma(x) \Sigma(y)}{n \Sigma\left(x^{2}\right)-(\Sigma(x))^{2}}, \quad b=\frac{\Sigma(y)-m \Sigma(x)}{n} .
$$

6. Use the results of the preceding exercise to calculate the coefficients of the linear function $P=m W+b$ in Example 2, Section 27, arranging the work with columns headed $x, y, x^{2}, x y$. Compare the results with those obtained in Section 27.

7. If the values $\left(x_{1}, y_{1}\right),\left(x_{2}, y_{2}\right) \ldots\left(x_{n}, y_{n}\right)$ of an empirical table are connected by a relation of the form $y=a x^{2}+b x+c$ show that the most probable values of the coefficients $a, b, c$, are given by the equations

$$
\begin{aligned}
& a \Sigma\left(x^{4}\right)+b \Sigma\left(x^{3}\right)+c \Sigma\left(x^{2}\right)=\Sigma\left(x^{2} y\right), \\
& a \Sigma\left(x^{3}\right)+b \Sigma\left(x^{2}\right)+c \Sigma(x)=\Sigma(x y) \text {, } \\
& a \Sigma\left(x^{2}\right)+b \Sigma(x)+c n \quad=\Sigma(y) \text {. }
\end{aligned}
$$

8. Use the results of the preceding exercise to calculate the most probable values of the coefficients of the quadratic function representing the 
empirical table in the example in Section 35. Arrange the work with columns headed $x, y, x y, x^{2}, x^{3}, x^{2} y, x^{4}$. Compare the results with the values obtained for the coefficients by the method of Section 35 .

9. If the values $\left(x_{1}, y_{1}\right),\left(x_{2}, y_{2}\right) \ldots\left(x_{n}, y_{n}\right)$ of an empirical table are connected by a relation of the form $y=k x^{m}$ find the most probable values of the coefficients, given that $D_{x} \log _{e} u=D_{x} u / u$. If the relation is of the form $y=k e^{b x}$ find the most probable values of the coefficients.

10. Determine the most probable values of the constants in an equation of the form $y=k x^{m}$ connecting the following values of $x$ and $y$, $(5,11),(10,31),(15,59),(20,88)$. Suggestion: Find $\log x$ and $\log y$, rounded off to two figures, and then proceed as for a linear function.

11. The temperature of a body cooling in air at zero temperature was $78^{\circ}, 60^{\circ}, 48^{\circ}, 36^{\circ}$ at the end of $1,2,3,4$, minute intervals respectively. Determine the most probable values of the rate of cooling and the original temperature.

12. Six measurements of a line with a steel tape were in feet 639.15 $639.08,639.11,639,19,639.10,639.14$, and with a chain 639.2, 639.1, 639.1, 639.4, 639.3, 639.0. Find the means of the two sets of measurements and their weights and the weighted mean.

13. Find the most probable value of the velocity of light from the following determinations in kilometers per second: $298,000 \pm 1000 ; 298,500 \pm$ $1000 ; 299,990 \pm 200 ; 300,100 \pm 1000 ; 299,930 \pm 100$.

14. A line is measured 10 times and the probable error of the mean is 0.012 foot. How many additional measurements of the same precision are required to reduce the probable error of the mean to 0.005 foot?

15. The parallax of the sun by two determinations is $8.883 \pm 0.034$ and $8.943 \pm 0.051$. What are the weights of the two observations? What is their weighted mean?

144. Correlation. In the experiment to determine the coefficient of friction of wood on wood, discussed in Section 27, the causal relation between the two weights is apparent. To a change in one weight corresponds a change in the other. An arbitrary value can be given to either weight and the corresponding value of the other determined, and the pairs of values found by the two methods are the same. That is, the direct and inverse relations are given by the same equation. In short, one variable is a function of the other.

When some variables are compared, for example, the price of roses and the amount of salt mined, no causal relation can be detected.

Between the extremes of two variables which are causal dependents or absolutely unrelated, there are varying degrees 
of dependence. In this section we shall consider the kind of relation given in the

Definition. Two variables are said to be correlated if an increase in one is accompanied either by an increase or by a decrease in the other. A measure of the degree of dependence is called a coefficient of correlation.

The coefficient of correlation measures the probability with which the value of one variable may be predicted from an assumed value of the other.

The correlation is said to be perfect if a causal relation is established between the two variables, that is, if one is a function of the other. The correlation is said to be positive or negative, according as an increase in one variable is accompanied by an increase or decrease in the other.

The values of two associated variables may be conveniently arranged in rows and columns. Such an array is called a correlation table.

Example 1. Represent the following correlation table graphically.

Correlation between July precipitation and yield of corn in Ohio for the years 1854-1913 inclusive.

\begin{tabular}{|c|c|c|c|c|c|c|c|c|c|c|}
\hline \multirow{2}{*}{\multicolumn{2}{|c|}{$\begin{array}{l}\text { Mid-values of } \\
\text { class intervals }\end{array}$}} & \multicolumn{8}{|c|}{ Precipitation in inches } & \multirow{2}{*}{$f_{y}$} \\
\hline & & 1.5 & 2.5 & 3.5 & 4.5 & 5.5 & 6.5 & 7.5 & 8.5 & \\
\hline \multirow{6}{*}{ 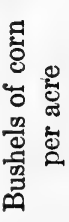 } & 42.5 & $\ldots$ & 1 & $\ldots$ & 1 & 2 & 1 & $\ldots$ & 1 & 6 \\
\hline & 37.5 & $\ldots$ & 1 & 7 & 8 & 7 & 1 & $\ldots$ & $\ldots$ & 24 \\
\hline & 32.5 & 1 & 5 & 8 & 4 & 1 & 1 & $\ldots$ & $\ldots$ & 20 \\
\hline & 27.5 & 1 & 5 & 1 & 2 & .. & ... & $\ldots$ & .. & 9 \\
\hline & 22.5 & $\ldots$ & $\ldots$ & $\ldots$ & 1 & $\ldots$ & $\ldots$ & $\ldots$ & $\ldots$ & 1 \\
\hline & $f_{x}$ & 2 & 12 & 16 & 16 & 10 & 3 & 0 & 1 & 60 \\
\hline
\end{tabular}

Each row is a frequency distribution for the class interval of $y$ given at the left, and each column for the class interval of $x$ given at the top.

The class interval for the precipitation is one inch, and for the corn yield five bushels per acre. 
The number 8 in the row and column headed respectively 37.5 and 4.5 means that in 8 different years a yield of 35 to 40 bushels of corn per acre was associated with a precipitation of 4 to 5 inches of rain. The other frequencies have similar meanings.

The column on the right and the row at the bottom of the table give respectively the sums of the frequencies for each class interval of $y$ and $x$. The sum of the right-hand column, or of the bottom row, is the total number of items, $n=60$.

A graphical representation of the table is obtained by plotting the tables below. Table 1 gives the mid-values of the $x$ classes

Table 1

\begin{tabular}{|c|c|c|c|}
\hline$x$ & Mean $y$ & $y$ & Mean $x$ \\
\hline 1.5 & 30 & 22.5 & 4.5 \\
\hline 2.5 & 31.7 & 27.5 & 2.9 \\
\hline 3.5 & 34.4 & 32.5 & 3.6 \\
\hline 4.5 & 34.4 & 37.5 & 4.5 \\
\hline 5.5 & 38 & 42.5 & 5.5 \\
\hline 6.5 & 37.5 & \multirow{3}{*}{\multicolumn{2}{|c|}{$\begin{array}{r}5 \overline{21.0} \\
M_{x}=4.2\end{array}$}} \\
\hline 8.5 & 42.5 & & \\
\hline & 244.5 & & \\
\hline$M_{y}$ & 34.9 & & \\
\hline
\end{tabular}
and the mean values of the corresponding vertical columns. The pairs of values in this table are plotted as small circles in Fig 224. In this instance, as in many cases, the circles lie approximately on a straight line $l_{1}$.

Table 2 gives the mid-values of the $y$ classes and the mean values of the corresponding horizontal rows. The pairs of values are plotted as small squares, and a straight line $l_{2}$ may be fitted to them approximately. In this case the small square at the point $(22.5,4.5)$ represents but one of $n=60$ items, and may be neglected in drawing $l_{2}$.

The table is said to be represented by these lines. As they do not coincide, the direct and inverse relations between the July precipitation and the yield of corn are not given by the same equation. That is, neither of these variables is a function of the other variable only.

As these lines have positive

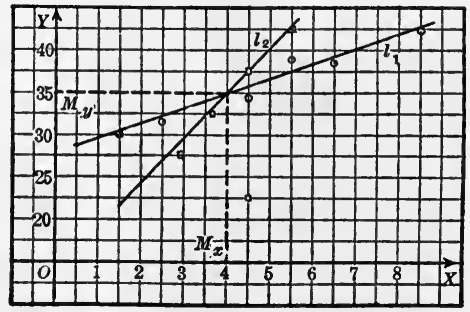

FIG. 224. slopes, to an increase in either variable corresponds an increase in the other. Hence the correlation is positive. 
The mean values of $x$ and $y$ for the entire correlation table are given at the ends of tables 1 and 2 . These values may be obtained also as the means of the row and column headed $f_{x}$ and $f_{y}$. In practice, they are computed in connection with other quantities, as in Example 3 below. Notice that the lines $l_{1}$ and $l_{2}$ appear to intersect at the point $M\left(M_{x}, M_{y}\right)$.

As in Example 1, the direct and inverse relations between two correlated variables are usually represented by two distinct lines. These lines coincide if the correlation is perfect, while $l_{1}$ and $l_{2}$ are parallel to the $x$ and $y$-axes respectively if the variables are unrelated. The correlation is positive or negative according as the slopes of these lines are positive or negative.

The line $l_{1}$ representing the means of the columns always makes a smaller angle with the $x$-axis than the line $l_{2}$ representing the means of the rows. If $x$ is the height of fathers and $y$ that of all their sons, the sons of men who are taller or shorter than the average tend to approach the average height more closely than their fathers. On this account Galton called the falling back of the lines toward the axes regression, and hence the lines are generally called lines of regression.

We shall assume the fact that the lines of regression pass through the point $M$ whose coördinates $M_{x}$ and $M_{y}$ are the mean values of the $x$ and $y$ distributions (see Example 1 ). Let $x^{\prime}$ and $y^{\prime}$ be new variables referred to axes through $M$ parallel to the old axes. Let the equations of $l_{1}$ and $l_{2}$ be

$$
y^{\prime}=m_{1} x^{\prime},
$$

where $m_{1}$ is the slope of $l_{1}$ referred to the $x$-axis, and

$$
x^{\prime}=m_{2} y^{\prime},
$$

where $m_{2}$ is the slope of $l_{2}$ referred to the $y$-axis.*

The most probable values of $m_{1}$ and $m_{2}$ can be determined by the principle of least squares.

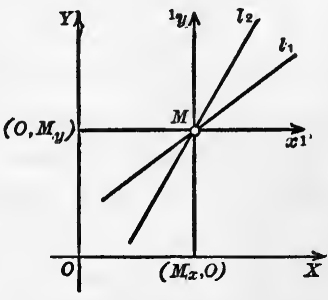

Fig. 225.

* If $P_{1}$ and $P_{2}$ are two points on $l_{2}$, and if $\theta_{2}$ is the inclination of $l_{2}$, then

$$
m_{2}=\frac{x_{1}^{\prime}-x_{2}^{\prime}}{y_{1}^{\prime}-y_{2}^{\prime}}=\cot \theta_{2} \text {. }
$$


For a given value, $x^{\prime}{ }_{1}$, the deviation of the mean value of the corresponding column, $y_{1}^{\prime}$, from the ordinate of the point on $l_{1}$ with the same abscissa, $m_{1} x^{\prime}{ }_{1}$, is $m_{1} x_{1}^{\prime}-y_{1}^{\prime}$. The sum of the squares of such deviations is

$$
\begin{aligned}
\Sigma\left(m_{1} x^{\prime}-y^{\prime}\right)^{2} & =\Sigma\left(m_{1}^{2} x^{\prime 2}-2 m_{1} x^{\prime} y^{\prime}+y^{\prime 2}\right) \\
& =m_{1}^{2} \Sigma x^{\prime 2}-2 m_{1} \Sigma x^{\prime} y^{\prime}+\Sigma y^{\prime 2}
\end{aligned}
$$

Differentiating with respect to $m_{1}$ and equating the derivative to zero, we get

$$
2 m_{1} \Sigma x^{\prime 2}-2 \Sigma x^{\prime} y^{\prime}=0 .
$$

The variables $x^{\prime}$ and $y^{\prime}$ are nothing but deviations from $M_{x}$ and $M_{y}$, and the particular values of $x$ and $y$ used in these summations may be suggestively denoted by $d_{x}$ and $d_{y}$. With this change in notation, $\Sigma x^{\prime} y^{\prime}=\Sigma d_{x} d_{y} ;$ and $\Sigma x^{\prime 2}=\Sigma d_{x}^{2}$.

Hence

$$
m_{1}=\frac{\Sigma x^{\prime} y^{\prime}}{\Sigma x^{\prime 2}}=\frac{\Sigma d_{x} d_{y}}{\Sigma d_{x}^{2}} \text {. }
$$

But the standard deviation of the $d_{x}$ 's is given by $\sigma_{x}^{2}=\frac{\Sigma d_{x}^{2}}{n}$, so that $\Sigma d_{x}^{2}=n \sigma_{x}^{2}$.

Hence the slope of $l_{1}$ is

$$
m_{1}=\frac{\sum d_{x} d_{y}}{n \sigma_{x}^{2}}, \quad \text { where } \quad \sigma_{x}^{2}=\frac{\sum d_{x}^{2}}{n} .
$$

Similarly, the slope of $l_{2}$ referred to the $y$-axis is

$$
m_{2}=\frac{\Sigma d_{x} d_{y}}{n \sigma_{y}{ }^{2}}, \quad \text { where } \quad \sigma_{y}{ }^{2}=\frac{\Sigma d_{u}{ }^{2}}{n} .
$$

Tle slopes $m_{1}$ and $m_{2}$ are respectively measures of the change of $y$ with respect to $x$ and of $x$ with respect to $y$. Their geometric mean, $r=\sqrt{m_{1} m_{2}}$, is usually chosen as the coefficient of correlation to represent these two measures of the direct and inverse relations between the variables. Hence the value of the coefficient of correlation used almost universally is

$$
r=\sqrt{m_{1} m_{2}}=\frac{\sum d_{x} d_{y}}{n \sigma_{y} \sigma_{x}} .
$$

Substituting the value of $\Sigma d_{x} d_{y}$ obtained from (3) in (1) and (2) we obtain

$$
m_{1}=r \frac{\sigma_{y}}{\sigma_{x}} \quad \text { and } \quad m_{2}=r \frac{\sigma_{x}}{\sigma_{y}}
$$


If $l_{1}$ and $l_{2}$ coincide, and if $\theta$ is their inclination, then

$$
r=\sqrt{m_{1} m_{2}}=\sqrt{\tan \theta \cot \theta}= \pm 1 \text {. }
$$

If $l_{1}$ and $l_{2}$ coincide with the $x^{\prime}$ and $y^{\prime}$-axes respectively, then

$$
r=\sqrt{m_{1} m_{2}}=\sqrt{0 \cdot 0}=0 .
$$

Hence for perfect correlution $r= \pm 1$, and for two unrelated variables $r=0$. Values of $r$ between +1 and -1 indicate various degrees of positive and negative correlation.

Example 2. Find the cuefficient of correlation for the table in Example 1 , graphically.

Reading from Fig. 224 the coördinates of two points on $l_{1}$, and dividing the difference of the ordinates by the difference of the abscissas, we find

$$
m_{1}=\frac{40-35}{7-4}=\frac{5}{3}=1.7 \text {. }
$$

Reading from the figure the coördinates of two points on $l_{2}$, and dividing the difference of the abscissas by the difference of the ordinates, we get

$$
m_{2}=\frac{5-4}{40-35}=\frac{1}{5}=0.2
$$

Hence

$$
r=\sqrt{m_{1} m_{2}}=\sqrt{1.7 \times 0.2}=\sqrt{0.34}=0.58 \text { approximately. }
$$

The calculation of the coefficient of correlation is simplified by the following considerations, which are analogous to those used in computing an arithmetic mean (Theorem, page 389), or a standard deviation (Theorem, page 401).

The notation employed, some of which has been used above, is as follows:

$M_{x}$ and $M_{y}$ denote the true means of the variables.

$d_{x}$ and $d_{y}$ denote deviations from the true means.

$\sigma_{x}$ and $\sigma_{y}$ denote the standard deviations of the deviations

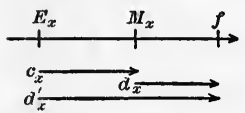

FIG. 226. from the true means.

$E_{x}$ and $E_{y}$ denote estimated values of the true means.

$d^{\prime}{ }_{x}$ and $d^{\prime}{ }_{y}$ denote deviations from the estimated means.

$c_{x}$ and $c_{y}$ denote the corrections used in calculating the arithmetic means, such that $M_{x}=E_{x}+c_{x}$ and $M_{y}=E_{y}+c_{y}$. The two sets of deviations are connected by the relations

$$
\begin{aligned}
& d^{\prime}{ }_{x}=d_{x}+c_{x} \\
& d^{\prime}{ }_{y}=d_{y}+c_{y} .
\end{aligned}
$$




$$
\text { Hence } \quad \begin{aligned}
\Sigma d_{x}^{\prime} d^{\prime}{ }_{y} & =\Sigma\left(d_{x}+c_{x}\right)\left(d_{y}+c_{y}\right) \\
& =\Sigma d_{x} d_{y}+c_{x} \Sigma d_{y}+c_{y} \Sigma d_{x}+\Sigma c_{x} c_{y} .
\end{aligned}
$$

But $\Sigma d_{x}=\Sigma d_{y}=0$, since the sum of the deviations from the mean is zero, and $\Sigma c_{x} c_{y}=n c_{x} c_{y}$, since $c_{x}$ and $c_{y}$ are constants. Therefore

or

$$
\begin{aligned}
\Sigma d^{\prime}{ }_{x} d^{\prime}{ }_{y} & =\Sigma d_{x} d_{y}+n c_{x} c_{y}, \\
\Sigma d_{x} d_{y} & =\Sigma d^{\prime}{ }_{x} d^{\prime}{ }_{y}-n c_{x} c_{y} .
\end{aligned}
$$

This equation is useful in calculating the coefficient of correlation $r$.

The probable error in $r$ is given by the formula, which we assume,

$$
P . E_{r}=0.67449 \frac{1-r^{2}}{\sqrt{n}} .
$$

The correlation between two variables is usually not considered as established unless $r$ is at least three times as great as the probable error.

Example 3. Calculate $r, M_{x}, M_{y}, m_{1}$, and $m_{2}$ for the table in Ex-

\begin{tabular}{|c|c|c|c|c|c|c|c|c|c|c|c|c|c|}
\hline & 1.5 & 2.5 & 3.5 & 4.5 & 5.5 & 6.5 & 7.5 & 8.5 & $f_{y}$ & $d^{\prime}{ }_{y}$ & $\left|f_{y} d^{\prime}{ }_{y}\right|$ & $f_{y} d^{\prime 2}{ }_{y}$ & $d_{x}^{\prime}{ }_{x}^{\prime}$ \\
\hline \begin{tabular}{l|l}
$\stackrel{0}{\Xi}$ & 42.5
\end{tabular} & & 1 & & 1 & 2 & 1 & . & 1 & 6 & 2 & 12 & 24 & 12 \\
\hline 37.5 & $\cdots$ & 1 & 7 & 8 & 7 & 1 & $\cdots$ & $\cdots$ & 24 & 1. & 24 & 24 & 0 \\
\hline 32.5 & 1 & 5 & 8 & 4 & 1 & 1 & $\underline{\cdots}$ & $\cdots$ & 20 & 0 & 0 & 0 & 0 \\
\hline 27.5 & 1 & 5 & 1 & 2 & $\cdots$ & $\ldots$ & ... & $\cdots$ & 9 & -1 & -9 & 9 & 14 \\
\hline 22.5 & $\ldots$ & $\ldots$ & $\ldots$. & 1 & $\ldots$ & $\ldots$ & $\cdots$ & $\ldots$ & 1 & -2 & $\mid-2$ & 4 & 0 \\
\hline$f_{x}$ & 2 & 12 & 16 & 16 & 10 & 3 & 0 & 1 & 60 & & & & \\
\hline$d_{x}^{\prime}$ & -3 & -2 & -1 & 0 & 1 & 2 & 3 & 4 & \multicolumn{5}{|c|}{$n=\Sigma f_{x}=\Sigma f_{y}=60$} \\
\hline$f_{x} d^{\prime \prime} x$ & -6 & -24 & -16 & 0 & 10 & 6 & 0 & 4 & \multirow{2}{*}{\multicolumn{3}{|c|}{$\begin{array}{l}\Sigma f_{x} d_{x}^{\prime}=-26 \\
\Sigma f_{x} d^{\prime 2}=120\end{array}$}} & $\sum f_{y} d^{\prime}{ }_{y}=$ & $=25$. \\
\hline$f_{x} d^{\prime 2}{ }_{x}$ & 18 & 48 & 16 & 0 & 10 & 12 & 0 & 16 & & & & $\Sigma f_{y} d^{\prime 2}{ }_{v}=$ & $=61$. \\
\hline$d_{x}^{\prime} d_{y}^{\prime}{ }_{y}$ & 3 & 4 & $|-6|$ & 0 & 11 & 6 & 0] & 8 & & & $\sum d_{x}^{\prime} d^{\prime}{ }_{y}$ & $y=26$. & \\
\hline
\end{tabular}
ample 1.

Let the class intervals be chosen as units. Assume that $E_{x}^{\prime}=4.5$ and $E_{y}=32.5$. The work can be conveniently arranged as follows:

Precipitation in inches 
The frequencies $f_{x}$ are the sums of the columns, and the frequencies $f_{y}$ are the sums of the rows.

The deviations $d_{x}^{\prime}$ are deviations from $E_{x}=4.5$ in class intervals of one inch. The deviations $d^{\prime}{ }_{y}$ are deviations from $E_{y}=32.5$ in class intervals of 5 bushels per acre. These deviations are analogous to those used in Example 1, page 390.

The products $f_{x} d^{\prime}{ }_{x}, f_{x} d^{\prime 2}{ }_{x}, f_{y} d^{\prime}{ }_{y}, f_{y} d^{\prime 2}{ }_{y}$ are obtained readily, and so also are the sums of the products.

The calculation of the sum $\Sigma d^{\prime}{ }_{x} d^{\prime}{ }_{y}$ requires elucidation. There are $n=60$ items in this sum, but they are not all distinct as several items may have the same deviations $d^{\prime}{ }_{x}$ and $d^{\prime}{ }_{y}$. For example, the precipitation was 2.5 inches, and the yield 27.5 bushels in 5 different years. There are therefore 5 items with deviations $d_{x}^{\prime}=-2$ and $d^{\prime}{ }_{y}=-1$. The sum of the products $d_{x}^{\prime} d^{\prime}{ }_{y}$ for these 5 items is therefore $5(-2)(-1)=10$. Hence the sum $\Sigma d_{x}{ }_{x} d_{y}{ }^{\prime}$ may be obtained by multiplying each frequency in the body of the given table by the product of the values of $d^{\prime}{ }_{x}$ and $d^{\prime}{ }_{y}$ for the column and row in which the frequency occurs, and then adding these products.

A more condensed method is worked out in the table. The column on the extreme right gives the partial sums of the products just described for each of the values of $d^{\prime}{ }_{y}$. To obtain the first partial sum, multiply each frequency in the row headed 42.5 by the corresponding value of $d^{\prime}$, add these products, and multiply the sum by $d^{\prime}{ }_{y}=2$. This gives

$$
[1(-2)+1 \cdot 0+2 \cdot 1+1 \cdot 2+1 \cdot 4] \times 2=12 .
$$

The other partial sums in the column erroneously but conveniently headed $d_{x}^{\prime} d_{y}^{\prime}$ are obtained in like manner. The sum of the partial sums in this column is $\Sigma d_{x}^{\prime} d^{\prime}{ }_{y}=26$.

This procedure may be varied by interchanging the words row and column, $d^{\prime}{ }_{x}$ and $d^{\prime}{ }_{y}$. The bottom row in the table gives the partial sums of terms containing $d_{x}^{\prime}=-3,-2$, etc. The sum of the numbers is 26 , which agrees with the result obtained above.

The rest of the calculation consists of substitution in known formulas, and is given below.

$$
\begin{aligned}
c_{x} & =\frac{\sum f_{x} d_{x}^{\prime}}{n}=\frac{-26}{60} \\
& =-0.43 \text { class intervals. } \\
\sigma_{x}{ }^{2} & =\frac{\Sigma f_{x} d^{\prime 2}{ }_{x}}{n}-c_{x}{ }^{2} \\
& =\frac{120}{60}-(-0.43)^{2} \\
& =1.82 .
\end{aligned}
$$

$$
\begin{aligned}
c_{y} & =\frac{\sum f_{y} d_{y}^{\prime}}{n}=\frac{25}{60} \quad \text { (Theorem, page 389) } \\
& =0.42 \text { class intervals. } \\
\sigma_{y}^{2} & =\frac{\sum f_{y} d_{y}^{\prime 2}}{n}-c_{y}^{2} \text { (Theorem, page 401) } \\
& =\frac{61}{60}-(0.42)^{2} \\
& =0.82 .
\end{aligned}
$$

$\therefore \sigma_{x}=1.3$ class intervals. $\quad \therefore \sigma_{y}=0.91$ class intervals.

$$
\begin{aligned}
& \text { By (5), } \\
& \Sigma d_{x} d_{y}=\Sigma d_{x}^{\prime} d^{\prime}{ }_{y}-n c_{x} c_{y} \\
& =26-60(-0.43)(0.42)=36.8 \text {. } \\
& r=\frac{\Sigma d_{x} d_{y}}{n \sigma_{x} \sigma_{y}}=\frac{36.8}{60 \times 1.3 \times .91}=0.52 \text {. }
\end{aligned}
$$


By (6) the probable error in $r$ is

Hence

Since

and

we have

and

$$
\begin{aligned}
P \cdot E_{r} & =0.67 \frac{1-0.52^{2}}{\sqrt{60}}=\frac{0.67(1+0.52)(1-0.52)}{\sqrt{60}} \\
& =\frac{0.67 \times 1.5 \times 0.48}{\sqrt{60}}=0.062 .
\end{aligned}
$$

The slopes of the lines of regression are, by (4),

$$
r=0.52 \pm 0.062 \text {. }
$$

$c_{x}=-0.43$ class intervals $=-0.43$ inches,

$$
\begin{aligned}
c_{y} & =0.42 \text { class intervals }=2.1 \text { bushels per acre, } \\
M_{x} & =E_{x}+c_{x}=4.5-0.43=4.07 \text { inches, }
\end{aligned}
$$

$M_{y}=E_{y}+c_{y}=32.5+2.1=34.6$ bushels per acre.

$$
\begin{aligned}
m_{1} & =r \frac{\sigma_{2}}{\sigma_{1}}=0.52 \frac{0.91}{1.3}=0.36, \\
\text { and } \quad m_{2} & =r \frac{\sigma_{1}}{\sigma_{2}}=0.52 \frac{1.3}{0.91}=0.74 .
\end{aligned}
$$

The entire computation has been conducted in terms of the class intervals as units. The class interval on the $x$-axis is one unit and that on the $y$-axis is 5 units. Hence the ratio of the class intervals is 5 . Then the slopes of the lines in the units used in the figure of Example 1 are

$$
m_{1}=0.36 \times 5=1.8 \text { and } m_{2}=\frac{0.74}{5}=0.15 \text {. }
$$

\begin{tabular}{|c|c|c|c|c|c|c|c|c|c|c|}
\hline \multirow{2}{*}{\multicolumn{2}{|c|}{$\begin{array}{c}\text { Lower ends of } \\
\text { intervals }\end{array}$}} & \multicolumn{9}{|c|}{ English } \\
\hline & & 55 & 60 & 65 & 70 & 75 & 80 & 85 & 90 & 95 \\
\hline \multirow{9}{*}{ 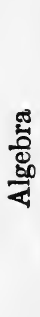 } & 95 & $\cdots$ & $\cdots$ & $\because$ & $\ddot{\theta}$ & 2 & 1 & 2 & $\cdots$ & $\cdots$ \\
\hline & $90^{\circ}$ & .. & . & 1 & 2 & 5 & 5 & 5 & $\cdots$ & . \\
\hline & 85 & 1 & .. & 1 & 4 & 1 & 10 & 1 & .. & .. \\
\hline & 80 & .. & 1 & 3 & 7 & 7 & 4 & 2 & $\ldots$ & $\ldots$ \\
\hline & 75 & .. & 1 & 8 & 4 & 7 & 3 & . & $\cdots$ & . \\
\hline & 70 & .. & 1 & 3 & 5 & 3 & 1 & . & .. & .. \\
\hline & 65 & . & . & 1 & 3 & 1 & 3 & .. & 2 & .. \\
\hline & 60 & . & 1 & 2 & .. & 3 & $\cdots$ & . & $\therefore$ & .. \\
\hline & 55 & . & . & 1 & 1 & 1 & 1 & . & . & . \\
\hline
\end{tabular}

The equations of the lines of regression, referred to the point (4.07, 34.6) as origin are therefore

$$
y^{\prime}=1.8 x^{\prime} \quad \text { and } \quad x^{\prime}=0.15 y^{\prime} .
$$

\section{EXERCISES}

1. Calculate the coefficient of correlation between the following grades in English and Algebra, and determine the probable error. Find the equations of the lines of regression and plot the lines. 
2. The following scores were made by the pupils of a fourth grade in a silent reading test. The first number in each set is the number of words read per minute, the second number is the number of questions answered in five minutes, the third number is the index of comprehension. $(170,39,74),(160,29,79),(281,31,80),(157,35,87),(146,27,87),(81$, $10.89),(345,47,90),(160,34,90),(88,26,91),(233,41,92),(191,35,93)$, $(157,32,93),(239,49,93),(142,42,94),(199,38,94),(254,51,95)$, $(194,44,95),(208,47,95),(155,33,96),(206,33,96),(508,45,97),(208$, $41,97),(180,45,97),(213,45,97),(259,51,98),(281,52,98),(224,34$, $100),(186,36,100),(141,31,100),(153,29,100),(147,21,100)$.

Let the class intervals for the three sets be 8-12, 13-17, etc., 70-89, 90-109, etc., 74-76, 77-79, etc., respectively.

Construct a correlation table with the words read as $x$, and the number of questions answered as $y$.

Construct a correlation table letting $x$ represent the number of questions answered in five minutes and $y$ the index of comprehension.

Calculate for each table the coefficient of correlation and its probable error. What can be inferred from the results?

3. The following table gives the correlation between weight of seeds and number of seeds per apple, for normal apples.

Determine whether a sufficiently high degree of correlation exists between the number and weights of seeds so that the latter may be used instead of the former in the study of the relation between seed and pulp development.

\begin{tabular}{|c|c|c|c|c|c|c|c|c|c|c|c|c|c|c|c|c|}
\hline \multirow{2}{*}{\multicolumn{2}{|c|}{ Mid-values }} & \multicolumn{15}{|c|}{ Seed weight in milligrams } \\
\hline & & & & & & & & & & & & & & & & \\
\hline \multirow{13}{*}{ 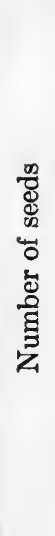 } & 16 & $\ldots$ & $\ldots$ & $\cdots$ & $\ldots$ & $\ldots$ & $\cdots$ & $\ldots$ & $\ldots$ & $\cdots$ & $\ldots$ & $\ldots$ & $\ldots$ & 1 & $\ldots$ & 1 \\
\hline & 15 & $\ldots$ & $\ldots$ & $\ldots$ & $\ldots$ & $\ldots$ & $\ldots$ & $\ldots$ & $\ldots$ & $\ldots$ & $\ldots$ & $\ldots$ & $\ldots$ & 2 & $\ldots$ & 1 \\
\hline & 14 & $\ldots$ & $\ldots$ & $\cdots$ & $\ldots$ & $\ldots$ & $\ldots$ & $\ldots$ & $\ldots$ & $\ldots$ & $\ldots$ & 2 & 2 & 4 & $\ldots$ & $\ldots$ \\
\hline & 13 & $\ldots$ & $\ldots$ & $\ldots$ & $\ldots$ & $\cdots$ & $\cdots$ & $\ldots$ & $\ldots$ & $\ldots$ & $\bar{\ldots}$ & 2 & 2 & 1 & 1 & $\ldots$ \\
\hline & 12 & $\ldots$ & $\ldots$ & $\cdots$ & $\ldots$ & $\cdots$ & $\ldots$ & $\ldots$ & $\ldots$ & 2 & 4 & 3 & 1 & $\ldots$ & $\ldots$ & $\ldots$ \\
\hline & 11 & $\ldots$ & $\ldots$ & $\ldots$ & $\ldots$ & $\cdots$ & $\ldots$ & 1 & 1 & 2 & 4 & 1 & 1 & $\ldots$ & $\ldots$ & $\ldots$ \\
\hline & 10 & $\ldots$ & $\ldots$ & $\ldots$ & $\ldots$ & $\cdots$ & 2 & 1 & 7 & 5 & 4 & $\cdots$ & $\ldots$ & $\ldots$ & $\ldots$ & $\therefore$ \\
\hline & 9 & $\ldots$ & $\cdots$ & $\ldots$ & $\ldots$ & 1 & 1 & 5 & 5 & $\cdots$ & $\cdots$ & $\cdots$ & $\cdots$ & $\cdots$ & $\cdots$ & $\ldots$ \\
\hline & 8 & $\ldots$ & $\ldots$ & $\ldots$ & $\ldots$ & $\ldots$ & 3 & 7 & 3 & $\cdots$ & $\cdots$ & $\ldots$ & $\ldots$ & $\cdots$ & $\ldots$ & $\ldots$ \\
\hline & 7 & $\ldots$ & $\ldots$ & $\ldots$ & 1 & 4 & 3 & $\therefore$ & $\ldots$ & $\ldots$ & $\ldots$ & $\ldots$ & $\ldots$ & $\ldots$ & $\ldots$ & $\ldots$ \\
\hline & 6 & $\ldots$ & $\ldots$ & $\ldots$ & 4 & 2 & $\ldots$ & $\cdots$ & $\ldots$ & $\ldots$ & $\ldots$ & $\ldots$ & $\ldots$ & $\cdots$ & $\ldots$ & $\ldots$ \\
\hline & 5 & $\cdots$ & $\cdots$ & 2 & $\ldots$ & $\cdots$ & $\cdots$ & $\ldots$ & $\ldots$ & $\ldots$ & $\ldots$ & $\cdots$ & $\ldots$ & $\ldots$ & $\ldots$ & 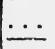 \\
\hline & 4 & 1 & $\ldots$ & $\ldots$ & $\ldots$ & $\ldots$ & $\ldots$ & $\ldots$ & $\ldots$ & $\cdots$ & $\ldots$ & $\cdots$ & .. & & & $\ldots$ \\
\hline
\end{tabular}


4. The following pairs of numbers are the scores made by pupils of a fourth grade in reading and spelling respectively.

$(20.7,65),(16.3,65),(15.1,100),(14.2,85),(14.2,100),(13,95),(12.5$, 70), (12.2, 85), (11.2, 75), $(10.5,100),(8.6,55),(7.8,35),(7.7,40),(7.5$, $50),(7.4,60),(7.4,75),(7.2,95),(6.8,95),(6.6,60),(5.6,40),(5.4,50)$, $(5.4,85),(5.4,45),(5.2,50),(5.2,75),(5.2,75),(5.2,95),(4.7,85),(4.4,55)$, $(4.3,45),(4.2,80),(3.8,55)$.

Find the coefficient of correlation and its probable error.

5. Solve Exercise 3 for the following table.

\begin{tabular}{|c|c|c|c|c|c|c|c|c|c|c|c|c|c|}
\hline \multirow{2}{*}{\multicolumn{2}{|c|}{ Mid-values }} & \multicolumn{12}{|c|}{ Seed weight in milligrams } \\
\hline & & 300 & 340 & 380 & 420 & 460 & 500 & 540 & 580 & 620 & $6 b^{\circ}$ & 700 & 740 \\
\hline \multirow{7}{*}{ 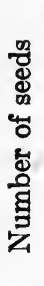 } & 10 & $\cdots$ & $\ldots$ & $\ldots$ & ... & 1 & 1 & 1 & 2 & 1 & 2 & 5 & $\ldots$ \\
\hline & 9 & $\ldots$ & 1 & ... & $\ldots$ & 1 & $\ldots$ & 4 & 1 & 4 & 1 & 1 & 2 \\
\hline & 8 & ... & ... & 1 & ... & 2 & 7 & 3 & 3 & 6 & 2 & $\ldots$ & $\ldots$ \\
\hline & 7 & ... & 1 & $\ldots$ & ... & 4 & 7 & 6 & 1 & ... & 2 & $\ldots$ & $\ldots$ \\
\hline & 6 & 1 & $\ldots$ & 3 & 2 & 5 & 1 & 1 & 1 & ... & $\ldots$ & $\ldots$ & $\ldots$ \\
\hline & 5 & $\ldots$ & 1 & 1 & 2 & ... & $\ldots$ & $\ldots$ & $\ldots$ & ... & $\ldots$ & $\ldots$ & $\ldots$ \\
\hline & 4 & 3 & 4 & $\ldots$ & $\ldots$ & $\ldots$ & ... & $\ldots$ & $\ldots$ & ... & $\ldots$ & $\ldots$ & $\ldots$ \\
\hline
\end{tabular}

6. Correlation between weight of seeds and weight of fruit, for normal apples. Find to what extent seed weight is related to weight of fruit.

\begin{tabular}{|c|c|c|c|c|c|c|c|c|c|c|c|c|c|}
\hline \multirow{2}{*}{\multicolumn{2}{|c|}{ Mid-values }} & \multicolumn{12}{|c|}{ Fruit weight in grams } \\
\hline & & 300 & 340 & 380 & 420 & 460 & 500 & 540 & 580 & 620 & 660 & 700 & 740 \\
\hline \multirow{11}{*}{ 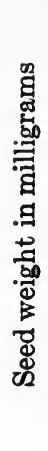 } & 195 & $\ldots$ & $\ldots$ & $\ldots$ & $\ldots$ & $\ldots$ & 1 & $\cdots$ & $\cdots$ & $\cdots$ & $\cdots$ & $\ldots$ & $\cdots$ \\
\hline & 185 & $\cdots$ & $\ldots$ & $\cdots$ & $\cdots$ & $\cdots$ & 1 & 1 & $\cdots$ & 1 & $\ldots$ & $\cdots$ & $\cdots$ \\
\hline & 175 & $\cdots$ & $\cdots$ & ... & . & $\cdots$ & 1 & ... & $\cdots$ & $\ldots$ & 1 & $\ldots$ & 1 \\
\hline & 165 & $\cdots$ & 1. & $\cdots$ & 1 & $\cdots$ & 2 & $\cdots$ & 1 & 2 & 1 & 1 & $\ldots$ \\
\hline & 155 & $\cdots$ & 1 & $\cdots$ & 1 & 1 & 1 & 4 & $\ldots$ & 1 & 1 & $\cdots$ & ... \\
\hline & 145 & ... & 1 & ... & 1 & 1 & 1 & 2 & 3 & 1 & 1 & 1 & $\cdots$ \\
\hline & 135 & 1 & 1 & 1 & $\ldots$ & 2 & 1 & 5 & 3 & 2 & 1 & 1 & 1 \\
\hline & 125 & 1 & ... & ... & 1 & ... & 3 & 1 & 2 & 1 & $\ldots$ & 1 & \\
\hline & 115 & 1 & 1 & 3 & ... & 5 & $\ldots$ & 1 & ... & 2 & $\ldots$ & 2 & $\ldots$ \\
\hline & 105 & 1 & 2 & 1 & ... & 4 & 4 & $\ldots$ & ... & 1 & 1 & $\ldots$ & $\ldots$ \\
\hline & 95 & $\cdots$ & ... & ... & . . & $\ldots$ & 2 & 1 & ... & ... & 1 & .. & $\ldots$ \\
\hline
\end{tabular}


7. Distribution of $\mathbf{1 0 0}$ pupils in each grade in a multiplication test.

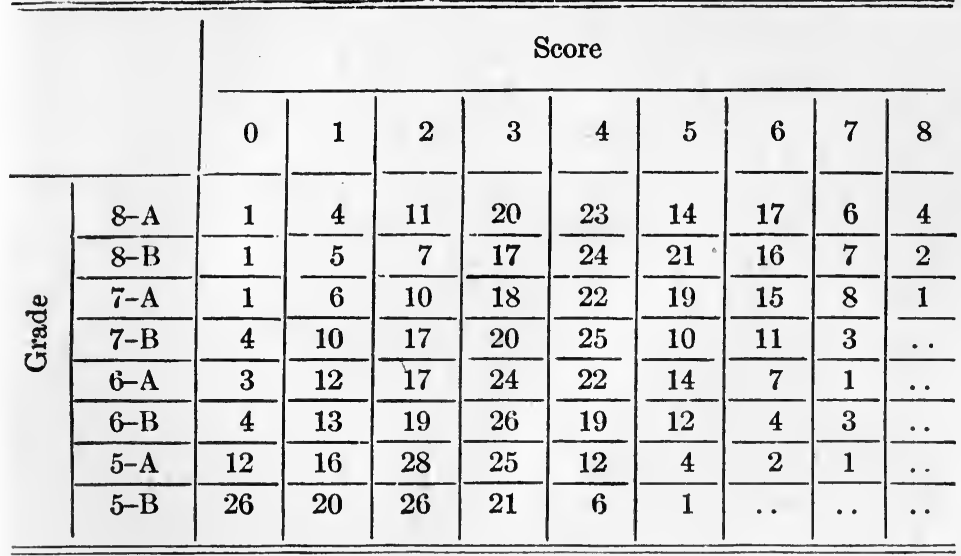

Determine the correlation coefficient and its probable error. Is increase in ability in multiplication closely related to progress through the grades?

8. The general average in all first year high school subjects of $\mathbf{1 2 1}$ students and the records made by them in English and algebra, two required subjects, are given in the table.

Lower ends of intervals $\quad 55,60,65,70,75,80,85,90,95$ $\begin{array}{llllllllll}\text { General average, frequencies, } & 0 & 5 & 12 & 28 & 25 & 26 & 17 & 8 & 0\end{array}$

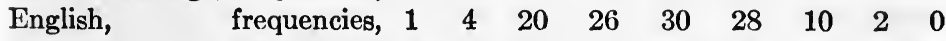

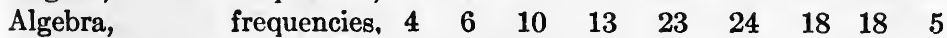

If one subject is taken as an index of the general ability of a student is it better to take the English or the algebra record?

9. Correlation of the average height of a plant of oats with the total yield of the plant in grams. 


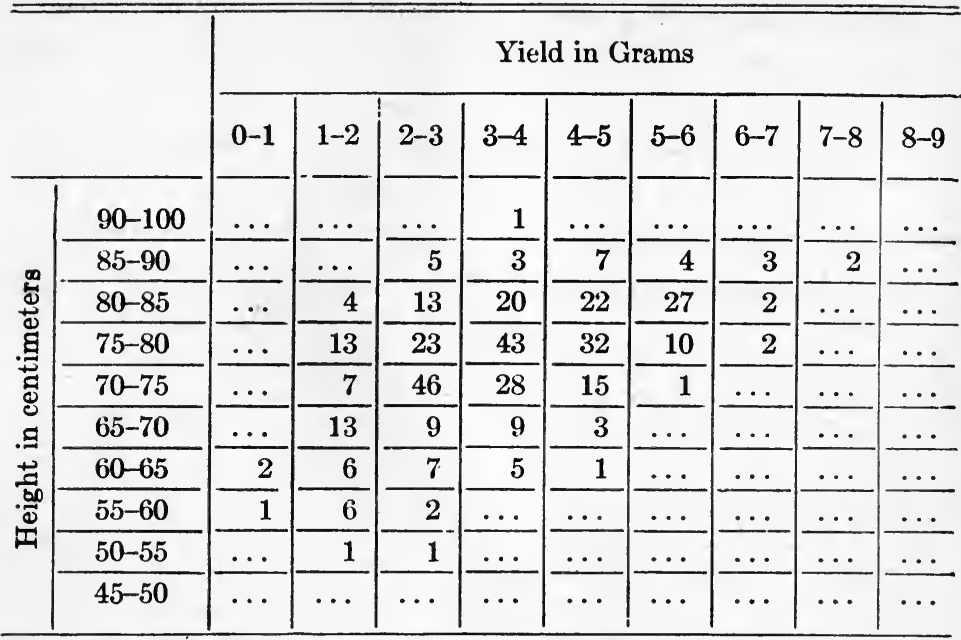

Find the coefficient of correlation and plot the lines of regression.

10. Correlation of the yield of a plant of oats with the number of kernels per plant.

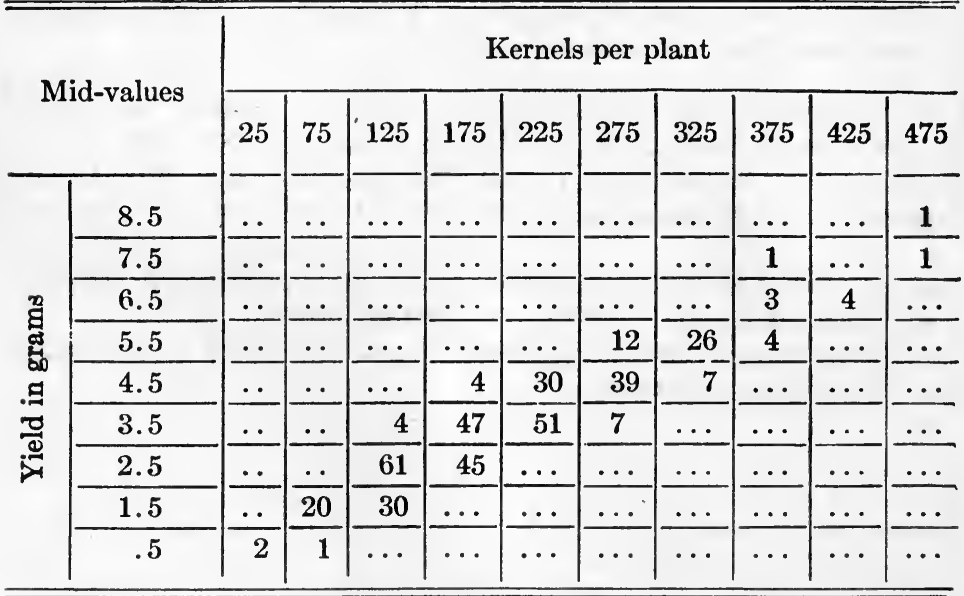

Find the coefficient of correlation, its probable error, and plot the lines of regression. What plants should be used for seeding purposes? 


\section{INDEX}

\section{THE NUMBERS REFER TO PAGES}

Abridged multiplication and division, 77.

Abscissa, 14.

Acceleration, 63, 276, 308, 312; composition and resolution of, 186.

Algebraic function, 38, 87.

American Experience Table, 380. Amplitude of harmonic function, 359.

Angle of elevation or depression, 183.

Annuities, 244.

Approximate error, 294, 414.

Approximate value of $f(x+\Delta x)$, 296.

Arc of circle, 171.

Area under a curve, 305; under probability curve, $409,412$.

Arithmetic mean, 388.

Astronomical exercises, 235.

Asymmetrical distribution, 385 .

Asymptotes, 24, 27.

Average, 83, 388; ordinate, 307 ; rate of change, 35 .

Axes of coördinates, 15.

Axis of symmetry, 22; of parabola, 88.

Bearing of line, 183.

Binomial expansion, 370 .

Biquadratic function, 39.

Center of symmetry, 22 .

Changes of function, 28, 272.
Characteristic of logarithm, 227; properties of function, 42 .

Circle, equation of, 327 .

Classification of functions, 38 .

Coefficient of correlation, 421, 425; of friction, 81, 189.

Cofunction, 177.

Combinations, 367, 371 .

Compound harmonic curve, 360 ; interest, 241; interest law, 215.

Components of acceleration, 187, 312 ; of forces, 187; of velocities, $187,311$.

Composition of accelerations, forces, and velocities, 186.

Cone, 325.

Concavity, 273.

Constant, 6; of integration, 302.

Construction of tables, 297.

Continuous function, 269.

Coördinates, 14.

Correlation, 420.

Cubic function, 39.

Curve of errors, 408.

Cylinder, 318.

Density, 61.

Dependent events, 375 ; variable, 5 .

Derivative, 267, 274; rules for, 269, $270,271,278,280,353,354$.

Difference of sines of two angles, 345.

Directed line, 12.

Discussion of table, 24.

Division, abridged,77; synthetic,136. 
Empirical data problems, by least squares, 418; exponential function, 257; harmonic function, 361 ; linear function, 78; power function, 127, 258; quadratic function, 104.

Equation of circle, 327; of straight line, 66; of probability curve, 407. Equilibrium of a particle, 188.

Excluded values, 22, 26.

Exponential equations, 237; function, 215; graph of, 216, 248, 255.

Forces, composition and resolution of, 186.

Frequency curve, 384, 385; distribution, 382 ; polygon, 382 .

Function, average rate of change of, 35 ; becoming infinite, 25 ; changes of, 28,272 ; classification of, 38 ; defined by an equation, 6 ; definition, 5 ; derivative of, 267 ; discussion of table, 24 ; fundamental problems of, 41; graph of, 17; inverse of, 40; maximum and minimum values, 29, 272; notation for, 8 ; rate of change of, 94 , 267, 272; zeros of, 23.

Fundamental trigonometric formulas, 332 .

Geometric mean, 395.

Graph of equation, 18; exponential function, 216, 248, 255; function, 17; harmonic function, 358; linear function, 57; ploynomial, 136; power function, 119, 254; quadratic function, 100 ; trigonometric function, 172.

Graph, interpretation of, 42.

Graphs of inverse functions, 114; reciprocal functions, 118; related functions, 151.
Harmonic curve, 358; mean, 396; motion, 362 .

Histogram, 383.

Horizontal angle, 183.

Horner's method, 147.

Hyperbola, 131.

\section{Identity, 134 .}

Identities, trigonometric, 336, 351. Inclination of straight line, 199.

Independent events, 375 ; variable, 5 . Infinite, function becoming, 25. Inflection, point of, 139, 273.

Instantaneous velocity, 93.

Integral, 301; rational function, 39, 133.

Integration, 301; rules for, 303, 354 . Intercepts, 23.

Interpolation, 121, 178.

Interpretation of graph, 42.

Inverse functions, 40; graphs of, 114; trigonometric functions, 209. Irrational function, 39 ; roots of equation, 147.

Law of sines, 201; of cosines, 202.

Least squares, 417.

Limit of a variable, 93, 265.

Linear equation, 57 ; fractional function, 131; function, 39, 57 . Lines of regression, 423.

Logarithmic function, 215, 221; graph of, 222; paper, 252; scale, 250 ; solution of triangles, 233, $346,348$.

Logarithms, common, 225.

Mantissa, 227.

Mathematical expectation, 374 .

Maximum point, 29, 272.

Maxima and minima, 285.

Mean, arithmetic, 388; deviation, 399; geometric, 395; harmonic, 396. 
Median, 391; deviation, 400

Minimum point, 29, 272.

Mode, 392.

Mortality table, 380.

Mutually exclusive events, 376.

Normal line, 281.

Oblique triangles, 203, 233, 346, 348.

Octahedron, regular, 330 .

Ordinate, 15.

Origin, 15.

Parallelogram law, 186.

Parallelopiped, 316.

Parabola, 88, 100.

Period of a function, 168; of harmonic function, 359 ; of $\sin \theta, 174$; of $\sin 2 \theta, 342$; of $\tan \theta, 195$.

Periodic function, 168.

Permutations, 366.

Point of inflection, 139, 273.

Point-slope equation of straight line, 67.

Polyhedron, 316.

Polynomial, 39, 133

Power function, 107; graph of, 119, 254; properties of, 153.

Present value, 242; of annuity, 245.

Principal value of inverse trigonometric function, 210.

Prism, 315, 317.

Probable error, 400, 411; of coefficient of correlation, :26.

Probability, 373; curve, 407.

Proportional variables, 61, 125.

Quadratic function, 39, 100.

Quartile deviation, 399.

Radian, 171.

Rate of change, 94 ; average, 35; of polynomial, 138, 271; uniform, 48 .
Rational function, 39 .

Rational roots of equations, 140.

Reciprocal function, 118; relations of trigonometric functions, 167.

Rectangular parallelopiped, 316.

Regular tetrahedron, 329; octahedron, 330.

Related functions, 151; rates, 289.

Relative error, 73, 291.

Remainder theorem, 134.

Resolution of accelerations, forces, velocities, 187.

Revolution, solid of, 323.

Right triangles, 180, 233.

Semi-logarithmic paper, 252.

Sign of function, 28.

Significant figures, 73.

Simple harmonic motion, 362 .

Slide rule, 251.

Slope of straight line, 50, 51, 199; of parallel lines, 52 ; of perpendicular lines, 200; of tangent line, 95, 281.

Slope-intercept equation of straight line, 66.

Small errors, 291.

Snow-ball law, 215.

Solid of revolution, 323 .

Solution of triangles, 181, 203, 233, $346,348$.

Sphere, 327.

Standard deviation, 400; form of numbers, 73.

Sum of sines of two angles, 345 .

Sum of two angles, 164; two lines, 13.

Symmetrical distribution, 385, 404. Symmetry with respect to point or line, 15, 22-24.

Synthetic division, 136.

Tables, construction of, 297.

Tabular difference, 121. 


\section{INDEX}

Tangent line, 95, 281.

Tetrahedron, regular, 329.

Translation of axes, 89 ; of $y$-axis, 144.

Transcendental functions, 39 .

Trigonometric equations, 334, 351 .

Trigonometric functions, definitions, 166; of $\theta+\phi, 338$; of $\theta-\phi, 341$; of $2 \theta, 342$; of $\theta / 2,343$; of $\mathrm{n} 90^{\circ} \pm \theta, 177,192$.

Trigonometric identities, 336, 351 . in a circle, 354; rate of change, 48 ; scale, 250; velocity, 46 .

Variable, 5; dependent, 5; independent, 5 .

Variation, 61, 125; of function, 31.

Velocity at an instant, 93, 276, 308, 312 ; composition and resolution of, 186; uniform, 46 .

Weighted arithmetic mean, 389.

Uniform acceleration, 63; motion _. Zeros of function, 23. 


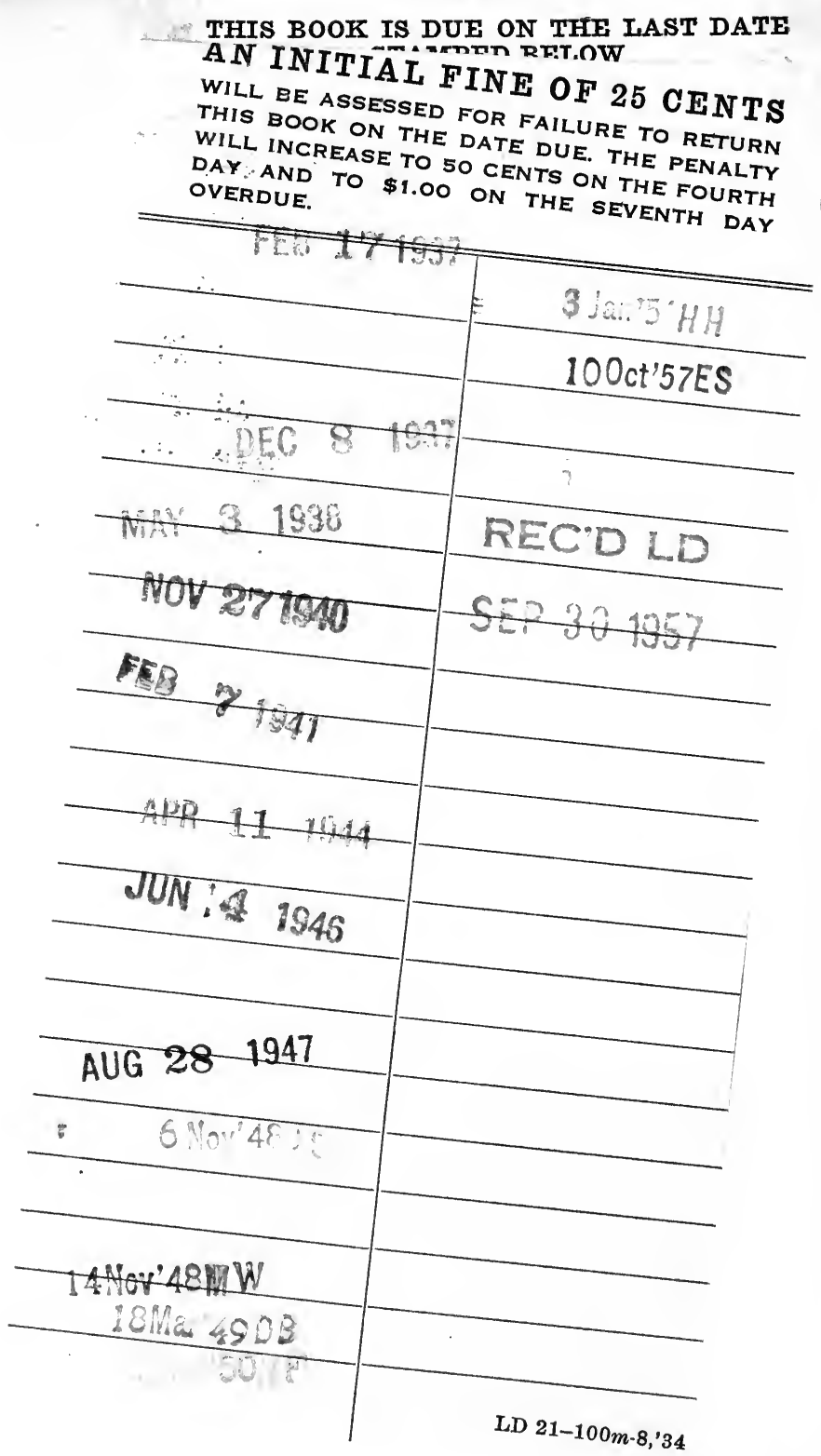




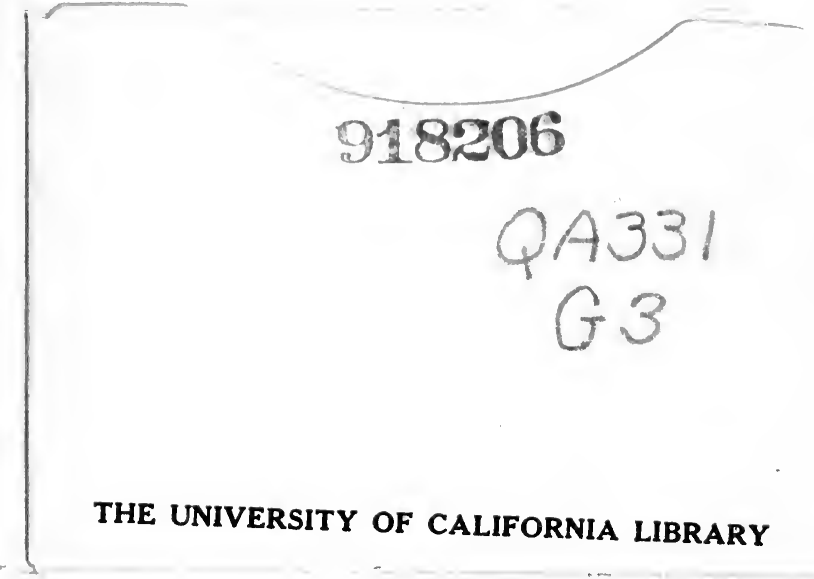


